\title{
Report No.310
}

\section{eparesearch}

Climate - Water - Sustainability

Identifying pressures $\bullet$ Informing policy $\bullet$ Developing solutions

\section{EC-Earth Global Climate Simulations - Ireland's Contributions to CMIP6}

Authors: Paul Nolan and Alastair McKinstry

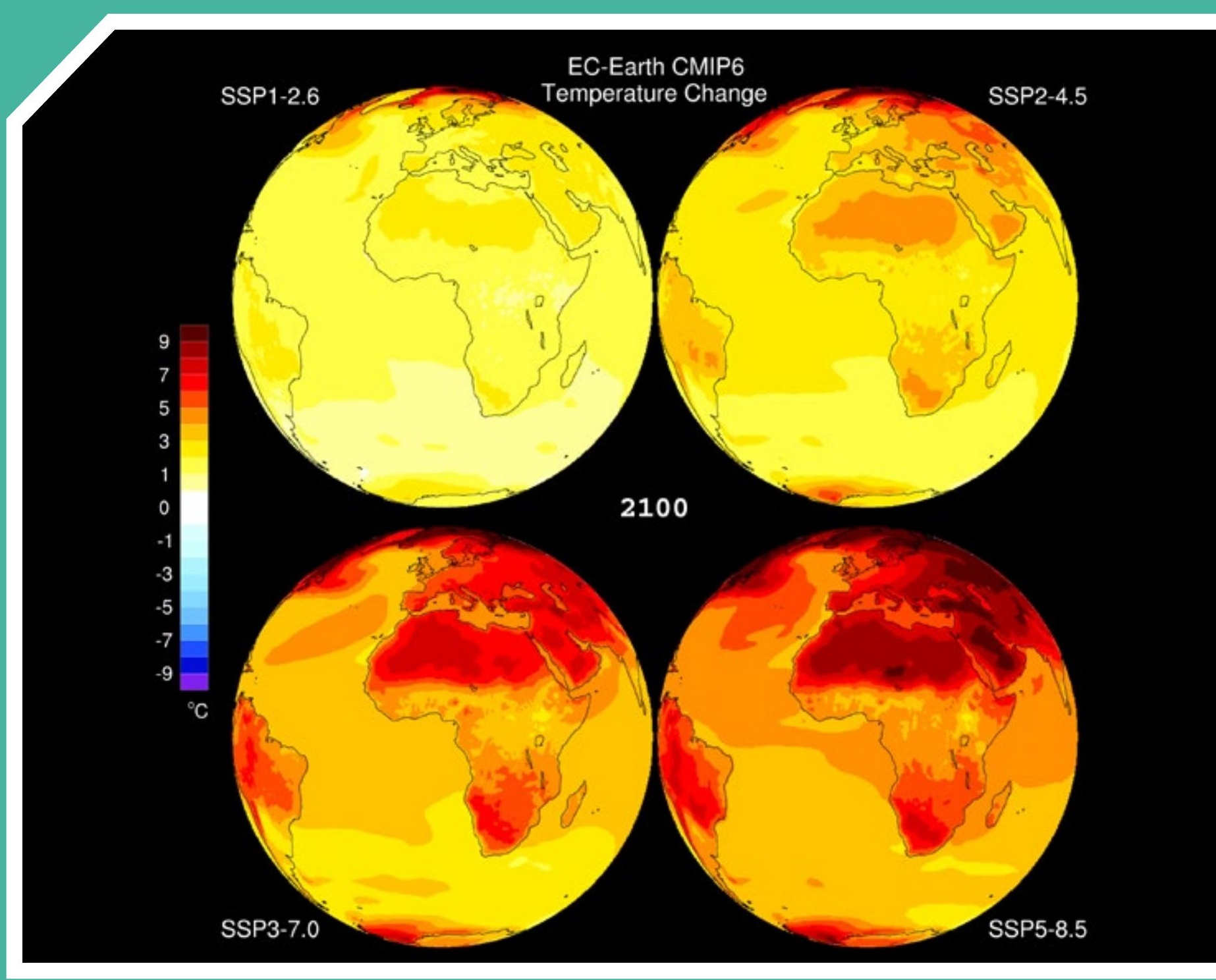

www.epa.ie 


\section{ENVIRONMENTAL PROTECTION AGENCY}

The Environmental Protection Agency (EPA) is responsible for protecting and improving the environment as a valuable asset for the people of Ireland. We are committed to protecting people and the environment from the harmful effects of radiation and pollution.

\section{The work of the EPA can be divided into three main areas:}

Regulation: We implement effective regulation and environmental compliance systems to deliver good environmental outcomes and target those who don't comply.

Knowledge: We provide high quality, targeted and timely environmental data, information and assessment to inform decision making at all levels.

Advocacy: We work with others to advocate for a clean, productive and well protected environment and for sustainable environmental behaviour.

\section{Our Responsibilities}

\section{Licensing}

We regulate the following activities so that they do not endanger human health or harm the environment:

- waste facilities (e.g. landfills, incinerators, waste transfer stations);

- large scale industrial activities (e.g. pharmaceutical, cement manufacturing, power plants);

- intensive agriculture (e.g. pigs, poultry);

- the contained use and controlled release of Genetically Modified Organisms (GMOs);

- sources of ionising radiation (e.g. $x$-ray and radiotherapy equipment, industrial sources);

- large petrol storage facilities;

- waste water discharges;

- dumping at sea activities.

\section{National Environmental Enforcement}

- Conducting an annual programme of audits and inspections of EPA licensed facilities.

- Overseeing local authorities' environmental protection responsibilities.

- Supervising the supply of drinking water by public water suppliers.

- Working with local authorities and other agencies to tackle environmental crime by co-ordinating a national enforcement network, targeting offenders and overseeing remediation.

- Enforcing Regulations such as Waste Electrical and Electronic Equipment (WEEE), Restriction of Hazardous Substances (RoHS) and substances that deplete the ozone layer.

- Prosecuting those who flout environmental law and damage the environment.

\section{Water Management}

- Monitoring and reporting on the quality of rivers, lakes, transitional and coastal waters of Ireland and groundwaters; measuring water levels and river flows.

- National coordination and oversight of the Water Framework Directive.

- Monitoring and reporting on Bathing Water Quality.

\section{Monitoring, Analysing and Reporting on the}

\section{Environment}

- Monitoring air quality and implementing the EU Clean Air for Europe (CAFÉ) Directive.

- Independent reporting to inform decision making by national and local government (e.g. periodic reporting on the State of Ireland's Environment and Indicator Reports).

\section{Regulating Ireland's Greenhouse Gas Emissions}

- Preparing Ireland's greenhouse gas inventories and projections.

- Implementing the Emissions Trading Directive, for over 100 of the largest producers of carbon dioxide in Ireland.

\section{Environmental Research and Development}

- Funding environmental research to identify pressures, inform policy and provide solutions in the areas of climate, water and sustainability.

\section{Strategic Environmental Assessment}

- Assessing the impact of proposed plans and programmes on the Irish environment (e.g. major development plans).

\section{Radiological Protection}

- Monitoring radiation levels, assessing exposure of people in Ireland to ionising radiation.

- Assisting in developing national plans for emergencies arising from nuclear accidents.

- Monitoring developments abroad relating to nuclear installations and radiological safety.

- Providing, or overseeing the provision of, specialist radiation protection services.

\section{Guidance, Accessible Information and Education}

- Providing advice and guidance to industry and the public on environmental and radiological protection topics.

- Providing timely and easily accessible environmental information to encourage public participation in environmental decision-making (e.g. My Local Environment, Radon Maps).

- Advising Government on matters relating to radiological safety and emergency response.

- Developing a National Hazardous Waste Management Plan to prevent and manage hazardous waste.

\section{Awareness Raising and Behavioural Change}

- Generating greater environmental awareness and influencing positive behavioural change by supporting businesses, communities and householders to become more resource efficient.

- Promoting radon testing in homes and workplaces and encouraging remediation where necessary.

\section{Management and structure of the EPA}

The EPA is managed by a full time Board, consisting of a Director General and five Directors. The work is carried out across five Offices:

- Office of Environmental Sustainability

- Office of Environmental Enforcement

- Office of Evidence and Assessment

- Office of Radiation Protection and Environmental Monitoring

- Office of Communications and Corporate Services

The EPA is assisted by an Advisory Committee of twelve members who meet regularly to discuss issues of concern and provide advice to the Board. 


\section{EC-Earth Global Climate Simulations: Ireland's Contributions to CMIP6}

\section{(2015-CCRP-FS.23) \\ EPA Research Report}

Prepared for the Environmental Protection Agency

by

Irish Centre for High-End Computing (ICHEC) and Met Éireann

\section{Authors:}

Paul Nolan and Alastair McKinstry

ENVIRONMENTAL PROTECTION AGENCY

An Ghníomhaireacht um Chaomhnú Comhshaoil

PO Box 3000, Johnstown Castle, Co. Wexford, Ireland

Telephone: +353 539160600 Fax: +353 539160699

Email: info@epa.ie Website: www.epa.ie 


\section{ACKNOWLEDGEMENTS}

This report is published as part of the EPA Research Programme 2014-2020. The EPA Research Programme is a Government of Ireland initiative funded by the Department of Communications, Climate Action and Environment. It is administered by the Environmental Protection Agency, which has the statutory function of co-ordinating and promoting environmental research.

The authors would like to acknowledge the members of the EPA project steering committee, namely Ray McGrath (University College Dublin), Philip O'Brien (EPA), Frank McGovern (EPA), Saji Varghese (Met Éireann), Anne Mason (EPA), Alice Wemaere (EPA), Keith Lambkin (Met Éireann), John O'Neill (Department of Communications, Climate Action and Environment), Patrick Fournet (Met Éireann) and Hazem Nagy (Marine Institute). In addition, the input of Jonathan McGovern, Met Éireann, is acknowledged.

The authors wish to acknowledge the Irish Centre for High-End Computing (ICHEC) for the provision of computational facilities and support. In particular, we are grateful to Eoin McHugh for his work on implementing the Earth System Grid Federation (ESGF) node at ICHEC and Marco Grossi and Niall Wilson, who assisted with the provision of ICHEC compute and storage resources. Finally, we wish to thank the European Centre for Medium-Range Weather Forecasts (ECMWF) (in particular, Paul Dando) for the provision of computational facilities and support.

\section{DISCLAIMER}

Although every effort has been made to ensure the accuracy of the material contained in this publication, complete accuracy cannot be guaranteed. The Environmental Protection Agency, the author and the steering committee members do not accept any responsibility whatsoever for loss or damage occasioned, or claimed to have been occasioned, in part or in full, as a consequence of any person acting, or refraining from acting, as a result of a matter contained in this publication. All or part of this publication may be reproduced without further permission, provided the source is acknowledged.

This report is based on research carried out/data from 2016 to 2019. More recent data may have become available since the research was completed.

The EPA Research Programme addresses the need for research in Ireland to inform policymakers and other stakeholders on a range of questions in relation to environmental protection. These reports are intended as contributions to the necessary debate on the protection of the environment.

EPA RESEARCH PROGRAMME 2014-2020

Published by the Environmental Protection Agency, Ireland 


\section{Project Partners}

\section{Dr Paul Nolan}

Irish Centre for High-End Computing (ICHEC)

National University of Ireland Galway

Galway

Ireland

Tel.: + 35315241608 (ext. 24)

Email: paul.nolan@ichec.ie

\author{
Alastair McKinstry \\ Irish Centre for High-End Computing (ICHEC) \\ National University of Ireland Galway \\ Galway \\ Ireland \\ Tel.: + 35315241608 (ext. 68) \\ Email: alastair.mckinstry@ichec.ie
}





\section{Contents}

$\begin{array}{ll}\text { Acknowledgements } & \text { ii }\end{array}$

Disclaimer $\quad$ ii

Project Partners

List of Figures $\quad$ vi

List of Tables and Boxes $\quad$ xvii

Executive Summary $\quad$ xix

1 Introduction $\quad 1$

1.1 The Coupled Model Intercomparison Project 3

1.2 EC-Earth Earth System Model 4

1.3 The SSP/RCP Scenario Matrix Framework 5

1.4 Ireland's Contributions to CMIP6 6

1.5 ESGF: The Earth System Grid Federation 11

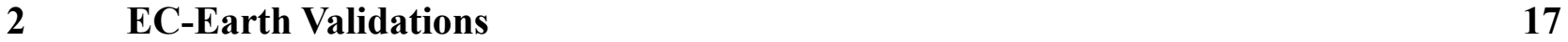

$\begin{array}{lll}2.1 & \text { Observational and Reanalysis Datasets } & 17\end{array}$

$\begin{array}{lll}2.2 & \text { Validation Methods and Metrics } & 17\end{array}$

$2.3 \quad$ EC-Earth Validation Results 18

$3 \quad$ EC-Earth Climate Projections $\quad 86$

$3.1 \quad 2-m$ Temperature Projections 86

$3.2 \quad$ Precipitation Projections 90

$3.3 \quad 10-\mathrm{m}$ Wind Speed Projections 96

3.4 Mean Sea Level Pressure Projections 100

3.5 Total Cloud Cover Projections 104

$\begin{array}{lll}3.6 & \text { Snowfall Projections } & 109\end{array}$

$\begin{array}{lll}3.7 & \text { Sea Surface Temperature Projections } & 114\end{array}$

$3.8 \quad$ Sea Ice Projections 119

4 Recommendations $\quad 143$

$\begin{array}{ll}\text { References } & 145\end{array}$

$\begin{array}{lr}\text { Abbreviations } & 149\end{array}$ 


\section{List of Figures}

Figure 1.1. The CMIP6 EC-Earth model components 5

Figure 1.2. The 21 CMIP6-endorsed MIPs 6

Figure 1.3. SSP-RCP scenario matrix illustrating ScenarioMIP simulations $\quad 8$

Figure 1.4. Docker-based architecture for a complete ICHEC ESGF node 14

Figure 2.1. Annual mean 2-m temperature, 1979-2014: (a) ERA5 reanalysis,

(b) CRU_ts4.03 observations, (c) EC-Earth r6i1p1f1, (d) EC-Earth r9i1p1f1,

(e) EC-Earth r11i1p1f1, (f) EC-Earth r13i1p1f1 and (g) EC-Earth r15i1p1f1

Figure 2.2. EC-Earth annual 2-m temperature bias, 1979-2014 (ERA5 reanalysis minus EC-Earth): (a) EC-Earth r6ilp1f1, (b) EC-Earth r9i1p1f1, (c) EC-Earth r11i1p1f1, (d) EC-Earth r13i1p1f1 and (e) EC-Earth r15i1p1f1

Figure 2.3. EC-Earth annual 2-m temperature bias, 1979-2014 (EC-Earth minus CRU_ts4.03): (a) EC-Earth r6i1p1f1, (b) EC-Earth r9ilp1f1, (c) EC-Earth r11i1p1f1, (d) EC-Earth r13i1p1f1 and (e) EC-Earth r15i1p1f1

Figure 2.4. Mean 2-m temperature for DJF, 1979-2014: (a) ERA5 reanalysis, (b) CRU_ts4.03 observations, (c) EC-Earth r6ilp1f1, (d) EC-Earth r9i1p1f1, (e) EC-Earth r11i1p1f1, (f) EC-Earth r13i1p1f1 and (g) EC-Earth r15ilp1f1

Figure 2.5. EC-Earth 2-m temperature bias for DJF, 1979-2014 (ERA5 reanalysis minus EC-Earth): (a) EC-Earth r6ilp1f1, (b) EC-Earth r9i1p1f1, (c) EC-Earth r11i1p1f1, (d) EC-Earth r13i1p1f1 and (e) EC-Earth r15i1p1f1

Figure 2.6. EC-Earth 2-m temperature bias for DJF, 1979-2014 (EC-Earth minus CRU_ts4.03): (a) EC-Earth r6i1p1f1, (b) EC-Earth r9i1p1f1, (c) EC-Earth r11i1p1f1, (d) EC-Earth r13i1p1f1 and (e) EC-Earth r15i1p1f1

Figure 2.7. Mean 2-m temperature for JJA, 1979-2014: (a) ERA5 reanalysis, (b) CRU_ts4.03 observations, (c) EC-Earth r6i1p1f1, (d) EC-Earth r9ilp1f1, (e) EC-Earth r11ilp1f1, (f) EC-Earth r13i1p1f1 and (g) EC-Earth r15ilp1f1

Figure 2.8. EC-Earth 2-m temperature bias for JJA, 1979-2014 (ERA5 reanalysis minus EC-Earth): (a) EC-Earth r6ilp1f1, (b) EC-Earth r9i1p1f1, (c) EC-Earth r11i1p1f1, (d) EC-Earth r13i1p1f1 and (e) EC-Earth r15i1p1f1

Figure 2.9. EC-Earth 2-m temperature bias for JJA, 1979-2014 (EC-Earth minus CRU_ts4.03): (a) EC-Earth r6i1p1f1, (b) EC-Earth r9i1p1f1, (c) EC-Earth r11i1p1f1, (d) EC-Earth r13i1p1f1 and (e) EC-Earth r15i1p1f1

Figure 2.10. Comparison of EC-Earth ensemble members with ERA5 reanalysis data for the period 1979-2014: (a) 2-m temperature and (b) 2-m temperature anomalies with respect to the 30 -year period 1981-2010 
Figure 2.11. Comparison of EC-Earth ensemble members with CRU_ts4.03 observations for the period 1901-2014: (a) 2-m land temperature and (b) 2-m land temperature anomalies with respect to the 30-year period 1981-2010

Figure 2.12. Annual mean daily precipitation (mm/day), 1979-2014: (a) ERA5 reanalysis, (b) CRU_ts4.03 observations, (c) EC-Earth r6ilp1f1,

(d) EC-Earth r9i1p1f1, (e) EC-Earth r11i1p1f1, (f) EC-Earth r13i1p1f1 and (g) EC-Earth r15i1p1f1

Figure 2.13. EC-Earth annual mean daily precipitation (mm/day) bias, 1979-2014 (ERA5 reanalysis minus EC-Earth): (a) EC-Earth r6i1p1f1, (b) EC-Earth r9ilp1f1, (c) EC-Earth r11i1p1f1, (d) EC-Earth r13i1p1f1 and (e) EC-Earth r15ilp1f1

Figure 2.14. EC-Earth annual mean daily precipitation (mm/day) bias, 1979-2014 (EC-Earth minus CRU_ts4.03): (a) EC-Earth r6i1p1f1, (b) EC-Earth r9i1p1f1, (c) EC-Earth r11i1p1f1, (d) EC-Earth r13i1p1f1 and (e) EC-Earth r15ilp1f1

Figure 2.15. Mean daily precipitation (mm/day) for DJF, 1979-2014: (a) ERA5 reanalysis, (b) CRU_ts4.03 observations, (c) EC-Earth r6ilp1f1,

(d) EC-Earth r9i1p1f1, (e) EC-Earth r11i1p1f1, (f) EC-Earth r13i1p1f1 and

(g) EC-Earth r15ilp1f1

Figure 2.16. EC-Earth mean daily precipitation (mm/day) bias for DJF, 1979-2014 (ERA5 reanalysis minus EC-Earth): (a) EC-Earth r6i1p1f1, (b) EC-Earth r9i1p1f1, (c) EC-Earth r11i1p1f1, (d) EC-Earth r13i1p1f1 and (e) EC-Earth r15ilp1f1

Figure 2.17. EC-Earth mean daily precipitation (mm/day) bias for DJF, 1979-2014 (EC-Earth minus CRU_ts4.03): (a) EC-Earth r6ilp1f1, (b) EC-Earth r9i1p1f1, (c) EC-Earth r11ilp1f1, (d) EC-Earth r13i1p1f1 and (e) EC-Earth r15ilp1f1

Figure 2.18. Mean daily precipitation (mm/day) for JJA, 1979-2014: (a) ERA5 reanalysis, (b) CRU_ts4.03 observations, (c) EC-Earth r6ilp1f1,

(d) EC-Earth r9i1p1f1, (e) EC-Earth r11i1p1f1, (f) EC-Earth r13i1p1f1 and (g) EC-Earth r15i1p1f1

Figure 2.19. EC-Earth mean daily precipitation (mm/day) bias for JJA, 1979-2014 (ERA5 reanalysis minus EC-Earth): (a) EC-Earth r6i1p1f1, (b) EC-Earth r9i1p1f1, (c) EC-Earth r11i1p1f1, (d) EC-Earth r13i1p1f1 and (e) EC-Earth r15ilp1f1

Figure 2.20. EC-Earth mean daily precipitation (mm/day) bias for JJA, 1979-2014 (EC-Earth minus CRU_ts4.03): (a) EC-Earth r6i1p1f1, (b) EC-Earth r9i1p1f1, (c) EC-Earth r11i1p1f1, (d) EC-Earth r13i1p1f1 and (e) EC-Earth r15ilp1f1

Figure 2.21. Comparison of EC-Earth ensemble members with ERA5 reanalysis data for the period 1979-2014: (a) precipitation and (b) precipitation anomalies (\%) with respect to the 30-year period 1981-2010 
Figure 2.22. Comparison of EC-Earth ensemble members with CRU_ts4.03 observational data for the period 1901-2014: (a) land precipitation and (b) land precipitation anomalies (\%) with respect to the 30-year period 1981-2010. A 5-year running mean was applied to all datasets

Figure 2.23. Annual mean 10-m wind speed (m/s), 1979-2014: (a) ERA5 reanalysis,

(b) EC-Earth r6ilp1f1, (c) EC-Earth r9i1p1f1, (d) EC-Earth r11i1p1f1,

(e) EC-Earth r13i1p1f1 and (f) EC-Earth r15i1p1f1

Figure 2.24. EC-Earth annual 10-m wind speed bias (m/s), 1979-2014 (ERA5 reanalysis minus EC-Earth): (a) EC-Earth r6ilp1f1, (b) EC-Earth r9i1p1f1, (c) EC-Earth r11i1p1f1, (d) EC-Earth r13i1p1f1 and (e) EC-Earth r15i1p1f1

Figure 2.25. Mean 10-m wind speed (m/s) for DJF, 1979-2014: (a) ERA5 reanalysis,

(b) EC-Earth r6ilp1f1, (c) EC-Earth r9i1p1f1, (d) EC-Earth r11i1p1f1,

(e) EC-Earth r13i1p1f1 and (f) EC-Earth r15i1p1f1

Figure 2.26. EC-Earth 10-m wind speed bias (m/s) for DJF, 1979-2014 (ERA5 reanalysis minus EC-Earth): (a) EC-Earth r6ilp1f1, (b) EC-Earth r9i1p1f1, (c) EC-Earth r11i1p1f1, (d) EC-Earth r13i1p1f1 and (e) EC-Earth r15i1p1f1

Figure 2.27. Mean 10-m wind speed (m/s) for JJA, 1979-2014: (a) ERA5 reanalysis,

(b) EC-Earth r6i1p1f1, (c) EC-Earth r9i1p1f1, (d) EC-Earth r11i1p1f1,

(e) EC-Earth r13i1p1f1 and (f) EC-Earth r15i1p1f1

Figure 2.28. EC-Earth 10-m wind speed bias (m/s) for JJA, 1979-2014 (ERA5 reanalysis minus EC-Earth): (a) EC-Earth r6ilp1f1, (b) EC-Earth r9i1p1f1, (c) EC-Earth r11i1p1f1, (d) EC-Earth r13i1p1f1 and (e) EC-Earth r15i1p1f1

Figure 2.29. Comparison of EC-Earth ensemble members with ERA5 reanalysis data for the period 1979-2014: (a) 10-m wind speed and (b) 10-m wind speed anomalies with respect to the 30-year period 1981-2010

Figure 2.30. Mean annual MSLP (hPa), 1979-2014: (a) ERA5 reanalysis, (b) EC-Earth r6ilp1f1, (c) EC-Earth r9i1p1f1, (d) EC-Earth r11i1p1f1, (e) EC-Earth r13ilp1f1 and (f) EC-Earth r15i1p1f1

Figure 2.31. EC-Earth mean annual MSLP bias (hPa), 1979-2014 (ERA5 reanalysis minus EC-Earth): (a) EC-Earth r6ilp1f1, (b) EC-Earth r9i1p1f1, (c) EC-Earth r11i1p1f1, (d) EC-Earth r13i1p1f1 and (e) EC-Earth r15i1p1f1

Figure 2.32. MSLP (hPa) for DJF, 1979-2014: (a) ERA5 reanalysis, (b) EC-Earth r6ilp1f1, (c) EC-Earth r9i1p1f1, (d) EC-Earth r11i1p1f1, (e) EC-Earth r13i1p1f1 and (f) EC-Earth r15i1p1f1

Figure 2.33. EC-Earth MSLP bias (hPa) for DJF, 1979-2014 (ERA5 reanalysis minus EC-Earth): (a) EC-Earth r6i1p1f1, (b) EC-Earth r9i1p1f1, (c) EC-Earth r11i1p1f1, (d) EC-Earth r13i1p1f1 and (e) EC-Earth r15i1p1f1

Figure 2.34. MSLP for JJA (hPa), 1979-2014: (a) ERA5 reanalysis, (b) EC-Earth r6ilp1f1, (c) EC-Earth r9i1p1f1, (d) EC-Earth r11i1p1f1, (e) EC-Earth r13ilp1f1 and (f) EC-Earth r15i1p1f1 
Figure 2.35. EC-Earth MSLP bias (hPa) for JJA, 1979-2014 (ERA5 reanalysis minus EC-Earth): (a) EC-Earth r6ilp1f1, (b) EC-Earth r9i1p1f1, (c) EC-Earth r11i1p1f1, (d) EC-Earth r13i1p1f1 and (e) EC-Earth r15i1p1f1

Figure 2.36. Comparison of EC-Earth ensemble members with ERA5 reanalysis data for the period 1979-2014: (a) MSLP and (b) MSLP anomalies with respect to the 30-year period 1981-2010

Figure 2.37. Annual mean cloud cover (\%), 1979-2014: (a) ERA5 reanalysis,

(b) EC-Earth r6ilp1f1, (c) EC-Earth r9i1p1f1, (d) EC-Earth r11i1p1f1,

(e) EC-Earth r13i1p1f1 and (f) EC-Earth r15i1p1f1

Figure 2.38. EC-Earth annual cloud cover bias (\%), 1979-2014 (ERA5 reanalysis minus

EC-Earth): (a) EC-Earth r6ilp1f1, (b) EC-Earth r9ilp1f1, (c) EC-Earth r11i1p1f1, (d) EC-Earth r13i1p1f1 and (e) EC-Earth r15i1p1f1

Figure 2.39. Mean cloud cover (\%) for DJF, 1979-2014: (a) ERA5 reanalysis,

(b) EC-Earth r6i1p1f1, (c) EC-Earth r9i1p1f1, (d) EC-Earth r11i1p1f1,

(e) EC-Earth r13i1p1f1 and (f) EC-Earth r15i1p1f1

Figure 2.40. EC-Earth cloud cover bias (\%) for DJF, 1979-2014 (ERA5 reanalysis minus EC-Earth): (a) EC-Earth r6ilp1f1, (b) EC-Earth r9i1p1f1, (c) EC-Earth r11i1p1f1, (d) EC-Earth r13i1p1f1 and (e) EC-Earth r15i1p1f1

Figure 2.41. Mean cloud cover (\%) for JJA, 1979-2014: (a) ERA5 reanalysis,

(b) EC-Earth r6ilp1f1, (c) EC-Earth r9i1p1f1, (d) EC-Earth r11i1p1f1,

(e) EC-Earth r13i1p1f1 and (f) EC-Earth r15i1p1f1

Figure 2.42. EC-Earth cloud cover bias (\%) for JJA, 1979-2014 (ERA5 reanalysis minus EC-Earth): (a) EC-Earth r6i1p1f1, (b) EC-Earth r9i1p1f1, (c) EC-Earth r11i1p1f1, (d) EC-Earth r13i1p1f1 and (e) EC-Earth r15i1p1f1

Figure 2.43. Comparison of EC-Earth ensemble members with ERA5 reanalysis data for the period 1979-2014: (a) cloud cover and (b) cloud cover anomalies with respect to the 30-year period 1981-2010

Figure 2.44. Annual mean daily snowfall (mm/day), 1979-2014: (a) ERA5 reanalysis,

(b) EC-Earth r6i1p1f1, (c) EC-Earth r9i1p1f1, (d) EC-Earth r11i1p1f1,

(e) EC-Earth r13i1p1f1 and (f) EC-Earth r15i1p1f1

Figure 2.45. EC-Earth annual mean daily snowfall bias (mm/day), 1979-2014 (ERA5 reanalysis minus EC-Earth): (a) EC-Earth r6i1p1f1, (b) EC-Earth r9i1p1f1, (c) EC-Earth r11i1p1f1, (d) EC-Earth r13i1p1f1 and (e) EC-Earth r15i1p1f1

Figure 2.46. Mean daily snowfall (mm/day) for DJF, 1979-2014: (a) ERA5 reanalysis,

(b) EC-Earth r6ilp1f1, (c) EC-Earth r9ilp1f1, (d) EC-Earth r11i1p1f1,

(e) EC-Earth r13i1p1f1 and (f) EC-Earth r15i1p1f1

Figure 2.47. EC-Earth mean daily snowfall bias (mm/day) for DJF, 1979-2014 (ERA5 reanalysis minus EC-Earth): (a) EC-Earth r6i1p1f1, (b) EC-Earth r9i1p1f1, (c) EC-Earth r11i1p1f1, (d) EC-Earth r13i1p1f1 and (e) EC-Earth r15i1p1f1 
Figure 2.48. Mean daily snowfall (mm/day) for JJA, 1979-2014: (a) ERA5 reanalysis,

(b) EC-Earth r6ilp1f1, (c) EC-Earth r9i1p1f1, (d) EC-Earth r11i1p1f1,

(e) EC-Earth r13i1p1f1 and (f) EC-Earth r15i1p1f1

Figure 2.49. EC-Earth mean daily snowfall bias (mm/day) for JJA, 1979-2014 (ERA5 reanalysis minus EC-Earth): (a) EC-Earth r6i1p1f1, (b) EC-Earth r9i1p1f1, (c) EC-Earth r11i1p1f1, (d) EC-Earth r13i1p1f1 and (e) EC-Earth r15i1p1f1

Figure 2.50. Comparison of EC-Earth ensemble members with ERA5 reanalysis data for the period 1979-2014: global annual snowfall anomalies (\%) with respect to the 30-year period, 1981-2010

Figure 2.51. Annual mean SST (K), 1979-2014: (a) ERA5 reanalysis, (b) EC-Earth r6ilp1f1, (c) EC-Earth r9i1p1f1, (d) EC-Earth r11i1p1f1, (e) EC-Earth r13ilp1f1 and (f) EC-Earth r15i1p1f1

Figure 2.52. EC-Earth annual mean SST bias $\left({ }^{\circ} \mathrm{C}\right), 1979-2014$ (ERA5 reanalysis minus EC-Earth): (a) EC-Earth r6i1p1f1, (b) EC-Earth r9i1p1f1, (c) EC-Earth r11i1p1f1, (d) EC-Earth r13i1p1f1 and (e) EC-Earth r15i1p1f1

Figure 2.53. Mean SST for DJF (K), 1979-2014: (a) ERA5 reanalysis, (b) EC-Earth r6ilp1f1, (c) EC-Earth r9i1p1f1, (d) EC-Earth r11i1p1f1, (e) EC-Earth r13i1p1f1 and (f) EC-Earth r15i1p1f1

Figure 2.54. EC-Earth mean SST bias $\left({ }^{\circ} \mathrm{C}\right)$ for DJF, 1979-2014 (ERA5 reanalysis minus EC-Earth): (a) EC-Earth r6i1p1f1, (b) EC-Earth r9i1p1f1, (c) EC-Earth r11i1p1f1, (d) EC-Earth r13i1p1f1 and (e) EC-Earth r15i1p1f1

Figure 2.55. Mean SST for JJA (K), 1979-2014: (a) ERA5 reanalysis, (b) EC-Earth r6ilp1f1, (c) EC-Earth r9i1p1f1, (d) EC-Earth r11i1p1f1, (e) EC-Earth r13ilp1f1 and (f) EC-Earth r15ilp1f1

Figure 2.56. EC-Earth mean SST bias $\left({ }^{\circ} \mathrm{C}\right)$ for JJA, 1979-2014 (ERA5 reanalysis minus EC-Earth): (a) EC-Earth r6ilp1f1, (b) EC-Earth r9i1p1f1, (c) EC-Earth r11i1p1f1, (d) EC-Earth r13i1p1f1 and (e) EC-Earth r15i1p1f1

Figure 2.57. Comparison of EC-Earth ensemble members with ERA5 reanalysis data for the period 1979-2014: (a) SST and (b) SST anomalies with respect to the 30-year period 1981-2010

Figure 2.58. Northern Hemisphere annual mean sea ice fraction (\%), 1979-2014:

(a) ERA5 reanalysis, (b) EC-Earth r6ilp1f1, (c) EC-Earth r9i1p1f1,

(d) EC-Earth r11i1p1f1, (e) EC-Earth r13i1p1f1 and (f) EC-Earth r15i1p1f1

Figure 2.59. Northern Hemisphere March mean sea ice fraction (\%), 1979-2014:

(a) ERA5 reanalysis, (b) EC-Earth r6ilp1f1, (c) EC-Earth r9i1p1f1,

(d) EC-Earth r11i1p1f1, (e) EC-Earth r13i1p1f1 and (f) EC-Earth r15i1p1f1

Figure 2.60. Northern Hemisphere September mean sea ice fraction (\%), 1979-2014:

(a) ERA5 reanalysis, (b) EC-Earth r6i1p1f1, (c) EC-Earth r9i1p1f1,

(d) EC-Earth r11i1p1f1, (e) EC-Earth r13i1p1f1 and (f) EC-Earth r15i1p1f1 
Figure 2.61. Southern Hemisphere annual mean sea ice fraction (\%), 1979-2014:

(a) ERA5 reanalysis, (b) EC-Earth r6ilp1f1, (c) EC-Earth r9i1p1f1,

(d) EC-Earth r11i1p1f1, (e) EC-Earth r13i1p1f1 and (f) EC-Earth r15i1p1f1

Figure 2.62. Southern Hemisphere March mean sea ice fraction (\%), 1979-2014:

(a) ERA5 reanalysis, (b) EC-Earth r6i1p1f1, (c) EC-Earth r9i1p1f1,

(d) EC-Earth r11i1p1f1, (e) EC-Earth r13i1p1f1 and (f) EC-Earth r15i1p1f1

Figure 2.63. Southern Hemisphere September mean sea ice fraction (\%), 1979-2014:

(a) ERA5 reanalysis, (b) EC-Earth r6ilp1f1, (c) EC-Earth r9i1p1f1,

(d) EC-Earth r11ilp1f1, (e) EC-Earth r13i1p1f1 and (f) EC-Earth r15i1p1f1

Figure 2.64. Comparison of the EC-Earth Northern Hemisphere sea ice fraction (\%) with ERA5 reanalysis data for the period 1979-2014: (a) annual, (b) March and

(c) September

Figure 2.65. Northern Hemisphere sea ice fraction anomalies with respect to the 30-year period 1981-2010: (a) annual, (b) March and (c) September

Figure 2.66. Comparison of EC-Earth Southern Hemisphere sea ice fraction (\%) with ERA5 reanalysis data for the period 1979-2014: (a) annual, (b) March and (c) September

Figure 2.67. Southern Hemisphere sea ice fraction anomalies with respect to the 30-year period 1981-2010: (a) annual, (b) March and (c) September

Figure 3.1. EC-Earth annual 2-m temperature projections $\left(2041-2070\right.$ vs $1981-2010,{ }^{\circ} \mathrm{C}$ change): (a) SSP1-2.6, (b) SSP2-4.5, (c) SSP3-7.0 and (d) SSP5-8.5

Figure 3.2. EC-Earth annual 2-m temperature projections $\left(2071-2100\right.$ vs $1981-2010,{ }^{\circ} \mathrm{C}$ change): (a) SSP1-2.6, (b) SSP2-4.5, (c) SSP3-7.0 and (d) SSP5-8.5

Figure 3.3. EC-Earth DJF 2-m temperature projections (2041-2070 vs 1981-2010, ${ }^{\circ} \mathrm{C}$ change): (a) SSP1-2.6, (b) SSP2-4.5, (c) SSP3-7.0 and (d) SSP5-8.5

Figure 3.4. EC-Earth DJF 2-m temperature projections (2071-2100 vs 1981-2010, ${ }^{\circ} \mathrm{C}$ change): (a) SSP1-2.6, (b) SSP2-4.5, (c) SSP3-7.0 and (d) SSP5-8.5

Figure 3.5. EC-Earth JJA 2-m temperature projections (2041-2070 vs $1981-2010,{ }^{\circ} \mathrm{C}$ change): (a) SSP1-2.6, (b) SSP2-4.5, (c) SSP3-7.0 and (d) SSP5-8.5

Figure 3.6. EC-Earth JJA 2-m temperature projections (2071-2100 vs 1981-2010, ${ }^{\circ} \mathrm{C}$ change): (a) SSP1-2.6, (b) SSP2-4.5, (c) SSP3-7.0 and (d) SSP5-8.5

Figure 3.7. Global mean annual 2-m temperature anomalies with respect to the 30 -year period 1981-2010: EC-Earth ensemble members r6ilp1f1, r9ilp1f1, r11i1p1f1, r13i1p1f1 and r15i1p1f1

Figure 3.8. Standard deviation of the ensemble of annual 2-m temperature projections (2071-2100): (a) SSP1-2.6, (b) SSP2-4.5, (c) SSP3-7.0 and (d) SSP5-8.5

Figure 3.9. EC-Earth annual precipitation projections (2041-2070 vs 1981-2010, \% change): (a) SSP1-2.6, (b) SSP2-4.5, (c) SSP3-7.0 and (d) SSP5-8.5 
Figure 3.10. EC-Earth annual precipitation projections (2071-2100 vs 1981-2010, \% change): (a) SSP1-2.6, (b) SSP2-4.5, (c) SSP3-7.0 and (d) SSP5-8.5

Figure 3.11. EC-Earth DJF precipitation projections (2041-2070 vs 1981-2010, \% change): (a) SSP1-2.6, (b) SSP2-4.5, (c) SSP3-7.0 and (d) SSP5-8.5

Figure 3.12. EC-Earth DJF precipitation projections (2071-2100 vs 1981-2010, \% change): (a) SSP1-2.6, (b) SSP2-4.5, (c) SSP3-7.0 and (d) SSP5-8.5

Figure 3.13. EC-Earth JJA precipitation projections (2041-2070 vs 1981-2010, \% change): (a) SSP1-2.6, (b) SSP2-4.5, (c) SSP3-7.0 and (d) SSP5-8.5

Figure 3.14. EC-Earth JJA precipitation projections (2071-2100 vs 1981-2010, \% change): (a) SSP1-2.6, (b) SSP2-4.5, (c) SSP3-7.0 and (d) SSP5-8.5

Figure 3.15. Global annual precipitation anomalies (\%) with respect to the 30-year period 1981-2010: EC-Earth ensemble members r6ilp1f1, r9i1p1f1, r11i1p1f1, r13i1p1f1 and r15i1p1f1

Figure 3.16. Standard deviation of the ensemble of annual precipitation projections (2071-2100): (a) SSP1-2.6, (b) SSP2-4.5, (c) SSP3-7.0 and (d) SSP5-8.5

Figure 3.17. EC-Earth annual mean 10-m wind speed projections (2041-2070 vs 1981-2010, m/s difference): (a) SSP1-2.6, (b) SSP2-4.5, (c) SSP3-7.0 and (d) SSP5-8.5

Figure 3.18. EC-Earth annual mean 10-m wind speed projections (2071-2100 vs 1981-2010, m/s difference): (a) SSP1-2.6, (b) SSP2-4.5, (c) SSP3-7.0 and (d) SSP5-8.5

Figure 3.19. EC-Earth DJF mean 10-m wind speed projections (2041-2070 vs 1981-2010, m/s difference): (a) SSP1-2.6, (b) SSP2-4.5, (c) SSP3-7.0 and (d) SSP5-8.5

Figure 3.20. EC-Earth DJF mean 10-m wind speed projections (2071-2100 vs 1981-2010, m/s difference): (a) SSP1-2.6, (b) SSP2-4.5, (c) SSP3-7.0 and (d) SSP5-8.5

Figure 3.21. EC-Earth JJA mean 10-m wind speed projections (2041-2070 vs 1981-2010, m/s difference): (a) SSP1-2.6, (b) SSP2-4.5, (c) SSP3-7.0 and (d) SSP5-8.5

Figure 3.22. EC-Earth JJA mean 10m wind speed projections (2071-2100 vs 1981-2010, $\mathrm{m} / \mathrm{s}$ difference): (a) SSP1-2.6, (b) SSP2-4.5, (c) SSP3-7.0 and (d) SSP5-8.5

Figure 3.23. Global annual mean 10-m wind speed anomalies with respect to the 30 -year period 1981-2010: EC-Earth ensemble members r6ilp1f1, r9i1p1f1, r11ilp1f1, r13i1p1f1 and r15i1p1f1

Figure 3.24. Standard deviation of the ensemble of annual mean 10-m wind speed projections (2071-2100): (a) SSP1-2.6, (b) SSP2-4.5, (c) SSP3-7.0 and (d) SSP5-8.5 
Figure 3.25. EC-Earth annual MSLP projections (2041-2070 vs 1981-2010, hPa difference): (a) SSP1-2.6, (b) SSP2-4.5, (c) SSP3-7.0 and (d) SSP5-8.5

Figure 3.26. EC-Earth annual MSLP projections (2071-2100 vs 1981-2010, hPa difference): (a) SSP1-2.6, (b) SSP2-4.5, (c) SSP3-7.0 and (d) SSP5-8.5

Figure 3.27. EC-Earth DJF MSLP projections (2041-2070 vs 1981-2010, hPa difference): (a) SSP1-2.6, (b) SSP2-4.5, (c) SSP3-7.0 and (d) SSP5-8.5

Figure 3.28. EC-Earth DJF MSLP projections (2071-2100 vs 1981-2010, hPa difference): (a) SSP1-2.6, (b) SSP2-4.5, (c) SSP3-7.0 and (d) SSP5-8.5

Figure 3.29. EC-Earth JJA MSLP projections (2041-2070 vs 1981-2010, hPa difference): (a) SSP1-2.6, (b) SSP2-4.5, (c) SSP3-7.0 and (d) SSP5-8.5

Figure 3.30. EC-Earth JJA MSLP projections (2071-2100 vs 1981-2010, hPa difference): (a) SSP1-2.6, (b) SSP2-4.5, (c) SSP3-7.0 and (d) SSP5-8.5

Figure 3.31. Global annual MSLP anomalies with respect to the 30-year period 1981-2010: EC-Earth ensemble members r6ilp1f1, r9i1p1f1, r11ilp1f1, r13i1p1f1 and r15i1p1f1

Figure 3.32. Standard deviation of the ensemble of Annual MSLP projections (2071-2100): (a) SSP1-2.6, (b) SSP2-4.5, (c) SSP3-7.0 and (d) SSP5-8.5

Figure 3.33. EC-Earth annual total cloud cover projections (2041-2070 vs 1981-2010, $\%$ difference); (a) SSP1-2.6, (b) SSP2-4.5, (c) SSP3-7.0 and (d) SSP5-8.5

Figure 3.34. EC-Earth annual total cloud cover projections (2071-2100 vs 1981-2010, \% difference): (a) SSP1-2.6, (b) SSP2-4.5, (c) SSP3-7.0 and (d) SSP5-8.5

Figure 3.35. EC-Earth DJF total cloud cover projections (2041-2070 vs 1981-2010, \% difference): (a) SSP1-2.6, (b) SSP2-4.5, (c) SSP3-7.0 and (d) SSP5-8.5

Figure 3.36. EC-Earth DJF total cloud cover projections (2071-2100 vs 1981-2010, \% difference): (a) SSP1-2.6, (b) SSP2-4.5, (c) SSP3-7.0 and (d) SSP5-8.5

Figure 3.37. EC-Earth JJA total cloud cover projections (2041-2070 vs 1981-2010, \% difference): (a) SSP1-2.6, (b) SSP2-4.5, (c) SSP3-7.0 and (d) SSP5-8.5

Figure 3.38. EC-Earth JJA total cloud cover projections (2071-2100 vs 1981-2010, \% difference): (a) SSP1-2.6, (b) SSP2-4.5, (c) SSP3-7.0 and (d) SSP5-8.5

Figure 3.39. Global annual total cloud cover anomalies (\%) with respect to the 30 -year period 1981-2010: EC-Earth ensemble members r6ilp1f1, r9i1p1f1, r11i1p1f1, r13i1p1f1 and r15i1p1f1

Figure 3.40. Standard deviation of the ensemble of annual total cloud cover projections (2071-2100): (a) SSP1-2.6, (b) SSP2-4.5, (c) SSP3-7.0 and (d) SSP5-8.5

Figure 3.41. EC-Earth annual snowfall projections (2041-2070 vs 1981-2010, mm/day difference): (a) SSP1-2.6, (b) SSP2-4.5, (c) SSP3-7.0 and (d) SSP5-8.5

Figure 3.42. EC-Earth annual snowfall projections (2071-2100 vs 1981-2010, mm/day difference): (a) SSP1-2.6, (b) SSP2-4.5, (c) SSP3-7.0 and (d) SSP5-8.5 
Figure 3.43. EC-Earth DJF snowfall projections (2041-2070 vs 1981-2010, mm/day difference): (a) SSP1-2.6, (b) SSP2-4.5, (c) SSP3-7.0 and (d) SSP5-8.5

Figure 3.44. EC-Earth DJF snowfall projections (2071-2100 vs 1981-2010, mm/day difference): (a) SSP1-2.6, (b) SSP2-4.5, (c) SSP3-7.0 and (d) SSP5-8.5

Figure 3.45. EC-Earth JJA snowfall projections (2041-2070 vs 1981-2010, mm/day difference): (a) SSP1-2.6, (b) SSP2-4.5, (c) SSP3-7.0 and (d) SSP5-8.5

Figure 3.46. EC-Earth JJA snowfall projections (2071-2100 vs 1981-2010, mm/day difference): (a) SSP1-2.6, (b) SSP2-4.5, (c) SSP3-7.0 and (d) SSP5-8.5

Figure 3.47. Annual snowfall anomalies (\%) with respect to the 30 -year period 1981-2010: EC-Earth ensemble members r6i1p1f1, r9i1p1f1, r11i1p1f1, r13i1p1f1 and r15ilp1f1 - (a) global, (b) Northern Hemisphere and (c) Southern Hemisphere

Figure 3.48. Standard deviation of the ensemble of annual snowfall projections (2071-2100): (a) SSP1-2.6, (b) SSP2-4.5, (c) SSP3-7.0 and (d) SSP5-8.5

Figure 3.49. EC-Earth annual SST projections (2041-2070 vs $1981-2010,{ }^{\circ} \mathrm{C}$ change):

(a) SSP1-2.6, (b) SSP2-4.5, (c) SSP3-7.0 and (d) SSP5-8.5

Figure 3.50. EC-Earth annual SST projections (2071-2100 vs 1981-2010, ${ }^{\circ} \mathrm{C}$ change):

(a) SSP1-2.6, (b) SSP2-4.5, (c) SSP3-7.0 and (d) SSP5-8.5

Figure 3.51. EC-Earth DJF SST projections (2041-2070 vs $1981-2010,{ }^{\circ} \mathrm{C}$ change):

(a) SSP1-2.6, (b) SSP2-4.5, (c) SSP3-7.0 and (d) SSP5-8.5

Figure 3.52. EC-Earth DJF SST projections (2071-2100 vs $1981-2010,{ }^{\circ} \mathrm{C}$ change):

(a) SSP1-2.6, (b) SSP2-4.5, (c) SSP3-7.0 and (d) SSP5-8.5

Figure 3.53. EC-Earth JJA SST projections (2041-2070 vs $1981-2010,{ }^{\circ} \mathrm{C}$ change);

(a) SSP1-2.6, (b) SSP2-4.5, (c) SSP3-7.0 and (d) SSP5-8.5

Figure 3.54. EC-Earth JJA SST projections (2071-2100 vs $1981-2010,{ }^{\circ} \mathrm{C}$ change):

(a) SSP1-2.6, (b) SSP2-4.5, (c) SSP3-7.0 and (d) SSP5-8.5

Figure 3.55. Global annual SST anomalies with respect to the 30-year period 1981-2010: EC-Earth ensemble members r6i1p1f1, r9i1p1f1, r11i1p1f1, r13i1p1f1 and r15ilp1f1

Figure 3.56. Standard deviation of the ensemble of annual SST projections (2071-2100):

(a) SSP1-2.6, (b) SSP2-4.5, (c) SSP3-7.0 and (d) SSP5-8.5

Figure 3.57. Annual mean Northern Hemisphere sea ice fraction (\%): (a) historical ensemble for the period 1981-2010 and (b) SSP1-2.6, (c) SSP2-4.5, (d) SSP3-7.0 and (e) SSP5-8.5 for the period 2041-2070

Figure 3.58. Annual Northern Hemisphere sea ice projections (\% change) (2041-2070 vs 1981-2010): (a) SSP1-2.6, (b) SSP2-4.5, (c) SSP3-7.0 and (d) SSP5-8.5

Figure 3.59. Annual mean Northern Hemisphere sea ice fraction (\%): (a) historical ensemble for the period 1981-2010 and (b) SSP1-2.6, (c) SSP2-4.5, (d) SSP3-7.0 and (e) SSP5-8.5 for the period 2071-2100 
Figure 3.60. Annual Northern Hemisphere sea ice projections (\% change) (2071-2100 vs 1981-2010): (a) SSP1-2.6, (b) SSP2-4.5, (c) SSP3-7.0 and (d) SSP5-8.5

Figure 3.61. Mean Northern Hemisphere March sea ice fraction (\%): (a) historical ensemble for the period 1981-2010 and (b) SSP1-2.6, (c) SSP2-4.5, (d) SSP3-7.0 and (e) SSP5-8.5 for the period 2041-2070

Figure 3.62. Northern Hemisphere March sea ice projections (\% change) (2041-2070 vs 1981-2010): (a) SSP1-2.6, (b) SSP2-4.5, (c) SSP3-7.0 and (d) SSP5-8.5

Figure 3.63. Mean Northern Hemisphere March sea ice fraction (\%): (a) historical ensemble for the period 1981-2010 and (b) SSP1-2.6, (c) SSP2-4.5, (d) SSP3-7.0 and (e) SSP5-8.5 for the period 2071-2100

Figure 3.64. Northern Hemisphere March sea ice projections (\% change) (2071-2100 vs 1981-2010): (a) SSP1-2.6, (b) SSP2-4.5, (c) SSP3-7.0 and (d) SSP5-8.5

Figure 3.65. Mean Northern Hemisphere September sea ice fraction (\%): (a) historical ensemble for the period 1981-2010 and (b) SSP1-2.6, (c) SSP2-4.5, (d) SSP3-7.0 and (e) SSP5-8.5 for the period 2041-2070

Figure 3.66. Northern Hemisphere September sea ice projections (\% change) (2041-2070 vs 1981-2010): (a) SSP1-2.6, (b) SSP2-4.5, (c) SSP3-7.0 and (d) SSP5-8.5

Figure 3.67. Northern Hemisphere September sea ice projections, 2071-2100:

(a) SSP1-2.6 sea ice fraction (\%) with green line showing the $15 \%$ contour line and (b) SSP1-2.6 anomaly relative to 1981-2010 (\% change)

Figure 3.68. Annual mean Southern Hemisphere sea ice fraction (\%): (a) historical ensemble for the period 1981-2010 and (b) SSP1-2.6, (c) SSP2-4.5, (d) SSP3-7.0 and (e) SSP5-8.5 for the period 2041-2070

Figure 3.69. Annual Southern Hemisphere sea ice projections (\% change) (2041-2070 vs 1981-2010): (a) SSP1-2.6, (b) SSP2-4.5, (c) SSP3-7.0 and (d) SSP5-8.5

Figure 3.70. Annual mean Southern Hemisphere sea ice fraction (\%): (a) historical ensemble for the period 1981-2010 and (b) SSP1-2.6, (c) SSP2-4.5, (d) SSP3-7.0 and (e) SSP5-8.5 for the period 2071-2100

Figure 3.71. Annual Southern Hemisphere sea ice projections (\% change) (2071-2100 vs 1981-2010): (a) SSP1-2.6, (b) SSP2-4.5, (c) SSP3-7.0 and (d) SSP5-8.5

Figure 3.72. Mean Southern Hemisphere March sea ice fraction (\%): (a) historical ensemble for the period 1981-2010 and (b) SSP1-2.6, (c) SSP2-4.5, (d) SSP3-7.0 and (e) SSP5-8.5 for the period 2041-2070

Figure 3.73. Southern Hemisphere March sea ice projections (\% change) (2041-2070 vs 1981-2010): (a) SSP1-2.6, (b) SSP2-4.5, (c) SSP3-7.0 and (d) SSP5-8.5

Figure 3.74. Mean Southern Hemisphere March sea ice projections, 2071-2100:

(a) SSP1-2.6 sea ice fraction (\%) with green line showing the $15 \%$ contour line, (b) SSP1-2.6 anomaly relative to 1981-2010 (\% change), (c) SSP2-4.5 sea ice fraction (\%) with green line showing the $15 \%$ contour line, (d) SSP2-4.5 anomaly relative to $1981-2010$ (\% change) 
Figure 3.75. Mean Southern Hemisphere September sea ice fraction (\%): (a) historical ensemble for the period 1981-2010 and (b) SSP1-2.6, (c) SSP2-4.5,

(d) SSP3-7.0 and (e) SSP5-8.5 for the period 2041-2070

Figure 3.76. Southern Hemisphere September sea ice projections ( $\%$ change) (2041-2070 vs 1981-2010): (a) SSP1-2.6, (b) SSP2-4.5, (c) SSP3-7.0 and (d) SSP5-8.5

Figure 3.77. Mean Southern Hemisphere September sea ice fraction (\%): (a) historical ensemble for the period 1981-2010 and (b) SSP1-2.6, (c) SSP2-4.5,

(d) SSP3-7.0 and (e) SSP5-8.5 for the period 2071-2100

Figure 3.78. Southern Hemisphere September sea ice projections ( $\%$ change) (2071-2100 vs 1981-2010): (a) SSP1-2.6, (b) SSP2-4.5, (c) SSP3-7.0 and (d) SSP5-8.5

Figure 3.79. Annual sea ice anomalies ( $\%$ change) with respect to the 30 -year period 1981-2010: EC-Earth ensemble members r6ilp1f1, r9i1p1f1, r11i1p1f1, r13i1p1f1 and r15i1p1f1 - (a) global, (b) Northern Hemisphere and (c) Southern Hemisphere 


\section{List of Tables and Boxes}

\section{Tables}

Table 1.1. Summary of SSP narratives

Table 1.2. Description of EC-Earth variables hosted on the ICHEC ESGF node with at least 6-hour temporal resolution

Table 1.3. Scaling results for a 1-month EC-Earth simulation on ECMWF/cca using the intel compilers

Table 1.4. Scaling results for a 1-month EC-Earth simulation on the ICHEC system using the intel compilers

Table 2.1. Mean global annual and seasonal 2-m temperature bias and MAE $\left({ }^{\circ} \mathrm{C}\right)$ for each of the five EC-Earth ensemble members

Table 2.2. Mean global annual and seasonal 2-m temperature bias and $\operatorname{MAE}\left({ }^{\circ} \mathrm{C}\right)$ for each of the five EC-Earth ensemble members

Table 2.3. Mean global annual and seasonal daily precipitation bias and MAE (mm/day) for each of the five EC-Earth ensemble members

Table 2.4. Mean global annual and seasonal daily precipitation percentage bias and MAE (\%) for each of the five EC-Earth ensemble members

Table 2.5. Mean global annual and seasonal daily precipitation bias and MAE (mm/ day) for each of the five EC-Earth ensemble members

Table 2.6. Mean global annual and seasonal 10-m wind speed bias and MAE (m/s) for each of the five EC-Earth ensemble members

Table 2.7. Mean global annual and seasonal MSLP bias and MAE (hPa) for each of the five EC-Earth ensemble members

Table 2.8. Mean global annual and seasonal cloud cover bias and MAE (\%) for each of the five EC-Earth ensemble members

Table 2.9. Mean and minimum bias $\left({ }^{\circ} \mathrm{C}\right)$ over the North Atlantic area $40-60^{\circ} \mathrm{N}$, $25-55^{\circ} \mathrm{W}$ for each of the five EC-Earth ensemble members

Table 2.10. Mean global annual and seasonal SST bias $\left({ }^{\circ} \mathrm{C}\right)$ for each of the five EC-Earth ensemble members

Table 3.1. Overview of the Scenario-MIP simulations

\section{Boxes}

Box 1.1. List of EC-Earth variables hosted on the ICHEC ESGF node with daily and monthly temporal resolutions 



\section{Executive Summary}

The global climate simulations described in this report constitute Ireland's contribution to the Coupled Model Intercomparison Project (CMIP) (phase 6) (CMIP6) and will be included for assessment in the United Nations Intergovernmental Panel on Climate Change (IPCC) Sixth Assessment Report (AR6).

Since 1995, CMIP has co-ordinated climate model experiments involving multiple international modelling teams. The CMIP project has led to a better understanding of past, present and future climate, and CMIP model experiments have routinely been the basis for future climate change assessments carried out by the IPCC. The CMIP phase 5 (CMIP5) simulations have demonstrated the added value of improved models and enhanced resolution when compared with outputs from the CMIP phase 3 (CMIP3) project. This improvement in skill is expected to continue with the CMIP6 simulations.

The EC-Earth consortium participated in CMIP5 and is currently participating in CMIP6 using a model that includes biogeochemical cycles and atmospheric chemistry. The current version of EC-Earth is based on the European Centre for Medium-Range Weather Forecasts (ECMWF) Integrated Forecast System (IFS) atmospheric model, the Nucleus for European Modelling of the Ocean (NEMO) model, the Louvainla-Neuve sea ice model (LIM3), the atmospheric Tracer Model version 5 (TM5), the Lund-PotsdamJena General Ecosystem Simulator (LPJ-GUESS) vegetation model and the Pelagic Interactions Scheme for Carbon and Ecosystem Studies (PISCES) ocean biogeochemistry model. Coupling is provided by OASIS3-MCT (the Ocean Atmosphere Sea Ice Soil OASIS - coupler interfaced with the Model Coupling Toolkit - MCT).

As part of the current project, the EC-Earth Atmosphere-Ocean General Circulation Model (AOGCM) configuration was employed. The atmosphere was simulated with $\sim 79-\mathrm{km}$ horizontal grid spacings (T255) and 91 vertical levels. The ocean was simulated with 1-degree horizontal resolution and 75 vertical levels. In total, five historical (1850-2014) and 20 Scenario Model Intercomparison Project (ScenarioMIP) simulations (2015-2100) were run.
The future climate was simulated under the full range of ScenarioMIP "tier 1" shared socioeconomic pathways (SSPs); SSP1-2.6, SSP2-4.5, SSP3-7.0 and SSP5-8.5. For one ensemble member, all model levels were archived, allowing for regional downscaling using regional climate models and participation in the CMIP6 Coordinated Regional Downscaling Experiment (CORDEX) Model Intercomparison Project (MIP).

The EC-Earth CMIP6 simulations were run on the Irish Centre for High-End Computing (ICHEC) "kay" and ECMWF supercomputing systems. All CMIP6 data were published on the ICHEC Earth System Grid Federation (ESGF) node. The ESGF is an international effort of climate centres with a mission to support CMIP6 and future IPCC assessments. It is expected that the CMIP6 data, produced as part of the current report, will be analysed by the international research community during 2019/2020 for inclusion in the upcoming IPCC AR6.

Chapter 2 provides an overview of validations of 2-m temperature, precipitation, 10-m wind speed, mean sea level pressure (MSLP), total cloud cover, snowfall, sea surface temperature and sea ice fraction. The EC-Earth historical data were compared with Climatic Research Unit observational datasets and ERA5 global reanalysis data (ERA5 is the fifth generation of the ECMWF global climate reanalysis dataset). Results confirm the ability of the EC-Earth model to simulate the global climate with a high level of accuracy.

Chapter 3 provides an overview of EC-Earth global climate projections. The future global climate was simulated to the year 2100 under each of the four SSPs (SSP1-2.6, SSP2-4.5, SSP3-7.0 and SSP5-8.5). This results in 20 future global climate experiments (five ensembles multiplied by four SSPs). Projections of climate change were assessed by comparing the two 30-year future periods 2041-2070 and 2071-2100 with the 30-year historical period 1981-2010. Climate projections are presented for the Northern Hemisphere winter (December, January and February), Northern Hemisphere summer (June, July and August) and over the full year. Results show large projected increases in temperature; the largest are 
noted over the land masses, in particular the northernmost regions and the Arctic. Projected temperature increases range from $\sim 0.5^{\circ} \mathrm{C}$ over the Southern Hemisphere oceans for SSP1-2.6 (2041-2070) to $\sim 18^{\circ} \mathrm{C}$ over the Arctic for SSP5-8.5 (2071-2100). By the year 2100 , the global mean temperature is projected to increase by approximately $1.5^{\circ} \mathrm{C}, 2.8^{\circ} \mathrm{C}$, $4.2^{\circ} \mathrm{C}$ and $5.5^{\circ} \mathrm{C}$ for SSP $1-2.6$, SSP2-4.5, SSP $3-7.0$ and SSP5-8.5, respectively. For precipitation, all ensemble members show a steady increase in mean global precipitation from around 2000, with a noticeable divergence between the SSPs around
2060. By the year 2100 , global mean precipitation is projected to increase by approximately $4 \%, 6 \%, 8 \%$ and $10 \%$ for SSP1-2.6, SSP2-4.5, SSP $3-7.0$ and SSP5-8.5, respectively. Northern Hemisphere sea ice is projected to disappear in the September months by 2071-2100 under SSP2-4.5, SSP3-7.0 and SSP58.5. Projections of $10-\mathrm{m}$ wind speed, MSLP, total cloud cover, snowfall and sea surface temperature are also presented in Chapter 3. 


\section{Introduction}

Increasing greenhouse gas emissions and changing land use are having a significant effect on the Earth's climate. The Intergovernmental Panel on Climate Change (IPCC) has concluded that "warming of the climate system is unequivocal, and since the 1950s, many of the observed changes are unprecedented over decades to millennia" (IPCC, 2013a). It is extremely likely (95-100\% probability) that human influence was the dominant cause of global warming between 1951 and 2010 (IPCC, 2013b) and was a contributor to global drought as early as 1900 (Marvel et al., 2019). The United Nations has declared that the world experienced more unprecedented highimpact climate extremes in the first decade of the 21 st century than in any previous decade (WMO, 2013). The IPCC estimates that there will be a rise in global mean surface temperatures of between 0.3 and $4.8^{\circ} \mathrm{C}$ by the late 21 st century (IPCC, 2013a), with European mean temperatures projected to exceed the global mean. The authors project that the median temperature over Ireland for the period 2046-2065 will increase by $1-1.5^{\circ} \mathrm{C}$ in future summers and by $0.5-1.5^{\circ} \mathrm{C}$ in future winters under the representative concentration pathway (RCP) 4.5 scenario (IPCC, 2013c). In 2018, the IPCC published a special report "on the impacts of global warming of $1.5^{\circ} \mathrm{C}$ above pre-industrial levels and related global greenhouse gas emission pathways, in the context of strengthening the global response to the threat of climate change, sustainable development, and efforts to eradicate poverty" (IPCC, 2018). The report concluded that "human-induced warming reached approximately $1^{\circ} \mathrm{C}$ (likely between $0.8^{\circ} \mathrm{C}$ and $1.2^{\circ} \mathrm{C}$ ) above pre-industrial levels in 2017 , increasing at $0.2^{\circ} \mathrm{C}$ (likely between $0.1^{\circ} \mathrm{C}$ and $0.3^{\circ} \mathrm{C}$ ) per decade (high confidence)". Furthermore, "warming greater than the global annual average is being experienced in many land regions and seasons, including two to three times higher in the Arctic". The authors concluded that global warming is likely to reach " $1.5^{\circ} \mathrm{C}$ between 2030 and 2052 if it continues to increase at the current rate (high confidence)" and "temperature extremes on land are projected to warm more than the global mean surface temperature (high confidence): extreme hot days in mid-latitudes warm by up to about $3^{\circ} \mathrm{C}$ at global warming of $1.5^{\circ} \mathrm{C}$ and about $4^{\circ} \mathrm{C}$ at $2^{\circ} \mathrm{C}$, and extreme cold nights in high latitudes warm by up to about $4.5^{\circ} \mathrm{C}$ at $1.5^{\circ} \mathrm{C}$ and about $6^{\circ} \mathrm{C}$ at $2^{\circ} \mathrm{C}$ (high confidence)". The number of hot days is projected to "increase in most land regions, with highest increases in the tropics (high confidence)". The global water cycle is also projected to change significantly, with increases in disparity between wet and dry regions, as well as wet and dry seasons, with some regional exceptions (IPCC, 2013b). In 2018, the IPCC concluded that "risks from droughts and precipitation deficits are projected to be higher at $2^{\circ} \mathrm{C}$ compared to $1.5^{\circ} \mathrm{C}$ of global warming in some regions (medium confidence). Risks from heavy precipitation events are projected to be higher at $2^{\circ} \mathrm{C}$ compared to $1.5^{\circ} \mathrm{C}$ of global warming in several northern hemisphere high-latitude and/or high-elevation regions, eastern Asia and eastern North America (medium confidence)". In addition, "heavy precipitation associated with tropical cyclones is projected to be higher at $2^{\circ} \mathrm{C}$ compared to $1.5^{\circ} \mathrm{C}$ global warming (medium confidence)" (IPCC, 2018).

The IPCC (2013a) has concluded that "the rate of sea level rise since the mid-19th century has been larger than the mean rate during the previous two millennia (high confidence)" (IPCC, 2013a), and "over the period 1901 to 2010, global mean sea level (GMSL) rose by 0.19 (0.17 to 0.21$) \mathrm{m}$ " (Church et al., 2013). Furthermore, results show that sea level rise is accelerating: "it is very likely that the mean rate of global averaged sea level rise was 1.7 (1.5 to 1.9$) \mathrm{mm} / \mathrm{yr}$ between 1901 and 2010, 2.0 (1.7 to 2.3) mm/yr between 1971 and 2010, and 3.2 (2.8 to 3.6 ) mm/yr between 1993 and 2010" (Church et al., 2013). These observed trends in global sea level rise (GSLR) were broadly confirmed by the IPCC Special Report on the Ocean and Cryosphere in a Changing Climate (IPCC, 2019; italics as in the original): "the total GMSL rise for $1902-2015$ is $0.16 \mathrm{~m}$ (likely range $0.12-0.21 \mathrm{~m}$ )" and "the rate of GMSL rise for 2006-2015 of $3.6 \mathrm{~mm} \mathrm{yr}^{-1}$ (3.1-4.1 $\mathrm{mm} \mathrm{yr}^{-1}$, very likely range), is unprecedented over the last century (high confidence), and about 2.5 times the rate for 1901-1990 of $1.4 \mathrm{~mm} \mathrm{yr}^{-1}\left(0.8-2.0 \mathrm{~mm} \mathrm{yr}^{-1}\right.$, very likely range)". 
The IPCC (2019) also concluded that GSLR is projected to rise at an increasing rate and that "extreme sea level events that are historically rare (once per century in the recent past) are projected to occur frequently (at least once per year) at many locations by 2050 in all RCP scenarios, especially in tropical regions (high confidence)". The GMSL rise "under RCP2.6 is projected to be $0.39 \mathrm{~m}(0.26-0.53 \mathrm{~m}$, likely range) for the period $2081-2100$, and $0.43 \mathrm{~m}$ $(0.29-0.59 \mathrm{~m}$, likely range) in 2100 with respect to 1986-2005. For RCP8.5, the corresponding GMSL rise is $0.71 \mathrm{~m}(0.51-0.92 \mathrm{~m}$, likely range) for $2081-$ 2100 and $0.84 \mathrm{~m}(0.61-1.10 \mathrm{~m}$, likely range) in $2100 "$ (IPCC, 2019). Furthermore, the average intensity of tropical cyclones, the proportion of category 4 and 5 tropical cyclones and the associated average precipitation rates are "projected to increase for a $2^{\circ} \mathrm{C}$ global temperature rise above any baseline period (medium confidence)" and "rising mean sea levels will contribute to higher extreme sea levels associated with tropical cyclones (very high confidence)" (italics as in the original).

Coastal areas are particularly vulnerable to the impacts of climate change. This is significant as coastal areas are home to a large proportion of the world's population; three-quarters of all large cities and 14 of the 17 largest cities lie on the coast and over 1.2 billion people globally live within $100 \mathrm{~km}$ of the coast in areas where the land height is less than $100 \mathrm{~m}$ above sea level (Small and Nichols, 2003). Rising sea levels, increased storminess and increases in extreme water levels will result in increased flooding, storm damage and erosion of coastal areas, which in turn pose risks to homes, buildings and other infrastructure, cause business and service interruptions, and have health and well-being effects. With regard to economic impact alone, a recent study by the European Union's Joint Research Centre (JRC) found that annual damages caused by coastal flooding in Europe, where one in three people live within $50 \mathrm{~km}$ of the coast, will see a significant increase to between $€ 93$ billion and $€ 961$ billion by 2100 because of climate change (Vousdoukas et al., 2018). The IPCC (2018) analysed the impact of global warming of $a 1.5^{\circ} \mathrm{C}$ and found that "projections of GMSL rise (relative to 1986-2005) suggest an indicative range of 0.26 to $0.77 \mathrm{~m}$ by 2100 for $1.5^{\circ} \mathrm{C}$ of global warming, $0.1 \mathrm{~m}(0.04-0.16 \mathrm{~m})$ less than for a global warming of $2^{\circ} \mathrm{C}$ (medium confidence)". The authors conclude that a "reduction of $0.1 \mathrm{~m}$ in GSLR implies that up to 10 million fewer people would be exposed to related risks, based on population in the year 2010 and assuming no adaptation (medium confidence)" (IPCC, 2018). The IPCC (2019) concluded that "coastal hazards will be exacerbated by an increase in the average intensity, magnitude of storm surge and precipitation rates of tropical cyclones".

The analysis of a large ensemble of high-resolution regional climate simulations show that temperature projections for Ireland are in line with global projections, with an expected increase in annual 2-m temperatures over Ireland of $1.3^{\circ} \mathrm{C}$ (RCP4.5 scenario) and $1.6^{\circ} \mathrm{C}$ (RCP8.5 scenario) by mid-century, with the strongest signals noted in the east (Nolan, 2015; O'Sullivan et al., 2015). Temperature increases are enhanced for the extremes; mid-century summer daytime and winter night-time temperatures are projected to increase by $0.7-2.6^{\circ} \mathrm{C}$ and $1.1-3.1^{\circ} \mathrm{C}$, respectively (Nolan, 2015; O'Sullivan et al., 2015). The number of frost and ice days is expected to decrease by approximately $50 \%$ by mid-century (Nolan, 2015). The rainfall climate is projected to become more variable, with an increase in extended dry periods during summer and an increase in the number of heavy rainfall events during autumn and winter (Nolan, 2015; Nolan et al., 2017). By mid-century, significant decreases in the mean wind speed and energy content of the wind are projected for the spring, summer and autumn months. Projected increases for winter were found to be statistically insignificant (Nolan et al., 2014; Nolan, 2015). Storms affecting Ireland are projected to decrease in frequency but increase in intensity, with increased risk of damage ${ }^{1}$ (Nolan, 2015). The National Adaptation Framework (DCCAE, 2018) estimated that the direct cost of flooding could rise from its current level of $€ 192$ million per annum to $€ 1.15$ billion if appropriate mitigation measures are not implemented.

1 It should be noted that the projections of storm tracks exhibit medium to high uncertainty, as reflected in a large spread (disagreement) between ensemble members. In addition, as extreme storms are rare events, a large ensemble is required for a robust statistical analysis of the projections. Future work will focus on analysing a larger ensemble of downscaled CMIP6 data (including the EC-Earth data analysed in the current report). 


\subsection{The Coupled Model Intercomparison Project}

Although the IPCC is regarded as the authoritative voice on climate change issues, it does not engage directly in climate research; its role is to assess the science based on the work of climate scientists through published peer-reviewed research. The Coupled Model Intercomparison Project (CMIP), an international group co-ordinating the running of simulations of the global climate system, has played a key role in supporting the IPCC in its previous assessment reports (e.g. Fifth Assessment Report - AR5) and is now fully engaged in its sixth phase (CMIP6) to support the IPCC with the next assessment report (Sixth Assessment Report - AR6), due to be released in 2021.

Since 1995, CMIP has co-ordinated climate model experiments involving multiple international modelling teams and provided a better understanding of past, present and future climate. The CMIP phase 5 (CMIP5) simulations have demonstrated the added value of enhanced resolution when compared with output from the CMIP phase 3 (CMIP3) project. There were significant improvements in the simulation of aspects of large-scale circulation, such as EI Niño Southern Oscillation (ENSO) (Shaffrey et al., 2009), tropical instability waves (Roberts et al., 2009), the Gulf Stream and its influence on the atmosphere (Chassignet and Marshall, 2008; Kuwano-Yoshida et al., 2010), the global water cycle (Demory et al., 2014), extratropical cyclones and storm tracks (Hodges et al., 2011) and Euro-Atlantic blocking (Jung et al., 2012). In addition, the increased resolution enables more realistic simulation of small-scale phenomena with potentially severe impacts, such as tropical cyclones (Zhao et al., 2009), tropical-extratropical interactions (Haarsma et al., 2013) and polar lows. The improved simulation of climate also results in better representation of extreme events, such as heat waves, droughts and floods. Studies have shown that, even at 50-km grid spacing, global climate models (GCMs) severely under-resolve tropical cyclones, resulting in a substantial truncation of the intensity spectrum of simulated storms (Zhao et al., 2009), and usually produce fewer events than the number observed (Camargo, 2013). The ability of CMIP5 models to simulate North Atlantic extratropical cyclones was assessed by Zappa et al. (2013). The authors found that "systematic biases affect the number and intensity of North Atlantic cyclones in CMIP5 models. In December, January and February (DJF), the North Atlantic storm track tends to be either too zonal or displaced southward, thus leading to too few and weak cyclones over the Norwegian Sea and too many cyclones in central Europe. In June, July and August (JJA), the position of the North Atlantic storm track is generally well captured but some CMIP5 models underestimate the total number of cyclones". Despite these biases, the representation of Northern Hemisphere storm tracks has improved from CMIP3 to CMIP5, with some CMIP5 models realistically representing both the number and the intensity of North Atlantic cyclones. In particular, some of the high-resolution atmospheric models tend to produce a better representation of the vertical tilt of the North Atlantic storm track and of the intensity of cyclones in DJF. This improvement in skill is expected to continue with the higher resolution CMIP6 simulations. This is particularly relevant for Ireland as improved skill in the simulation of CMIP6 storms will lead to sharper projections of North Atlantic storms, as simulated by regional climate models ( $\mathrm{RCMs}$ ), and a reduction in the uncertainty of these projections (see footnote 4). Sillmann et al. (2013) assessed the performance of CMIP5 GCMs in simulating climate extreme indices defined by the Expert Team on Climate Change Detection and Indices (ETCCDI), and compared it with that of the previous model generation (CMIP3). They found that, "for the precipitation indices, the intermodel uncertainty in the CMIP3 and CMIP5 ensembles is comparable, but the CMIP5 models tend to simulate more intense precipitation and fewer consecutive wet days than the CMIP3 models, and thus are closer to the observations". This improvement is partly attributed to the generally higher spatial resolution of CMIP5 models than that of CMIP3 models (the effect of increasing resolution on precipitation extremes has been discussed, for instance, in Wehner et al., 2010). Results indicate that, for the temperature indices, the performance of the CMIP3 and CMIP5 multi-model ensembles is similar with regard to their ensemble mean and median, but that the spread among CMIP3 models tends to be larger than among CMIP5 models, despite the larger number of models in the CMIP5 ensemble (Sillmann et al., 2013). Again, this improvement in skill is expected to continue with the higher resolution CMIP6 simulations. 
As part of the EC-Earth consortium, researchers at Met Éireann and the Irish Centre for High-End Computing (ICHEC) have implemented the EC-Earth model on various supercomputer platforms and have contributed to CMIP5 experiments, which formed an essential part of the IPCC AR5. The CMIP6 EC-Earth contributions (outlined in this report) will improve on the CMIP5 contributions and enhance the overall understanding of anthropogenic climate change on a global scale. The simulations will be included for assessment in the upcoming IPCC AR6 report.

\subsubsection{The Coupled Model Intercomparison Project phase 6}

The fundamental objective of CMIP is to improve the understanding of past, present and future climate change arising from natural variability or in response to changes in radiative forcing, principally linked to greenhouse gas emissions. The co-ordination feature of CMIP6 is very important as it enables the scientific community to focus more effectively on knowledge gaps in the understanding of the Earth's climate system, the themes of which are summarised under the Grand Science Challenges of the World Climate Research Programme (WCRP). In particular, CMIP6 will attempt to address the questions:

- How does the Earth system respond to forcing (e.g. from greenhouse gas emissions)?

- What are the origins and consequences of systematic climate model biases?

- How can we assess future climate changes given internal climate variability, predictability and uncertainties in emission scenarios?

More particularly, CMIP6 lays out a framework of climate simulations that supporting groups may choose to pursue. It includes the DECK (Diagnostic, Evaluation and Characterization of Klima) and historical simulations (1850 to near present), with the latter documenting the basic features of model performance across different phases of CMIP (Eyring et al., 2015).

An agreed ensemble of Model Intercomparison Projects (MIPs), 21 in total, provides further refinement to address specific science topics. Of relevance to the current report is the Scenario Model Intercomparison Project (ScenarioMIP), which provides multi-model climate projections based on alternative scenarios of future emissions and land use changes for at least the 21st century (Eyring et al., 2015). Others include the Coordinated Regional Downscaling Experiment (CORDEX) diagnostic MIP, which focuses on regional climate, and the Decadal Climate Prediction Project (DCPP) MIP, which will investigate the ability to skilfully predict climate variations from a year to a decade ahead.

Irish participation in CMIP6 comes through the EC-Earth climate modelling consortium (see sections 1.3 and 1.5). EC-Earth participated in CMIP5 with EC-Earth v2.3 and in CMIP6 with a model that includes biogeochemical cycles and atmospheric chemistry (see section 1.2).

Crucially, CMIP6 requires that data outputs comply with common standards and are made publicly available, with supporting documentation, for analysis by the science community through the Earth System Grid Federation (ESGF) (see section 1.5).

\subsection{EC-Earth Earth System Model}

EC-Earth is an IPCC-class Earth system model (ESM) developed by a European consortium of which ICHEC and Met Éireann are members. CMIP6 (v3.3.1.1) is based on a newer cycle of the European Centre for Medium-Range Weather Forecast (ECMWF) Integrated Forecast System (IFS) atmospheric model (c36r4), the Nucleus for European Modelling of the Ocean (NEMO) model (v3.6), the Louvain-la-Neuve sea ice model (LIM3), the Tracer Model version 5 (TM5) atmospheric composition model, the Lund-Potsdam-Jena General Ecosystem Simulator (LPJ-GUESS) vegetation model and the Pelagic Interactions Scheme for Carbon and Ecosystem Studies (PISCES) ocean biogeochemistry model. Coupling is provided by OASIS3-MCT (the Ocean Atmosphere Sea Ice Soil - OASIS - coupler interfaced with the Model Coupling Toolkit - MCT) (see Figure 1.1). EC-Earth is optimised for a standard horizontal resolution of T255 with 91 vertical layers for the atmosphere, and for 1 degree with 75 layers for the ocean. In addition, high-resolution configurations are available: 0.25 degrees and 75 layers in the ocean, and T511 $(\sim 39 \mathrm{~km})$ and T799 $(\sim 25 \mathrm{~km})$ in the atmosphere.

The atmosphere component of the EC-Earth system is based on the IFS cy36r4 model, which has been used 


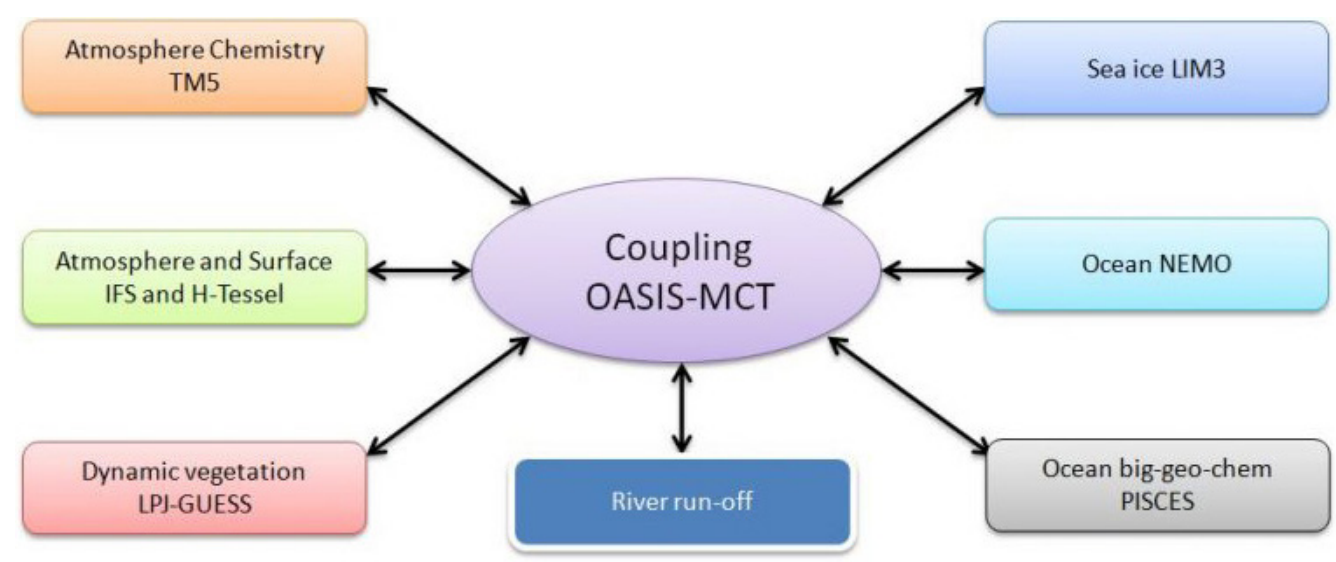

Figure 1.1. The CMIP6 EC-Earth model components.

operationally by the ECMWF for making seasonal predictions (Molteni et al., 2011). The IFS is a spectral model of the atmosphere with a semi-Lagrangian advection scheme. A number of updates have been necessary to transform the IFS into a physical atmosphere model suitable as a GCM or ESM. The IFS is jointly developed and maintained by the ECMWF, based in Reading, England, and MétéoFrance, based in Toulouse. The Hydrology-Tiled ECMWF Scheme for Surface Exchanges over Land (H-TESSEL) is used within the IFS for describing soil run-off and the evolution of soil, vegetation and snow over the continents at diverse spatial resolutions (Balsamo et al., 2009).

The ocean component of the EC-Earth model is NEMO (Madec and the NEMO team, 2008). For CMIP6 version of EC-Earth, NEMO3.6 is used, which includes the ocean model OPA (Ocean PArallelise) and LIM3 (Rousset et al., 2015). Ocean biogeochemical fluxes are represented with PISCES-v2 (Aumont et al., 2015).

The atmospheric composition model of EC-Earth is TM5 (Huijnen et al., 2010). It can be used for the interactive simulation of carbon dioxide $\left(\mathrm{CO}_{2}\right)$, methane $\left(\mathrm{CH}_{4}\right)$, ozone $\left(\mathrm{O}_{3}\right)$ and tropospheric aerosols.

The LPJ-GUESS dynamic vegetation and biogeochemistry model (Smith et al., 2014) is the terrestrial biosphere component of EC-Earth, simulating vegetation dynamics, land use and land management.

CMIP6 has a much increased set of model intercomparisons than previous generations. Figure 1.2 outlines the 21 CMIP6-approved MIPs. Of these, the EC-Earth community contributes to 18 using eight configurations of the EC-Earth model: EC-EARTH3 (the coupled AtmosphereOcean Ceneral Circulation Model - AOGCM; this configuration was used for the EC-Earth simulations in the current report), EC-EARTH3-HR (high resolution), EC-EARTH3-LR (low resolution), EC-EARTH3-CC (carbon cycle), EC-EARTH3-GrIS (Greenland ice sheet), EC-EARTH3-AerChem (air chemistry), EC-EARTH3-Veg (interactive vegetation) and EC-EARTH3-Veg-LR (low-resolution interactive vegetation).

\subsection{The SSP/RCP Scenario Matrix Framework $^{2}$}

The CMIP6 ScenarioMIP utilises a parallel process of combining future socioeconomic pathways with forcing pathways to assess climate change (Moss et al., 2010; Riahi et al., 2017). This process includes the RCPs, which cover the climate forcing dimension of different possible futures (van Vuuren et al., 2011), and served as the basis for the development of new climate change projections assessed in the IPCC AR5

2 Text and figures in section 1.3 are taken from Riahi et al. (2017) and https://www.carbonbrief.org/explainer-how-sharedsocioeconomic-pathways-explore-future-climate-change (accessed 11 February 2020). The article by Riahi et al. (2017) is an open access article distributed under the terms of the Creative Commons CC-BY license, which permits unrestricted use, distribution, and reproduction in any medium, provided the original work is properly cited. Material from https://www.carbonbrief.org/explainerhow-shared-socioeconomic-pathways-explore-future-climate-change can be reproduced unadapted under a Creative Commons Attribution-NonCommercial-NoDerivatives 4.0 International Public License. 


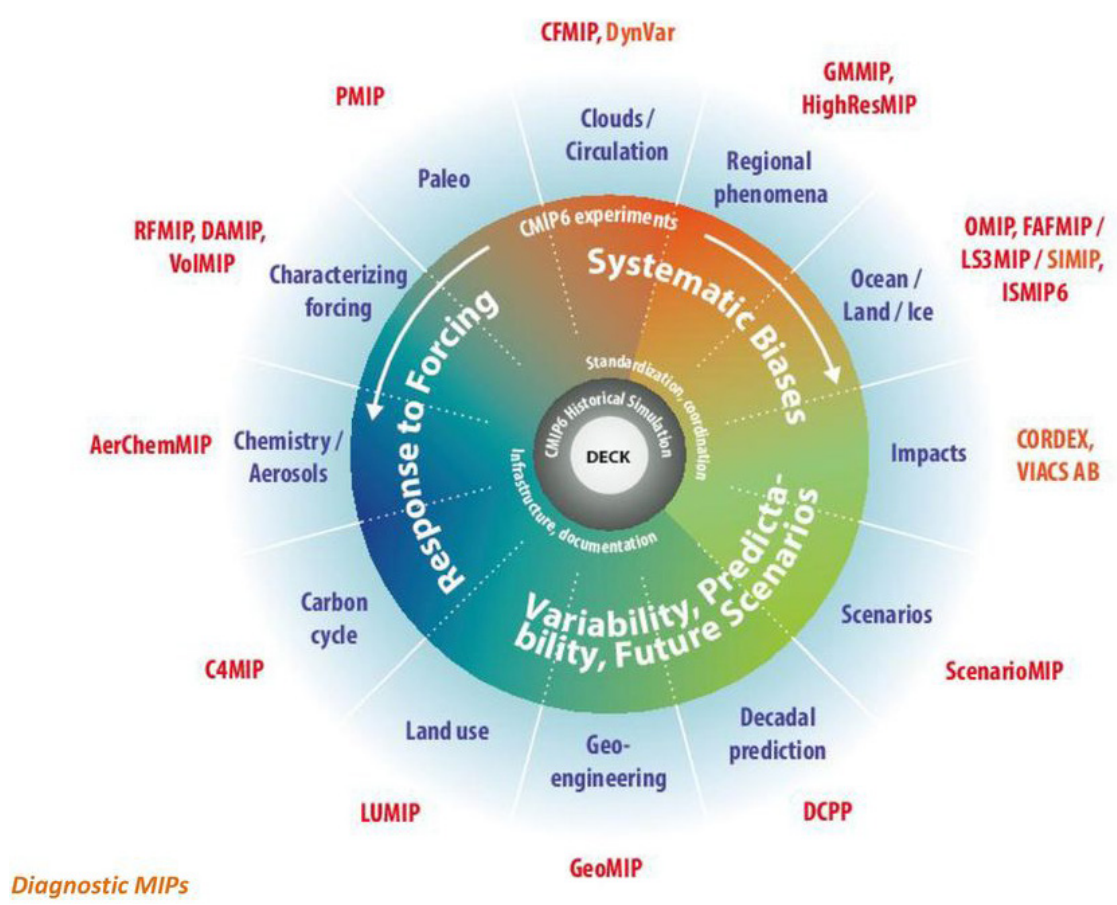

Figure 1.2. The 21 CMIP6-endorsed MIPs. Source: Eyring et al. (2016). This work is distributed under the Creative Commons Attribution 3.0 License.

(Taylor et al., 2012; IPCC, 2013b). The RCPs describe different levels of greenhouse gases and likely radiative forcings. Four pathways were developed, spanning a broad range of forcing in $2100(2.6,4.5$, 6.0 and $8.5 \mathrm{~W} / \mathrm{m}^{2}$ ), but purposefully did not include any socioeconomic "narratives" to go alongside them.

Other groups have focused on modelling how socioeconomic factors may change over the next century (Ebi et al., 2014, Kriegler et al., 2014, O’Neill et al., 2014, van Vuuren et al., 2014). These "shared socioeconomic pathways" (SSPs) look at five different ways in which the world might evolve in the absence of climate policy and how different levels of climate change mitigation could be achieved. The SSPS are based on five narratives describing alternative socioeconomic developments, including sustainable development, regional rivalry, inequality, fossil-fuelled development and middle-of-the-road development. The narrative for each of the five SSPs is described in Table 1.1.

The RCPs and SSPs were designed to be complementary. The RCPs set pathways for greenhouse gas concentrations and, effectively, the amount of warming that could occur by the end of the century, whereas the SSPs set the stage on which reductions in emissions will (or will not) be achieved. The new framework employed by the CMIP6
ScenarioMIP combines the SSPs and the RCPs in a scenario matrix architecture (see Figure 1.3).

\subsection{Ireland's Contributions to CMIP6}

The EC-Earth simulations described in this report comprise Ireland's contribution to CMIP6 and will be included for assessment in the United Nations IPCC AR6 report.

To date, CMIP6 participation is in the form of DECK CMIP contributions (historical) and ScenarioMIP contributions (Eyring et al., 2015). Specifically, the following CMIP6 EC-Earth contributions were run:

- five T255L91-ORCA1L75 AOGCM CMIP6 historical simulations, 1850-2014;

- 20 ScenarioMIP simulations 2015-2100 - five T255L91-ORCA1L75 AOGCM CMIP6 simulations for each ScenarioMIP "tier 1" SSP-RCP (SSP1-2.6, SSP2-4.5, SSP3-7.0 and SSP5-8.5).

The EC-Earth AOGCM configuration was employed. The atmosphere was simulated with $\sim 79-\mathrm{km}$ horizontal grid spacings (T255) and 91 vertical levels. The ocean was simulated with 1-degree horizontal resolution and 75 vertical levels. In total, five historical and 20 "tier 1" ScenarioMIP simulations were run. 
Table 1.1. Summary of SSP narratives

\begin{tabular}{|c|c|}
\hline SSP & Narrative \\
\hline \multirow[t]{2}{*}{ SSP1 } & "Sustainability - Taking the Green Road (Low challenges to mitigation and adaptation) \\
\hline & $\begin{array}{l}\text { The world shifts gradually, but pervasively, toward a more sustainable path, emphasising more inclusive development } \\
\text { that respects perceived environmental boundaries. Management of the global commons slowly improves, educational } \\
\text { and health investments accelerate the demographic transition, and the emphasis on economic growth shifts toward a } \\
\text { broader emphasis on human well-being. Driven by an increasing commitment to achieving development goals, inequality } \\
\text { is reduced both across and within countries. Consumption is oriented toward low material growth and lower resource and } \\
\text { energy intensity." }\end{array}$ \\
\hline \multirow[t]{2}{*}{ SSP2 } & "Middle of the Road (Medium challenges to mitigation and adaptation) \\
\hline & $\begin{array}{l}\text { The world follows a path in which social, economic, and technological trends do not shift markedly from historical patterns. } \\
\text { Development and income growth proceeds unevenly, with some countries making relatively good progress while others } \\
\text { fall short of expectations. Global and national institutions work toward but make slow progress in achieving sustainable } \\
\text { development goals. Environmental systems experience degradation, although there are some improvements and overall } \\
\text { the intensity of resource and energy use declines. Global population growth is moderate and levels off in the second half } \\
\text { of the century. Income inequality persists or improves only slowly and challenges to reducing vulnerability to societal and } \\
\text { environmental changes remain." }\end{array}$ \\
\hline \multirow[t]{2}{*}{ SSP3 } & "Regional Rivalry - A Rocky Road (High challenges to mitigation and adaptation) \\
\hline & $\begin{array}{l}\text { A resurgent nationalism, concerns about competitiveness and security, and regional conflicts push countries to increasingly } \\
\text { focus on domestic or, at most, regional issues. Policies shift over time to become increasingly oriented toward national } \\
\text { and regional security issues. Countries focus on achieving energy and food security goals within their own regions at the } \\
\text { expense of broader-based development. Investments in education and technological development decline. Economic } \\
\text { development is slow, consumption is material-intensive, and inequalities persist or worsen over time. Population growth is } \\
\text { low in industrialised and high in developing countries. A low international priority for addressing environmental concerns } \\
\text { leads to strong environmental degradation in some regions." }\end{array}$ \\
\hline \multirow[t]{2}{*}{ SSP4 } & "Inequality - A Road Divided (Low challenges to mitigation, high challenges to adaptation) \\
\hline & $\begin{array}{l}\text { Highly unequal investments in human capital, combined with increasing disparities in economic opportunity and political } \\
\text { power, lead to increasing inequalities and stratification both across and within countries. Over time, a gap widens between } \\
\text { an internationally-connected society that contributes to knowledge- and capital-intensive sectors of the global economy, } \\
\text { and a fragmented collection of lower-income, poorly educated societies that work in a labor intensive, low-tech economy. } \\
\text { Social cohesion degrades and conflict and unrest become increasingly common. Technology development is high in } \\
\text { the high-tech economy and sectors. The globally connected energy sector diversifies, with investments in both carbon- } \\
\text { intensive fuels like coal and unconventional oil, but also low-carbon energy sources. Environmental policies focus on local } \\
\text { issues around middle and high income areas." }\end{array}$ \\
\hline \multirow[t]{2}{*}{ SSP5 } & "Fossil-fueled Development - Taking the Highway (High challenges to mitigation, low challenges to adaptation) \\
\hline & $\begin{array}{l}\text { This world places increasing faith in competitive markets, innovation and participatory societies to produce rapid } \\
\text { technological progress and development of human capital as the path to sustainable development. Global markets are } \\
\text { increasingly integrated. There are also strong investments in health, education, and institutions to enhance human and } \\
\text { social capital. At the same time, the push for economic and social development is coupled with the exploitation of abundant } \\
\text { fossil fuel resources and the adoption of resource and energy intensive lifestyles around the world. All these factors lead } \\
\text { to rapid growth of the global economy, while global population peaks and declines in the } 21 \text { st century. Local environmental } \\
\text { problems like air pollution are successfully managed. There is faith in the ability to effectively manage social and ecological } \\
\text { systems, including by geo-engineering if necessary." }\end{array}$ \\
\hline
\end{tabular}

Source: Riahi et al. (2017).

The ensemble members (historical and ScenarioMIP) are named r6i1p1f1, r9i1p1f1, r11i1p1f1, r13i1p1f1 and r15i1p1f1 (see section 1.4.1 for an overview of the ensemble naming convention). For ensemble member r11i1p1f1, all model levels are archived, allowing for regional downscaling using RCMs and participation in the CMIP6 CORDEX MIP. The choice of this particular ensemble member for downscaling was purely practical; sufficient storage resources for archiving of model-level data were available on the ICHEC systems when the r11i1p1f1 simulation commenced.
The raw EC-Earth datasets were post-processed ("cmorised") and hosted on the ICHEC ESGF node (see section 1.5). In total, CMIP6 runs comprise over 2500 years of simulated data, over 500,000 files and 500 TB of data (1 PB when including the backup of datasets on the ICHEC system). Table 1.2 provides an overview of variables archived at 3-hour and/or 6-hour temporal resolution. Additional variables, archived at daily and/or monthly temporal resolution, are listed in Box 1.1. Numerous fixed variables are also archived, such as land area fraction, surface altitude, sea floor depth and thickness of soil layers. 


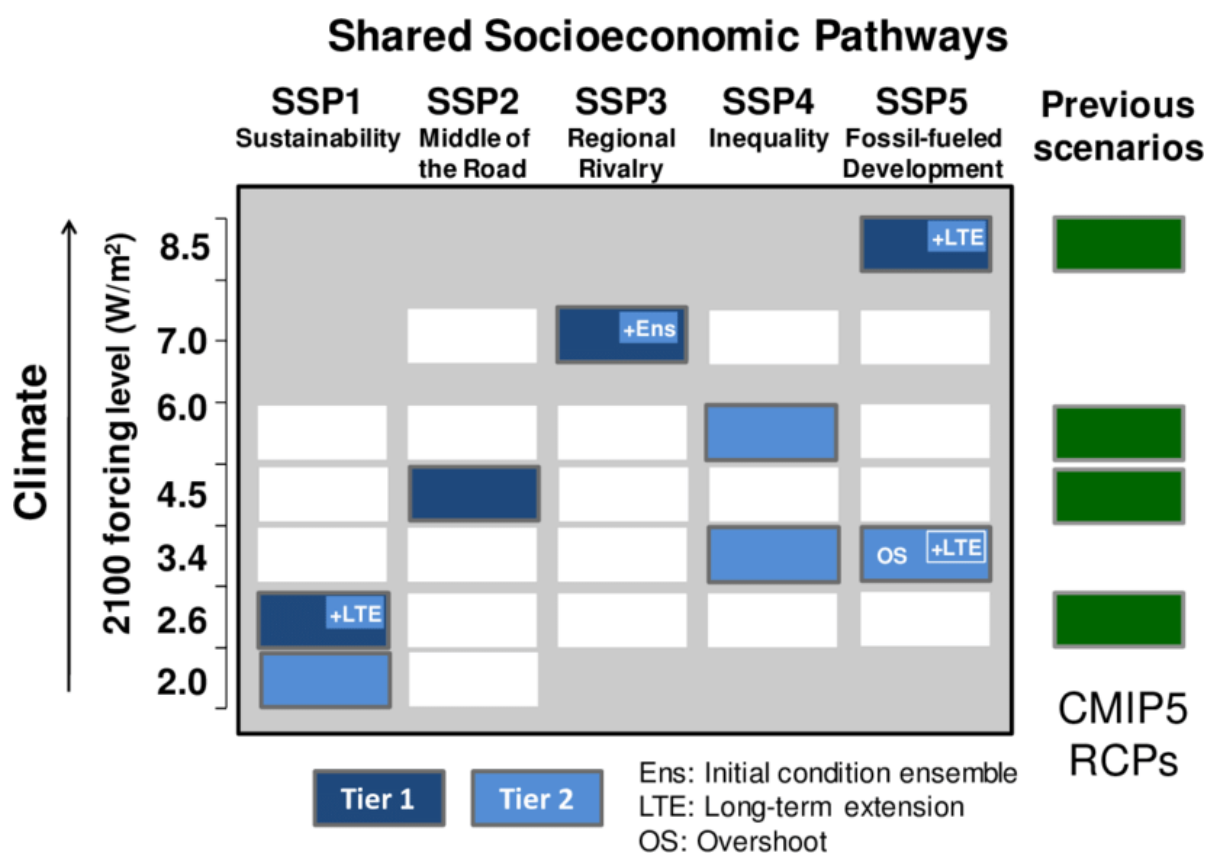

Figure 1.3. SSP-RCP scenario matrix illustrating ScenarioMIP simulations. Each cell in the matrix indicates a combination of a socioeconomic development pathway (i.e. an SSP) and a climate outcome based on a particular forcing pathway that current integrated assessment model runs have shown to be feasible (Riahi et al., 2017). Dark blue cells indicate scenarios that will serve as the basis for climate model projections in tier 1 of ScenarioMIP; light blue cells indicate scenarios in Tier 2. Source: O'Neill et al. (2016). This work is distributed under the Creative Commons Attribution 3.0 License.

Current/future CMIP6 work will involve participation in the following MIPs: CORDEX regional downscaling, high-resolution (T511L91-ORCA025L75) HighResMIP and additional historical/ScenarioMIP simulations using the EC-Earth-Veg interactive vegetation configuration. Currently, the authors are running EC-Earth-Veg simulations comprising:

- two T255L91-ORCA1L75 EC-Earth-Veg historical simulations, 1850-2014;

- eight ScenarioMIP simulations, 2015-2100 - two T255L91-ORCA1L75 EC-Earth-Veg simulations for each ScenarioMIP "tier 1" SSP-RCP (SSP1-2.6, SSP2-4.5, SSP3-7.0 \& SSP5-8.5).

These EC-Earth-Veg simulations are complete and will be hosted on the ICHEC ESGF node in March 2020.

\subsubsection{CMIP6 ensemble member naming convention}

For a given experiment, the realisation_index, initialisation_index, physics_index and forcing_index are used to uniquely identify each simulation of an ensemble of runs contributed by a single model.
These indices are defined as follows (Taylor et al., 2018):

- realisation_index=an integer $(\geq 1)$ distinguishing among members of an ensemble of simulations that differ only in their initial conditions (e.g. initialised from different points in a control run). Also, each so-called RCP (future scenario) simulation should normally be assigned the same realisation integer as the historical run from which it was initiated. This will allow users to easily splice together the appropriate historical and future runs.

- initialisation_index=an integer $(\geq 1)$, which should be assigned a value of 1 except to distinguish simulations performed under the same conditions but with different initialisation procedures. In CMIP6 this index should invariably be assigned the value "1" except for some hindcast and forecast experiments called for by the DCPP activity. The initialisation_index can be used either to distinguish between different algorithms used to impose initial conditions on a forecast or to distinguish between different observational datasets used to initialise a forecast. 
Table 1.2. Description of EC-Earth variables hosted on the ICHEC ESGF node with at least 6-hour temporal resolution

\begin{tabular}{|c|c|c|c|c|}
\hline Variable & Units & Description & $\begin{array}{l}\text { Variable } \\
\text { name }\end{array}$ & $\begin{array}{l}\text { Time } \\
\text { interval } \\
\text { archived }\end{array}$ \\
\hline $\begin{array}{l}\text { Total cloud cover } \\
\text { percentage }\end{array}$ & $\%$ & $\begin{array}{l}\text { Total cloud area fraction (reported as a percentage) for the } \\
\text { whole atmospheric column, as seen from the surface or the top } \\
\text { of the atmosphere. Includes both large-scale and convective } \\
\text { cloud }\end{array}$ & clt & $\begin{array}{l}3 \text { hours, day, } \\
\text { month }\end{array}$ \\
\hline $\begin{array}{l}\text { Surface upward latent } \\
\text { heat flux }\end{array}$ & $\mathrm{W} / \mathrm{m}^{2}$ & $\begin{array}{l}\text { The surface called "surface" means the lower boundary of } \\
\text { the atmosphere. "Upward" indicates a vector component, } \\
\text { which is positive when directed upward (negative downward). } \\
\text { The surface latent heat flux is the exchange of heat between } \\
\text { the surface and the air on account of evaporation (including } \\
\text { sublimation) }\end{array}$ & hfls & $\begin{array}{l}3 \text { hour, day, } \\
\text { month }\end{array}$ \\
\hline $\begin{array}{l}\text { Surface upward sensible } \\
\text { heat flux }\end{array}$ & $\mathrm{W} / \mathrm{m}^{2}$ & $\begin{array}{l}\text { The surface sensible heat flux, also called turbulent heat flux, } \\
\text { is the exchange of heat between the surface and the air by } \\
\text { motion of air }\end{array}$ & hfss & $\begin{array}{l}3 \text { hour, day, } \\
\text { month }\end{array}$ \\
\hline $\begin{array}{l}\text { Near-surface specific } \\
\text { humidity }\end{array}$ & 1 & Near-surface (usually 2-m) specific humidity & huss & $\begin{array}{l}3 \text { hours, day, } \\
\text { month }\end{array}$ \\
\hline Total run-off & $\mathrm{kg} / \mathrm{m}^{2} / \mathrm{s}$ & $\begin{array}{l}\text { The total run-off (including drainage through the base of the } \\
\text { soil model) per unit area leaving the land portion of the grid cell }\end{array}$ & mrro & $\begin{array}{l}3 \text { hours, day, } \\
\text { month }\end{array}$ \\
\hline $\begin{array}{l}\text { Moisture in upper portion } \\
\text { of soil column }\end{array}$ & $\mathrm{kg} / \mathrm{m}^{2}$ & $\begin{array}{l}\text { The mass of water in all phases in the upper } 10 \mathrm{~cm} \text { of the soil } \\
\text { layer }\end{array}$ & mrsos & $\begin{array}{l}3 \text { hours, day, } \\
\text { month }\end{array}$ \\
\hline Precipitation & $\mathrm{kg} / \mathrm{m}^{2} / \mathrm{s}$ & Precipitation; includes both liquid and solid phases & $\mathrm{pr}$ & $\begin{array}{l}3 \text { hours, day, } \\
\text { month }\end{array}$ \\
\hline Convective precipitation & $\mathrm{kg} / \mathrm{m}^{2} / \mathrm{s}$ & $\begin{array}{l}\text { Convective precipitation at the surface; includes both liquid and } \\
\text { solid phases }\end{array}$ & prc & $\begin{array}{l}3 \text { hours, day, } \\
\text { month }\end{array}$ \\
\hline Snowfall flux & $\mathrm{Kg} / \mathrm{m}^{2} / \mathrm{s}$ & $\begin{array}{l}\text { At the surface; includes precipitation of all forms of water in the } \\
\text { solid phase; 3-hour mean snowfall flux }\end{array}$ & prsn & $\begin{array}{l}3 \text { hours, day, } \\
\text { month }\end{array}$ \\
\hline Surface air pressure & $\mathrm{Pa}$ & $\begin{array}{l}\text { Surface pressure; two-dimensional field to calculate the three- } \\
\text { dimensional pressure field from hybrid co-ordinates }\end{array}$ & ps & $\begin{array}{l}3 \text { hours, } \\
6 \text { hours, day, } \\
\text { month }\end{array}$ \\
\hline $\begin{array}{l}\text { Surface downwelling } \\
\text { longwave radiation }\end{array}$ & $\mathrm{W} / \mathrm{m}^{2}$ & $\begin{array}{l}\text { The surface called "surface" means the lower boundary of } \\
\text { the atmosphere. "Longwave" means longwave radiation. } \\
\text { Downwelling radiation is radiation from above; it does not mean } \\
\text { "net downward" }\end{array}$ & rlds & $\begin{array}{l}3 \text { hours, day, } \\
\text { month }\end{array}$ \\
\hline $\begin{array}{l}\text { Surface downwelling } \\
\text { clear-sky longwave } \\
\text { radiation }\end{array}$ & $\mathrm{W} / \mathrm{m}^{2}$ & $\begin{array}{l}\text { The surface called "surface" means the lower boundary of } \\
\text { the atmosphere. "Longwave" means longwave radiation. } \\
\text { Downwelling radiation is radiation from above; it does not mean } \\
\text { "net downward" }\end{array}$ & rldscs & $\begin{array}{l}3 \text { hours, } \\
\text { month }\end{array}$ \\
\hline $\begin{array}{l}\text { Surface upwelling } \\
\text { longwave radiation }\end{array}$ & $\mathrm{W} / \mathrm{m}^{2}$ & $\begin{array}{l}\text { The surface called "surface" means the lower boundary of the } \\
\text { atmosphere. "Longwave" means longwave radiation. Upwelling } \\
\text { radiation is radiation from below; it does not mean "net upward" }\end{array}$ & rlus & $\begin{array}{l}3 \text { hours, day, } \\
\text { month }\end{array}$ \\
\hline $\begin{array}{l}\text { Surface downwelling } \\
\text { shortwave radiation }\end{array}$ & $\mathrm{W} / \mathrm{m}^{2}$ & Surface solar irradiance for ultraviolet radiation calculations & rsds & $\begin{array}{l}3 \text { hours, day, } \\
\text { month }\end{array}$ \\
\hline $\begin{array}{l}\text { Surface downwelling } \\
\text { clear-sky shortwave } \\
\text { radiation }\end{array}$ & $\mathrm{W} / \mathrm{m}^{2}$ & $\begin{array}{l}\text { Surface solar clear-sky irradiance for ultraviolet radiation } \\
\text { calculations }\end{array}$ & rsdscs & $\begin{array}{l}3 \text { hours, } \\
\text { month }\end{array}$ \\
\hline $\begin{array}{l}\text { Surface upwelling } \\
\text { shortwave radiation }\end{array}$ & $\mathrm{W} / \mathrm{m}^{2}$ & $\begin{array}{l}\text { The surface called "surface" means the lower boundary of } \\
\text { the atmosphere. "Shortwave" means shortwave radiation. } \\
\text { Upwelling radiation is radiation from below; it does not mean } \\
\text { "net upward" }\end{array}$ & rsus & $\begin{array}{l}3 \text { hours, day, } \\
\text { month }\end{array}$ \\
\hline $\begin{array}{l}\text { Surface upwelling clear- } \\
\text { sky shortwave radiation }\end{array}$ & $\mathrm{W} / \mathrm{m}^{2}$ & $\begin{array}{l}\text { The surface called "surface" means the lower boundary of } \\
\text { the atmosphere. "Shortwave" means shortwave radiation. } \\
\text { Upwelling radiation is radiation from below; it does not mean } \\
\text { "net upward" }\end{array}$ & rsuscs & $\begin{array}{l}3 \text { hours, } \\
\text { month }\end{array}$ \\
\hline $\begin{array}{l}\text { Near-surface air } \\
\text { temperature }\end{array}$ & $\mathrm{K}$ & Near-surface (usually $2 \mathrm{~m}$ ) air temperature & tas & $\begin{array}{l}3 \text { hours, day, } \\
\text { month }\end{array}$ \\
\hline $\begin{array}{l}\text { Surface temperature } \\
\text { where land or sea Ice }\end{array}$ & $\mathrm{K}$ & Surface temperature of all surfaces except the open ocean & tslsi & 3 hours, day \\
\hline
\end{tabular}


Table 1.2. Continued

\begin{tabular}{|c|c|c|c|c|}
\hline Variable & Units & Description & $\begin{array}{l}\text { Variable } \\
\text { name }\end{array}$ & $\begin{array}{l}\text { Time } \\
\text { interval } \\
\text { archived }\end{array}$ \\
\hline $\begin{array}{l}\text { Eastward component of } \\
\text { the } 10-\mathrm{m} \text { wind }\end{array}$ & $\mathrm{m} / \mathrm{s}$ & Sampled synoptically & uas & $\begin{array}{l}3 \text { hours, day, } \\
\text { month }\end{array}$ \\
\hline $\begin{array}{l}\text { Northward component of } \\
\text { the } 10-\mathrm{m} \text { wind }\end{array}$ & $\mathrm{m} / \mathrm{s}$ & Sampled synoptically & vas & $\begin{array}{l}3 \text { hours, day, } \\
\text { month }\end{array}$ \\
\hline Sea surface temperature & ${ }^{\circ} \mathrm{C}$ & $\begin{array}{l}\text { Temperature of the upper boundary of the liquid ocean, } \\
\text { including temperatures below the sea ice and floating ice } \\
\text { shelves }\end{array}$ & tos & $\begin{array}{l}3 \text { hours, day, } \\
\text { month }\end{array}$ \\
\hline Surface temperature & $\mathrm{K}$ & Surface/skin temperature & ts & $\begin{array}{l}3 \text { hours, } \\
\text { month }\end{array}$ \\
\hline $\begin{array}{l}\text { Near-surface relative } \\
\text { humidity }\end{array}$ & $\%$ & $\begin{array}{l}\text { The relative humidity with respect to liquid water for } \mathrm{T}>0^{\circ} \mathrm{C} \text {, } \\
\text { and with respect to ice for } \mathrm{T}<0^{\circ} \mathrm{C}\end{array}$ & hurs & $\begin{array}{l}6 \text { hours, day, } \\
\text { month }\end{array}$ \\
\hline $\begin{array}{l}\text { Relative vorticity at } \\
850 \mathrm{hPa}\end{array}$ & /s & The upward component of the vorticity vector & rv850 & 6 hours \\
\hline Eastward wind at $100 \mathrm{~m}$ & $\mathrm{~m} / \mathrm{s}$ & Zonal wind at $100-\mathrm{m}$ height & ua100m & 6 hours \\
\hline Northward wind at $100 \mathrm{~m}$ & $\mathrm{~m} / \mathrm{s}$ & Meridional wind at $100-\mathrm{m}$ height & va100m & 6 hours \\
\hline $\begin{array}{l}\text { Maximum } 10-\mathrm{m} \text { wind } \\
\text { speed of gust }\end{array}$ & $\mathrm{m} / \mathrm{s}$ & Wind speed gust maximum at $10 \mathrm{~m}$ above the surface & wsgmax10m & 6 hours \\
\hline Sea level pressure & $\mathrm{Pa}$ & Sea level pressure & psl & $\begin{array}{l}6 \text { hours, day, } \\
\text { month }\end{array}$ \\
\hline Air temperature & $\mathrm{K}$ & Air temperature on pressure levels & ta & $\begin{array}{l}6 \text { hours, day, } \\
\text { month }\end{array}$ \\
\hline Eastward wind & $\mathrm{m} / \mathrm{s}$ & Zonal wind (positive in a eastward direction) on pressure levels & ua & $\begin{array}{l}6 \text { hours, day, } \\
\text { month }\end{array}$ \\
\hline Northward wind & $\mathrm{m} / \mathrm{s}$ & $\begin{array}{l}\text { Meridional wind (positive in a northward direction) on pressure } \\
\text { levels }\end{array}$ & va & $\begin{array}{l}6 \text { hours, day, } \\
\text { month }\end{array}$ \\
\hline Geopotential height & $\mathrm{m}$ & $\begin{array}{l}\text { The geopotential divided by the standard acceleration due to } \\
\text { gravity (on pressure tropospheric levels) }\end{array}$ & $\mathrm{zg}$ & $\begin{array}{l}6 \text { hours, day, } \\
\text { month }\end{array}$ \\
\hline $\begin{array}{l}\text { Geopotential height at } \\
500 \mathrm{hPa}\end{array}$ & $\mathrm{m}$ & Geopotential height on the $500-\mathrm{hPa}$ surface & zg500 & 6 hours, day \\
\hline Temperature of soil & $\mathrm{K}$ & $\begin{array}{l}\text { Temperature of the soil. Reported as missing for grid cells with } \\
\text { no land }\end{array}$ & tsl & $\begin{array}{l}6 \text { hours, } \\
\text { month }\end{array}$ \\
\hline
\end{tabular}

\section{Box 1.1. List of EC-Earth variables hosted on the ICHEC ESGF node with daily and monthly temporal resolutions}

\section{Daily variables (52 in total)}

clt, hfls, hfss, hur, hurs, hursmax, hursmin, hus, huss, mrro, mrso, mrsos, omldamax, pr, prc, prsn, psl, rlds, rlus, rlut, rsds, rsus, sfcWind, sfcWindmax, siconc, siconca, sisnthick, sispeed, sitemptop, sithick, sitimefrac, siu, siv, snc, snw, ta, ta850, tas, tasmax, tasmin, tauu, tauv, tos, tossq, ts/si, ua, uas, va, vas, wap, zg and zg500

\section{Monthly variables (147 in total)}

bigthetao, bigthetaoga, clivi, clt, clwvi, evs, evspsbl, ficeberg, friver, fsitherm, hfbasin, hfbasinpmadv, hfds, hfdsn, hfevapds, hfgeou, hfibthermds, hfls, hfrainds, hfss, hfx, hfy, htovgyre, htovovrt, hur, hurs, hus, huss, Iwsnl, masscello, masso, mlotst, mlotstmax, mlotstmin, mlotstsq, mrfso, mrro, ros, mrso, mrsol, mrsos, msftbarot, ps, psl, pso, rlds, rldscs, rls, rlus, rlut, rlutcs, rsdo, rsds, rsdscs, rsdt, rsntds, rss, rsus, rsuscs, rsut, rsutcs, rtmt, sbl, sfcWind, sfcWindmax, sfdsi, siage, sicompstren, siconc, siconca, sidivvel, sidmassevapsubl, sidmassgrowthbot, sidmassgrowthwat, sidmassmeltbot, sidmassmelttop, sidmasssi, sidmasstranx, sidmasstrany, siflswdtop, sihc, simass, sisali, sisnmass, sisnthick, sispeed, sitemptop, sithick, sitimefrac, siu, siv, sivol, sltovgyre, sltovovrt, snc, snd, sndmassmelt, sndmasssnf, snm, snw, so, sob, soga, sos, sosga, t20d, ta, tas, tasmax, tasmin, tauu, tauuo, tauv, tauvo, thetao, thetaoga, thetaot, thetaot2000, thetaot300, thetaot700, thkcello, tob, tos, tosga, tossq, ts, tsl, tsn, ua, uas, umo, uo, va, vas, vmo, vo, volo, wap, wfo, wfonocorr, wmo, wo, zg, zhalfo, zos, zossq and zostoga

For a full description of the variables see https://earthsystemcog.org/projects/wip/CMIP6DataRequest (accessed 11 February 2020). 
- physics_index =an integer $(\geq 1)$ identifying the physics version used by the model. In the usual case of a single physics version of a model, this argument should normally be assigned the value 1 , but it is essential that a consistent assignment of physics_index be used across all simulations performed by a particular model. Use of "physics_index" is reserved for closely-related model versions (e.g., as in a "perturbed physics" ensemble) or for the same model run with slightly different parameterisations (e.g., of cloud physics). Model versions that are substantially different from one another should be given a different "source_ id" (rather than simply assigning a different value of the physics_index).

- forcing_index=an integer $(\geq 1)$ used to distinguish runs conforming to the protocol of a single CMIP6 experiment, but with different variants of forcing applied. One can, for example, distinguish between two historical simulations, one forced with the CMIP6-recommended forcing data sets and another forced by a different dataset, which might yield information about how forcing uncertainty affects the simulation.

For example, the r6i1p1f1 simulation of the current research is the sixth EC-Earth consortium ensemble member (r6) and uses standard initialisation procedures (i1) and standard EC-Earth physics ( $p 1$ ) and CMIP6-recommended forcings (f1). The choice of ensemble members of the current project (i.e. r6, r9, r11, r13 and r15) has no specific research significance; initial ensemble members were assigned to the consortium members by alphabetical order of institute.

\subsubsection{Implementation of EC-Earth on ICHEC and ECMWF supercomputing systems}

The EC-Earth CMIP6 simulations were run on both ICHEC "kay" and ECMWF supercomputing systems. EC-Earth passed the "reproducibility experiments" and demonstrated that the system is stable and that the results are robust, regardless of high-performance computing (HPC) platform used. These experiments involved running hundreds of years of simulation of both IFS-only and AOGCM configurations on (1) ECMWF cca with intel compilers, (2) ECMWF cca with cray compilers and (3) ICHEC kay with intel compilers.
The results of the reproducibility tests demonstrated the following:

- Using the cray compilers on the ECMWF system is significantly more computationally efficient. However, the cray reproducibility tests did not pass and therefore the EC-Earth consortium was advised to use the intel compilers in place of cray compliers on the ECMWF system. The EC-Earth reproducibility protocol was designed by the EC-Earth community (Massonnet et al., 2019) to assess the reproducibility of the EC-Earth model system. This protocol is based on a statistical comparison of standard climate metrics derived from multi-ensembles of multi-decadal control integrations executed in different HPC environments.

- The ICHEC kay machine (using intel compilers) passed the reproducibility tests and the runs were found to be computationally efficient. The results informed the decision to run the majority of the CMIP6 runs on the ICHEC machine.

- The ELPiN (Exclude Land Processes in NEMO) software greatly improved the efficiency of EC-Earth.

EC-Earth was found to run relatively slowly on the ECMWF system and so a careful scale testing was completed to determine the optimal configuration. Table 1.3 presents scaling statistics for the EC-Earth AOGCM on the ECMWF cca machine using intel compilers. The simulated time was 1 month. Three "forking" strategies were considered: no forking (not shown), all nodes shared (ShareAll) and dedicated nodes for IFS with other components sharing nodes (not shown). rnf_numproc (river run-off module) was set to 1 in all cases and the ELPiN land removal tool was implemented. The configuration highlighted in blue was found to be optimal and was implemented for the CMIP6 production runs. Table 1.4 presents similar scaling results for the ICHEC kay machine and demonstrates that EC-Earth runs approximately twice as fast at ICHEC compared with the ECMWF.

\subsection{ESGF: The Earth System Grid Federation}

The ESGF (Cinquini et al., 2014) is a peer-to-peer collaborative system that develops, deploys and maintains software infrastructure for the management, 
Table 1.3. Scaling results for a 1-month EC-Earth simulation on ECMWF/cca using the intel compilers ${ }^{a}$

$\begin{array}{llllllll}\text { Fork } & \text { No. of IFS cores } & \begin{array}{l}\text { No. of NEMO } \\ \text { cores }\end{array} & \begin{array}{l}\text { No. of } \\ \text { nodes }\end{array} & \begin{array}{l}\text { Time } \\ \text { (mm:ss) }\end{array} & \text { SBUs } & \text { SYPD } & \text { CHPSY } \\ \text { ShareAll } & 222 & 136 & 10 & 18: 08 & 1795.2433 & 6.74 & 1281 \\ \text { ShareAll } & 240 & 118 & 10 & 19: 54 & 1951.7020 & 6.14 & 1405 \\ \text { ShareAll } & 210 & 148 & 10 & 17: 57 & 1772.6616 & 6.81 & 1268 \\ \text { ShareAll } & 209(\text { xios }=2) & 148 & 10 & 17: 13 & 1717.8204 & 7.10 & 1216 \\ \text { ShareAll } & 208(\text { xios }=3) & 148 & 10 & 19: 04 & 1871.0532 & 6.41 & 1346 \\ \text { ShareAll } & 207(\text { xios }=4) & 148 & 10 & 17: 11 & 1737.1761 & 7.11 & 1213 \\ \text { ShareAll } & 200 & 158 & 10 & 18: 37 & 1833.9547 & 6.56 & 1315 \\ \text { ShareAll } & 246 & 148 & 11 & 16: 23 & 1777.8231 & 7.46 & 1273 \\ \text { ShareAll } & 246 & 148 & 11 & 16: 10 & 1752.9833 & 7.56 & 1256 \\ \text { ShareAll } & 246 & 148 & 11 & 17: 30 & 1932.1850 & 6.98 & 1359 \\ \text { ShareAll } & 245(\text { xios=2) } & 148 & 11 & 15: 16 & 1667.8181 & 8.01 & 1186 \\ \text { ShareAll } & 245(\text { xios }=2) & 148 & 11 & 16: 52 & 1818.6314 & 7.25 & 1310 \\ \text { ShareAll } & 245(\text { xios }=2) & 148 & 11 & 16: 08 & 1749.4347 & 7.58 & 1253 \\ \text { ShareAll } & 243(\text { xios }=4) & 148 & 11 & 18: 07 & 1996.0589 & 6.75 & 1407 \\ \text { ShareAll } & 265(\text { xios }=2) & 165 & 12 & 16: 47 & 2074.9335 & 7.28 & 1422 \\ \text { ShareAll } & 265 & 165 & 12 & 16: 29 & 1945.5017 & 7.41 & 1397 \\ \text { ShareAll } & 282 & 148 & 12 & 16: 42 & 1989.7683 & 7.32 & 1415\end{array}$

aThe configuration highlighted in blue (repeated for confirmation of results) was found to be the most efficient and was used for the CMIP6 EC-Earth production runs. The system billing unit (SBU) is used for accounting of computing resources on the ECMWF machines; the SYPD metric is the number of simulated years per day; and CHPSY is a measure of the core hours per simulated year.

dissemination and analysis of model output and observational data (Observatons for Model Intercomparison Project or Obs4MIPS; Ferraro et al., 2015). It is an international effort of climate centres with a mission to support CMIP6 and future assessments, support the dissemination of data and improve the automated processing of the data. Model simulations, satellite observations and reanalysis products are all being served from the ESGF Peer-toPeer (P2P) distributed data archive.

ESGF P2P is a component architecture expressly designed to handle large-scale data management for worldwide distribution. The architecture was developed by a team of computer and climate scientists as an operational system for serving climate data from multiple locations and sources.

An ESGF node consists of a web front-end, allowing users to search for data, along with a data node and, optionally, an identity provider, an index and compute components. In current releases (available at https:// github.com/ESGF), the ESGF front-end has been rewritten to use CoG, a collaborative environment allowing the hosting of multiple projects, providing links not just to the data but also to project files and various wiki resources. It now also interoperates directly with ES-DOC (n.d.), to provide automated documentation for ESMs using the Common Information Model (CIM).

\subsubsection{Implementation of the ICHEC ESGF node}

At ICHEC, we use esgf.ichec.ie to host the CMIP6 datasets. In addition, the ICHEC ESGF node hosts the national CMIP5 EC-Earth contributions, along with EC-Earth output from the PMIP3 experiment at the Royal Netherlands Meteorological Institute (KNMI), CORDEX downscaling from Maynooth University (Fealy et al., 2018) and "HiReslreland" high-resolution $(\sim 4 \mathrm{~km})$ regional downscaled CMIP5 projections data from ICHEC/Environmental Protection Agency (Nolan, 2015).

ICHEC rebuilt the ESGF node in 2019 on a virtual machine running CentOS 7.6; the virtual machine host is directly connected to the storage system, providing $10 \mathrm{Gbit} / \mathrm{s}$ throughput to the underlying storage hardware. The underlying storage system (iRODS, https://www.irods.org/) was reinstalled, with iRODS software upgraded to client 4.1.11. We use the iRODS 
Table 1.4. Scaling results for a 1-month EC-Earth simulation on the ICHEC system using the intel compilers $^{\mathrm{a}}$

\begin{tabular}{|c|c|c|c|c|c|c|}
\hline Elphin & Nodes & $\begin{array}{l}\text { No. of IFS } \\
\text { cores }\end{array}$ & $\begin{array}{l}\text { No. of NEMO } \\
\text { cores }\end{array}$ & xios & Time (mm:ss) & SYPD \\
\hline No (yes) & 6 & 120 & 118 & 1 & $17: 12(15: 32)$ & $7.11(7.87)$ \\
\hline No (yes) & 7 & 160 & 118 & 1 & $16: 00(13: 52)$ & $7.64(8.81)$ \\
\hline No (yes) & 8 & 200 & 118 & 1 & $15: 10(13: 14)$ & $8.06(9.24)$ \\
\hline No (yes) & 9 & 240 & 118 & 1 & $14: 44(12: 47)$ & $8.30(9.56)$ \\
\hline No (yes) & 10 & 280 & 118 & 1 & $14: 47(12: 22)$ & $8.27(9.88)$ \\
\hline No (yes) & 11 & 320 & 118 & 1 & $14: 47(12: 26)$ & $8.27(9.83)$ \\
\hline No (yes) & 12 & 360 & 118 & 1 & $14: 37(12: 16)$ & $8.36(9.97)$ \\
\hline Yes & 9 & 231 & 127 & 1 & $12: 13$ & 10.01 \\
\hline Yes & 12 & 351 & 127 & 1 & $11: 13$ & 10.90 \\
\hline Yes & 7 & 142 & 136 & 1 & $13: 52$ & 8.81 \\
\hline Yes & 8 & 182 & 136 & 1 & $12: 17$ & 9.95 \\
\hline Yes & 9 & 222 & 136 & 1 & $11: 38$ & 10.51 \\
\hline Yes & 10 & 262 & 136 & 1 & $11: 24$ & 10.72 \\
\hline Yes & 11 & 302 & 136 & 1 & $11: 10$ & 10.95 \\
\hline Yes & 12 & 342 & 136 & 1 & $10: 41$ & 11.44 \\
\hline Yes & 8 & 174 & 144 & 1 & $12: 17$ & 9.95 \\
\hline Yes & 9 & 214 & 144 & 1 & $11: 48$ & 10.36 \\
\hline Yes & 10 & 254 & 144 & 1 & $11: 31$ & 10.61 \\
\hline Yes & 11 & 294 & 144 & 1 & $11: 15$ & 10.87 \\
\hline Yes & 12 & 334 & 144 & 1 & $11: 13$ & 10.90 \\
\hline Yes & 8 & 170 & 148 & 1 & $12: 07$ & 10.09 \\
\hline Yes & 9 & 210 & 148 & 1 & $11: 22$ & 10.75 \\
\hline Yes & 10 & 250 & 148 & 1 & $11: 15$ & 10.87 \\
\hline Yes & 11 & 290 & 148 & 1 & $10: 49$ & 11.30 \\
\hline Yes & 12 & 330 & 148 & 1 & $10: 27$ & 11.70 \\
\hline Yes & 8 & 160 & 158 & 1 & $12: 42$ & 9.63 \\
\hline Yes & 9 & 200 & 158 & 1 & $11: 02$ & 11.08 \\
\hline Yes & 10 & 240 & 158 & 1 & $10: 43$ & 11.41 \\
\hline Yes & 11 & 280 & 158 & 1 & $10: 27$ & 11.70 \\
\hline Yes & 12 & 320 & 158 & 1 & $10: 16$ & 11.91 \\
\hline Yes & 9 & 184 & 174 & 1 & $11: 17$ & 10.83 \\
\hline Yes & 10 & 224 & 174 & 1 & $10: 10$ & 12.02 \\
\hline Yes & 11 & 264 & 174 & 1 & 09:58 & 12.27 \\
\hline Yes & 12 & 304 & 174 & 1 & 09:39 & 12.67 \\
\hline Yes & 10 & 218 & 180 & 1 & $10: 16$ & 11.91 \\
\hline Yes & 11 & 258 & 180 & 1 & $09: 44$ & 12.56 \\
\hline Yes & 12 & 298 & 180 & 1 & 09:34 & 12.78 \\
\hline Yes & 10 & 206 & 192 & 1 & $10: 22$ & 11.79 \\
\hline Yes & 11 & 246 & 192 & 1 & 09:38 & 12.69 \\
\hline Yes & 12 & 286 & 192 & 1 & 09:28 & 12.91 \\
\hline
\end{tabular}

aThe configuration highlighted in blue was found to be the most efficient and was used for the CMIP6 EC-Earth production runs. The SYPD (simulated days per day) is the number of EC-Earth-simulated years that complete in 24 hours. 
FUSE (Filesystem in Userspace) mount to provide a filesystem view of the iRODS storage as /esg/data on the virtual machine node. We also moved to the Docker version of ESGF (ESGF_DOCKER, n.d.). The ESGF Docker project aims to provide Docker containers for each of the components of the ESGF software stack, as an alternative to the traditional installer. This means that the search component (esgf-solr) runs in a different container from the database component (esgf-postgres) or publisher component (esgf-publisher); serious or security bugs in one will not affect the other containers, and components can be rebuilt and launched independently. At the time of decision, this looked likely to become the default production version; however, since then, most existing nodes have opted to continue with the traditional install version, making ICHEC's node the only operational Dockerised implementation at this time (see Figure 1.4). Although some bugfixes were required, the Dockerised version has been stable at ICHEC and so we have proceeded to use it for production.

The ESGF node at ICHEC is now automatically deployed from ICHEC's continuous integration system, allowing new instances to be built from scratch in minutes. This has allowed for test instances (esgf-test.ichec.ie) to be trivially built to test out new configuration changes, and ESGF system stability has been greatly improved. Containerisation has also meant that multiple instances of the publisher component can be run in parallel, which is crucial as the publisher contains a data scan step that is time-consuming. Furthermore, the file system can be mounted differently in each Docker component, allowing for different IO (input/output) strategies to be deployed. This was useful as different configurations are optimal for the different components; the main THREDDS component, publishing files to the outside world, benefits from large block sizes and consumes multiple file handles. THREDDS, by default, keeps multiple files open, presuming that there are multiple readers, each downloading the whole file. Alternatively, the publish/scan step is tuned for small block sizes as the publisher reads a single file at a time, but does many small reads and seeks as it validates the metadata within a NetCDF file. As of January 2020, we are seeing sustained download rates of over $1 \mathrm{Gbit} / \mathrm{s}$ from the ICHEC ESGF node; $10 \mathrm{Gbit} / \mathrm{s}$ should be possible on the underlying hardware.

ICHEC runs an identity provider container, allowing users to create logins on the node. However, we recommend that users login from the main ESGF

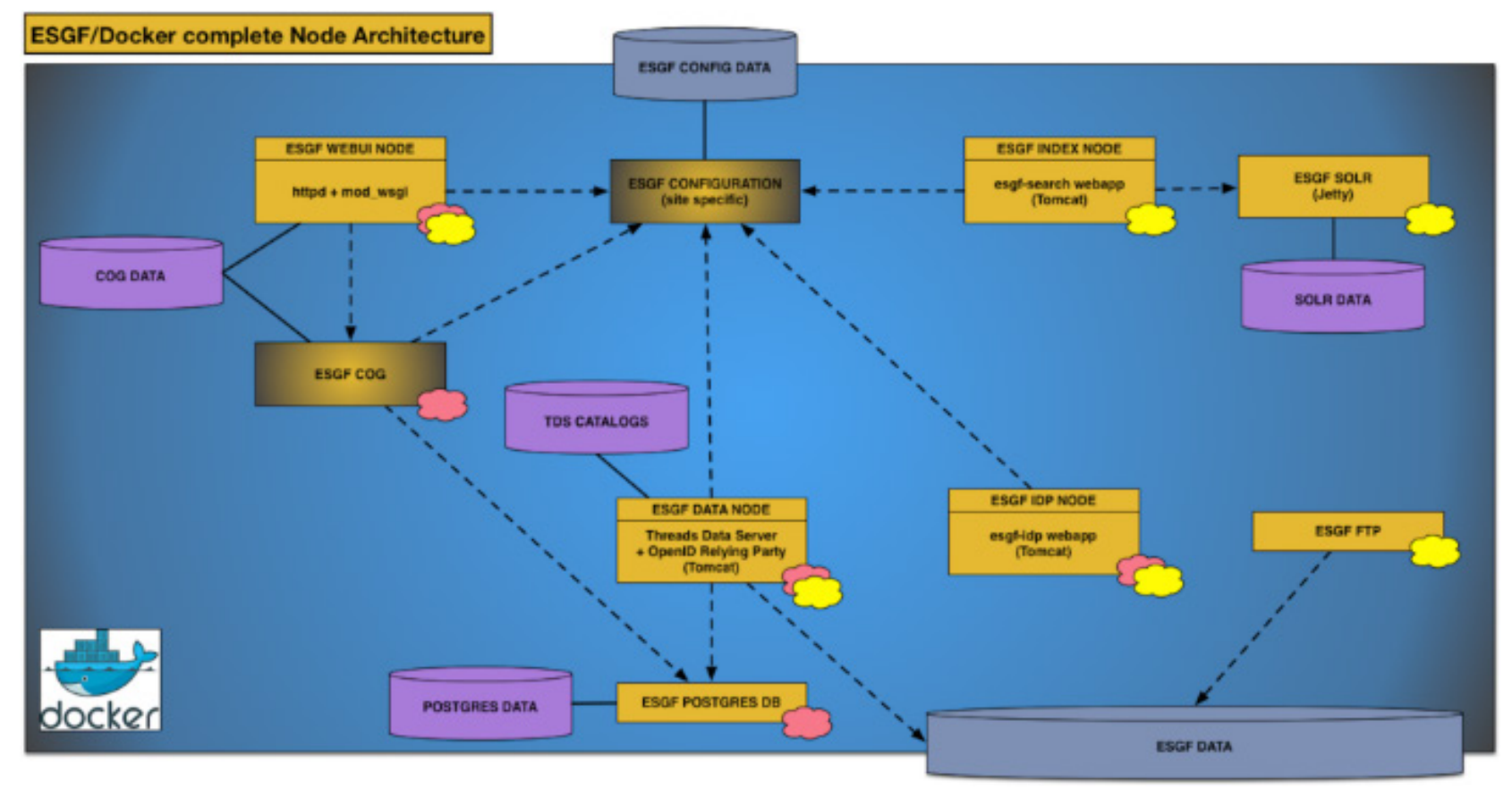

Figure 1.4. Docker-based architecture for a complete ICHEC ESGF node. Orange boxes are components hosted in virtual machines within ICHEC; purple containers are internal databases. See https://cedadev. github.io/esgf-dockerl (accessed 11 February 2020) for further details. 
nodes. The ESGF architecture also includes serverside compute components but these are not currently enabled at ICHEC.

\subsubsection{Publishing to the ESGF}

All of the "cmorised" simulation output from EC-Earth is in the form of NetCDF files. Each experiment produces $\sim 250$ datasets, each consisting of between 80 and 160 files. Each dataset is a single variable for a given simulation for various temporal resolutions (e.g. 3 hours, 6 hours, daily, monthly). In total, the CMIP6 work at ICHEC currently amounts to over 10,000 files shared on the ESGF.

CMIP specifies in detail how data are organised and published (Eyring et al., 2015). The NetCDF files have standardised metadata (such as processing history, tracking IDs, institutions) and variables defined with standardised units and specified in agreed controlled vocabularies. Although weather and climate data are typically published to standards using the "CF" (climate and forecasting) convention, CMIP goes beyond this to define agreed time-averaging and grid specification recommendations. These are then enforced with the software CMOR (Climate Model Output Rewriter) version 3 (CMOR3; https://github.com/PCMDI/cmor).

Compared with CMIP5, CMIP6 has tighter standards for data organisation: files on the server are better organised (agreed directory structures; better naming conventions for models within consortiums, such as EC-Earth; conventions on storing versioned sets of model outputs to enable better retraction and updates where necessary). The EC-Earth community standardised on "EC-Earth-Consortium" as institution_id. In comparison, for CMIP5, ICHEC was registered as an institution.

Each data node manager is then registered with ES-DOC (n.d.) with publishing rights. EC-Earth uses multiple data nodes (ICHEC, the National Supercomputer Centre at Linköping University in Sweden, KNMI in the Netherlands, the Barcelona Supercomputing Center and the Danish Meteorological Institute). Once the data are present on the data nodes, the metadata are sent to the search indexes. ICHEC currently uses the DKRZ (German Climate Computing Centre) as the index node. DKRZ then forwards this indexing information to the rest of the ESGF.
Publication consists of the following steps:

- Data are converted from EC-Earth output formats (GRIB and NetCDF for different atmosphere and ocean components) using ece 2 cmor. This postprocessing took approximately 1 hour for 1 year of data using 20 parallel cores.

- A new Pre-Publication Attribute Reviewer for ESGF (PrePARE) is used to check attributes.

- Data are uploaded to the ESGF node, with agreed directory structures. These are set in esg.ini in the ESGF configuration for each model. Each file contains one variable for a given experiment, with filenames specified and checked in esg.ini.

- Mapfiles are generated for each dataset using esgmapfile. This checks file structures (including directories, names and attributes) and checksums.

- A python library, CDF2CIM, is used to extract metadata from the NetCDF files during the map generation phase. A CIM2 (JSON format) file documenting the metadata is then uploaded to ES-DOC on the publication of datasets to the ESGF.

- Datasets are published on the ESGF node with esgpublish. The datasets are recorded in the node dataset and THREDDs database and are available locally for checking.

- The metadata are then pushed to the index node (using esgpublish - publish) to make the files visible beyond the data node.

Data availability can be limited by local policies to allow visibility to certain users only or enforced groups of users, for example the "CMIP_Research" group can have greater access to files during validation. Such group membership is enforced by the identity provider, which provides the OpenID logins that are authenticated within the ESGF using SAML (Security Assertion Markup Language) authentication. Hence, we recommend that users register for an OpenID at the main sites (e.g. DKRZ) so that they have the appropriate group memberships.

The CMIP and ESGF projects have a defined process in place for updates if required; every file has a tracking_id and every dataset has a version number. In the event of errors and corrections, the existing dataset is retracted (unpublished) on the ESGF node and a replacement with a new version number published; by convention, in CMIP6 a date-type version number is used (e.g. "20190710" is currently 
used). On the ESGF node, it is possible to search and view both current and previous versions in the index.

The tracking_id in each file is a unique hash of the file, allowing a backwards search on the portals to be carried out to discover where a given file came from (although the filename can also be used for this, the filename may be changed over time as it is copied from place to place). The tracking_id can also be used to discover if the file has been retracted or replaced.

\subsubsection{Further ESGF work}

As of August 2019, all EC-Earth AOGCM CMIP6 datasets, produced as part of the current report, are published on the ICHEC ESGF node. It is expected that the CMIP6 data will be analysed by the international research community over the coming months for inclusion in the upcoming IPCC AR6 report. From previous experience, downloads will continue until data from a follow-on CMIP7 become available over the next decade. ICHEC will continue to publish the data until then, including additional CMIP6 contributions and follow-on downscaling work, and will work on integrating CMIP6 outputs into other projects, such as the Climate Ireland portal (www.climateireland. ie).

\subsubsection{ESGF acknowledgements}

We gratefully acknowledge Eoin McHugh at ICHEC for working through iRODS and ESGF installation bugs, making possible the reproducible installation of the ESGF at ICHEC, and Katharina Berger at DKRZ and Prashanth Dwarakanath at Linköping University for testing and resolving multiple ESGF issues. 


\section{EC-Earth Validations}

The EC-Earth ensemble members were validated by comparing the historical datasets with Climatic Research Unit (CRU) observational datasets and ERA5 global reanalysis data (ERA5 is the fifth generation of the ECMWF global climate reanalysis dataset; C3S, 2017). As outlined in Chapter 1, the historical climate was simulated using the EC-Earth T255L91-ORCA1L75 AOGCM configuration. The atmosphere was simulated with $\sim 79-\mathrm{km}$ horizontal grid spacings (T255) and 91 vertical levels. The ocean was simulated with 1-degree horizontal resolution and 75 vertical levels. In total, the historical climate was simulated using five ensemble members ( $r 6 i 1 p 1 f 1$, r9i1p1f1, r11i1p1f1, r13i1p1f1 and r15i1p1f1) for the period 1850-2014.

\subsection{Observational and Reanalysis Datasets}

\subsubsection{ERA5 global reanalysis}

Reanalysis provides a numerical description of the recent climate by combining models with observations. ERA5 is currently available for the period 1979 to within 3 months of real time. Subsequent releases of ERA5 will cover the earlier decades from 1950 and will be available to within 7 days of real time. ERA5 was produced using 4D-Var data assimilation in CY41R2 of the ECMWF IFS, with 137 hybrid sigma/ pressure (model) levels in the vertical, with the top level at $0.01 \mathrm{hPa}$. Atmospheric data are available on these levels and are also interpolated to 37 pressure, 16 potential temperature and one potential vorticity level(s). "Surface or single-level" data are also available, containing two-dimensional parameters such as precipitation, 2-m temperature, top of atmosphere radiation and vertical integrals over the entire atmosphere. The IFS is coupled to a soil model, the parameters of which are also designated as surface parameters, and an ocean wave model. For the current report, ERA5 data were used for the validation of 2-m temperature, precipitation, 10-m wind speed, mean sea level pressure (MSLP), total cloud cover, snowfall, sea surface temperature (SST) and sea ice fraction. Note that, although all ERA5 climate fields are directly comparable to observations, assimilated fields (e.g. temperature, wind speed and pressure) are expected to be more accurate than non-assimilated fields (e.g. precipitation and snowfall).

\subsubsection{CRU Global Gridded Observational Datasets}

The CRU high-resolution observational time series dataset is produced by the CRU, University of East Anglia (Harris et al., 2014). The dataset contains monthly time series of precipitation, daily maximum and minimum temperatures, cloud cover and other variables covering all land areas (excluding Antarctica) for 1901-2015. The dataset is gridded to $0.5 \times 0.5$ degree resolution, based on analysis of over 4000 individual weather station records. For the current report, CRU time series data (version 4.03) were used for the validation of EC-Earth 2-m temperature and precipitation variables for the periods 1979-2014 (for comparison with ERA5) and 1901-2014.

\subsection{Validation Methods and Metrics}

The EC-Earth raw data were post-processed ("cmorised") using international best practices (see Chapter 1) and are archived on a reduced Gaussian grid $(512 \times 256, N=128)$. The CRU and ERA5 datasets were interpolated onto the lower resolution EC-Earth grid using the method of bilinear interpolation and Climate Data Operators (CDO) software. The EC-Earth data were then directly compared with the observational and reanalysis datasets using the bias and mean absolute error (MAE) statistics. The bias statistic is given by:

$$
\operatorname{bias}_{(i, j)}=\overline{\operatorname{GCM}}_{(i, j)}-\overline{\mathrm{OBS}}_{(i, j)}
$$

where the $\overline{G C M}_{(i, j)}$ and $\overline{O B S}_{(i, j)}$ terms represent the GCM and observed/reanalysis values, respectively, at grid point $(i, j)$, averaged over the time period of interest. To quantify the overall global bias, the mean was calculated over all $N$ grid points using:

$$
\text { global_bias }=\frac{1}{N} \sum_{i, j}\left(\overline{\operatorname{GCM}}_{(i, j)}-\overline{\mathrm{OBS}}_{(i, j)}\right)
$$


The global mean is calculated using weighted averages derived from each grid cell area.

The bias metric allows for the evaluation of the systematic errors of the EC-Earth model but can hide large errors, as positive and negative values can cancel. For this reason, the MAE metric was also used to evaluate the overall global performance of EC-Earth. The MAE statistic is given by:

$$
\text { global_MAE }=\frac{1}{N} \sum_{i, j}\left|\overline{\operatorname{GCM}}_{(i, j)}-\overline{\mathrm{OBS}}_{(i, j)}\right|
$$

The percentage bias and MAE are also considered for precipitation, given by:

$$
p e r \_g l o b a l \_b i a s=\frac{100}{N} \sum_{i, j} \frac{\left(\overline{G C M}_{(i, j)}-\overline{O B S}_{(i, j)}\right)}{\overline{O B S}_{(i, j)}}
$$

and:

$$
p e r \_g l o b a l \_M A E=\frac{100}{N} \sum_{i, j} \frac{\left|\overline{G C M}_{(i, j)}-\overline{\mathrm{OBS}}_{(i, j)}\right|}{\overline{\mathrm{OBS}}_{(i, j)}}
$$

The validation presented here considers the following time periods; annual, DJF, MAM (March, April and May), JJA and SON (September, October and November).

\subsection{EC-Earth Validation Results}

\subsubsection{2-m temperature validations}

Figure 2.1 presents the spatial distribution of annual mean 2-m temperature for the ERA5 dataset, CRU dataset and each of the five EC-Earth ensemble members for the 36-year period 1979-2014. ${ }^{3}$ All EC-Earth ensemble members accurately capture the magnitude and spatial characteristics of the historical temperature climate. This is confirmed in Figures 2.2 and 2.3, which present the ERA5 and CRU bias, respectively. The general trend is for EC-Earth to underestimate the temperature over land areas, with some exceptions, such as southern Australia. Over oceans, EC-Earth exhibits a strong warm bias in the Southern Hemisphere. Small differences are noted elsewhere with the exception of the North Atlantic, which exhibits a cold bias, the magnitude of which varies between ensemble members; the strongest bias is noted for ensemble member r11i1p1f1 (Figure 2.2c).

Validations for DJF are presented in Figures 2.4-2.6 and show a similar signal to the annual results, with a diminished warm bias over the Southern Hemisphere oceans and Antarctica and an enhanced cold bias over most land areas. Conversely, JJA validations exhibit an enhanced warm bias over the southern oceans and Antarctica and a diminished cold bias over land (Figures 2.7-2.9).

The annual and seasonal overall global bias and MAE statistics relative to ERA5 data (1979-2014) for each of the five ensemble members are presented in Table 2.1. The bias statistics are all positive and range from $0.02^{\circ} \mathrm{C}$ (DJF, r11i1p1f1) to $0.78^{\circ} \mathrm{C}$ (JJA, r9i1p1f1). The MAE statistics range from $1.21^{\circ} \mathrm{C}$ (SON, $\mathrm{r} 15 \mathrm{i} 1 \mathrm{p} 1 \mathrm{f} 1)$ to $2.01^{\circ} \mathrm{C}$ (MAM, r11i1p1f1). Similarly, the CRU statistics for the period 1901-2014 are presented in Table 2.2. The bias values are all negative (recall that the CRU dataset excludes Antarctica, for which the EC-Earth dataset exhibits a warm bias compared with ERA5, and the oceans), with values ranging from $-1.70^{\circ} \mathrm{C}$ (MAM, r6i1p1f1) to $-0.51^{\circ} \mathrm{C}$ (JJA, r9i1p1f1). The MAE statistics range from $1.55^{\circ} \mathrm{C}$ (SON, $\mathrm{r} 13 \mathrm{i} 1 \mathrm{p} 1 \mathrm{f1}$ ) to $2.66^{\circ} \mathrm{C}$ (DJF, r6i1p1f1). The largest bias and MAE statistics are noted for DJF and MAM; the spatial characteristics of the bias for MAM are similar to those for DJF (Figure 2.6) with the exception that the warm bias over North-East Asia is not evident.

The ERA5 and EC-Earth mean global annual 2-m temperature time series (1979-2014), presented in Figure 2.10a, demonstrate a consistent overestimation of EC-Earth temperatures. Figure $2.10 \mathrm{~b}$, the annual anomalies with respect to the 1981-2010 mean, shows good agreement between ERA5 reanalysis data and EC-Earth ensemble members. However, the EC-Earth temperature rise is overestimated for the later years. The CRU and EC-Earth mean global annual 2-m temperature time series (1901-2014), presented in Figure 2.11a, demonstrate a consistent underestimation of EC-Earth land surface temperatures. The temperate anomalies for the period 1901-2014 (Figure 2.11b) again show a slight overestimation of temperature rise for the later years.

3 Note that the 1979-2014 period was chosen as it is the longest common time period of the ERA5, CRU and EC-Earth datasets. CRU datasets cover the period 1901-2014; analyses over this time period are considered in subsequent validations. 
(a)

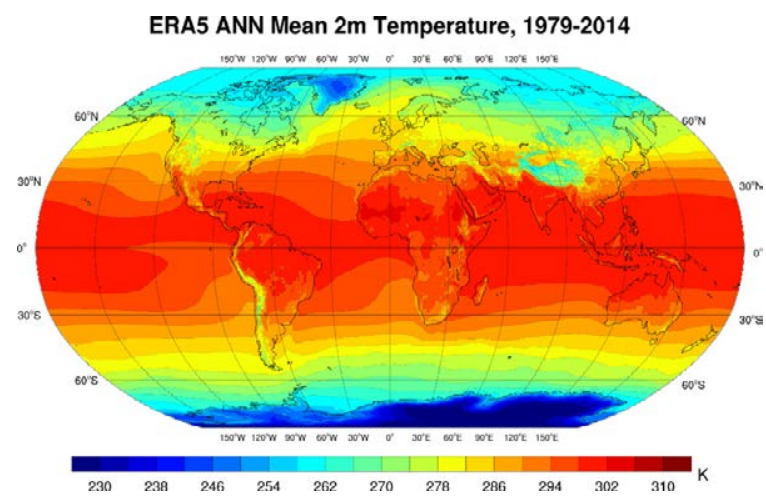

(c)

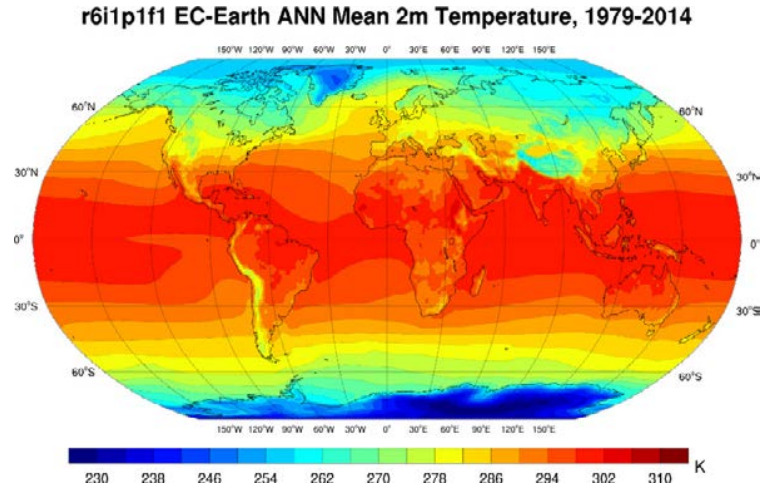

(e)

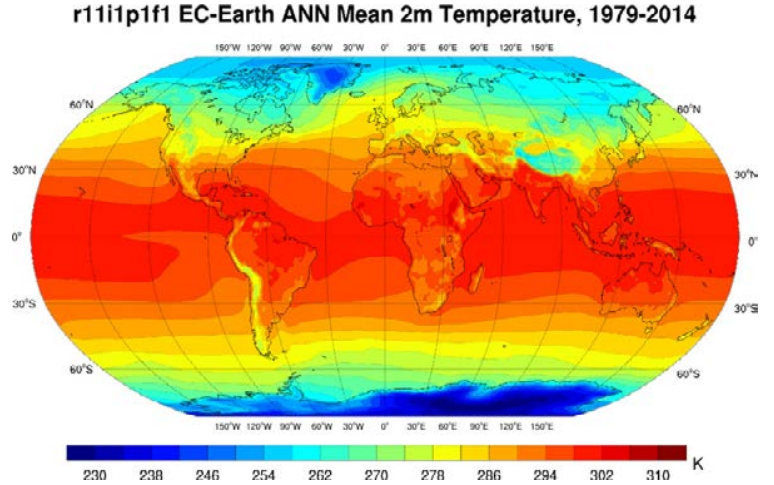

(g)

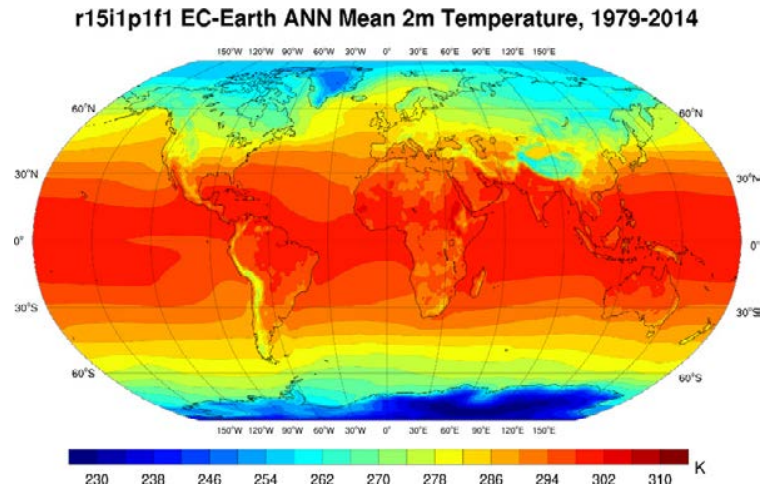

(b)

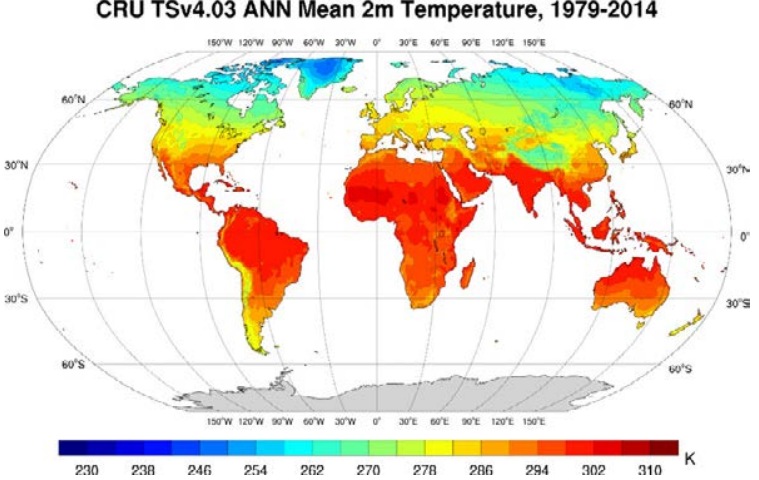

(d)

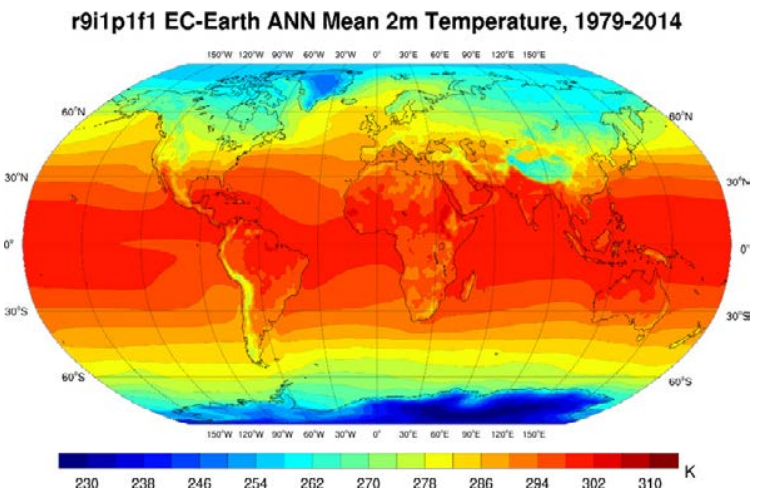

(f)

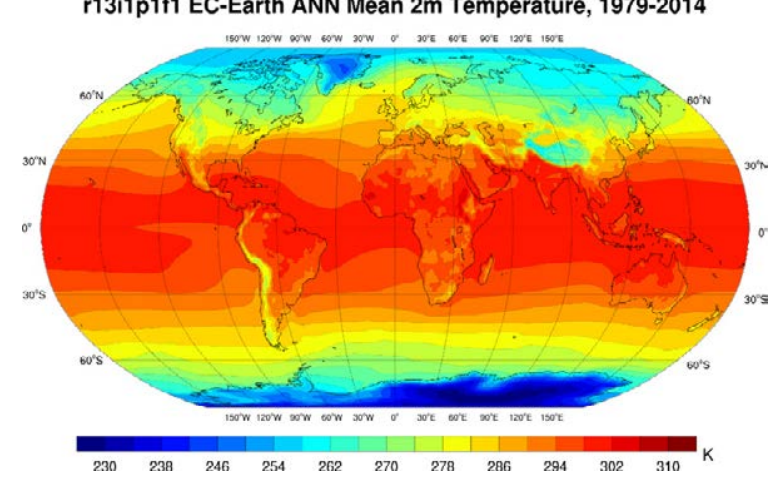

Figure 2.1. Annual mean 2-m temperature, 1979-2014: (a) ERA5 reanalysis, (b) CRU_ts4.03 observations, (c) EC-Earth r6i1p1f1, (d) EC-Earth r9i1p1f1, (e) EC-Earth r11i1p1f1, (f) EC-Earth r13i1p1f1 and (g) EC-Earth r15i1p1f1. 
(a)

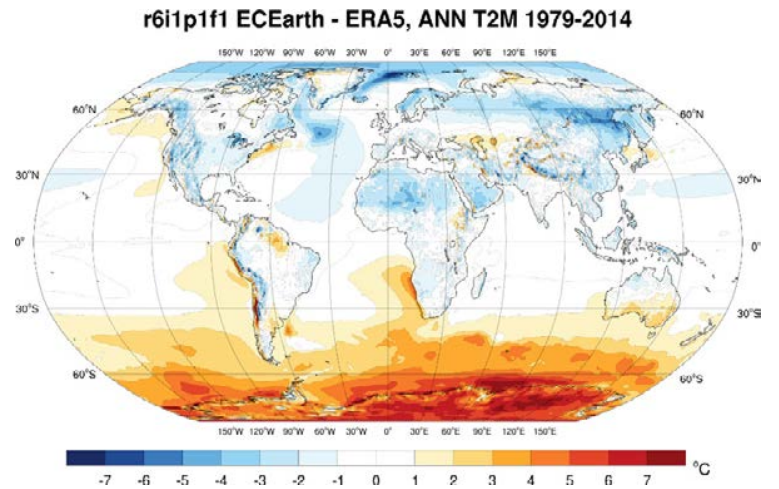

(c)

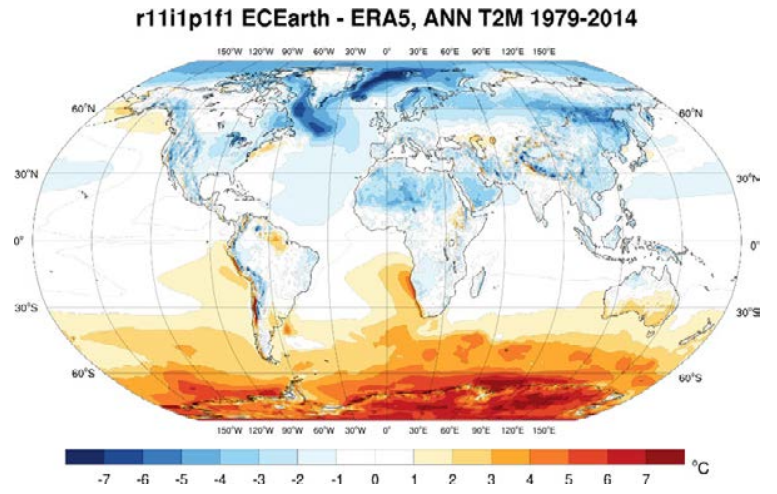

(e)

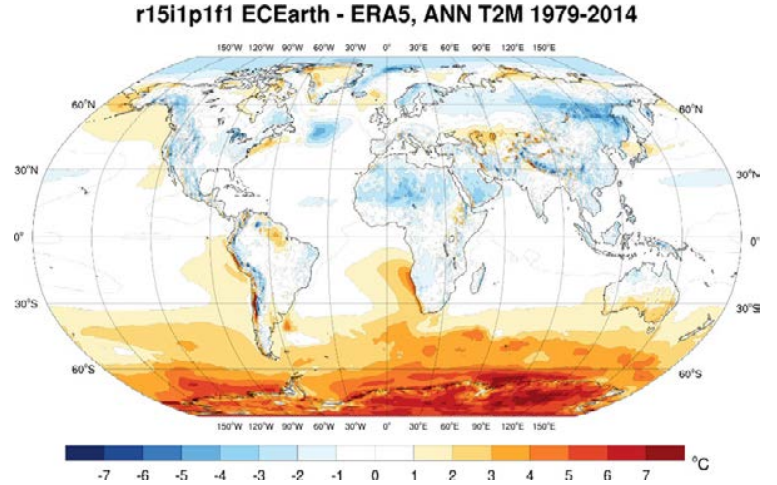

(b)

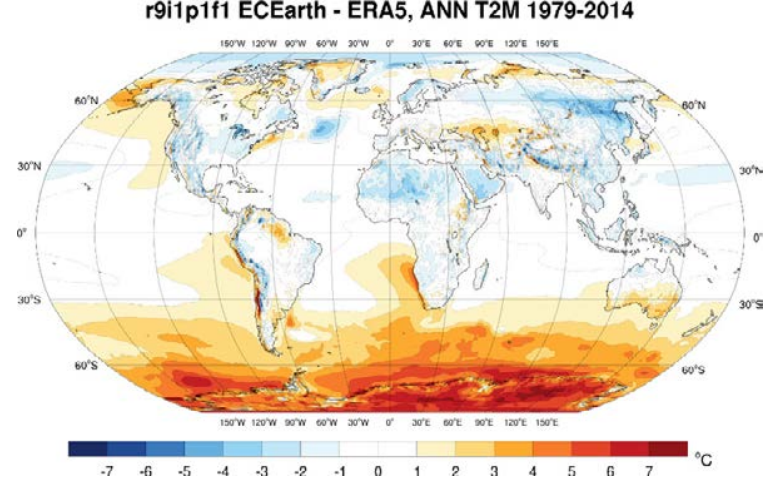

(d)

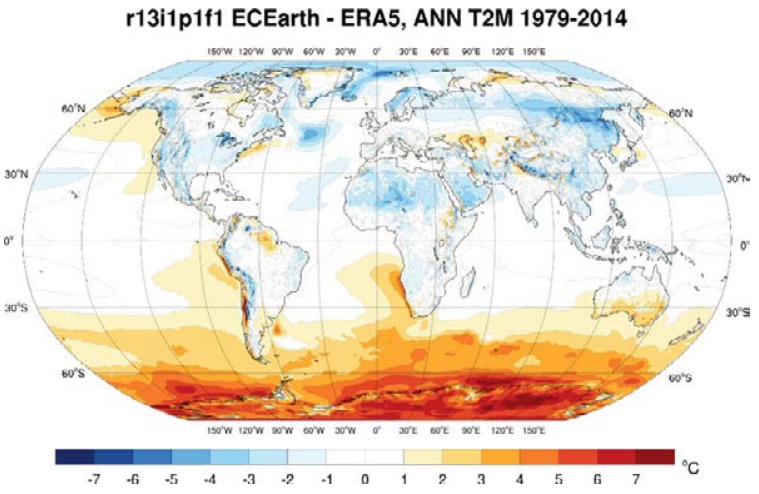

Figure 2.2. EC-Earth annual 2-m temperature bias, 1979-2014 (ERA5 reanalysis minus EC-Earth): (a) EC-Earth r6i1p1f1, (b) EC-Earth r9i1p1f1, (c) EC-Earth r11i1p1f1, (d) EC-Earth r13i1p1f1 and (e) EC-Earth r15i1p1f1. 
(a)

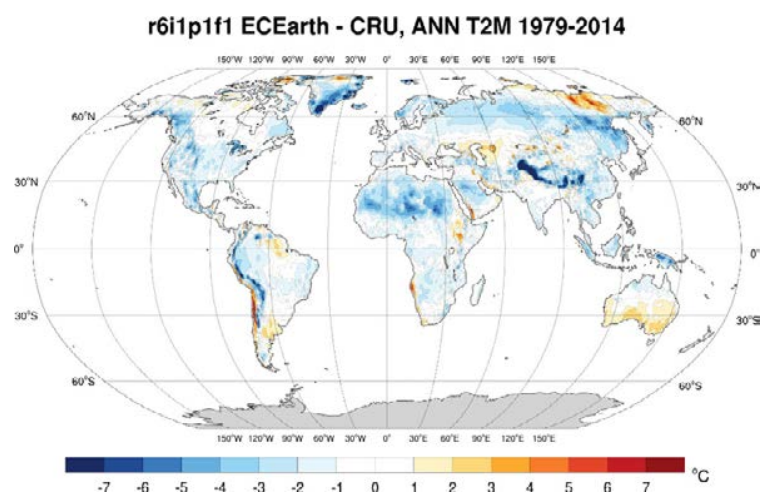

(c)

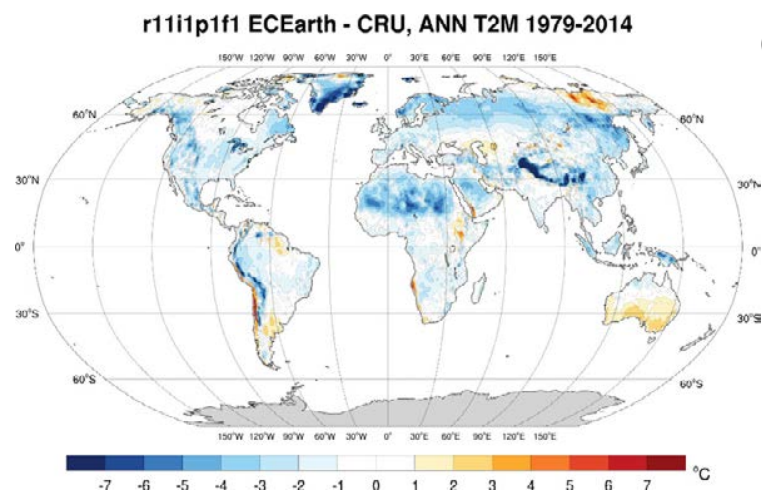

(b)

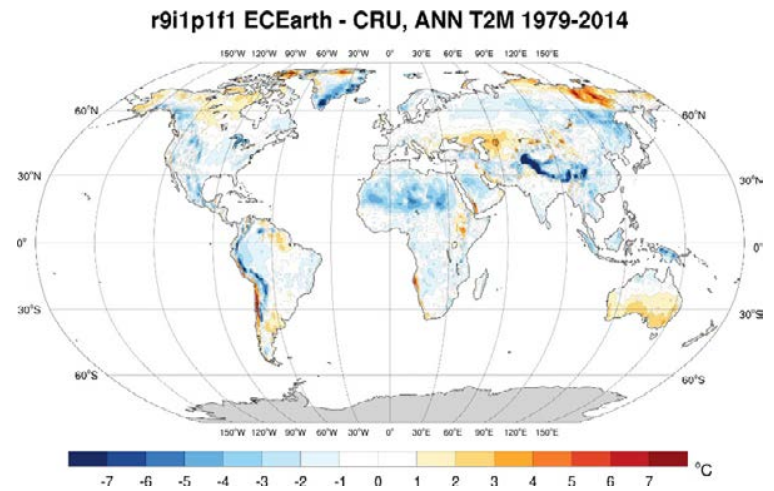

(d)

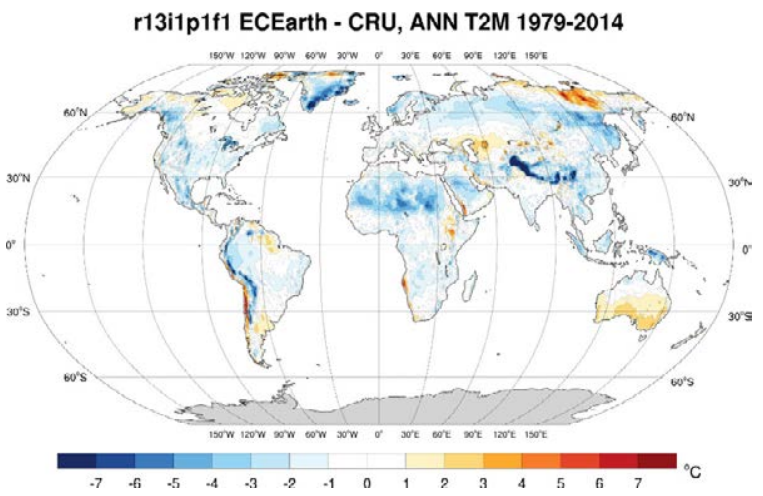

(e)

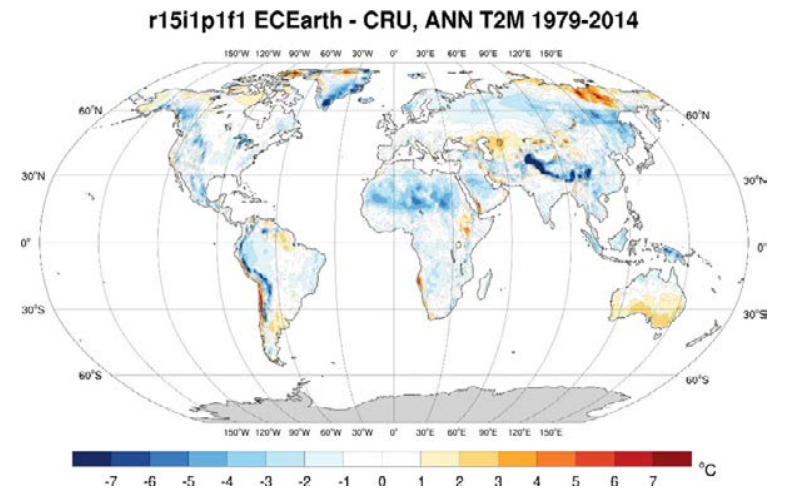

Figure 2.3. EC-Earth annual 2-m temperature bias, 1979-2014 (EC-Earth minus CRU_ts4.03): (a) EC-Earth r6i1p1f1, (b) EC-Earth r9i1p1f1, (c) EC-Earth r11i1p1f1, (d) EC-Earth r13i1p1f1 and (e) EC-Earth r15i1p1f1. 
(a)

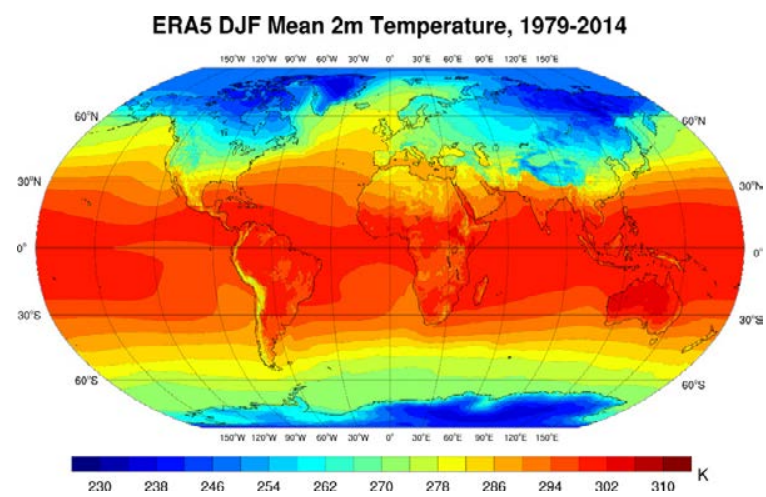

(c)

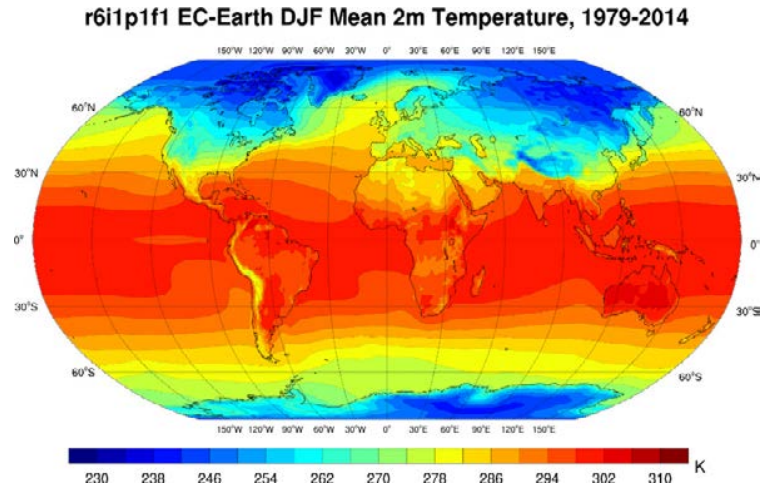

(e)

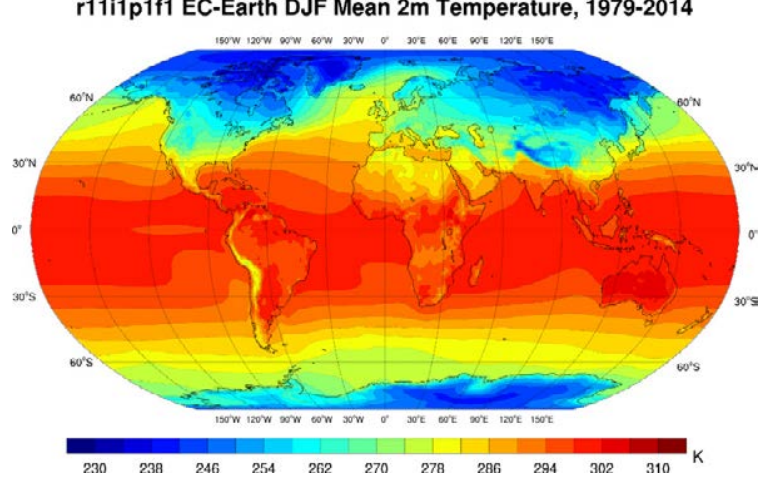

(g)

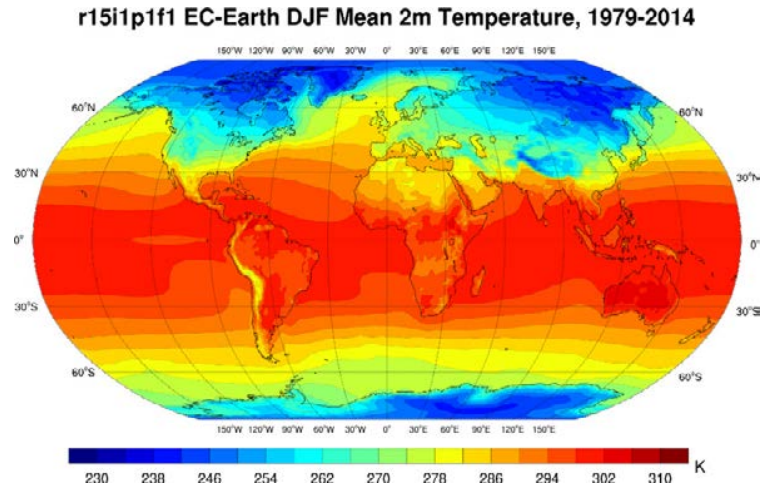

(b)

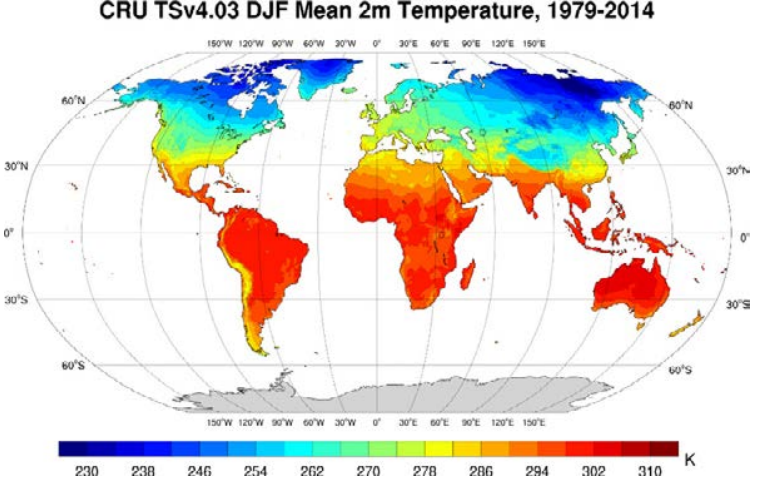

(d)

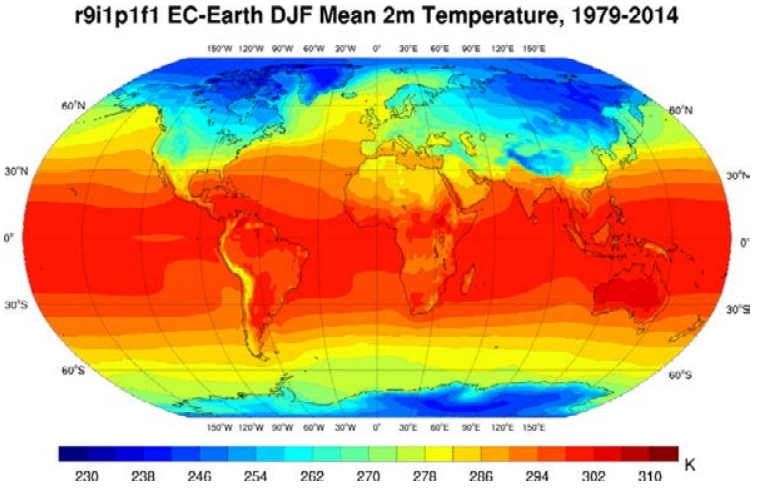

(f)

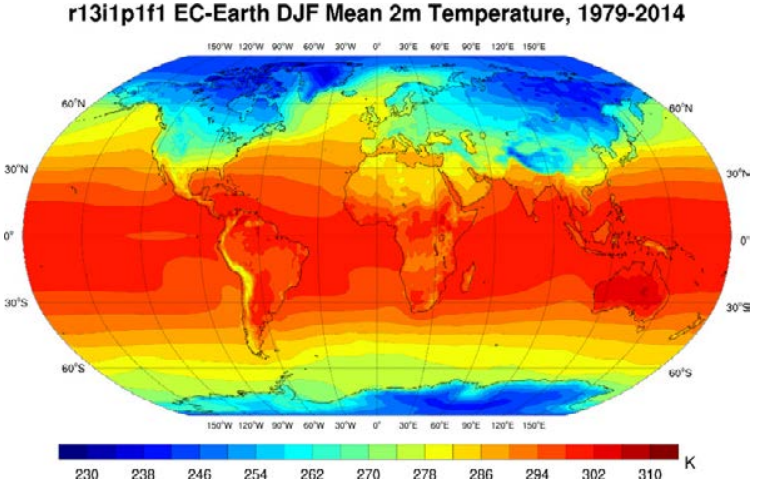

Figure 2.4. Mean 2-m temperature for DJF, 1979-2014: (a) ERA5 reanalysis, (b) CRU_ts4.03 observations, (c) EC-Earth r6i1p1f1, (d) EC-Earth r9i1p1f1, (e) EC-Earth r11i1p1f1, (f) EC-Earth r13i1p1f1 and (g) EC-Earth r15i1p1f1. 
(a)

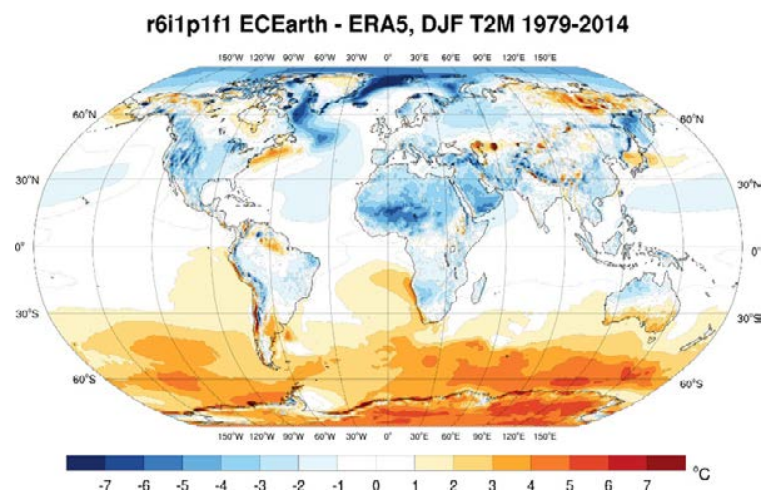

(c)

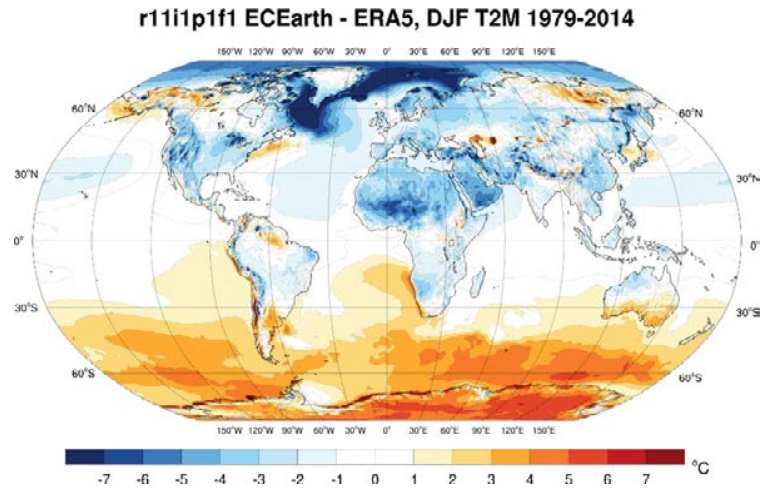

(e)

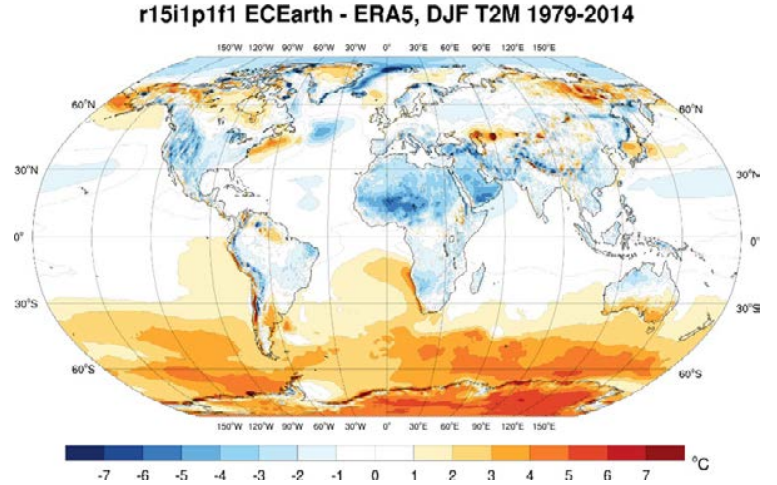

(b)

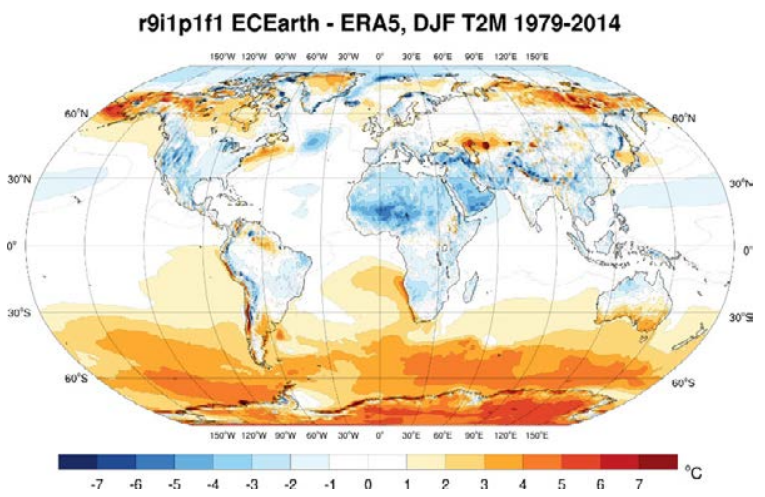

(d)

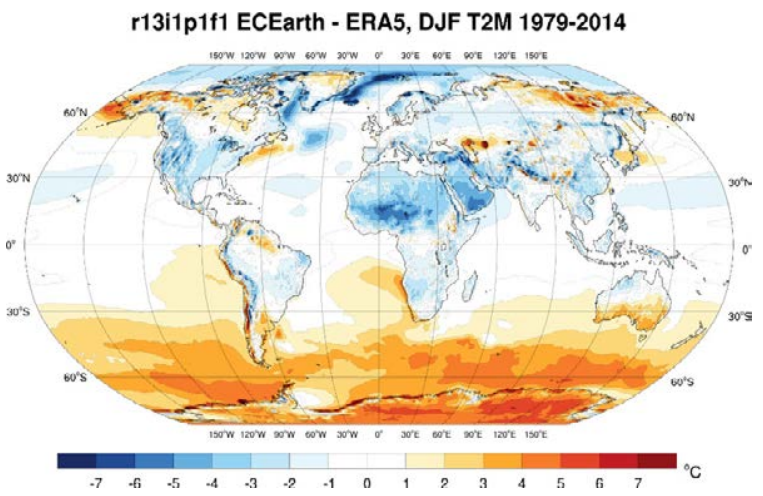

Figure 2.5. EC-Earth 2-m temperature bias for DJF, 1979-2014 (ERA5 reanalysis minus EC-Earth): (a) EC-Earth r6i1p1f1, (b) EC-Earth r9i1p1f1, (c) EC-Earth r11i1p1f1, (d) EC-Earth r13i1p1f1 and (e) EC-Earth r15i1p1f1. 
(a)

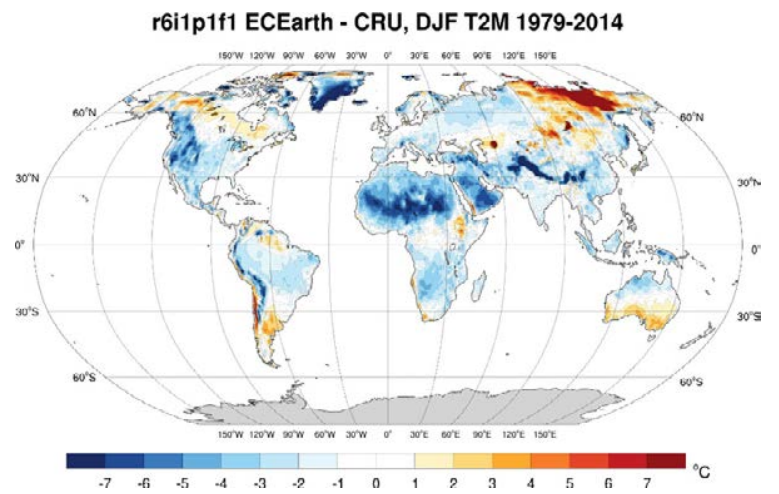

(c)

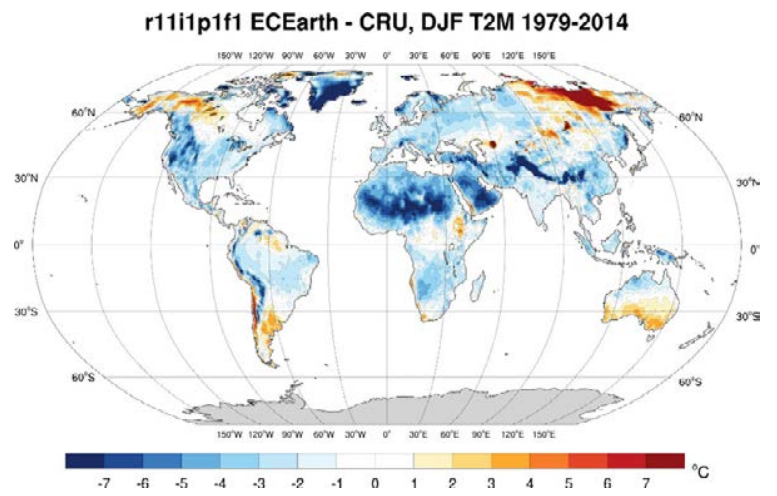

(e)

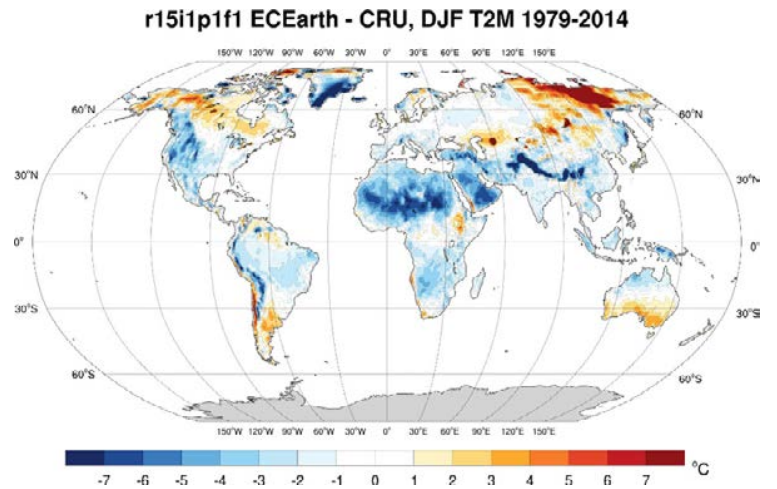

(b)

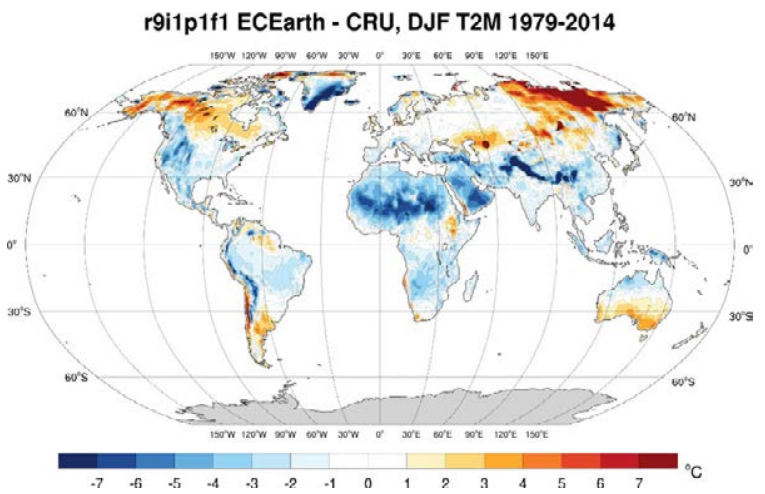

(d)

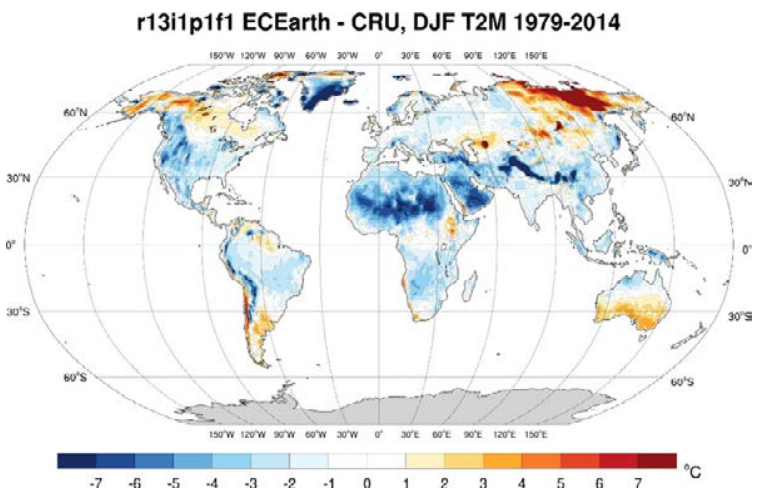

Figure 2.6. EC-Earth 2-m temperature bias for DJF, 1979-2014 (EC-Earth minus CRU_ts4.03): (a) EC-Earth r6i1p1f1, (b) EC-Earth r9i1p1f1, (c) EC-Earth r11i1p1f1, (d) EC-Earth r13i1p1f1 and (e) EC-Earth r15i1p1f1. 
(a)

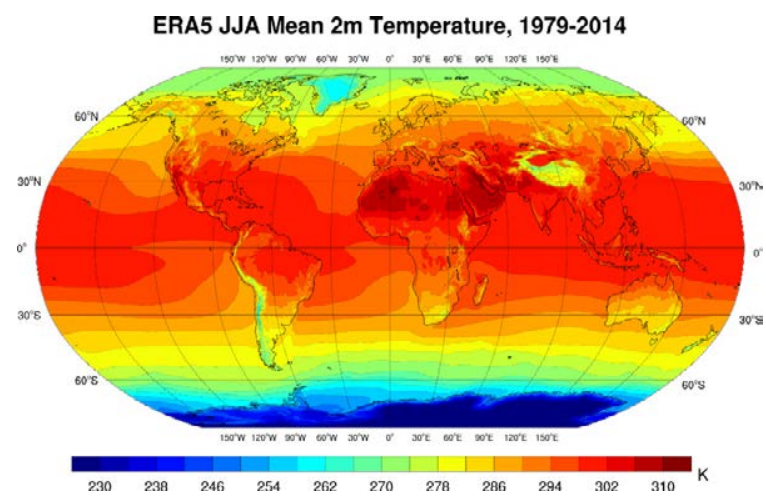

(c)

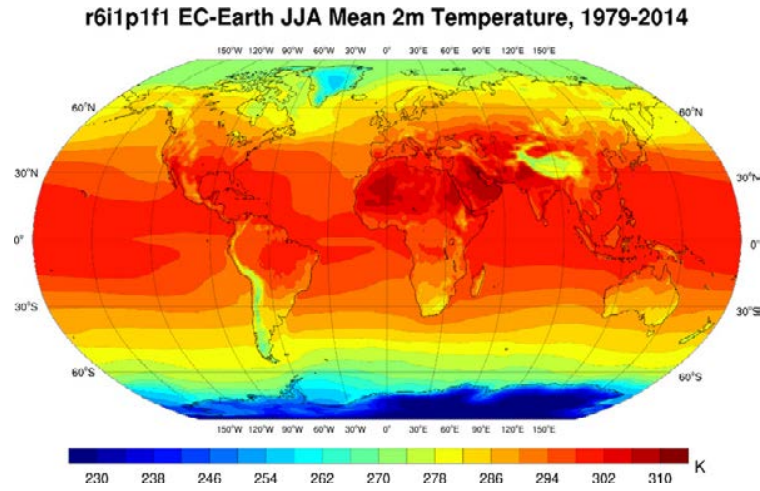

(e)

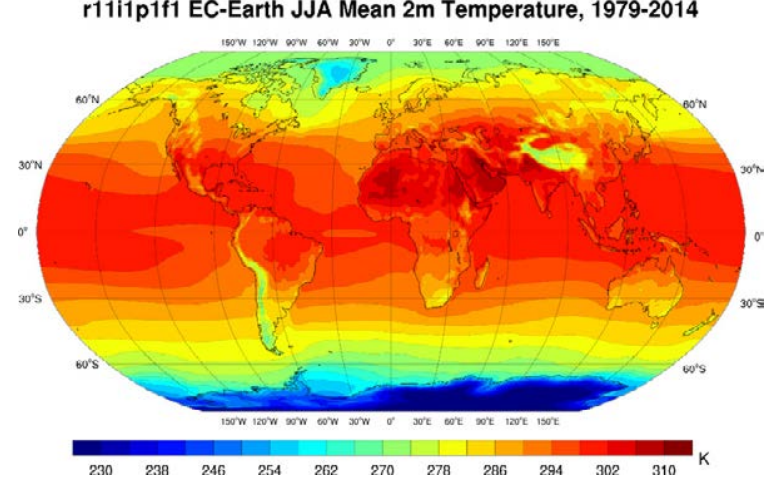

(g)

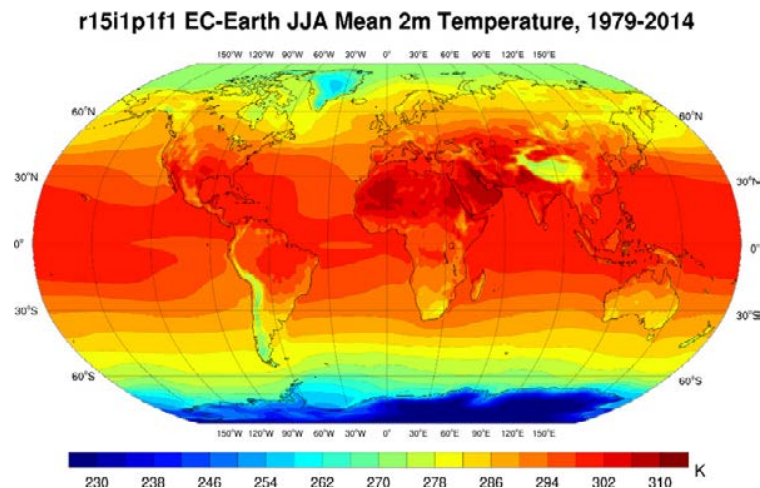

(b)

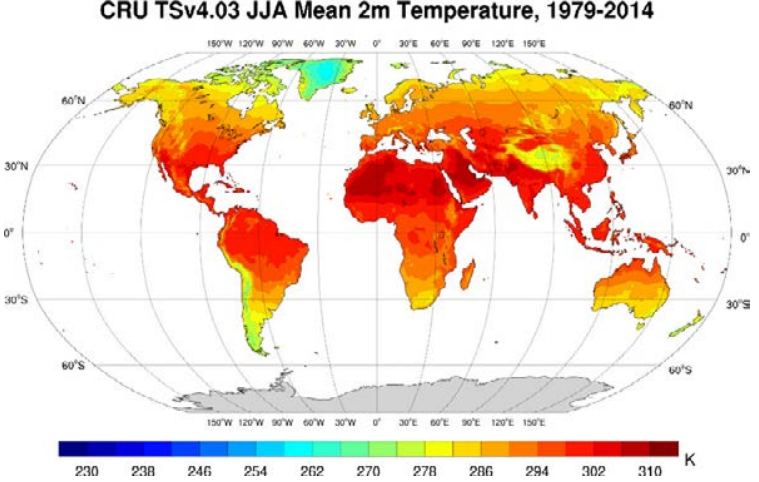

(d)

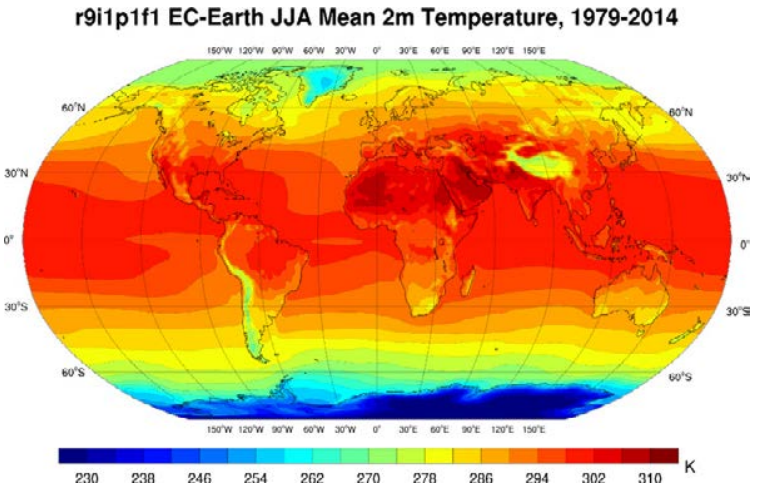

(f)

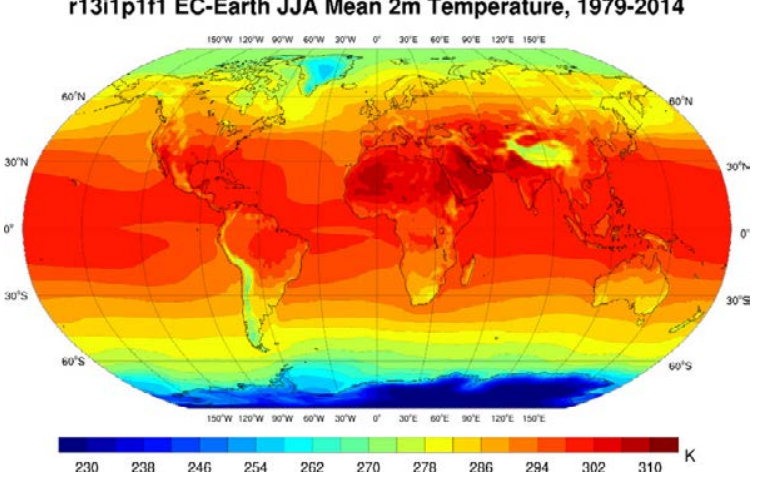

Figure 2.7. Mean 2-m temperature for JJA, 1979-2014: (a) ERA5 reanalysis, (b) CRU_ts4.03 observations, (c) EC-Earth r6i1p1f1, (d) EC-Earth r9i1p1f1, (e) EC-Earth r11i1p1f1, (f) EC-Earth r13i1p1f1 and (g) EC-Earth r15i1p1f1. 
(a)

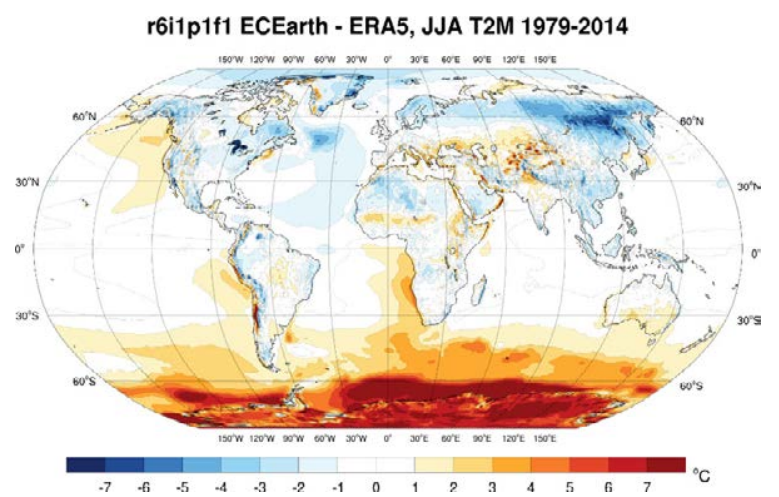

(c)

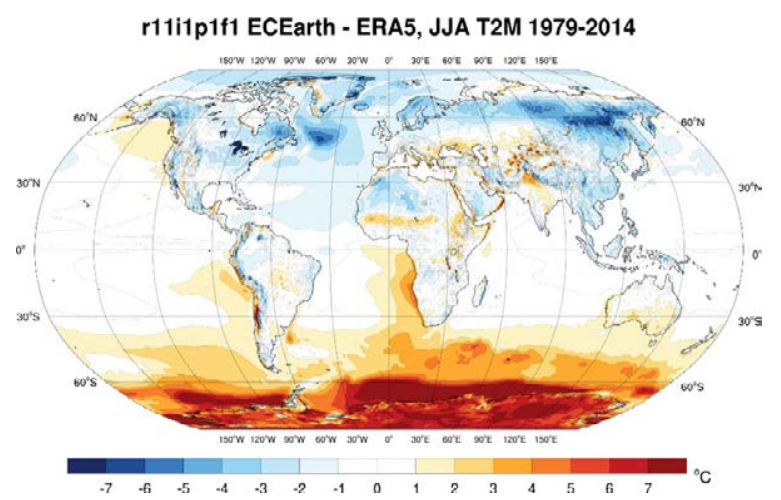

(e)

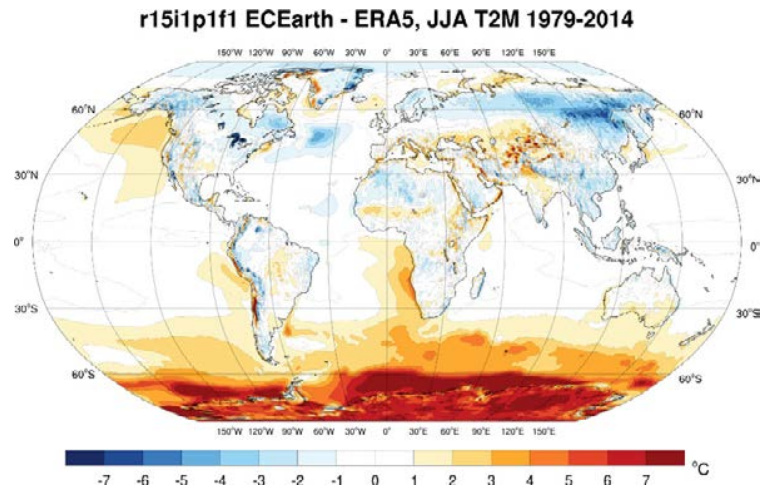

(b)

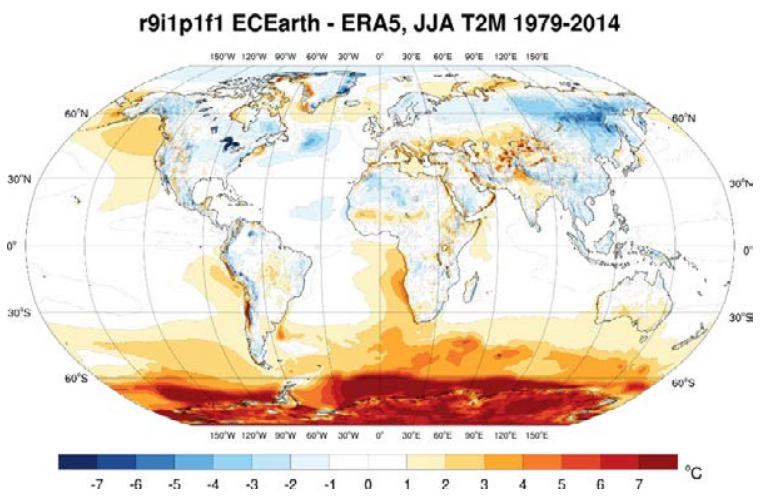

(d)

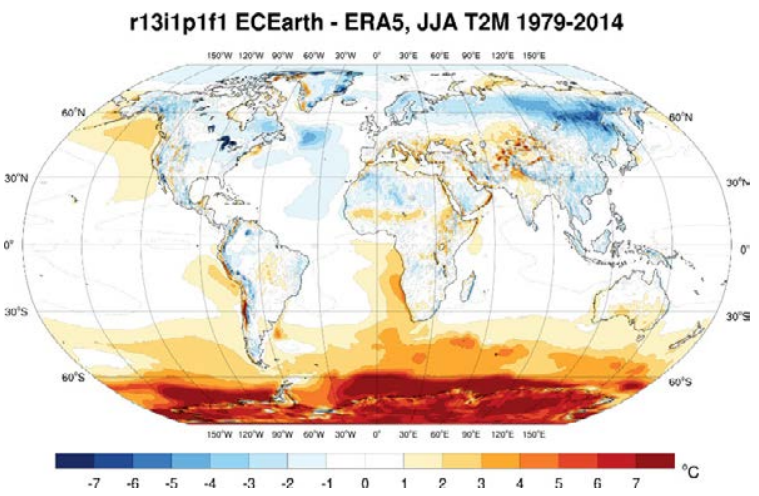

Figure 2.8. EC-Earth 2-m temperature bias for JJA, 1979-2014 (ERA5 reanalysis minus EC-Earth): (a) EC-Earth r6i1p1f1, (b) EC-Earth r9i1p1f1, (c) EC-Earth r11i1p1f1, (d) EC-Earth r13i1p1f1 and (e) EC-Earth r15i1p1f1. 
(a)

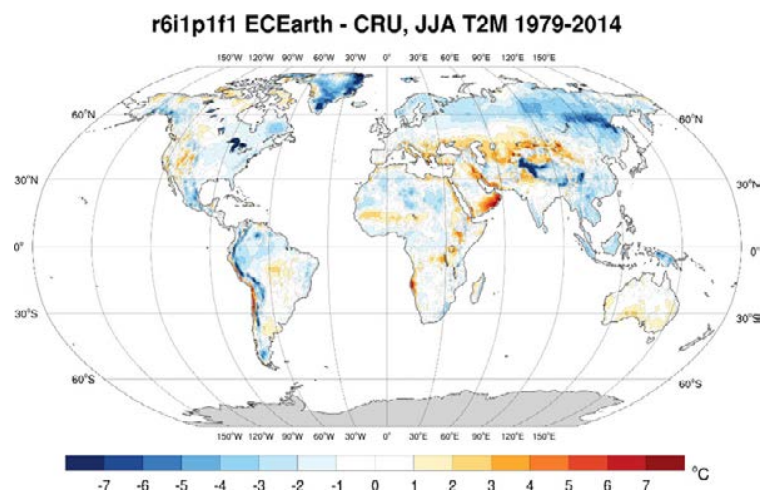

(c)

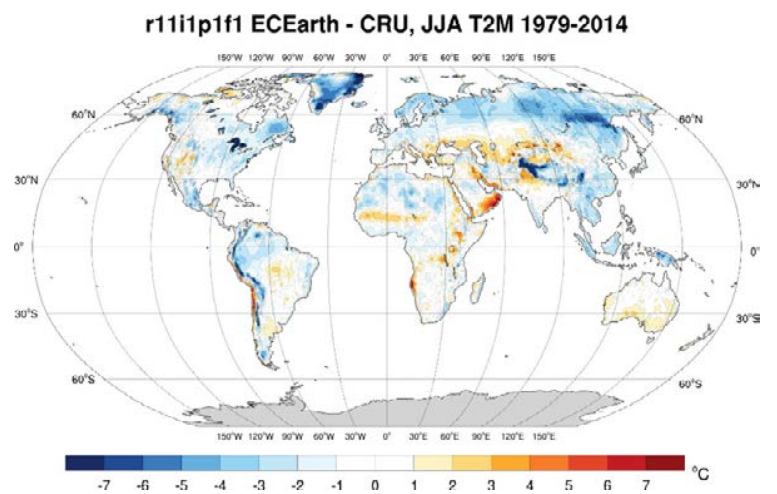

(e)

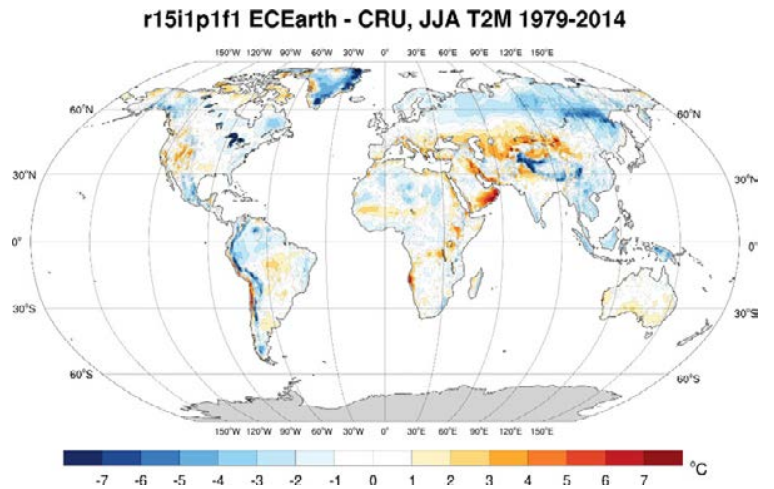

(b)

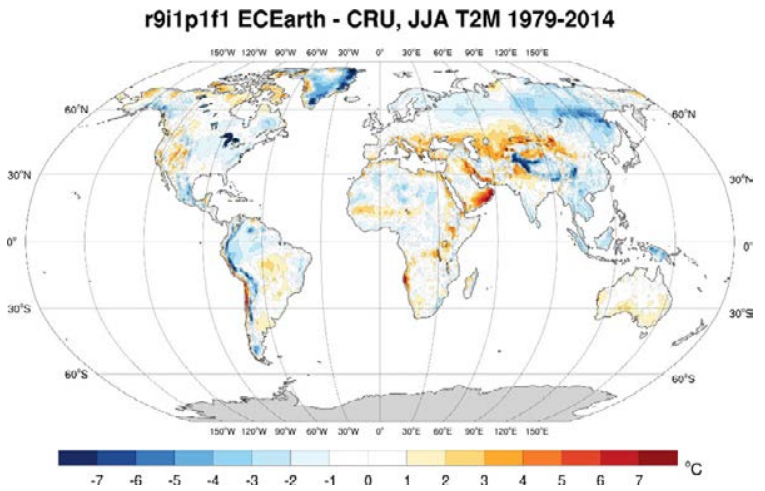

(d)

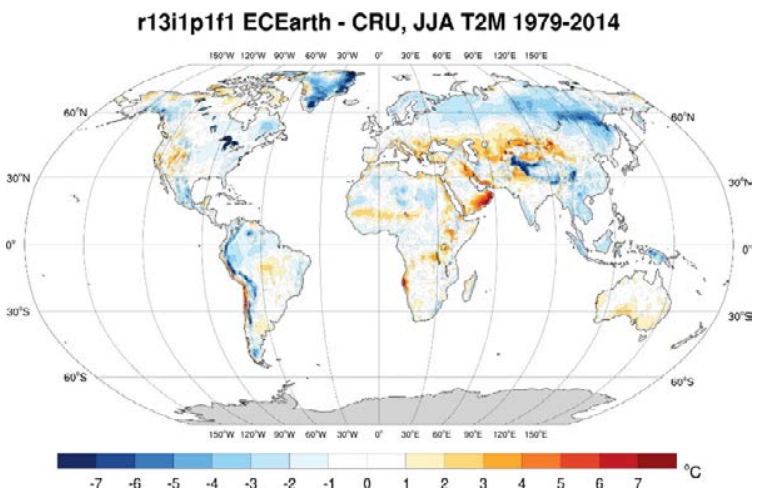

Figure 2.9. EC-Earth 2-m temperature bias for JJA, 1979-2014 (EC-Earth minus CRU_ts4.03): (a) EC-Earth r6i1p1f1, (b) EC-Earth r9i1p1f1, (c) EC-Earth r11i1p1f1, (d) EC-Earth r13i1p1f1 and (e) EC-Earth r15i1p1f1. 
Table 2.1. Mean global annual and seasonal 2-m temperature bias and MAE $\left({ }^{\circ} \mathrm{C}\right)$ for each of the five ECEarth ensemble members ${ }^{a}$

\begin{tabular}{|c|c|c|c|c|c|c|c|c|c|c|}
\hline \multirow{2}{*}{$\begin{array}{l}\text { Time } \\
\text { period }\end{array}$} & \multicolumn{2}{|c|}{ r6i1p1f1 } & \multicolumn{2}{|c|}{ r9i1p1f1 } & \multicolumn{2}{|c|}{ r11i1p1f1 } & \multicolumn{2}{|c|}{ r13i1p1f1 } & \multicolumn{2}{|c|}{ r15i1p1f1 } \\
\hline & Bias & MAE & Bias & MAE & Bias & MAE & Bias & MAE & Bias & MAE \\
\hline Annual & 0.33 & 1.42 & 0.67 & 1.38 & 0.18 & 1.59 & 0.49 & 1.40 & 0.54 & 1.35 \\
\hline DJF & 0.21 & 1.60 & 0.62 & 1.56 & 0.02 & 1.80 & 0.39 & 1.58 & 0.45 & 1.51 \\
\hline MAM & 0.31 & 1.83 & 0.61 & 1.79 & 0.15 & 2.01 & 0.44 & 1.82 & 0.48 & 1.76 \\
\hline JJA & 0.49 & 1.42 & 0.78 & 1.43 & 0.37 & 1.54 & 0.63 & 1.43 & 0.66 & 1.39 \\
\hline SON & 0.33 & 1.24 & 0.67 & 1.23 & 0.17 & 1.39 & 0.51 & 1.23 & 0.55 & 1.21 \\
\hline
\end{tabular}

aln each case the model data are compared with ERA5 reanalysis data for the period 1979-2014.

Table 2.2. Mean global annual and seasonal 2-m temperature bias and MAE $\left({ }^{\circ} \mathrm{C}\right)$ for each of the five ECEarth ensemble members ${ }^{a}$

\begin{tabular}{|c|c|c|c|c|c|c|c|c|c|c|}
\hline \multirow{2}{*}{$\begin{array}{l}\text { Time } \\
\text { period }\end{array}$} & \multicolumn{2}{|c|}{ r6i1p1f1 } & \multicolumn{2}{|c|}{ r9i1p1f1 } & \multicolumn{2}{|c|}{ r11i1p1f1 } & \multicolumn{2}{|c|}{ r13i1p1f1 } & \multicolumn{2}{|c|}{ r15i1p1f1 } \\
\hline & Bias & MAE & Bias & MAE & Bias & MAE & Bias & MAE & Bias & MAE \\
\hline Annual & -1.32 & 1.81 & -0.89 & 1.60 & -1.11 & 1.71 & -0.91 & 1.60 & -0.97 & 1.63 \\
\hline DJF & -1.62 & 2.66 & -1.07 & 2.48 & -1.37 & 2.55 & -1.12 & 2.46 & -1.17 & 2.47 \\
\hline MAM & -1.70 & 2.36 & -1.29 & 2.14 & -1.48 & 2.25 & -1.30 & 2.14 & -1.38 & 2.18 \\
\hline JJA & -0.83 & 1.74 & -0.51 & 1.60 & -0.66 & 1.66 & -0.54 & 1.61 & -0.58 & 1.61 \\
\hline SON & -1.12 & 1.78 & -0.69 & 1.57 & -0.91 & 1.67 & -0.66 & 1.55 & -0.77 & 1.60 \\
\hline
\end{tabular}

aln each case the model data are compared with CRU_ts4.03 observational data for the period 1901-2014. The temperature data are confined to land points and exclude Antarctica.

(a)

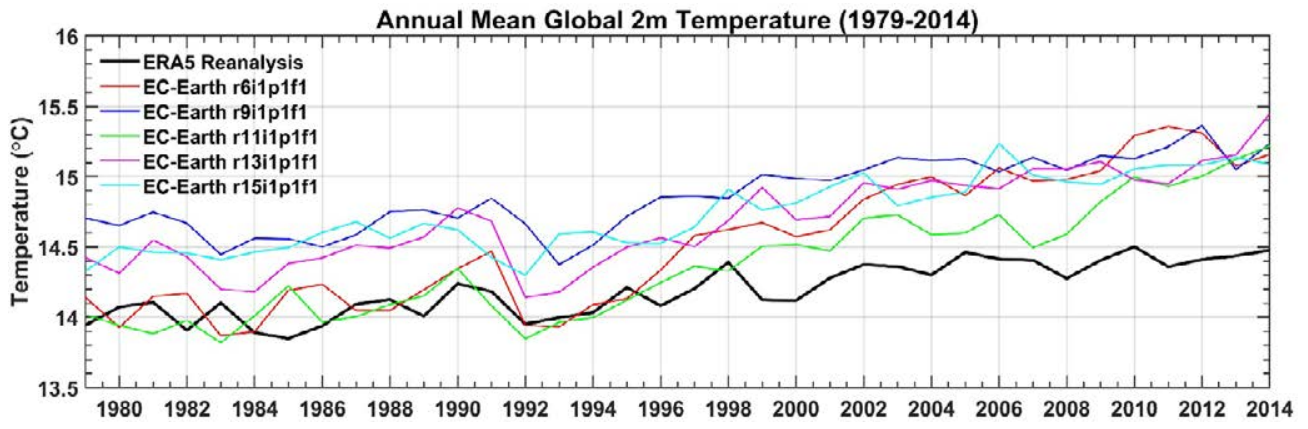

(b)

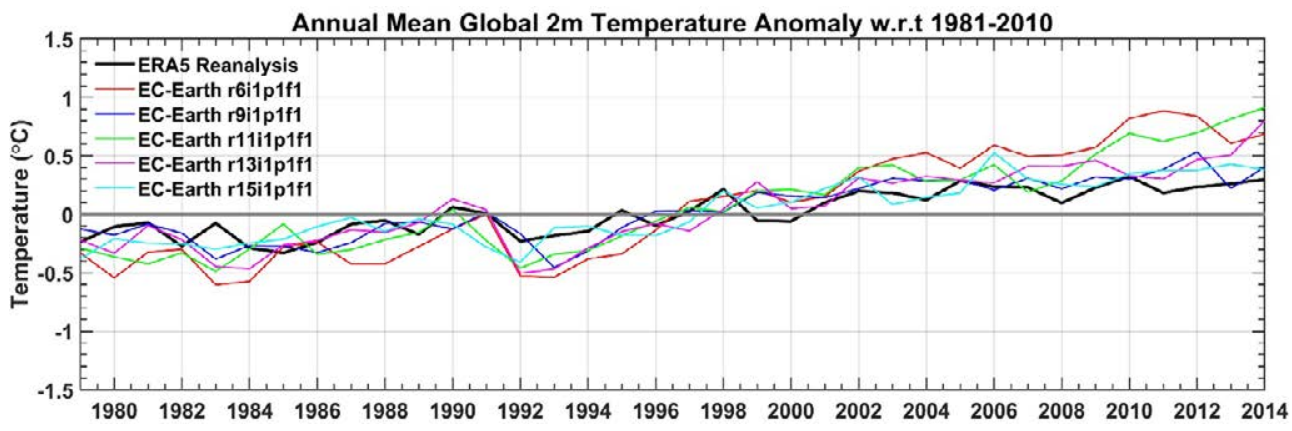

Figure 2.10. Comparison of EC-Earth ensemble members with ERA5 reanalysis data for the period 1979-2014: (a) 2-m temperature and (b) 2-m temperature anomalies with respect to the 30-year period 1981-2010. 
(a)

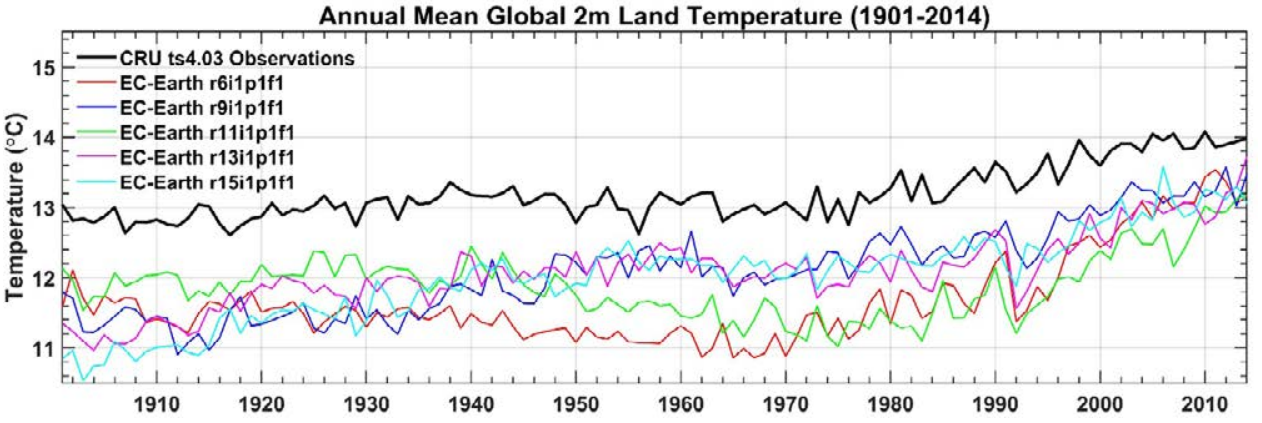

(b)

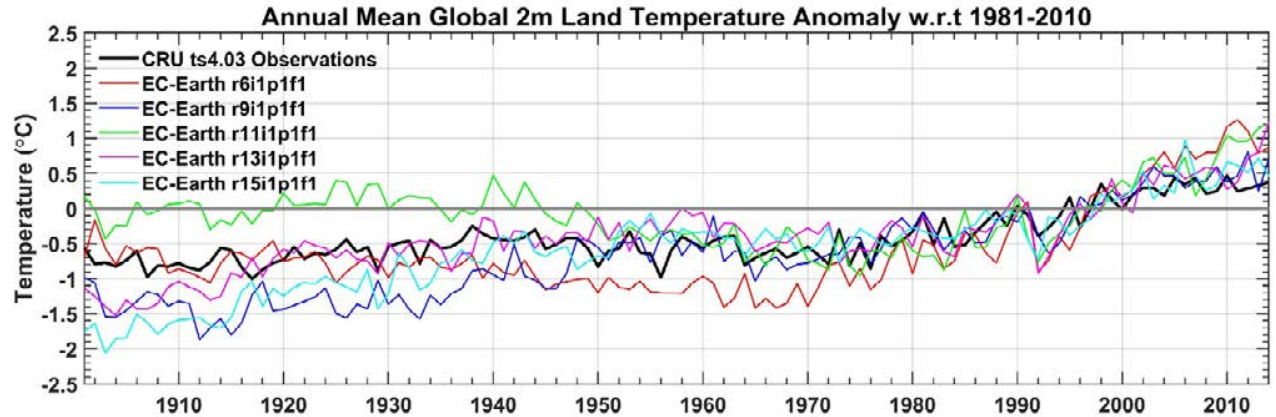

Figure 2.11. Comparison of EC-Earth ensemble members with CRU_ts4.03 observations for the period 1901-2014: (a) 2-m land temperature and (b) 2-m land temperature anomalies with respect to the 30-year period 1981-2010. The temperature data are confined to land points and exclude Antarctica.

\subsubsection{Precipitation validations}

Figure 2.12 presents the spatial distribution of annual precipitation ( $\mathrm{mm} /$ day) for the ERA5 dataset, the CRU dataset and each of the five EC-Earth ensemble members for the 36-year period 1979-2014. All EC-Earth ensemble members accurately capture the magnitude and spatial characteristics of the historical precipitation climate. This is confirmed in Figures 2.13 and 2.14, showing the ERA5 and CRU bias, respectively. The largest differences are noted around the Intertropical Convergence Zone (ITCZ), in particular over the ITCZ ocean regions, the north-east region of South America (dry bias), the remaining regions of South America (small wet bias) and south/ central Africa (small wet bias). Validations for DJF and JJA are presented in Figures 2.15-2.20 and show a similar signal to the annual results, with enhanced biases noted over the ICTZ regions. The wet/dry bands around the ICTZ (e.g. Figures 2.13, 2.16 and 2.19) suggest that the precipitation biases result from a slight incorrect positioning of the ICTZ as opposed to an error in the magnitude of precipitation amounts. All EC-Earth ensemble members are similar, with small variations between members.
The annual and seasonal overall global bias and MAE statistics relative to ERA5 data (1979-2014) for each of the five ensemble members are presented in Table 2.3. The bias statistics range from $-0.055 \mathrm{~mm} /$ day (JJA, r11i1p1f1) to $0.061 \mathrm{~mm} /$ day (DJF, r15i1p1f1). The MAE statistics range from $0.537 \mathrm{~mm} /$ day (annual, r15i1p1f1) to $0.879 \mathrm{~mm} /$ day (MAM, r11i1p1f1). The percentage bias and MAE statistics are presented in Table 2.4. The percentage bias statistics range from $2.5 \%$ (SON, r11i1p1f1) to 16.4\% (MAM, r9i1p1f1 and r11i1p1f1). The percentage MAE statistics range from $26.2 \%$ (SON, r15i1p1f1) to 44.3\% (MAM, r11i1p1f1). Similarly, the CRU statistics for the period 1901-2014 are presented in Table 2.5. The bias values range from $-0.006 \mathrm{~mm} /$ day (JJA, r13i1p1f1) to $0.26 \mathrm{~mm} /$ day (DJF, r9i1p1f1). The MAE statistics range from $0.662 \mathrm{~mm} /$ day (annual, r13i1p1f1) to $0.858 \mathrm{~mm} /$ day (JJA, r11i1p1f1 and r15i1p1f1). The percentage bias and MAE statistics are not presented for CRU data as these datasets are made available with units of $\mathrm{mm} / \mathrm{month}$ with a precision of one decimal place. This rounding results in zero values over many dry areas; hence, a quotient cannot be calculated. 
(a)

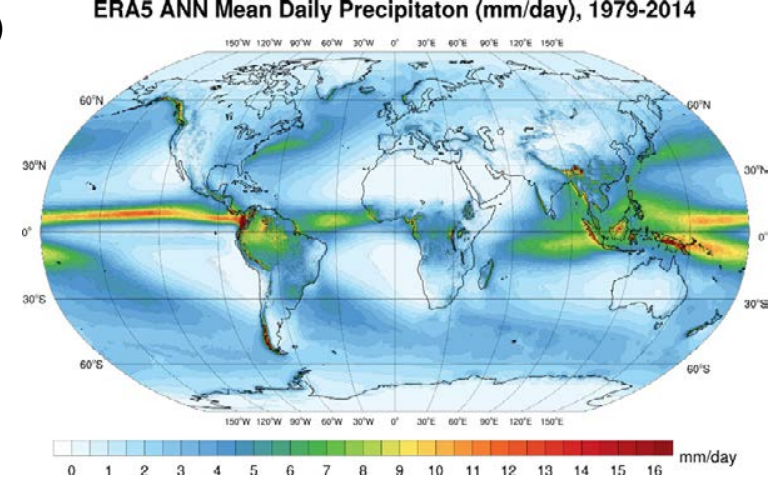

(c)

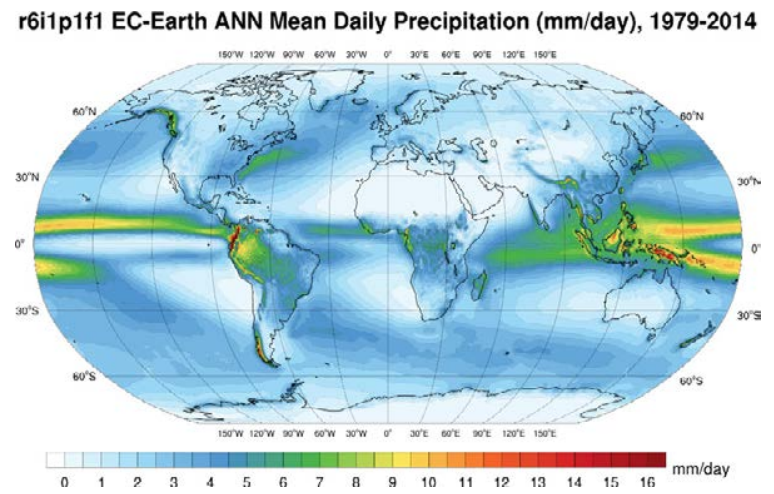

(e)

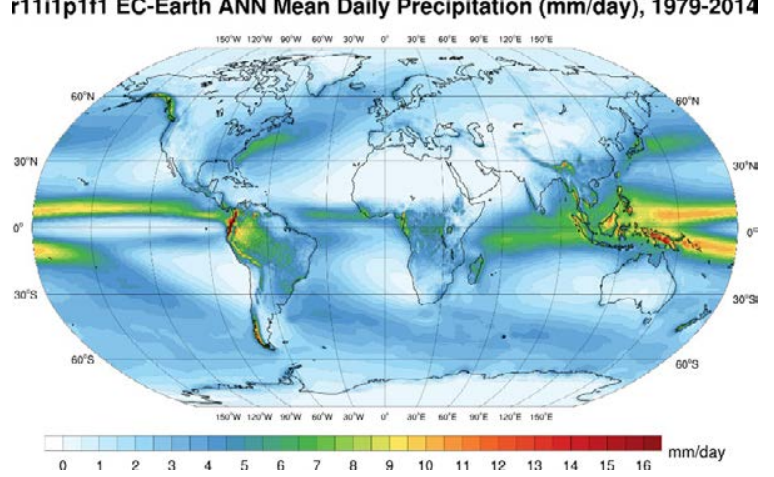

(g)

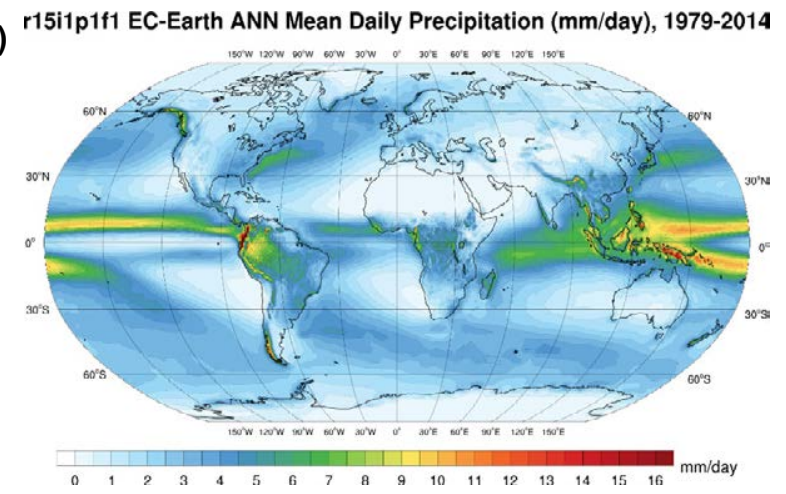

(b)

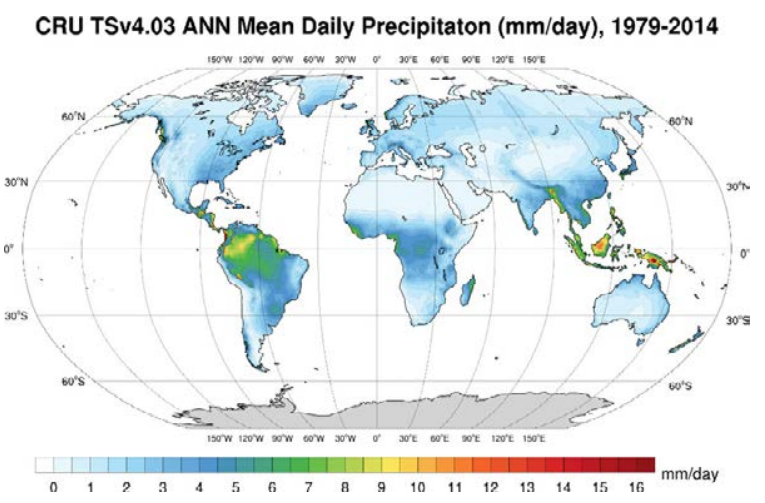

(d)
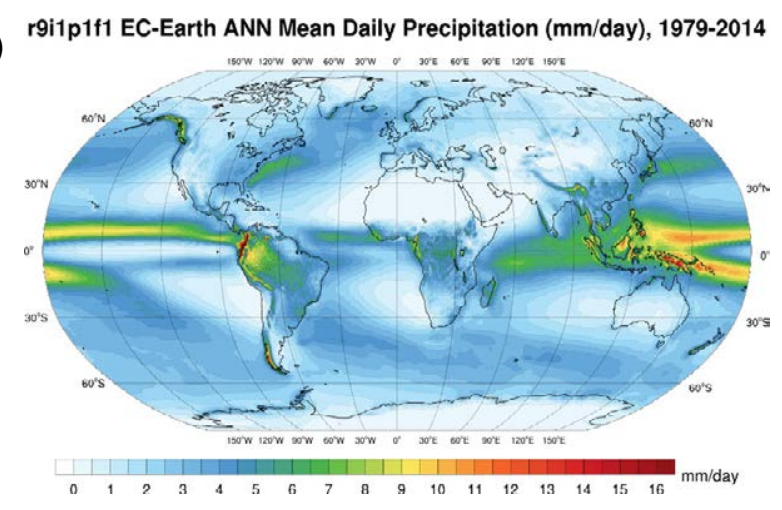

(f)

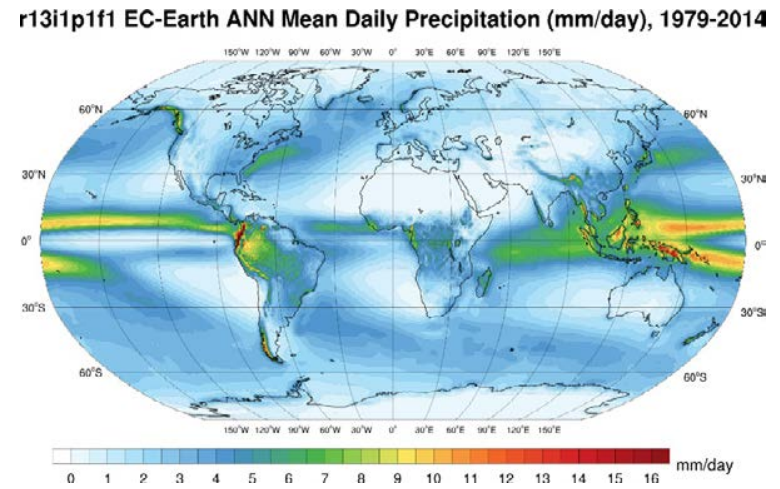

Figure 2.12. Annual mean daily precipitation (mm/day), 1979-2014: (a) ERA5 reanalysis, (b) CRU_ts4.03 observations, (c) EC-Earth r6i1p1f1, (d) EC-Earth r9i1p1f1, (e) EC-Earth r11i1p1f1, (f) EC-Earth r13i1p1f1 and (g) EC-Earth r15i1p1f1. 
(a)

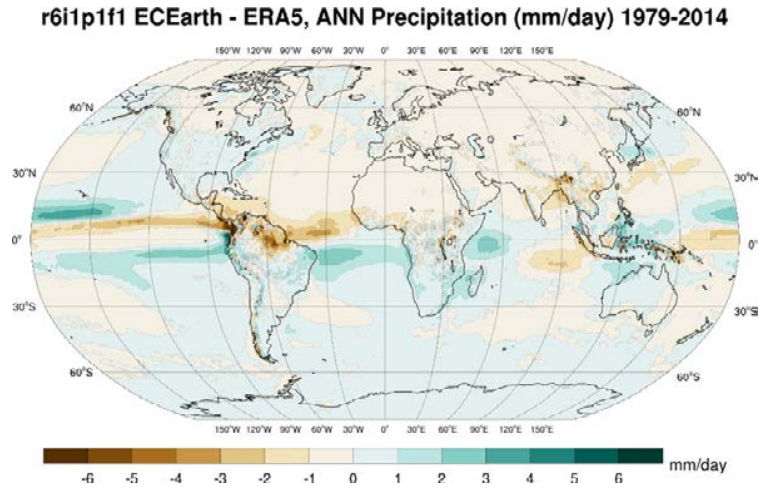

(c)

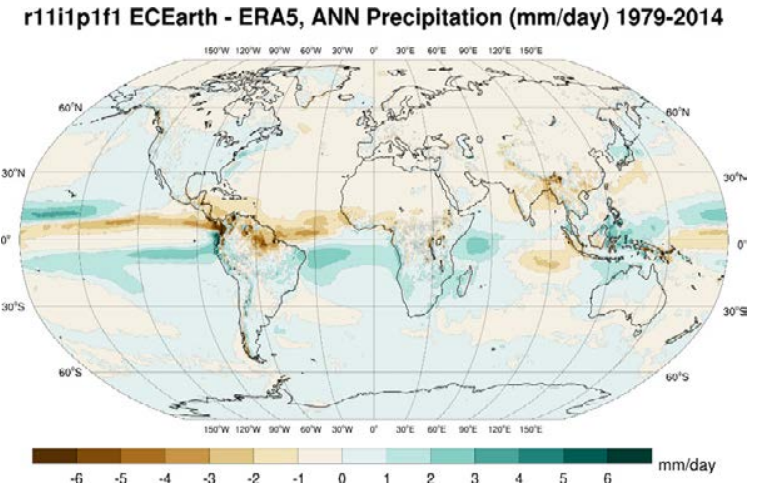

(e)

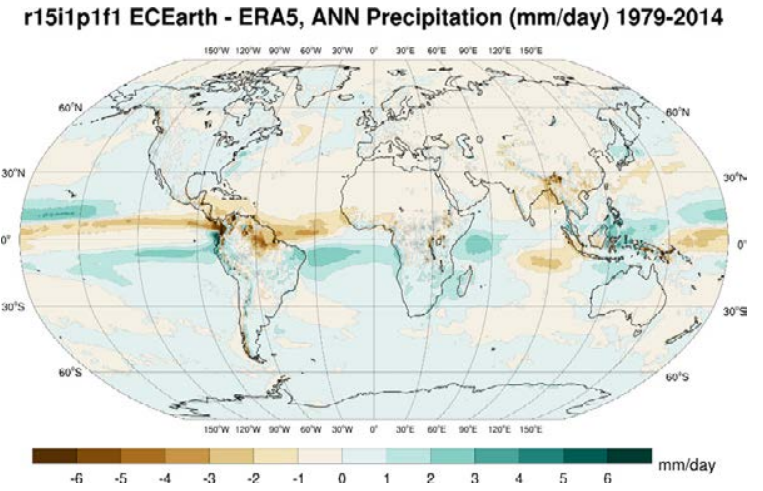

(b)

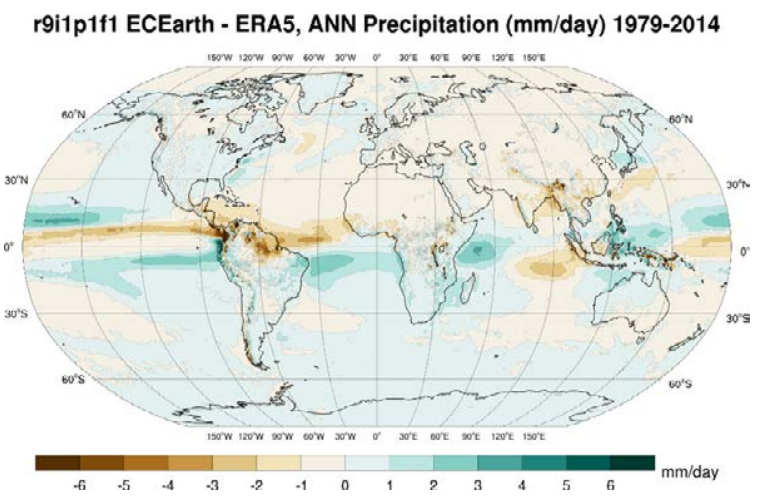

(d)

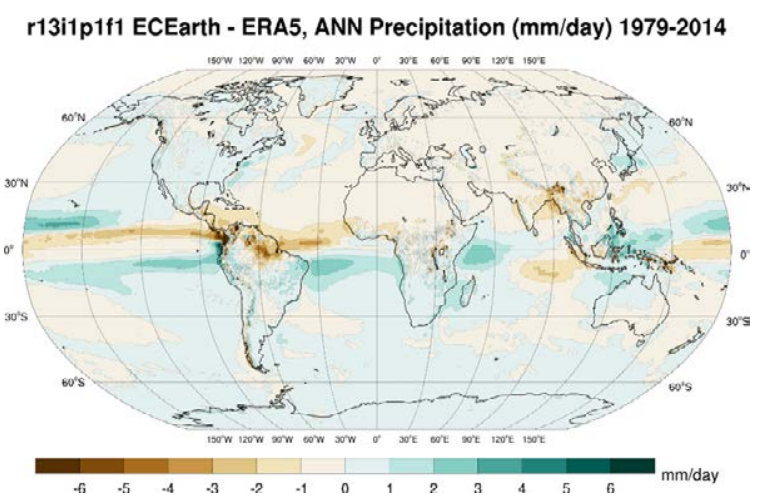

Figure 2.13. EC-Earth annual mean daily precipitation (mm/day) bias, 1979-2014 (ERA5 reanalysis minus EC-Earth): (a) EC-Earth r6i1p1f1, (b) EC-Earth r9i1p1f1, (c) EC-Earth r11i1p1f1, (d) EC-Earth r13i1p1f1 and (e) EC-Earth r15i1p1f1. 
(a)

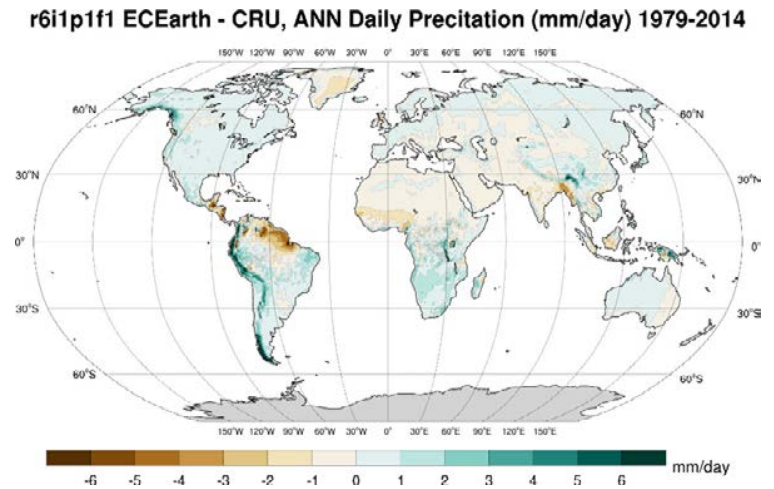

(c)
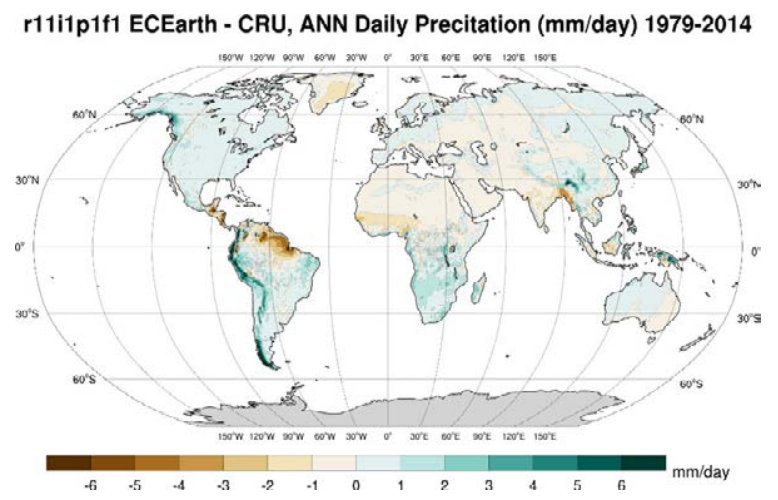

(e)

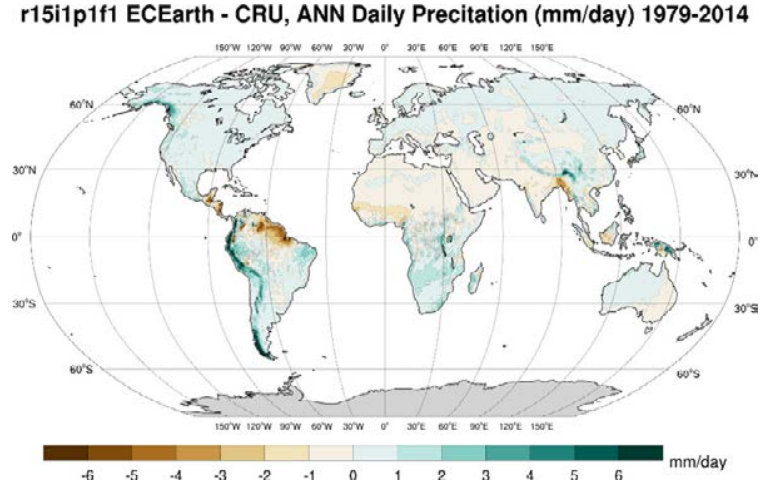

(b)

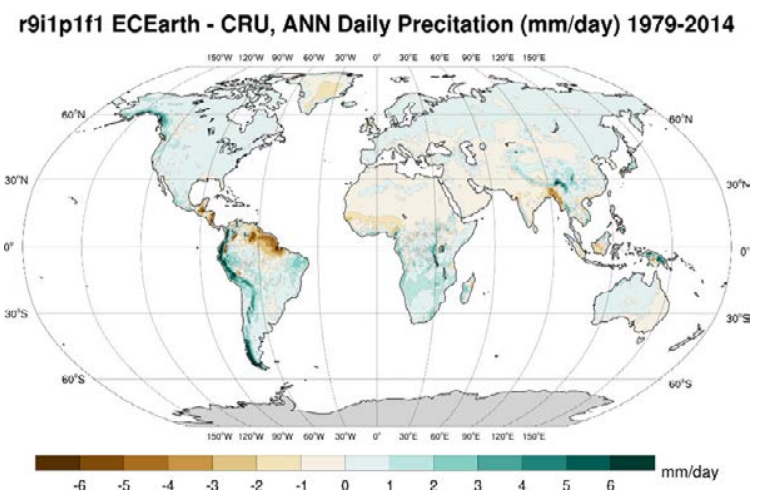

(d)

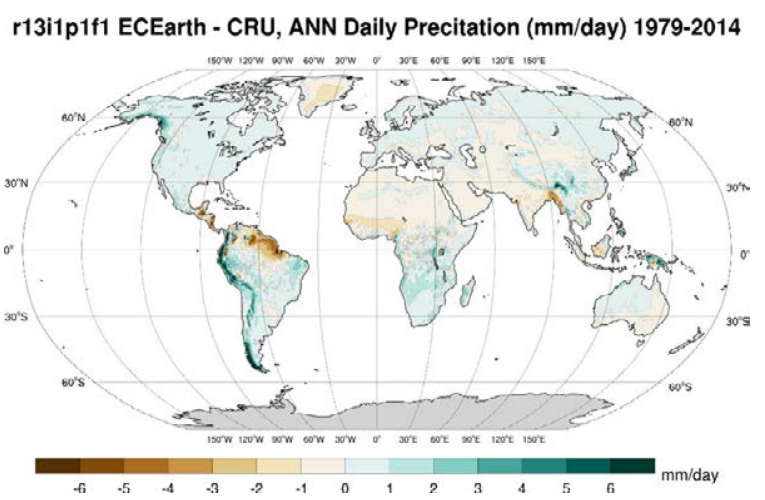

Figure 2.14. EC-Earth annual mean daily precipitation (mm/day) bias, 1979-2014 (EC-Earth minus CRU_ts4.03): (a) EC-Earth r6i1p1f1, (b) EC-Earth r9i1p1f1, (c) EC-Earth r11i1p1f1, (d) EC-Earth r13i1p1f1 and (e) EC-Earth r15i1p1f1. 
(a)

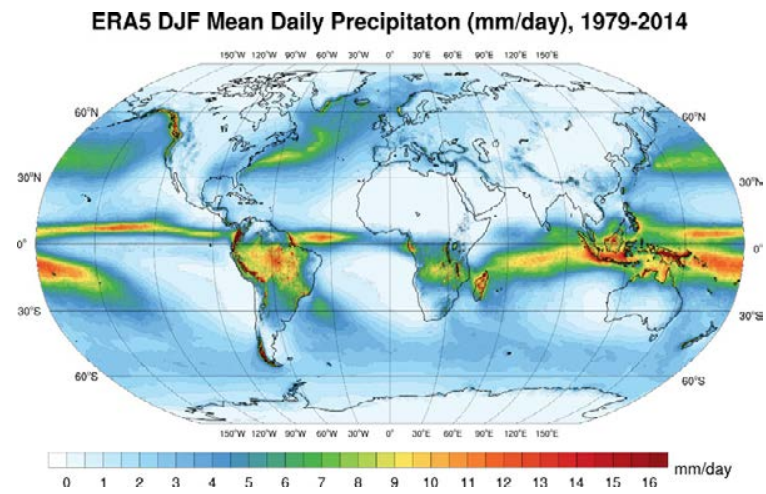

(c)

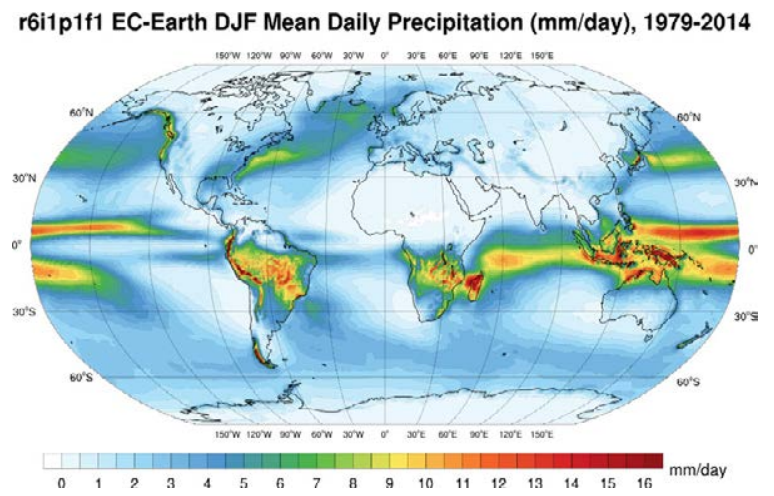

(e)

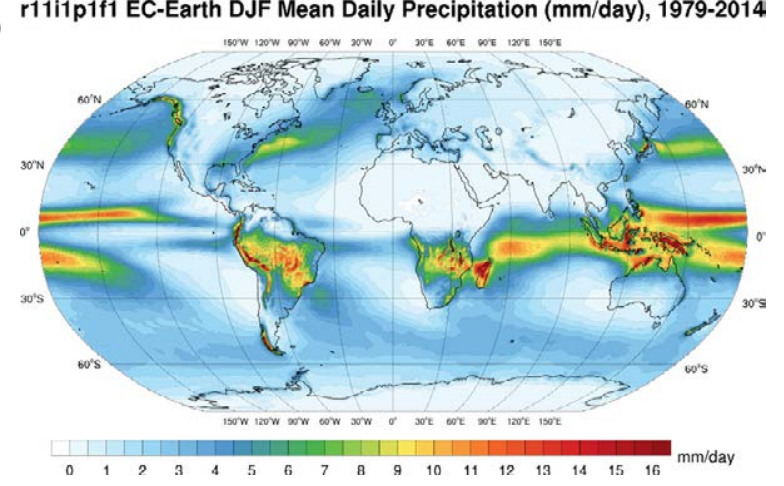

(g)

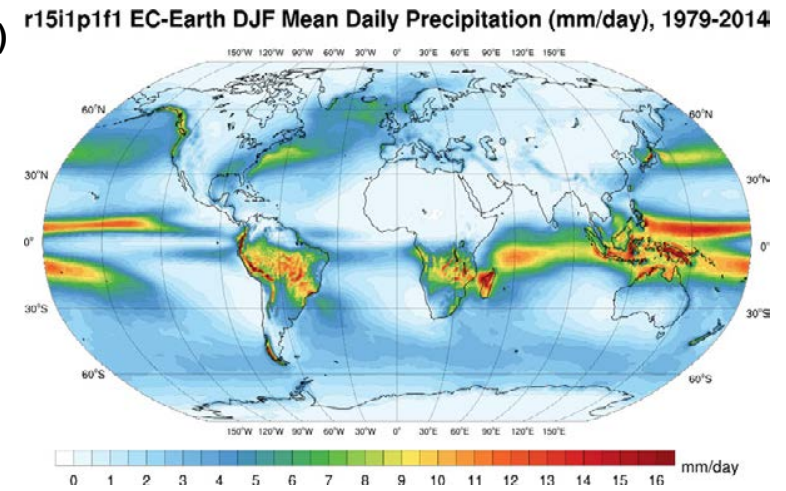

(b)

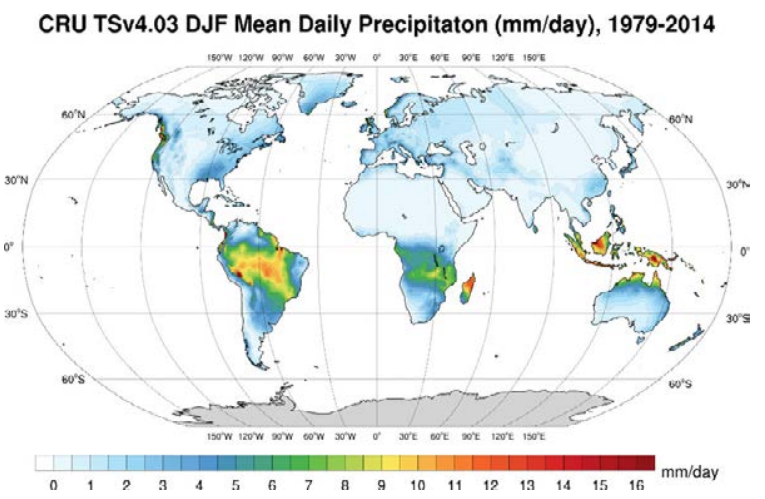

(d)
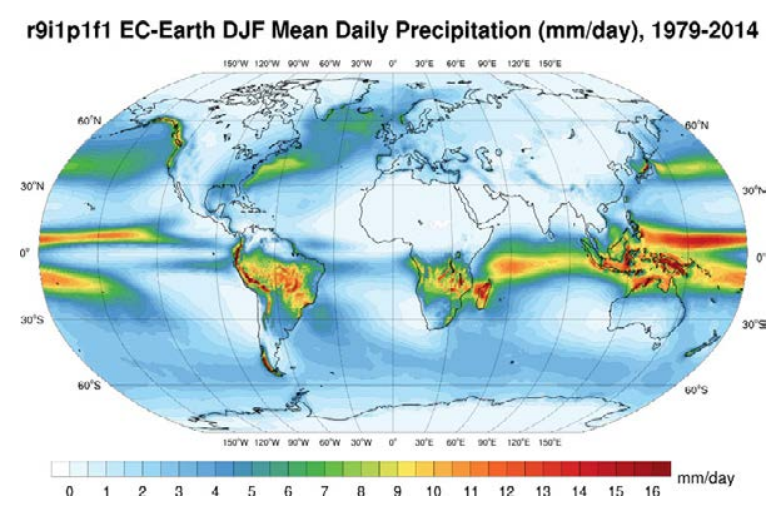

(f)

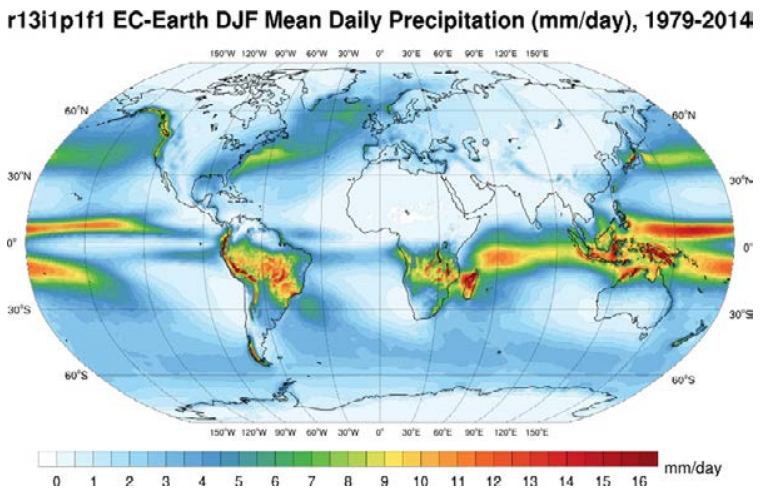

Figure 2.15. Mean daily precipitation (mm/day) for DJF, 1979-2014: (a) ERA5 reanalysis, (b) CRU_ts4.03 observations, (c) EC-Earth r6i1p1f1, (d) EC-Earth r9i1p1f1, (e) EC-Earth r11i1p1f1, (f) EC-Earth r13i1p1f1 and (g) EC-Earth r15i1p1f1. 
(a)

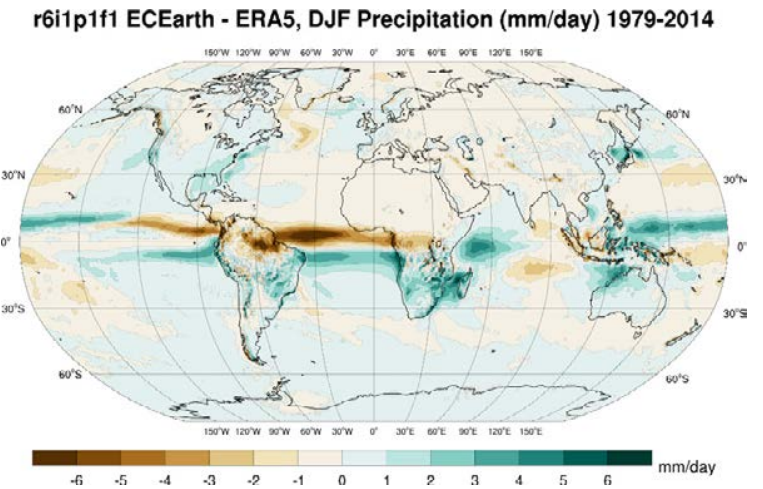

(c)

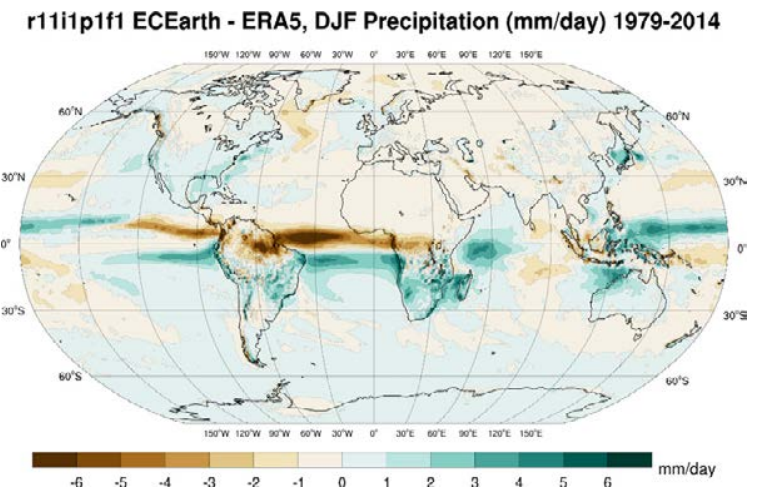

(b)

r9i1p1f1 ECEarth - ERA5, DJF Precipitation (mm/day) 1979-2014

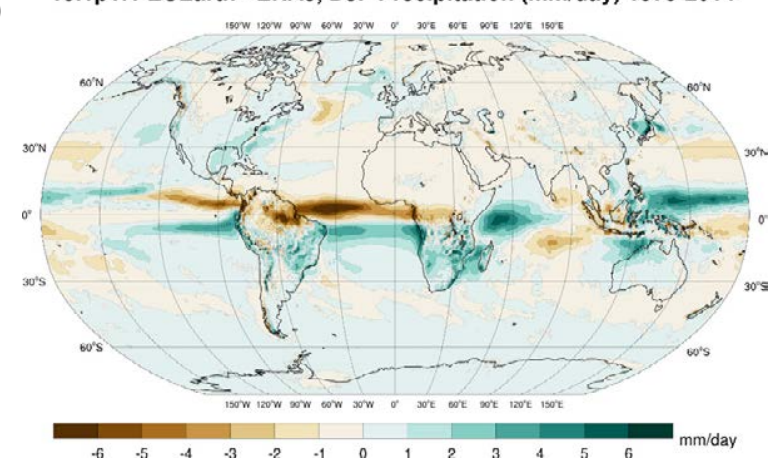

(d)

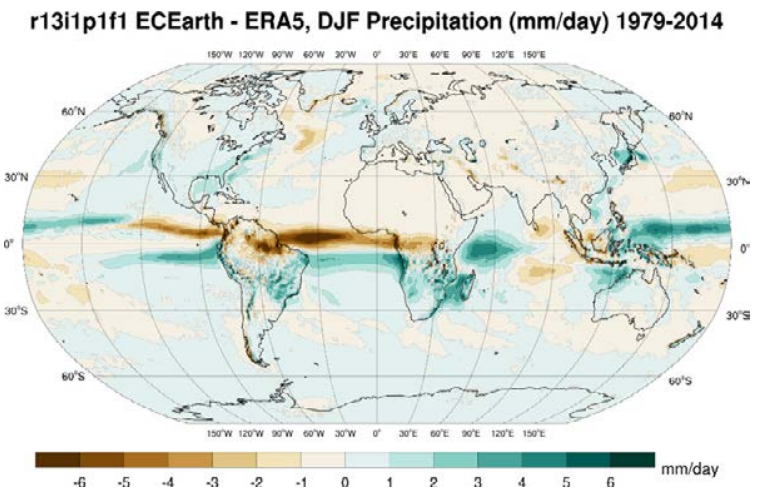

(e)

r15i1p1f1 ECEarth - ERA5, DJF Precipitation (mm/day) 1979-2014

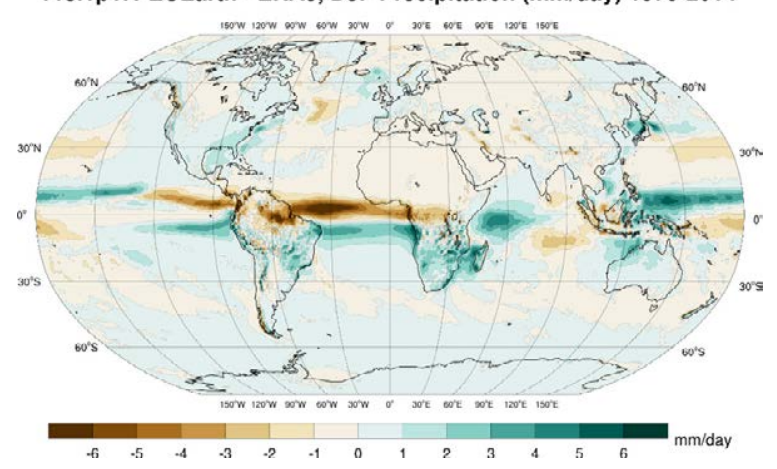

Figure 2.16. EC-Earth mean daily precipitation ( $\mathrm{mm} /$ day) bias for DJF, 1979-2014 (ERA5 reanalysis minus EC-Earth): (a) EC-Earth r6i1p1f1, (b) EC-Earth r9i1p1f1, (c) EC-Earth r11i1p1f1, (d) EC-Earth r13i1p1f1 and (e) EC-Earth r15i1p1f1. 
(a)

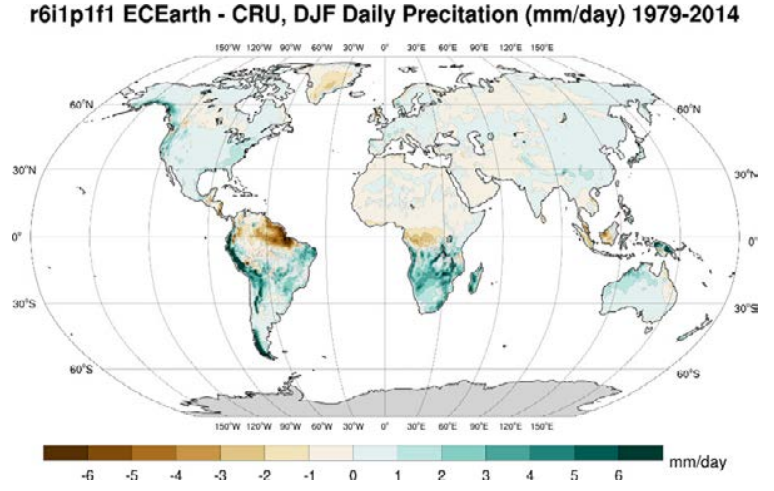

(c)
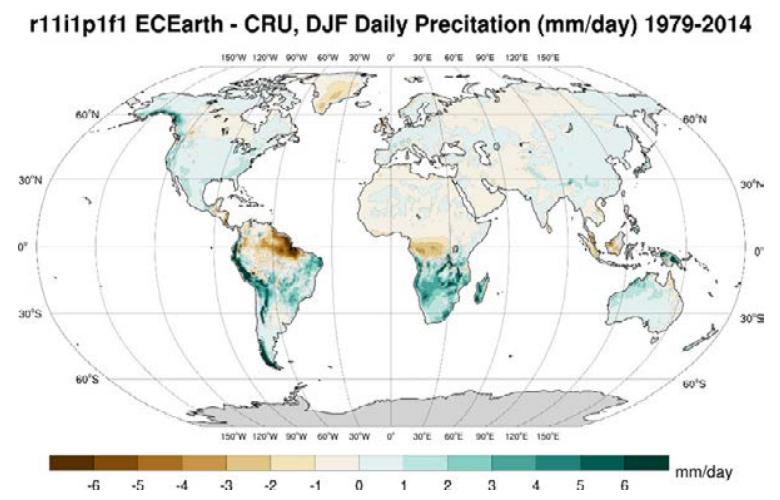

(e)

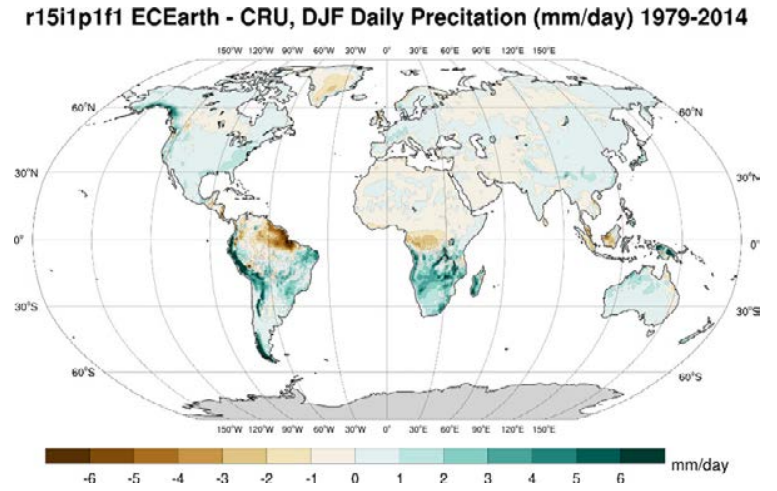

(b)

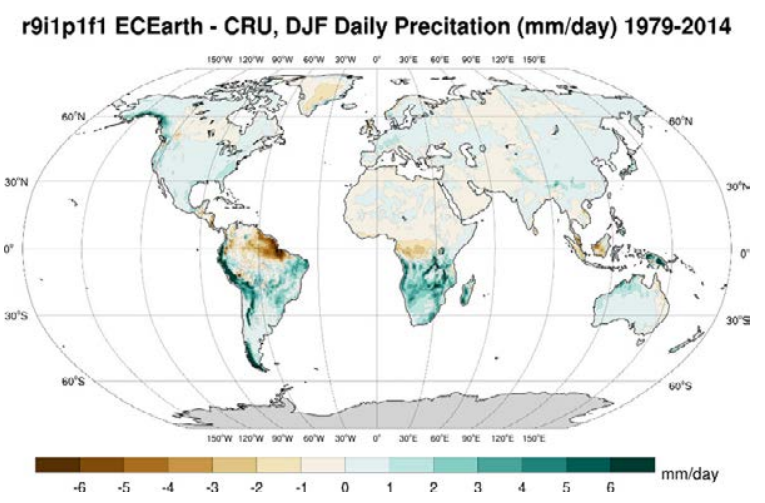

(d)
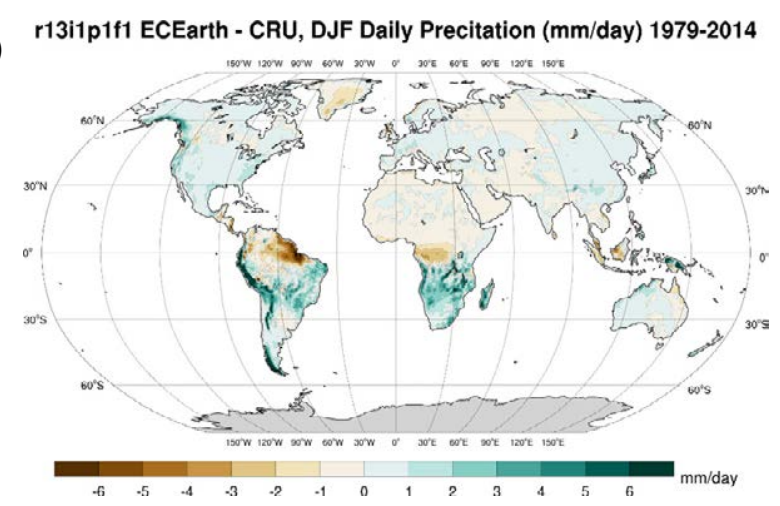

Figure 2.17. EC-Earth mean daily precipitation (mm/day) bias for DJF, 1979-2014 (EC-Earth minus CRU_ts4.03): (a) EC-Earth r6i1p1f1, (b) EC-Earth r9i1p1f1, (c) EC-Earth r11i1p1f1, (d) EC-Earth r13i1p1f1 and (e) EC-Earth r15i1p1f1. 
(a)

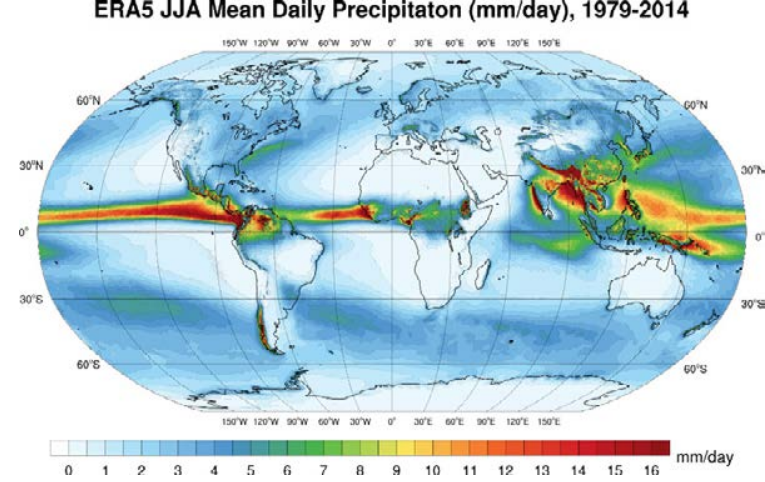

(c)

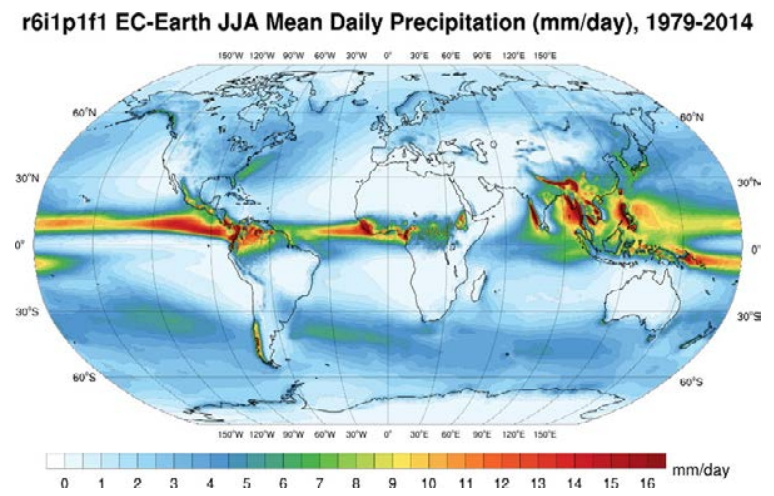

(e)

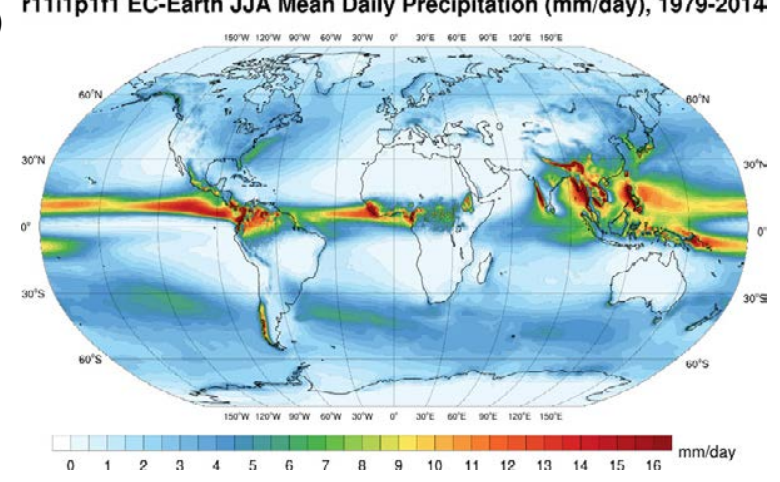

(g)

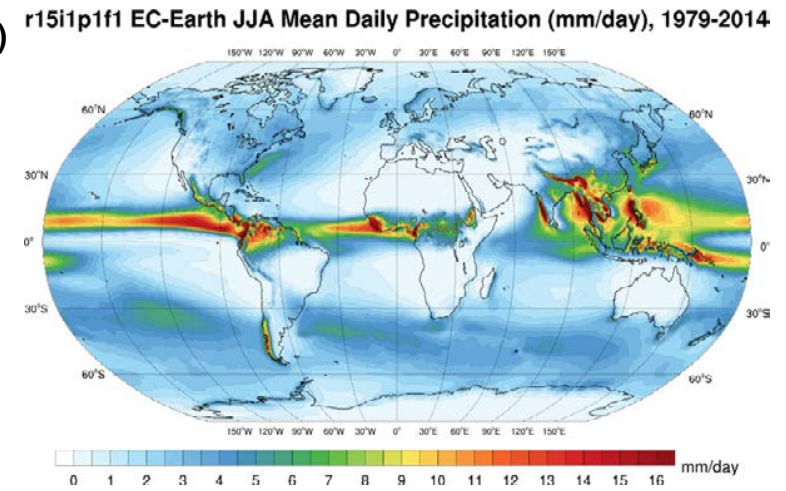

(b)

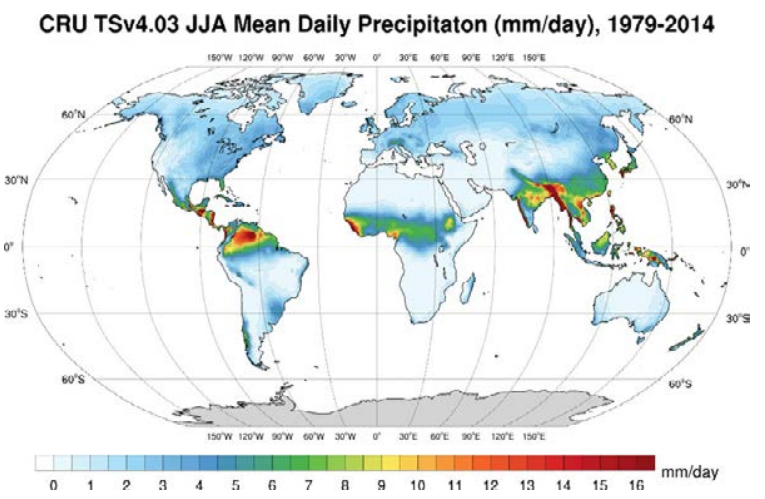

(d)
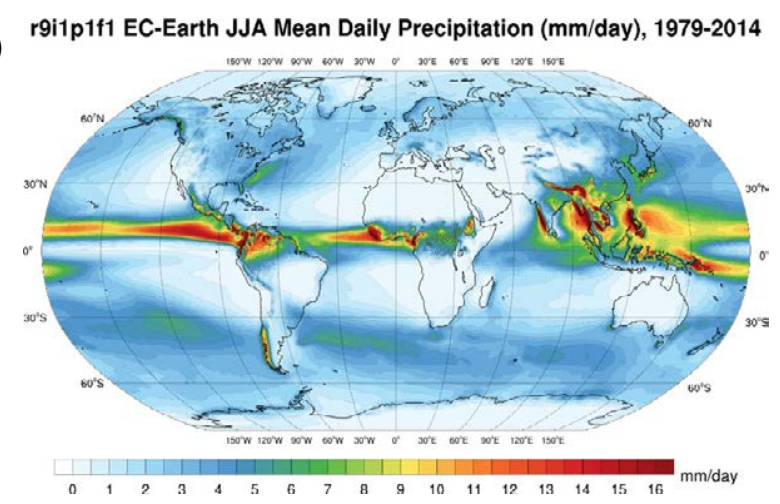

(f)

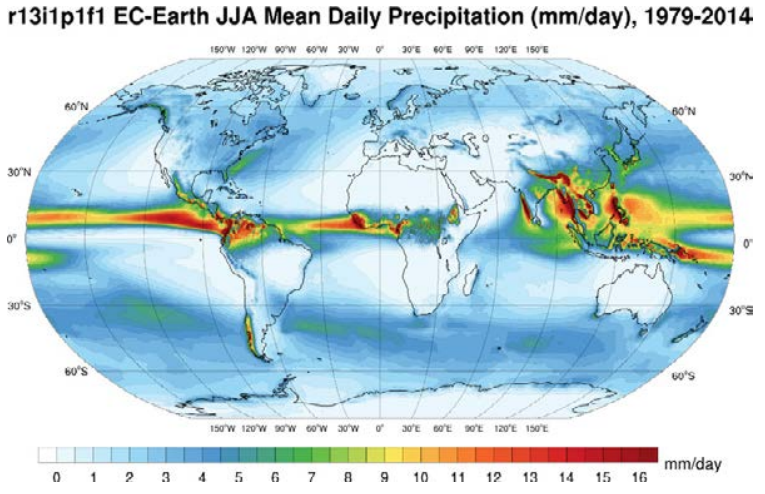

Figure 2.18. Mean daily precipitation (mm/day) for JJA, 1979-2014: (a) ERA5 reanalysis, (b) CRU_ts4.03 observations, (c) EC-Earth r6i1p1f1, (d) EC-Earth r9i1p1f1, (e) EC-Earth r11i1p1f1, (f) EC-Earth r13i1p1f1 and (g) EC-Earth r15i1p1f1. 
(a)

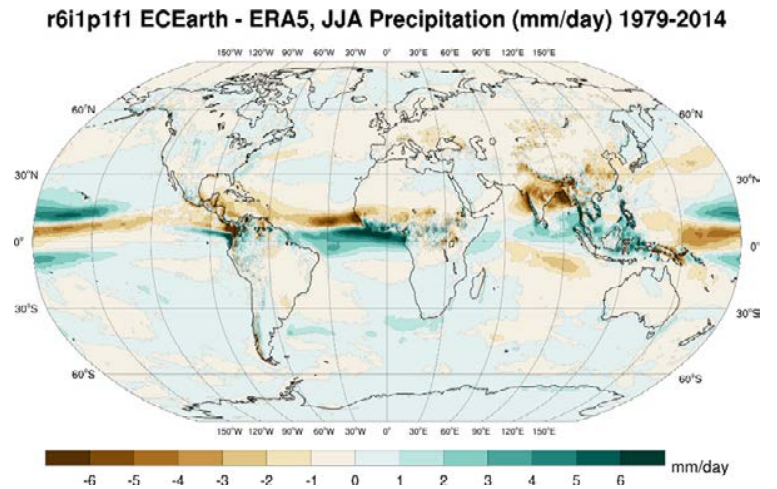

(c)

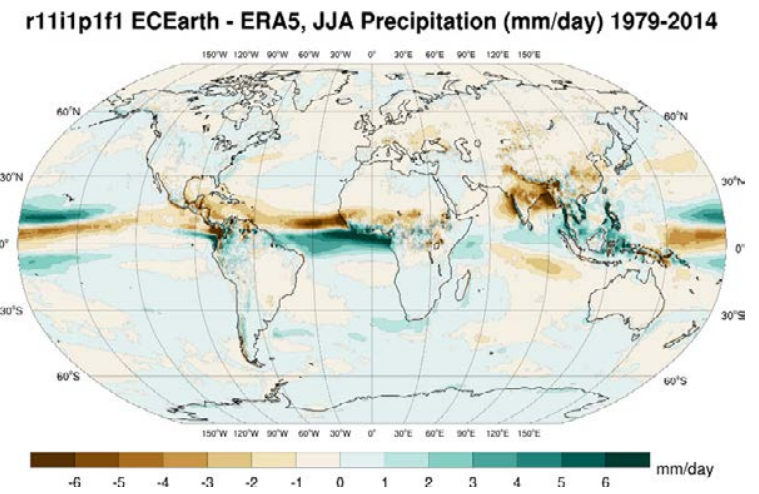

(b)

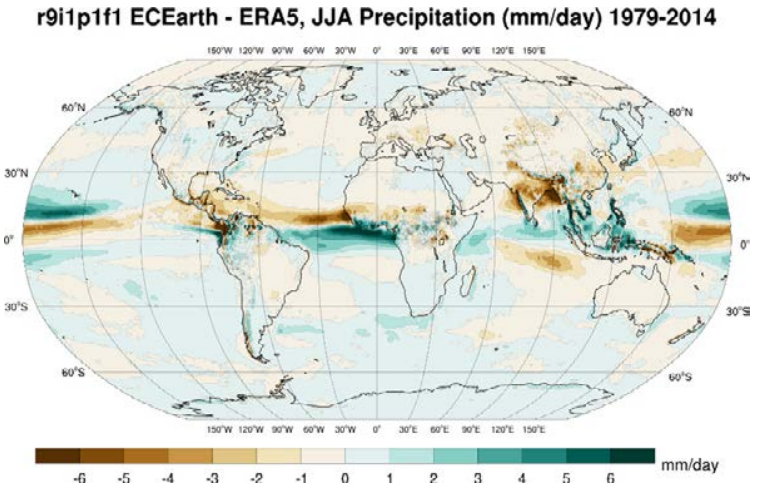

(d)

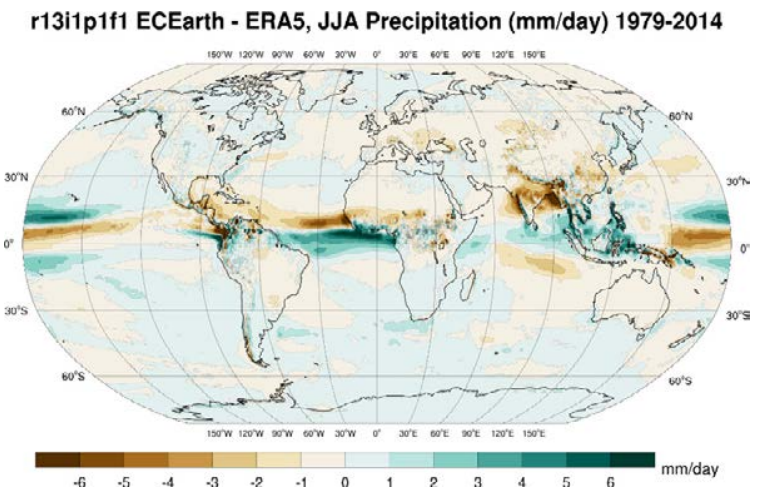

(e)

r15i1p1f1 ECEarth - ERA5, JJA Precipitation (mm/day) 1979-2014

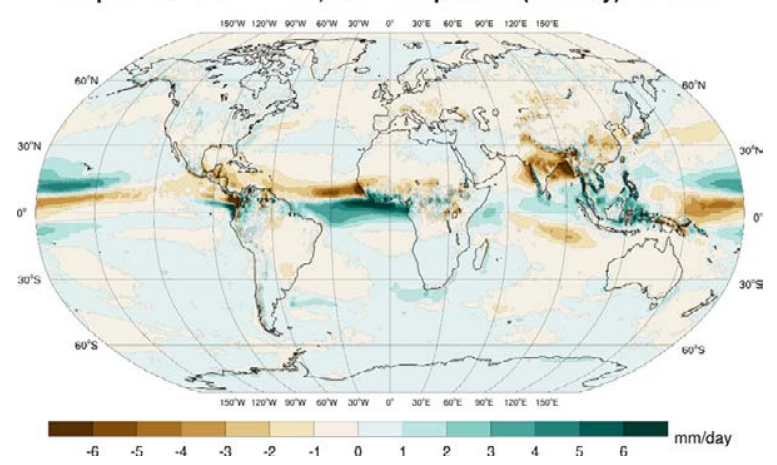

Figure 2.19. EC-Earth mean daily precipitation ( $\mathrm{mm} /$ day) bias for JJA, 1979-2014 (ERA5 reanalysis minus EC-Earth): (a) EC-Earth r6i1p1f1, (b) EC-Earth r9i1p1f1, (c) EC-Earth r11i1p1f1, (d) EC-Earth r13i1p1f1 and (e) EC-Earth r15i1p1f1. 
(a)

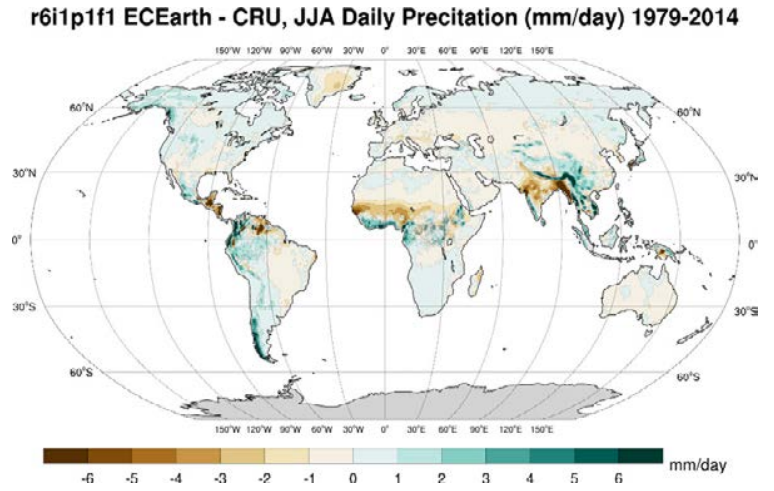

(c)

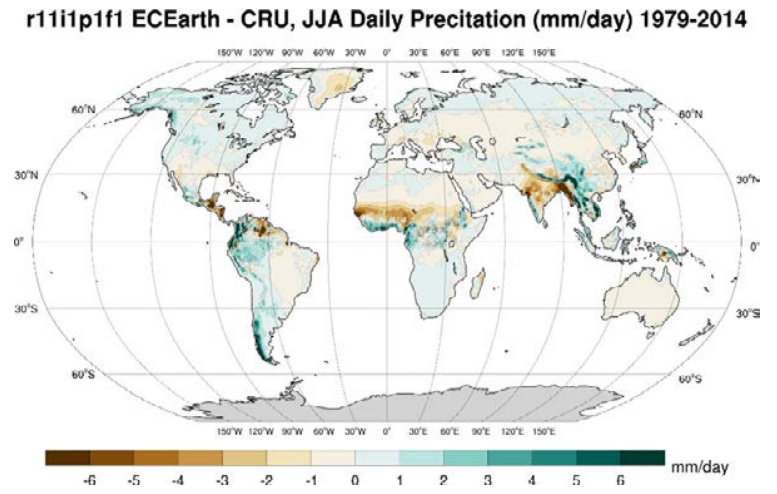

(e)

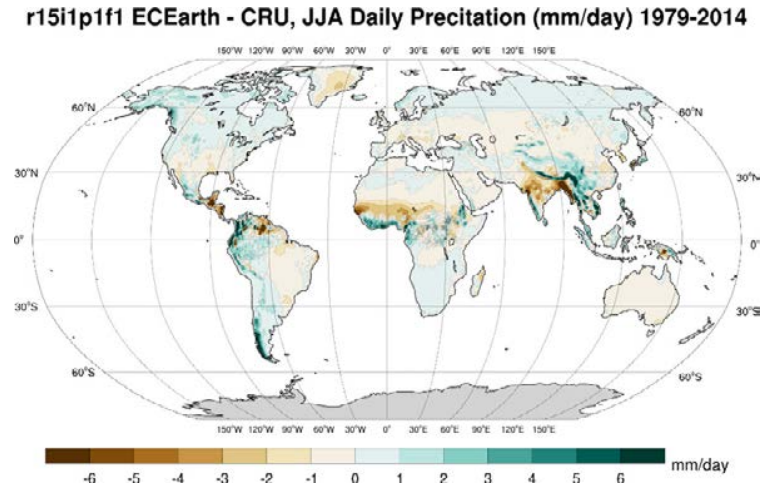

(b)

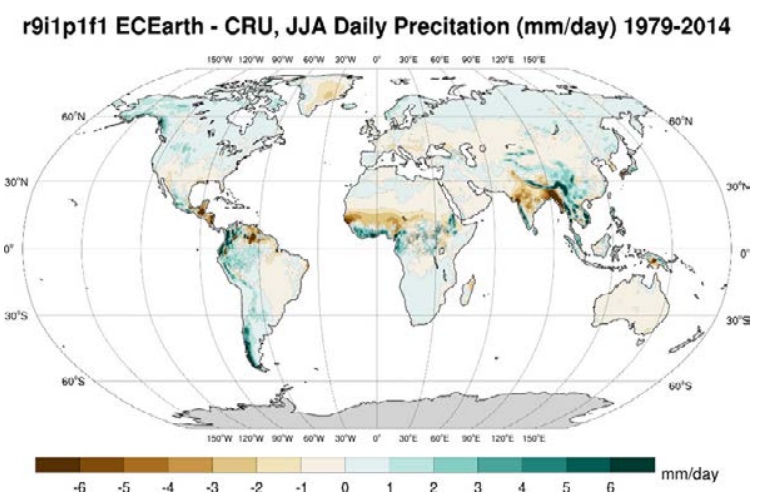

(d)

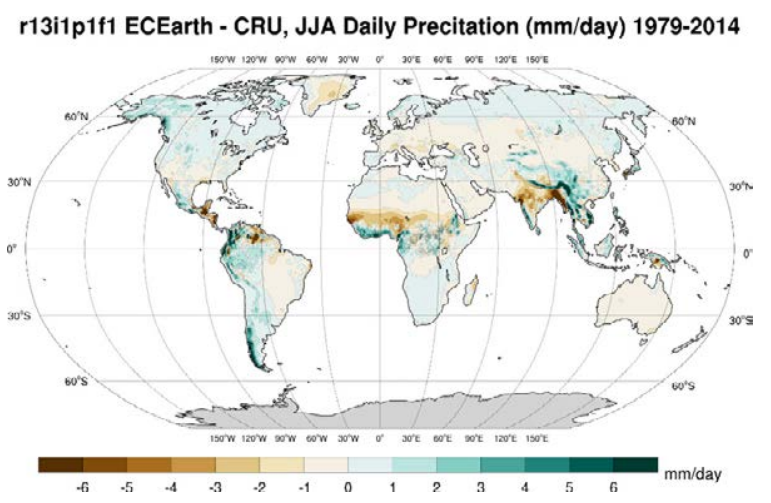

Figure 2.20. EC-Earth mean daily precipitation (mm/day) bias for JJA, 1979-2014 (EC-Earth minus CRU_ts4.03): (a) EC-Earth r6i1p1f1, (b) EC-Earth r9i1p1f1, (c) EC-Earth r11i1p1f1, (d) EC-Earth r13i1p1f1 and (e) EC-Earth r15i1p1f1.

Table 2.3. Mean global annual and seasonal daily precipitation bias and MAE ( $\mathrm{mm} / \mathrm{day})$ for each of the five EC-Earth ensemble members ${ }^{a}$

\begin{tabular}{|c|c|c|c|c|c|c|c|c|c|c|}
\hline & \multicolumn{2}{|c|}{ r6i1p1f1 } & \multicolumn{2}{|c|}{ r9i1p1f1 } & \multicolumn{2}{|c|}{ r11i1p1f1 } & \multicolumn{2}{|c|}{ r13i1p1f1 } & \multicolumn{2}{|c|}{ r15i1p1f1 } \\
\hline & Bias & MAE & Bias & MAE & Bias & MAE & Bias & MAE & Bias & MAE \\
\hline Annual & -0.001 & 0.549 & 0.018 & 0.556 & -0.012 & 0.567 & 0.004 & 0.540 & 0.008 & 0.537 \\
\hline DJF & 0.051 & 0.666 & 0.071 & 0.685 & 0.037 & 0.677 & 0.054 & 0.655 & 0.061 & 0.666 \\
\hline MAM & 0.027 & 0.859 & 0.042 & 0.857 & 0.017 & 0.879 & 0.029 & 0.845 & 0.033 & 0.840 \\
\hline JJA & -0.043 & 0.678 & -0.018 & 0.678 & -0.055 & 0.695 & -0.032 & 0.667 & -0.028 & 0.659 \\
\hline SON & -0.040 & 0.616 & -0.022 & 0.640 & -0.048 & 0.630 & -0.032 & 0.622 & -0.031 & 0.602 \\
\hline
\end{tabular}

an each case the model data are compared with ERA5 reanalysis data for the period 1979-2014. 
Table 2.4. Mean global annual and seasonal daily precipitation percentage bias and MAE (\%) for each of the five EC-Earth ensemble members ${ }^{a}$

\begin{tabular}{|c|c|c|c|c|c|c|c|c|c|c|}
\hline & \multicolumn{2}{|c|}{ r6i1p1f1 } & \multicolumn{2}{|c|}{ r9i1p1f1 } & \multicolumn{2}{|c|}{ r11i1p1f1 } & \multicolumn{2}{|c|}{ r13i1p1f1 } & \multicolumn{2}{|c|}{ r15i1p1f1 } \\
\hline & Bias & MAE & Bias & MAE & Bias & MAE & Bias & MAE & Bias & MAE \\
\hline Annual & 9.1 & 27.5 & 10.1 & 27.6 & 8.8 & 28.9 & 8.5 & 27.1 & 9.0 & 26.8 \\
\hline DJF & 12.0 & 34.1 & 12.8 & 34.7 & 12.1 & 35.8 & 10.9 & 33.2 & 11.8 & 33.6 \\
\hline MAM & 16.3 & 42.7 & 16.4 & 42.1 & 16.4 & 44.3 & 15.2 & 42.2 & 15.5 & 41.5 \\
\hline JJA & 9.1 & 32.2 & 9.0 & 32.2 & 8.5 & 33.5 & 6.5 & 30.8 & 8.9 & 31.9 \\
\hline SON & 3.2 & 26.9 & 4.6 & 27.5 & 2.5 & 27.8 & 2.7 & 27.0 & 3.2 & 26.2 \\
\hline
\end{tabular}

aln each case the model data are compared with ERA5 reanalysis data for the period 1979-2014.

Table 2.5. Mean global annual and seasonal daily precipitation bias and MAE (mm/day) for each of the five EC-Earth ensemble members ${ }^{a}$

\begin{tabular}{|c|c|c|c|c|c|c|c|c|c|c|}
\hline & \multicolumn{2}{|l|}{ r6i1p1f1 } & \multicolumn{2}{|l|}{ r9i1p1f1 } & \multicolumn{2}{|c|}{ r11i1p1f1 } & \multicolumn{2}{|c|}{ r13i1p1f1 } & \multicolumn{2}{|c|}{ r15i1p1f1 } \\
\hline & Bias & MAE & Bias & MAE & Bias & MAE & Bias & MAE & Bias & MAE \\
\hline Annual & 0.124 & 0.667 & 0.147 & 0.669 & 0.130 & 0.664 & 0.143 & 0.662 & 0.138 & 0.663 \\
\hline DJF & 0.245 & 0.777 & 0.260 & 0.772 & 0.240 & 0.778 & 0.251 & 0.761 & 0.256 & 0.775 \\
\hline MAM & 0.143 & 0.840 & 0.157 & 0.851 & 0.134 & 0.839 & 0.160 & 0.842 & 0.147 & 0.850 \\
\hline JJA & -0.040 & 0.856 & -0.011 & 0.861 & -0.027 & 0.858 & -0.006 & 0.855 & -0.014 & 0.858 \\
\hline SON & 0.160 & 0.739 & 0.193 & 0.749 & 0.183 & 0.742 & 0.178 & 0.741 & 0.176 & 0.735 \\
\hline
\end{tabular}

aln each case the model data are compared with CRU_ts4.03 observational data for the period 1901-2014. The data are confined to land points and exclude Antarctica.

The ERA5 and EC-Earth mean global annual precipitation time series (1979-2014), presented in Figure 2.21a, demonstrate good agreement except for the period 1989-1996, when all EC-Earth ensemble members overestimate precipitation. In particular, the dry period around 1992 is not accurately resolved by EC-Earth. Figure $2.21 \mathrm{~b}$, the annual anomalies with respect to the 1981-2010 mean, shows good agreement between ERA5 and EC-Earth, with a clear rise in global precipitation evident from the early 1990s. The CRU and EC-Earth mean global annual precipitation time series (1901-2014), presented in Figure 2.22a, demonstrate a consistent overestimation of EC-Earth precipitation over land during 1850-1945 and 1985-2014. The precipitation anomalies for the period 1901-2014 (Figure 2.22b) all show a clear increase in precipitation over land from around 1995.

\subsubsection{0-m wind speed validations}

Figures 2.23-2.28 compare the EC-Earth 10-m wind speed $(\mathrm{m} / \mathrm{s})$ data with ERA5 data for the period 1979-2014. The figures demonstrate that all EC-Earth ensemble members accurately capture the magnitude and spatial characteristics of the historical 10-m wind speed climate for the annual, DJF and JJA time periods. The general trend is for EC-Earth to slightly overestimate wind speeds over land and underestimate wind speeds in the equatorial ocean regions.

The annual and seasonal overall global bias and MAE statistics relative to ERA5 data (1979-2014) for each of the five ensemble members are presented in Table 2.6. The bias statistics are all positive, ranging from $0.01 \mathrm{~m} / \mathrm{s}$ (JJA, r6i1p1f1) to $0.158 \mathrm{~m} / \mathrm{s}$ (DJF, r6i1p1f1). The MAE statistics range from $0.393 \mathrm{~m} / \mathrm{s}$ (annual, r15i1p1f1) to $0.537 \mathrm{~m} / \mathrm{s}$ (DJF, r11i1p1f1).

The ERA5 and EC-Earth mean global annual 10-m wind speed time series (1979-2014), presented in Figure 2.29a, demonstrate good agreement. However, all EC-Earth ensemble members exhibit a slight overestimation of $\sim 0.1 \mathrm{~m} / \mathrm{s}$ during the period 1979-1997. Figure 2.29b shows the annual anomalies with respect to the 1981-2010 mean; all ERA5 and EC-Earth annual values are within the range of $\pm 0.1 \mathrm{~m} / \mathrm{s}$. 
(a)

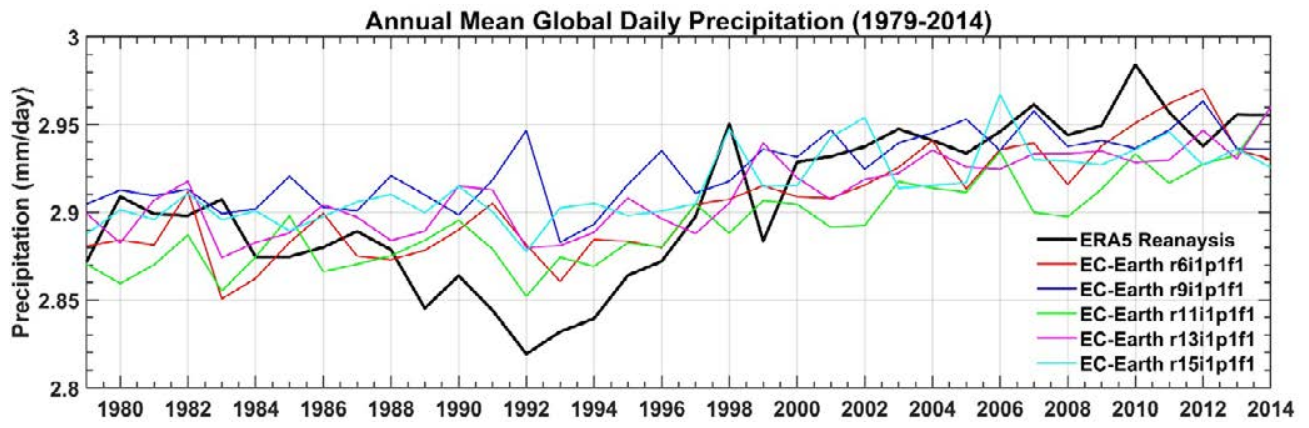

(b)

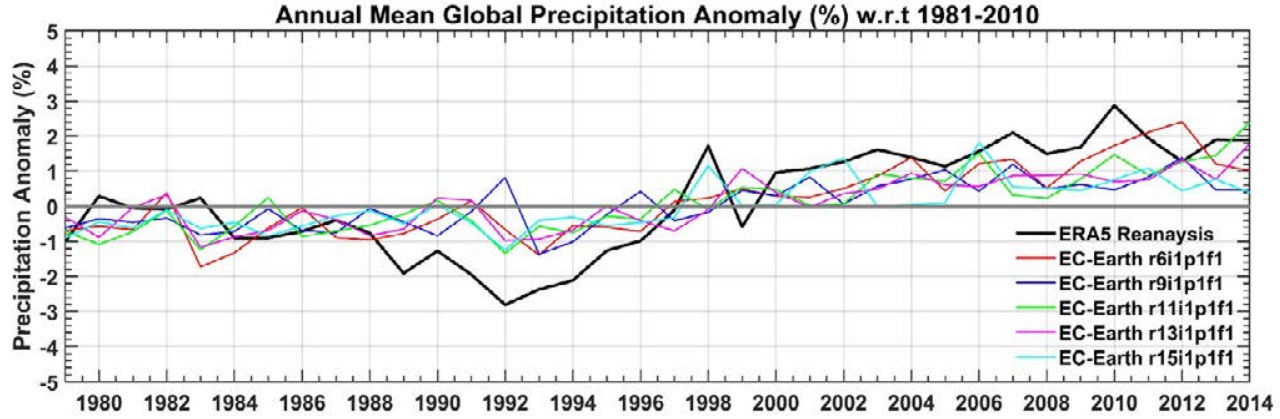

Figure 2.21. Comparison of EC-Earth ensemble members with ERA5 reanalysis data for the period 1979-2014: (a) precipitation and (b) precipitation anomalies (\%) with respect to the 30-year period 1981-2010.

(a)

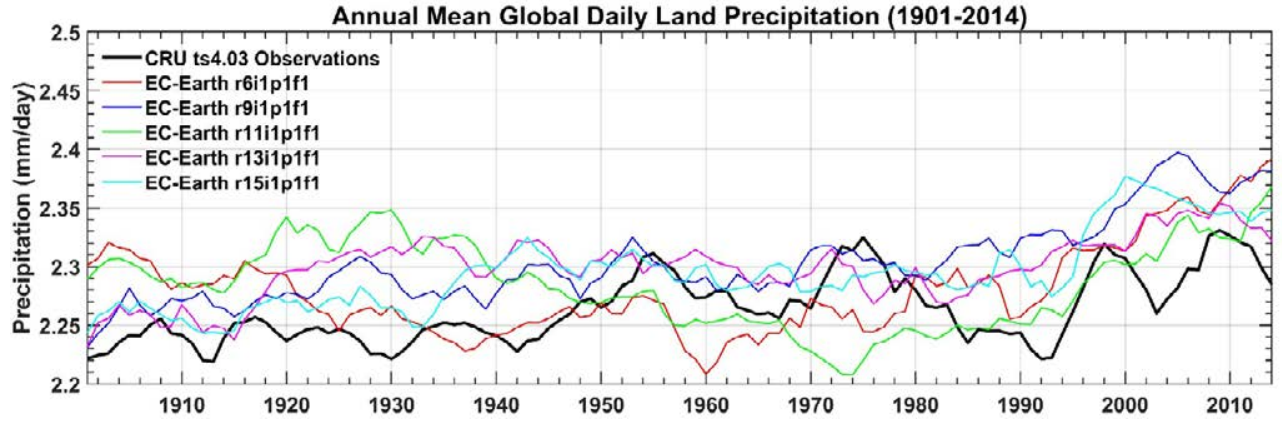

(b)

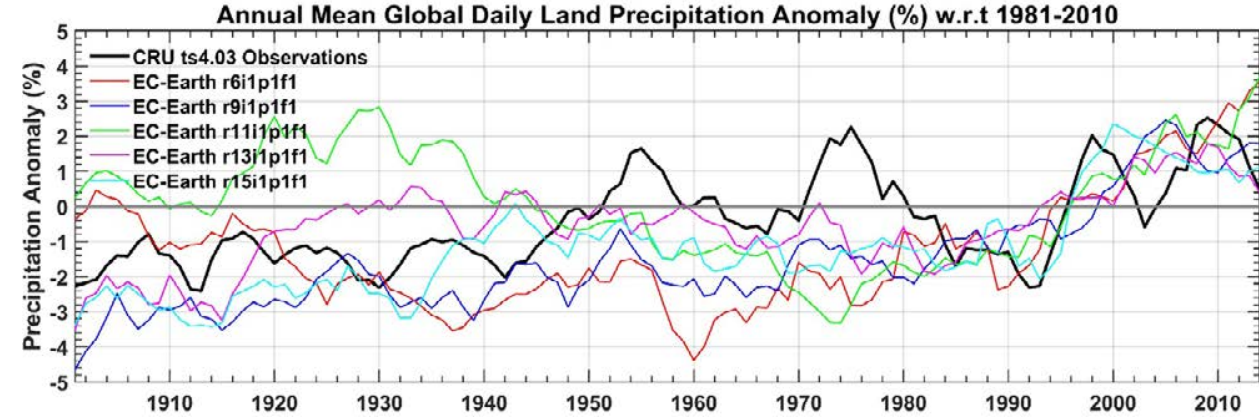

Figure 2.22. Comparison of EC-Earth ensemble members with CRU_ts4.03 observational data for the period 1901-2014: (a) land precipitation and (b) land precipitation anomalies (\%) with respect to the 30-year period 1981-2010. A 5-year running mean was applied to all datasets. The temperature data are confined to land points and exclude Antarctica. 
(a)

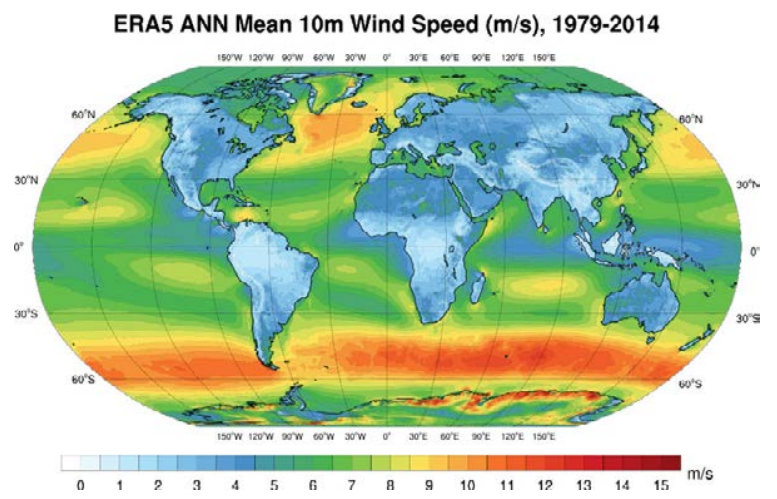

(c)

r9i1p1f1 EC-Earth ANN Mean 10m Wind Speed (m/s), 1979-2014

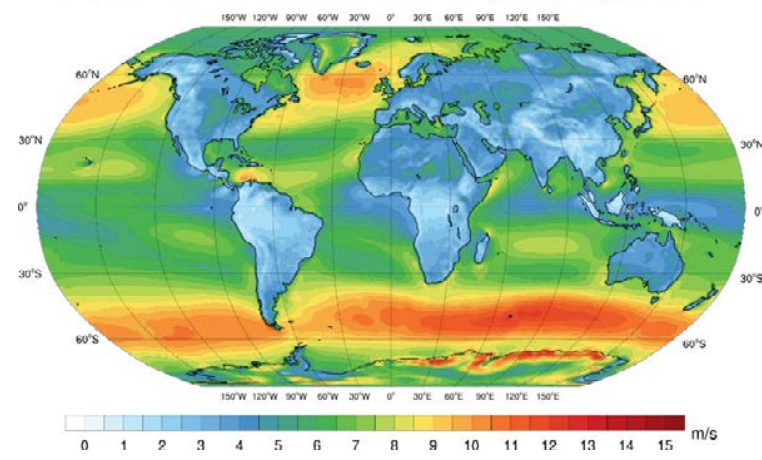

(e)

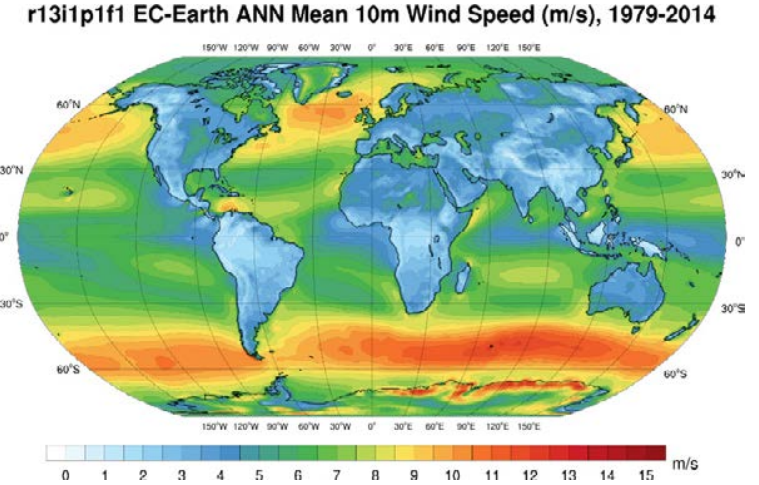

(b)

r6i1p1f1 EC-Earth ANN Mean 10m Wind Speed (m/s), 1979-2014

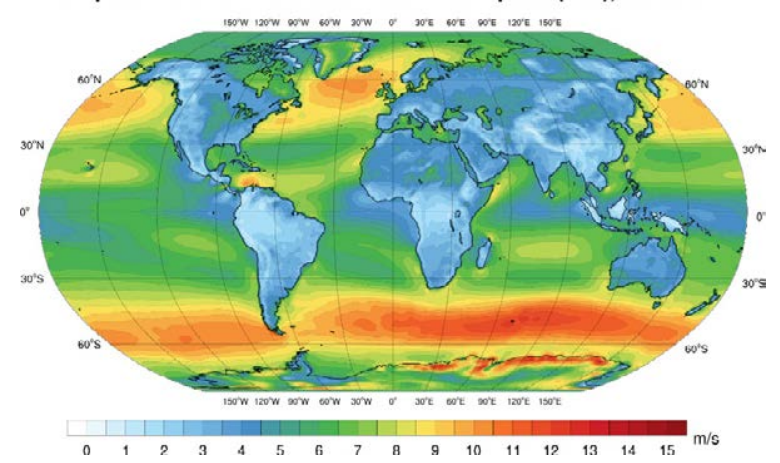

(d)

r11i1p1f1 EC-Earth ANN Mean 10m Wind Speed (m/s), 1979-2014

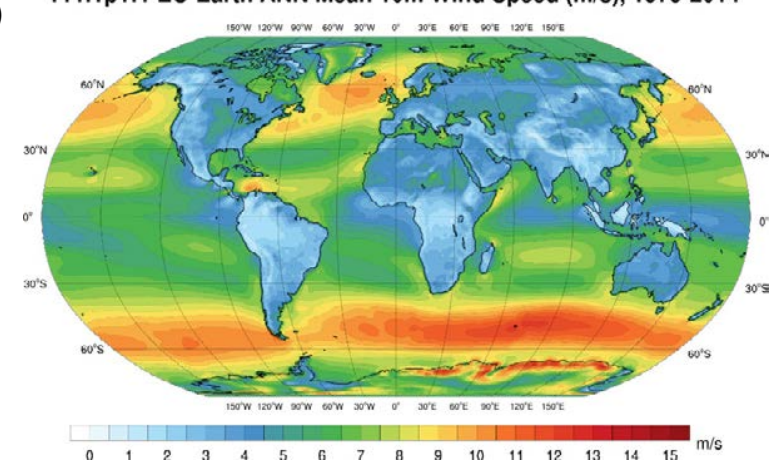

(f)

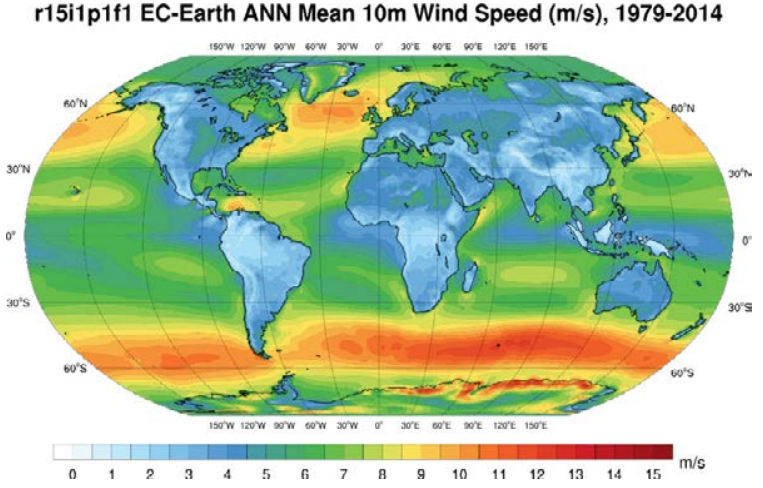

Figure 2.23. Annual mean 10-m wind speed (m/s), 1979-2014: (a) ERA5 reanalysis, (b) EC-Earth r6i1p1f1, (c) EC-Earth r9i1p1f1, (d) EC-Earth r11i1p1f1, (e) EC-Earth r13i1p1f1 and (f) EC-Earth r15i1p1f1. 
(a)

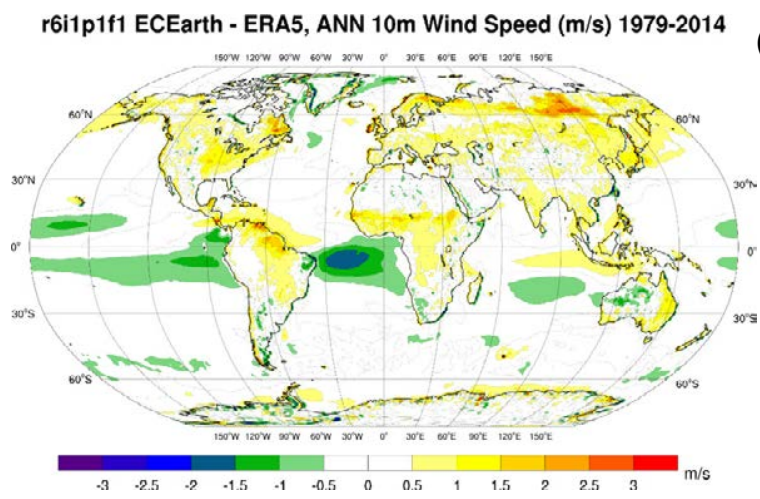

(c)

r11i1p1f1 ECEarth - ERA5, ANN 10m Wind Speed (m/s) 1979-2014

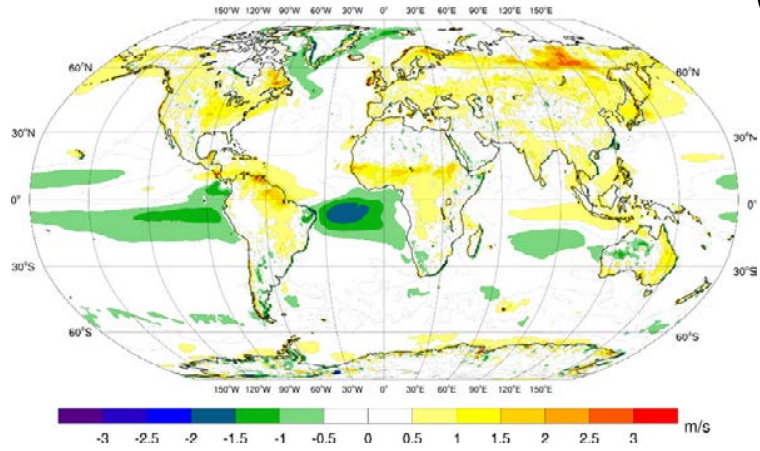

(d)

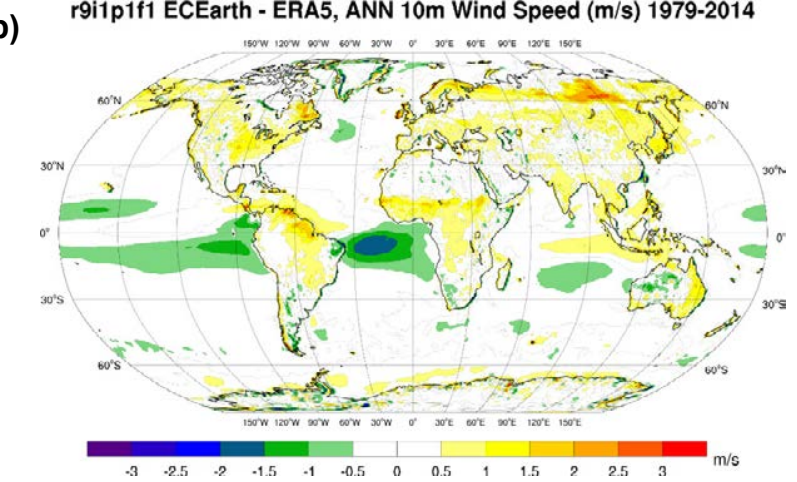

r13i1p1f1 ECEarth - ERA5, ANN 10m Wind Speed (m/s) 1979-2014

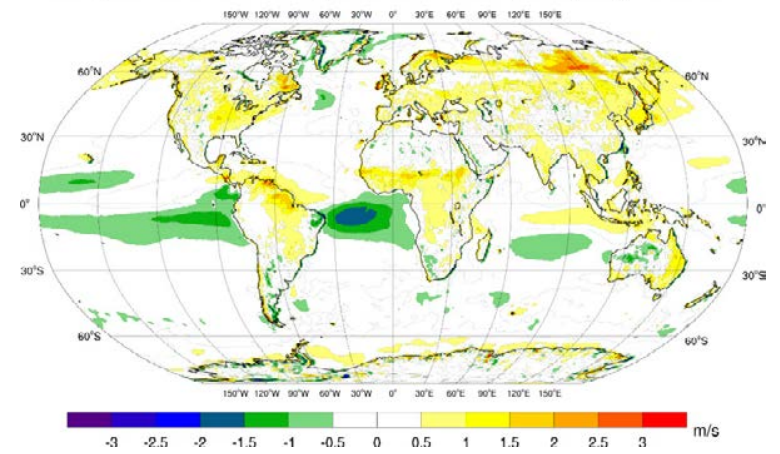

(e)

r15i1p1f1 ECEarth - ERA5, ANN 10m Wind Speed (m/s) 1979-2014

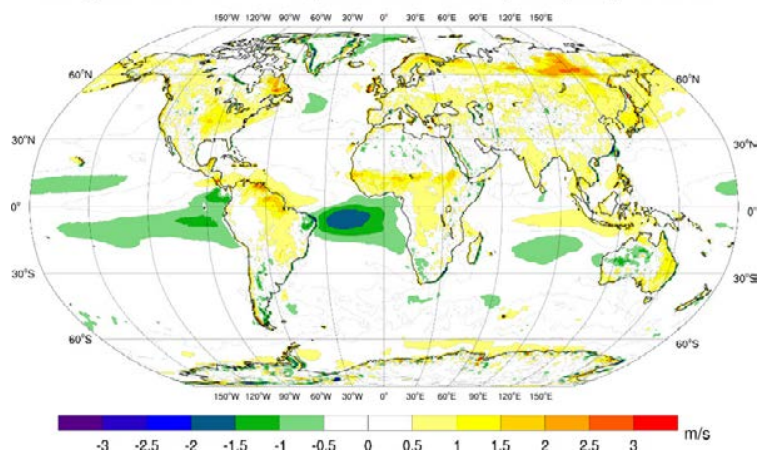

Figure 2.24. EC-Earth annual 10-m wind speed bias (m/s), 1979-2014 (ERA5 reanalysis minus EC-Earth):

(a) EC-Earth r6i1p1f1, (b) EC-Earth r9i1p1f1, (c) EC-Earth r11i1p1f1, (d) EC-Earth r13i1p1f1 and

(e) EC-Earth r15i1p1f1. 
(a)

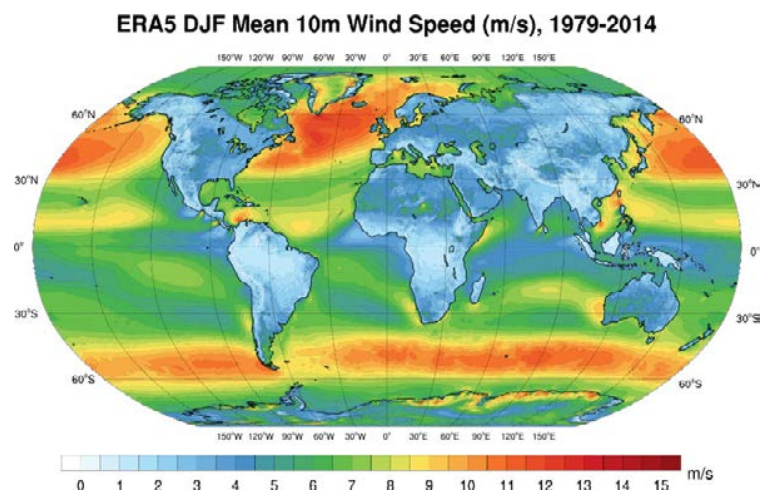

(c)

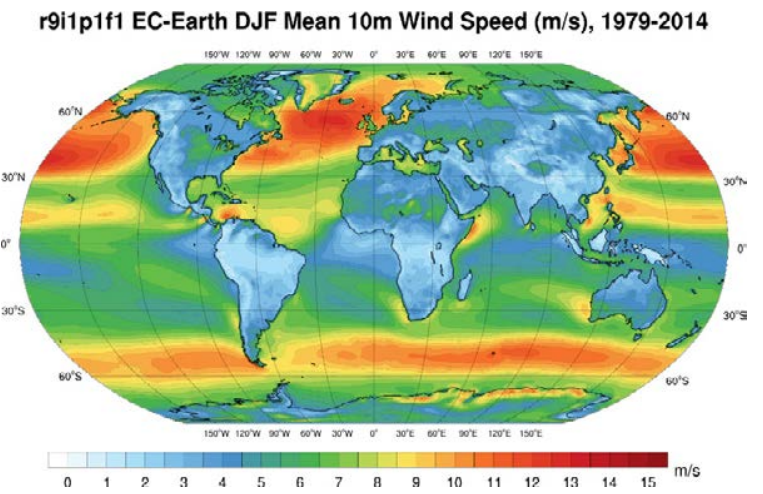

(e)

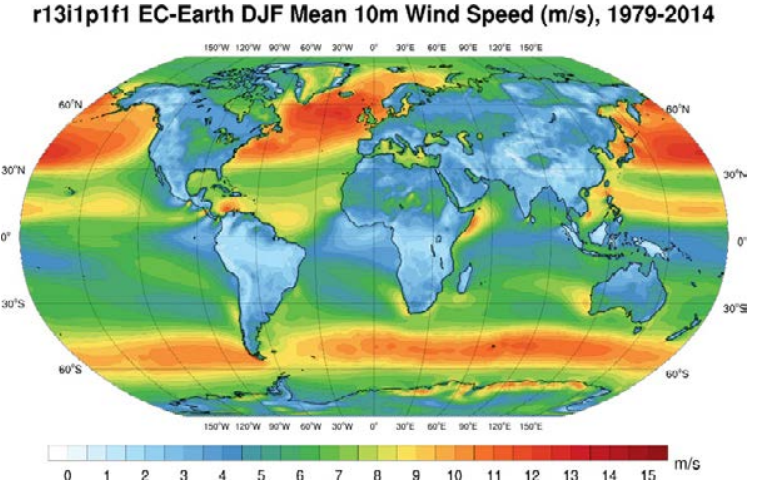

(b)

r6i1p1f1 EC-Earth DJF Mean 10m Wind Speed (m/s), 1979-2014

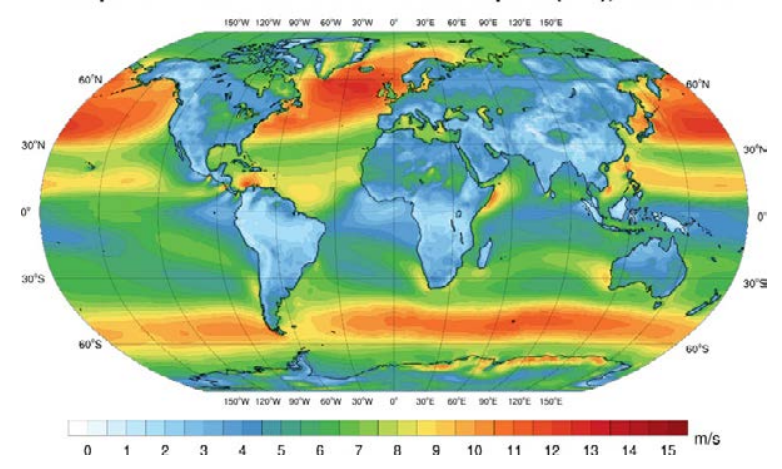

(d)

r11i1p1f1 EC-Earth DJF Mean 10m Wind Speed (m/s), 1979-2014

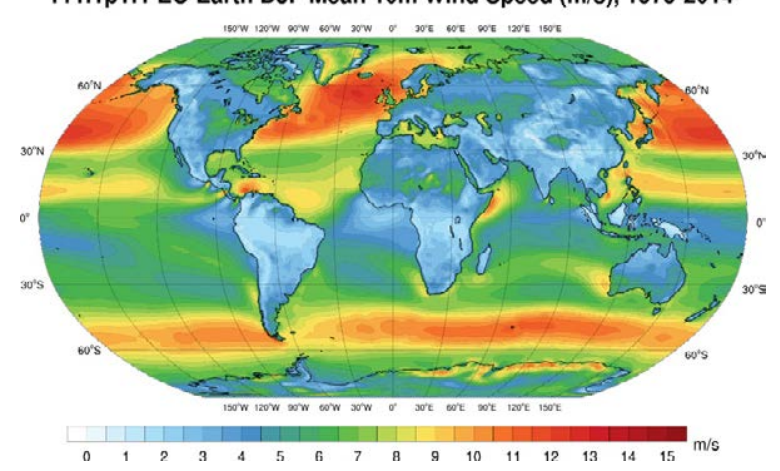

(f)

r15i1p1f1 EC-Earth DJF Mean 10m Wind Speed (m/s), 1979-2014

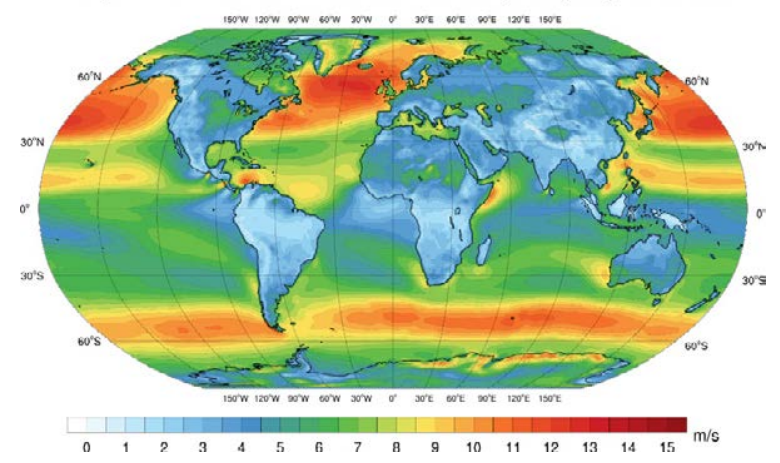

Figure 2.25. Mean 10-m wind speed (m/s) for DJF, 1979-2014: (a) ERA5 reanalysis, (b) EC-Earth r6i1p1f1, (c) EC-Earth r9i1p1f1, (d) EC-Earth r11i1p1f1, (e) EC-Earth r13i1p1f1 and (f) EC-Earth r15i1p1f1. 
(a)

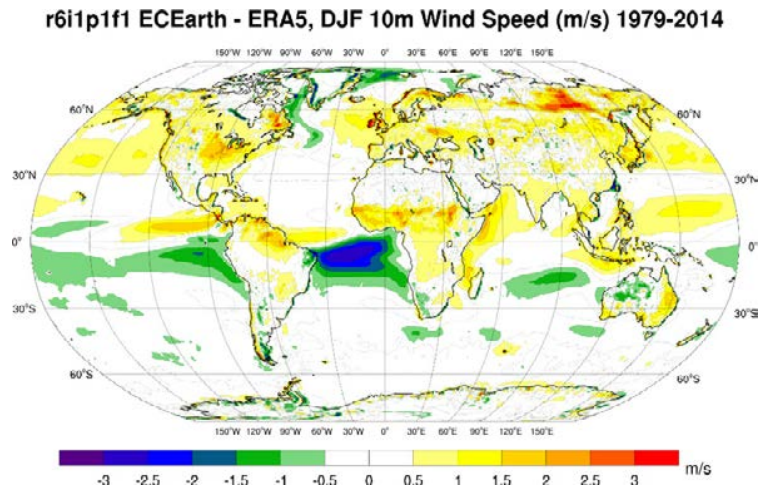

(c)

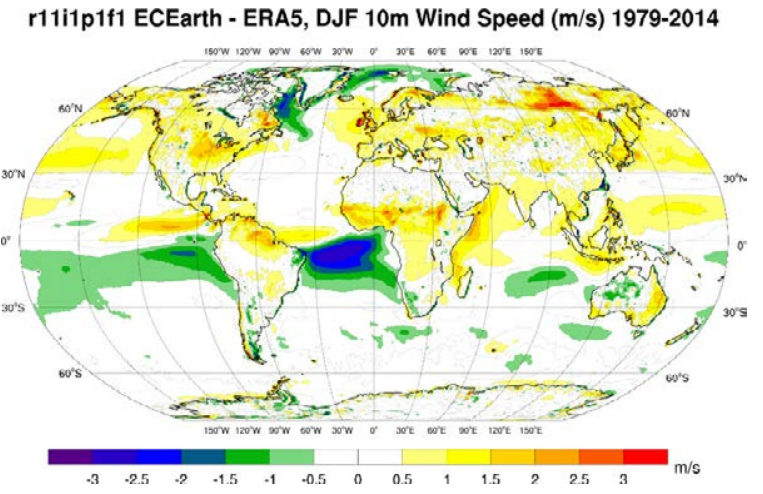

(e)

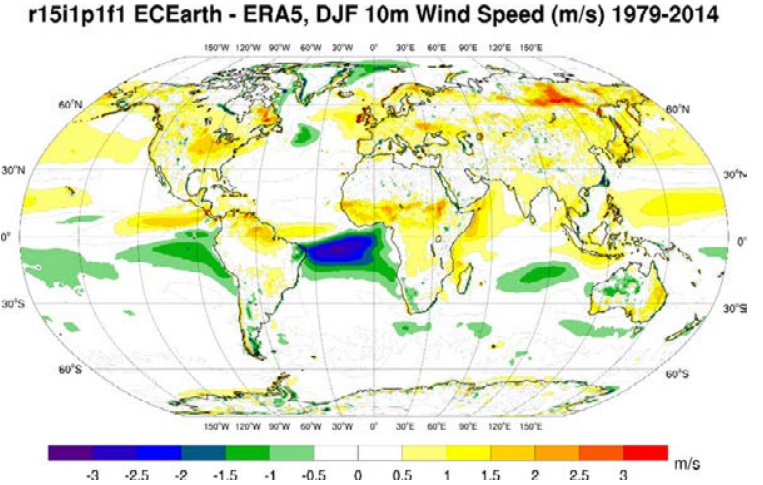

(b)

r9i1p1f1 ECEarth - ERA5, DJF 10m Wind Speed (m/s) 1979-2014

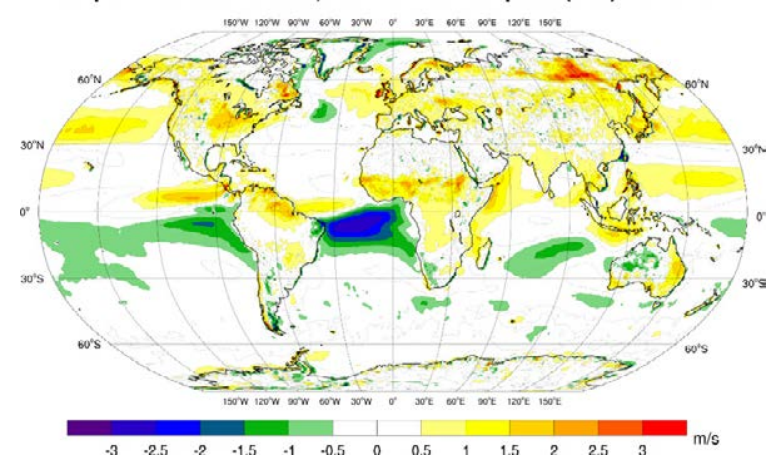

(d)

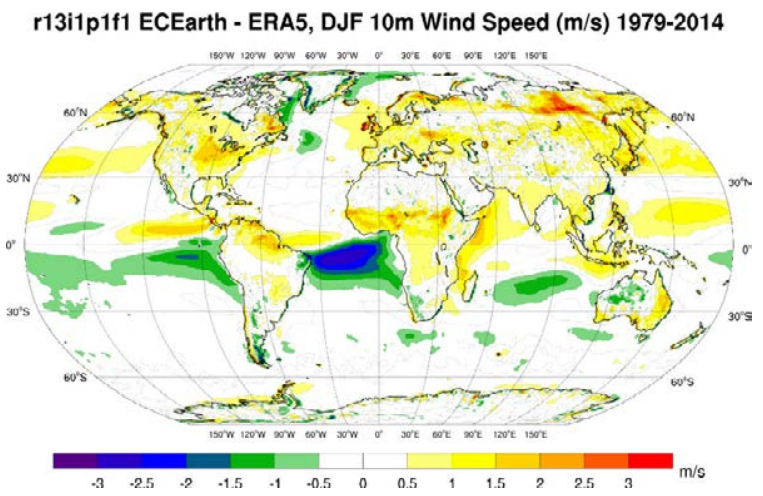

Figure 2.26. EC-Earth 10-m wind speed bias (m/s) for DJF, 1979-2014 (ERA5 reanalysis minus EC-Earth): (a) EC-Earth r6i1p1f1, (b) EC-Earth r9i1p1f1, (c) EC-Earth r11i1p1f1, (d) EC-Earth r13i1p1f1 and (e) EC-Earth r15i1p1f1. 
(a)

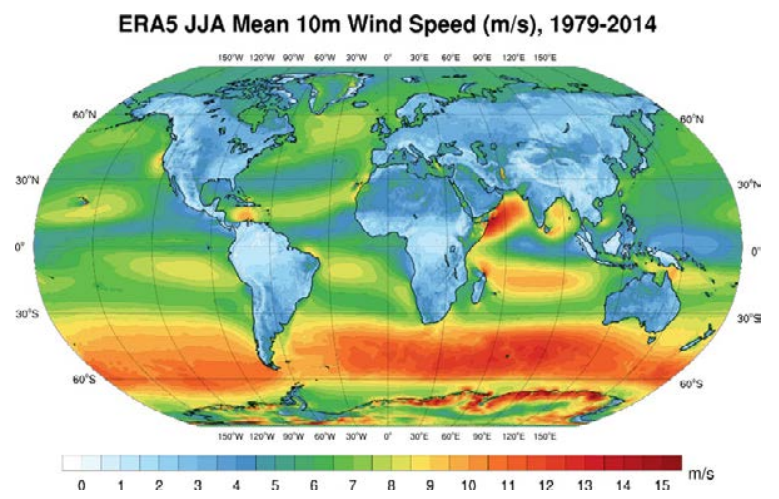

(c)

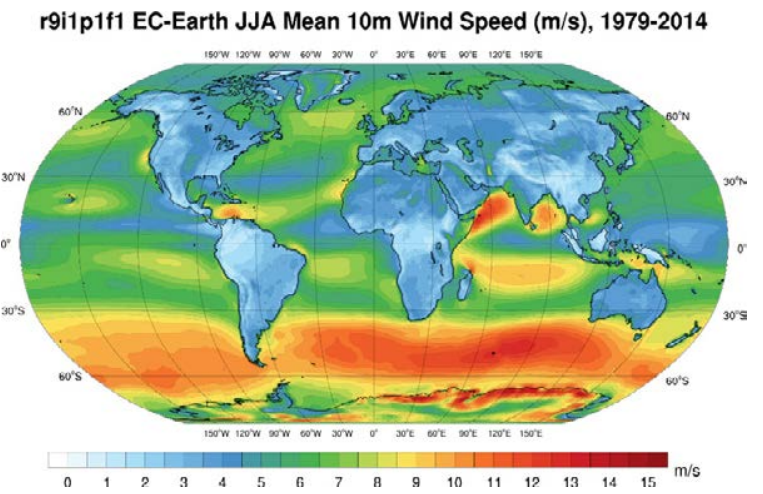

(e)

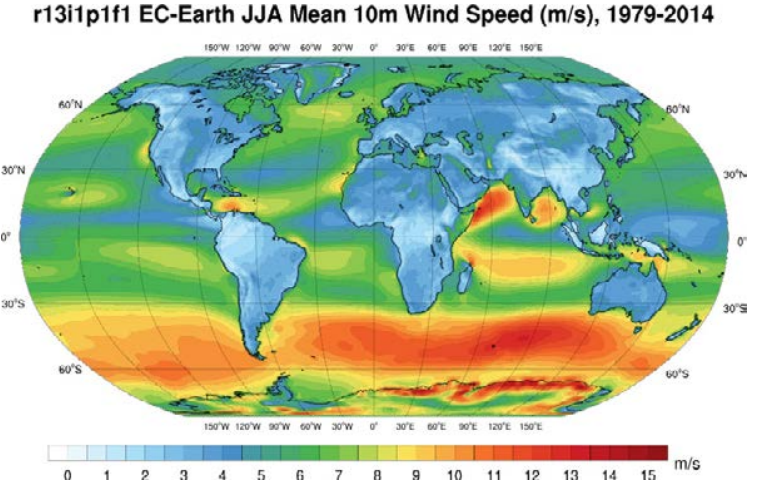

(b)

r6i1p1f1 EC-Earth JJA Mean 10m Wind Speed (m/s), 1979-2014

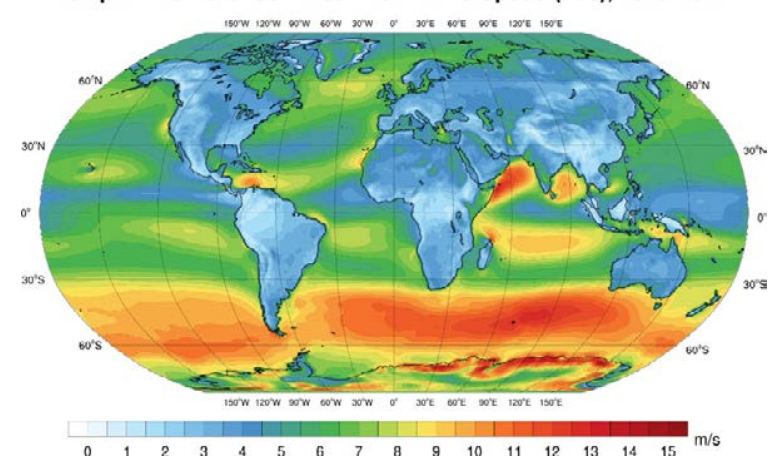

(d)
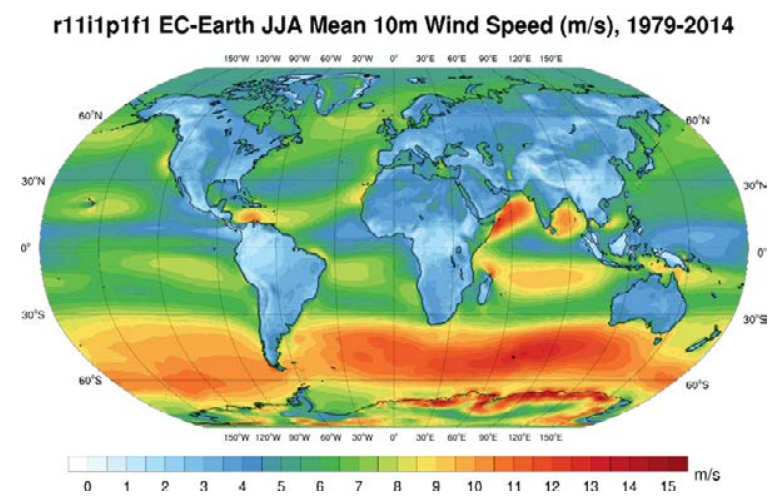

(f)

r15i1p1f1 EC-Earth JJA Mean 10m Wind Speed (m/s), 1979-2014

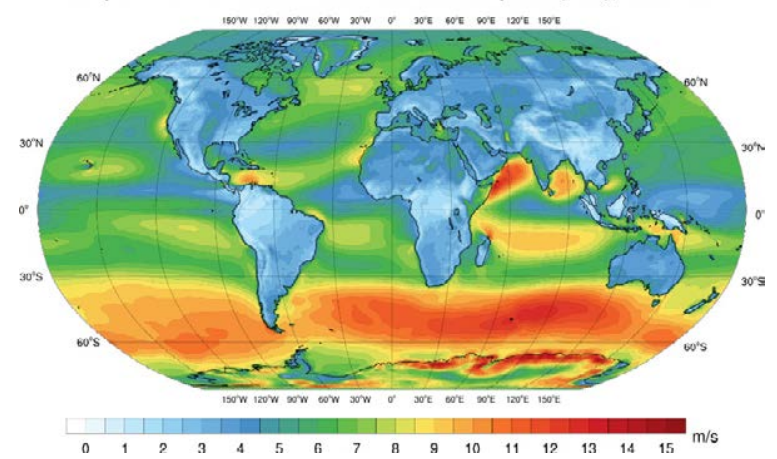

Figure 2.27. Mean 10-m wind speed (m/s) for JJA, 1979-2014: (a) ERA5 reanalysis, (b) EC-Earth r6i1p1f1, (c) EC-Earth r9i1p1f1, (d) EC-Earth r11i1p1f1, (e) EC-Earth r13i1p1f1 and (f) EC-Earth r15i1p1f1. 
(a)

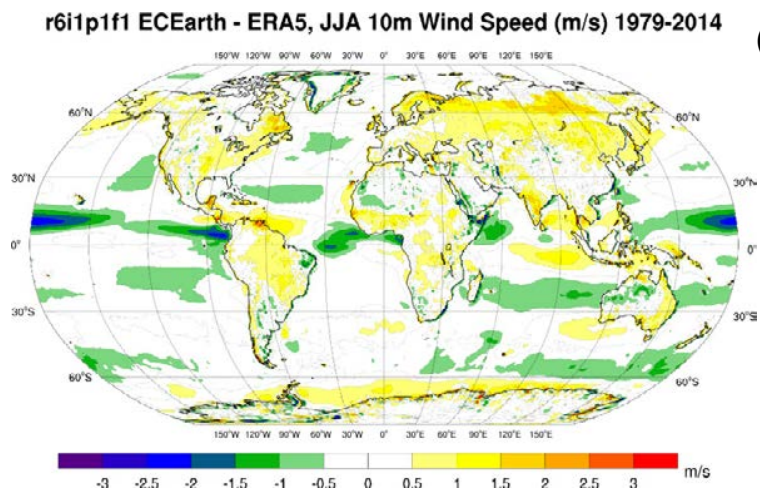

(c)

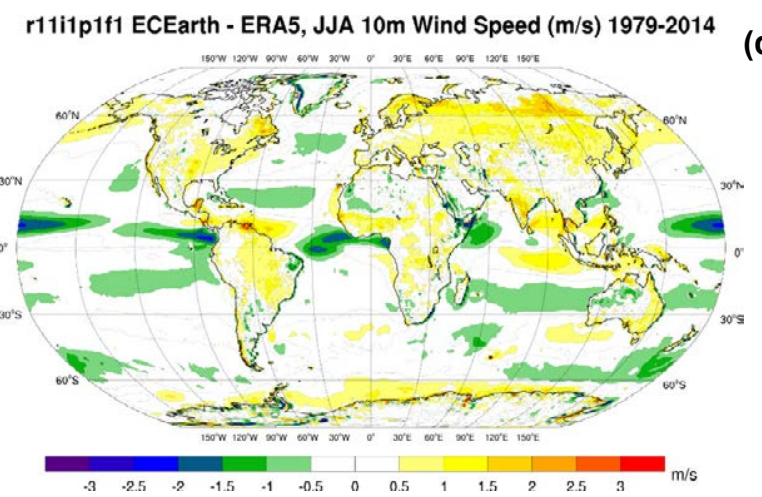

(e)

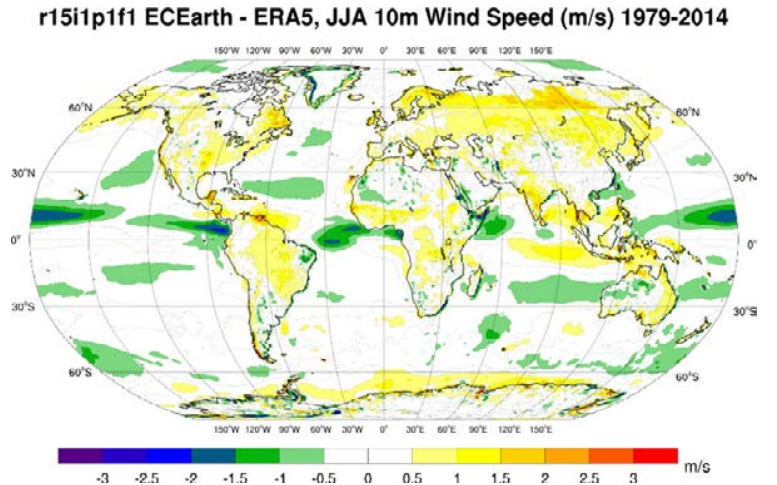

(b)

r9i1p1f1 ECEarth - ERA5, JJA 10m Wind Speed (m/s) 1979-2014

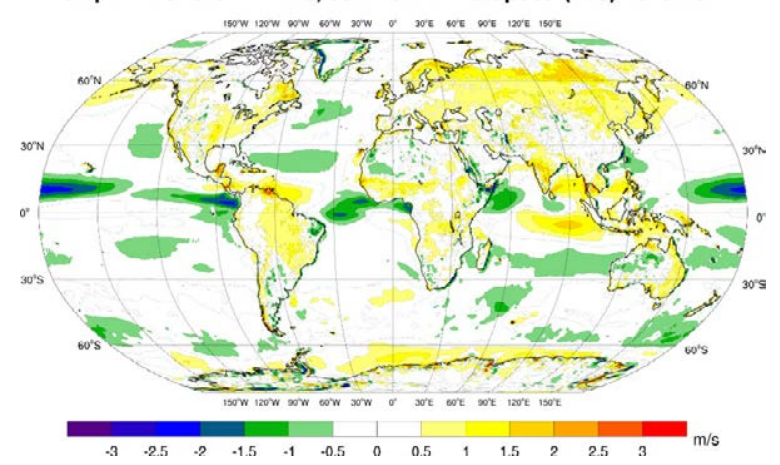

(d)

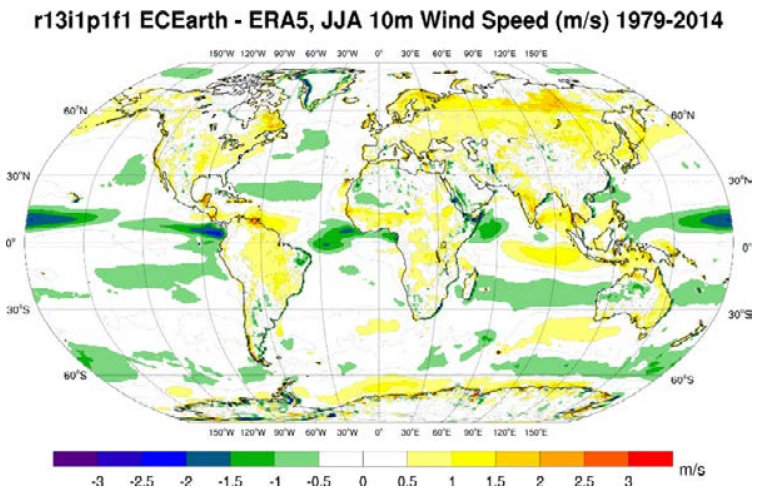

Figure 2.28. EC-Earth 10-m wind speed bias (m/s) for JJA, 1979-2014 (ERA5 reanalysis minus EC-Earth): (a) EC-Earth r6i1p1f1, (b) EC-Earth r9i1p1f1, (c) EC-Earth r11i1p1f1, (d) EC-Earth r13i1p1f1 and (e) EC-Earth r15i1p1f1.

Table 2.6. Mean global annual and seasonal 10-m wind speed bias and MAE $(\mathrm{m} / \mathrm{s})$ for each of the five EC-Earth ensemble members ${ }^{a}$

\begin{tabular}{|c|c|c|c|c|c|c|c|c|c|c|}
\hline \multirow{2}{*}{$\begin{array}{l}\text { Time } \\
\text { period }\end{array}$} & \multicolumn{2}{|c|}{ r6i1p1f1 } & \multicolumn{2}{|c|}{ r9i1p1f1 } & \multicolumn{2}{|c|}{ r11i1p1f1 } & \multicolumn{2}{|c|}{ r13i1p1f1 } & \multicolumn{2}{|c|}{ r15i1p1f1 } \\
\hline & Bias & MAE & Bias & MAE & Bias & MAE & Bias & MAE & Bias & MAE \\
\hline Annual & 0.082 & 0.408 & 0.064 & 0.403 & 0.087 & 0.425 & 0.072 & 0.406 & 0.077 & 0.393 \\
\hline DJF & 0.158 & 0.517 & 0.137 & 0.505 & 0.150 & 0.537 & 0.145 & 0.500 & 0.154 & 0.498 \\
\hline MAM & 0.086 & 0.499 & 0.058 & 0.495 & 0.093 & 0.524 & 0.069 & 0.505 & 0.059 & 0.483 \\
\hline JJA & 0.010 & 0.461 & 0.016 & 0.454 & 0.023 & 0.470 & 0.018 & 0.461 & 0.026 & 0.446 \\
\hline SON & 0.073 & 0.456 & 0.046 & 0.460 & 0.081 & 0.460 & 0.057 & 0.451 & 0.069 & 0.443 \\
\hline
\end{tabular}

aln each case the model data are compared with ERA5 reanalysis data for the period 1979-2014. 
(a)

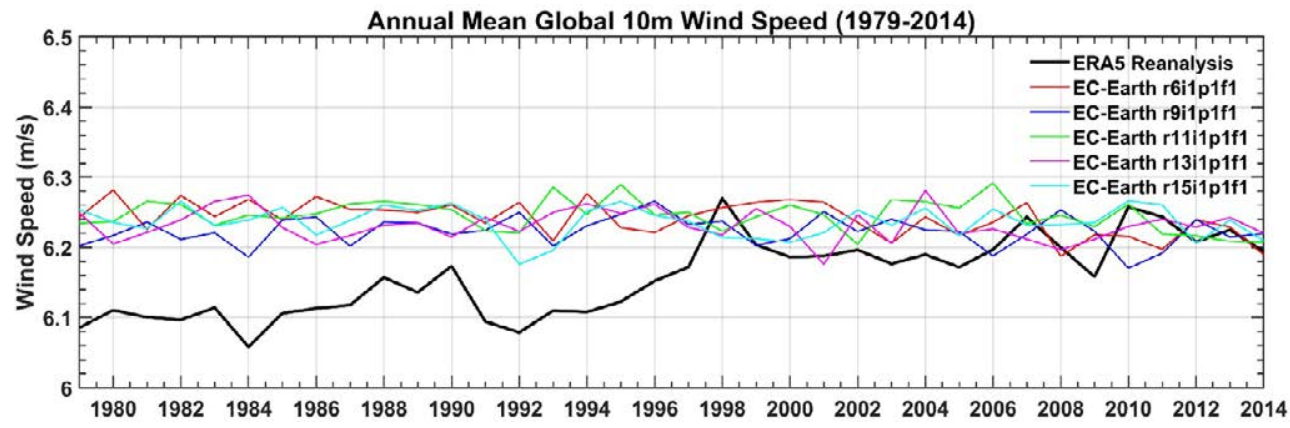

(b)

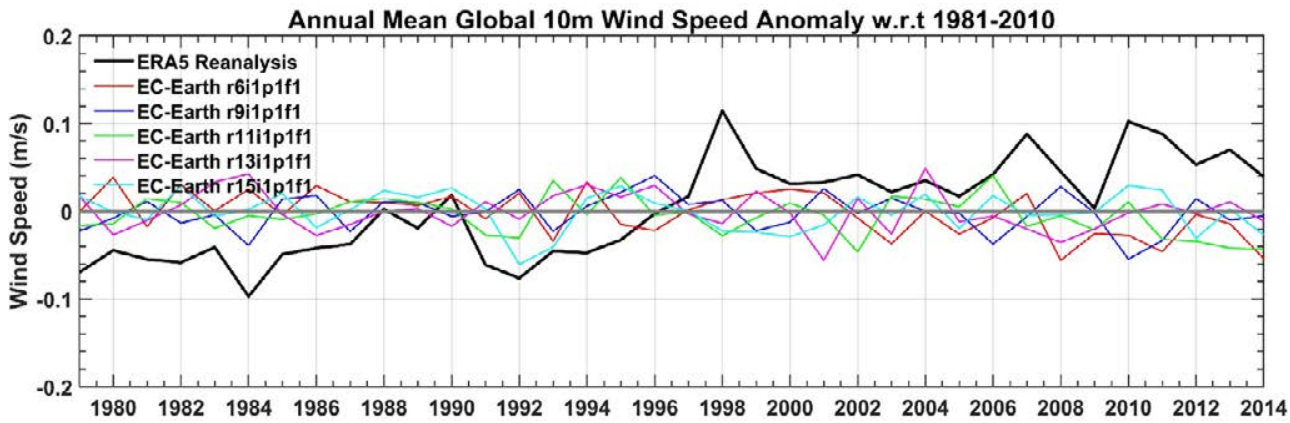

Figure 2.29. Comparison of EC-Earth ensemble members with ERA5 reanalysis data for the period 1979-2014: (a) 10-m wind speed and (b) 10-m wind speed anomalies with respect to the 30-year period 1981-2010.

\subsubsection{Mean sea level pressure validations}

Figure 2.30 presents the spatial distribution of mean annual MSLP for ERA5 and each of the five EC-Earth ensemble members for the 36-year period 1979-2014. All EC-Earth ensemble members accurately capture the magnitude and spatial characteristics of the historical MSLP climate. Figure 2.31 demonstrates that the differences relative to ERA5 data are similar for all EC-Earth ensemble members - an underestimate over the southern subtropical and North Pacific regions. All other regions exhibit a small positive bias (or small differences). MSLP validations for DJF (Figures 2.32 and 2.33) follow a similar (but enhanced) trend as the annual biases. In addition, a negative bias is evident over the UK and Ireland. MSLP validations for JJA (Figures 2.34 and 2.35) show a negative bias over north-east Europe and the southern subtropical regions, and a positive bias over the poles and the UK and Ireland, the magnitude of which varies between ensemble members; the strongest bias is noted for ensemble member r11i1p1f1 (Figure 2.35c).

The annual and seasonal overall global bias and MAE statistics relative to ERA5 data (1979-2014) for each of the five ensemble members are presented in Table 2.7. The bias statistics are all negative, ranging from $-0.16 \mathrm{hPa}$ (JJA, r11i1p1f1) to $-0.05 \mathrm{hPa}$ (DJF, r11i1p1f1). The MAE statistics range from $0.68 \mathrm{hPa}$ (annual, r15i1p1f1) to $1.29 \mathrm{hPa}$ (DJF, r11i1p1f1).

The ERA5 and EC-Earth mean global annual MSLP time series (1979-2014), presented in Figure 2.36a, demonstrate good agreement. However, all EC-Earth ensemble members exhibit a slight underestimation of MSLP of $\sim 0.2 \mathrm{hPa}$ from about 1990. Figure $2.36 \mathrm{~b}$ shows the annual anomalies with respect to the 1981-2010 mean; all ERA5 and EC-Earth annual values are within the range of $-0.2 \mathrm{hPa}$ to $0.1 \mathrm{hPa}$. 
(a)

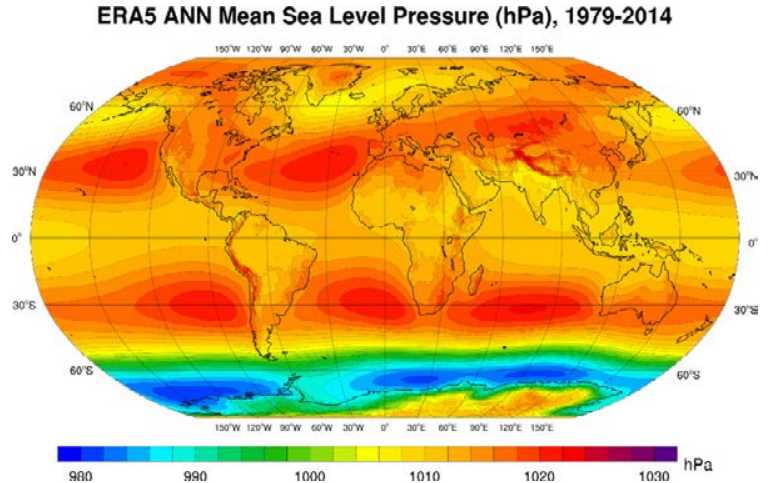

(c)

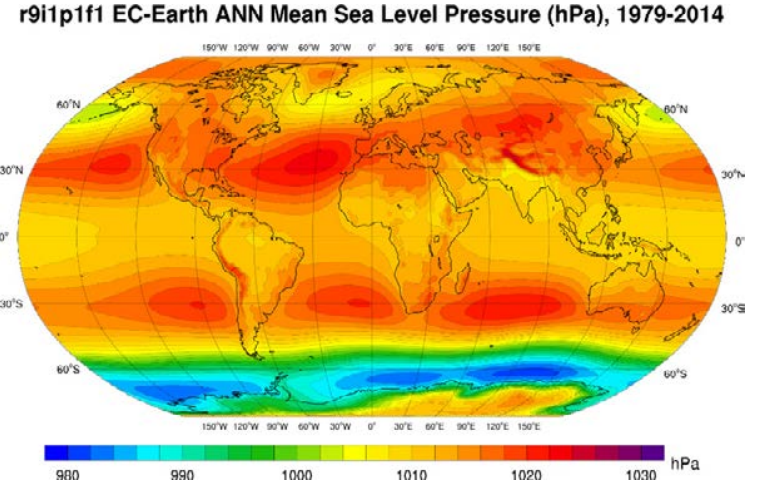

(e)

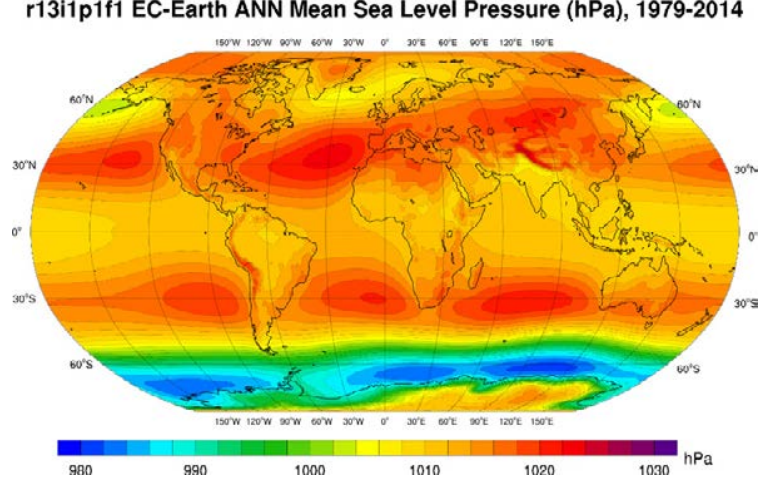

(b)

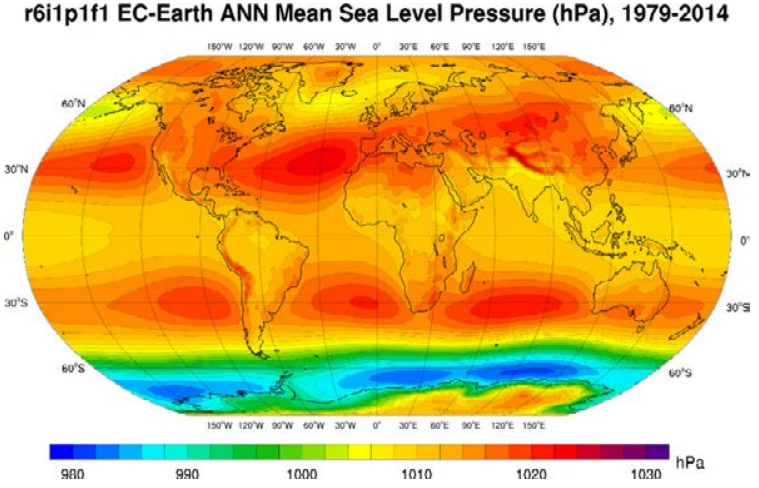

(d)
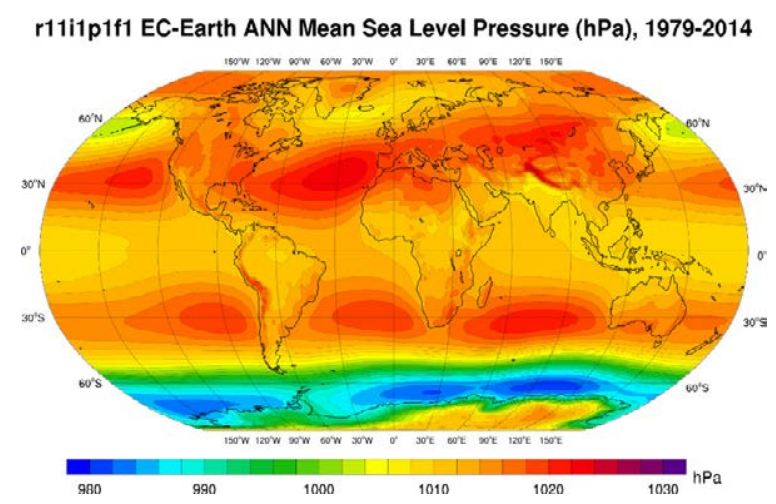

(f)

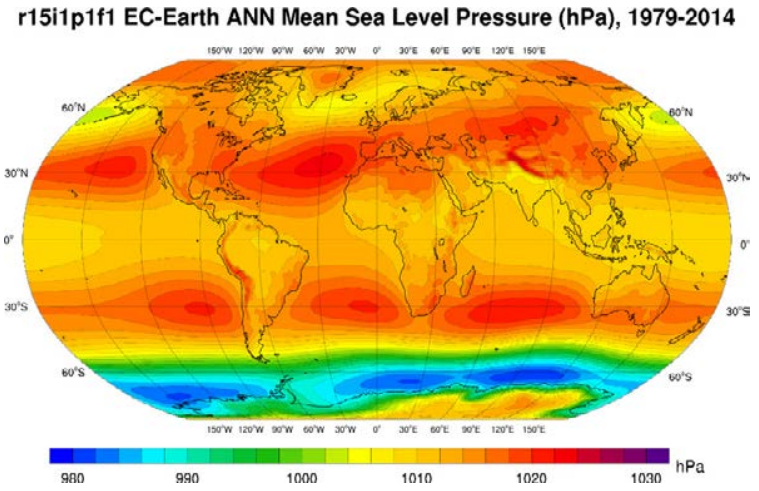

Figure 2.30. Mean annual MSLP (hPa), 1979-2014: (a) ERA5 reanalysis, (b) EC-Earth r6i1p1f1, (c) EC-Earth r9i1p1f1, (d) EC-Earth r11i1p1f1, (e) EC-Earth r13i1p1f1 and (f) EC-Earth r15i1p1f1. 
(a)

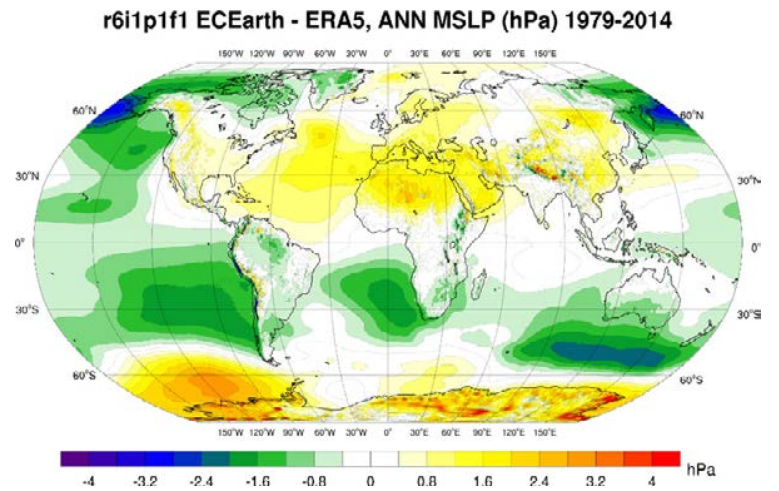

(c)

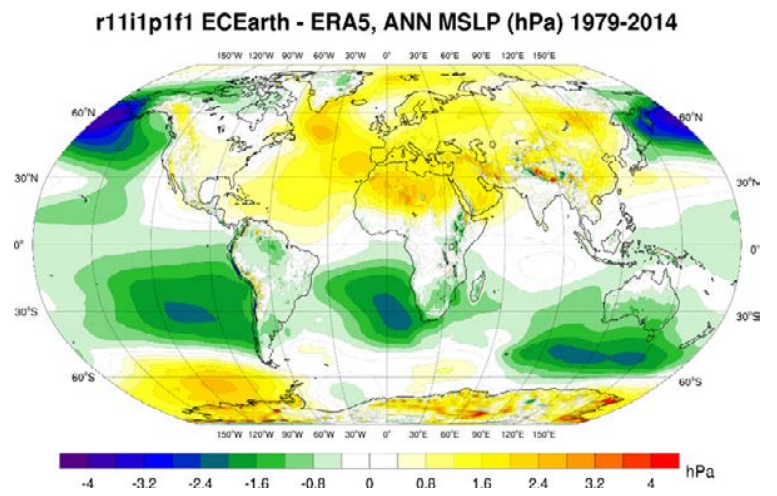

(e)

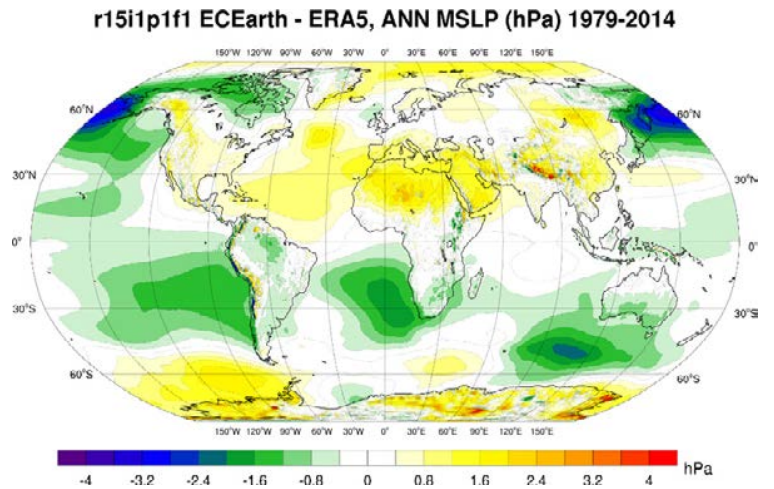

(b)

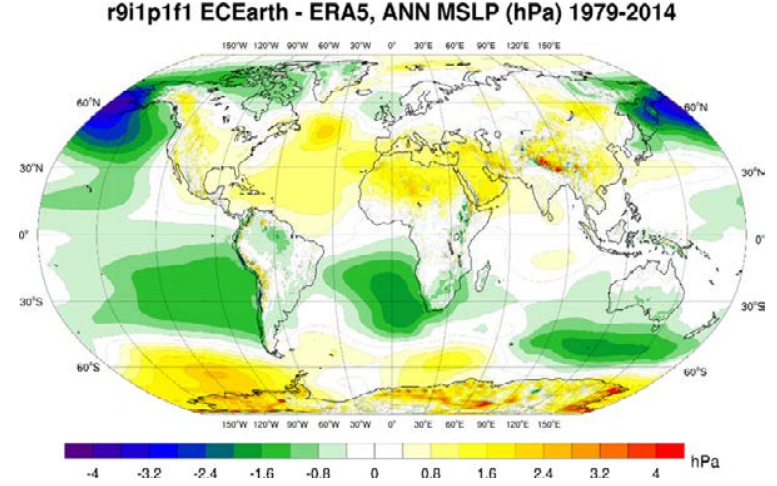

(d)

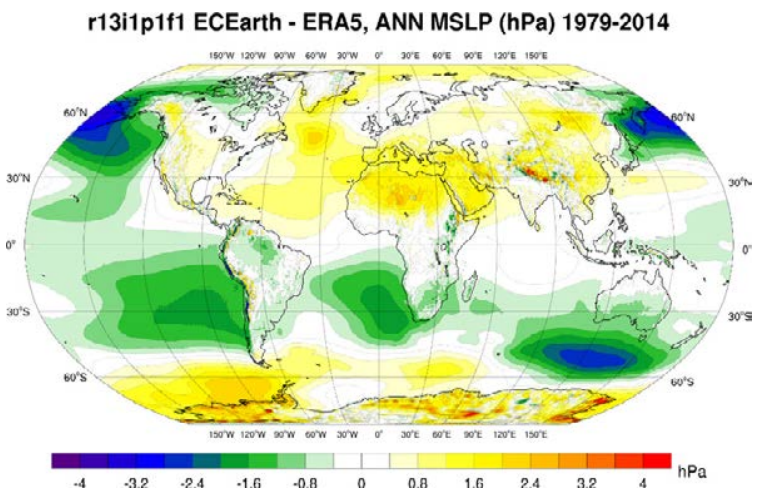

Figure 2.31. EC-Earth mean annual MSLP bias (hPa), 1979-2014 (ERA5 reanalysis minus EC-Earth):

(a) EC-Earth r6i1p1f1, (b) EC-Earth r9i1p1f1, (c) EC-Earth r11i1p1f1, (d) EC-Earth r13i1p1f1 and (e) EC-Earth r15i1p1f1. 
(a)

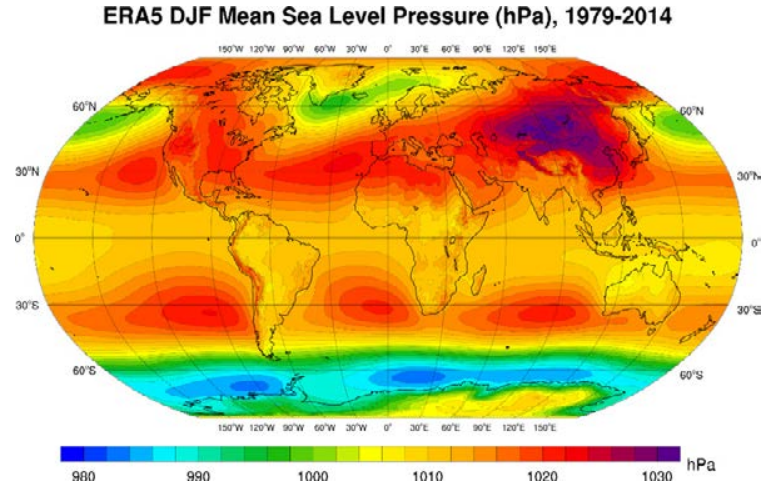

(c)

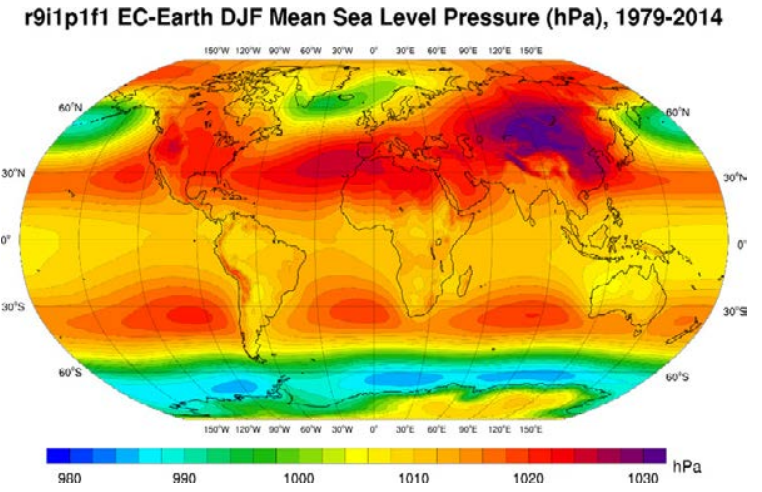

(e)

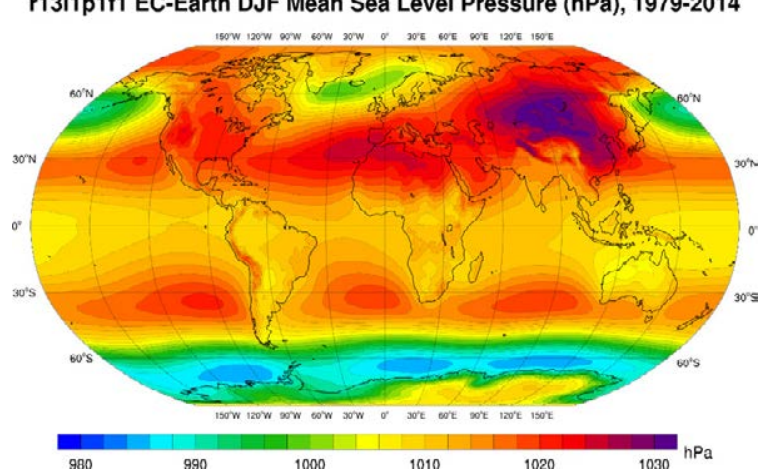

(b)

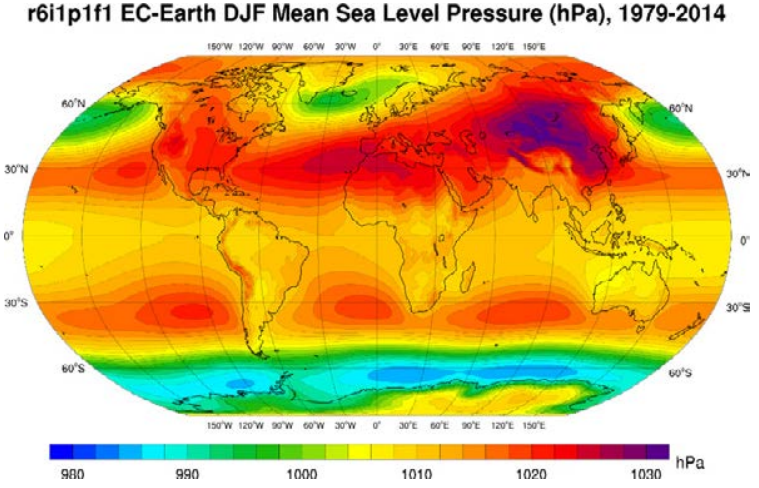

(d)

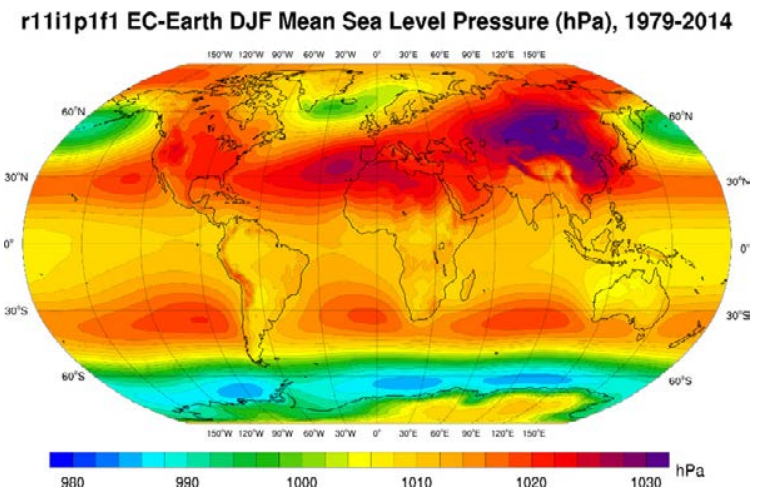

(f)

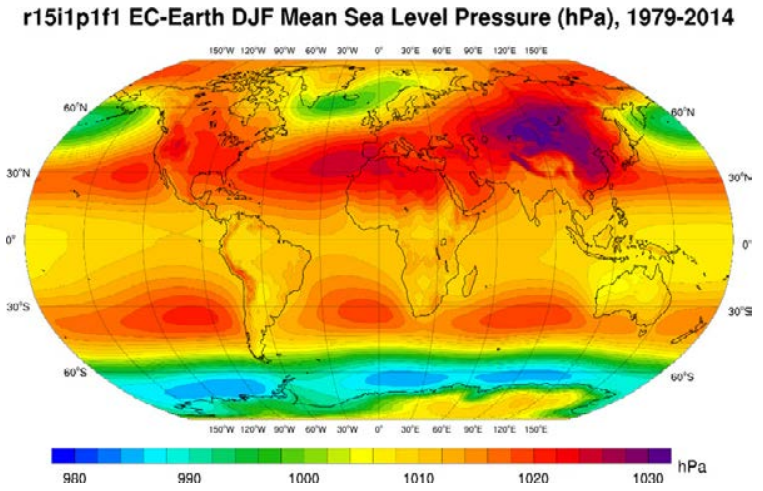

Figure 2.32. MSLP (hPa) for DJF, 1979-2014: (a) ERA5 reanalysis, (b) EC-Earth r6i1p1f1, (c) EC-Earth r9i1p1f1, (d) EC-Earth r11i1p1f1, (e) EC-Earth r13i1p1f1 and (f) EC-Earth r15i1p1f1. 
(a)

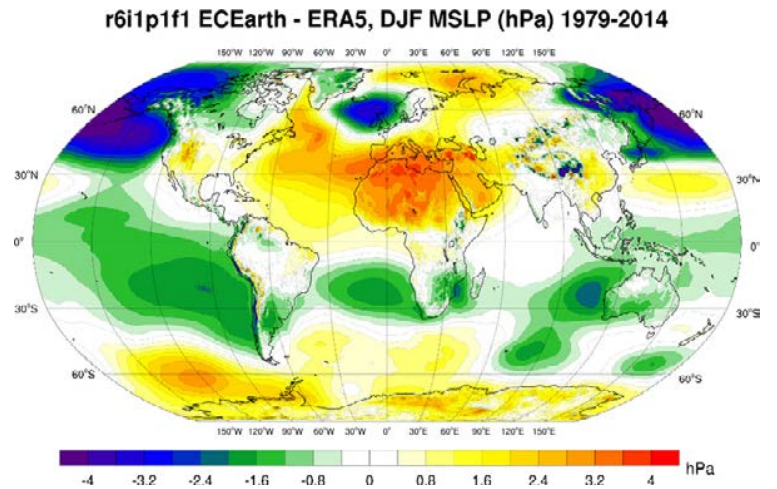

(c)

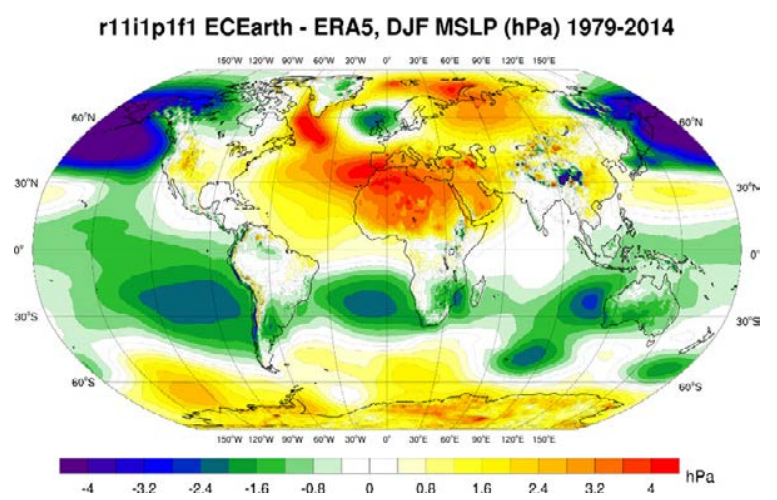

(e)

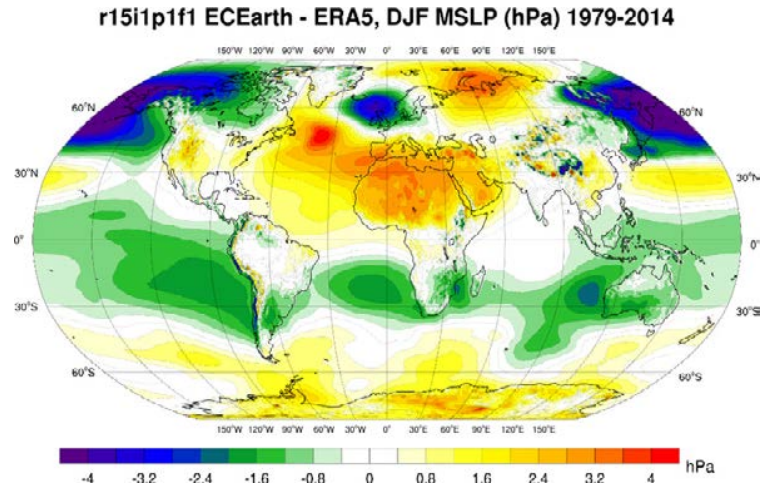

(b)

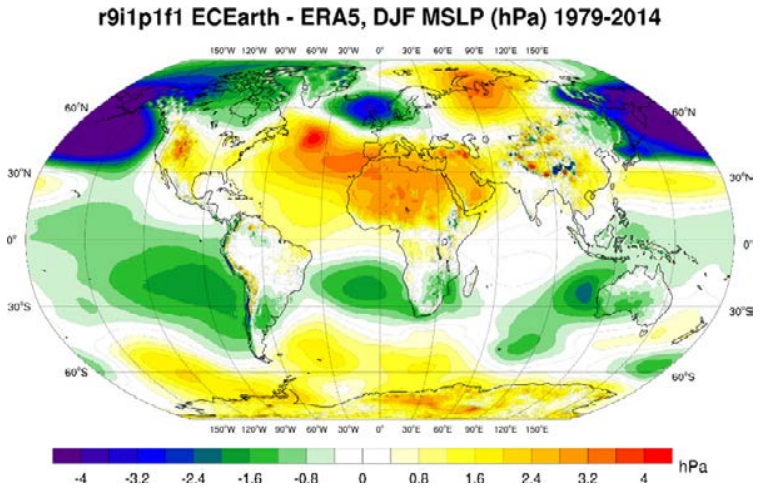

(d)

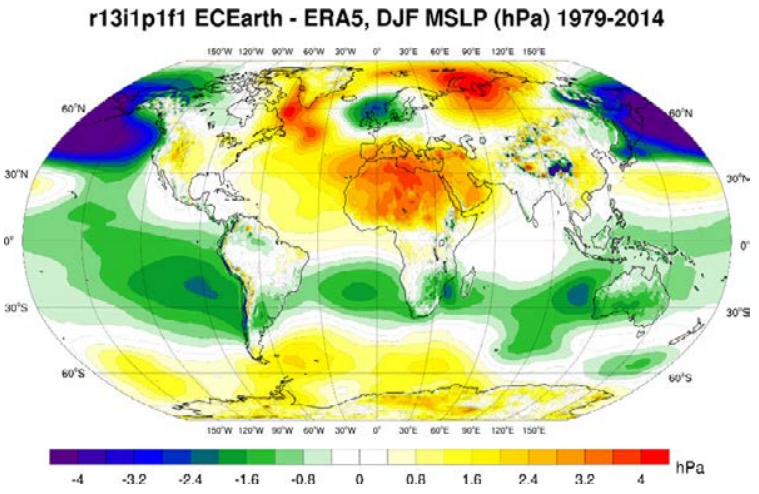

Figure 2.33. EC-Earth MSLP bias (hPa) for DJF, 1979-2014 (ERA5 reanalysis minus EC-Earth): (a) EC-Earth r6i1p1f1, (b) EC-Earth r9i1p1f1, (c) EC-Earth r11i1p1f1, (d) EC-Earth r13i1p1f1 and (e) EC-Earth r15i1p1f1. 
(a)

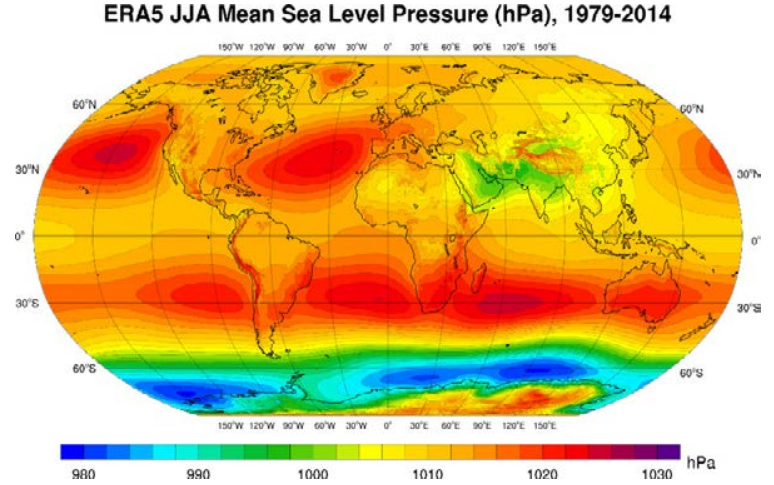

(c)

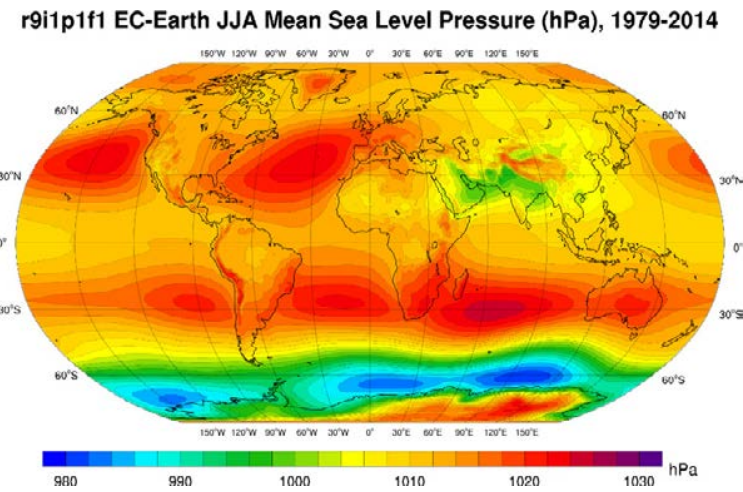

(e)

r13i1p1f1 EC-Earth JJA Mean Sea Level Pressure (hPa), 1979-2014

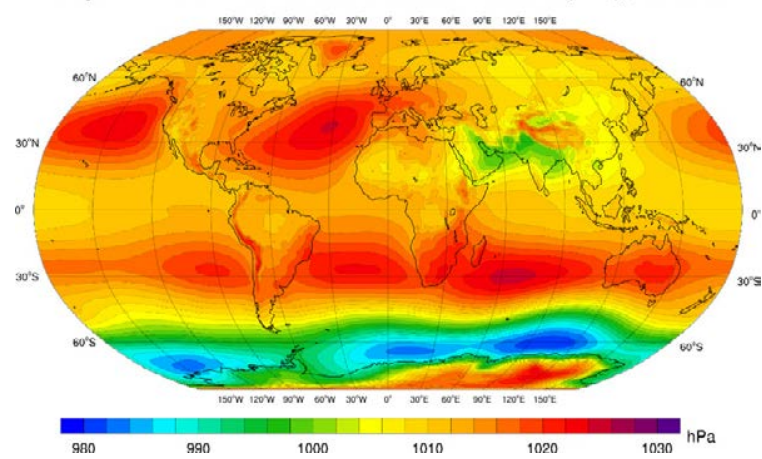

(b)

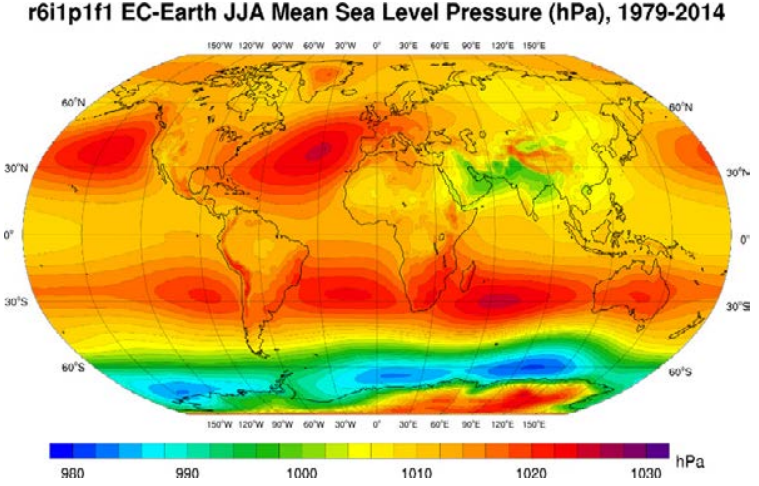

(d)
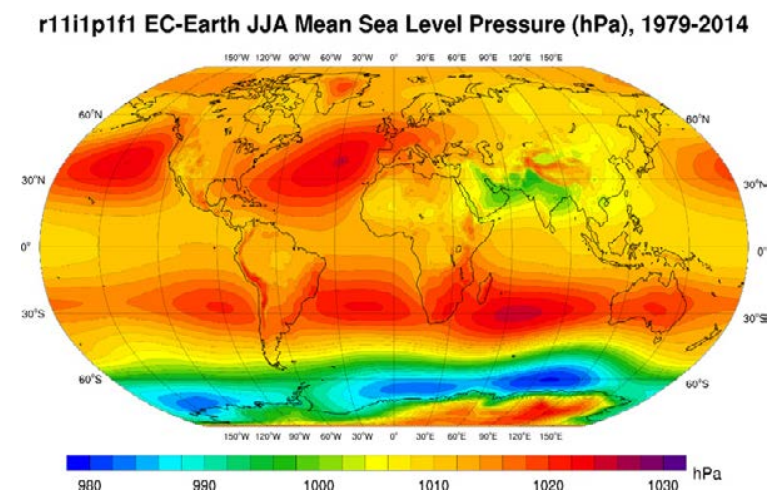

(f)

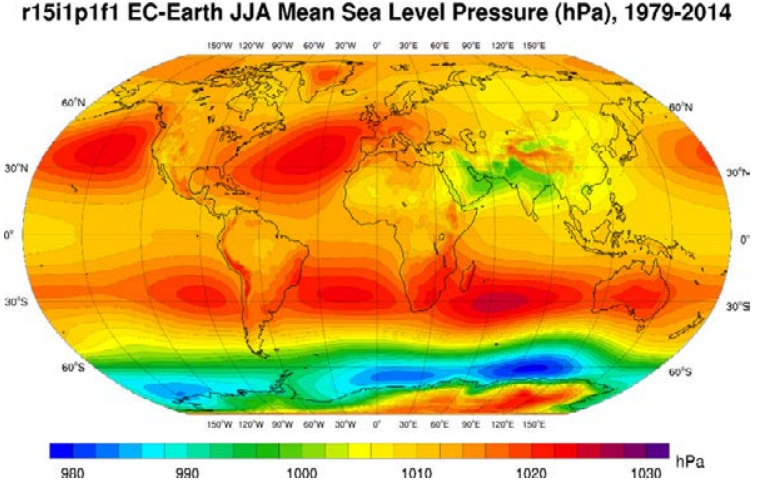

Figure 2.34. MSLP for JJA (hPa), 1979-2014: (a) ERA5 reanalysis, (b) EC-Earth r6i1p1f1, (c) EC-Earth r9i1p1f1, (d) EC-Earth r11i1p1f1, (e) EC-Earth r13i1p1f1 and (f) EC-Earth r15i1p1f1. 
(a)

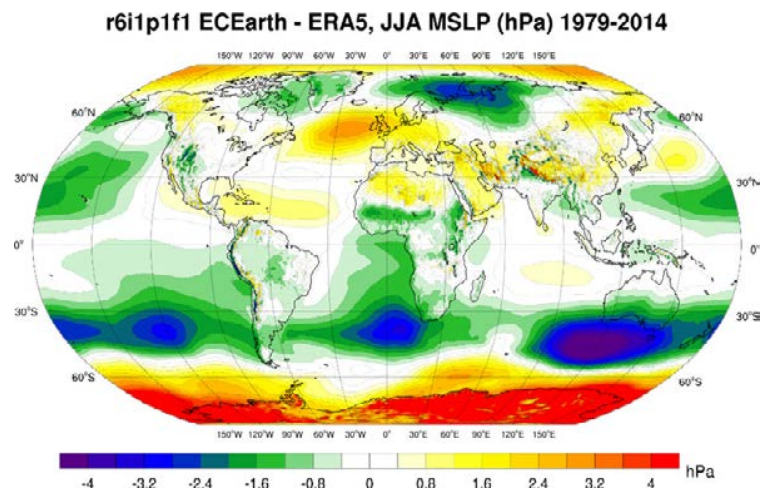

(c)

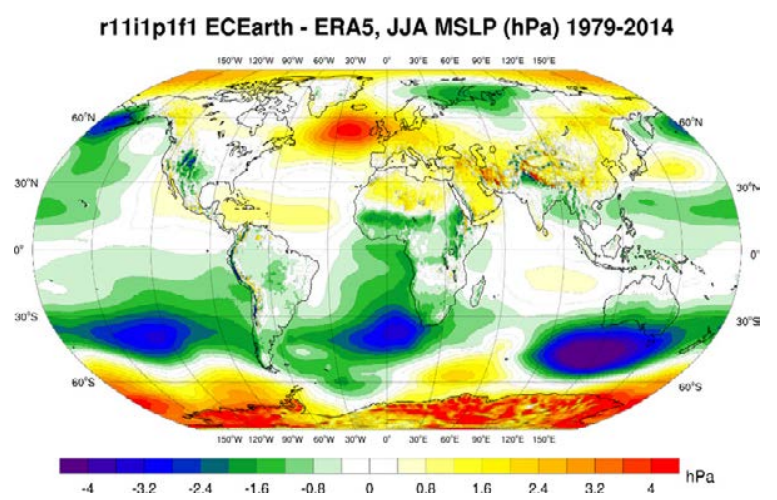

(e)

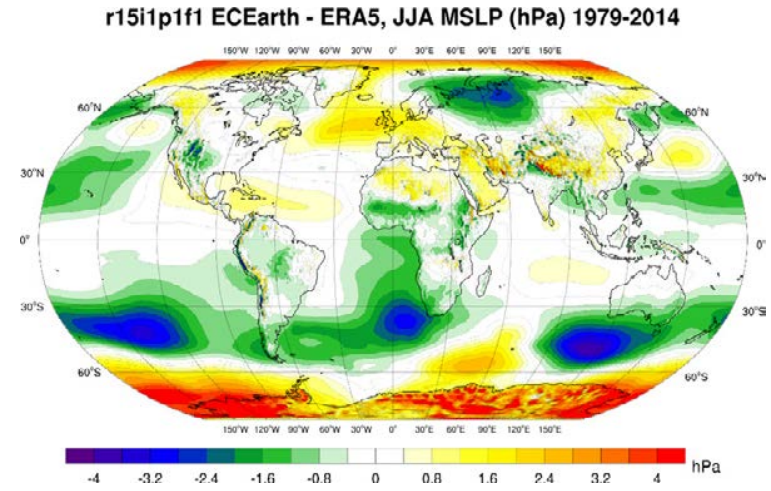

(b)

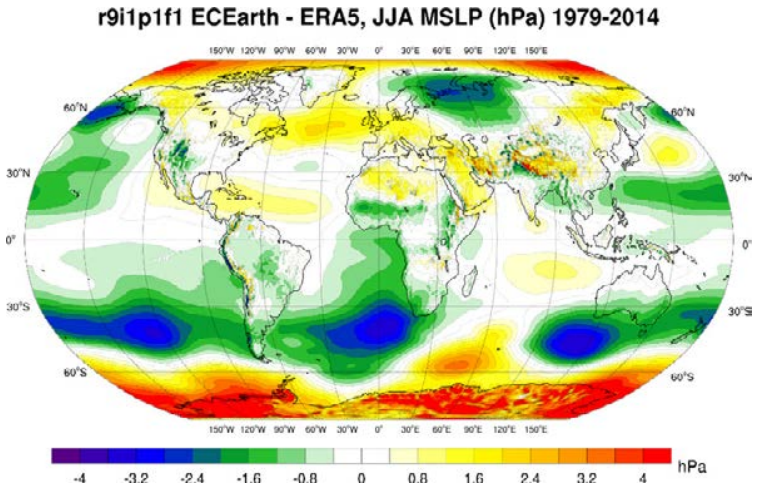

(d)

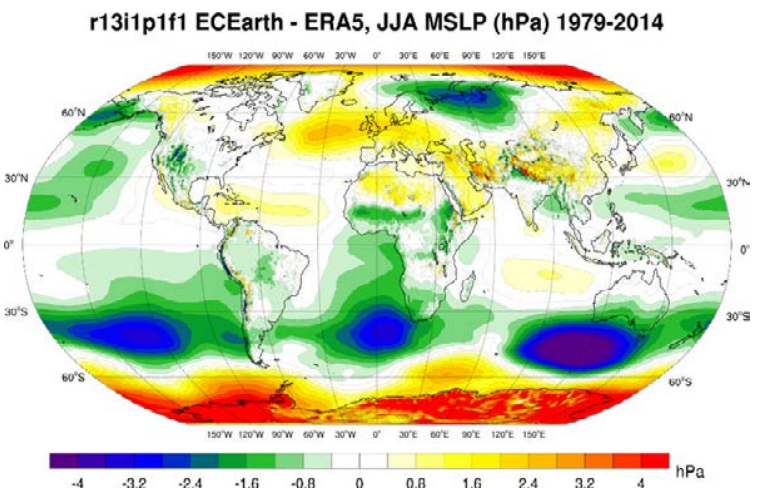

Figure 2.35. EC-Earth MSLP bias (hPa) for JJA, 1979-2014 (ERA5 reanalysis minus EC-Earth): (a) EC-Earth r6i1p1f1, (b) EC-Earth r9i1p1f1, (c) EC-Earth r11i1p1f1, (d) EC-Earth r13i1p1f1 and (e) EC-Earth r15i1p1f1. 
Table 2.7. Mean global annual and seasonal MSLP bias and MAE (hPa) for each of the five EC-Earth ensemble members ${ }^{a}$

\begin{tabular}{|c|c|c|c|c|c|c|c|c|c|c|}
\hline \multirow{2}{*}{$\begin{array}{l}\text { Time } \\
\text { period }\end{array}$} & \multicolumn{2}{|c|}{ r6i1p1f1 } & \multicolumn{2}{|c|}{ r9i1p1f1 } & \multicolumn{2}{|c|}{ r11i1p1f1 } & \multicolumn{2}{|c|}{ r13i1p1f1 } & \multicolumn{2}{|c|}{ r15i1p1f1 } \\
\hline & Bias & MAE & Bias & MAE & Bias & MAE & Bias & MAE & Bias & MAE \\
\hline Annual & -0.12 & 0.82 & -0.10 & 0.74 & -0.12 & 0.90 & -0.12 & 0.78 & -0.12 & 0.68 \\
\hline DJF & -0.07 & 1.13 & -0.06 & 1.17 & -0.05 & 1.29 & -0.07 & 1.15 & -0.07 & 1.08 \\
\hline MAM & -0.11 & 0.94 & -0.09 & 0.88 & -0.10 & 1.06 & -0.11 & 0.98 & -0.11 & 0.87 \\
\hline JJA & -0.14 & 1.04 & -0.11 & 0.99 & -0.16 & 1.05 & -0.14 & 1.0 & -0.13 & 0.89 \\
\hline SON & -0.14 & 1.06 & -0.13 & 1.02 & -0.15 & 1.03 & -0.15 & 0.98 & -0.15 & 0.89 \\
\hline
\end{tabular}

an each case the model data are compared with ERA5 reanalysis data for the period 1979-2014.

(a)

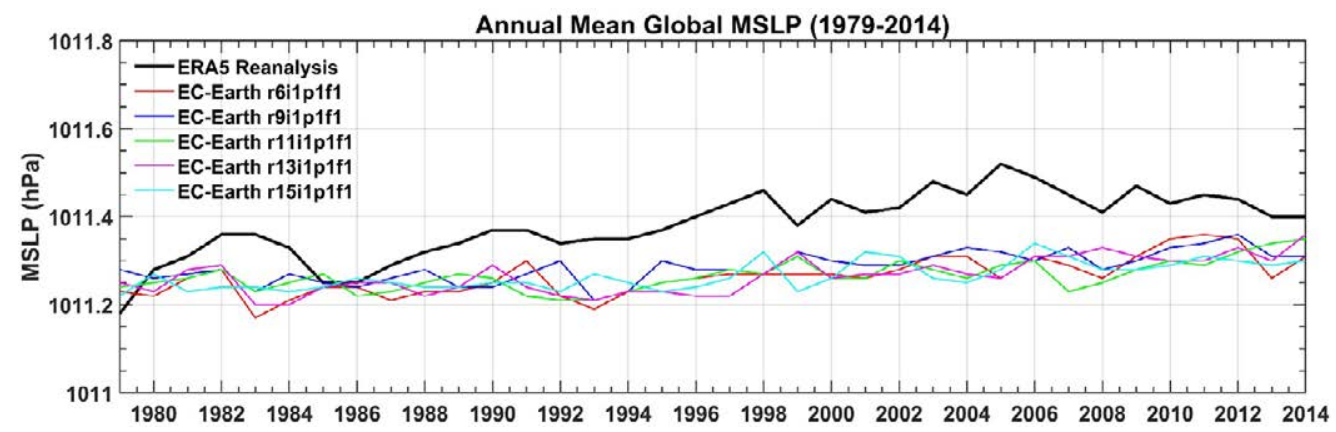

(b)

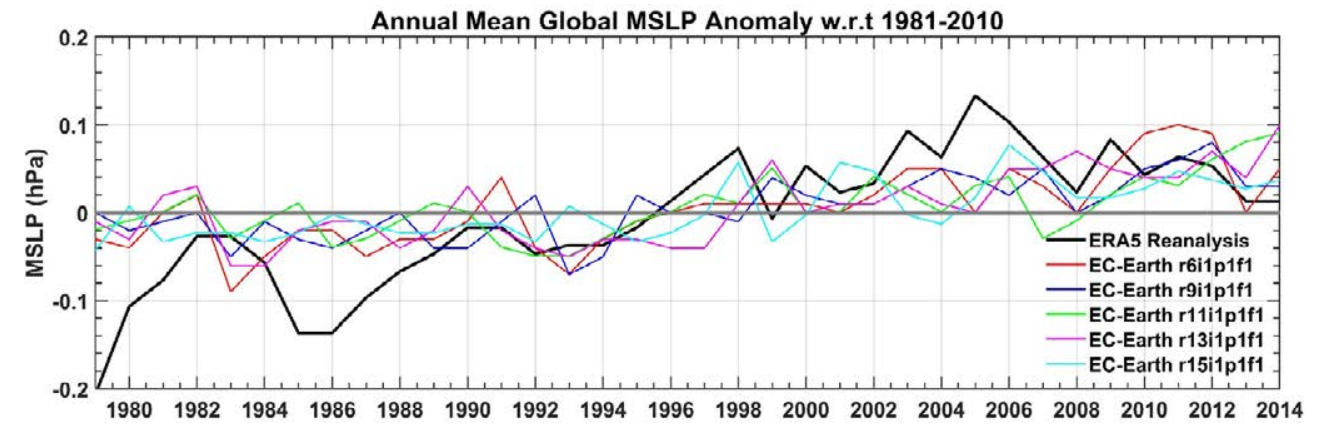

Figure 2.36. Comparison of EC-Earth ensemble members with ERA5 reanalysis data for the period 1979-2014: (a) MSLP and (b) MSLP anomalies with respect to the 30-year period 1981-2010.

\subsubsection{Total cloud cover validations}

Figure 2.37, which shows the ERA5 and EC-Earth spatial distribution of annual mean total cloud cover, demonstrates that all EC-Earth ensemble members accurately capture the magnitude and spatial characteristics of the historical cloud climate.

Figure 2.38 demonstrates that the differences relative to ERA5 data are similar for all EC-Earth ensemble members - an underestimate over the Arctic, Central America, Central Africa and the southern subtropical ocean regions. All other regions exhibit a slight positive bias (or small differences). Cloud cover validations for DJF (Figures 2.39 and 2.40) follow a similar (but enhanced) trend as the annual validations. In addition, negative (positive) biases are noted over the Middle East and India (Australia, South Africa, Indian Ocean and western Pacific) regions during DJF. Total cloud validations for JJA (Figures 2.41 and 2.42) show a positive bias over North America, Russia and the northern subtropical and equatorial ocean regions, and a negative bias over the southern subtropical regions, the Indian Ocean, the north-east Pacific Ocean and India. 
(a)

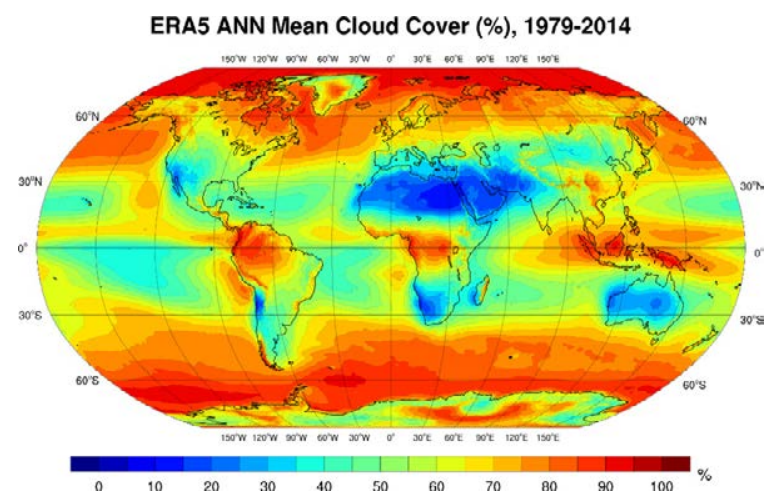

(c)

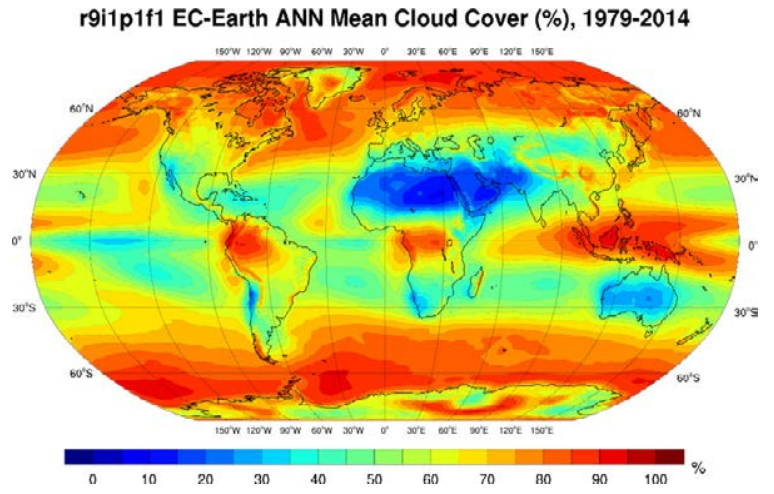

(e)

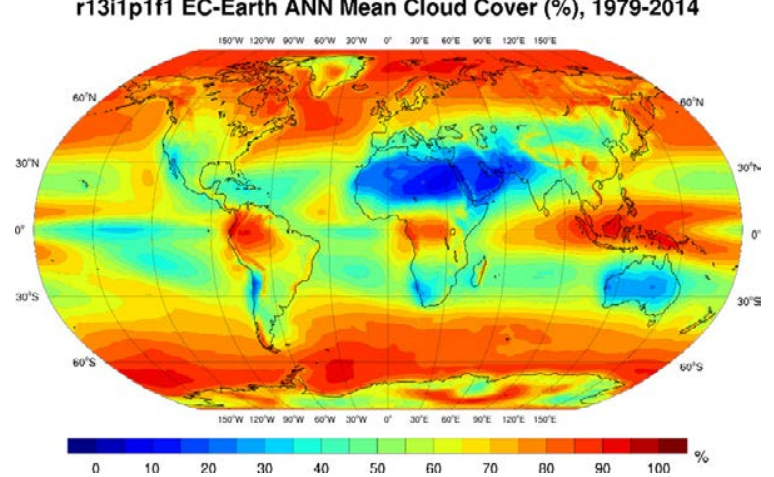

(b)

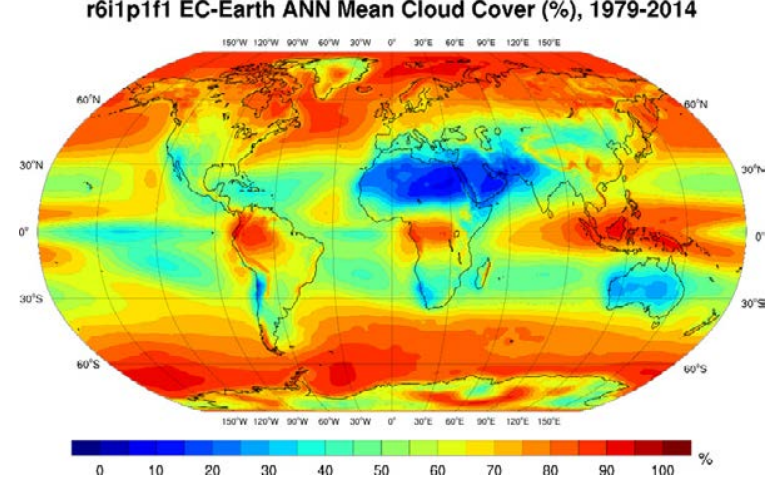

(d)

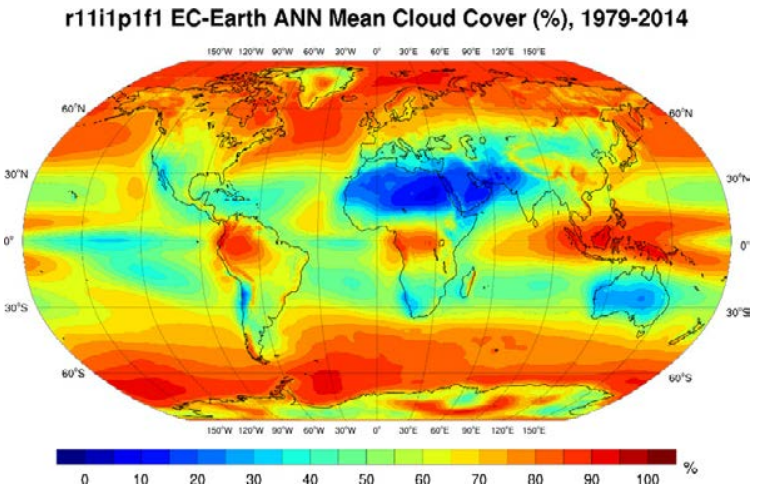

(f)

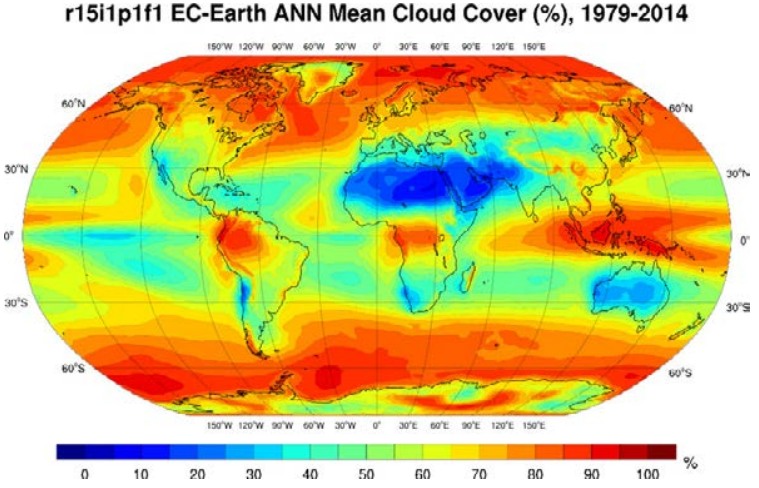

Figure 2.37. Annual mean cloud cover (\%), 1979-2014: (a) ERA5 reanalysis, (b) EC-Earth r6i1p1f1, (c) EC-Earth r9i1p1f1, (d) EC-Earth r11i1p1f1, (e) EC-Earth r13i1p1f1 and (f) EC-Earth r15i1p1f1. 
(a)

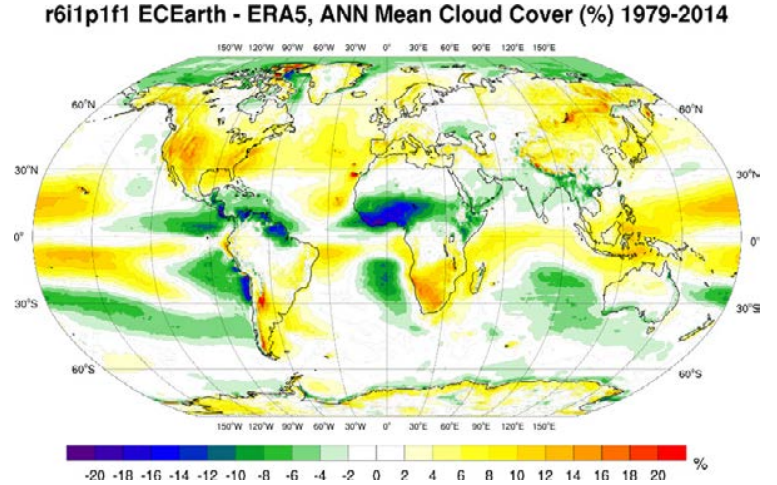

(c)

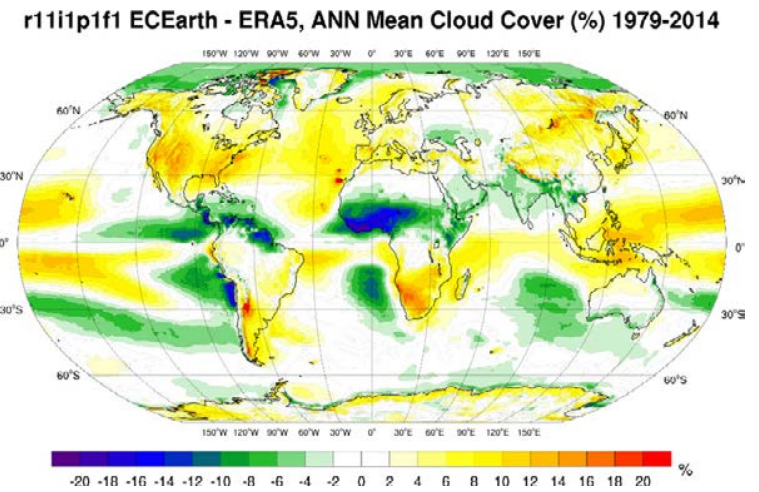

(e)

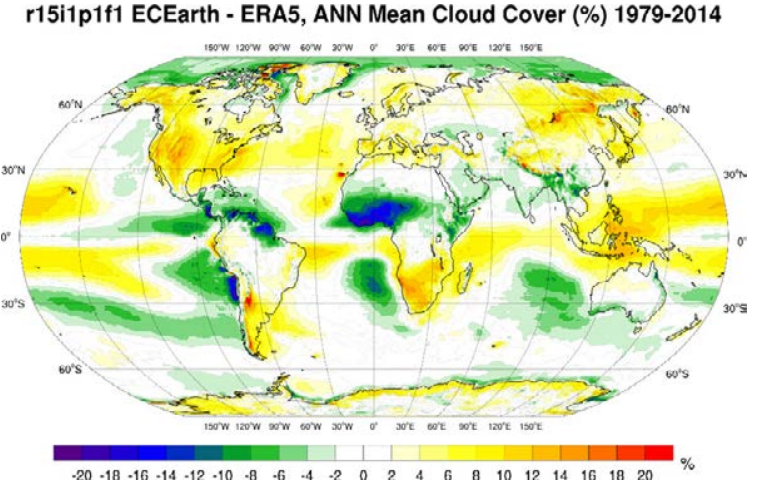

(b)

r9i1p1f1 ECEarth - ERA5, ANN Mean Cloud Cover (\%) 1979-2014

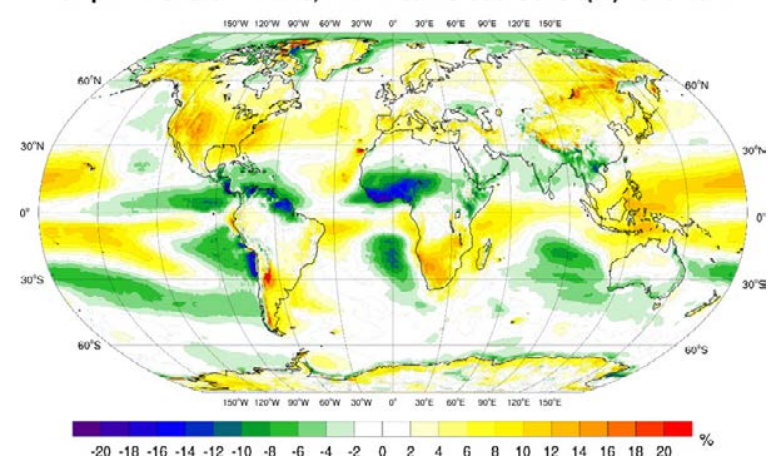

(d)

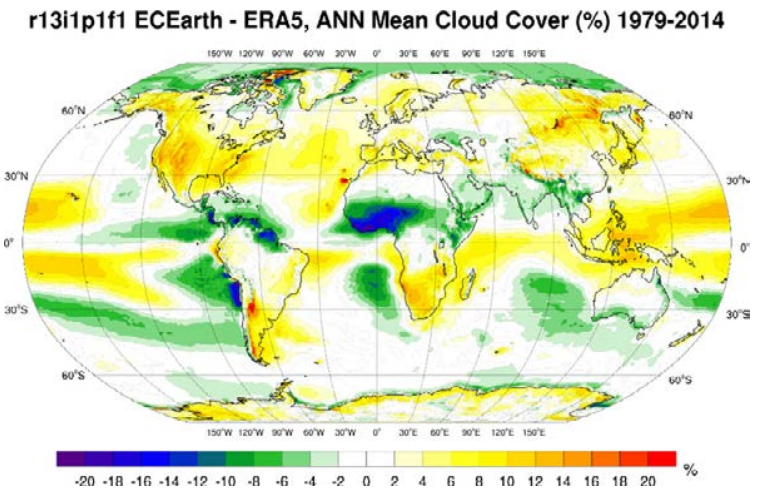

Figure 2.38. EC-Earth annual cloud cover bias (\%), 1979-2014 (ERA5 reanalysis minus EC-Earth):

(a) EC-Earth r6i1p1f1, (b) EC-Earth r9i1p1f1, (c) EC-Earth r11i1p1f1, (d) EC-Earth r13i1p1f1 and (e) EC-Earth r15i1p1f1. 
(a)

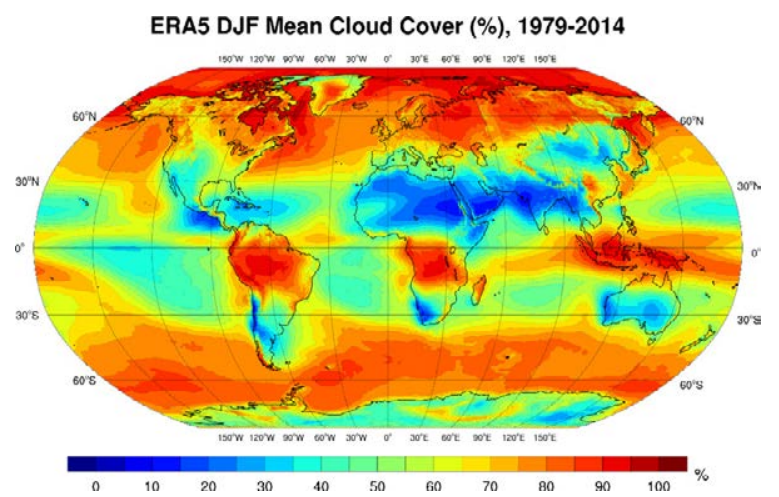

(c)

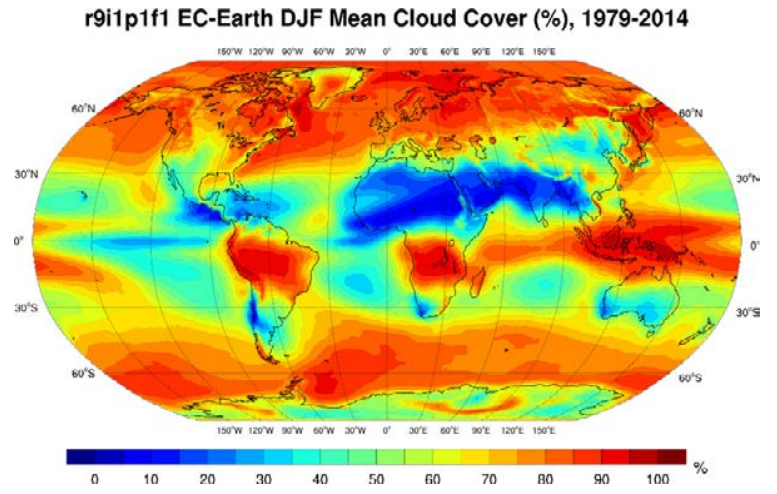

(e)

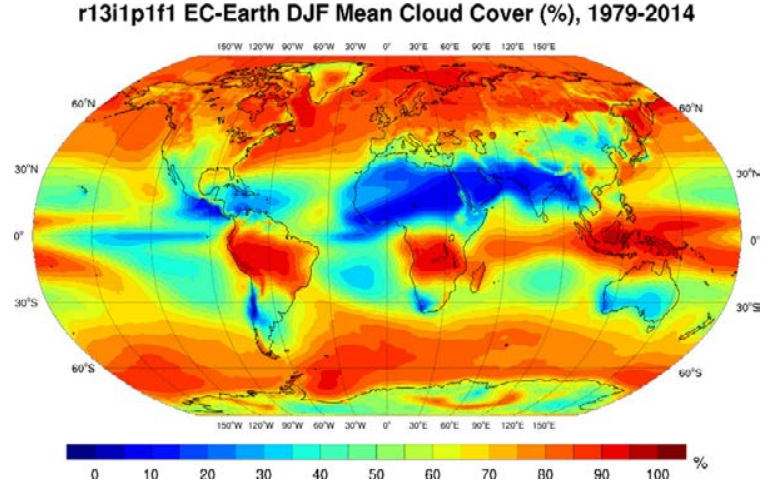

(b)

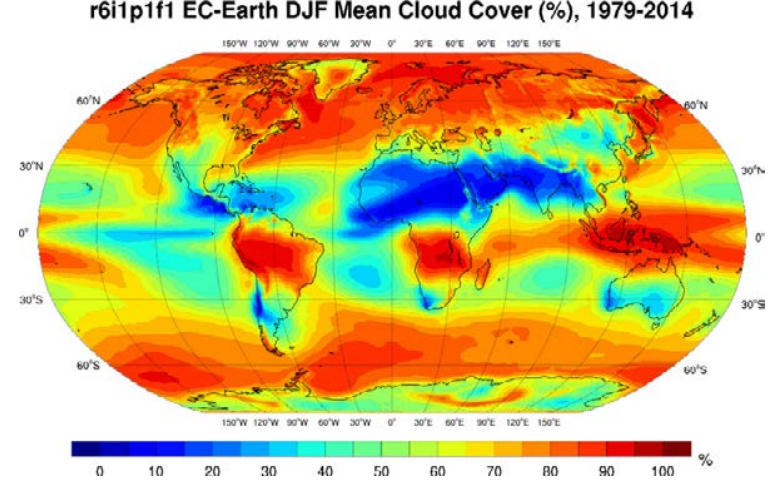

(d)

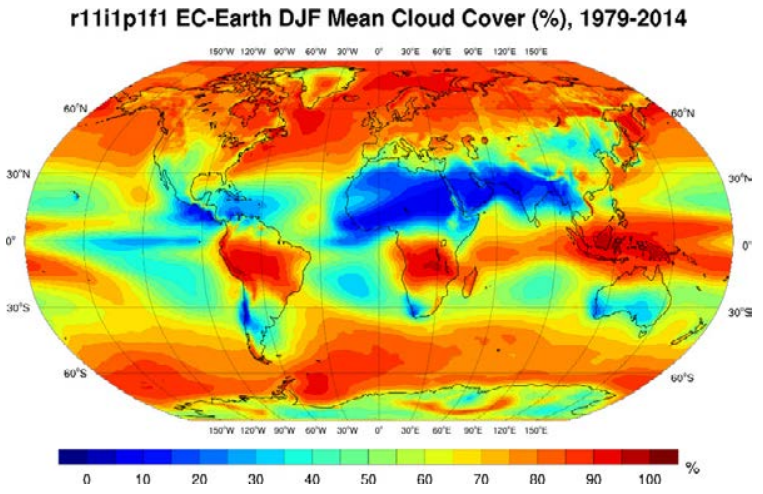

(f)

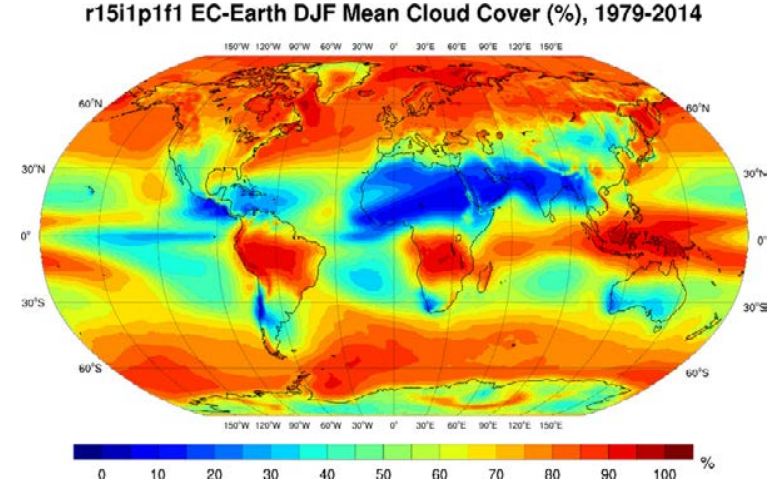

Figure 2.39. Mean cloud cover (\%) for DJF, 1979-2014: (a) ERA5 reanalysis, (b) EC-Earth r6i1p1f1, (c) EC-Earth r9i1p1f1, (d) EC-Earth r11i1p1f1, (e) EC-Earth r13i1p1f1 and (f) EC-Earth r15i1p1f1. 
(a)

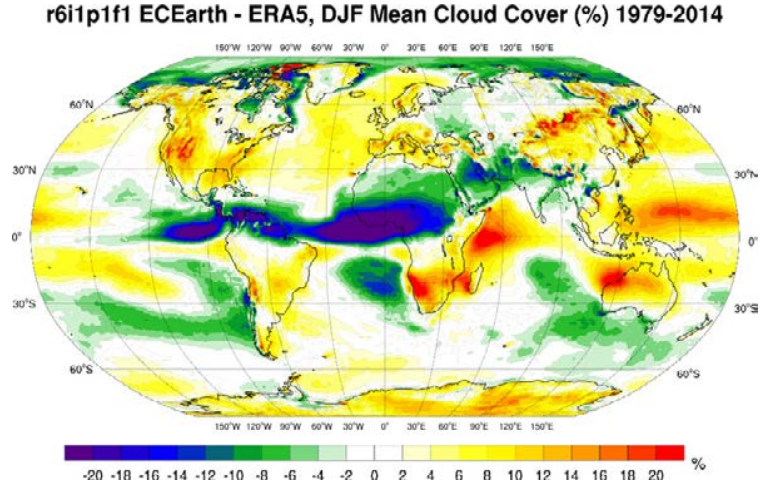

(c)

r1111p1f1 ECEarth - ERA5, DJF Mean Cloud Cover (\%) 1979-2014

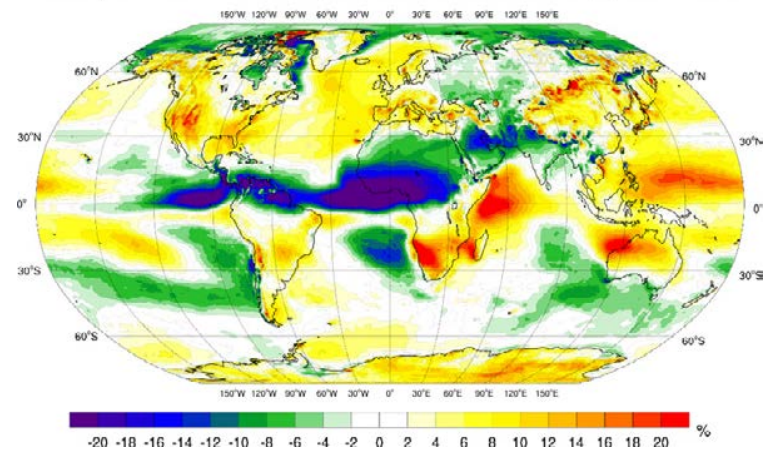

(e)

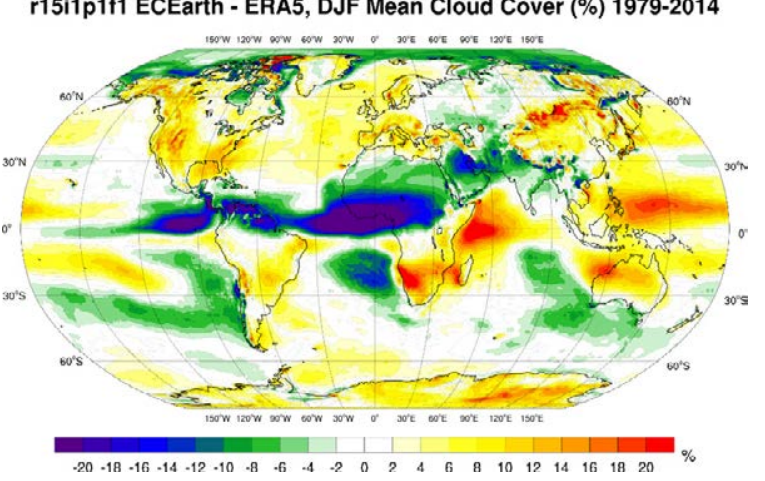

(b)

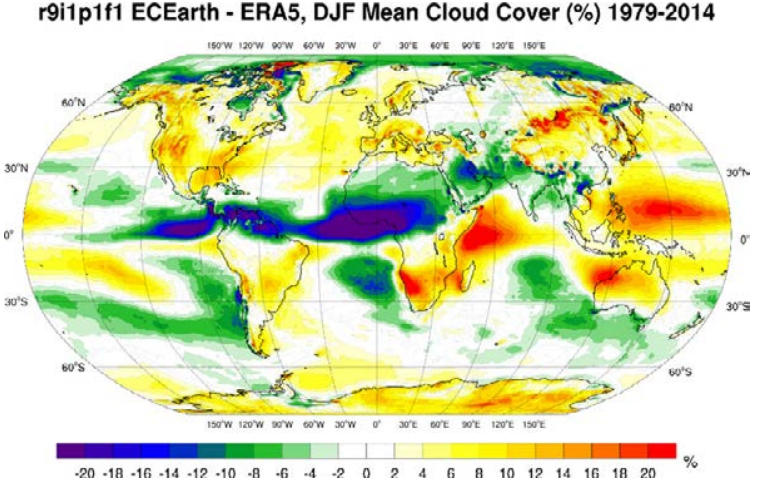

(d)
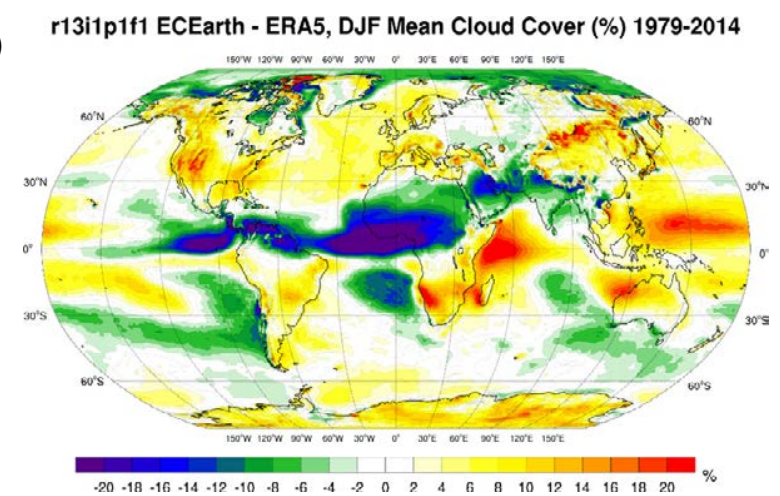

Figure 2.40. EC-Earth cloud cover bias (\%) for DJF, 1979-2014 (ERA5 reanalysis minus EC-Earth):

(a) EC-Earth r6i1p1f1, (b) EC-Earth r9i1p1f1, (c) EC-Earth r11i1p1f1, (d) EC-Earth r13i1p1f1 and

(e) EC-Earth r15i1p1f1. 
(a)

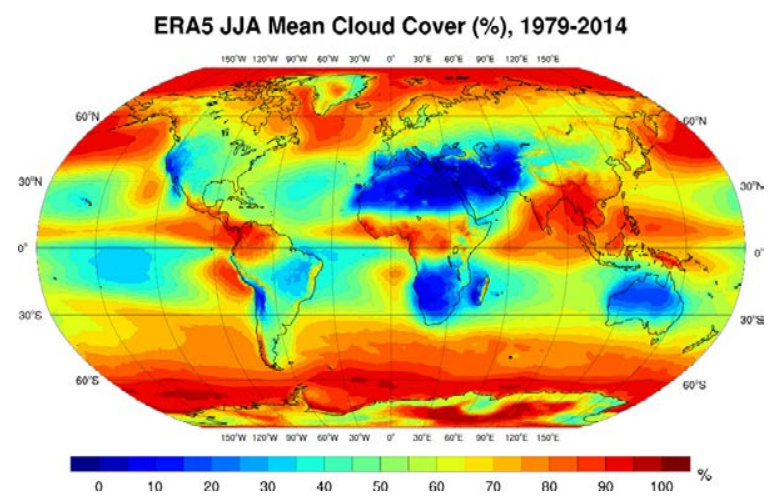

(c)

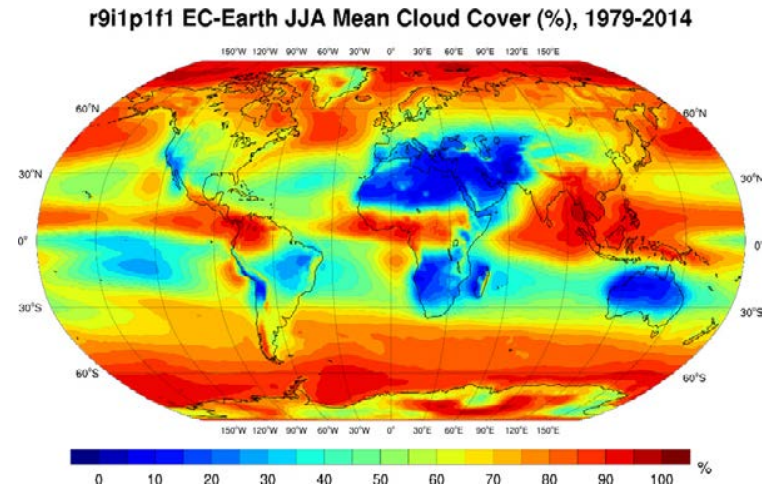

(e)

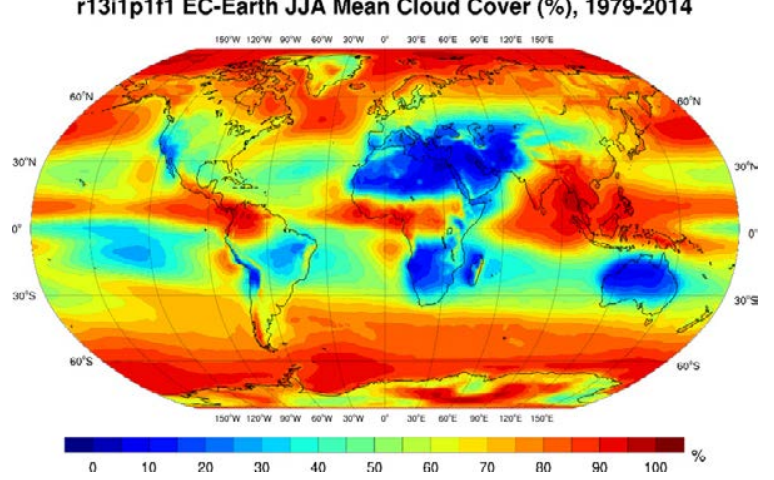

(b)

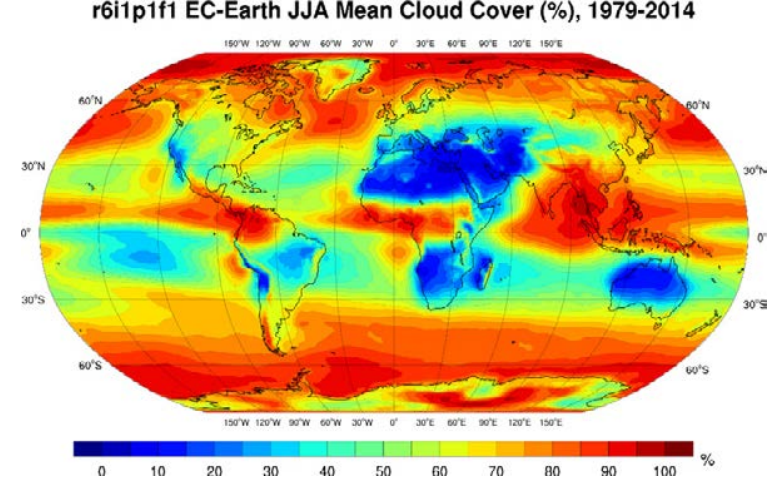

(d)

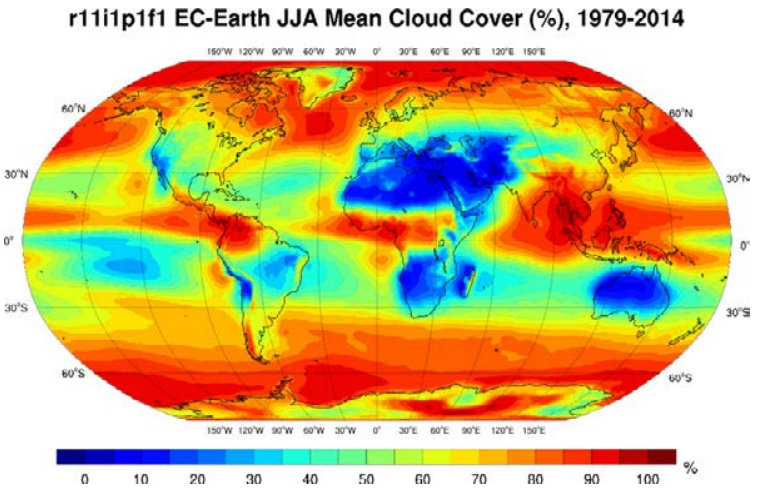

(f)

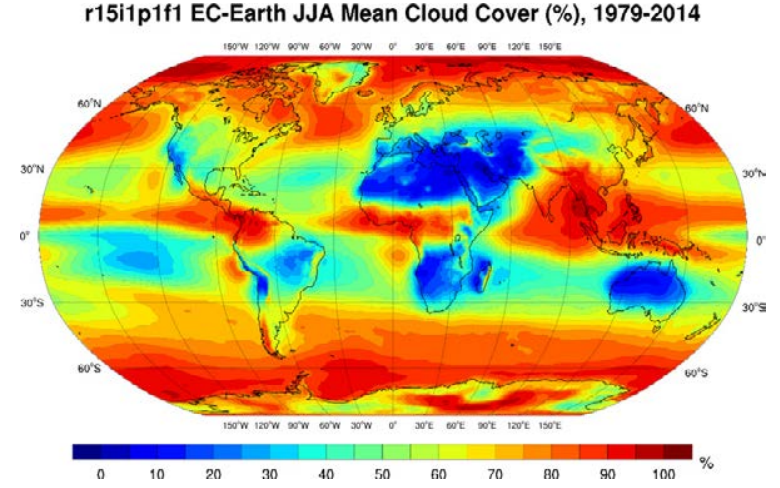

Figure 2.41. Mean cloud cover (\%) for JJA, 1979-2014: (a) ERA5 reanalysis, (b) EC-Earth r6i1p1f1, (c) EC-Earth r9i1p1f1, (d) EC-Earth r11i1p1f1, (e) EC-Earth r13i1p1f1 and (f) EC-Earth r15i1p1f1. 
(a)

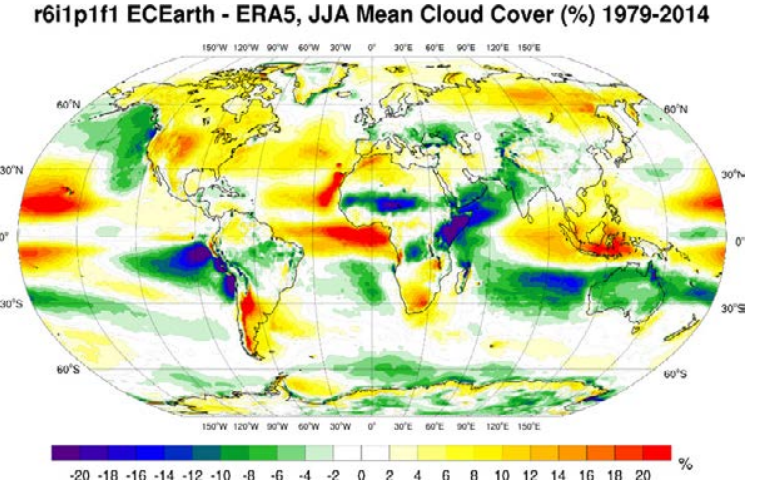

(c)

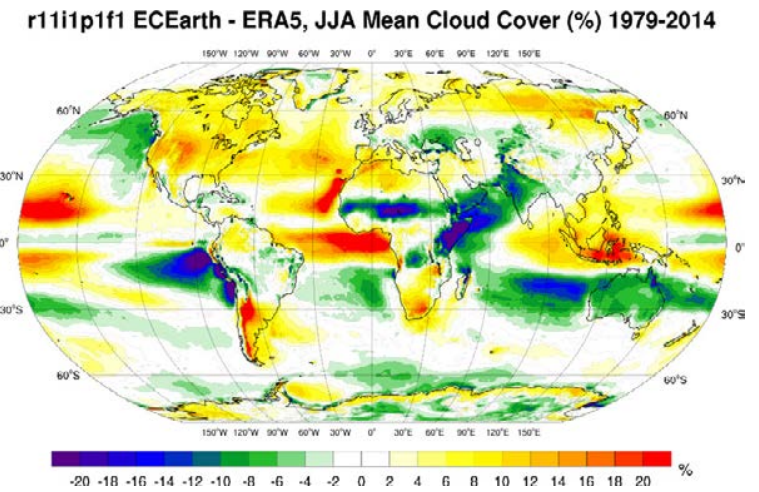

(e)

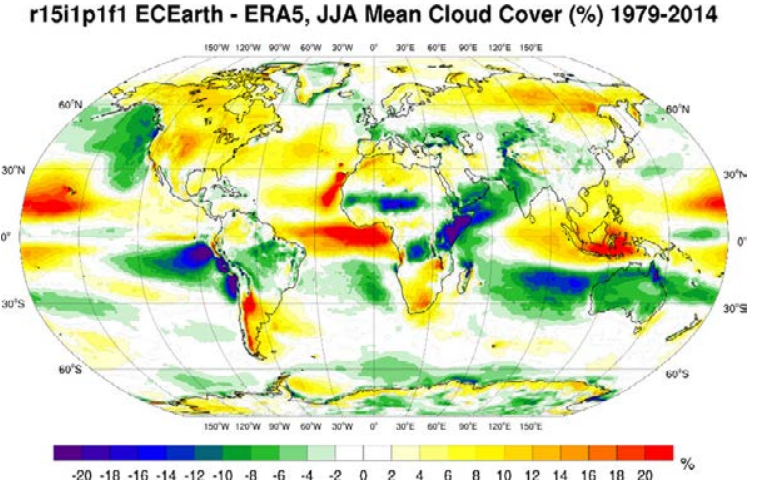

(b)
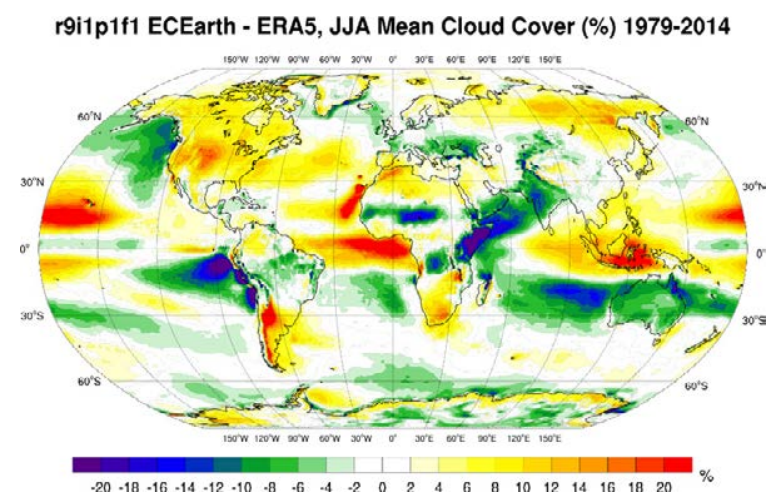

(d)

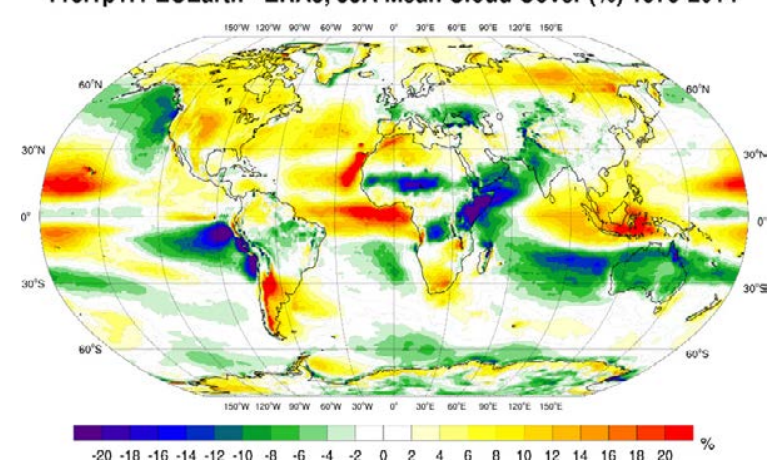

Figure 2.42. EC-Earth cloud cover bias (\%) for JJA, 1979-2014 (ERA5 reanalysis minus EC-Earth): (a) EC-Earth r6i1p1f1, (b) EC-Earth r9i1p1f1, (c) EC-Earth r11i1p1f1, (d) EC-Earth r13i1p1f1 and (e) EC-Earth r15i1p1f1. 
The annual and seasonal overall global cloud cover bias and MAE statistics relative to ERA5 data (1979-2014) for each of the five ensemble members are presented in Table 2.8. The bias statistics are all positive, ranging from $0.95 \%$ (DJF, r15i1p1f1) to $1.55 \%$ (SON, r11i1p1f1). The MAE statistics range from $3.91 \%$ (annual, r15i1p1f1) to $6.36 \%$ (MAM, r11i1p1f1).
The ERA5 and EC-Earth mean global annual total cloud cover time series (1979-2014), presented in Figure 2.43a, demonstrate good agreement. However, all EC-Earth ensemble members exhibit a slight overestimation of cloud cover of $\sim 1.5 \%$ relative to ERA5 data. Figure 2.43b shows the annual anomalies with respect to the 1981-2010 mean; all ERA5 and EC-Earth annual values are within the range of $\pm 0.6 \%$.

Table 2.8. Mean global annual and seasonal cloud cover bias and MAE (\%) for each of the five EC-Earth ensemble members ${ }^{a}$

\begin{tabular}{|c|c|c|c|c|c|c|c|c|c|c|}
\hline \multirow{2}{*}{$\begin{array}{l}\text { Time } \\
\text { period }\end{array}$} & \multicolumn{2}{|c|}{ r6i1p1f1 } & \multicolumn{2}{|c|}{ r9i1p1f1 } & \multicolumn{2}{|c|}{ r11i1p1f1 } & \multicolumn{2}{|c|}{ r13i1p1f1 } & \multicolumn{2}{|c|}{ r15i1p1f1 } \\
\hline & Bias & MAE & Bias & MAE & Bias & MAE & Bias & MAE & Bias & MAE \\
\hline Annual & 1.25 & 4.03 & 1.12 & 4.0 & 1.27 & 4.24 & 1.21 & 4.02 & 1.12 & 3.91 \\
\hline DJF & 1.07 & 5.74 & 1.01 & 5.75 & 1.0 & 6.03 & 1.03 & 5.82 & 0.95 & 5.60 \\
\hline MAM & 1.17 & 6.08 & 0.97 & 6.22 & 1.11 & 6.36 & 1.10 & 6.08 & 1.01 & 6.05 \\
\hline JJA & 1.31 & 5.12 & 1.15 & 5.12 & 1.43 & 5.32 & 1.27 & 5.21 & 1.19 & 5.04 \\
\hline SON & 1.48 & 4.53 & 1.36 & 4.61 & 1.55 & 4.68 & 1.43 & 4.55 & 1.32 & 4.42 \\
\hline
\end{tabular}

aln each case the model data are compared with ERA5 reanalysis data for the period 1979-2014.

(a)

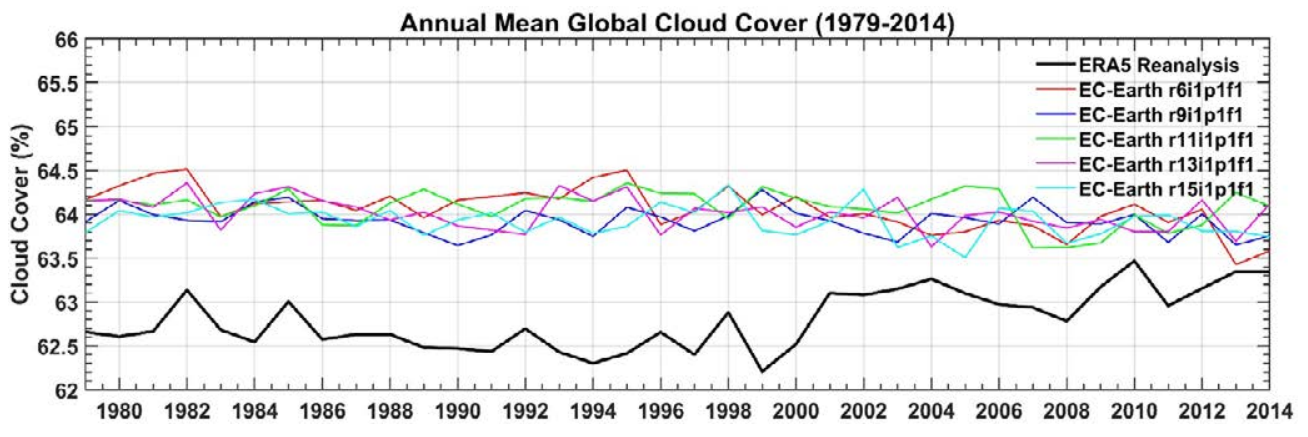

(b)

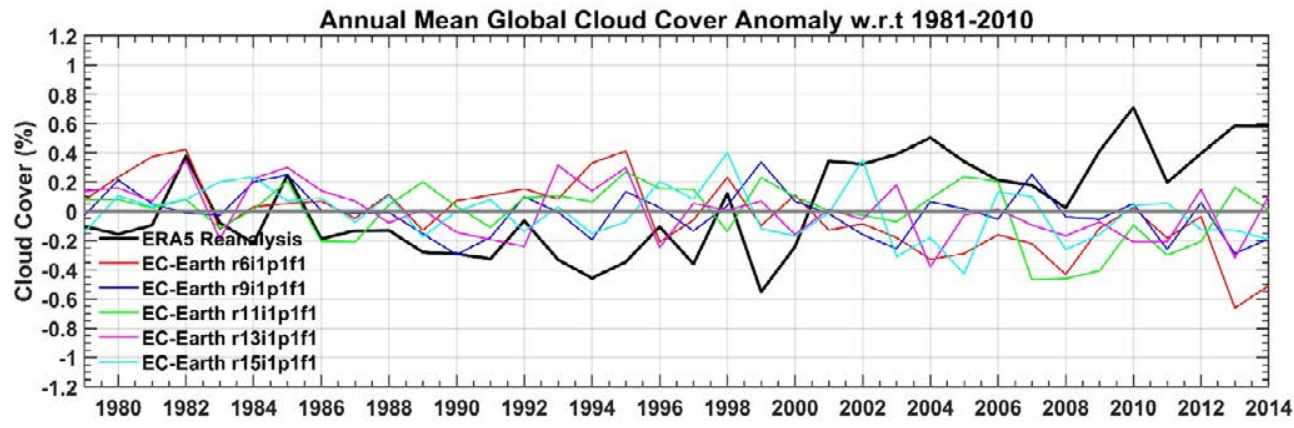

Figure 2.43. Comparison of EC-Earth ensemble members with ERA5 reanalysis data for the period 1979-2014: (a) cloud cover and (b) cloud cover anomalies with respect to the 30-year period 1981-2010. 


\subsubsection{Snowfall validations}

Figure 2.44 presents the annual mean snowfall (mm/day) for ERA5 and the five EC-Earth ensemble members for the period 1979-2014. The EC-Earth ensemble members perform well relative to ERA5 data, showing similar spatial magnitudes and spatial distributions of snowfall. Figure 2.45 demonstrates that the differences relative to ERA5 data are similar for all EC-Earth ensemble members - an underestimation over the Southern and Arctic Oceans and a slight overestimation over Antarctica. A slight overestimation is also noted over the north-west Atlantic for ensemble members r6i1p1f1 and r11i1p1f1. Snowfall validations for DJF (Figures 2.46 and 2.47) show a similar (but enhanced) signal to the annual validations. In addition, a negative bias is noted over much of Europe and Russia. Snowfall validations for JJA (Figures 2.48 and 2.49) show a positive bias over Antarctica and a negative bias over the Southern Hemisphere oceans.

The global annual snowfall anomalies with respect to the 1981-2010 mean, presented in Figure 2.50, demonstrate good agreement between the EC-Earth ensemble members and ERA5; all ERA5 and EC-Earth annual values are within the range of $-6 \%$ to $4 \%$.

\subsubsection{Sea surface temperature validations}

Figure 2.51 shows the ERA5 and EC-Earth mean annual SST for 1979-2014. All EC-Earth ensemble members accurately capture the magnitude and spatial characteristics of SST. Figure 2.52 demonstrates that the differences relative to ERA5 data are similar for all EC-Earth ensemble members, with a warm bias over most regions, in particular the Southern Hemisphere oceans. A cold bias is noted over the North Atlantic, the magnitude of which varies between ensemble members; the largest cold bias is noted for ensemble member r11i1p1f1 (Figure 2.52c). SST validations for DJF (Figures 2.53 and 2.54) and JJA (Figures 2.55 and 2.56) exhibit a similar signal to the annual validations.

In order to quantify the EC-Earth cold bias over the North Atlantic, the mean and minimum bias values were calculated over the area $40-60^{\circ} \mathrm{N}, 25-55^{\circ} \mathrm{W}$. The results, presented in Table 2.9, show that the cold anomaly exists for all ensemble members and all seasons, with the largest biases noted for r11i1p1f1.
The annual and seasonal overall global bias and MAE statistics relative to ERA5 data (1979-2014) for each of the five ensemble members are presented in Table 2.10. The bias statistics are all positive, ranging from $0.67^{\circ} \mathrm{C}$ (SON, r11ip1fi) to $1.32^{\circ} \mathrm{C}$ (DJF, r9i1p1f1). The MAE statistics range from $1.03^{\circ} \mathrm{C}(\mathrm{SON}$, r6i1p1f1) to $1.63^{\circ} \mathrm{C}$ (MAM, r11i1p1f1).

The ERA5 and EC-Earth mean global annual SST time series (1979-2014), presented in Figure 2.57a, show that all EC-Earth ensemble members exhibit an overestimation of SST of $\sim 1^{\circ} \mathrm{C}$. Figure $2.57 \mathrm{~b}$ shows the annual SST anomalies with respect to the 1981-2010 mean; there is a slight overestimation of the EC-Earth SST rise during the later years.

\subsubsection{Sea ice fraction validations}

The Northern Hemisphere mean annual sea ice fraction (1979-2014) for ERA5 and each of the five EC-Earth ensemble members is presented in Figure 2.58. The green line shows the $50 \%$ contour line. All EC-Earth ensemble members are similar to ERA5 but show an overestimation of the extent of the sea ice. The Northern Hemisphere sea ice fractions for March and September are presented in Figures 2.59 and 2.60, respectively. Again, all ensemble members are similar to ERA5 but show a slight overestimation of the extent of the sea ice. Similarly, the Southern Hemisphere sea ice fraction is presented in Figure 2.61 (annual), Figure 2.62 (March) and Figure 2.63 (September). In the Southern Hemisphere, all EC-Earth ensemble members underestimate sea ice extent (and fraction; see below), particularly during March.

Figure 2.64 presents the ERA5 and EC-Earth Northern Hemisphere sea ice fraction time series (1979-2014) over the full year and for March and September. A close agreement is noted for all EC-Earth ensemble members relative to ERA5 data, with EC-Earth performing best during September and over the full year. Figure 2.65 shows the sea ice fraction anomalies with respect to the 1981-2010 mean over the full year and for March and September; all EC-Earth ensemble members accurately resolve the downwards trend in Northern Hemisphere sea ice fraction. 
Figure 2.66 presents the ERA5 and EC-Earth Southern Hemisphere sea ice fraction time series (1979-2014) over the full year and for March and September. An underestimation is noted for all EC-Earth ensemble members relative to ERA5 data. Figure 2.67 shows the annual sea ice fraction anomalies with respect to the 1981-2010 mean over the full year and for March and September; the EC-Earth anomalies are in close agreement with ERA5. (a)

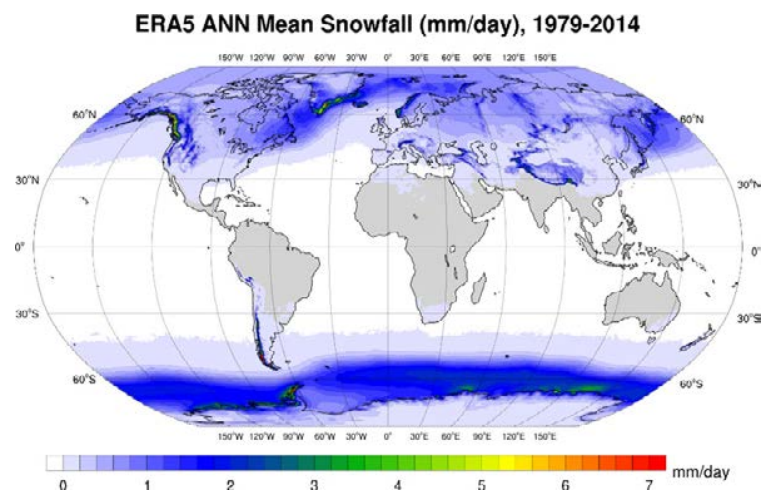

(c)

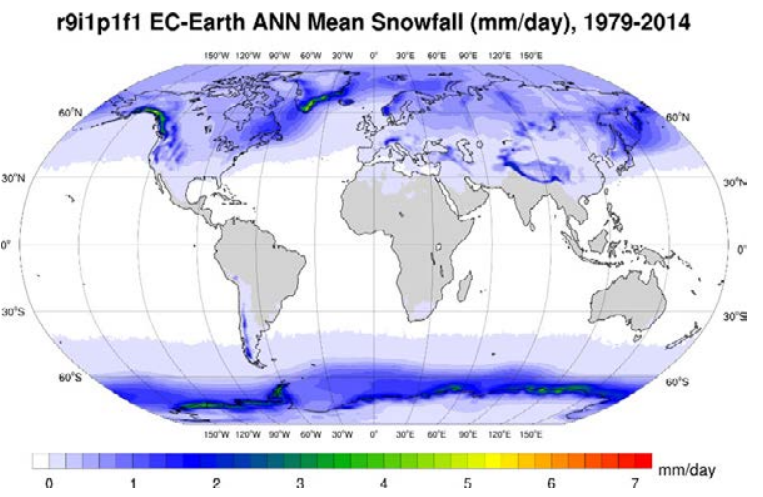

(e)

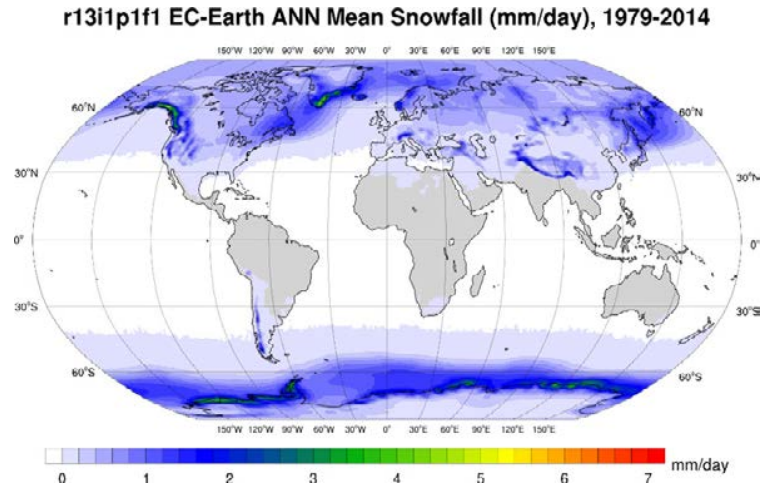

(b)

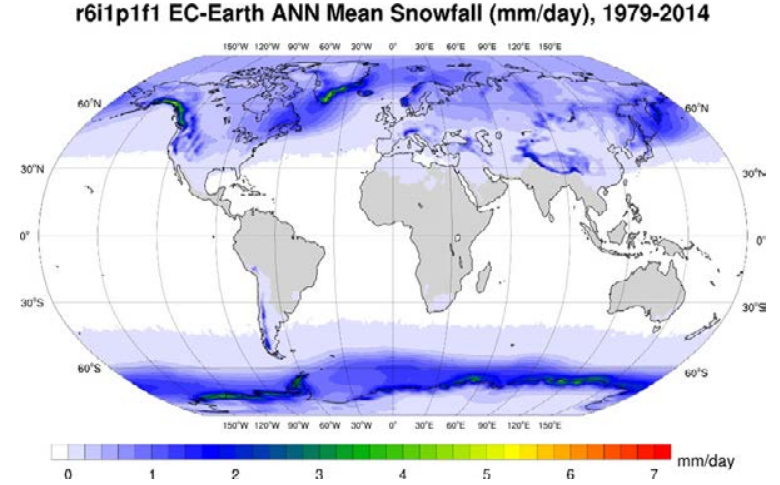

(d)

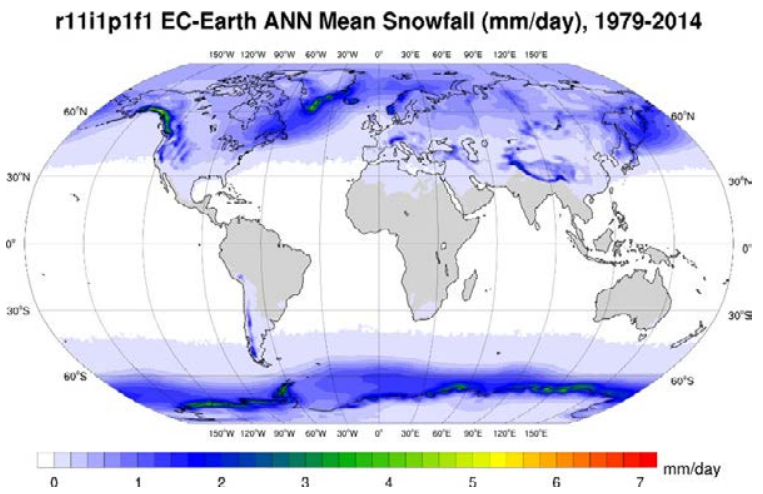

(f)

r15i1p1f1 EC-Earth ANN Mean Snowfall (mm/day), 1979-2014

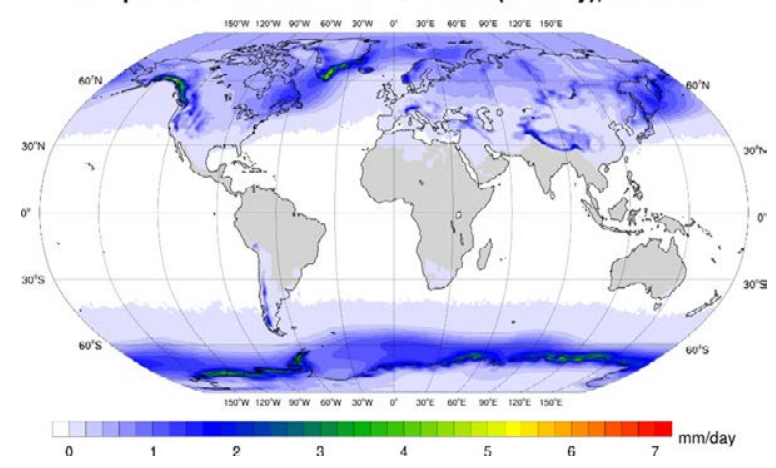

Figure 2.44. Annual mean daily snowfall (mm/day), 1979-2014: (a) ERA5 reanalysis, (b) EC-Earth r6i1p1f1, (c) EC-Earth r9i1p1f1, (d) EC-Earth r11i1p1f1, (e) EC-Earth r13i1p1f1 and (f) EC-Earth r15i1p1f1. 
(a)

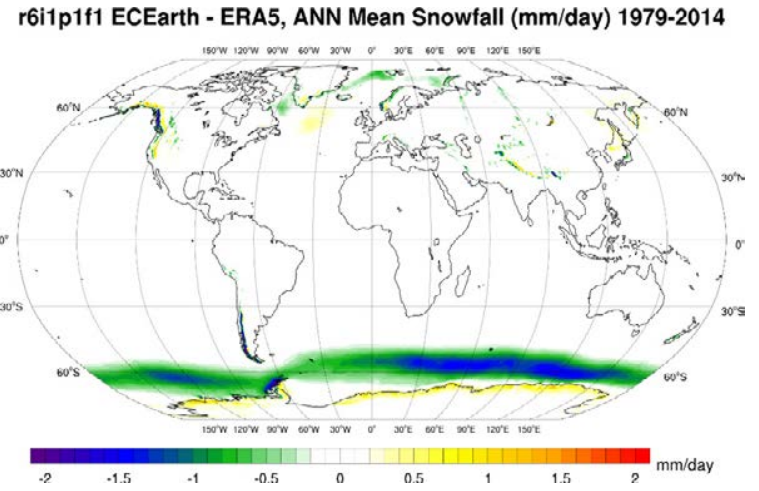

(c)
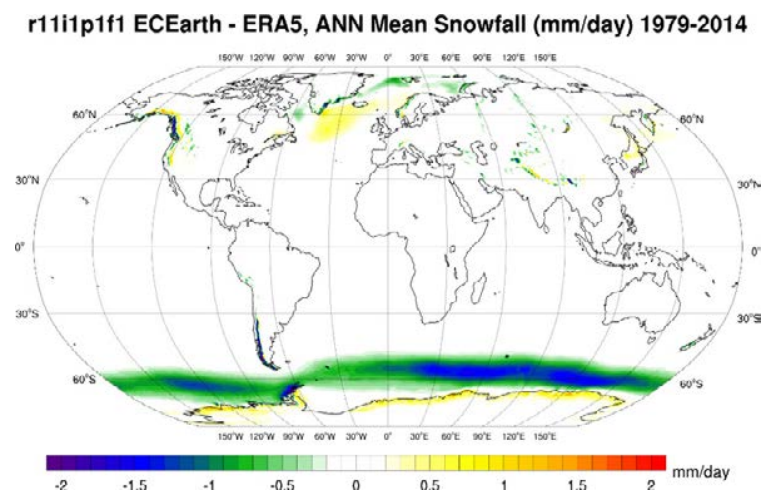

(e)

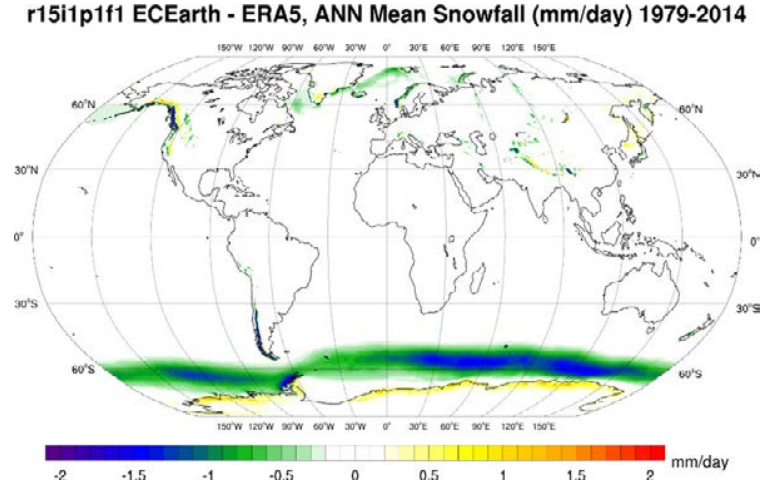

(b)

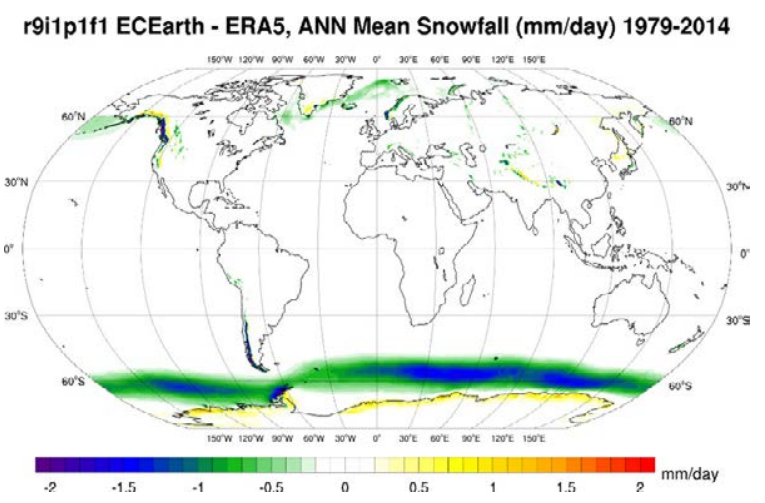

(d)

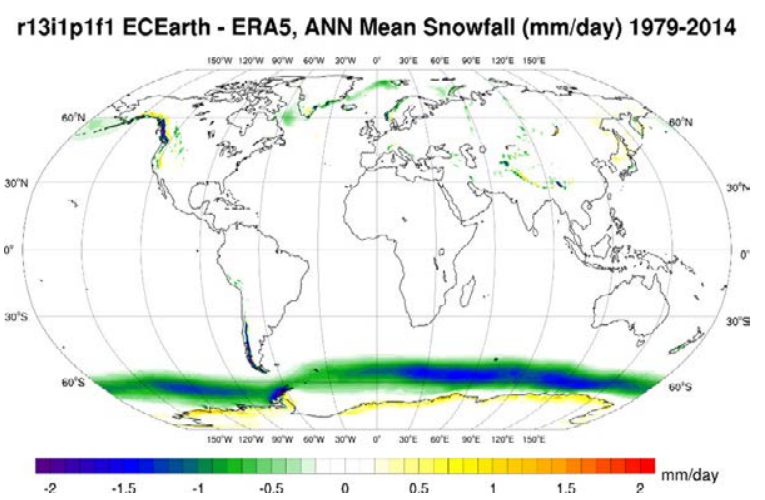

Figure 2.45. EC-Earth annual mean daily snowfall bias (mm/day), 1979-2014 (ERA5 reanalysis minus EC-Earth): (a) EC-Earth r6i1p1f1, (b) EC-Earth r9i1p1f1, (c) EC-Earth r11i1p1f1, (d) EC-Earth r13i1p1f1 and (e) EC-Earth r15i1p1f1. 
(a)

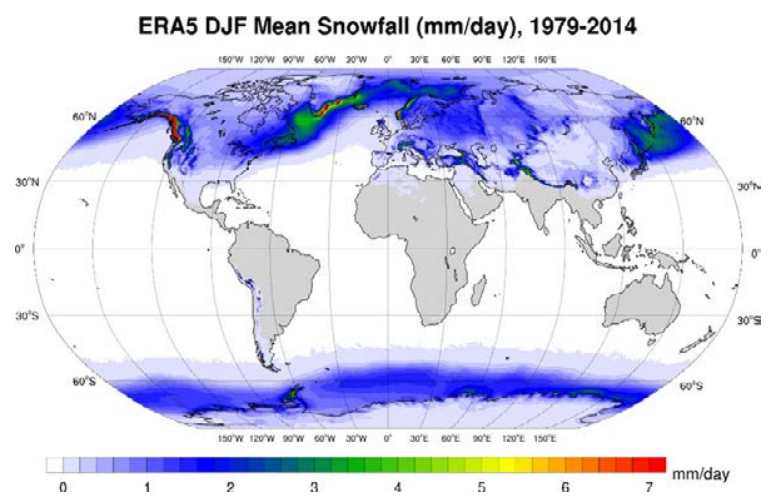

(c)

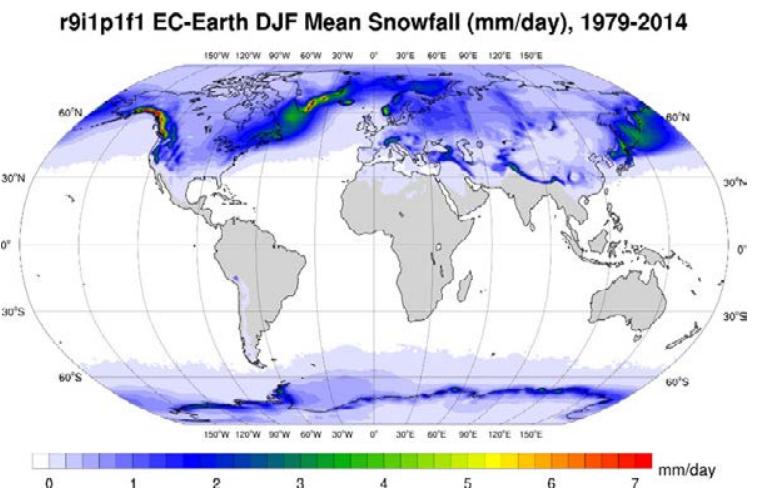

(e)

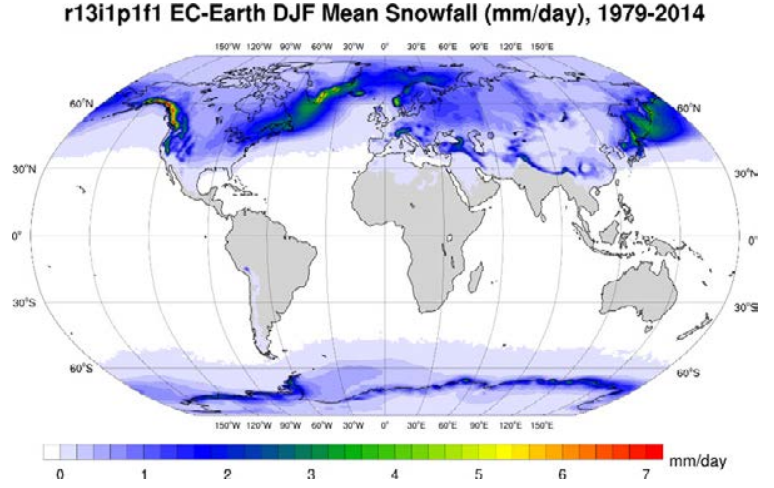

(b)

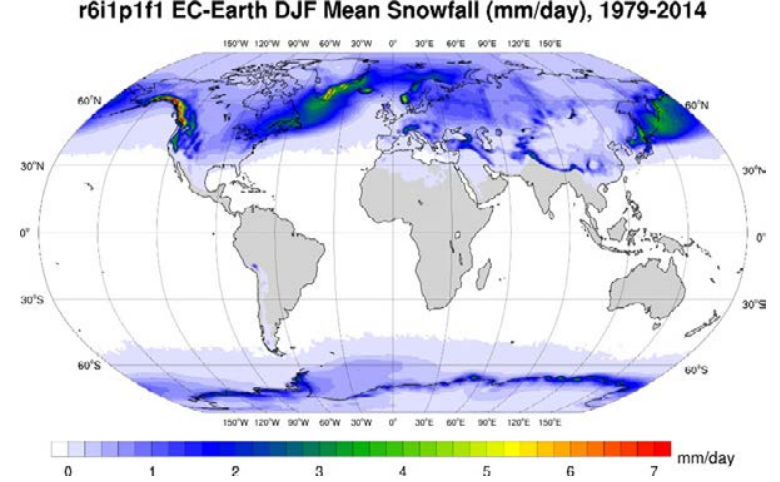

(d)

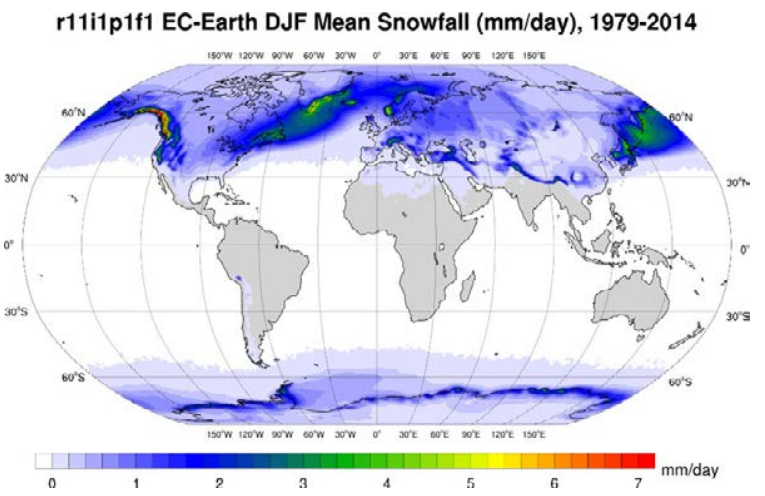

(f)

r15i1p1f1 EC-Earth DJF Mean Snowfall (mm/day), 1979-2014

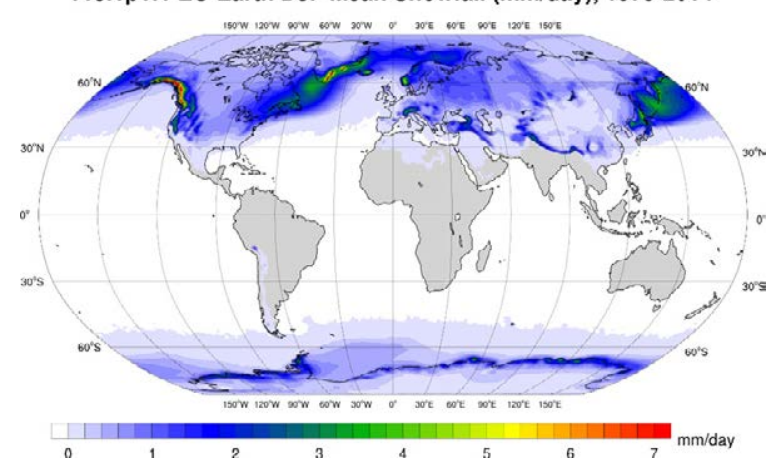

Figure 2.46. Mean daily snowfall (mm/day) for DJF, 1979-2014: (a) ERA5 reanalysis, (b) EC-Earth r6i1p1f1, (c) EC-Earth r9i1p1f1, (d) EC-Earth r11i1p1f1, (e) EC-Earth r13i1p1f1 and (f) EC-Earth r15i1p1f1. 
(a)

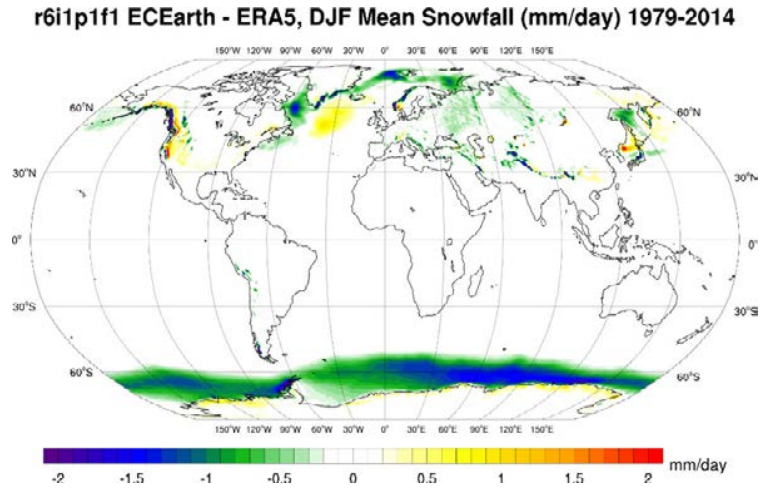

(c)
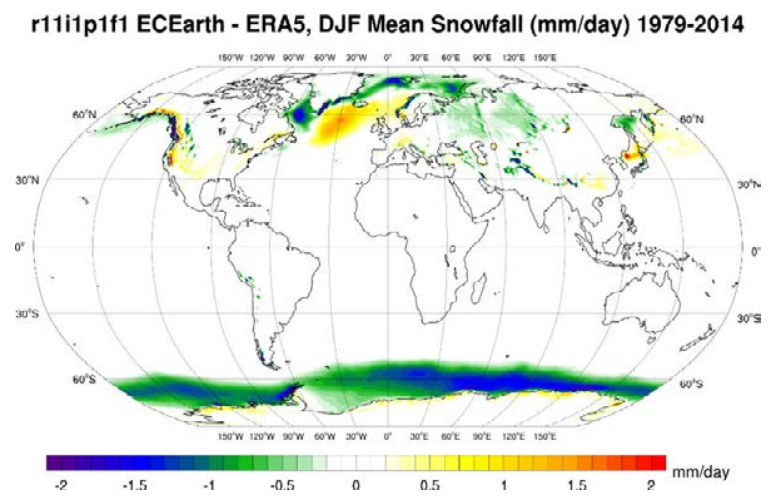

(e)

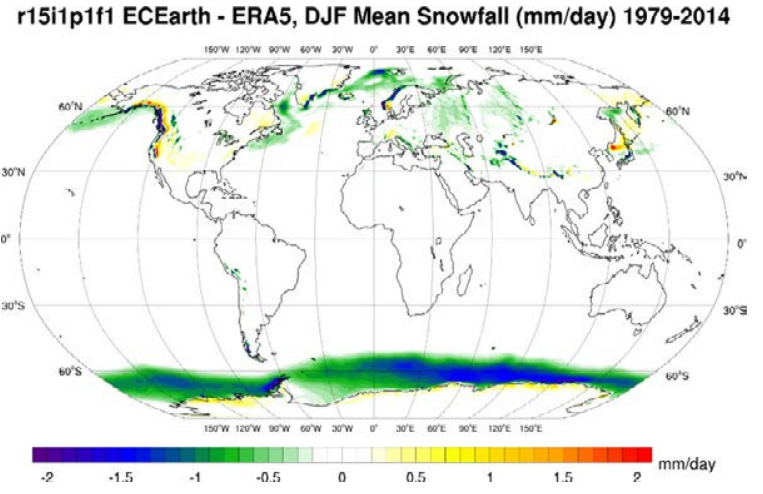

(b)

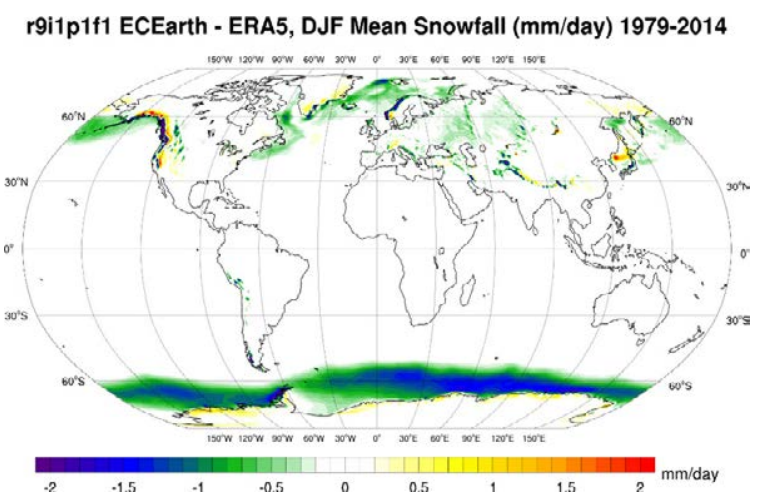

(d)

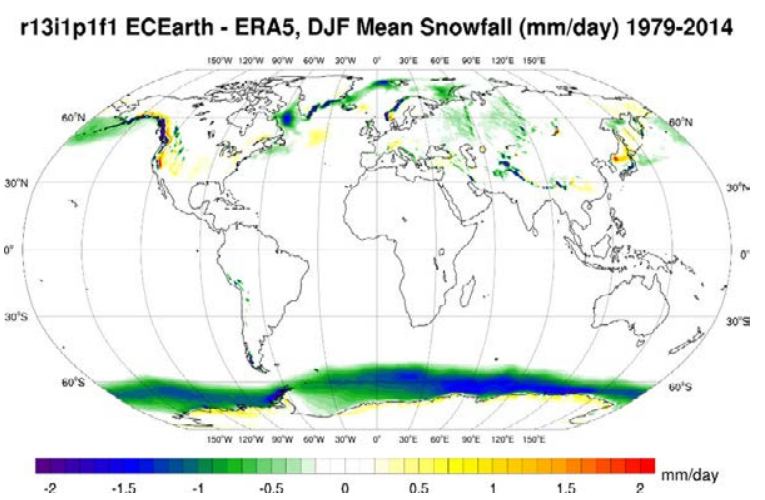

Figure 2.47. EC-Earth mean daily snowfall bias (mm/day) for DJF, 1979-2014 (ERA5 reanalysis minus EC-Earth): (a) EC-Earth r6i1p1f1, (b) EC-Earth r9i1p1f1, (c) EC-Earth r11i1p1f1, (d) EC-Earth r13i1p1f1 and (e) EC-Earth r15i1p1f1. 
(a)

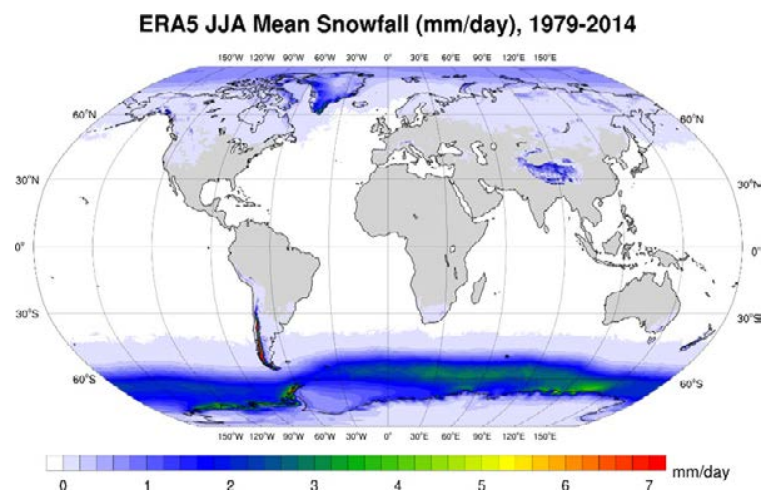

(c)

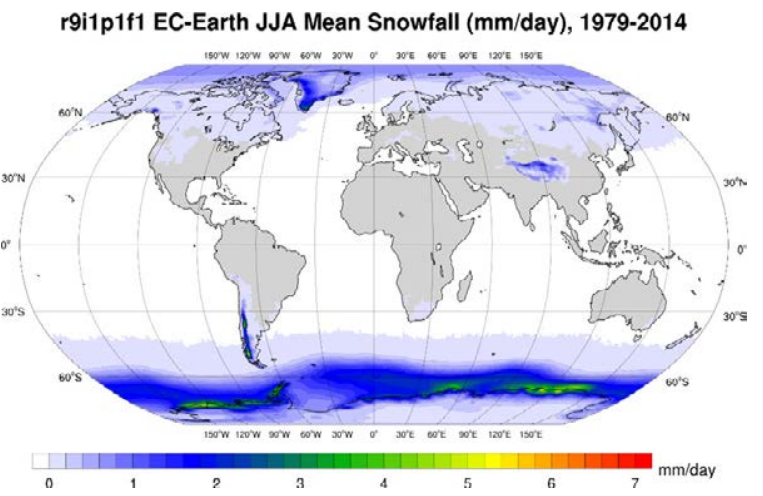

(e)

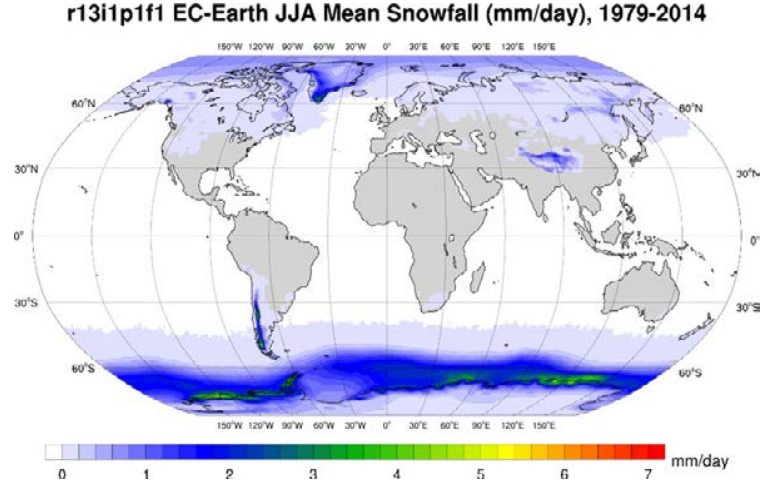

(b)

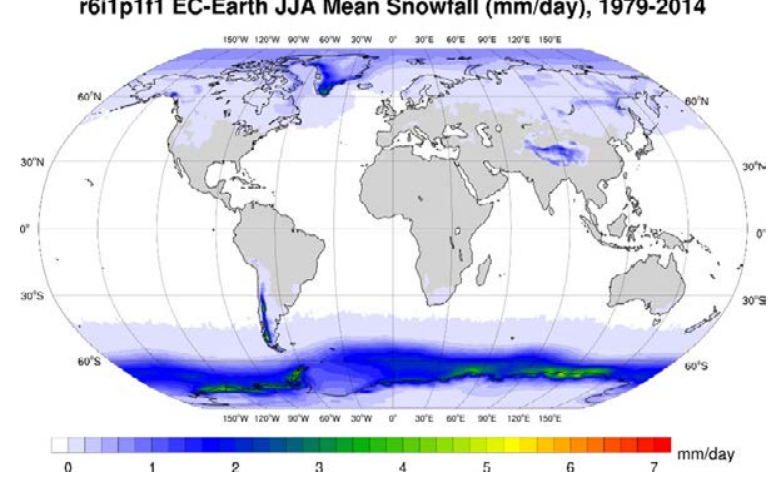

(d)

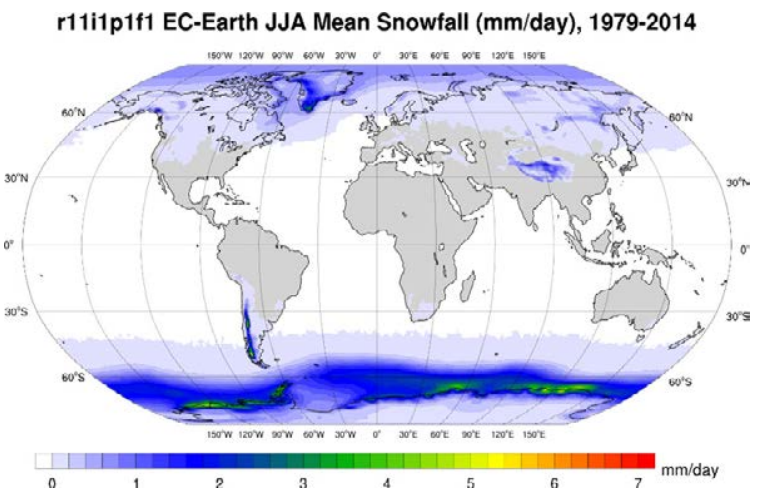

(f)

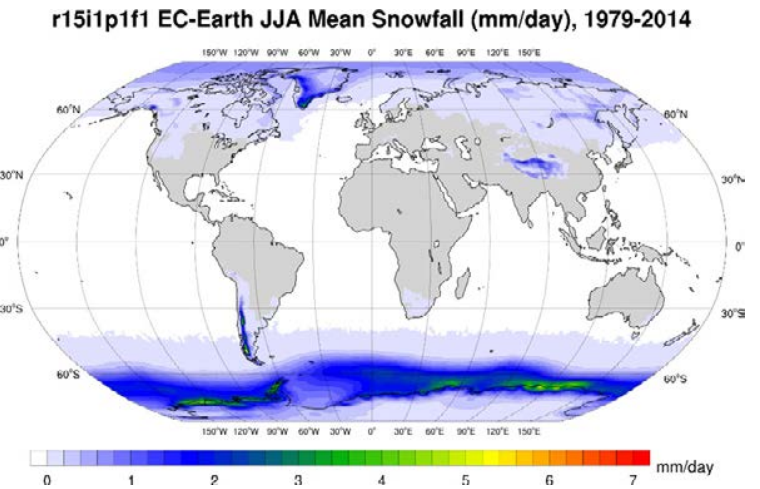

Figure 2.48. Mean daily snowfall (mm/day) for JJA, 1979-2014: (a) ERA5 reanalysis, (b) EC-Earth r6i1p1f1, (c) EC-Earth r9i1p1f1, (d) EC-Earth r11i1p1f1, (e) EC-Earth r13i1p1f1 and (f) EC-Earth r15i1p1f1. 
(a)

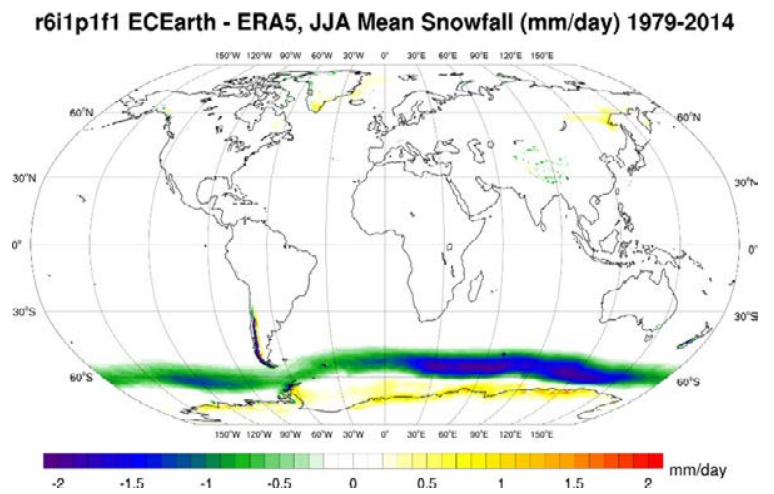

(c)

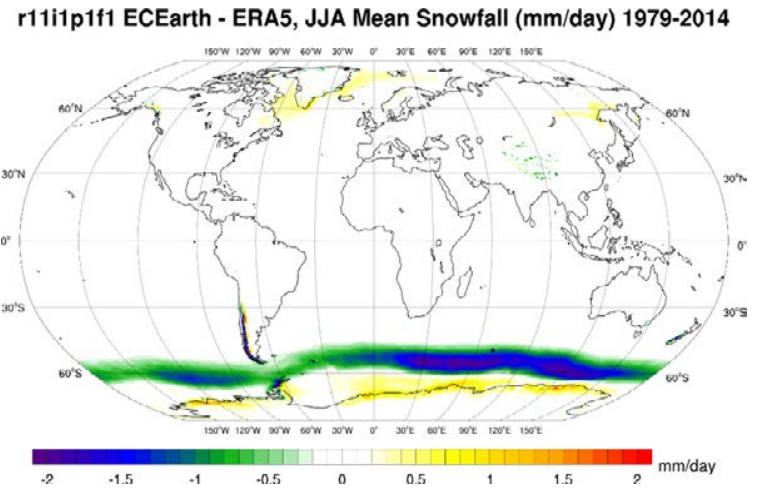

(e)

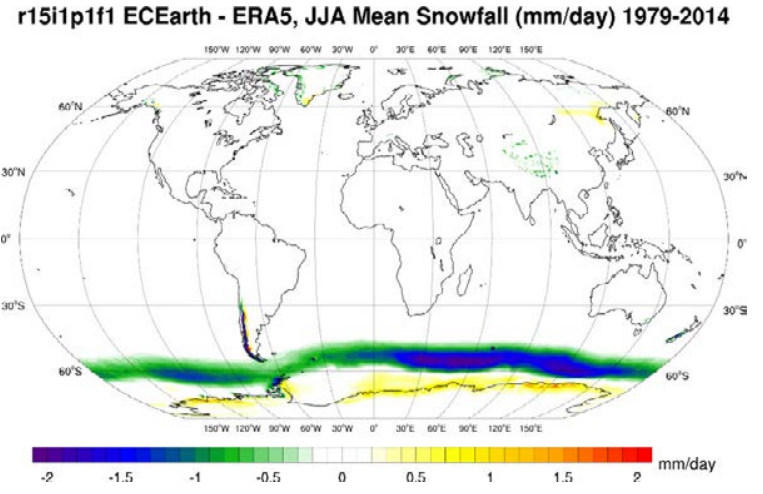

(b)

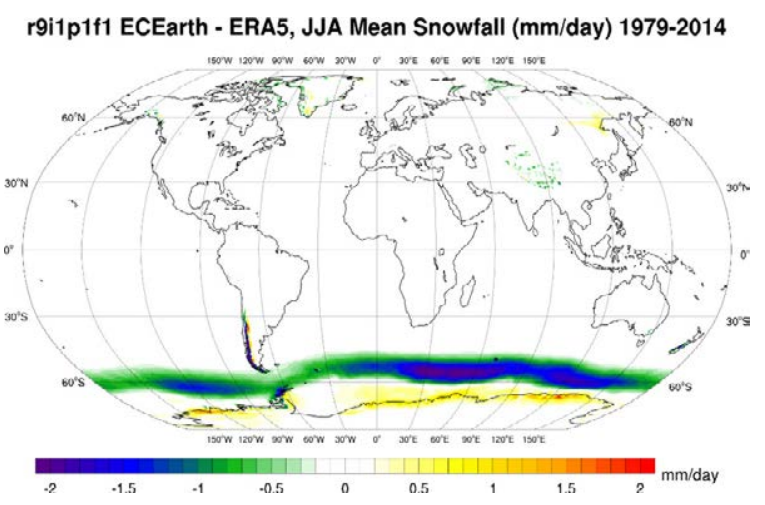

(d)

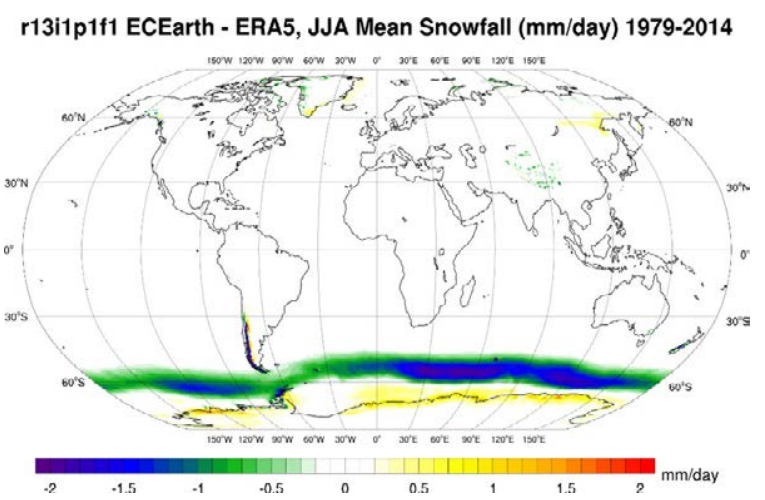

Figure 2.49. EC-Earth mean daily snowfall bias (mm/day) for JJA, 1979-2014 (ERA5 reanalysis minus EC-Earth): (a) EC-Earth r6i1p1f1, (b) EC-Earth r9i1p1f1, (c) EC-Earth r11i1p1f1, (d) EC-Earth r13i1p1f1 and (e) EC-Earth r15i1p1f1.

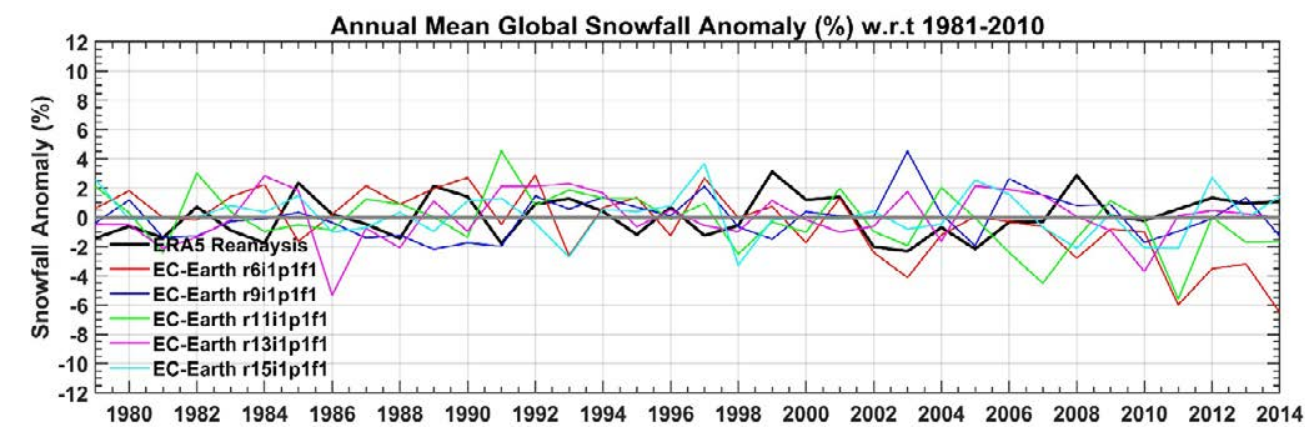

Figure 2.50. Comparison of EC-Earth ensemble members with ERA5 reanalysis data for the period 1979-2014: global annual snowfall anomalies (\%) with respect to the 30-year period, 1981-2010. 
(a)

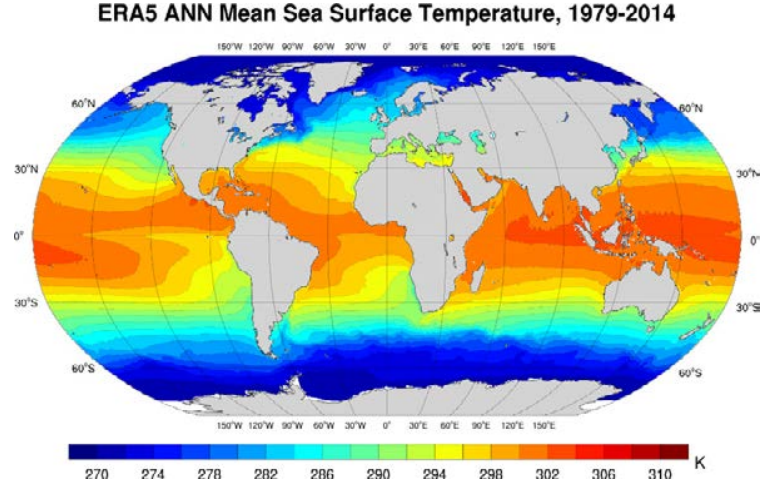

(c)

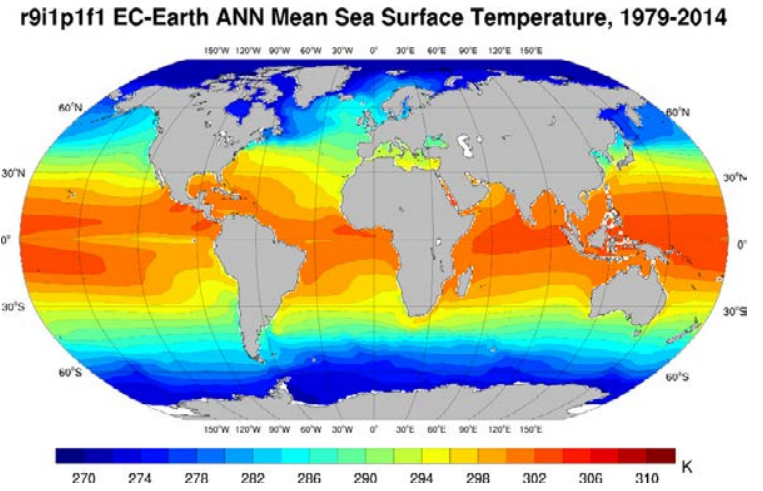

(e)

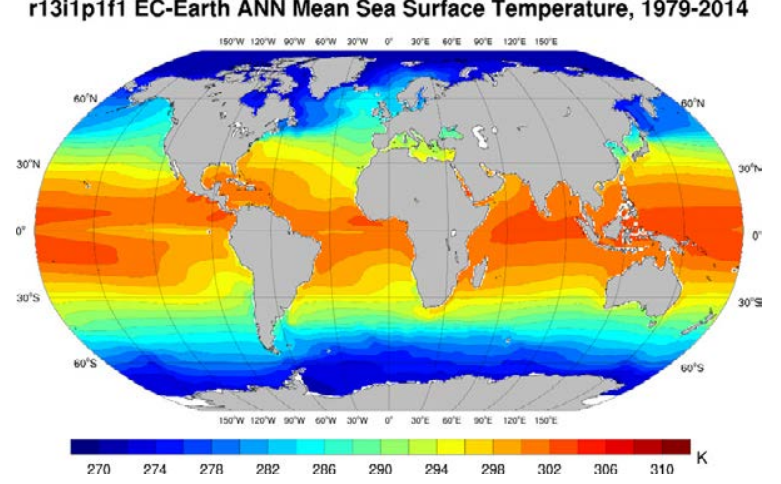

(b)

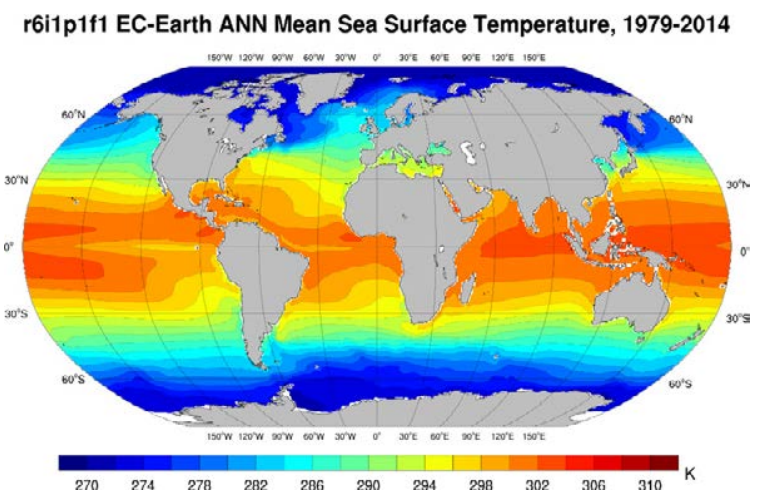

(d)
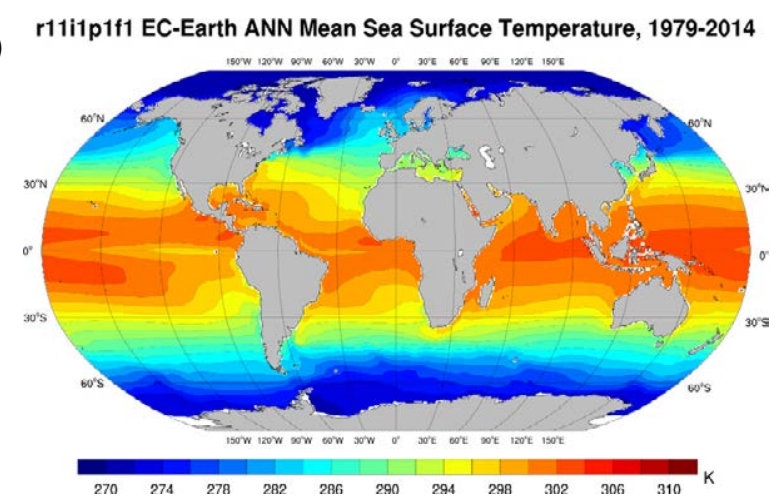

(f)

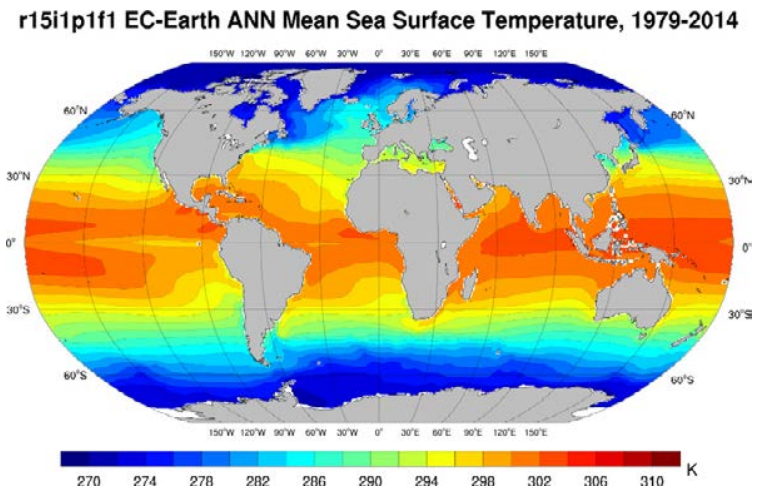

Figure 2.51. Annual mean SST (K), 1979-2014: (a) ERA5 reanalysis, (b) EC-Earth r6i1p1f1, (c) EC-Earth r9i1p1f1, (d) EC-Earth r11i1p1f1, (e) EC-Earth r13i1p1f1 and (f) EC-Earth r15i1p1f1. 
(a)

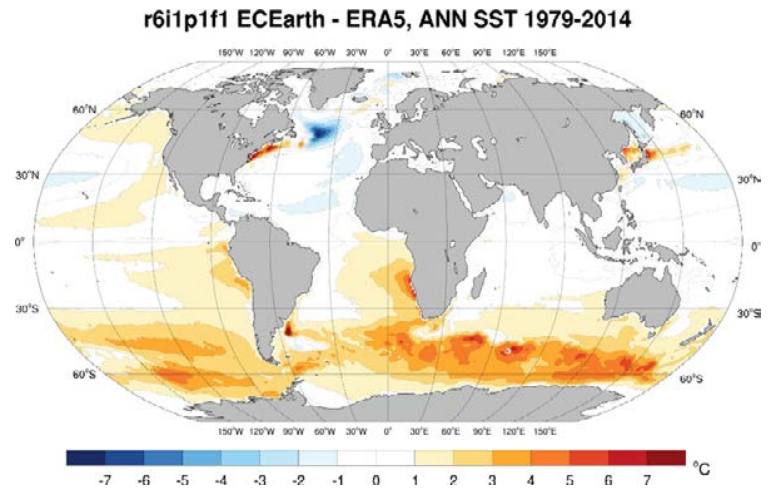

(c)

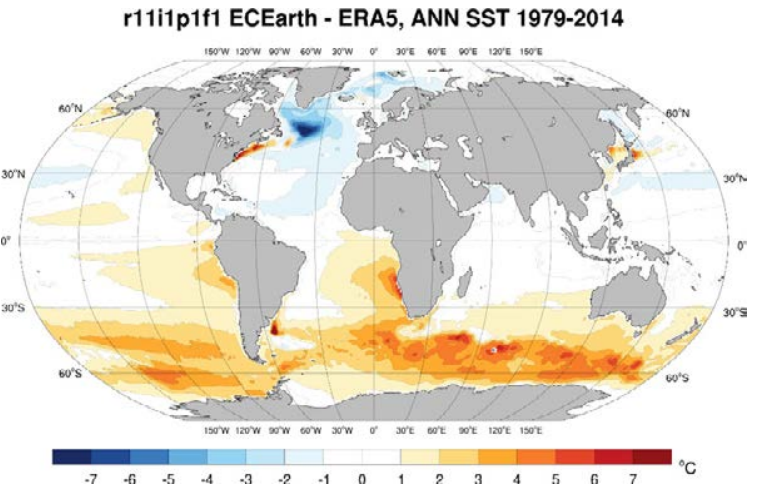

(e)

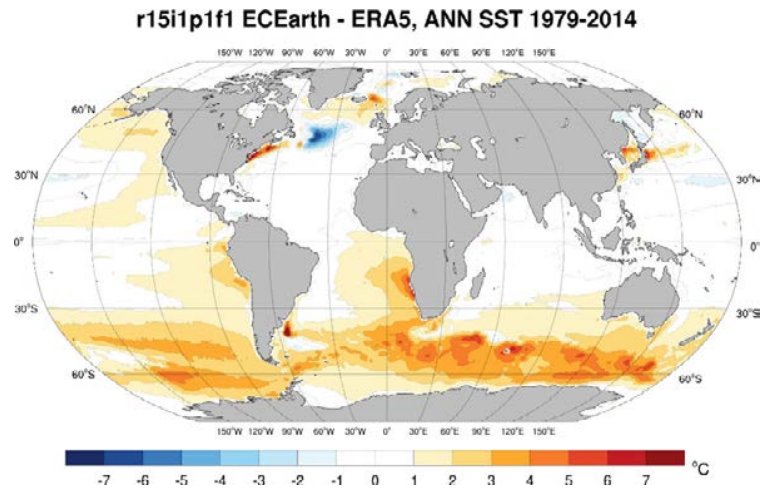

(b)

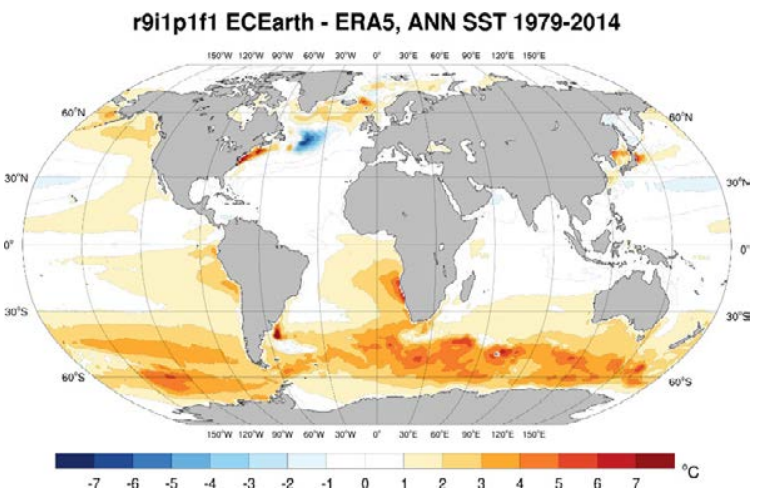

(d)

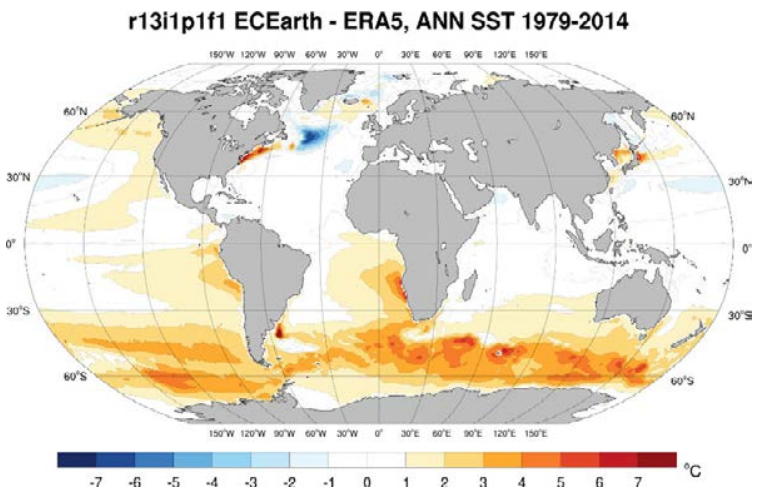

Figure 2.52. EC-Earth annual mean SST bias $\left({ }^{\circ} \mathrm{C}\right), 1979-2014$ (ERA5 reanalysis minus EC-Earth): (a) EC-Earth r6i1p1f1, (b) EC-Earth r9i1p1f1, (c) EC-Earth r11i1p1f1, (d) EC-Earth r13i1p1f1 and (e) EC-Earth r15i1p1f1. 
(a)

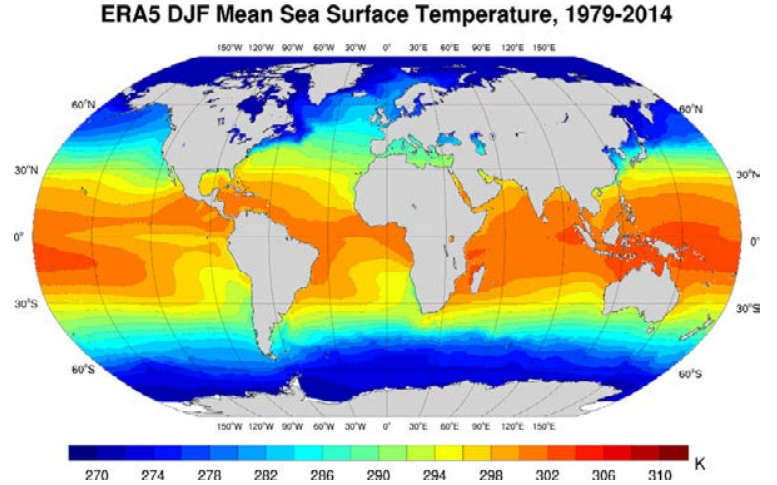

(c)

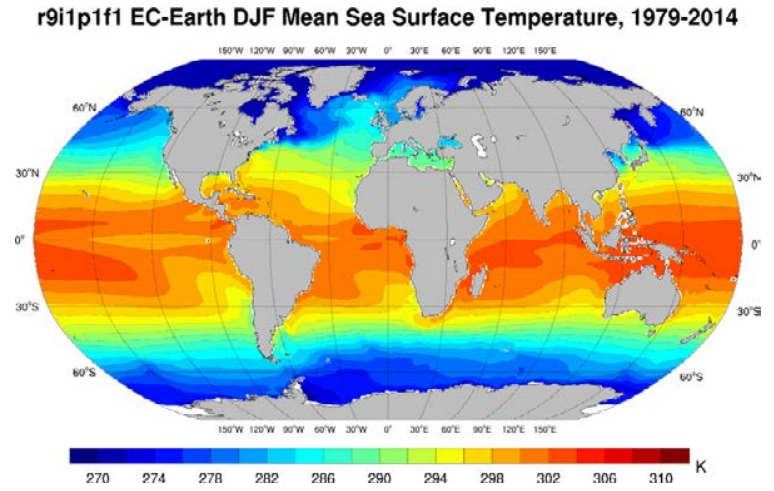

(e)

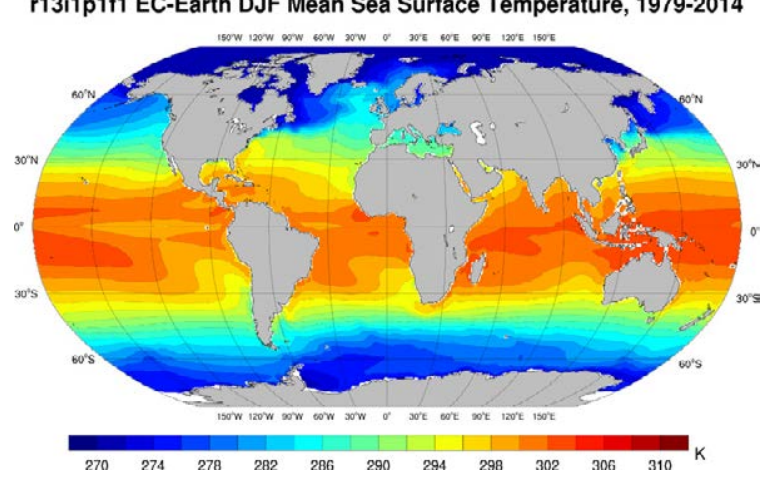

(b)
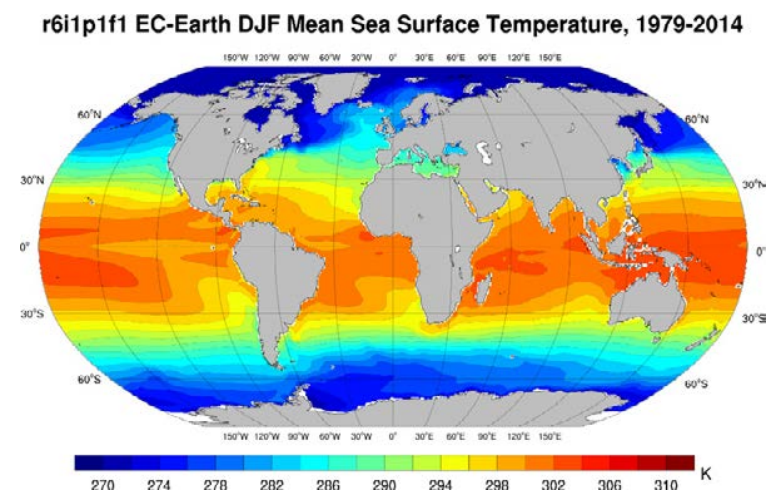

(d)
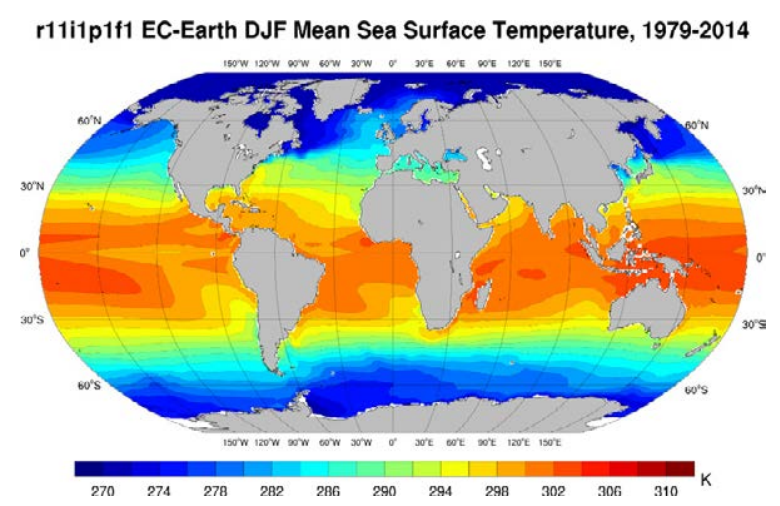

(f)

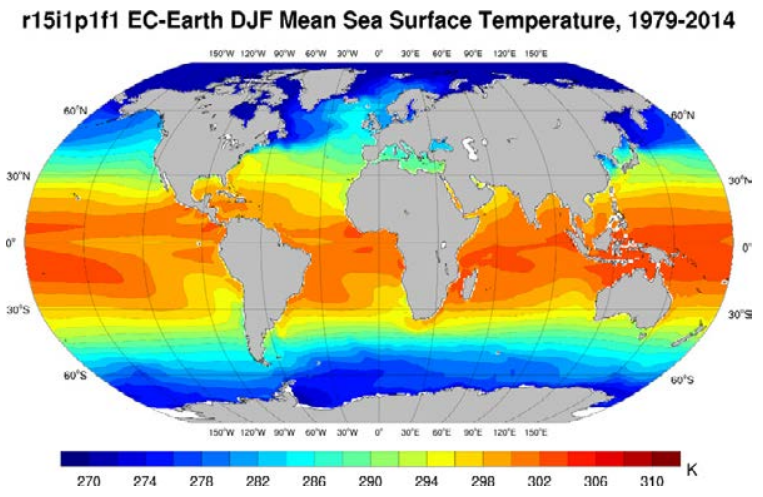

Figure 2.53. Mean SST for DJF (K), 1979-2014: (a) ERA5 reanalysis, (b) EC-Earth r6i1p1f1, (c) EC-Earth r9i1p1f1, (d) EC-Earth r11i1p1f1, (e) EC-Earth r13i1p1f1 and (f) EC-Earth r15i1p1f1. 
(a)

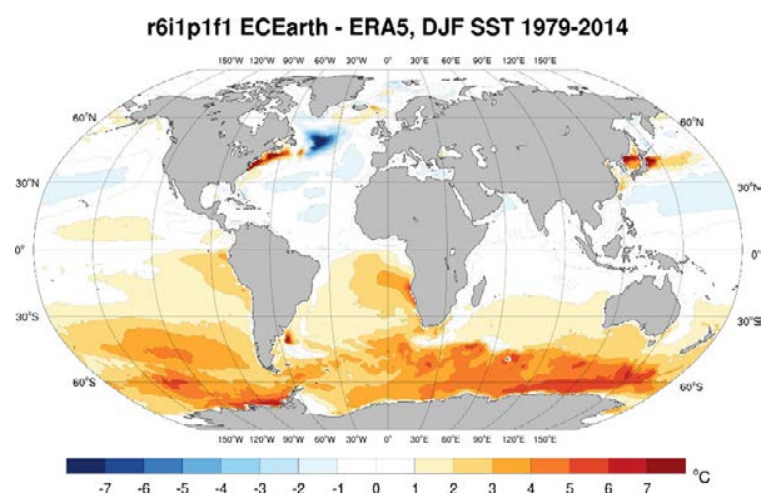

(c)

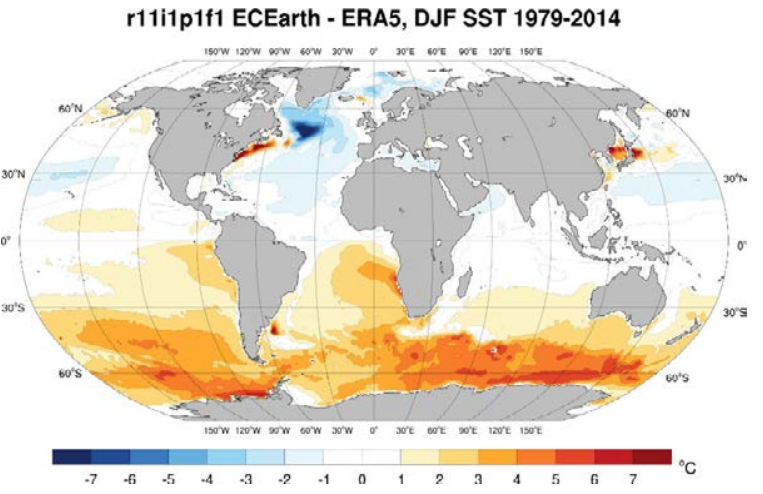

(e)

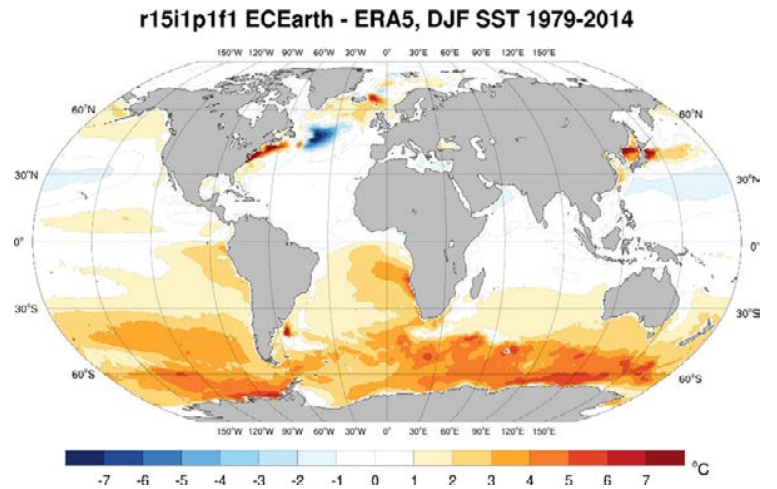

(b)

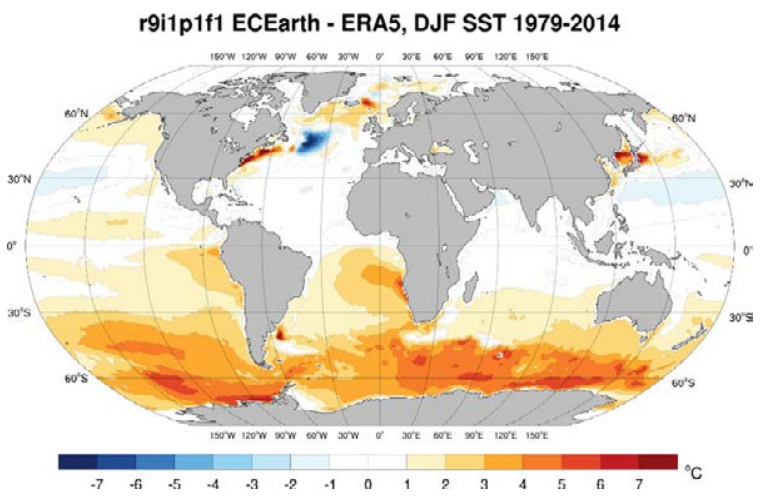

(d)

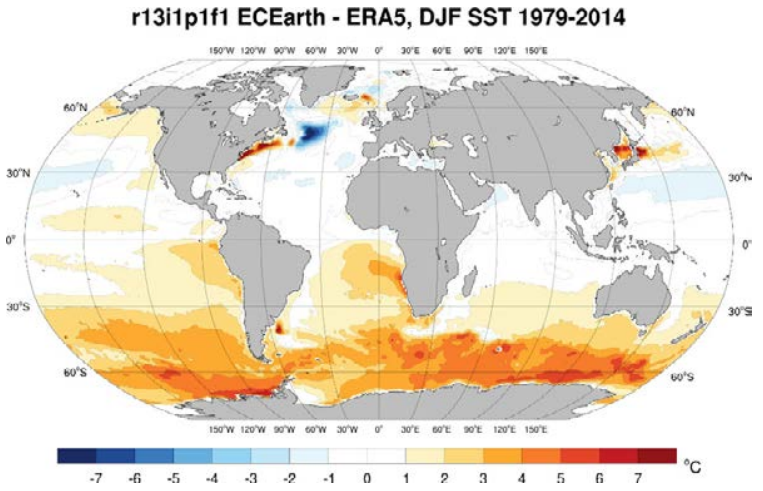

Figure 2.54. EC-Earth mean SST bias $\left({ }^{\circ} \mathrm{C}\right)$ for DJF, 1979-2014 (ERA5 reanalysis minus EC-Earth): (a) EC-Earth r6i1p1f1, (b) EC-Earth r9i1p1f1, (c) EC-Earth r11i1p1f1, (d) EC-Earth r13i1p1f1 and (e) EC-Earth r15i1p1f1. 
(a)

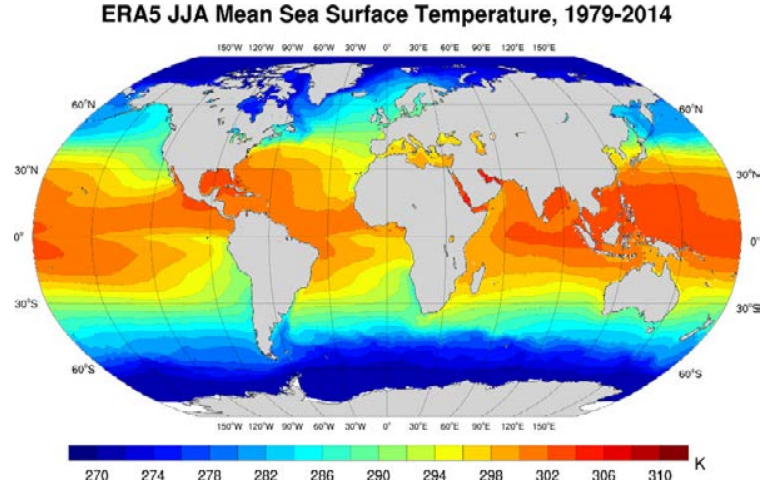

(c)

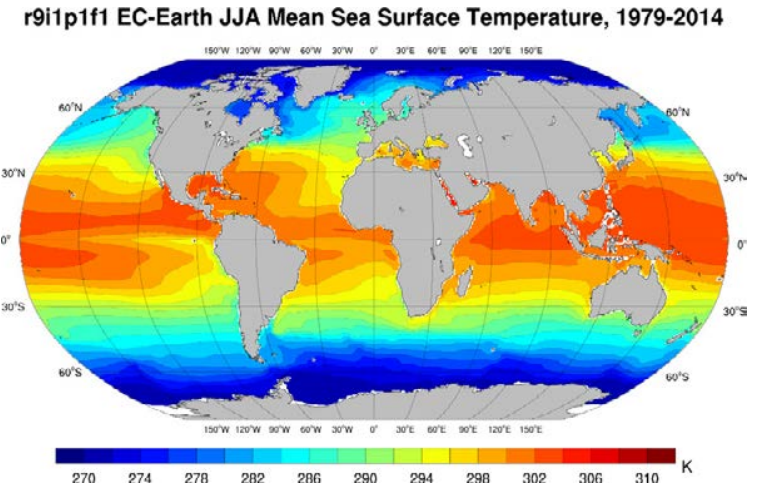

(e)

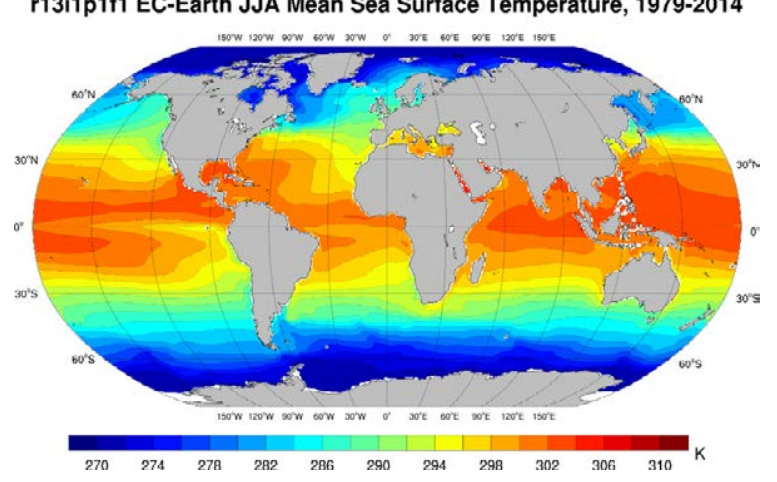

(b)

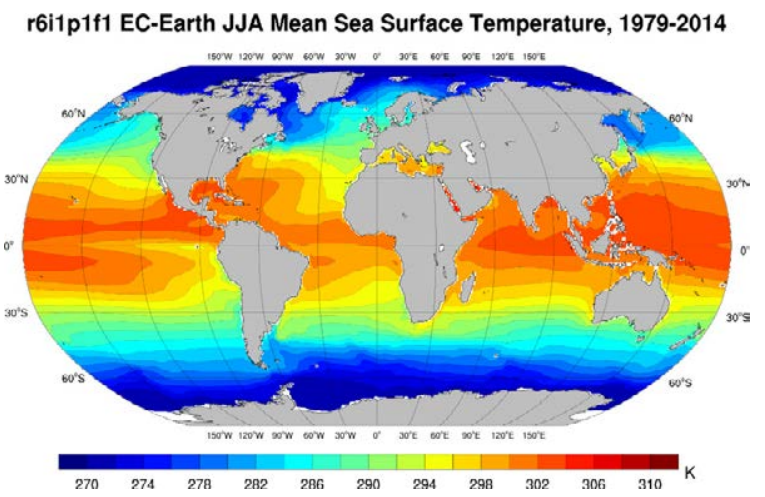

(d)

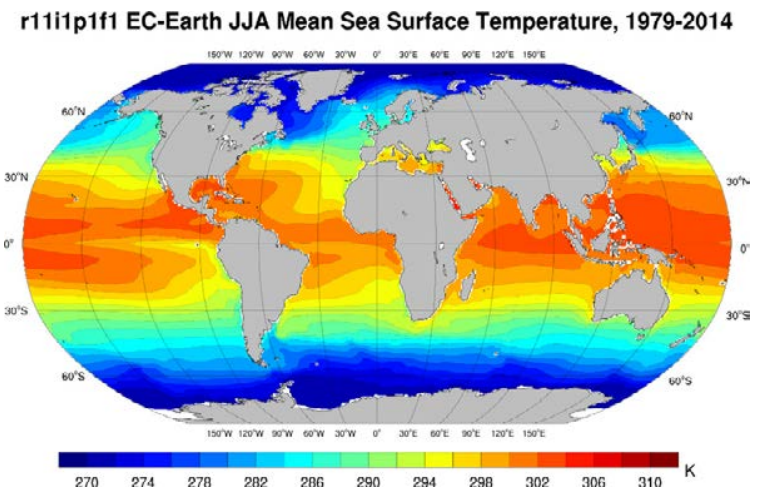

(f)

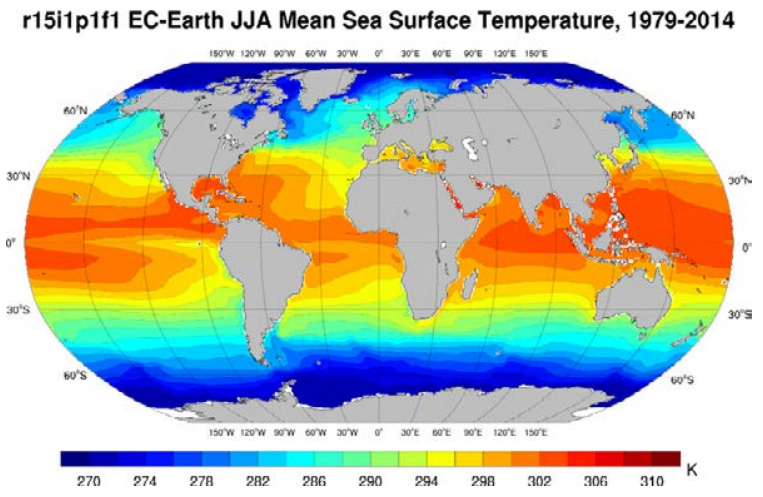

Figure 2.55. Mean SST for JJA (K), 1979-2014: (a) ERA5 reanalysis, (b) EC-Earth r6i1p1f1, (c) EC-Earth r9i1p1f1, (d) EC-Earth r11i1p1f1, (e) EC-Earth r13i1p1f1 and (f) EC-Earth r15i1p1f1. 
(a)

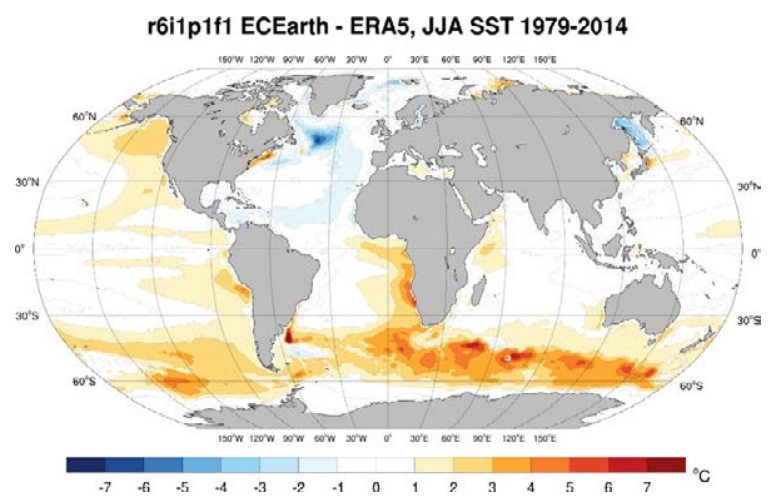

(c)

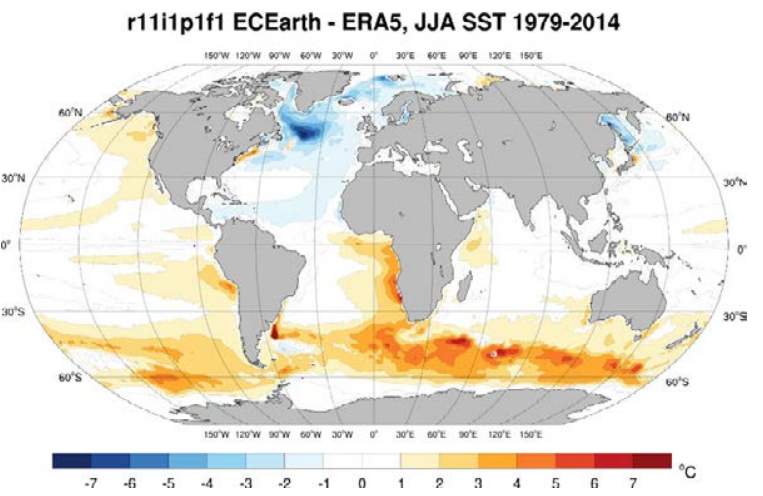

(e)

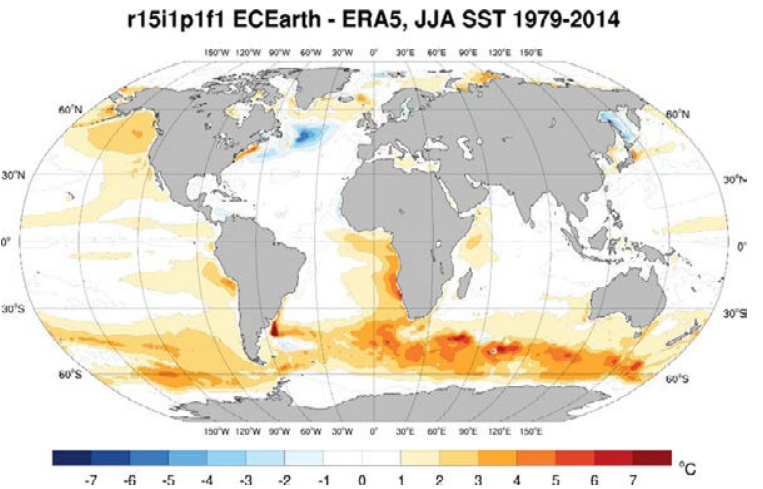

(b)

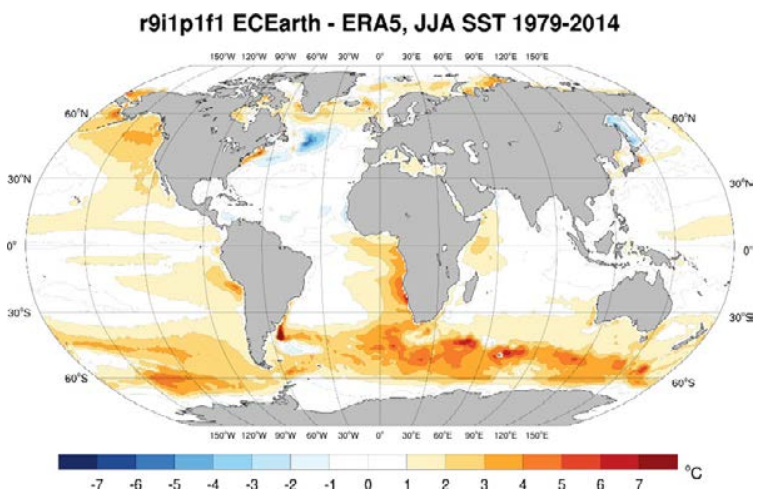

(d)

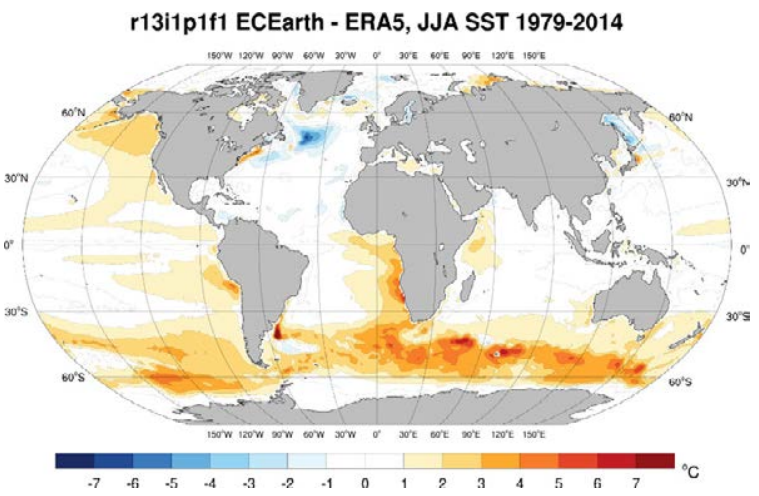

Figure 2.56. EC-Earth mean SST bias $\left({ }^{\circ} \mathrm{C}\right)$ for JJA, 1979-2014 (ERA5 reanalysis minus EC-Earth): (a) EC-Earth r6i1p1f1, (b) EC-Earth r9i1p1f1, (c) EC-Earth r11i1p1f1, (d) EC-Earth r13i1p1f1 and (e) EC-Earth r15i1p1f1. 
Table 2.9. Mean and minimum bias $\left({ }^{\circ} \mathrm{C}\right)$ over the North Atlantic area $40-60^{\circ} \mathrm{N}, 25-55^{\circ} \mathrm{W}$ for each of the five EC-Earth ensemble members ${ }^{a}$

\begin{tabular}{|c|c|c|c|c|c|c|c|c|c|c|}
\hline \multirow{2}{*}{$\begin{array}{l}\text { Time } \\
\text { period }\end{array}$} & \multicolumn{2}{|c|}{ r6i1p1f1 } & \multicolumn{2}{|c|}{ r9i1p1f1 } & \multicolumn{2}{|c|}{ r11i1p1f1 } & \multicolumn{2}{|c|}{ r13i1p1f1 } & \multicolumn{2}{|c|}{ r15i1p1f1 } \\
\hline & Mean & Min. & Mean & Min. & Mean & Min. & Mean & Min. & Mean & Min. \\
\hline Annual & -1.54 & -7.45 & -0.58 & -6.83 & -2.76 & -8.70 & -1.20 & -6.99 & -0.92 & -6.83 \\
\hline DJF & -1.69 & -8.54 & -0.76 & -8.23 & -2.77 & -9.67 & -1.31 & -8.32 & -1.07 & -8.18 \\
\hline MAM & -1.87 & -9.14 & -0.92 & -8.15 & -3.14 & -10.7 & -1.51 & -8.30 & -1.19 & -7.84 \\
\hline JJA & -1.61 & -6.55 & -0.53 & -5.66 & -3.15 & -7.89 & -1.32 & -6.25 & -1.05 & -5.99 \\
\hline SON & -0.98 & -5.59 & -0.12 & -5.38 & -2.00 & -6.53 & -0.67 & -5.40 & -0.38 & -5.30 \\
\hline
\end{tabular}

aln each case the model data are compared with ERA5 reanalysis data for the period 1979-2014.

Table 2.10. Mean global annual and seasonal SST bias $\left({ }^{\circ} \mathrm{C}\right)$ for each of the five EC-Earth ensemble members $^{a}$

\begin{tabular}{|c|c|c|c|c|c|c|c|c|c|c|}
\hline \multirow{2}{*}{$\begin{array}{l}\text { Time } \\
\text { period }\end{array}$} & \multicolumn{2}{|c|}{ r6i1p1f1 } & \multicolumn{2}{|c|}{ r9i1p1f1 } & \multicolumn{2}{|c|}{ r11i1p1f1 } & \multicolumn{2}{|c|}{ r13i1p1f1 } & \multicolumn{2}{|c|}{ r15i1p1f1 } \\
\hline & Bias & MAE & Bias & MAE & Bias & MAE & Bias & MAE & Bias & MAE \\
\hline Annual & 0.91 & 1.22 & 1.13 & 1.32 & 0.84 & 1.30 & 1.01 & 1.26 & 1.03 & 1.24 \\
\hline DJF & 1.10 & 1.48 & 1.32 & 1.59 & 1.04 & 1.54 & 1.19 & 1.51 & 1.20 & 1.47 \\
\hline MAM & 1.02 & 1.52 & 1.23 & 1.62 & 0.96 & 1.63 & 1.13 & 1.58 & 1.15 & 1.54 \\
\hline JJA & 0.78 & 1.09 & 1.0 & 1.19 & 0.68 & 1.17 & 0.87 & 1.13 & 0.91 & 1.12 \\
\hline SON & 0.75 & 1.03 & 0.96 & 1.13 & 0.67 & 1.08 & 0.85 & 1.07 & 0.88 & 1.06 \\
\hline
\end{tabular}

aln each case the model data are compared with ERA5 reanalysis data for the period 1979-2014

(a)

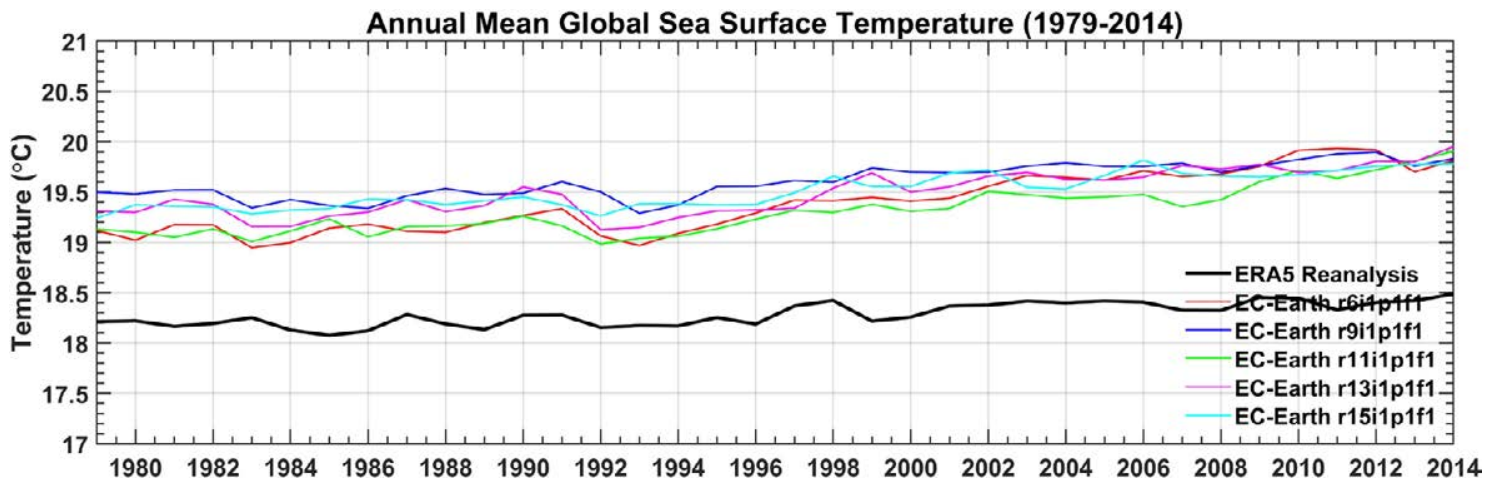

(b)

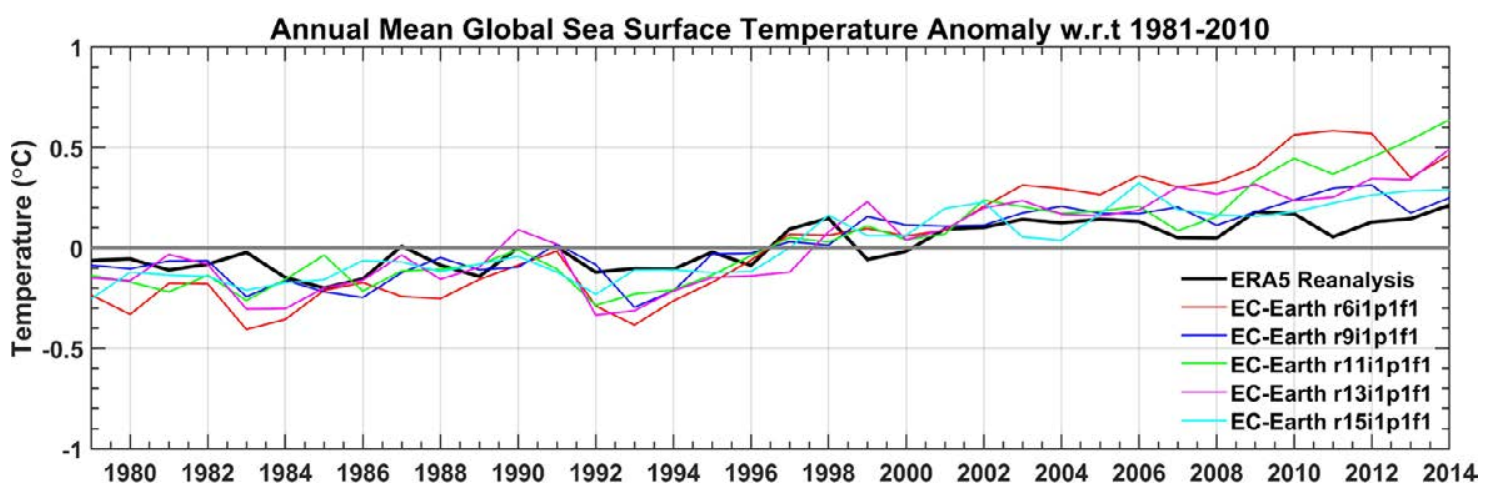

Figure 2.57. Comparison of EC-Earth ensemble members with ERA5 reanalysis data for the period 1979-2014: (a) SST and (b) SST anomalies with respect to the 30-year period 1981-2010. 
(a)

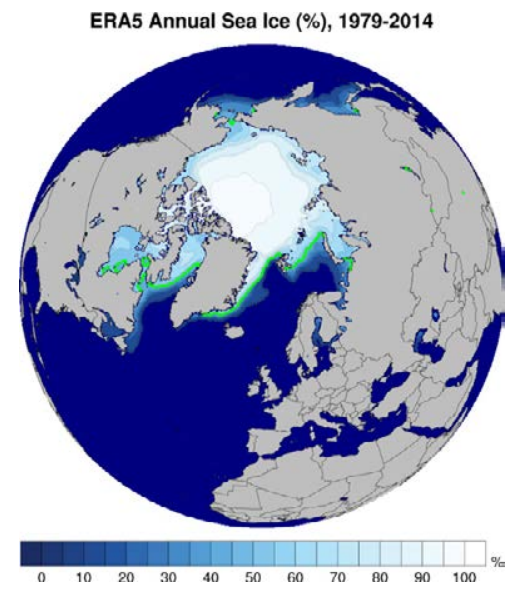

(c)

EC-Earth r9i1 Annual Sea Ice (\%), 1979-2014

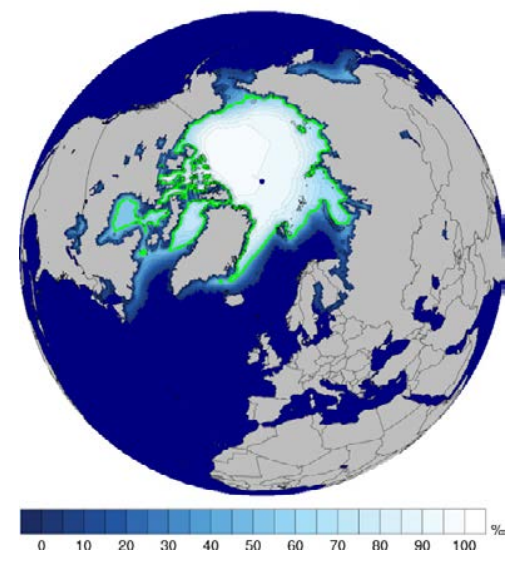

(e)

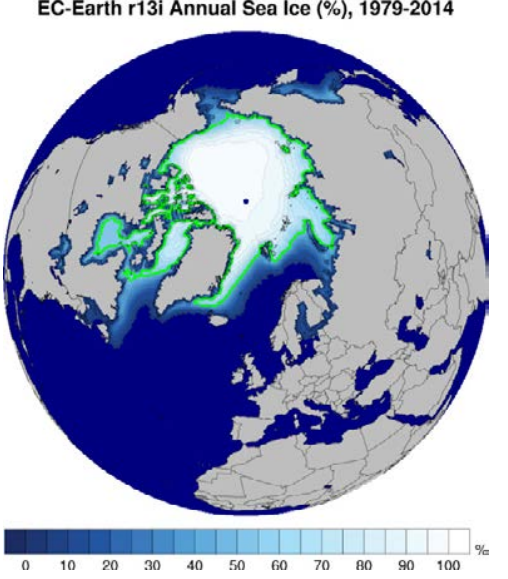

(b)

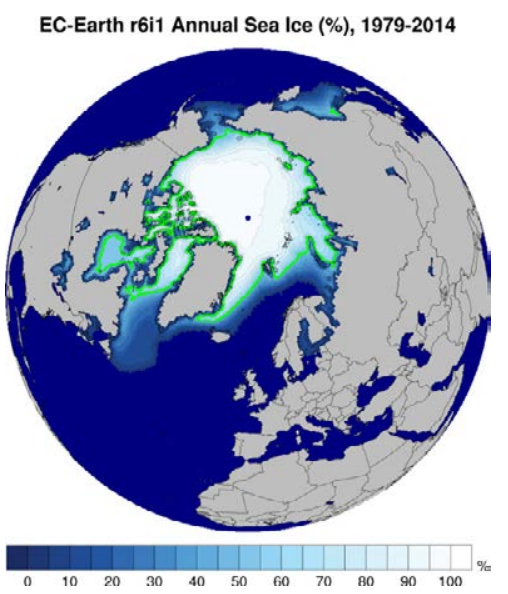

(d)

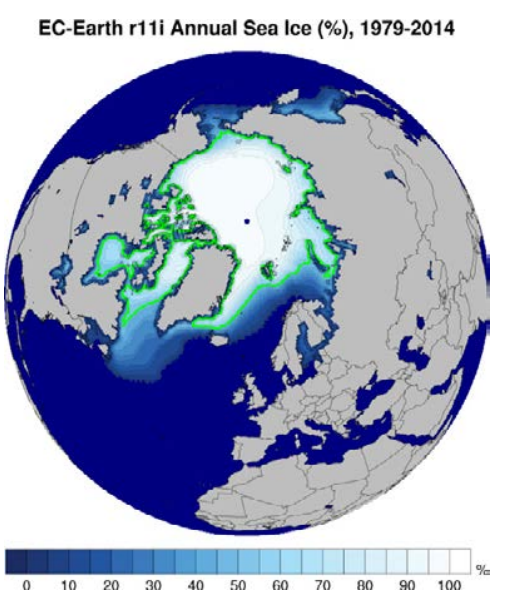

(f)

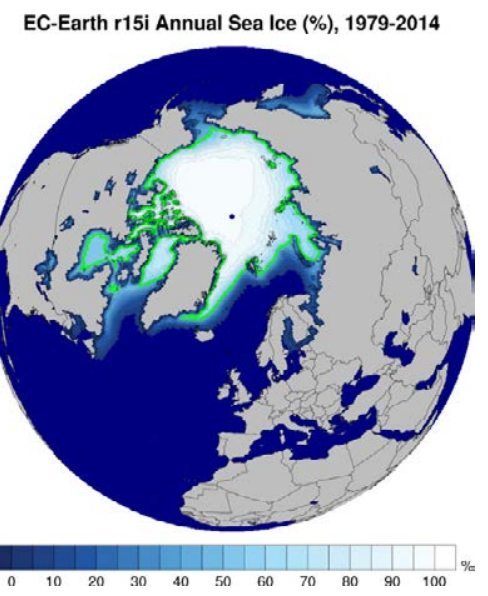

Figure 2.58. Northern Hemisphere annual mean sea ice fraction (\%), 1979-2014: (a) ERA5 reanalysis, (b) EC-Earth r6i1p1f1, (c) EC-Earth r9i1p1f1, (d) EC-Earth r11i1p1f1, (e) EC-Earth r13i1p1f1 and (f) EC-Earth r15i1p1f1. The green line shows the $50 \%$ contour line. 
(a)

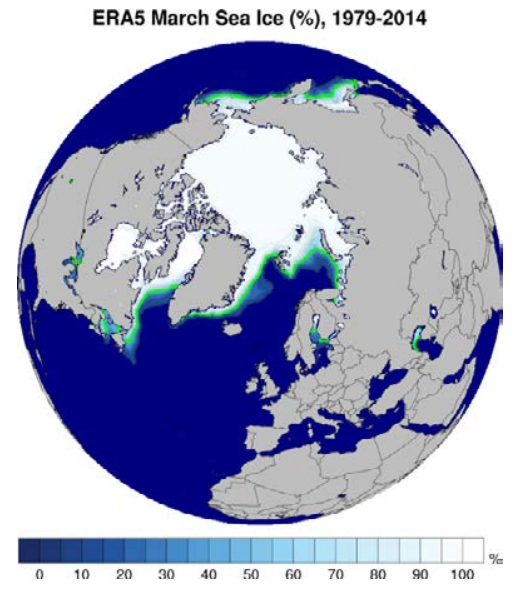

(c)

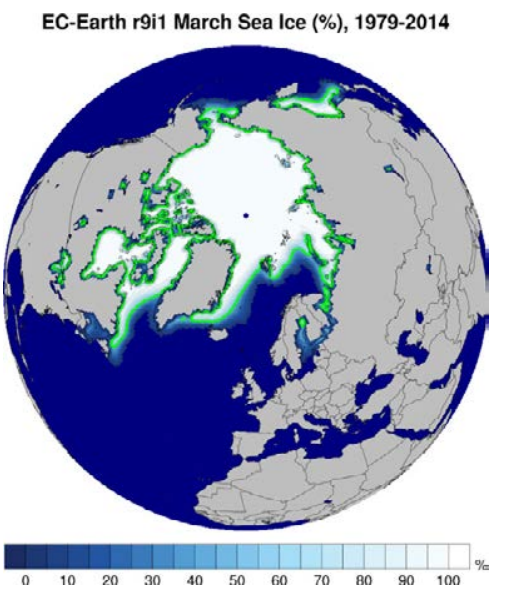

(e)

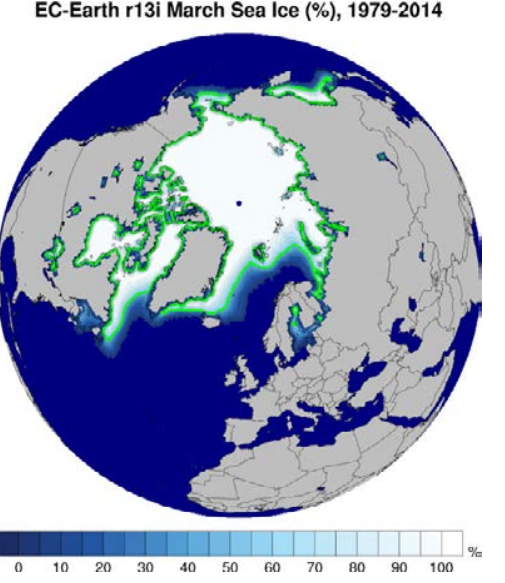

(b)

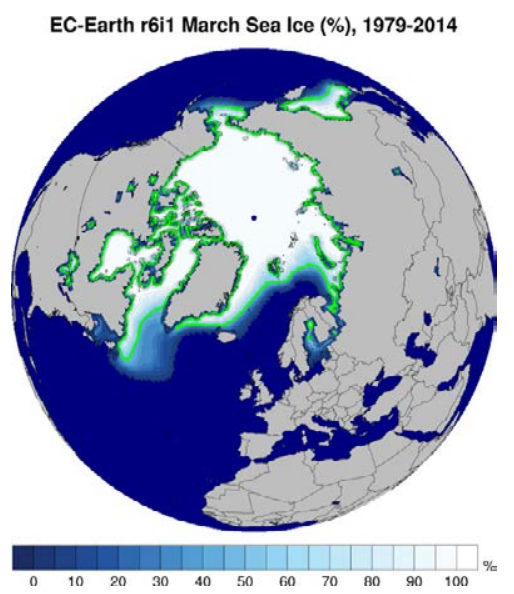

(d)

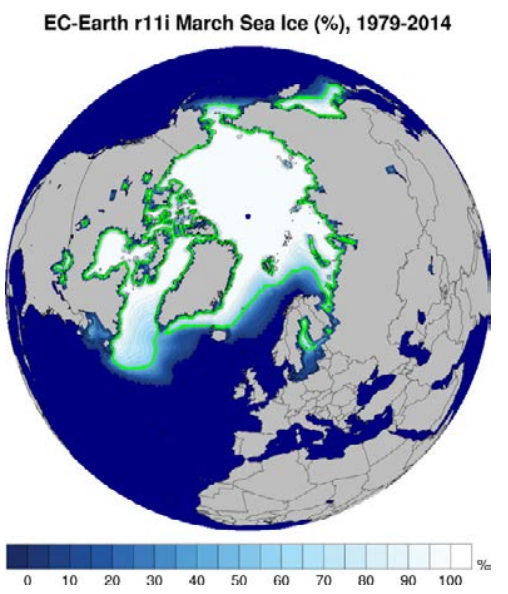

(f)

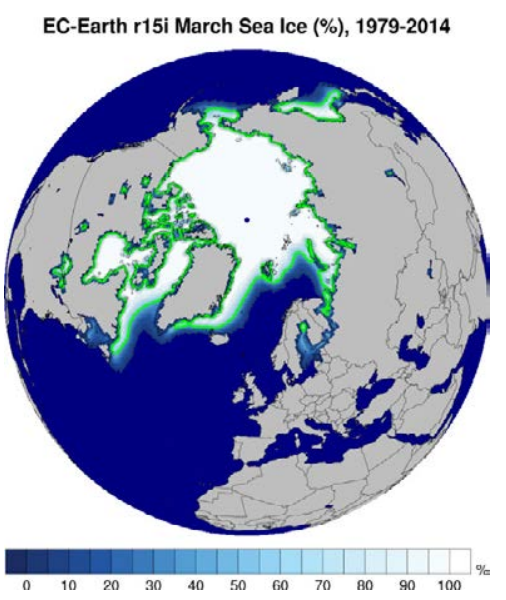

Figure 2.59. Northern Hemisphere March mean sea ice fraction (\%), 1979-2014: (a) ERA5 reanalysis, (b) EC-Earth r6i1p1f1, (c) EC-Earth r9i1p1f1, (d) EC-Earth r11i1p1f1, (e) EC-Earth r13i1p1f1 and (f) EC-Earth r15i1p1f1. The green line shows the $50 \%$ contour line. 
(a)

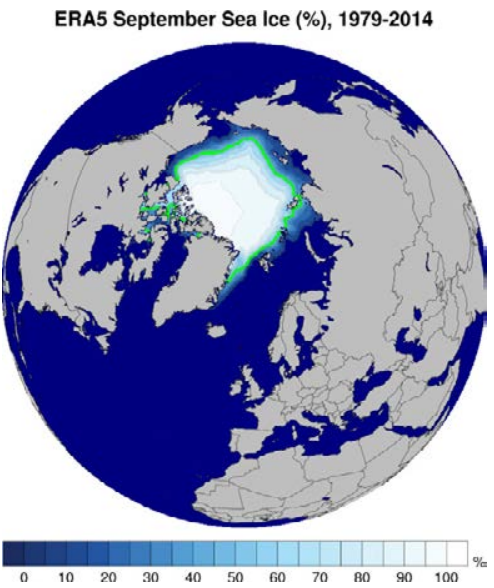

(c)

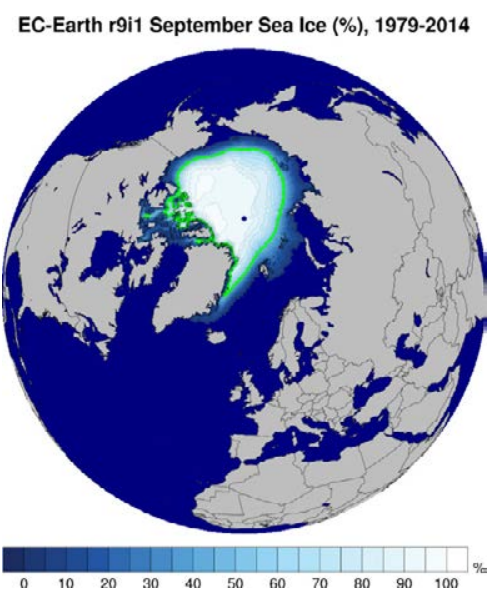

(e)

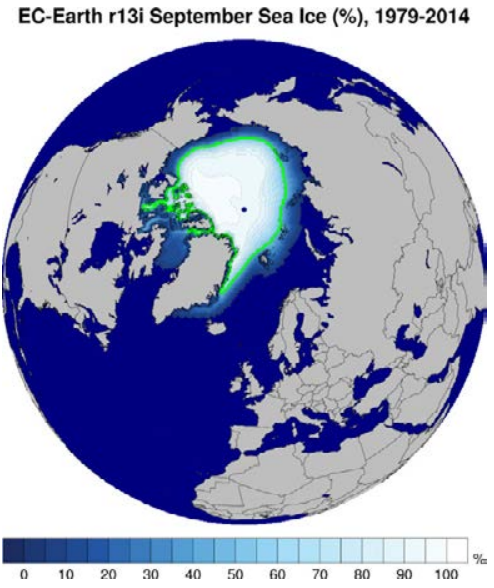

(b)

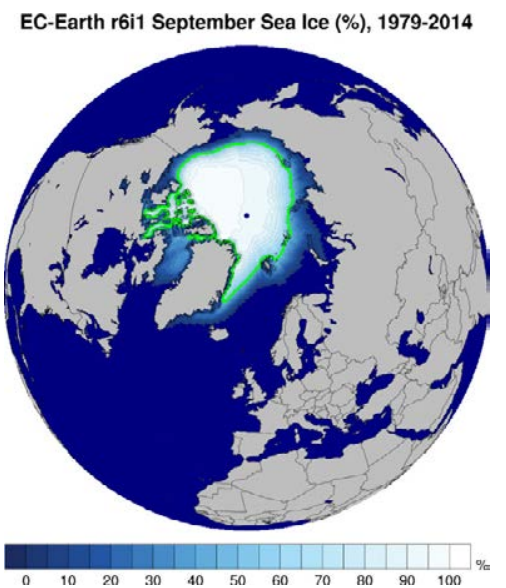

(d)

EC-Earth r11i September Sea Ice (\%), 1979-2014

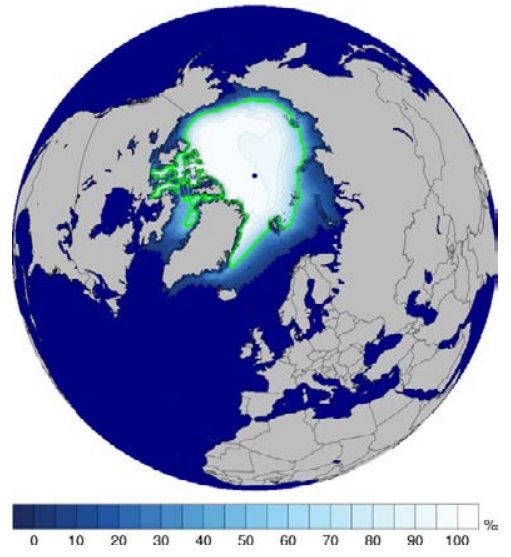

(f)

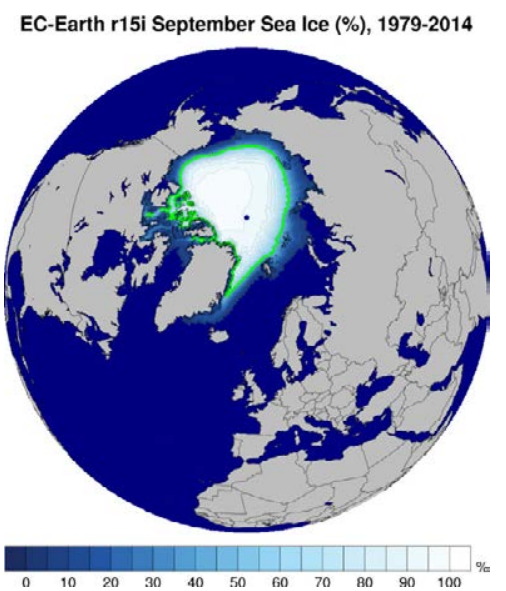

Figure 2.60. Northern Hemisphere September mean sea ice fraction (\%), 1979-2014: (a) ERA5 reanalysis, (b) EC-Earth r6i1p1f1, (c) EC-Earth r9i1p1f1, (d) EC-Earth r11i1p1f1, (e) EC-Earth r13i1p1f1 and (f) EC-Earth r15i1p1f1. The green line shows the $50 \%$ contour line. 
(a)

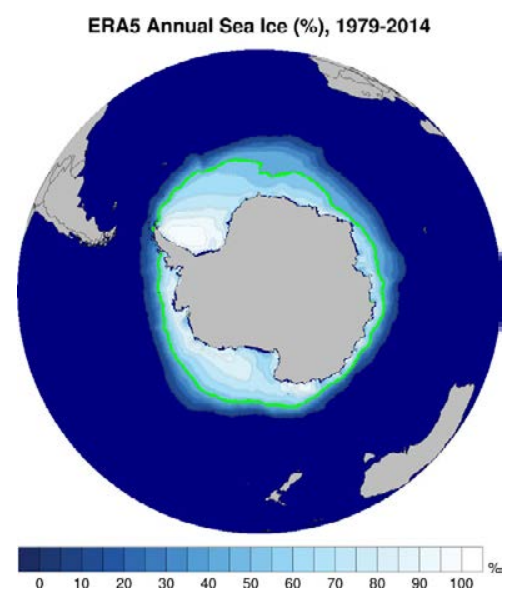

(c)

EC-Earth r9i1 Annual Sea Ice (\%), 1979-2014

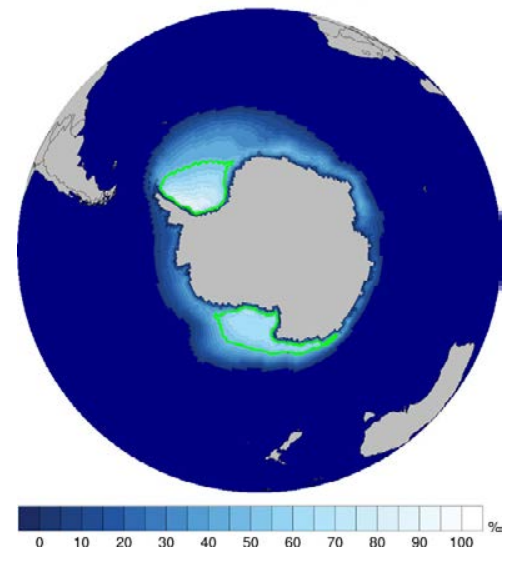

(e)

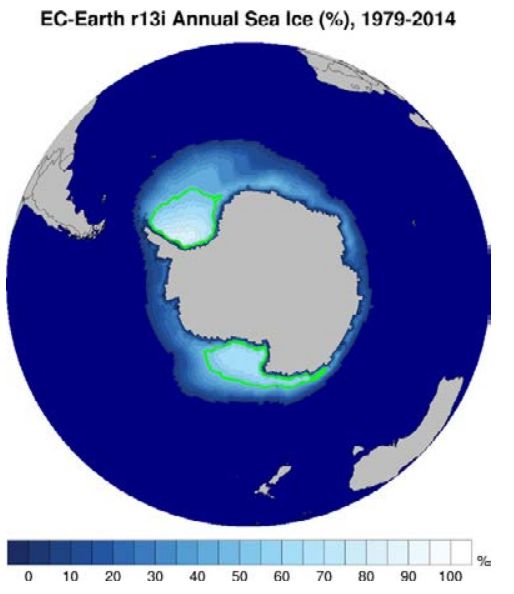

(b)

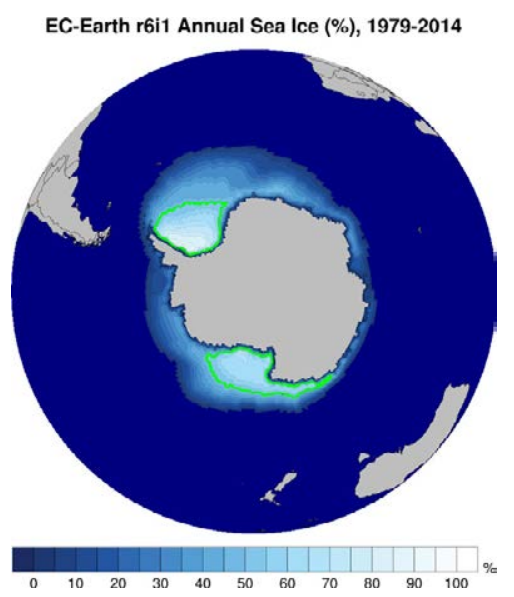

(d)

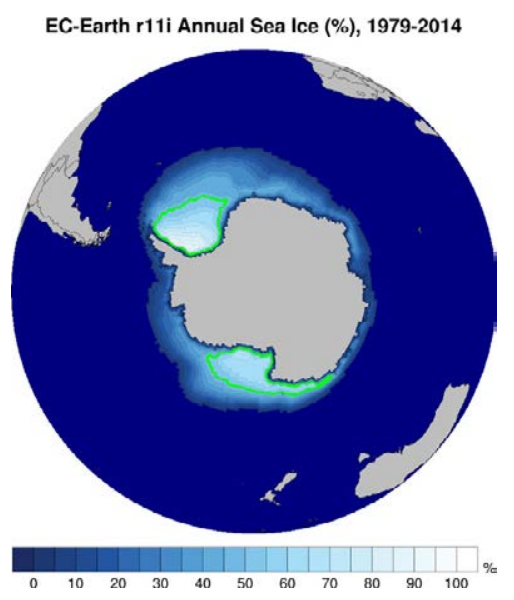

(f)

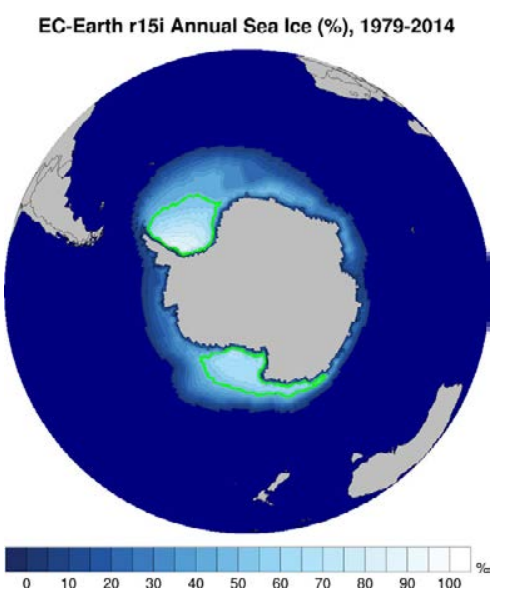

Figure 2.61. Southern Hemisphere annual mean sea ice fraction (\%), 1979-2014: (a) ERA5 reanalysis, (b) EC-Earth r6i1p1f1, (c) EC-Earth r9i1p1f1, (d) EC-Earth r11i1p1f1, (e) EC-Earth r13i1p1f1 and (f) EC-Earth r15i1p1f1. The green line shows the $50 \%$ contour line. 
(a)

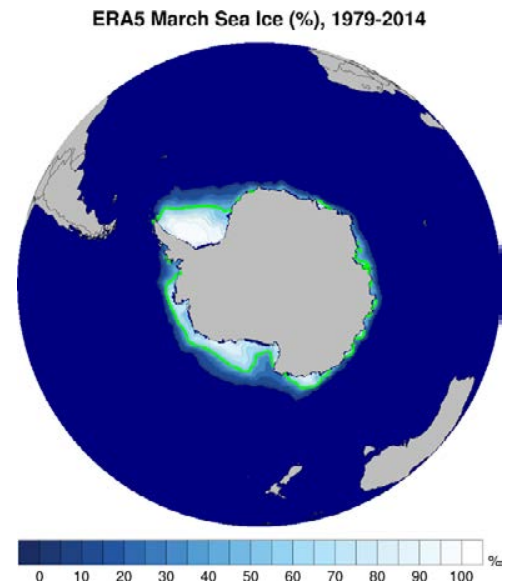

(c)

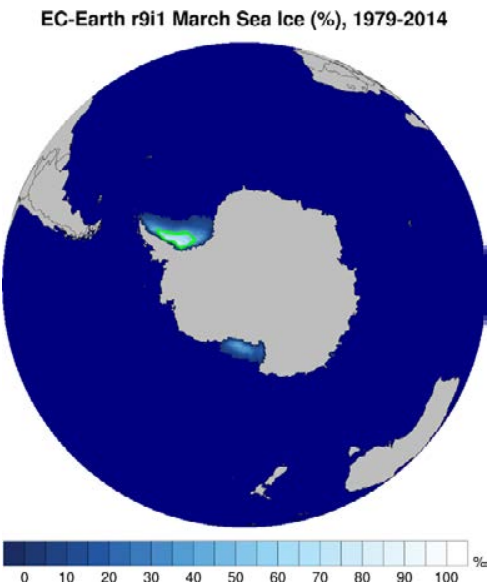

(e)

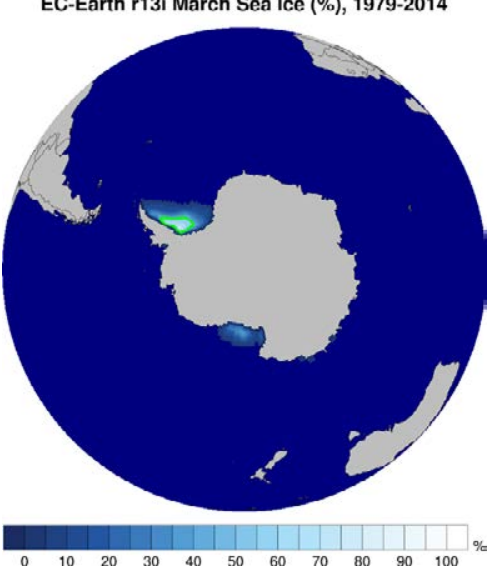

(b)

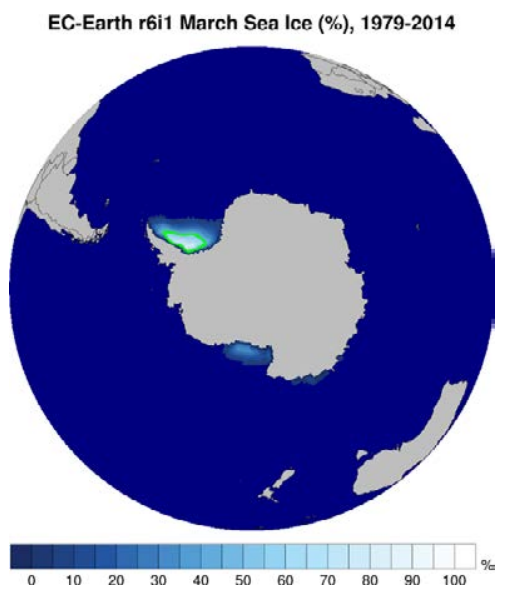

(d)

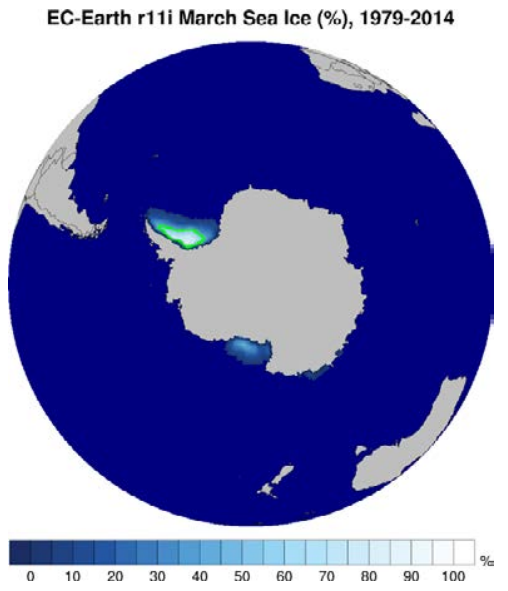

(f)

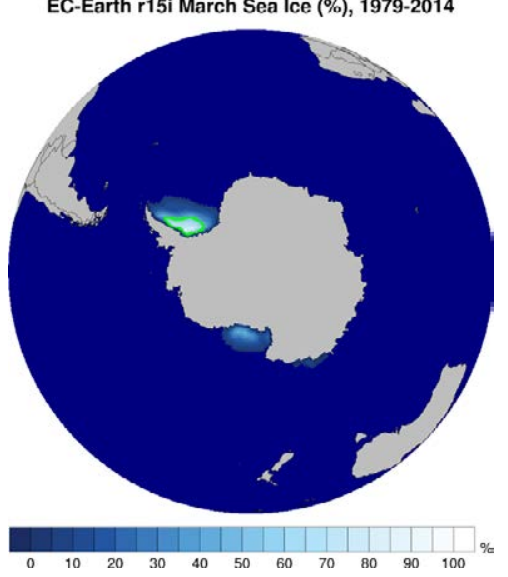

Figure 2.62. Southern Hemisphere March mean sea ice fraction (\%), 1979-2014: (a) ERA5 reanalysis, (b) EC-Earth r6i1p1f1, (c) EC-Earth r9i1p1f1, (d) EC-Earth r11i1p1f1, (e) EC-Earth r13i1p1f1 and (f) EC-Earth r15i1p1f1. The green line shows the $50 \%$ contour line. 
(a)

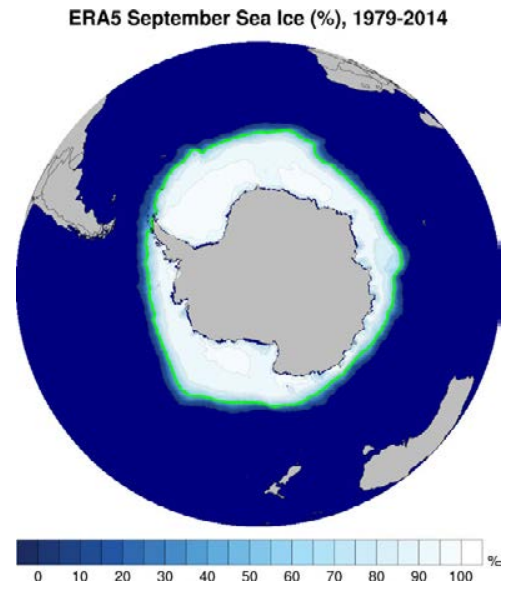

(c)

EC-Earth r9i1 September Sea Ice (\%), 1979-2014

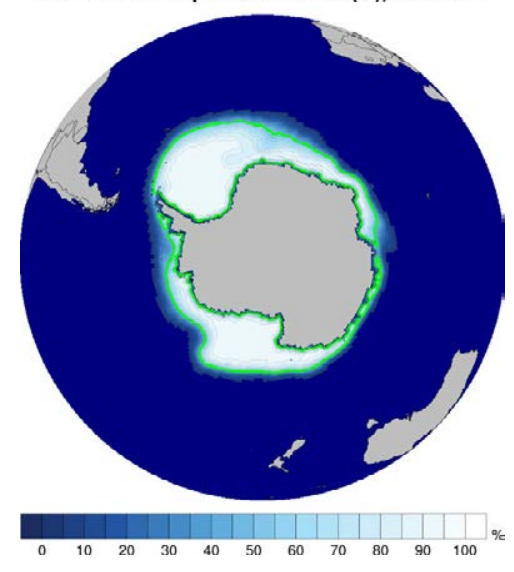

(e)

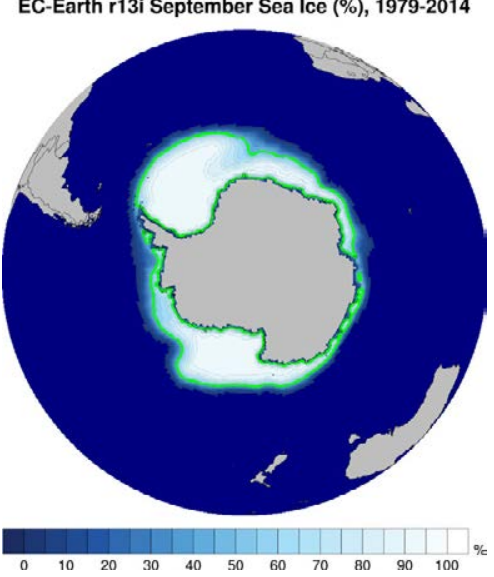

(b)

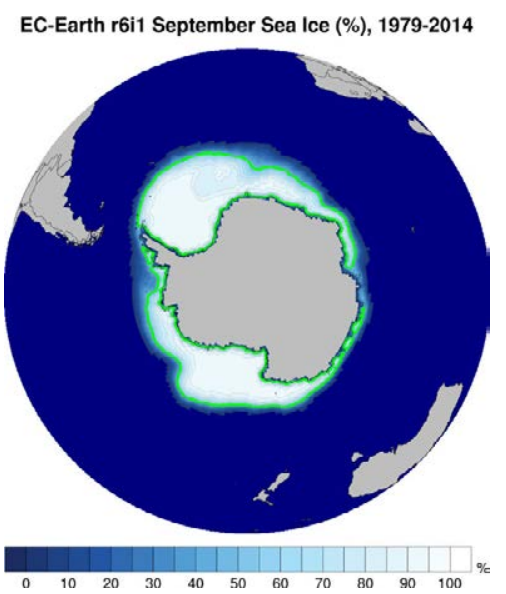

(d)

EC-Earth r11i September Sea Ice (\%), 1979-2014

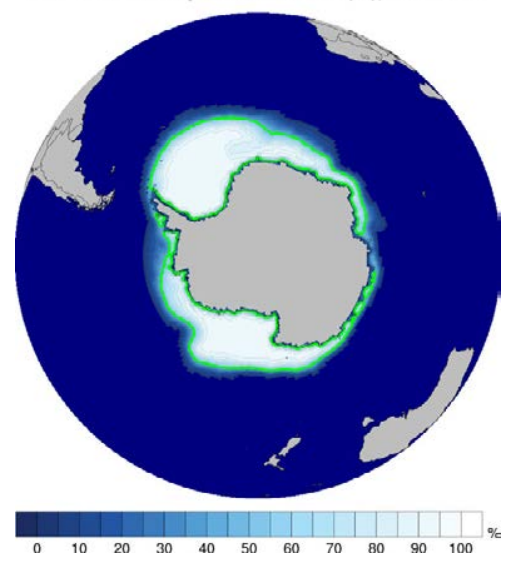

EC-Earth r15i September Sea Ice (\%), 1979-2014

(f)

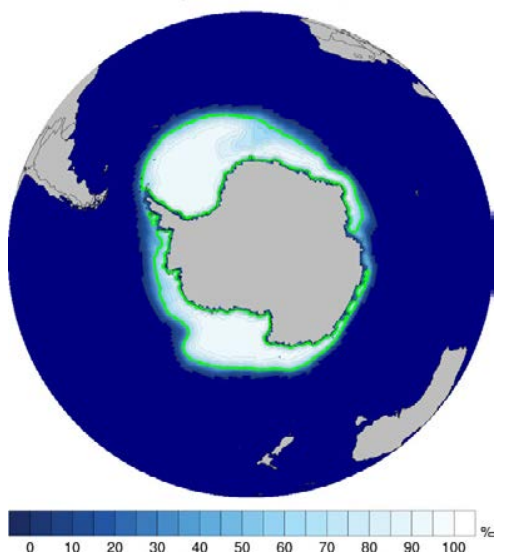

Figure 2.63. Southern Hemisphere September mean sea ice fraction (\%), 1979-2014: (a) ERA5 reanalysis, (b) EC-Earth r6i1p1f1, (c) EC-Earth r9i1p1f1, (d) EC-Earth r11i1p1f1, (e) EC-Earth r13i1p1f1 and (f) EC-Earth r15i1p1f1. The green line shows the $50 \%$ contour line. 
(a)

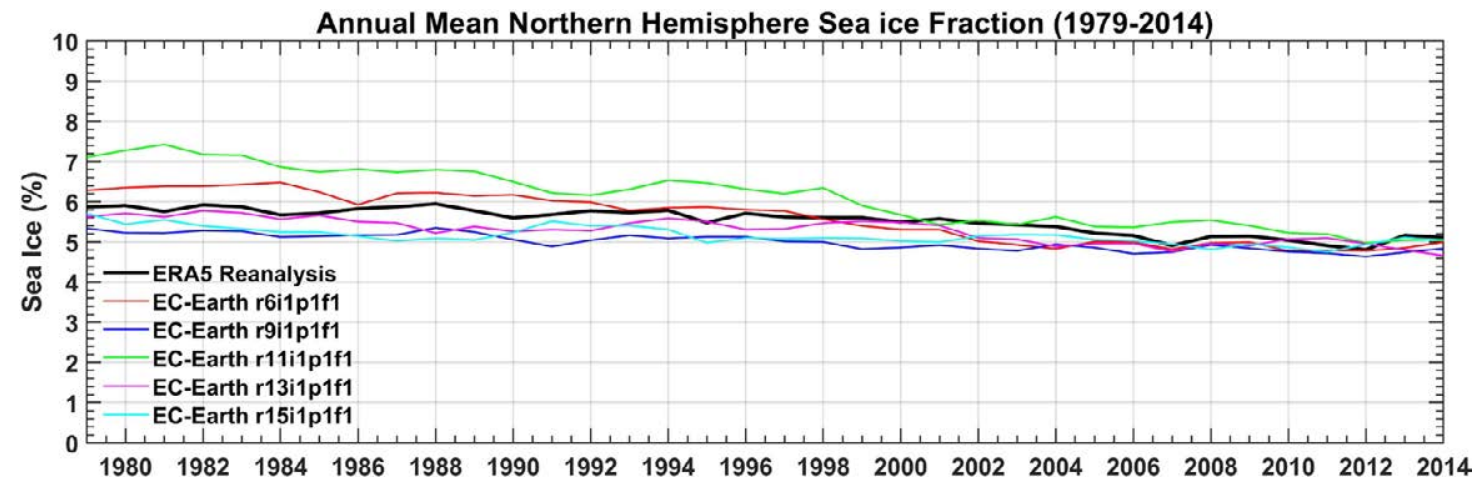

(b)

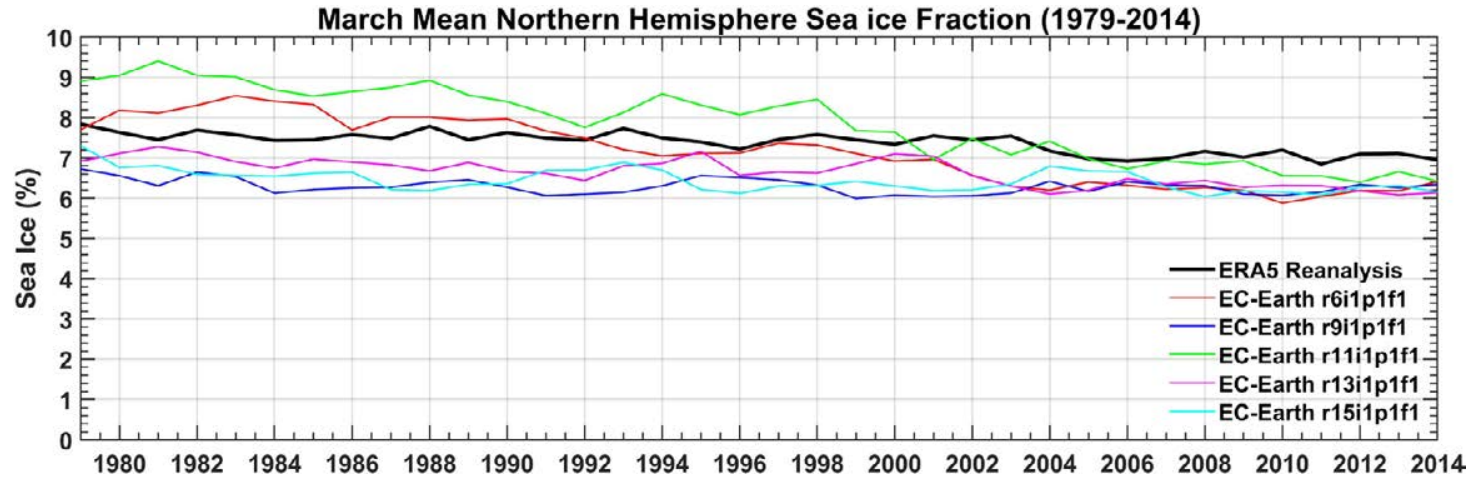

(c)

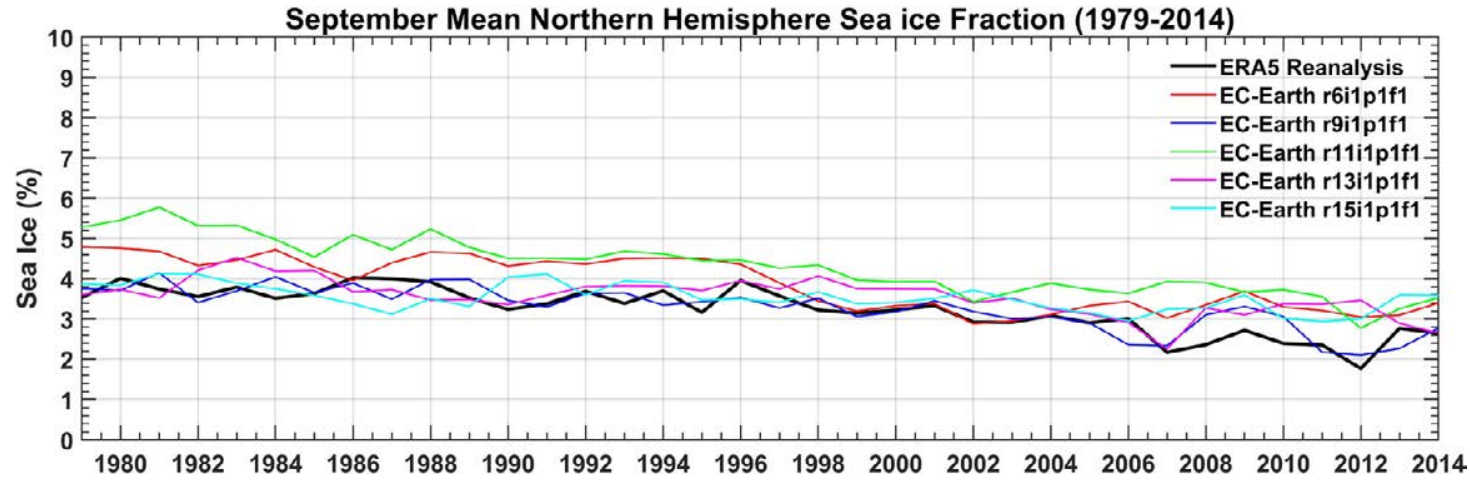

Figure 2.64. Comparison of the EC-Earth Northern Hemisphere sea ice fraction (\%) with ERA5 reanalysis data for the period 1979-2014: (a) annual, (b) March and (c) September. 
(a)

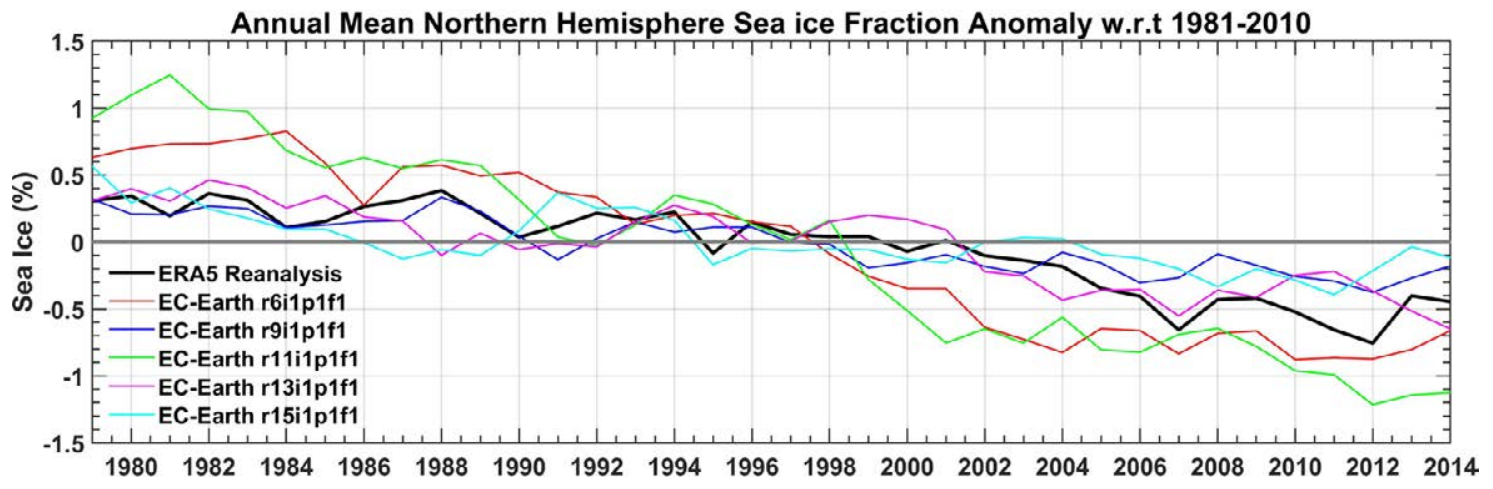

(b)

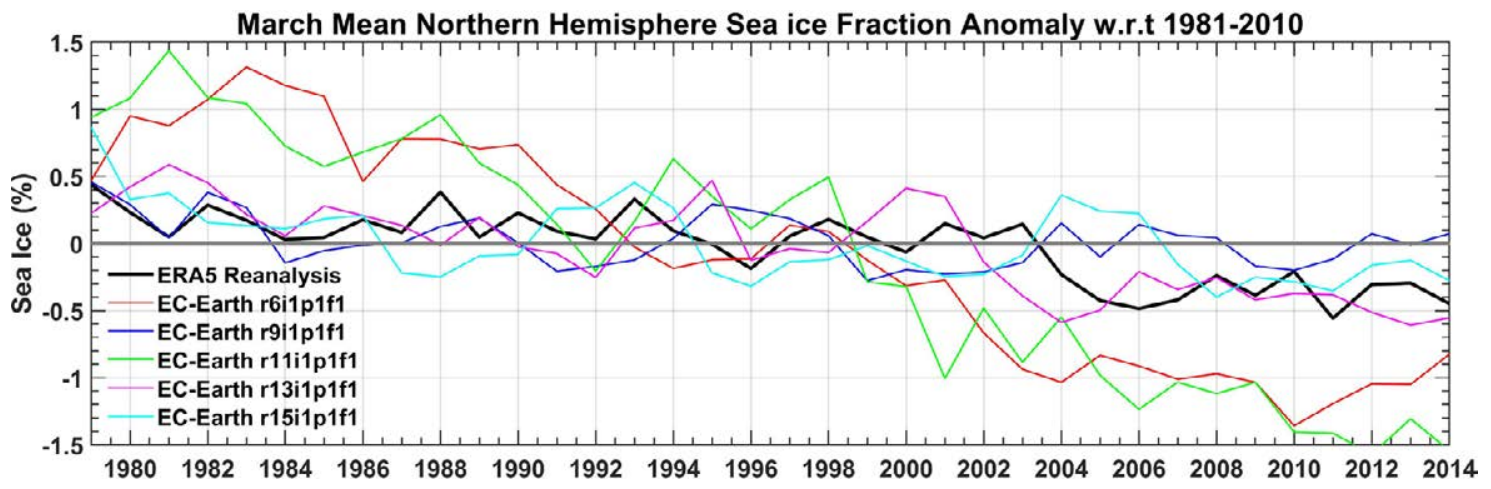

(c)

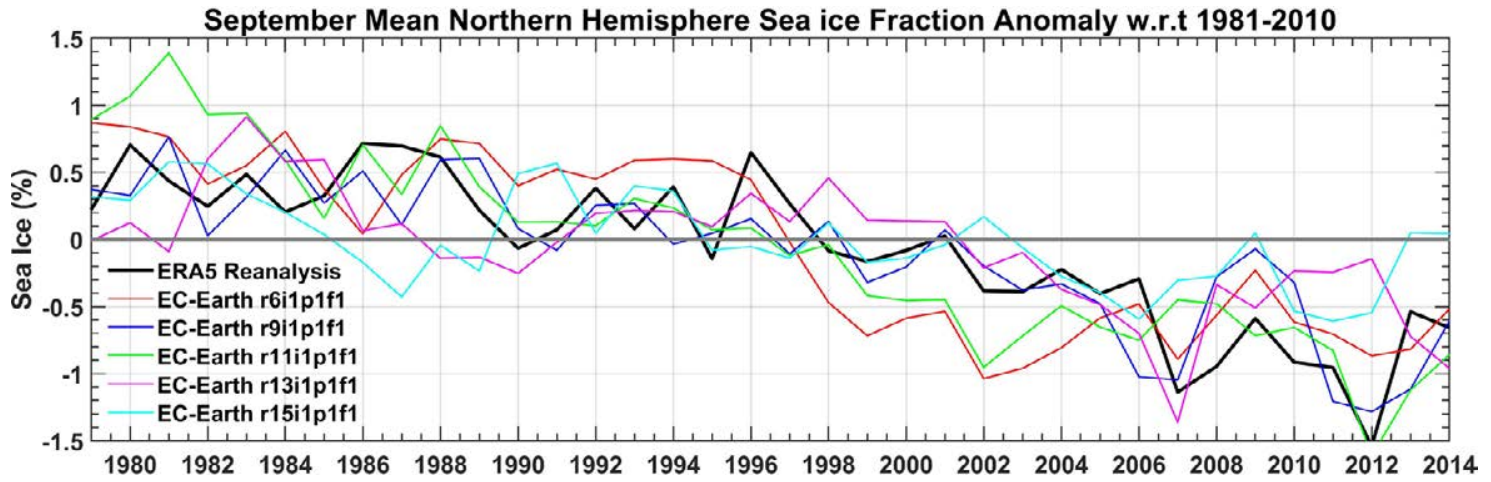

Figure 2.65. Northern Hemisphere sea ice fraction anomalies with respect to the 30-year period 19812010: (a) annual, (b) March and (c) September. 
(a)

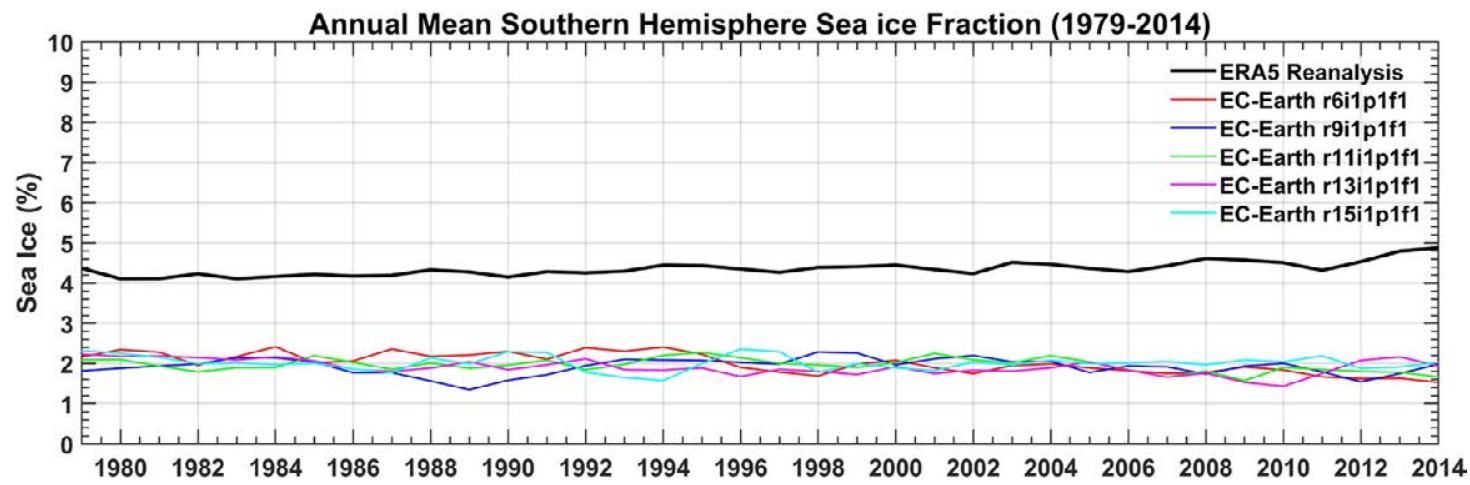

(b)

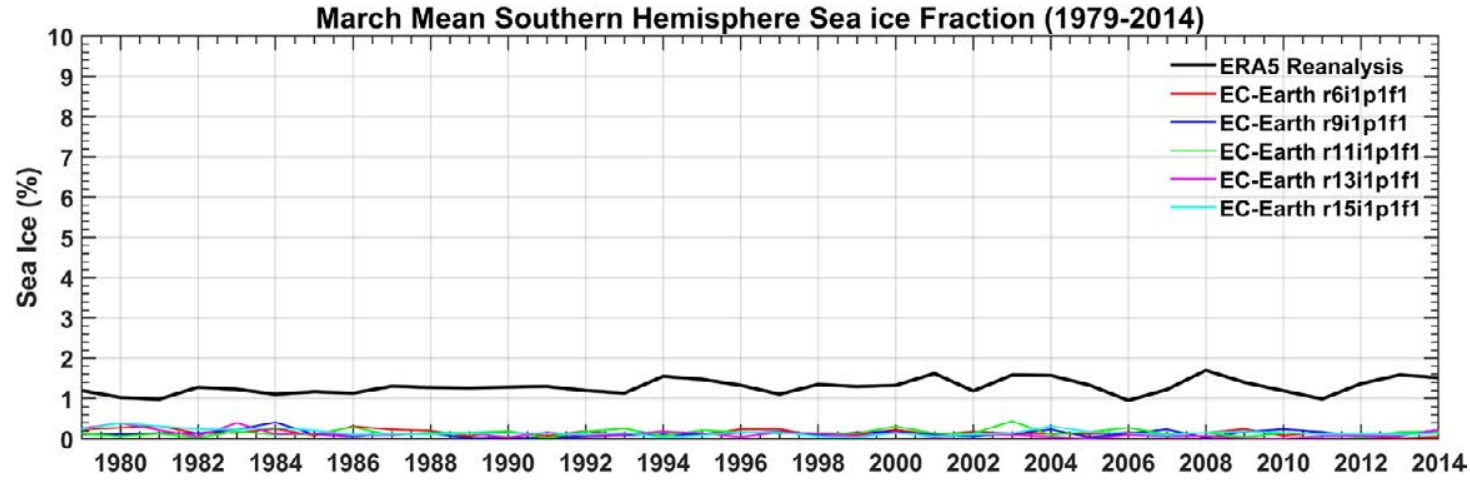

(c)

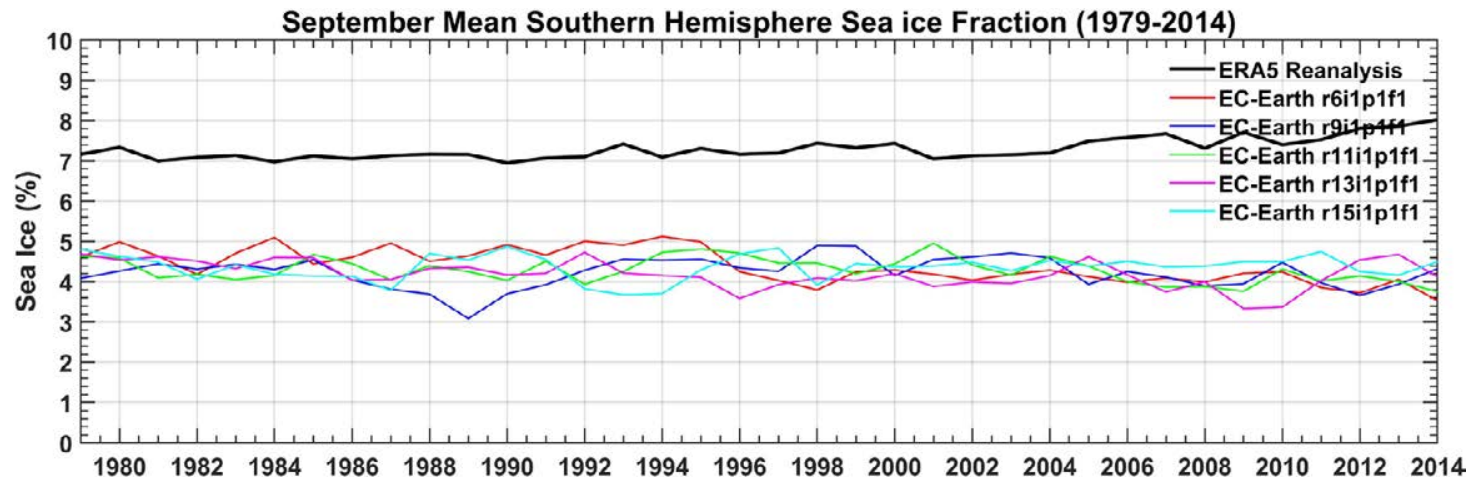

Figure 2.66. Comparison of EC-Earth Southern Hemisphere sea ice fraction (\%) with ERA5 reanalysis data for the period 1979-2014: (a) annual, (b) March and (c) September. 
(a)

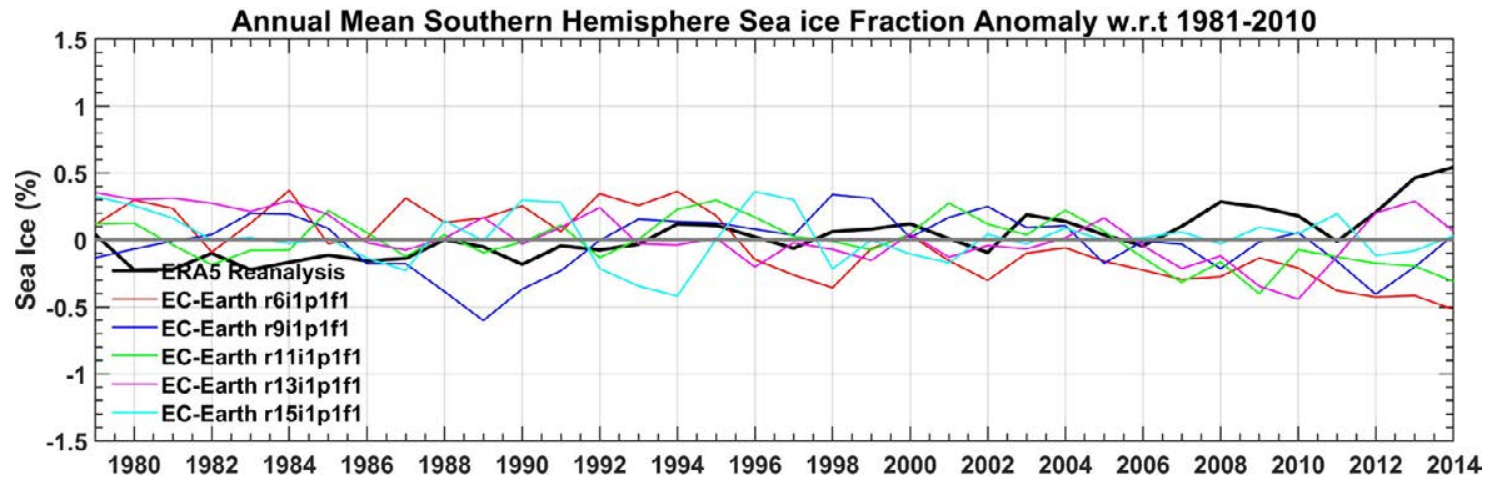

(b)

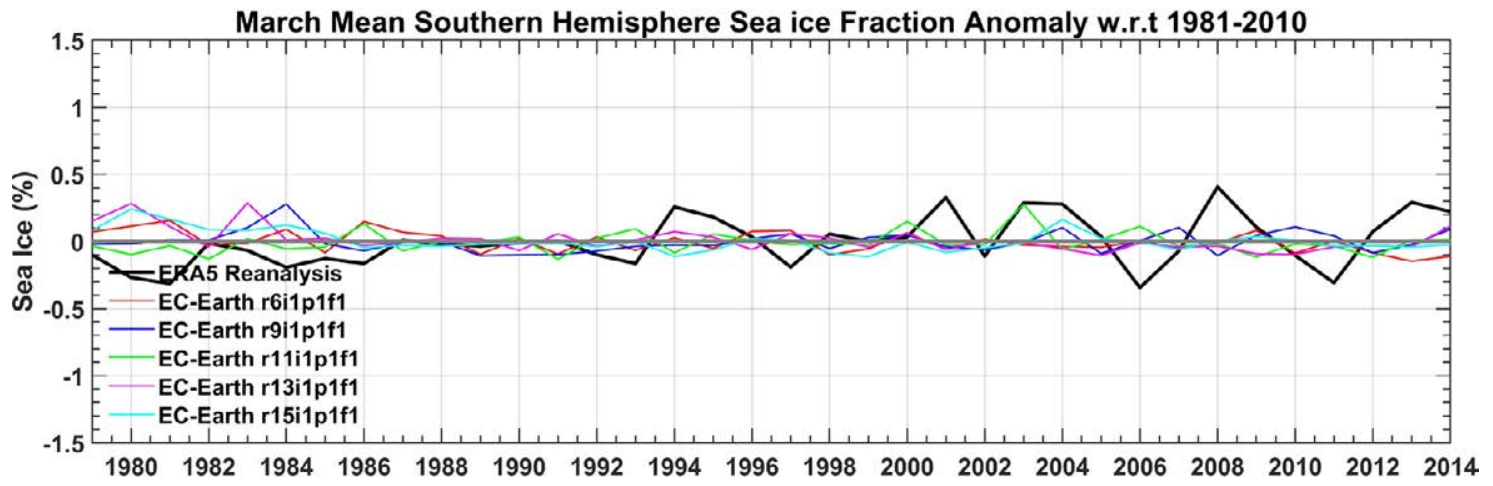

(c)

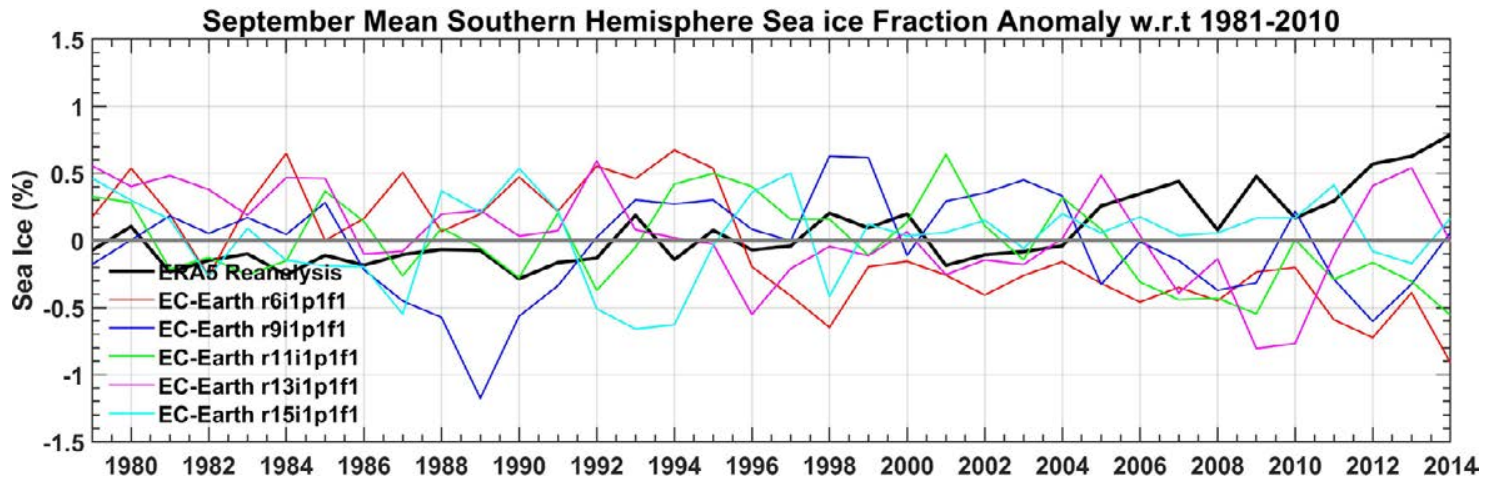

Figure 2.67. Southern Hemisphere sea ice fraction anomalies with respect to the 30-year period 19812010: (a) annual, (b) March and (c) September. 


\section{EC-Earth Climate Projections}

The future global climate was simulated by extending each of the five historical (1850-2014) EC-Earth T255ORCA1L75 experiments to the year 2100 under each of the four ScenarioMIP "tier 1" SSPs. This results in 20 future global climate experiments (five ensembles multiplied by four scenarios). Table 3.1 provides an overview of these simulations.

Projections of climate change were assessed by comparing the two 30-year future periods 2041-2070 and 2071-2100 with the 30-year historical period 1981-2010. Climate projections are presented for the Northern Hemisphere winter (DJF), the Northern Hemisphere summer (JJA) and over the full year. For the climate projections presented here, the mean of the five ensemble members for each SSP was analysed. For example, Figure 3.1a presents the mean of the five SSP1-2.6 ensemble projections for the period 2041-2070.

To quantify the spread (or disagreement) between ensemble members, the standard deviation of the ensemble of climate projections was analysed.

For example, Figure 3.8a (the standard deviation of the five SSP1-2.6 ensemble projections of 2-m temperature for the period 2071-2100) demonstrates a small spread (high agreement) between ensemble members except for the regions south of Greenland and the Arctic, north of Scandinavia. This analysis provides a measure of climate projection uncertainty and highlights areas where the ensemble members agree/disagree. ${ }^{4}$ It was found that the spread remains consistent between seasons and so the standard deviation statistical figures are limited to the annual projections. Furthermore, only the period 2071-2100 was analysed as it was found that the corresponding statistics for 2041-2070 typically showed similar but smaller values.

\subsection{2-m Temperature Projections}

Figure 3.1 presents the spatial distribution of annual mean 2-m temperature projections for each of the four SSPs for the 30-year period 2041-2070 (relative to 1981-2010). The corresponding 2071-2100 projections are presented in Figure 3.2. Note that for each figure, the mean of the five ensemble members is considered. The largest increases in temperatures are seen over the land masses, in particular the northern-most regions and the Arctic. Projections of temperature increase range from $\sim 0.5^{\circ} \mathrm{C}$ over the Southern Hemisphere oceans for 2041-2070 SSP1-2.6 (Figure $3.1 \mathrm{a}$ ) to $\sim 18^{\circ} \mathrm{C}$ over the Arctic for 2071-2100 SSP5-8.5 (Figure 3.2d).

Projections for DJF (Figures 3.3 and 3.4 ) follow a similar trend except that increases in temperature over the northern land masses and the Arctic are enhanced. The projections for JJA (Figures 3.5 and 3.6) follow a similar trend to the annual projections except that increases in temperature over the northern land mases and the Arctic are diminished whereas increases in temperature over Antarctica are enhanced.

\section{Table 3.1. Overview of the Scenario-MIP simulations}

$\begin{array}{lll}\text { EC-Earth ensemble member } & \text { SSPs } & \text { Model levels archived } \\ \text { r6i1p1f1 } & \text { SSP1-2.6, SSP2-4.5, SSP3-7.0 and SSP5-8.5 } & \text { No } \\ \text { r9i1p1f1 } & \text { SSP1-2.6, SSP2-4.5, SSP3-7.0 and SSP5-8.5 } & \text { No } \\ \text { r11i1p1f1 } & \text { SSP1-2.6, SSP2-4.5, SSP3-7.0 and SSP5-8.5 } & \text { Yes } \\ \text { r13i1p1f1 } & \text { SSP1-2.6, SSP2-4.5, SSP3-7.0 and SSP5-8.5 } & \text { No } \\ \text { r15i1p1f1 } & \text { SSP1-2.6, SSP2-4.5, SSP3-7.0 and SSP5-8.5 } & \text { No }\end{array}$

4 To obtain a robust quantification of the uncertainty of the EC-Earth climate projections, a large ensemble is required. Future work will extend this analysis to include the full ensemble of EC-Earth CMIP6 simulations produced by the consortium. 
(a)

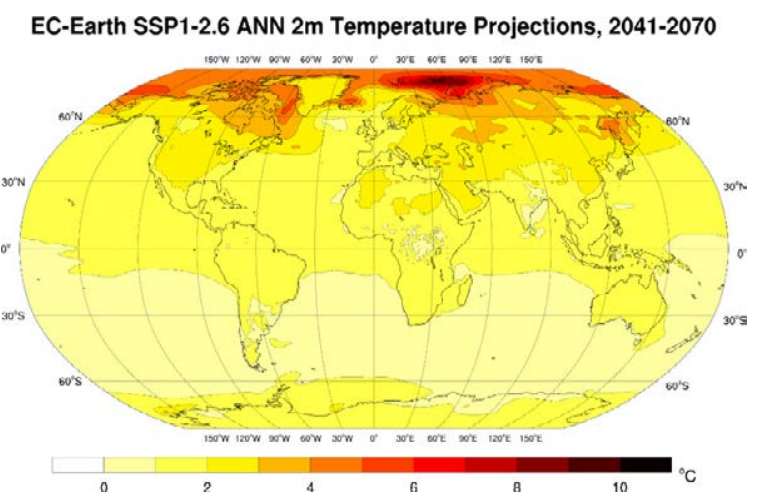

(c)

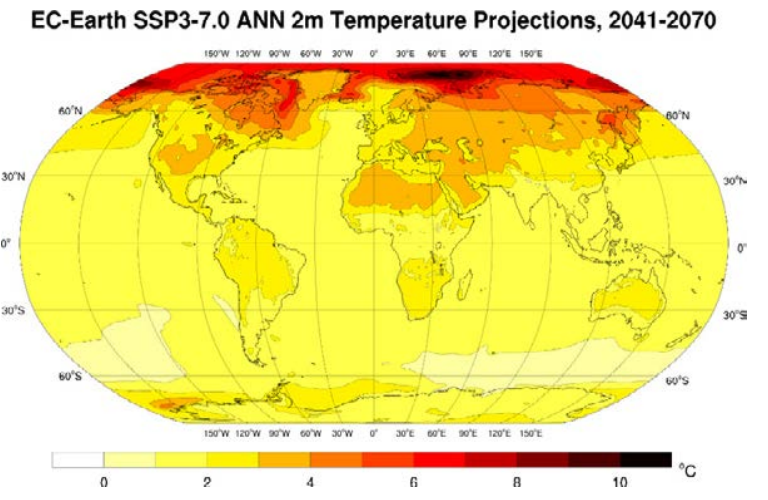

(b)

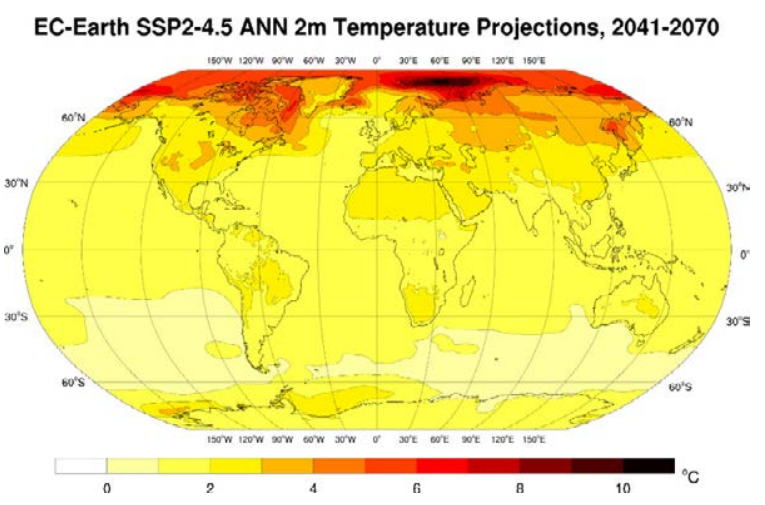

(d)

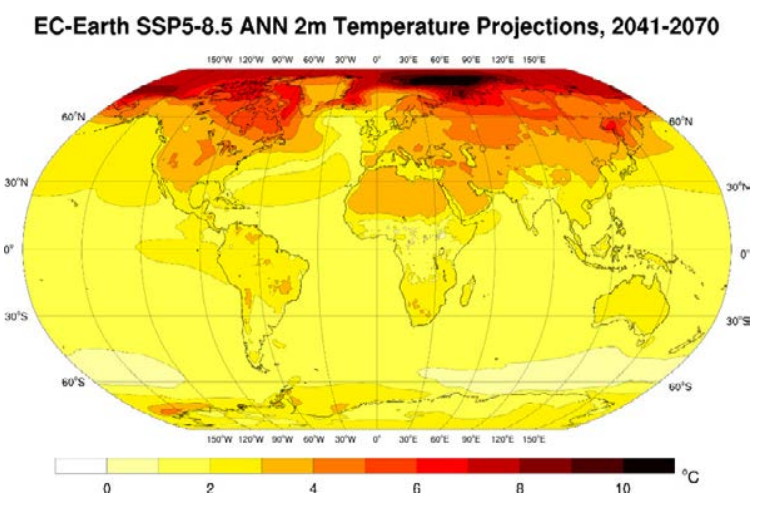

Figure 3.1. EC-Earth annual $2-\mathrm{m}$ temperature projections $\left(2041-2070\right.$ vs $1981-2010,{ }^{\circ} \mathrm{C}$ change): (a) SSP1-2.6, (b) SSP2-4.5, (c) SSP3-7.0 and (d) SSP5-8.5. In each case, an average is taken of the ensemble members r6i1p1f1, r9i1p1f1, r11i1p1f1, r13i1p1f1 and r15i1p1f1.

(a)

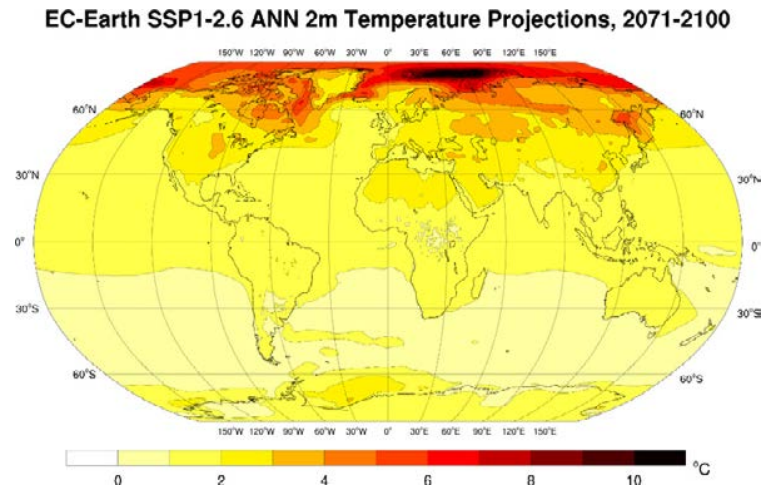

(c)

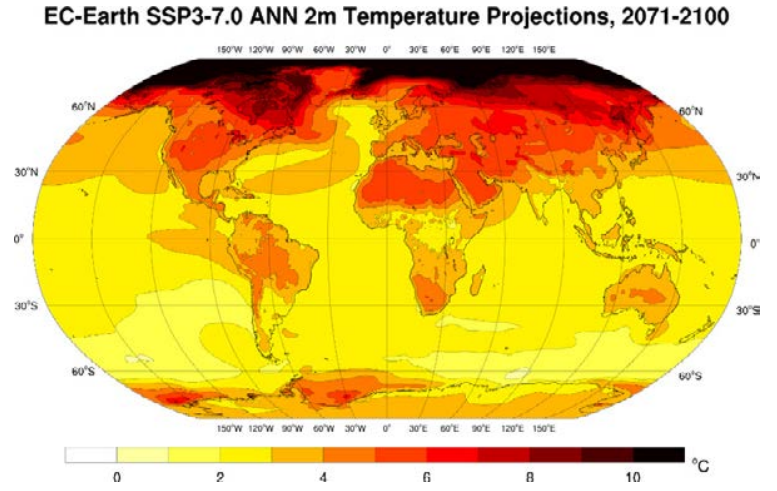

(b)

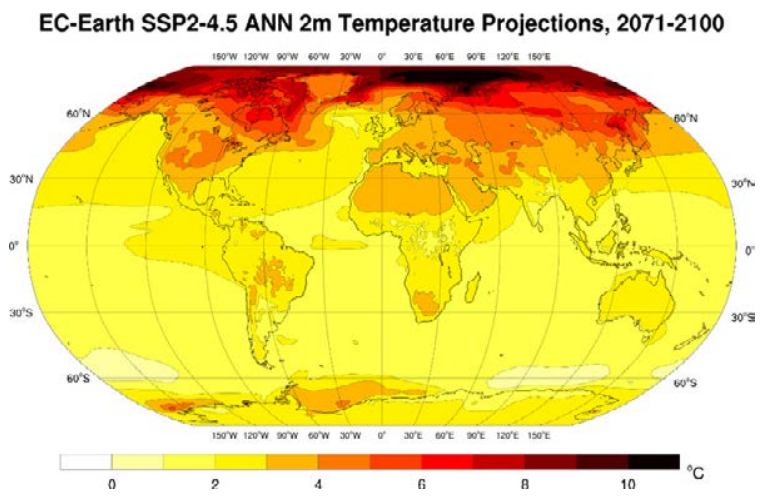

(d)

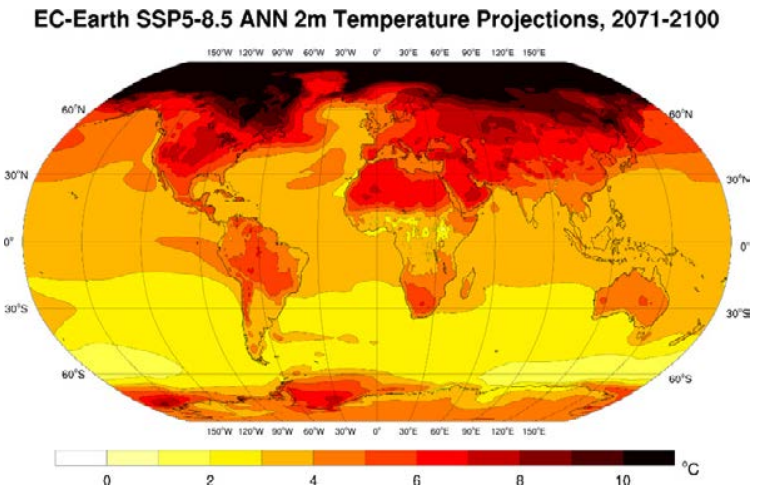

Figure 3.2. EC-Earth annual $2-\mathrm{m}$ temperature projections (2071-2100 vs $1981-2010,{ }^{\circ} \mathrm{C}$ change): (a) SSP1-2.6, (b) SSP2-4.5, (c) SSP3-7.0 and (d) SSP5-8.5. In each case, an average is taken of the ensemble members r6i1p1f1, r9i1p1f1, r11i1p1f1, r13i1p1f1 and r15i1p1f1. 
(a)

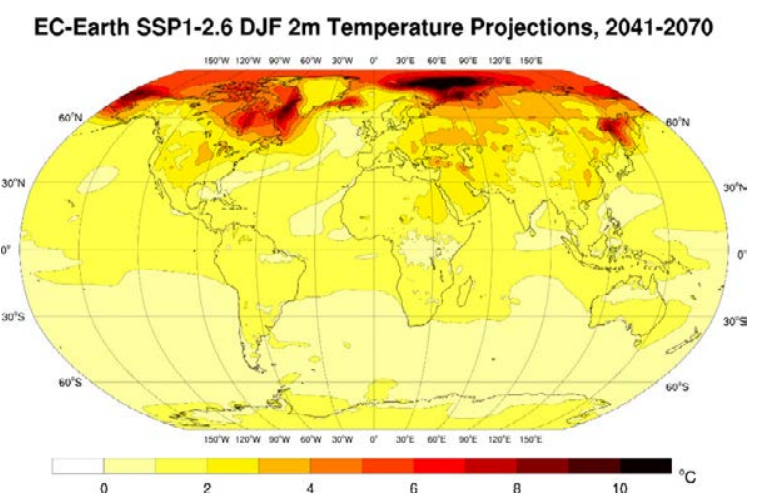

(c)

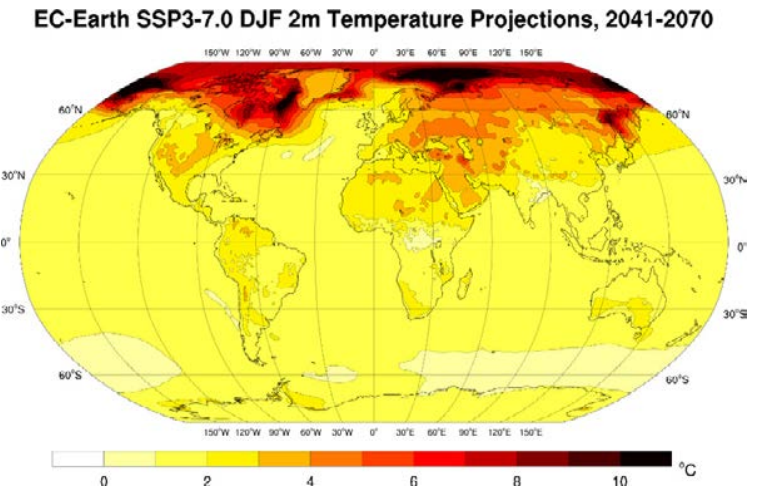

(b)

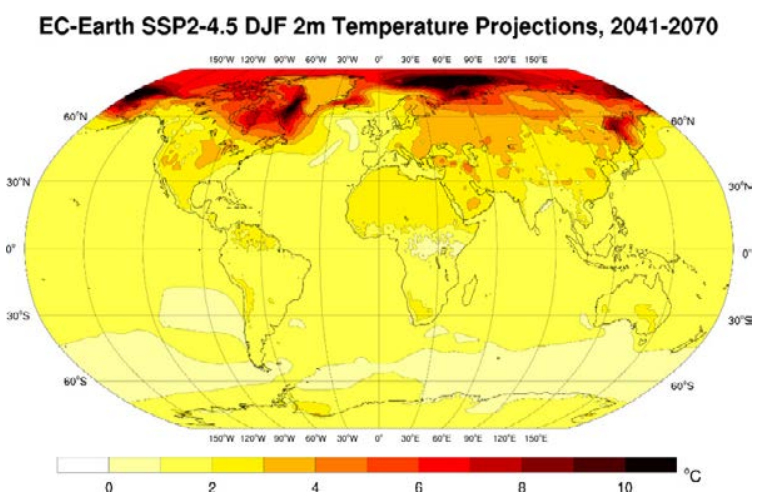

(d)

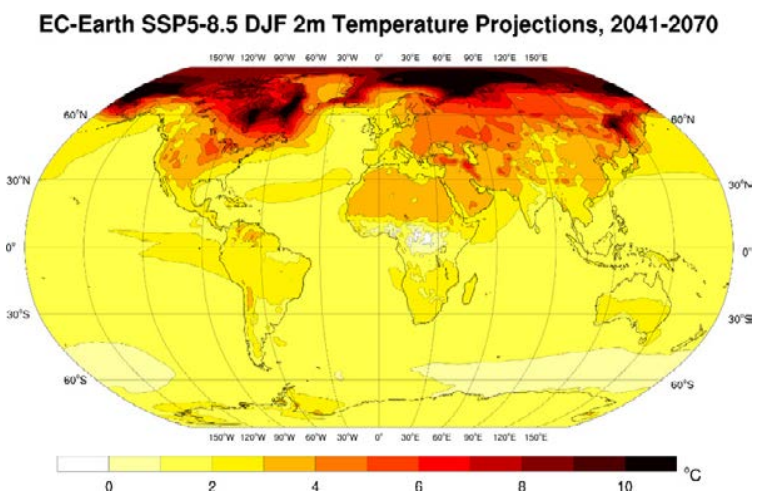

Figure 3.3. EC-Earth DJF 2-m temperature projections (2041-2070 vs $1981-2010,{ }^{\circ} \mathrm{C}$ change): (a) SSP1-2.6, (b) SSP2-4.5, (c) SSP3-7.0 and (d) SSP5-8.5. In each case, an average is taken of the ensemble members r6i1p1f1, r9i1p1f1, r11i1p1f1, r13i1p1f1 and r15i1p1f1.

(a)

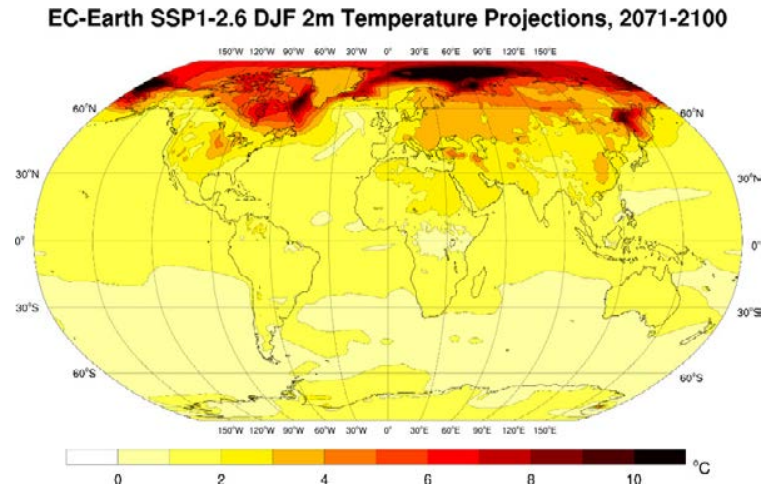

(c)

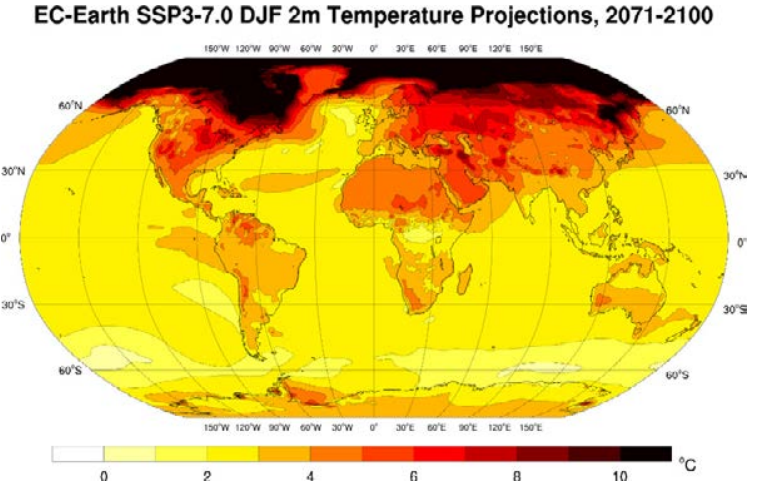

(b)

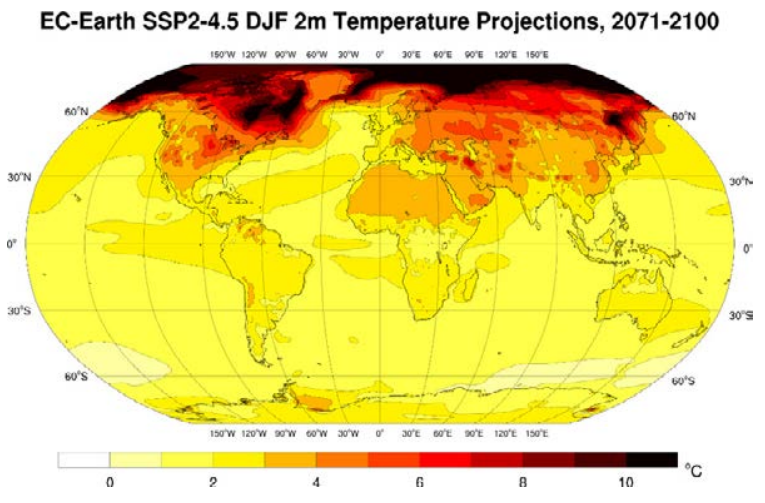

(d)

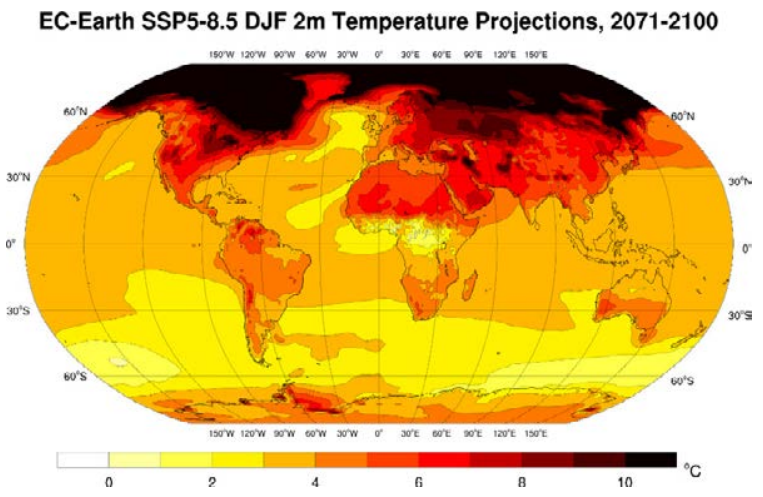

Figure 3.4. EC-Earth DJF 2-m temperature projections (2071-2100 vs $1981-2010,{ }^{\circ} \mathrm{C}$ change): (a) SSP1-2.6, (b) SSP2-4.5, (c) SSP3-7.0 and (d) SSP5-8.5. In each case, an average is taken of the ensemble members r6i1p1f1, r9i1p1f1, r11i1p1f1, r13i1p1f1 and r15i1p1f1. 
(a)

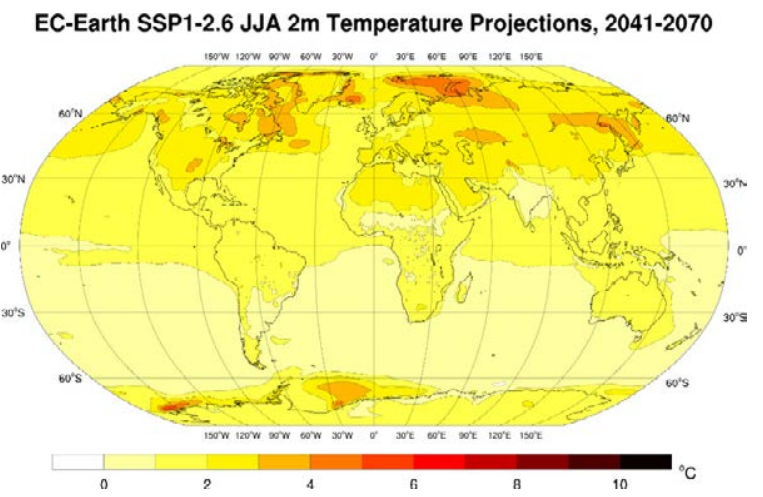

(c)

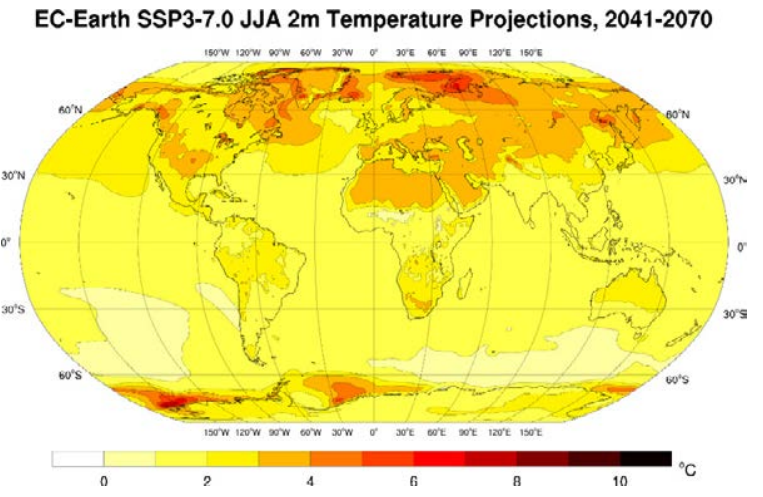

(b)

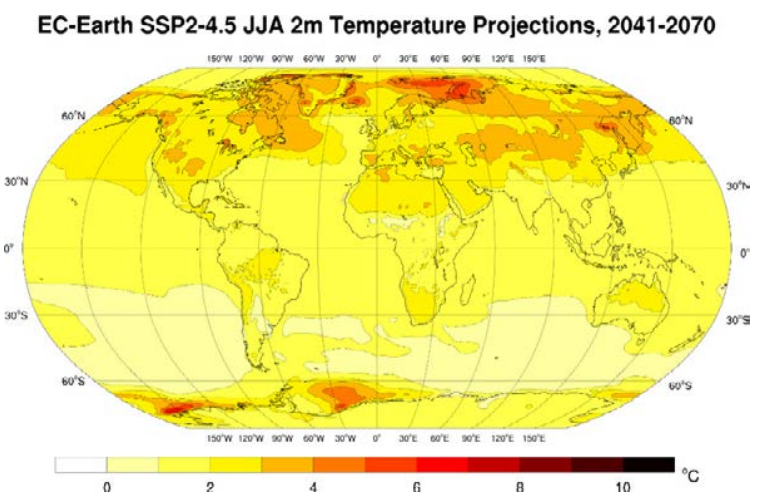

(d)

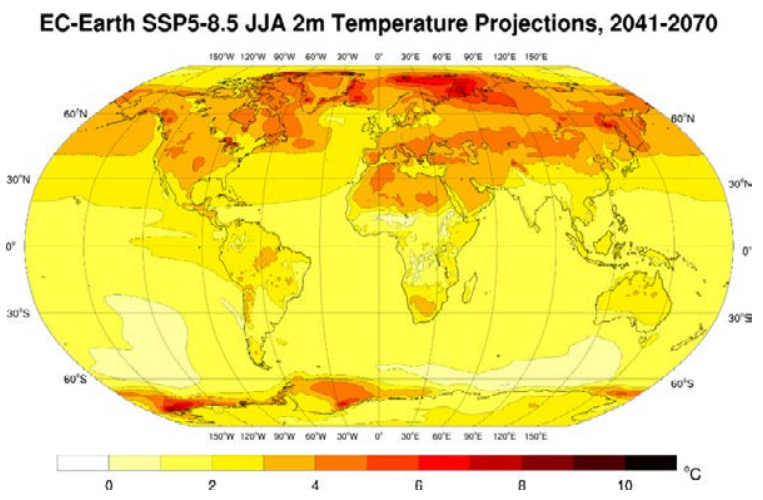

Figure 3.5. EC-Earth JJA 2-m temperature projections (2041-2070 vs $1981-2010,{ }^{\circ} \mathrm{C}$ change): (a) SSP1-2.6, (b) SSP2-4.5, (c) SSP3-7.0 and (d) SSP5-8.5. In each case, an average is taken of the ensemble members r6i1p1f1, r9i1p1f1, r11i1p1f1, r13i1p1f1 and r15i1p1f1.

(a)

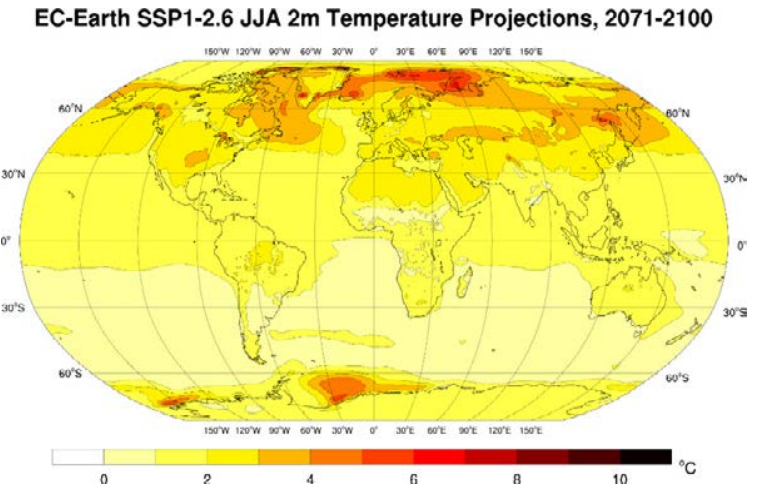

(c)

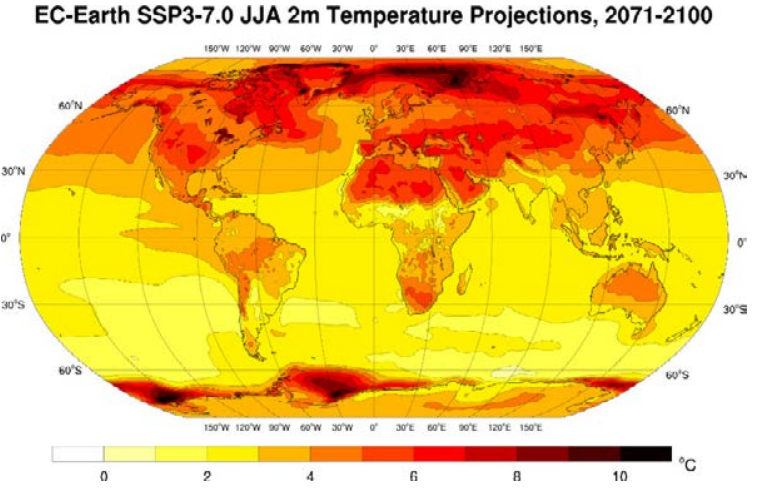

(b)

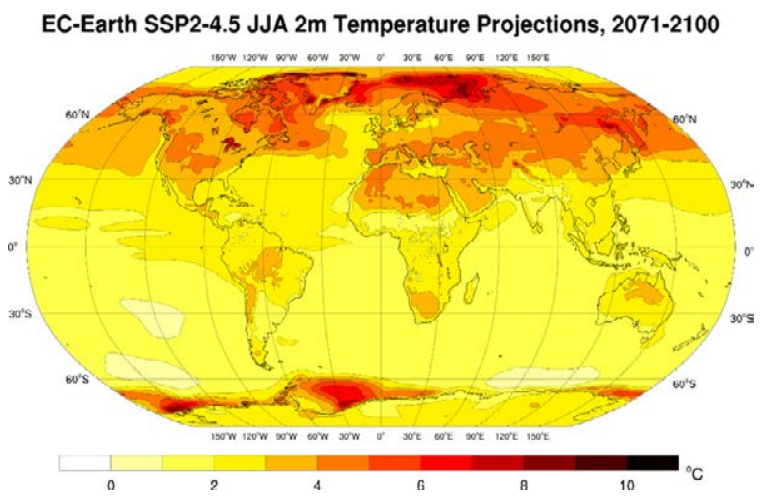

(d)

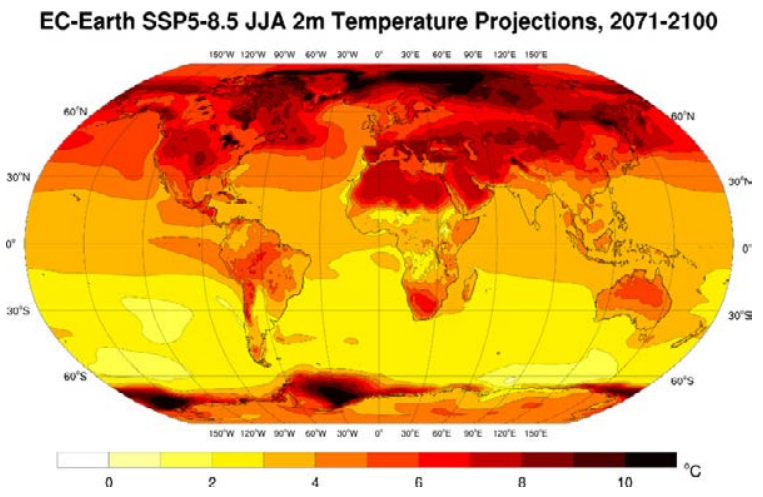

Figure 3.6. EC-Earth JJA 2-m temperature projections (2071-2100 vs $1981-2010,{ }^{\circ} \mathrm{C}$ change): (a) SSP1-2.6, (b) SSP2-4.5, (c) SSP3-7.0 and (d) SSP5-8.5. In each case, an average is taken of the ensemble members r6i1p1f1, r9i1p1f1, r11i1p1f1, r13i1p1f1 and r15i1p1f1. 
Note that the large projected increases in the Arctic region should be viewed in the context of the low mean temperatures in this region. The mean annual 2-m temperature in the Arctic Circle (latitudes above $66.5^{\circ}$ ), derived from ERA5 $1979-2014$ data, is $-10.9^{\circ} \mathrm{C}$, with values ranging from $-28.2^{\circ} \mathrm{C}$ over Greenland to $6.7^{\circ} \mathrm{C}$ over the Greenland Sea. Similarly, the mean DJF and JJA 2-m temperatures in the Arctic Circle are $-22.9^{\circ} \mathrm{C}$ (ranging from $-40.9^{\circ} \mathrm{C}$ to $3.7^{\circ} \mathrm{C}$ ) and $2.7^{\circ} \mathrm{C}$ (ranging from $-15.2^{\circ} \mathrm{C}$ to $16.5^{\circ} \mathrm{C}$ ), respectively.

The mean global annual temperature anomalies (relative to 1981-2010) for all five historical simulations (1850-2014) and 20 SSPs (2015-2100) are presented in Figure 3.7. The bold lines represent the ensemble means. All ensemble members show a steady increase in temperature from around 2000, with a noticeable divergence between the SSPs from around 2050. By the year 2100, the global mean temperature is projected to increase by approximately $1.5^{\circ} \mathrm{C}, 2.8^{\circ} \mathrm{C}, 4.2^{\circ} \mathrm{C}$ and $5.5^{\circ} \mathrm{C}$ for SSP $1-2.6$, SSP2-4.5, SSP3-7.0 and SSP5-8.5, respectively. The small spread between the individual ensemble members demonstrates a high level of agreement. This is confirmed by Figure 3.8, which shows the standard deviation of each SSP ensemble of climate projections. High levels of agreement between ensembles are noted for all regions except south of Greenland and the Arctic region north of Scandinavia. A closer analysis of the individual ensemble members shows that the disagreement in the northern regions is the result of a difference between two groups - the r6i1p1f1 and r11i1p1f1 ensemble members project higher (lower) temperature rises than the r9i1p1f1, r13i1p1f1 and r15i1p1f1 ensemble members in the regions roughly north (south) of Iceland. Assigning attribution to these features is beyond the scope of the current report. Future work will address this issue by extending the analysis to include the full ensemble of EC-Earth CMIP6 simulations produced by the consortium and investigating factors such as the relative skill of ensemble members (note from Figure 2.2 that r6i1p1f1 and r11i1p1f1 have an enhanced cold bias in the area of interest) and the uncertainty in projections of sea ice extent and SST.

\subsection{Precipitation Projections}

Figure 3.9 presents the spatial distribution of annual precipitation projections (\% change) for each of the four SSPs for the 2041-2070 period. The corresponding projections for 2071-2100 are presented in Figure 3.10. The general trend is for an increase in precipitation except in the North Atlantic region south of Iceland and regions just north and south of the equator, including North Africa and large parts of South America and South Africa. Southern Europe and the Mediterranean show a drying for the end-of-century SSP3-7.0 and SSP5-8.5 projections. Note that the large projected changes over the Sahara and the Middle East should be considered in the context of small increases/decreases in precipitation in dry regions, resulting in large percentage changes (see Figures 2.12a and b for observed global precipitation data).

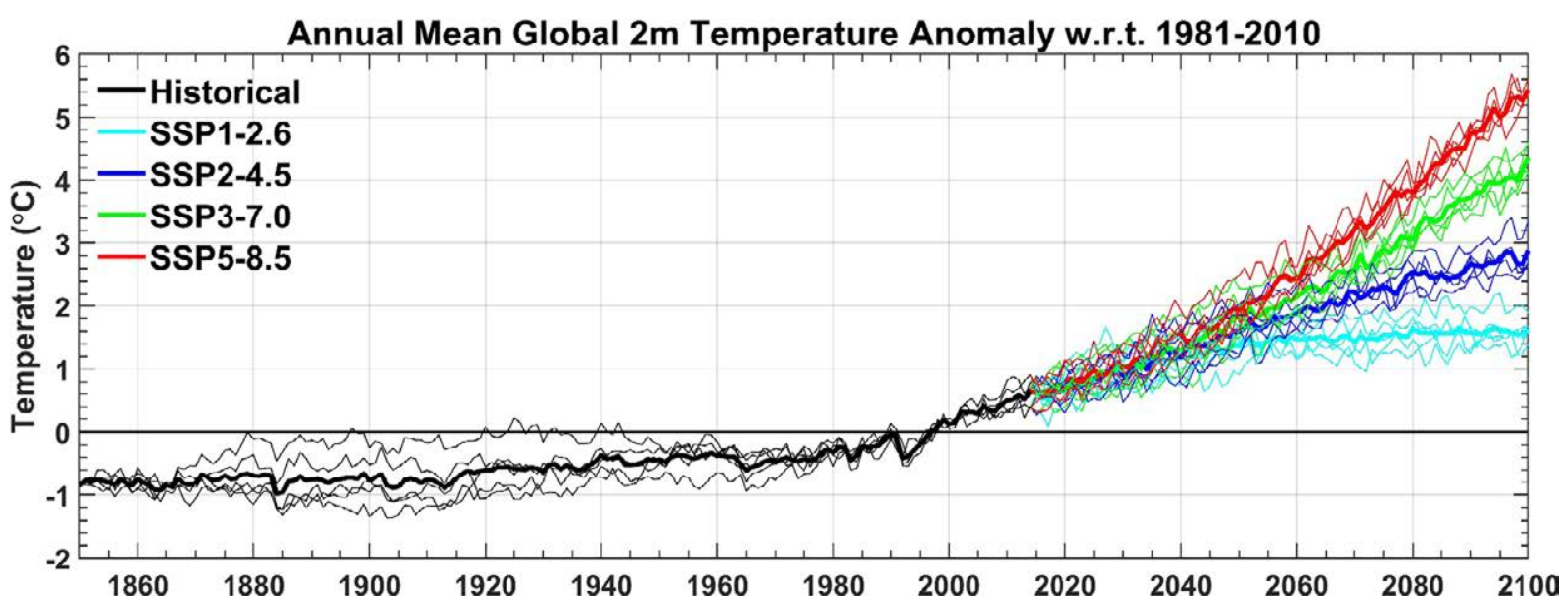

Figure 3.7. Global mean annual 2-m temperature anomalies with respect to the 30-year period 1981-2010: EC-Earth ensemble members r6i1p1f1, r9i1p1f1, r11i1p1f1, r13i1p1f1 and r15i1p1f1. The bold lines represent the ensemble means. 
(a)

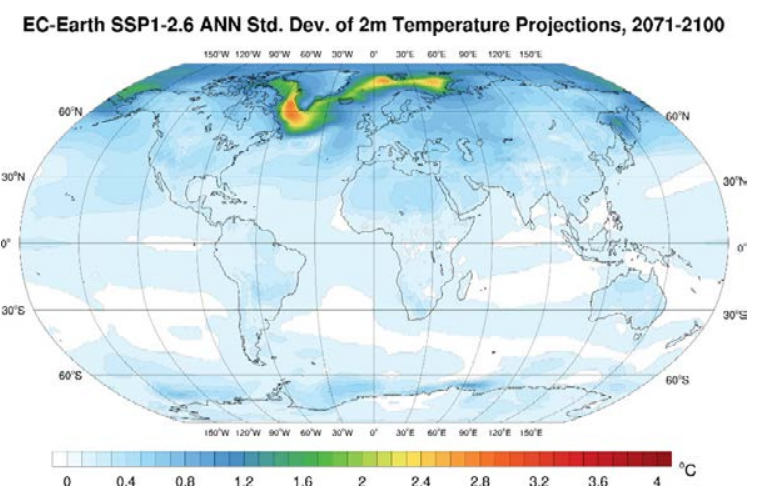

(c)
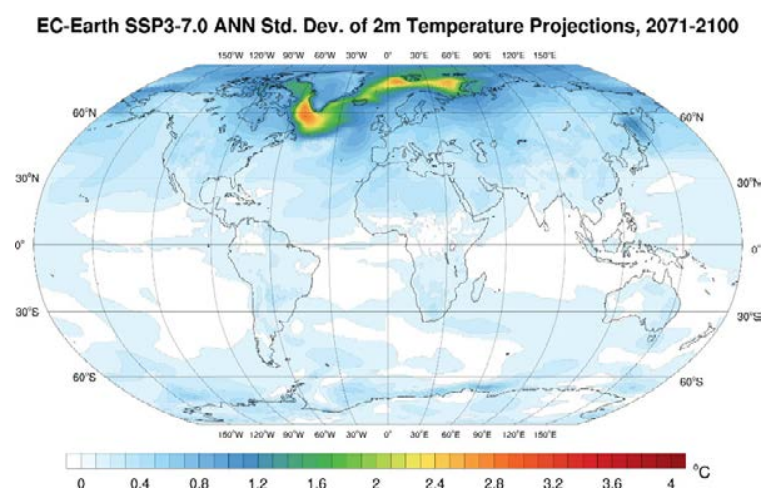

(b)

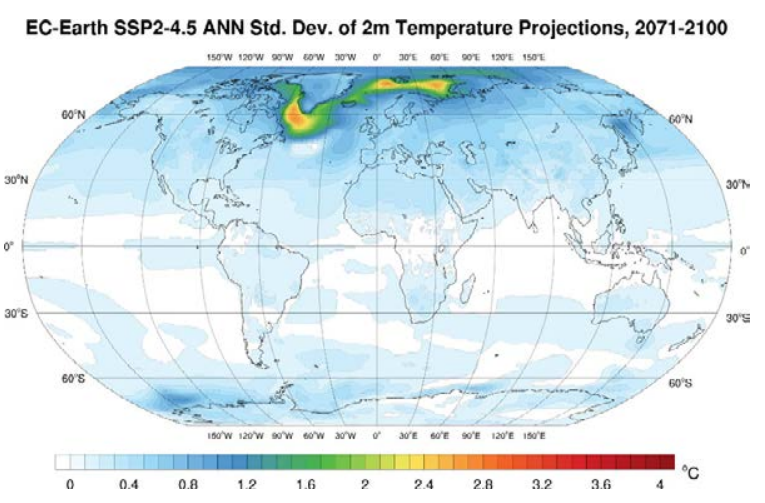

(d)

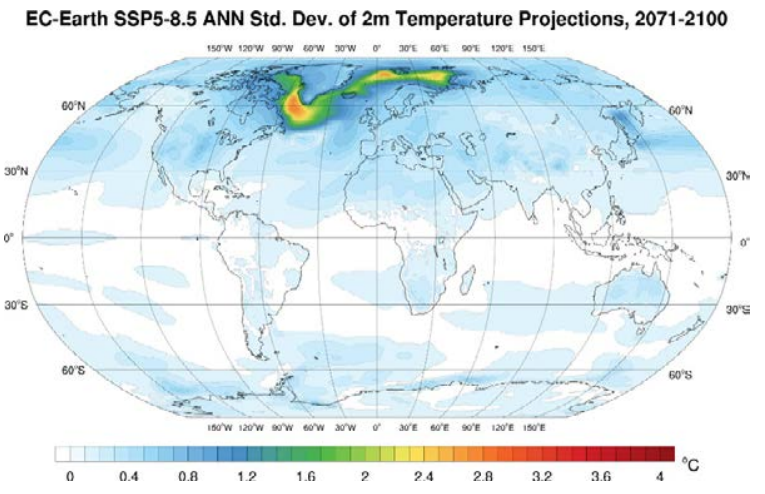

Figure 3.8. Standard deviation of the ensemble of annual 2-m temperature projections (2071-2100): (a) SSP1-2.6, (b) SSP2-4.5, (c) SSP3-7.0 and (d) SSP5-8.5.

(a)

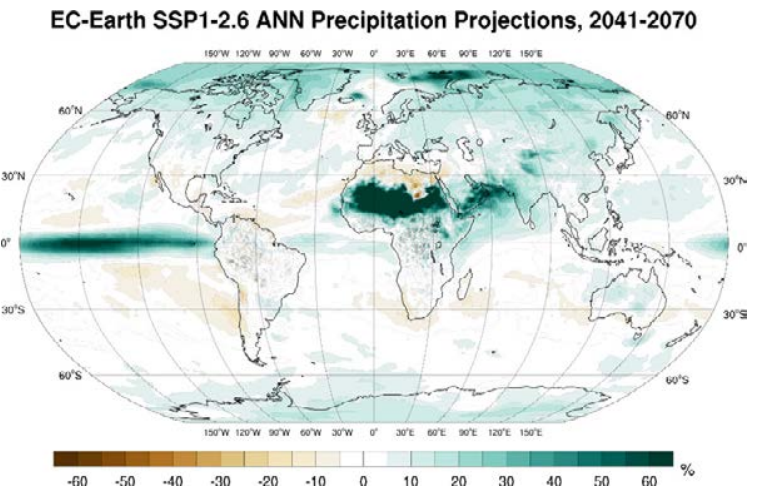

(c)

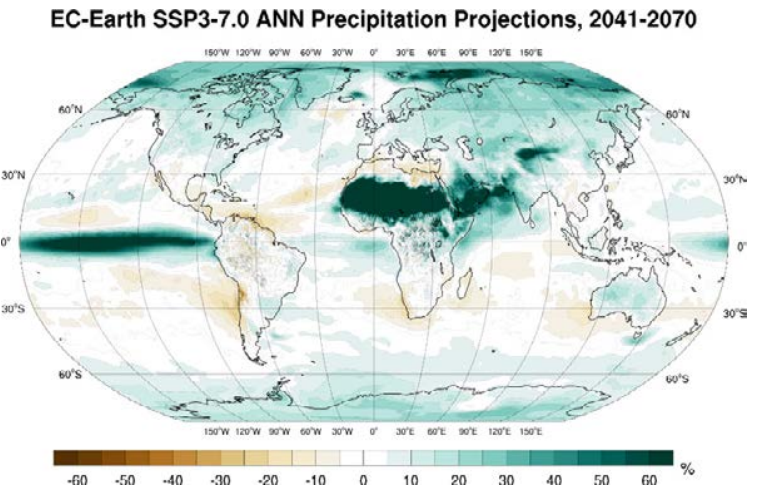

(b)

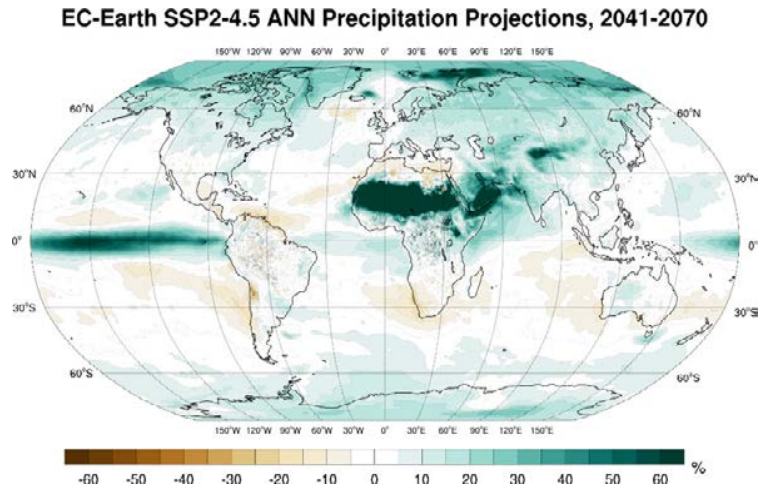

(d)

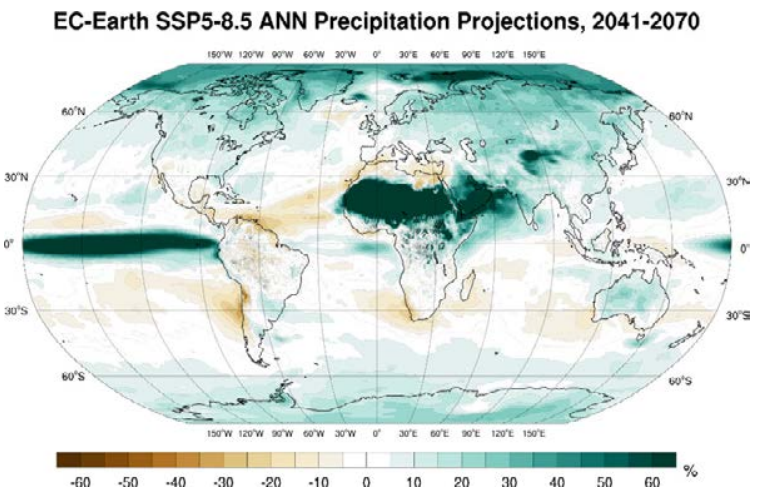

Figure 3.9. EC-Earth annual precipitation projections (2041-2070 vs 1981-2010, \% change): (a) SSP1-2.6, (b) SSP2-4.5, (c) SSP3-7.0 and (d) SSP5-8.5. In each case, an average is taken of the ensemble members r6i1p1f1, r9i1p1f1, r11i1p1f1, r13i1p1f1 and r15i1p1f1. 
(a)

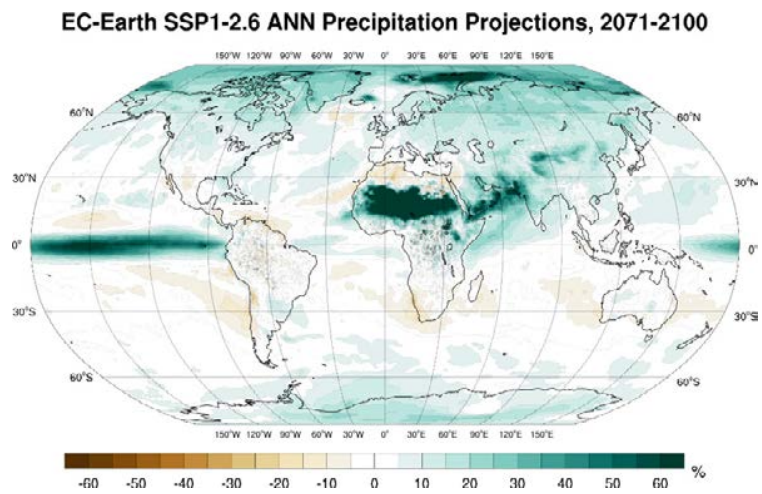

(c)

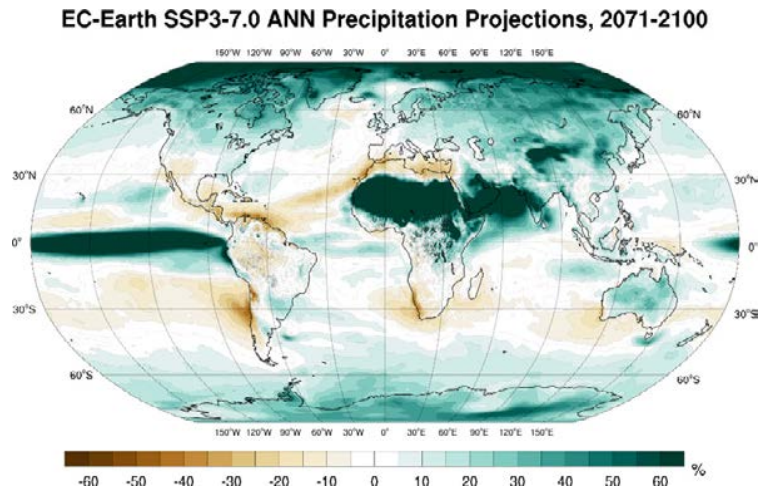

(b)

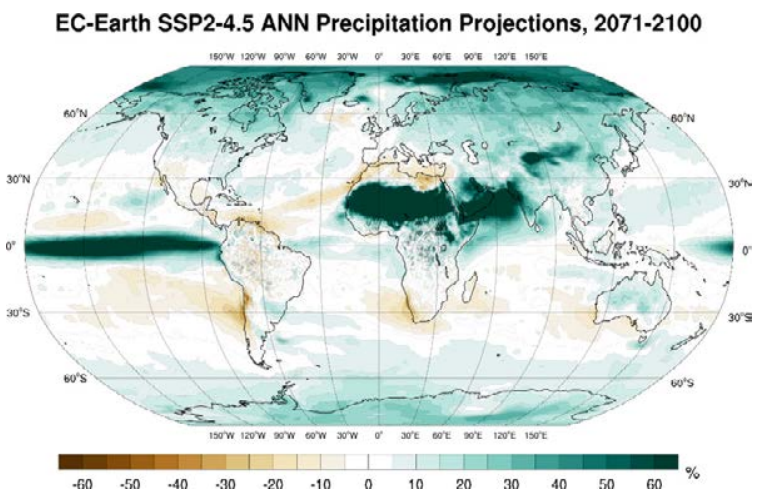

(d)

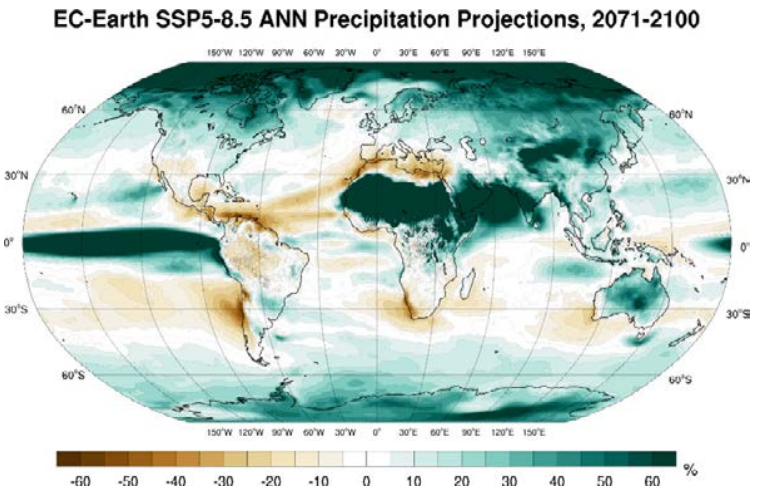

Figure 3.10. EC-Earth annual precipitation projections (2071-2100 vs $1981-2010$, \% change): (a) SSP1-2.6, (b) SSP2-4.5, (c) SSP3-7.0 and (d) SSP5-8.5. In each case, an average is taken of the ensemble members r6i1p1f1, r9i1p1f1, r11i1p1f1, r13i1p1f1 and r15i1p1f1.

Precipitation projections for DJF (Figures 3.11 and 3.12) follow a similar (but enhanced) trend to the annual projections. However, Europe and the Mediterranean are projected to be wetter under all SSPs. The projections for JJA (Figures 3.13 and 3.14) follow a similar trend to the annual projections, with a general increase in precipitation in most regions and an enhanced drying over southern Europe, North America, South America and South Africa. For JJA, there is no drying projected in the Atlantic region south of Iceland.

The mean global annual precipitation anomalies (relative to 1981-2010) for all five historical simulations (1850-2014) and 20 SSPs (2015-2100) are presented in Figure 3.15. The bold lines represent the ensemble means. All ensemble members show a steady increase in precipitation from around 2000, with a noticeable divergence between the SSPs around
2060. By the year 2100, global mean precipitation is projected to increase by approximately $4 \%$ $(0.1 \mathrm{~mm} /$ day $), 6 \%(0.16 \mathrm{~mm} /$ day $), 8 \%(0.25 \mathrm{~mm} /$ day $)$ and $10 \%(0.3 \mathrm{~mm} /$ day $)$ for SSP1-2.6, SSP2-4.5, SSP3-7.0 and SSP5-8.5, respectively.

Compared with temperature (see Figure 3.7), the spread between ensemble members is enhanced for precipitation. However, the spread between ensemble members is greatly decreased compared with CMIP5 (Gleeson et al., 2013), suggesting an added measure of confidence for the CMIP6 precipitation projections. Figure 3.16 shows the standard deviation of each SSP ensemble of climate projections; there is a high level of agreement between ensembles for most regions except North Africa and the Middle East (see note 6 regarding percentage changes in dry regions), the equatorial Pacific region and, to a lesser extent, over Australia and the Arctic. 
(a)

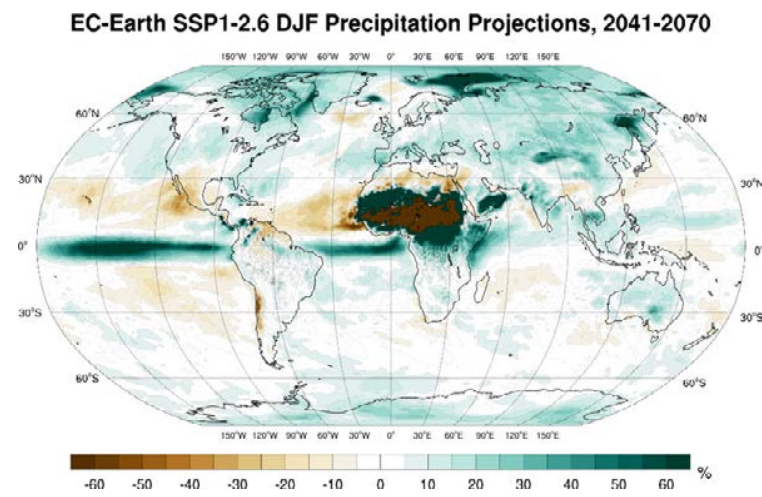

(c)

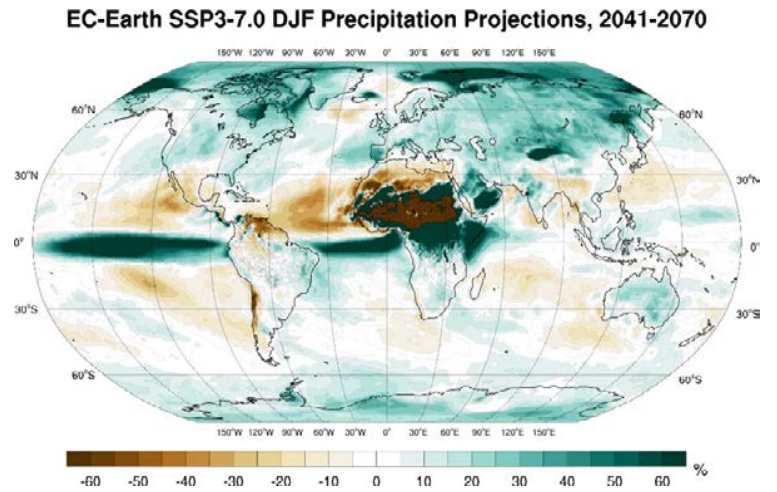

(b)

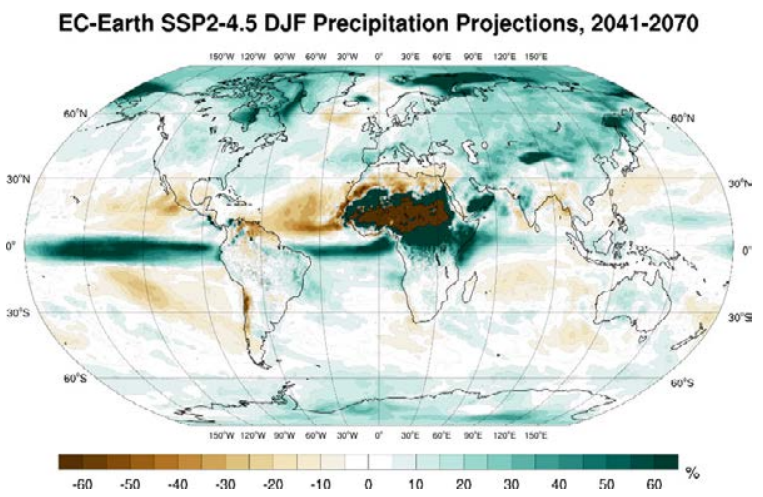

(d)

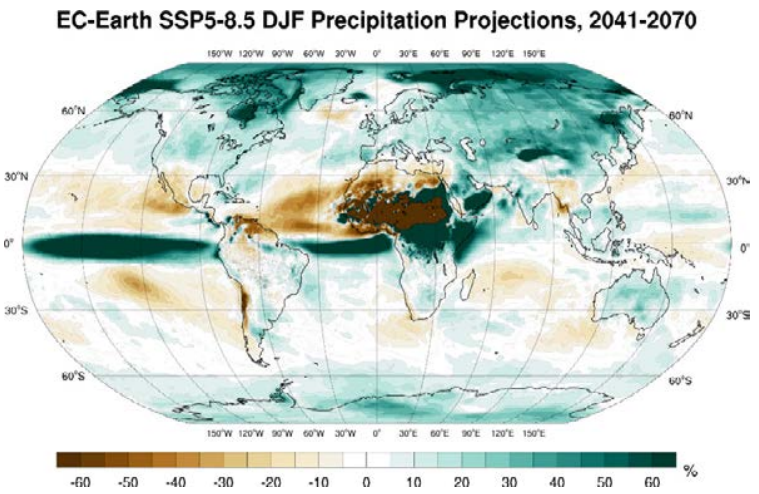

Figure 3.11. EC-Earth DJF precipitation projections (2041-2070 vs 1981-2010, \% change): (a) SSP1-2.6, (b) SSP2-4.5, (c) SSP3-7.0 and (d) SSP5-8.5. In each case, an average is taken of the ensemble members r6i1p1f1, r9i1p1f1, r11i1p1f1, r13i1p1f1 and r15i1p1f1.

(a)

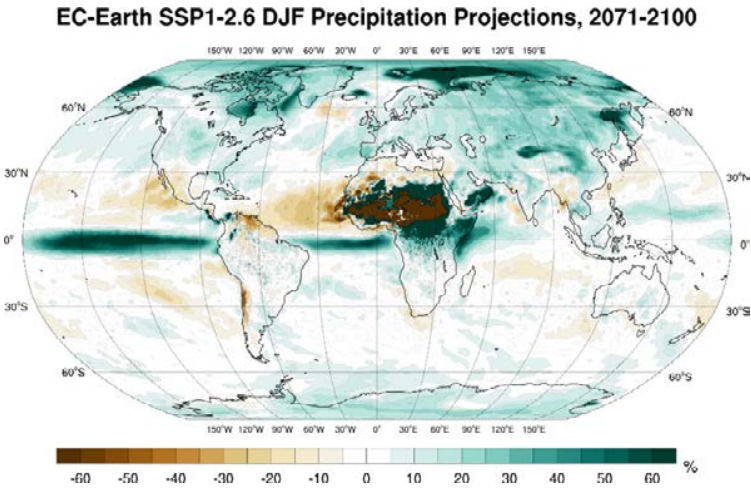

(c)

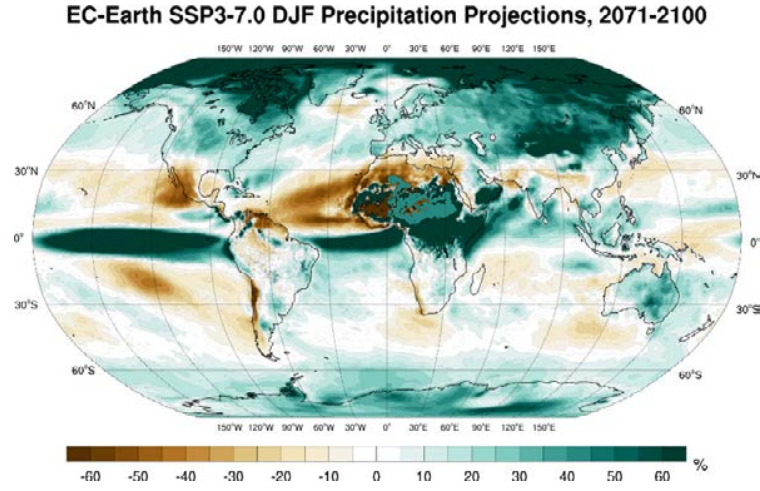

(b)

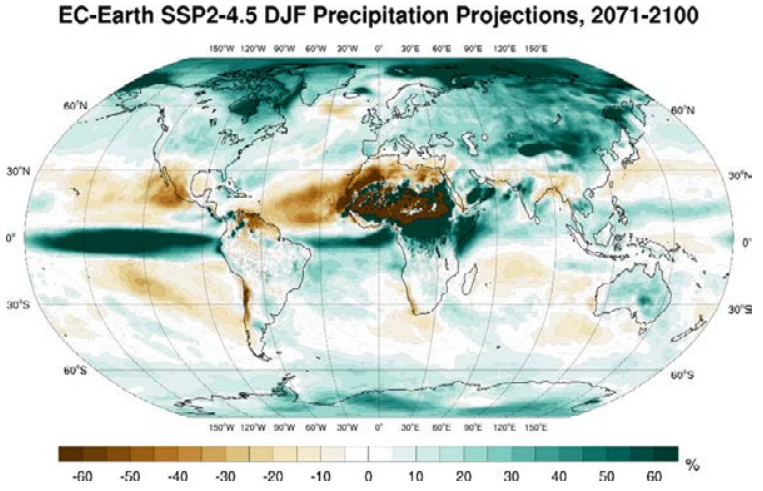

(d)

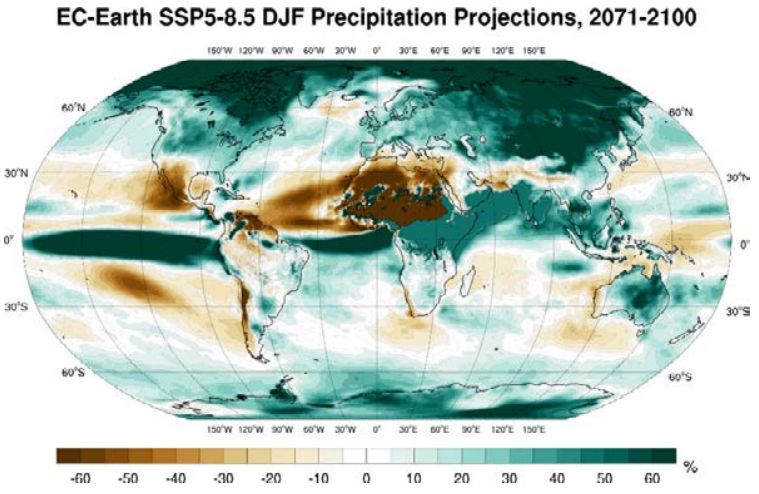

Figure 3.12. EC-Earth DJF precipitation projections (2071-2100 vs 1981-2010, \% change): (a) SSP1-2.6, (b) SSP2-4.5, (c) SSP3-7.0 and (d) SSP5-8.5. In each case, an average is taken of the ensemble members r6i1p1f1, r9i1p1f1, r11i1p1f1, r13i1p1f1 and r15i1p1f1. 
(a)

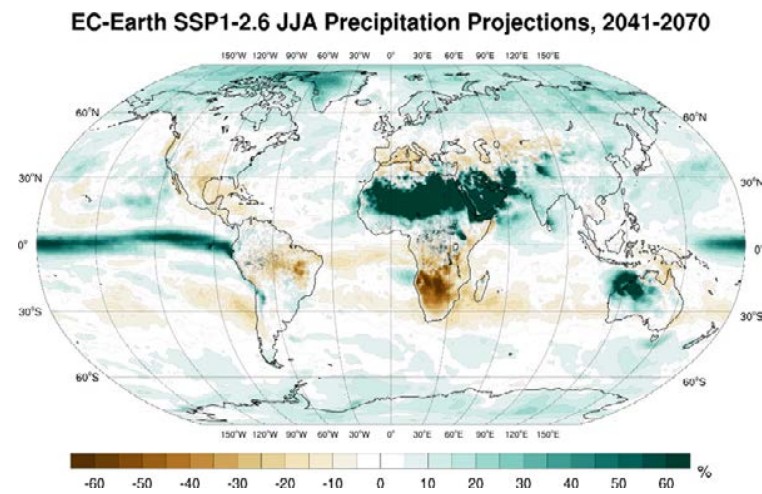

(c)

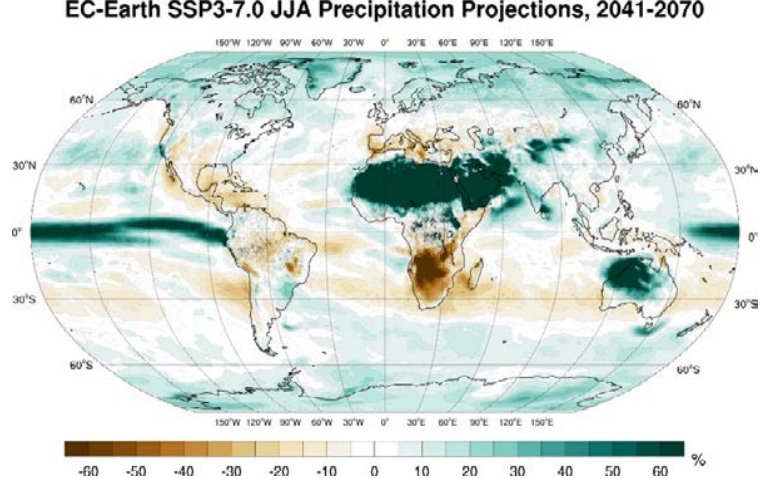

(b)

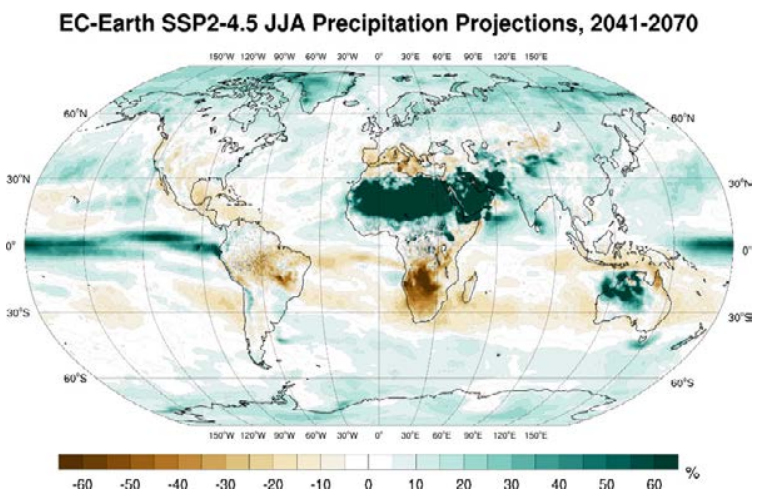

(d)

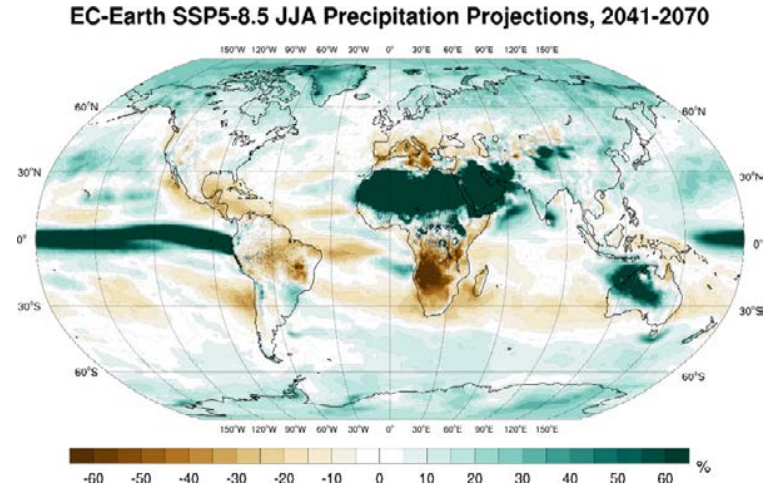

Figure 3.13. EC-Earth JJA precipitation projections (2041-2070 vs 1981-2010, \% change): (a) SSP1-2.6, (b) SSP2-4.5, (c) SSP3-7.0 and (d) SSP5-8.5. In each case, an average is taken of the ensemble members r6i1p1f1, r9i1p1f1, r11i1p1f1, r13i1p1f1 and r15i1p1f1.

(a)

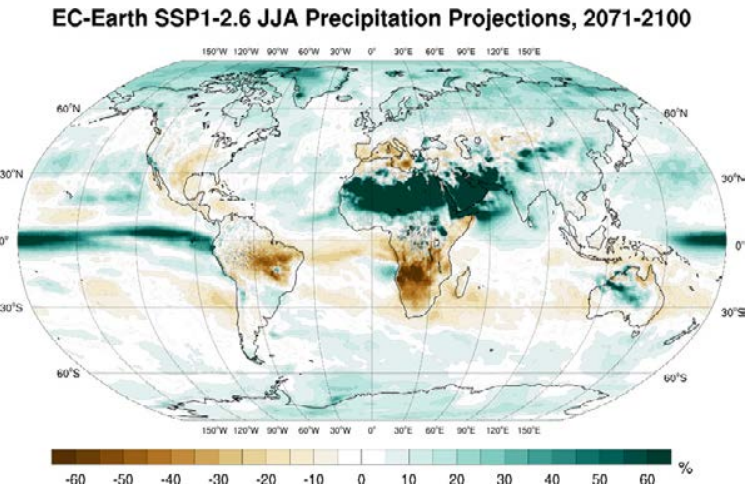

(c)

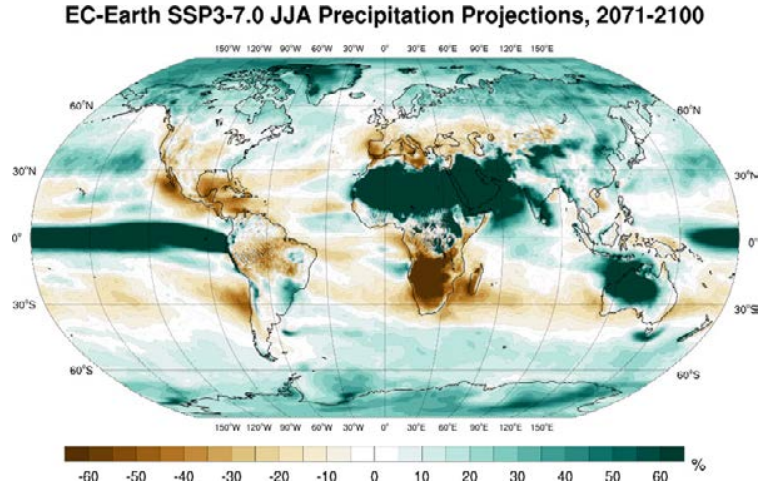

(b)

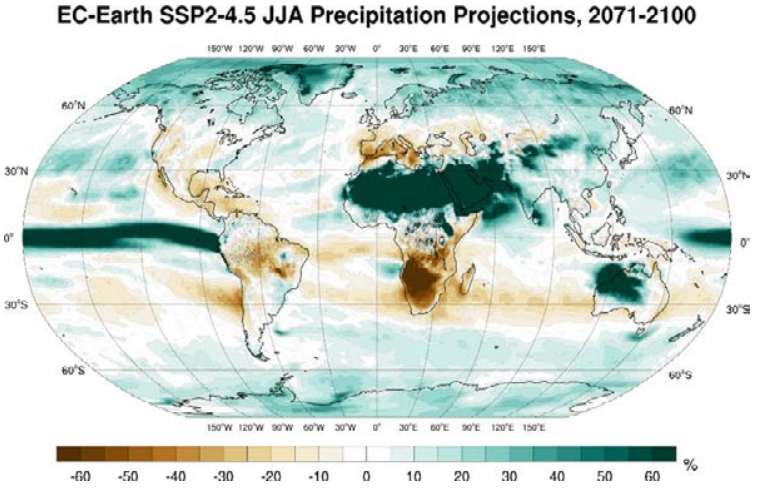

(d)

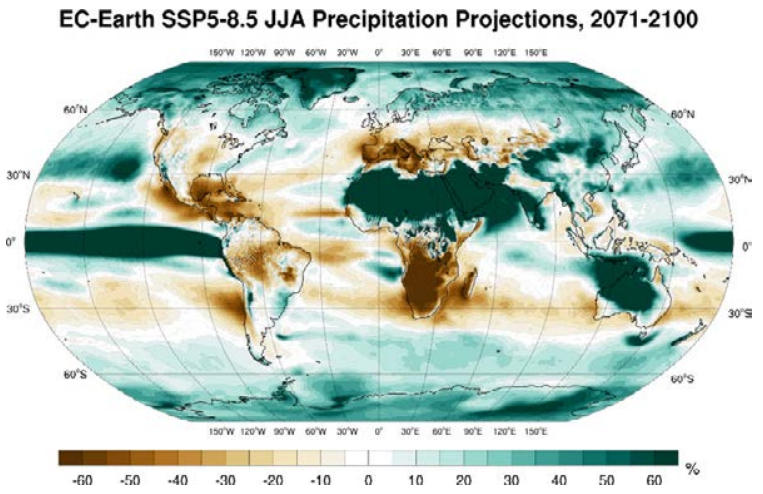

Figure 3.14. EC-Earth JJA precipitation projections (2071-2100 vs 1981-2010, \% change): (a) SSP1-2.6, (b) SSP2-4.5, (c) SSP3-7.0 and (d) SSP5-8.5. In each case, an average is taken of the ensemble members r6i1p1f1, r9i1p1f1, r11i1p1f1, r13i1p1f1 and r15i1p1f1. 


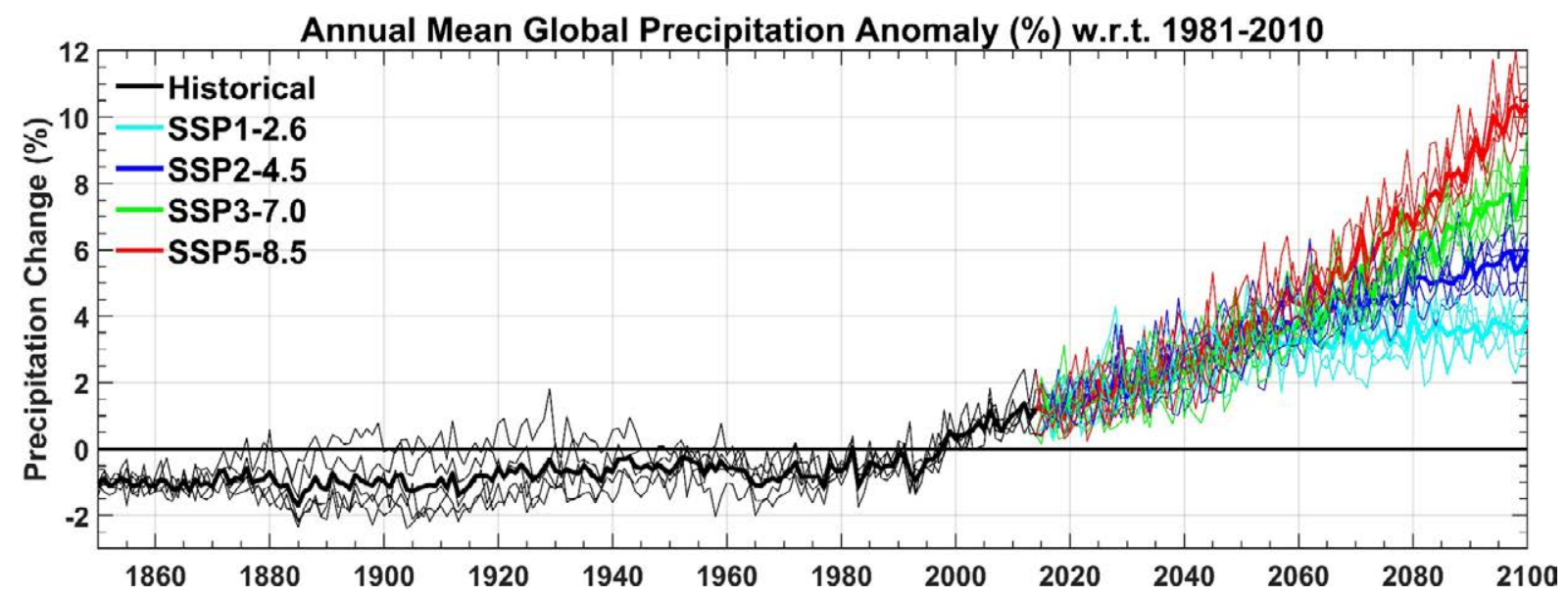

Figure 3.15. Global annual precipitation anomalies (\%) with respect to the 30-year period 1981-2010: EC-Earth ensemble members r6i1p1f1, r9i1p1f1, r11i1p1f1, r13i1p1f1 and r15i1p1f1.The bold lines represent the ensemble means.

(a)

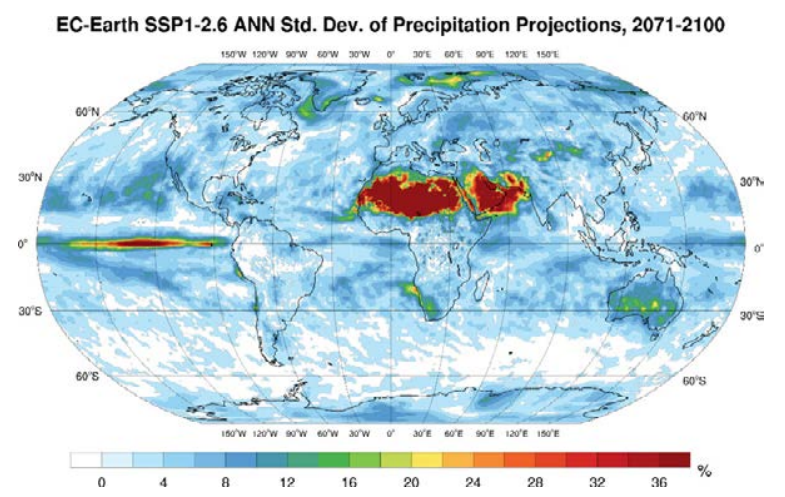

(c)

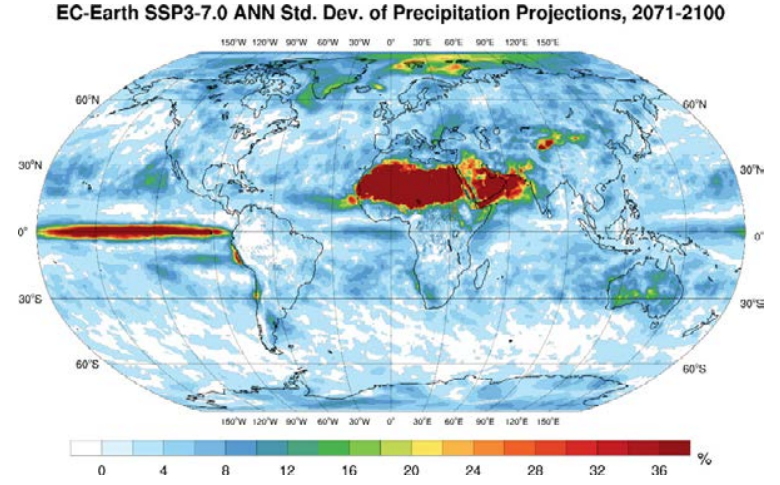

(b)

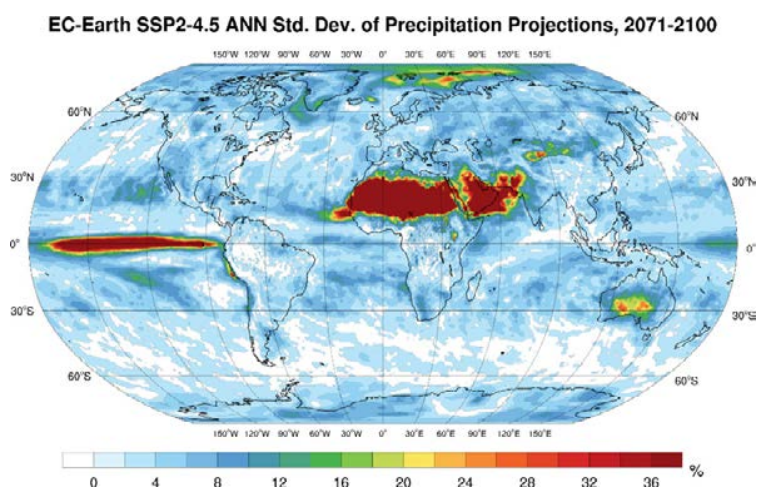

(d)

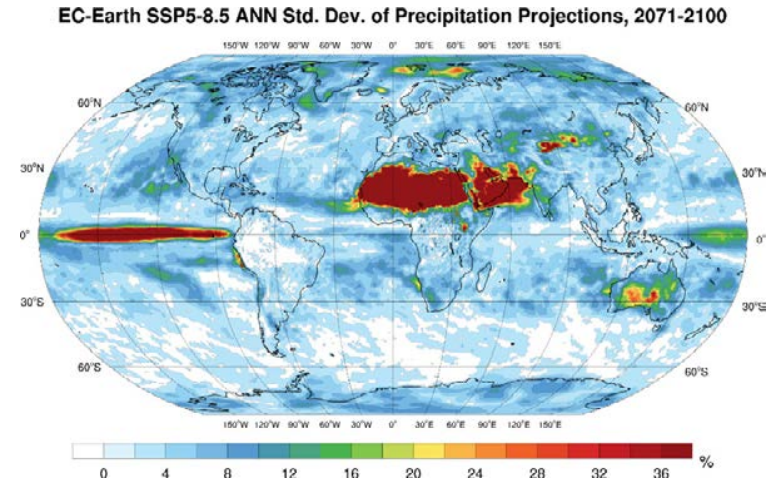

Figure 3.16. Standard deviation of the ensemble of annual precipitation projections (2071-2100):

(a) SSP1-2.6, (b) SSP2-4.5, (c) SSP3-7.0 and (d) SSP5-8.5. 


\subsection{0-m Wind Speed Projections}

In general, mean 10-m wind speeds are projected to decrease slightly (or show no change) over all regions except the Arctic and the Southern Hemisphere oceans surrounding Antarctica. This general trend is evident for annual (Figures 3.17 and 3.18), DJF (Figures 3.19 and 3.20) and JJA (Figures 3.21 and 3.22) projections. The trend is enhanced for the 2071-2100 period and the higher SSPs (e.g. Figures $3.18 \mathrm{c}$ and d). For JJA, small increases in wind speed are evident over extended regions of the Southern Hemisphere oceans (e.g. Figures 3.21 and 3.22).

The mean global annual 10-m wind speed anomalies (relative to 1981-2010) for all five historical simulations (1850-2014) and 20 SSPs (2015-2100) are presented in Figure 3.23. The bold lines represent the ensemble means. All ensemble members show a small steady decrease in 10-m wind speed from around 2010. Although a small divergence between the SSPs is evident from around 2070, the differences are small. By the end of the century, the global mean 10-m wind speed is projected to decrease by approximately $0.05 \mathrm{~m} / \mathrm{s}(1 \%), 0.1 \mathrm{~m} / \mathrm{s}(1.5 \%), 0.15 \mathrm{~m} / \mathrm{s}(2.2 \%)$ and $0.2 \mathrm{~m} / \mathrm{s}(3 \%)$ for SSP1-2.6, SSP2-4.5, SSP3-7.0 and SSP5-8.5, respectively. It should be noted that, although there is a consistent downwards trend in wind speed on a global scale, the numbers are small, ranging from $1 \%$ to $3 \%$. Figure 3.24 shows the standard deviation of each SSP ensemble of climate projections; there is a high level of agreement between ensembles, particularly over land. (a)

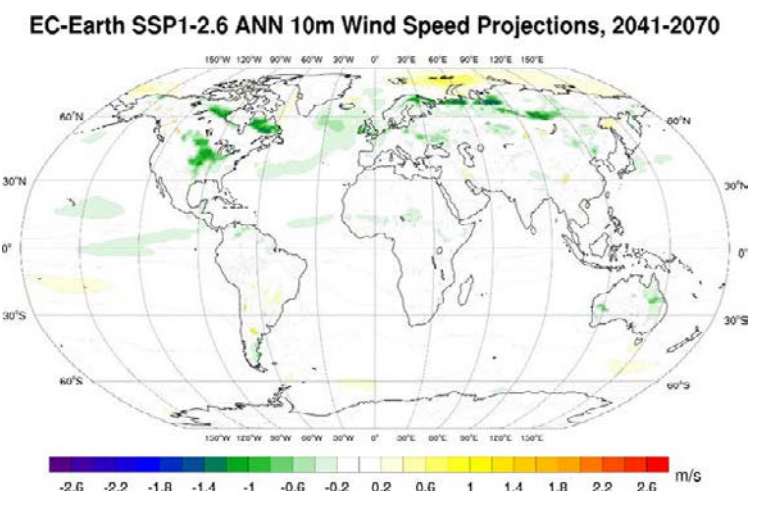

(c)

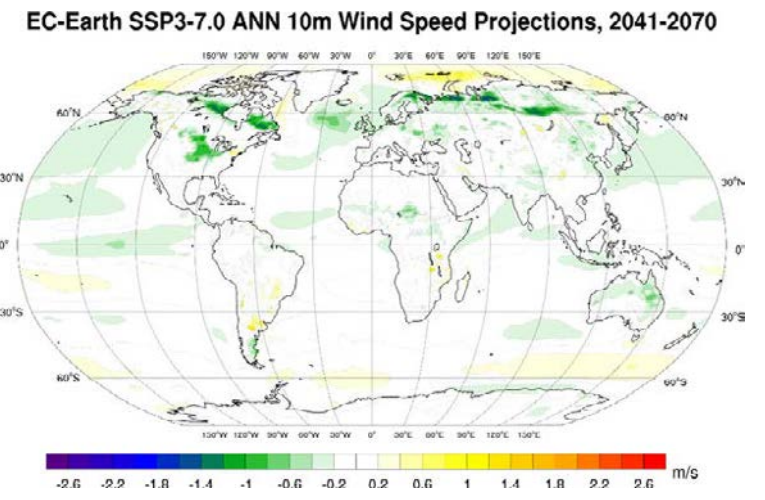

(b)

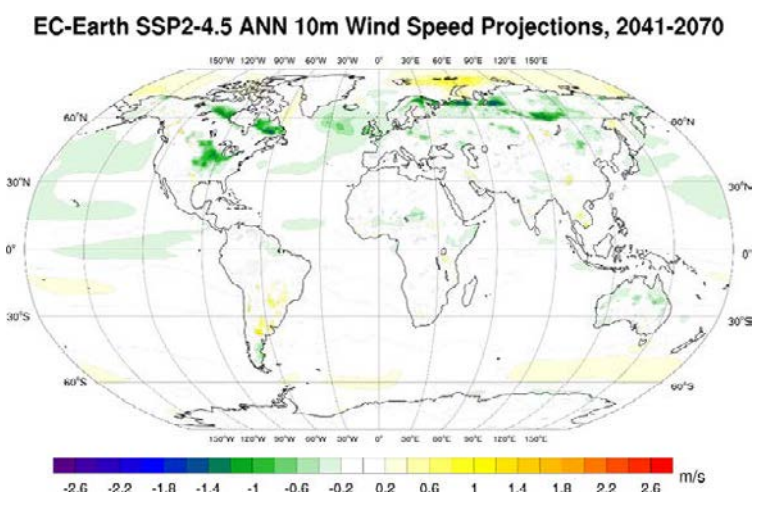

(d)

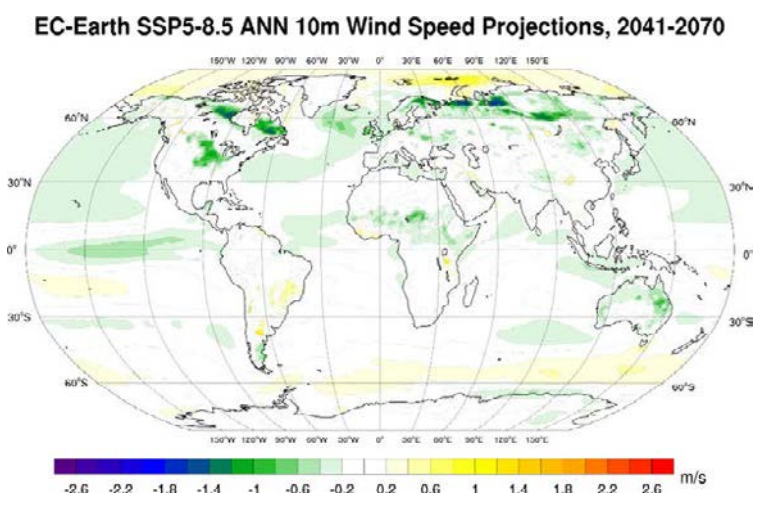

Figure 3.17. EC-Earth annual mean 10-m wind speed projections $(2041-2070$ vs $1981-2010, \mathrm{~m} / \mathrm{s}$ difference): (a) SSP1-2.6, (b) SSP2-4.5, (c) SSP3-7.0 and (d) SSP5-8.5. In each case, an average is taken of the ensemble members r6i1p1f1, r9i1p1f1, r11i1p1f1, r13i1p1f1 and r15i1p1f1. 
(a)

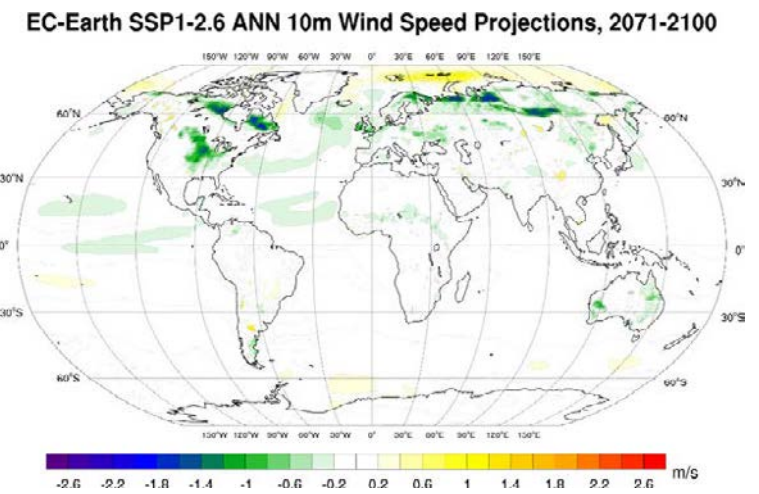

(c)

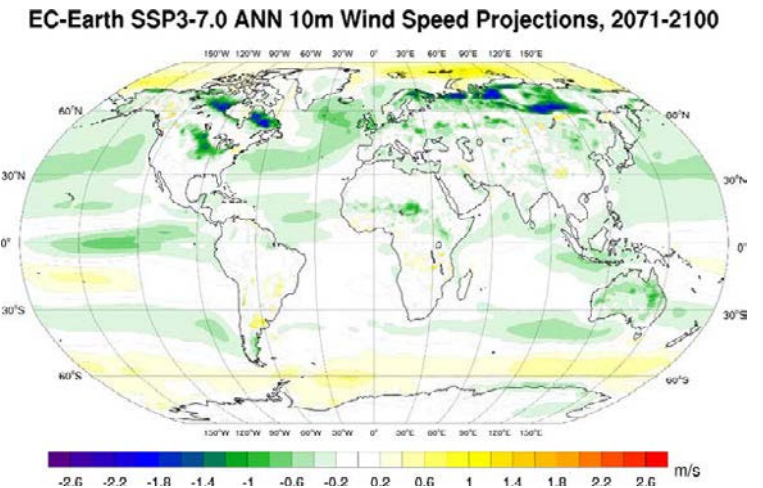

(b)

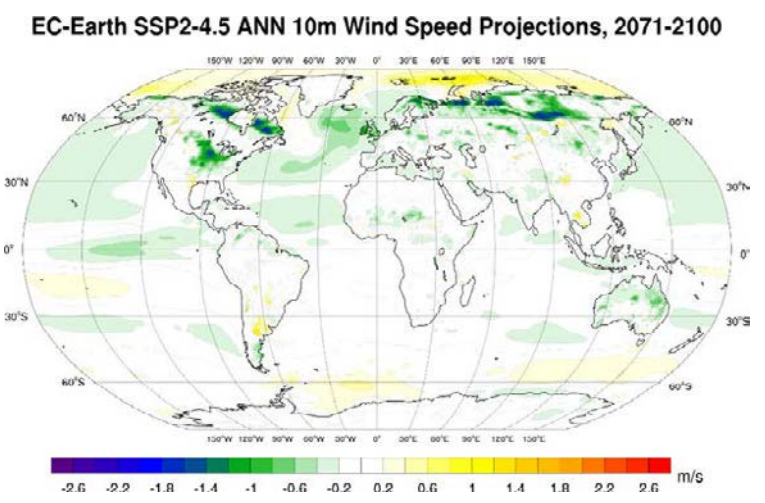

(d)

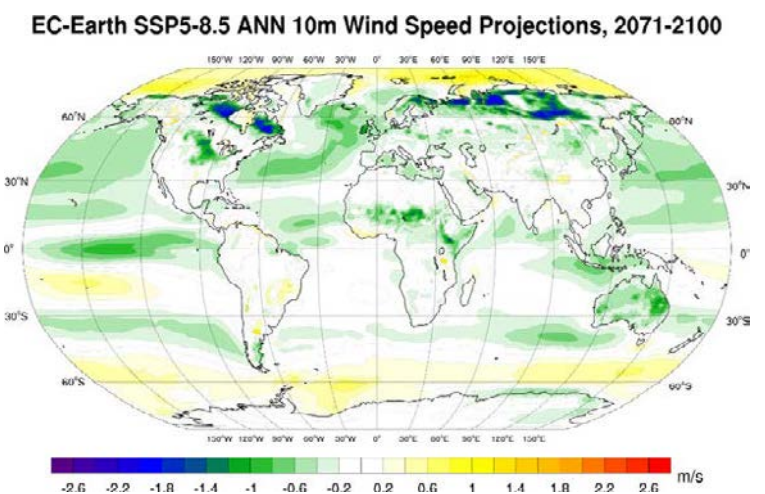

Figure 3.18. EC-Earth annual mean 10-m wind speed projections $(2071-2100$ vs $1981-2010, \mathrm{~m} / \mathrm{s}$ difference): (a) SSP1-2.6, (b) SSP2-4.5, (c) SSP3-7.0 and (d) SSP5-8.5. In each case, an average is taken of the ensemble members r6i1p1f1, r9i1p1f1, r11i1p1f1, r13i1p1f1 and r15i1p1f1.

(a)

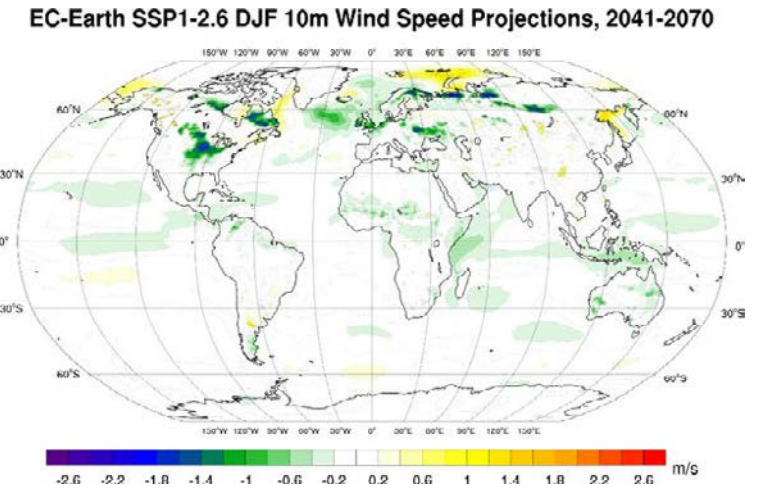

(c)

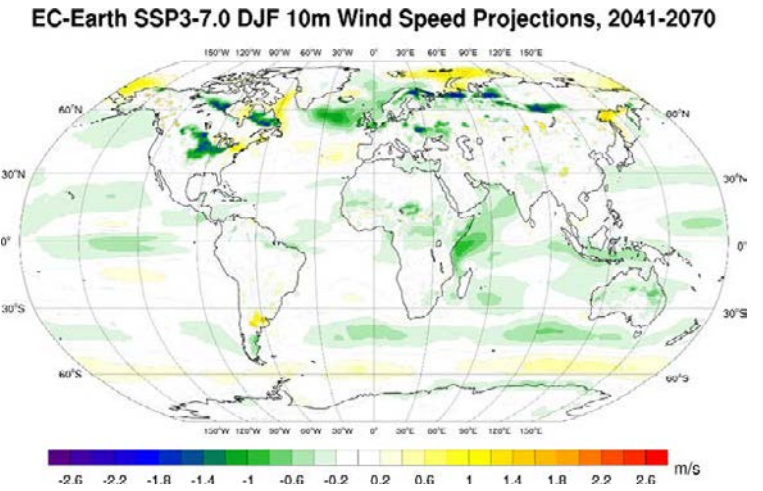

(b)

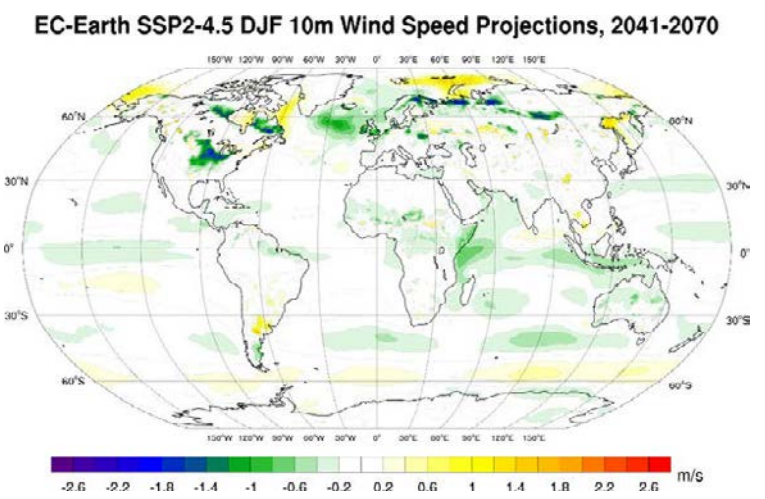

(d)

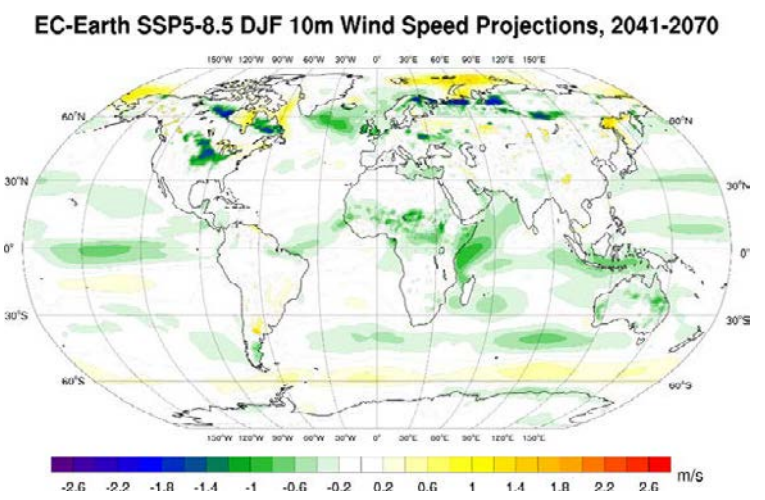

Figure 3.19. EC-Earth DJF mean 10-m wind speed projections (2041-2070 vs 1981-2010, $\mathrm{m} / \mathrm{s}$ difference): (a) SSP1-2.6, (b) SSP2-4.5, (c) SSP3-7.0 and (d) SSP5-8.5. In each case, an average is taken of the ensemble members r6i1p1f1, r9i1p1f1, r11i1p1f1, r13i1p1f1 and r15i1p1f1. 
(a)

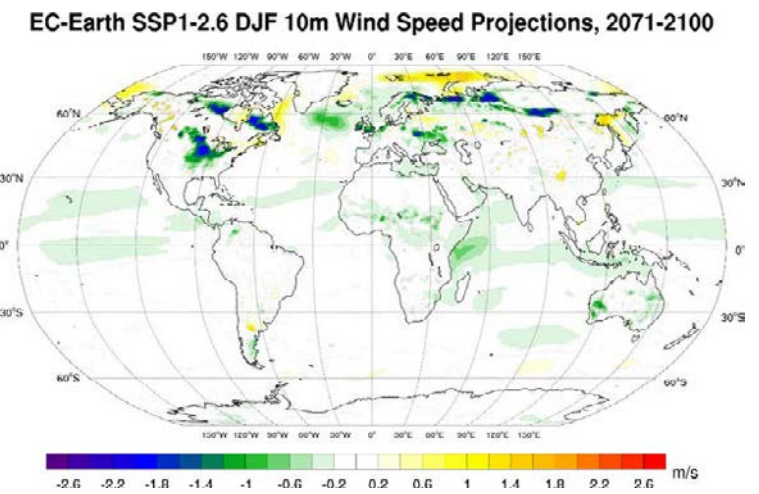

(c)

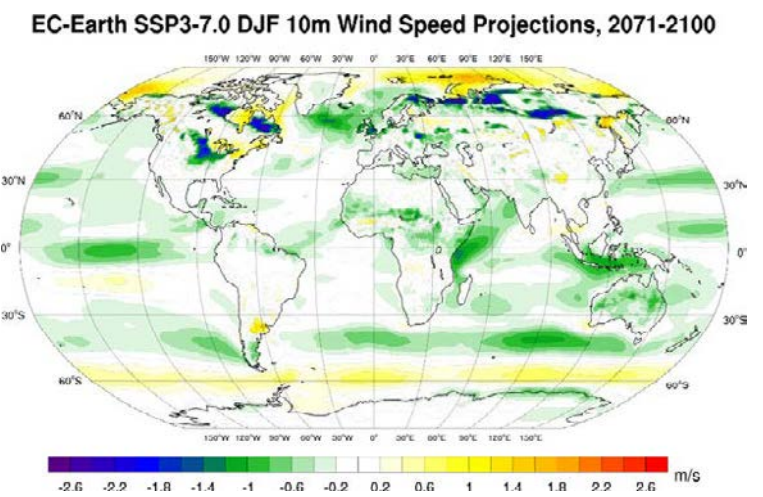

(b)

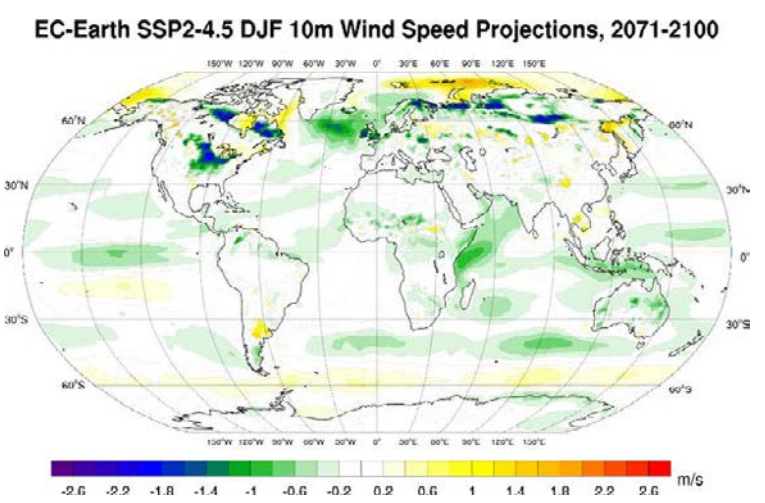

(d)

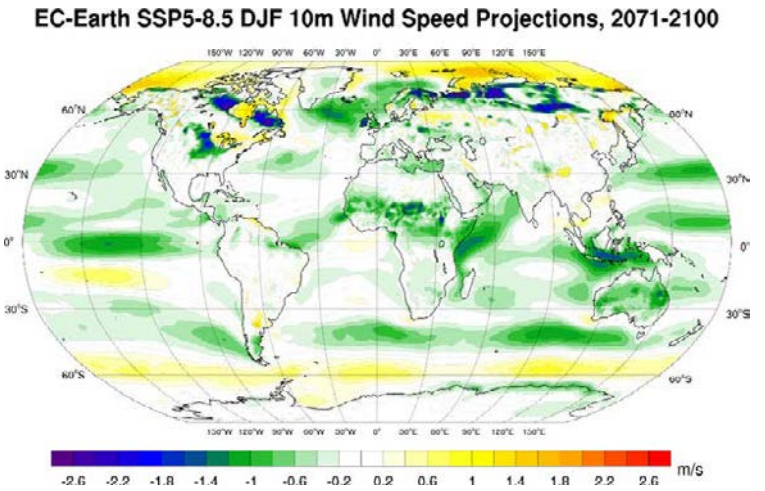

Figure 3.20. EC-Earth DJF mean 10-m wind speed projections (2071-2100 vs 1981-2010, m/s difference): (a) SSP1-2.6, (b) SSP2-4.5, (c) SSP3-7.0 and (d) SSP5-8.5. In each case, an average is taken of the ensemble members r6i1p1f1, r9i1p1f1, r11i1p1f1, r13i1p1f1 and r15i1p1f1.

(a)

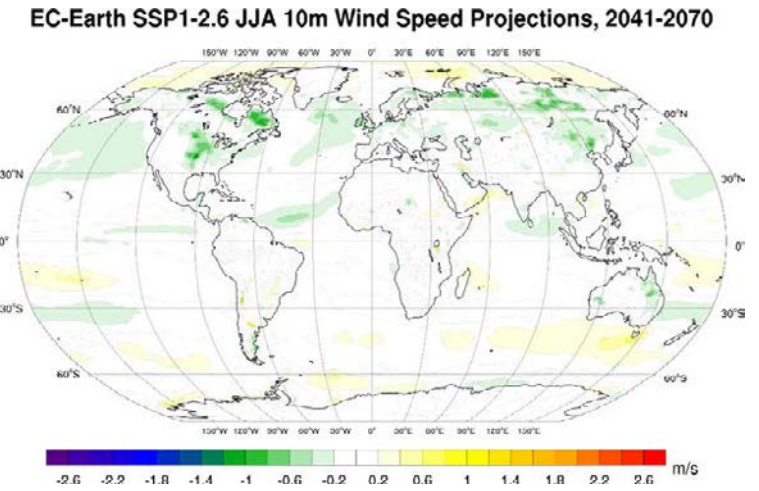

(c)

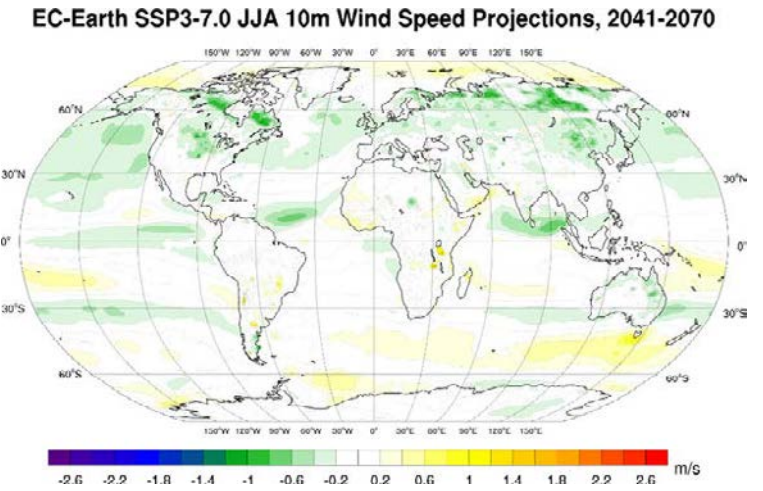

(b)

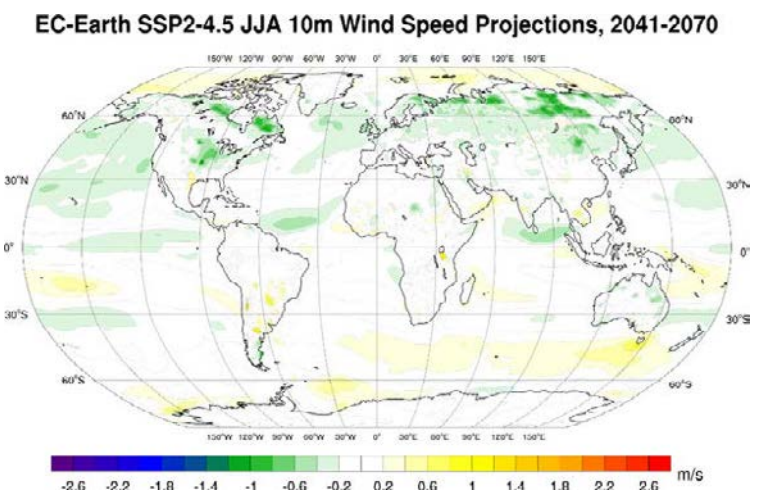

(d)

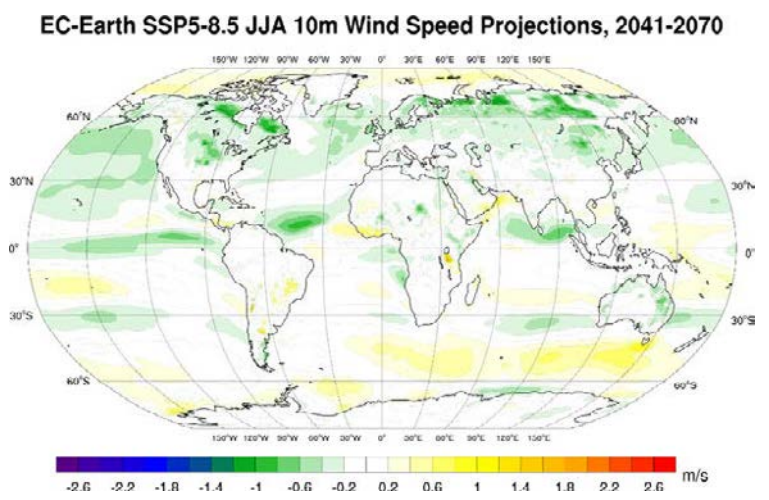

Figure 3.21. EC-Earth JJA mean 10-m wind speed projections (2041-2070 vs 1981-2010, m/s difference): (a) SSP1-2.6, (b) SSP2-4.5, (c) SSP3-7.0 and (d) SSP5-8.5. In each case, an average is taken of the ensemble members r6i1p1f1, r9i1p1f1, r11i1p1f1, r13i1p1f1 and r15i1p1f1. 
(a)

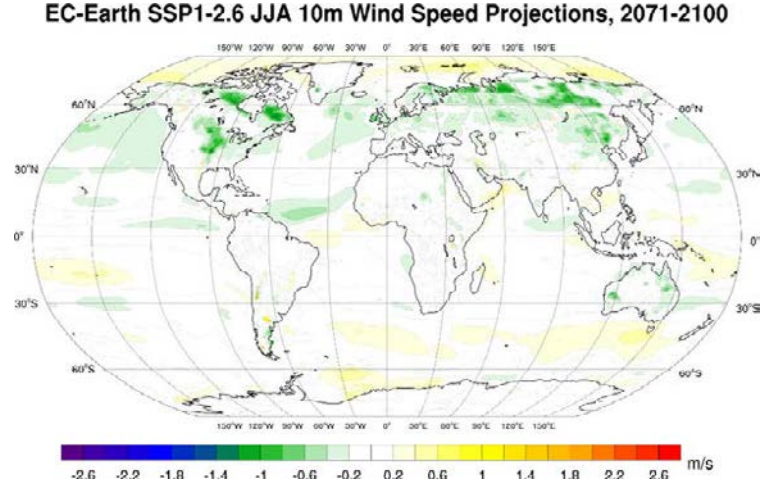

(c)

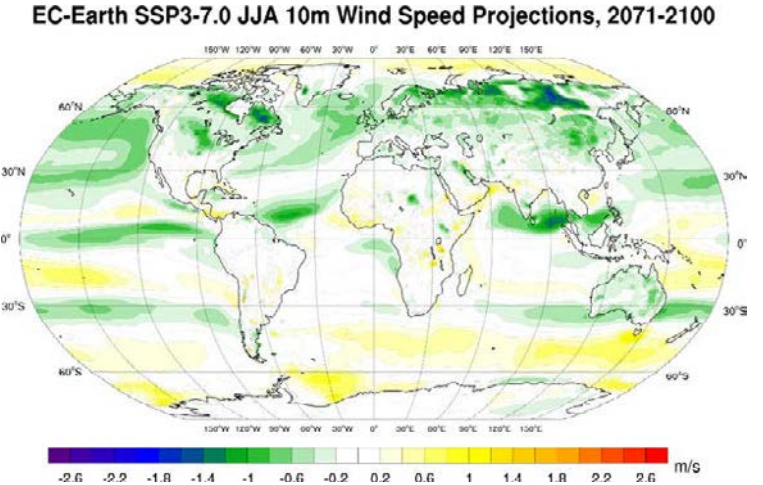

(b)

EC-Earth SSP2-4.5 JJA 10m Wind Speed Projections, 2071-2100

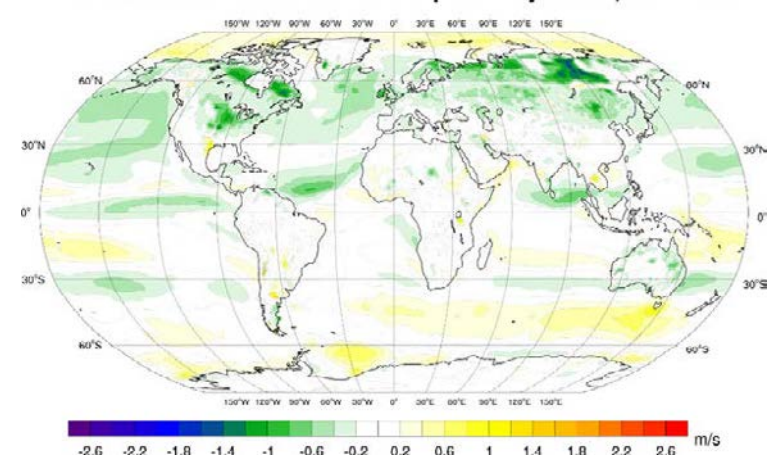

(d)

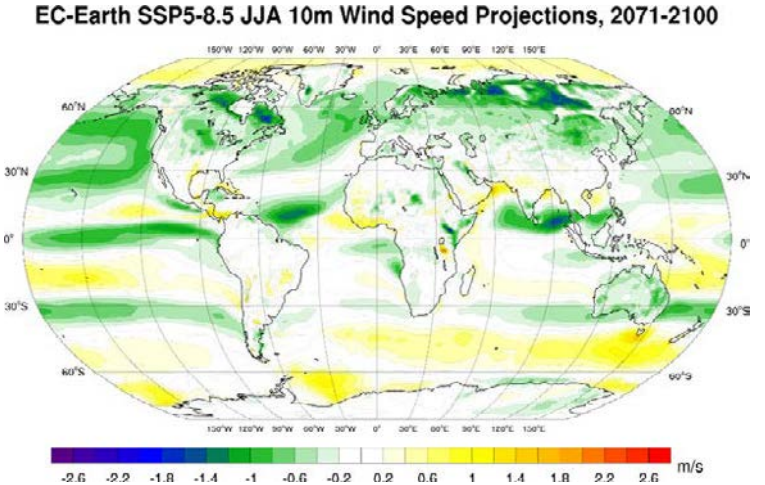

Figure 3.22. EC-Earth JJA mean 10m wind speed projections (2071-2100 vs 1981-2010, m/s difference): (a) SSP1-2.6, (b) SSP2-4.5, (c) SSP3-7.0 and (d) SSP5-8.5. In each case, an average is taken of the ensemble members r6i1p1f1, r9i1p1f1, r11i1p1f1, r13i1p1f1 and r15i1p1f1.

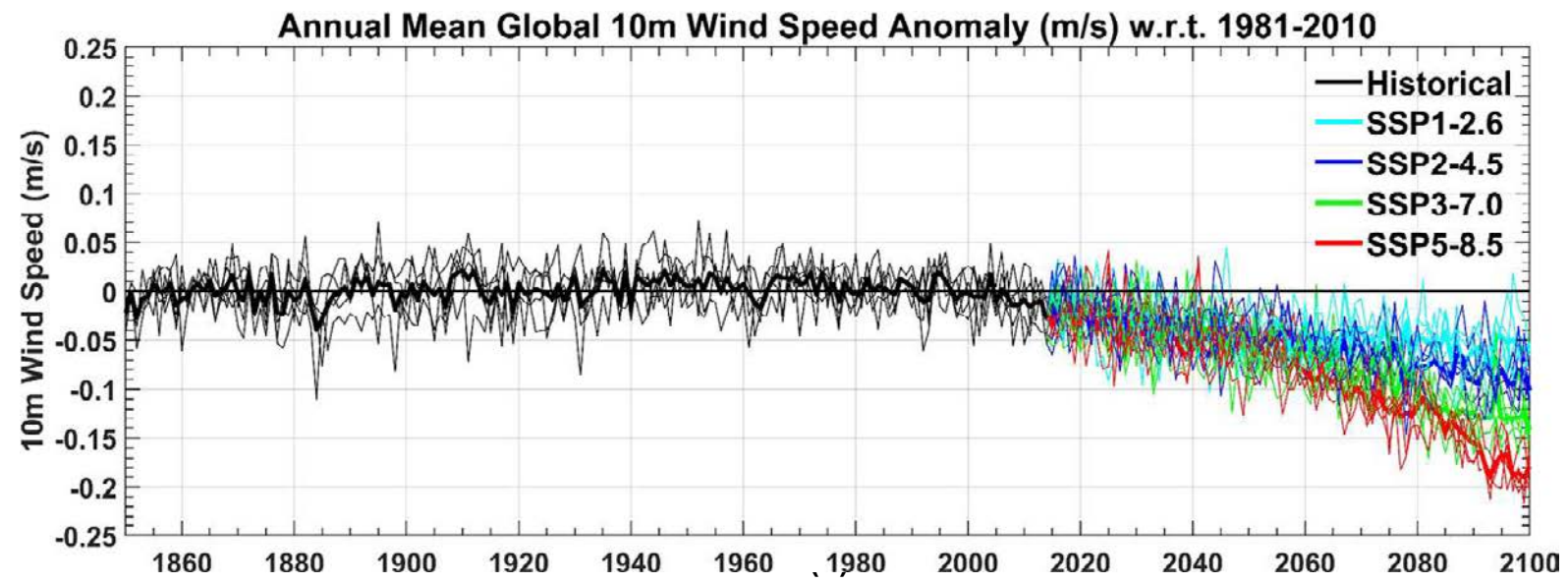

Figure 3.23. Global annual mean 10-m wind speed anomalies with respect to the 30-year period 19812010: EC-Earth ensemble members r6i1p1f1, r9i1p1f1, r11i1p1f1, r13i1p1f1 and r15i1p1f1. The bold lines represent the ensemble means. 
(a)

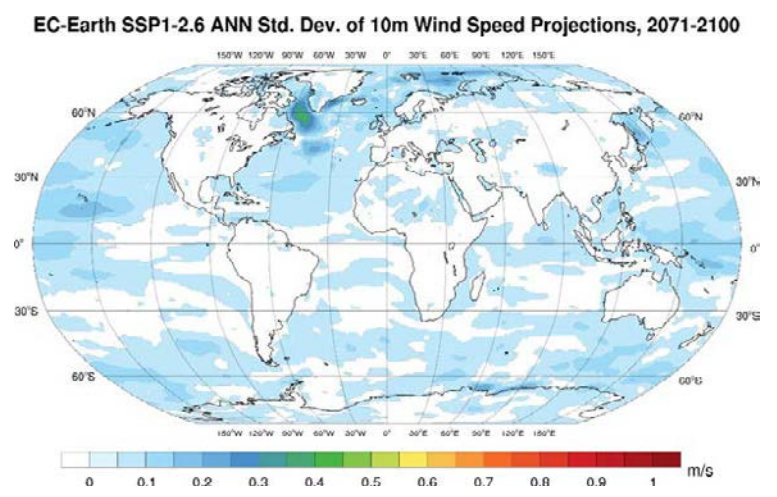

(c)

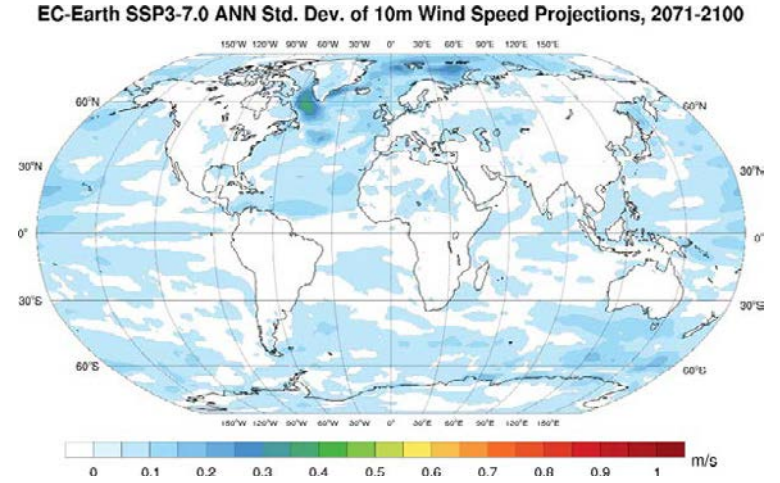

(b)

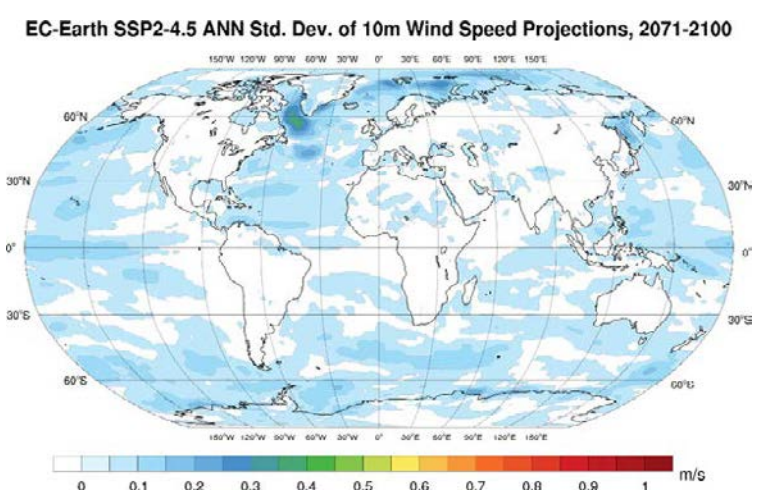

(d)

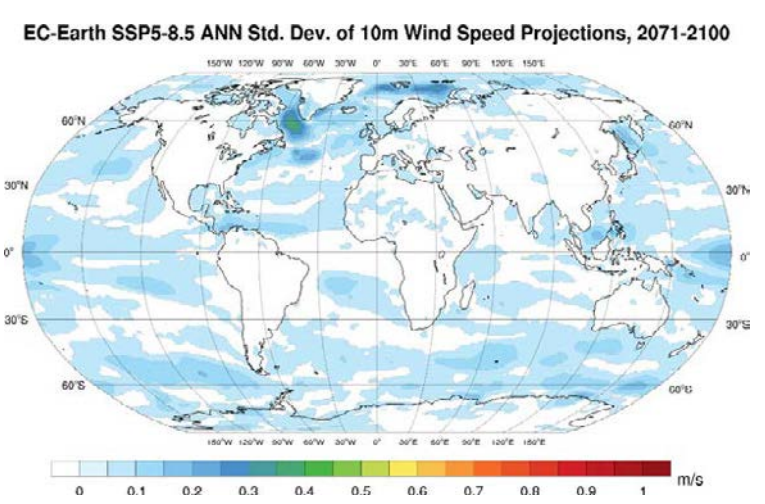

Figure 3.24. Standard deviation of the ensemble of annual mean 10-m wind speed projections (20712100): (a) SSP1-2.6, (b) SSP2-4.5, (c) SSP3-7.0 and (d) SSP5-8.5.

\subsection{Mean Sea Level Pressure Projections}

Figures 3.25 and 3.26 present the spatial distribution of annual projections of MSLP for each of the four SSPs for the 2041-2070 and 2071-2100 periods, respectively. The general trend is for a small increase (or no change) in MSLP in all regions except the Arctic, Antarctic, North African and East European regions. The trend is enhanced for the 2071-2100 period and the higher SSPs (e.g. Figure 3.26c and d).

The MSLP projections for DJF (Figures 3.27 and 3.28) show decreases over Antarctica, North Africa and most of the northern-most latitudes. During DJF, MSLP is projected to decrease (increase) in the North Atlantic Ocean south (north) of Ireland. These results suggest a weakening of both the subpolar low and the subtropical high and a trend towards a more negative North Atlantic Oscillation during future winters. Future work will fully investigate this issue by analysing the full ensemble of CMIP6 simulations. Elsewhere, increases in MSLP are projected over the majority of the oceans. MSLP projections for JJA (Figures 3.29 and 3.30) follow a similar (but enhanced) trend to the annual projections.

The mean global annual MSLP anomalies (relative to 1981-2010) for all five historical simulations (1850-2014) and 20 SSPs (2015-2100) are presented in Figure 3.31. The bold lines represent the ensemble means. All ensemble members show a steady increase in MSLP from around 2015, with a noticeable divergence between the SSPs around 2060. By the year 2100, the global mean MSLP is projected to increase by approximately $0.15,0.3,0.6$ and $0.7 \mathrm{hPa}$ for SSP1-2.6, SSP2-4.5, SSP3-7.0 and SSP5-8.5, respectively. The spread between ensemble members is small. This is reflected in Figure 3.32, which shows the standard deviation of each SSP ensemble of climate projections; there is a high level of agreement between ensemble members for all regions except regions in the high latitudes. 
(a)

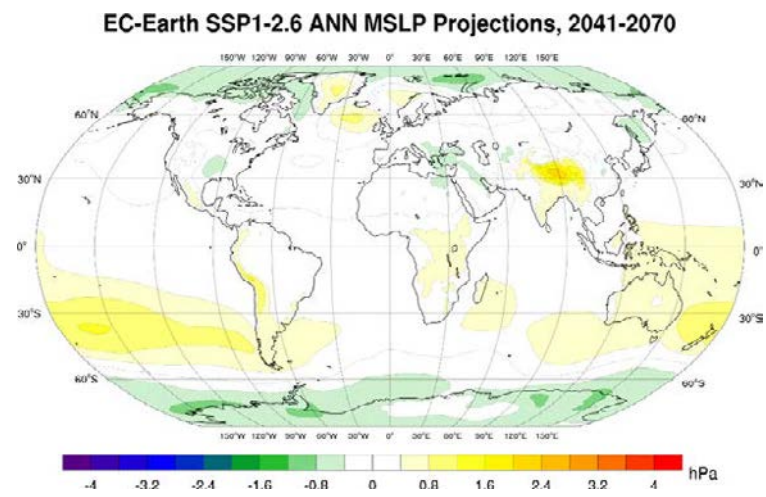

(c)

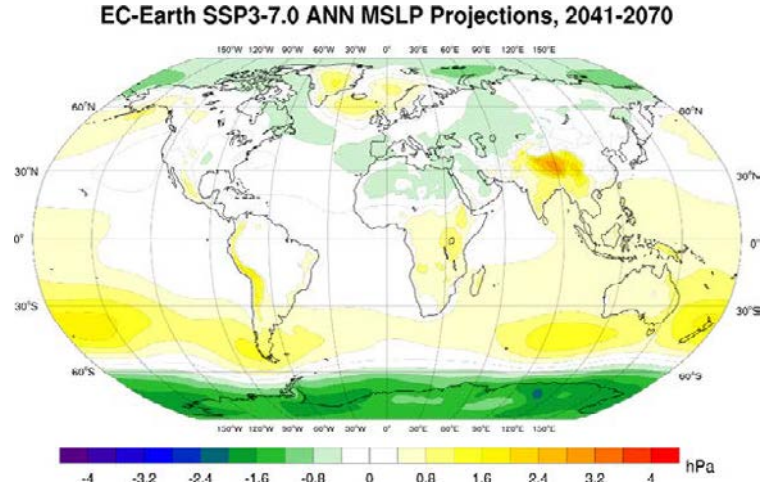

(b)

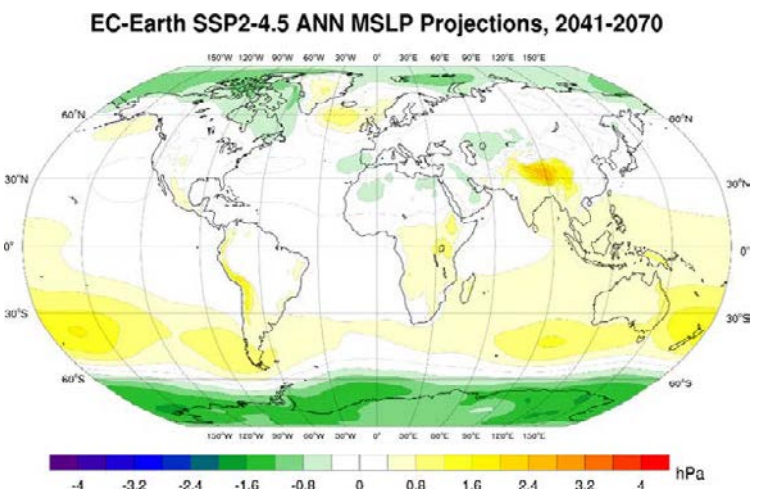

(d)

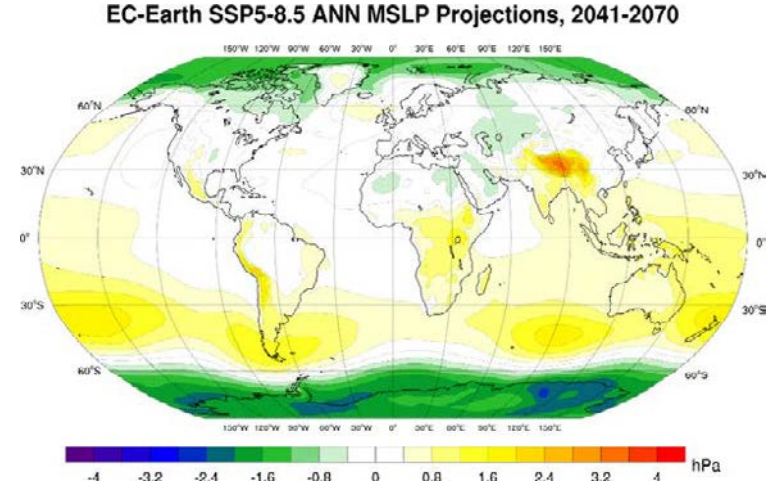

Figure 3.25. EC-Earth annual MSLP projections (2041-2070 vs 1981-2010, hPa difference): (a) SSP1-2.6, (b) SSP2-4.5, (c) SSP3-7.0 and (d) SSP5-8.5. In each case, an average is taken of the ensemble members r6i1p1f1, r9i1p1f1, r11i1p1f1, r13i1p1f1 and r15i1p1f1.

(a)

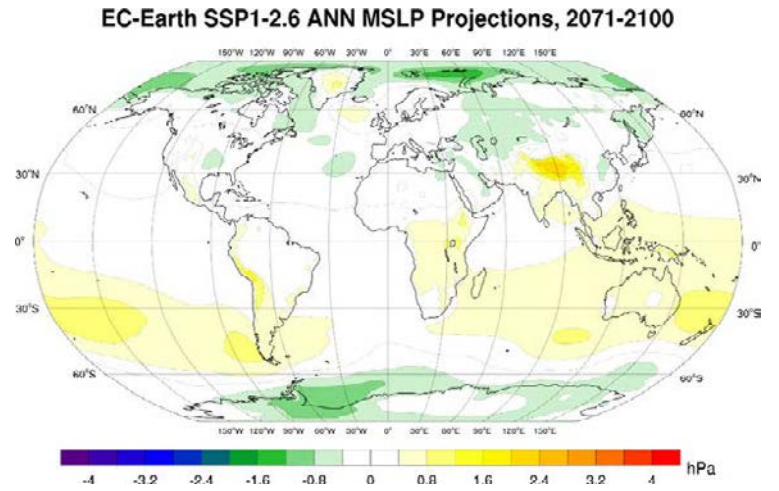

(c)

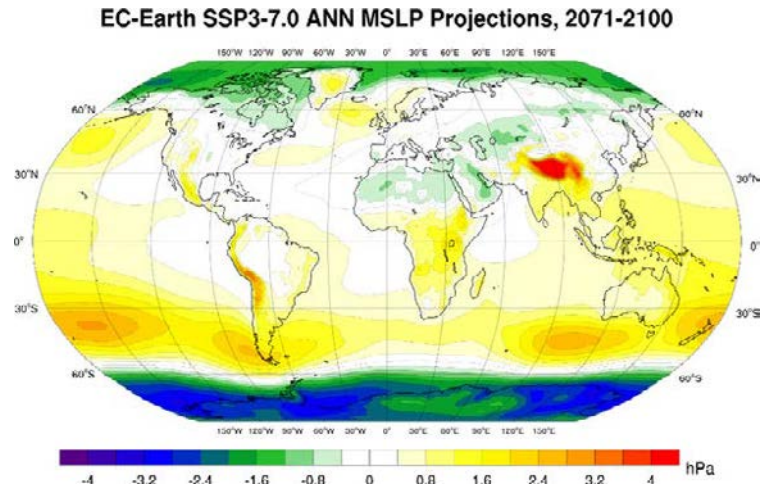

(b)

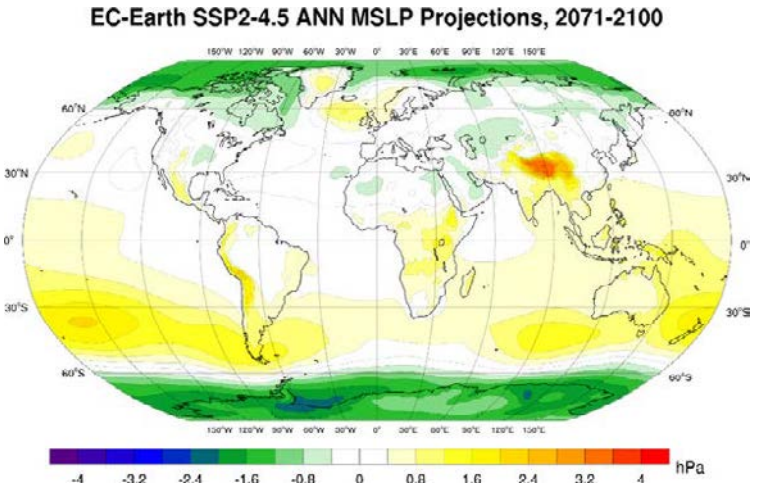

(d)

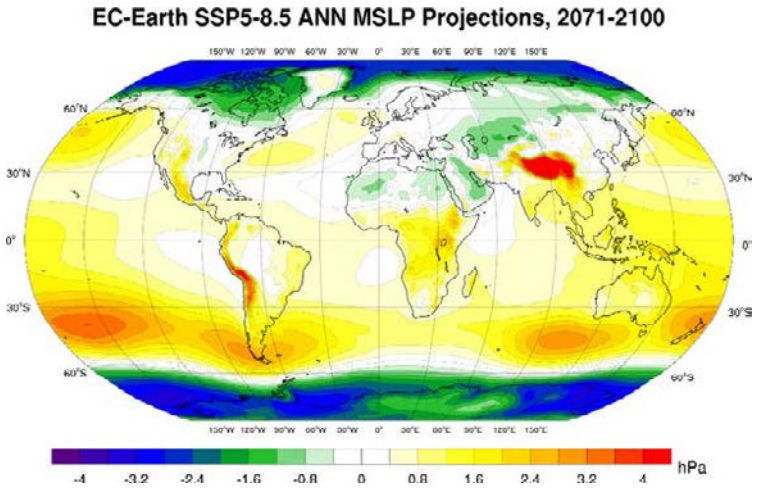

Figure 3.26. EC-Earth annual MSLP projections (2071-2100 vs 1981-2010, hPa difference): (a) SSP1-2.6, (b) SSP2-4.5, (c) SSP3-7.0 and (d) SSP5-8.5. In each case, an average is taken of the ensemble members r6i1p1f1, r9i1p1f1, r11i1p1f1, r13i1p1f1 and r15i1p1f1. 
(a)

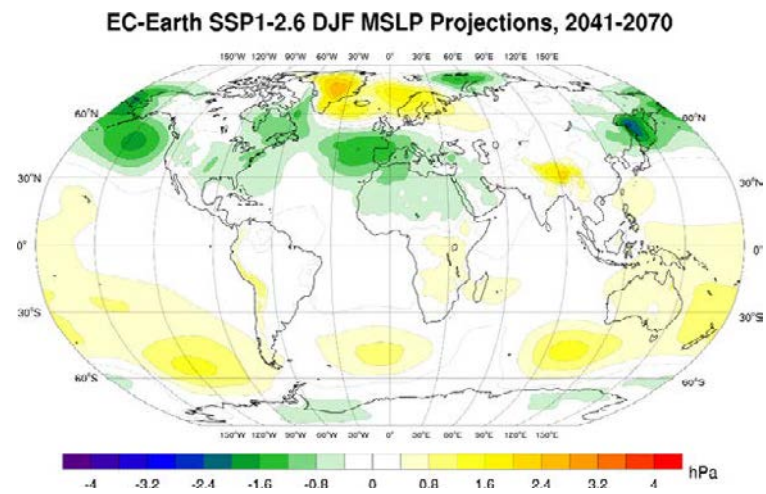

(c)

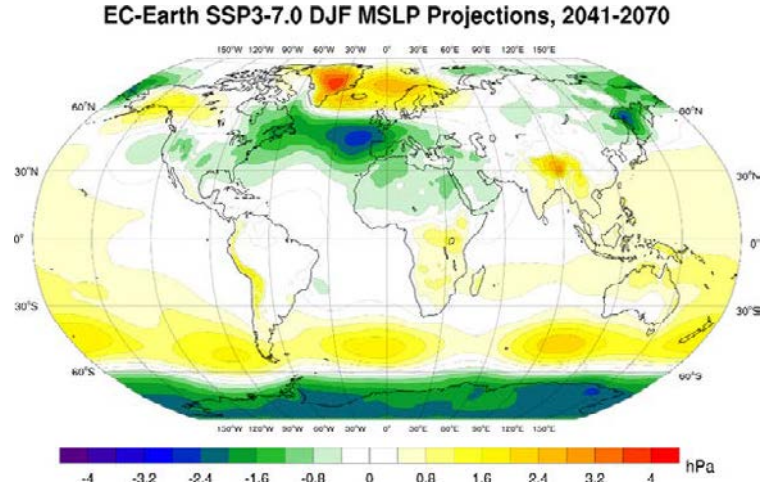

(b)

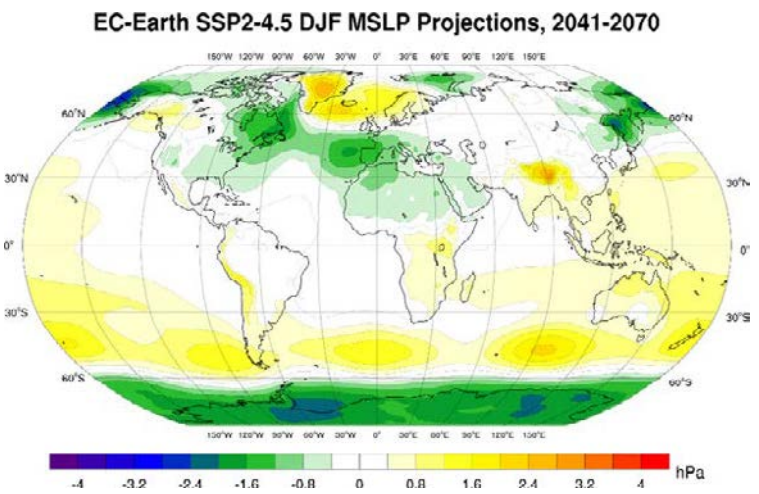

(d)

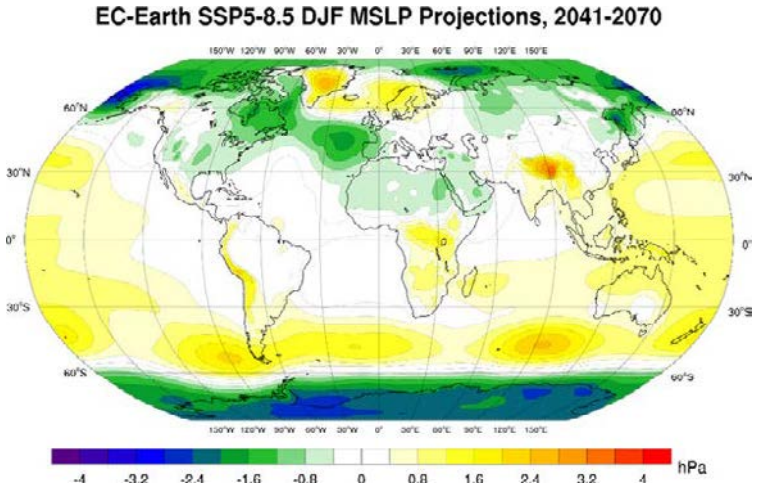

Figure 3.27. EC-Earth DJF MSLP projections (2041-2070 vs 1981-2010, hPa difference): (a) SSP1-2.6, (b) SSP2-4.5, (c) SSP3-7.0 and (d) SSP5-8.5. In each case, an average is taken of the ensemble members r6i1p1f1, r9i1p1f1, r11i1p1f1, r13i1p1f1 and r15i1p1f1.

(a)

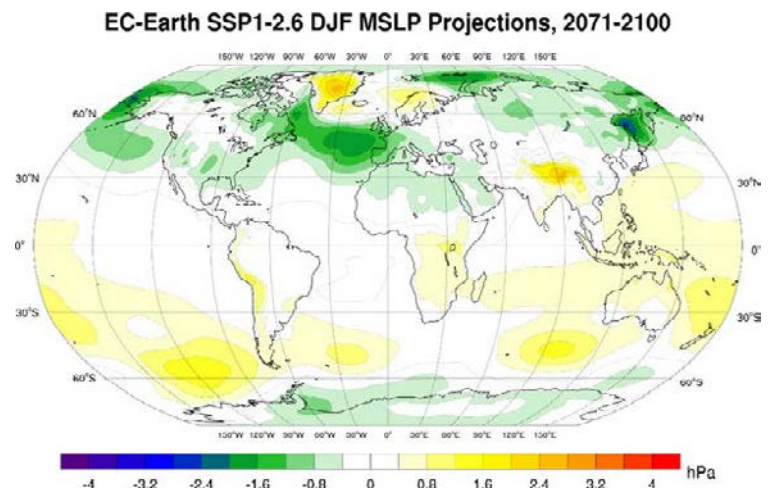

(c)

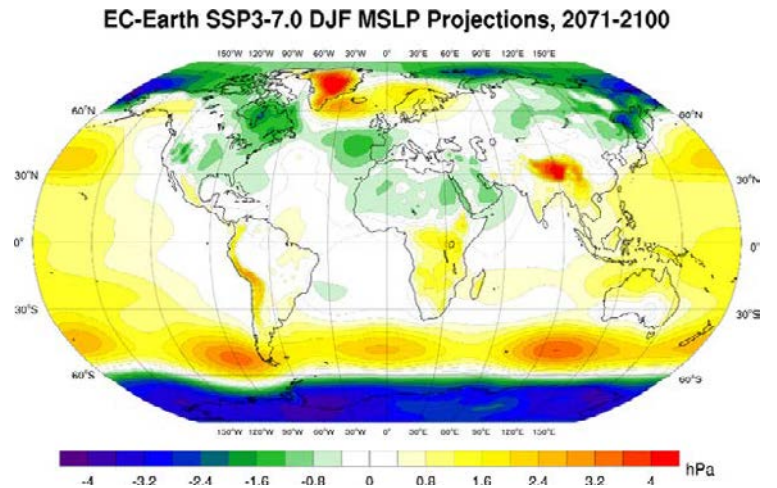

(b)

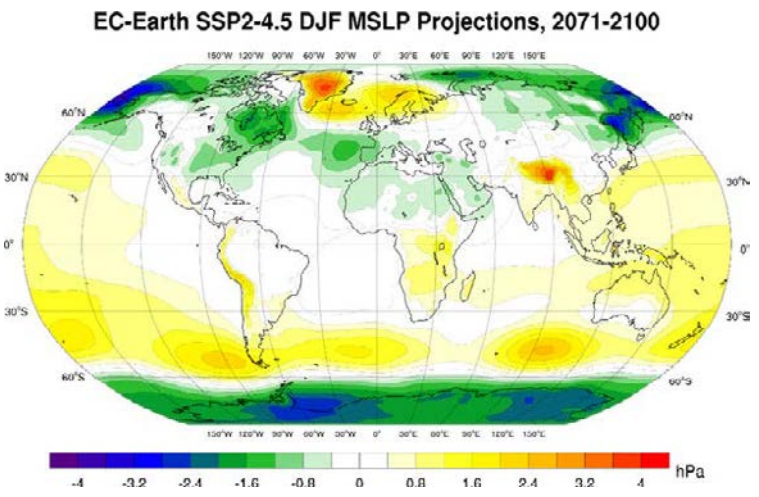

(d)

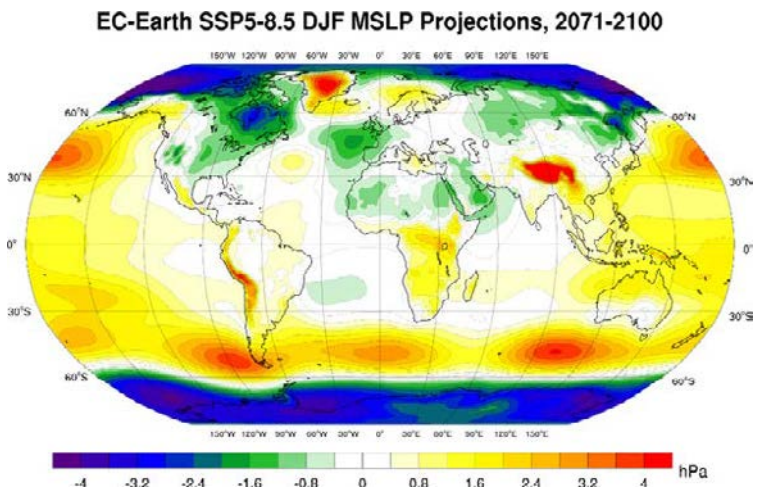

Figure 3.28. EC-Earth DJF MSLP projections (2071-2100 vs 1981-2010, hPa difference): (a) SSP1-2.6, (b) SSP2-4.5, (c) SSP3-7.0 and (d) SSP5-8.5. In each case, an average is taken of the ensemble members r6i1p1f1, r9i1p1f1, r11i1p1f1, r13i1p1f1 and r15i1p1f1. 
(a)

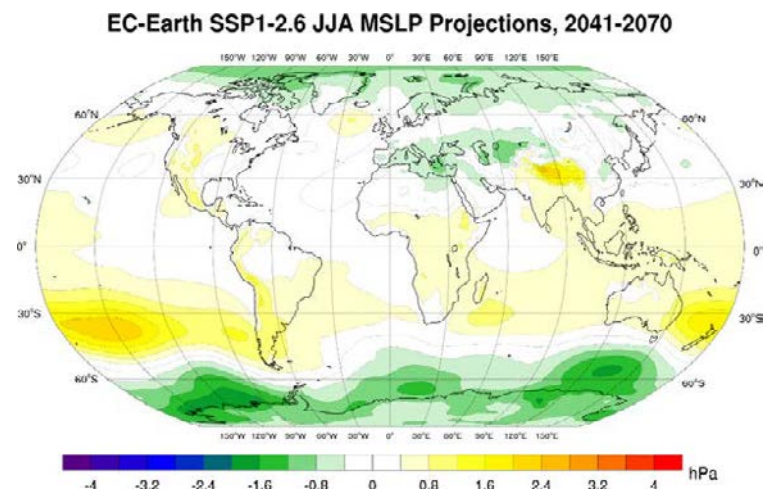

(c)

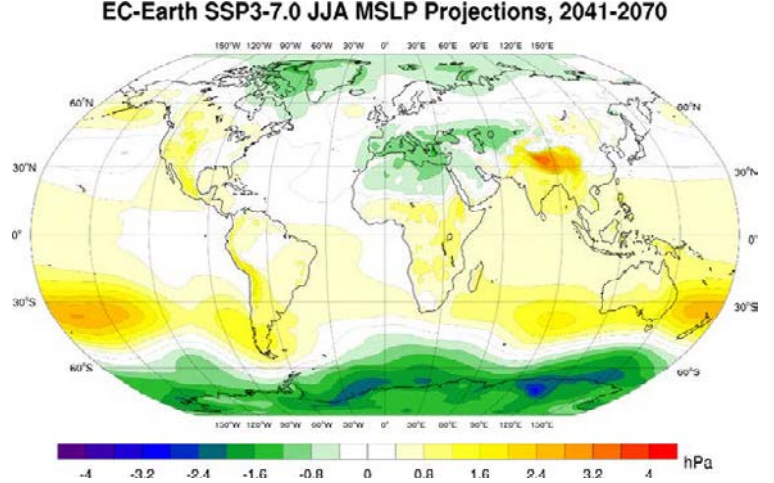

(b)

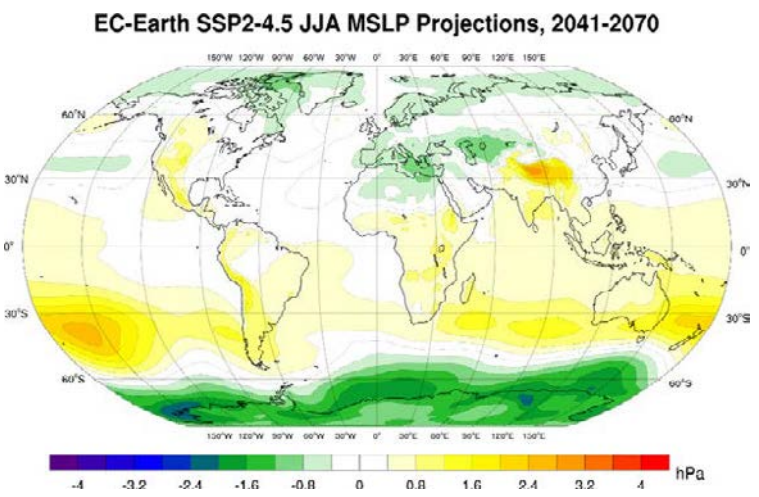

(d)

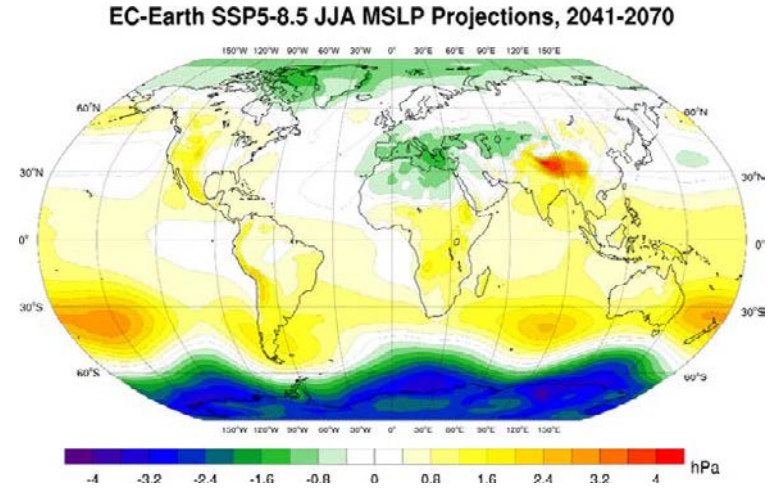

Figure 3.29. EC-Earth JJA MSLP projections (2041-2070 vs 1981-2010, hPa difference): (a) SSP1-2.6, (b) SSP2-4.5, (c) SSP3-7.0 and (d) SSP5-8.5. In each case, an average is taken of the ensemble members r6i1p1f1, r9i1p1f1, r11i1p1f1, r13i1p1f1 and r15i1p1f1.

(a)

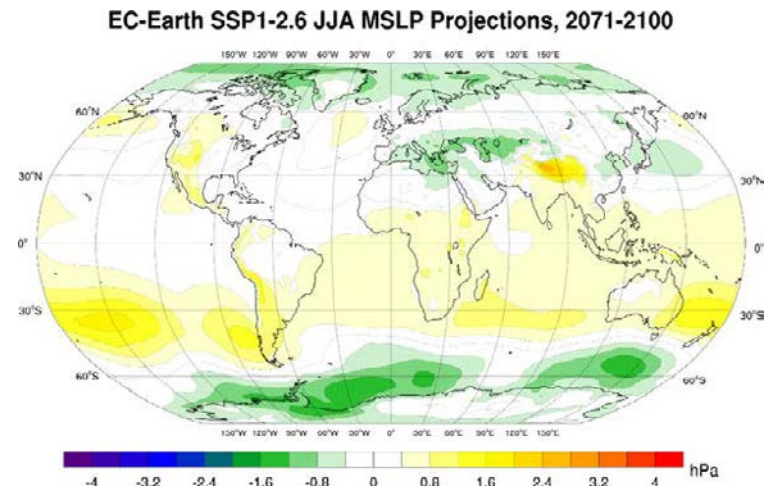

(c)

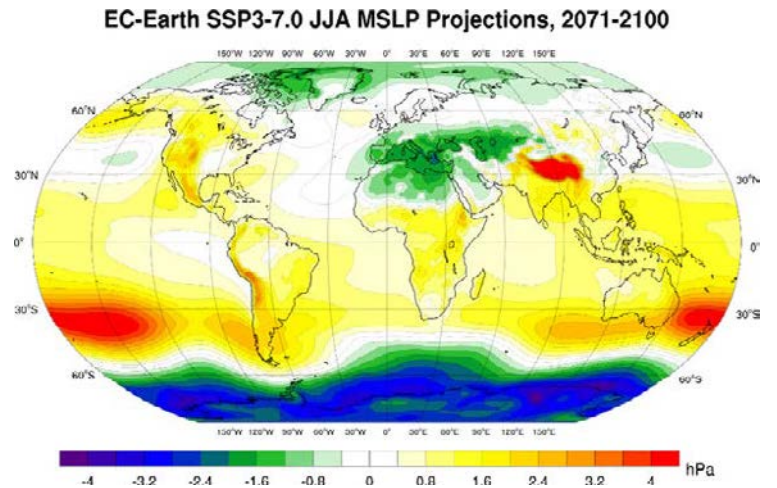

(b)

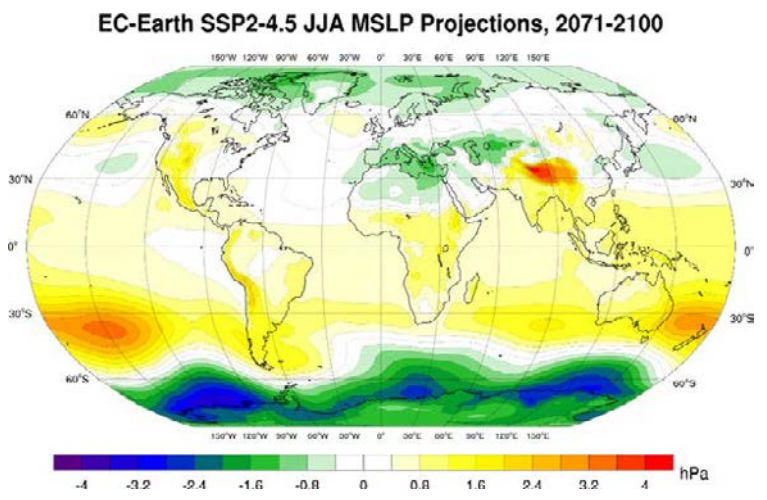

(d)

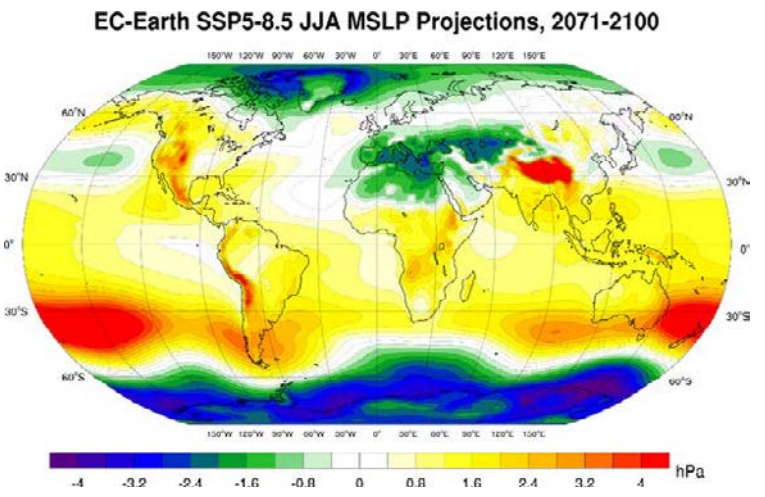

Figure 3.30. EC-Earth JJA MSLP projections (2071-2100 vs 1981-2010, hPa difference): (a) SSP1-2.6, (b) SSP2-4.5, (c) SSP3-7.0 and (d) SSP5-8.5. In each case, an average is taken of the ensemble members r6i1p1f1, r9i1p1f1, r11i1p1f1, r13i1p1f1 and r15i1p1f1. 


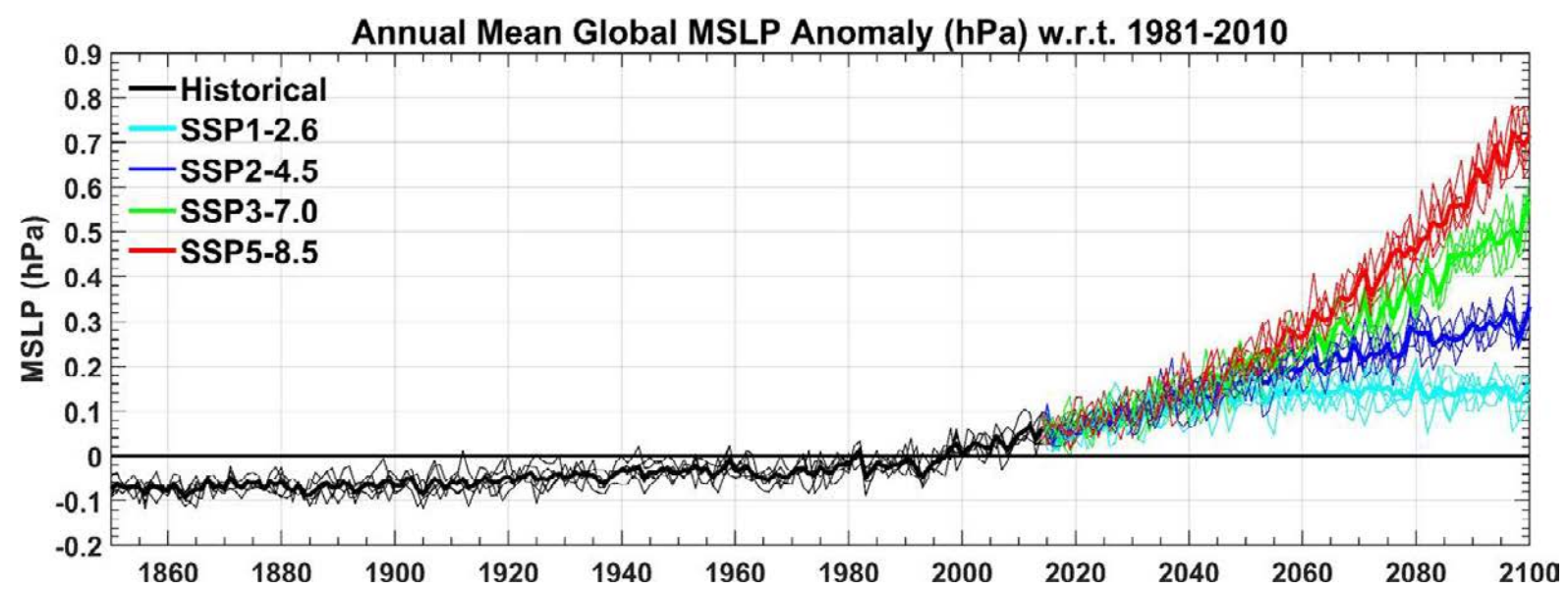

Figure 3.31. Global annual MSLP anomalies with respect to the 30-year period 1981-2010: EC-Earth ensemble members r6i1p1f1, r9i1p1f1, r11i1p1f1, r13i1p1f1 and r15i1p1f1.The bold lines represent the ensemble means.

(a)

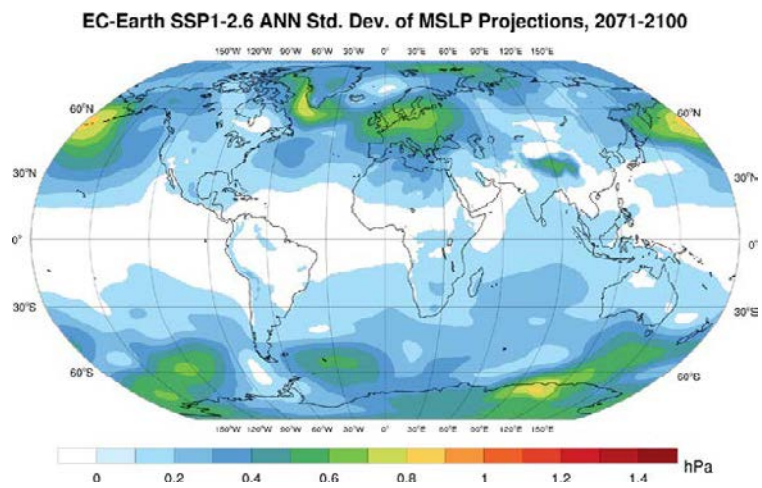

(c)

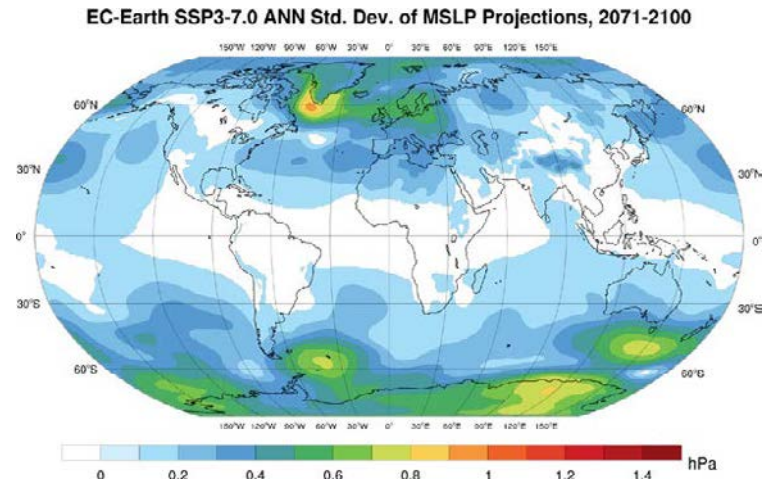

(b)

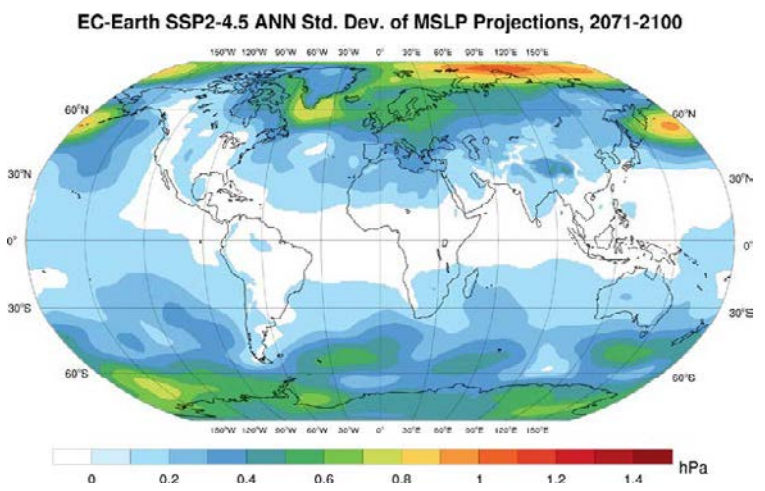

(d)

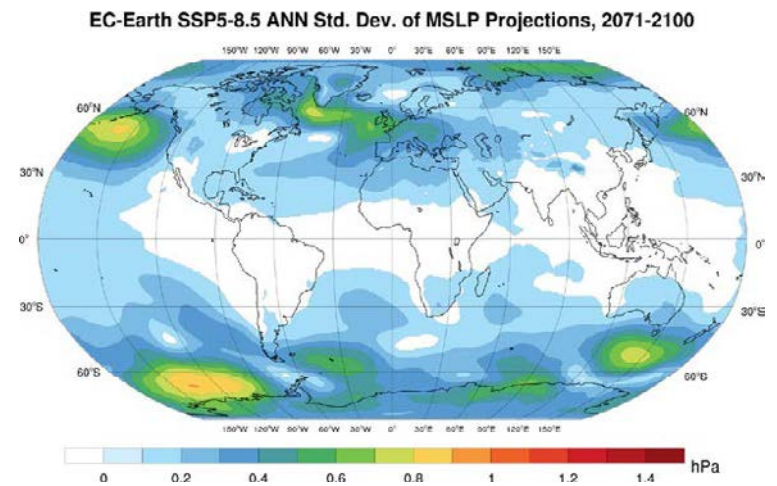

Figure 3.32. Standard deviation of the ensemble of annual MSLP projections (2071-2100): (a) SSP1-2.6, (b) SSP2-4.5, (c) SSP3-7.0 and (d) SSP5-8.5.

\subsection{Total Cloud Cover Projections}

In general, mean total cloud cover is projected to decrease slightly (or exhibit no change) over all regions except Central Africa, the Arabian Peninsula, India, the eastern equatorial Pacific and the equatorial Atlantic regions, where small increases are projected. This general trend is evident for annual (Figures 3.33 and 3.34) and DJF (Figures 3.35 and 3.36) projections. For DJF, a general small increase is also noted over Eurasia (e.g. Figure 3.36d). For JJA, the trend is similar except that cloud cover is projected to decrease over all of Eurasia and larger decreases are projected over the North Atlantic (Figures 3.37 and 3.38). The trends are enhanced for the 2071-2100 period and the higher SSPs (e.g. Figure 3.38c and d). 
(a)

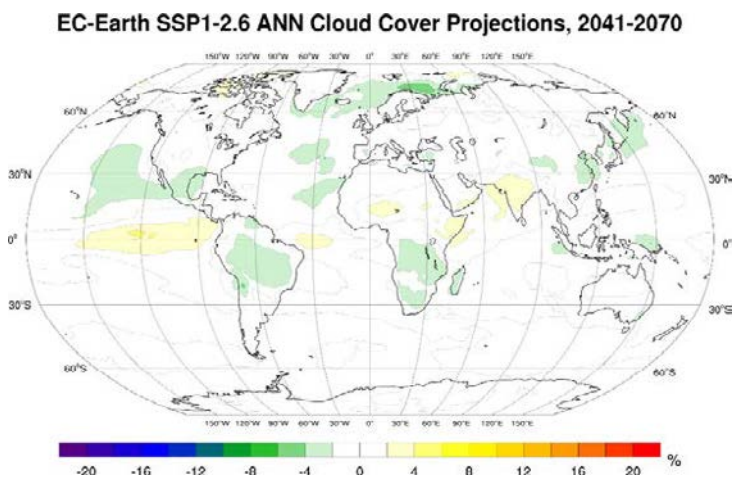

(c)

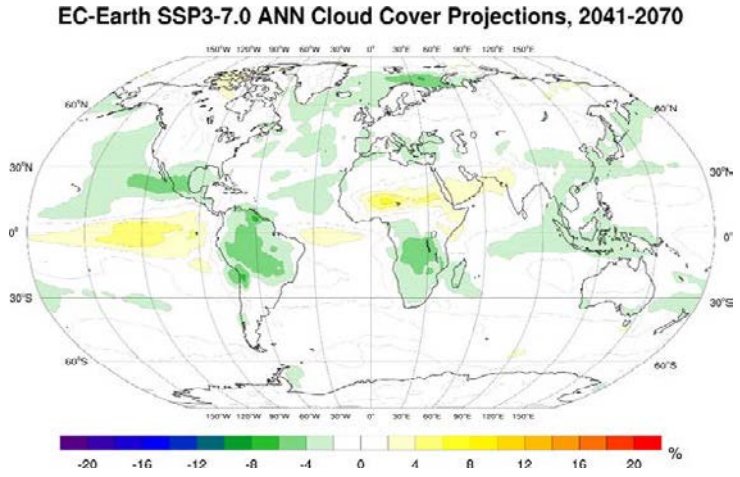

(b)

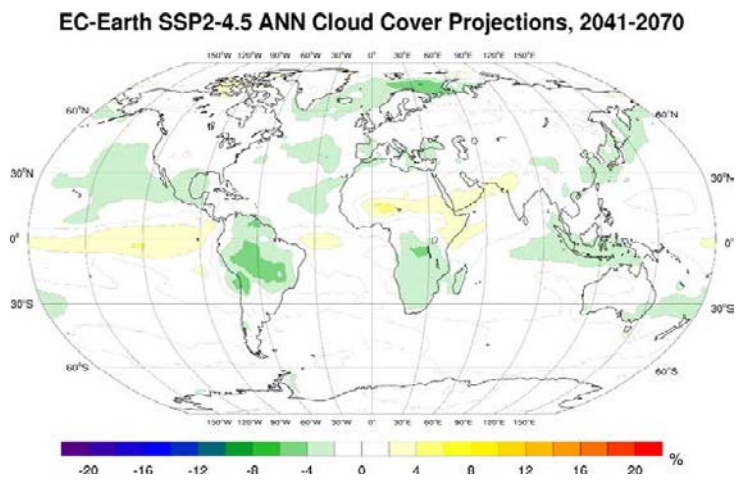

(d)

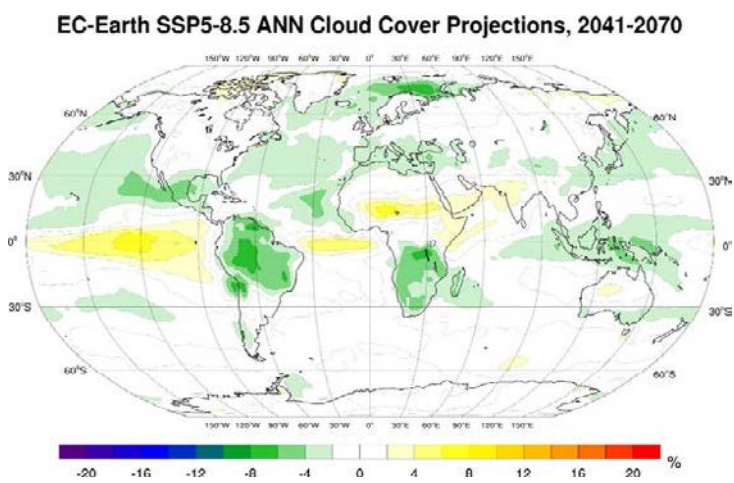

Figure 3.33. EC-Earth annual total cloud cover projections (2041-2070 vs $1981-2010$, \% difference): (a) SSP1-2.6, (b) SSP2-4.5, (c) SSP3-7.0 and (d) SSP5-8.5. In each case, an average is taken of the ensemble members r6i1p1f1, r9i1p1f1, r11i1p1f1, r13i1p1f1 and r15i1p1f1. The anomalies (\%) are calculated as "future (\%) minus past (\%)" as opposed to a percentage change.

(a)

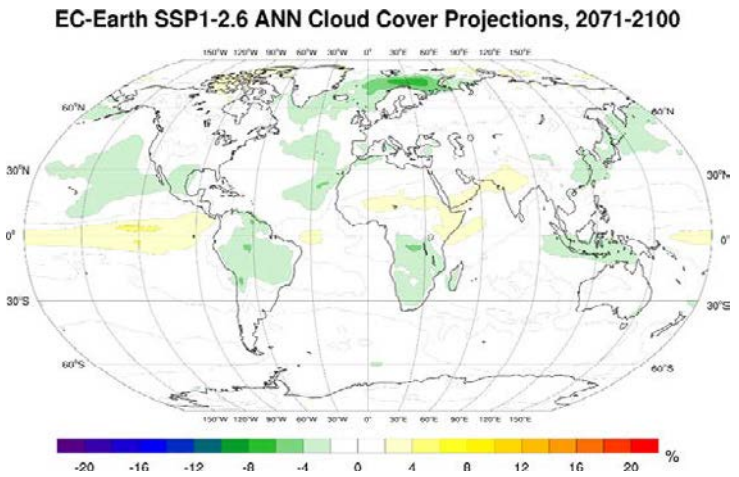

(c)

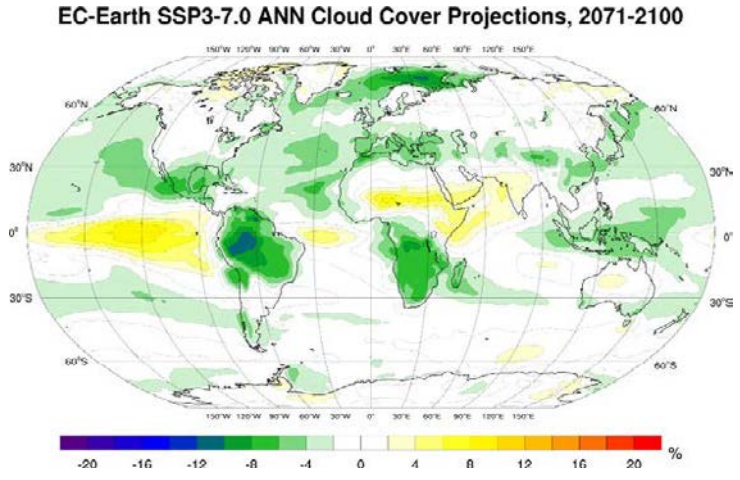

(b)

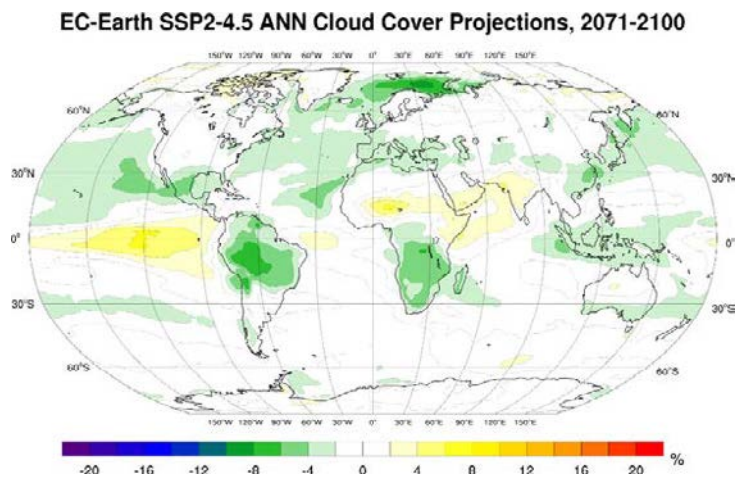

(d)

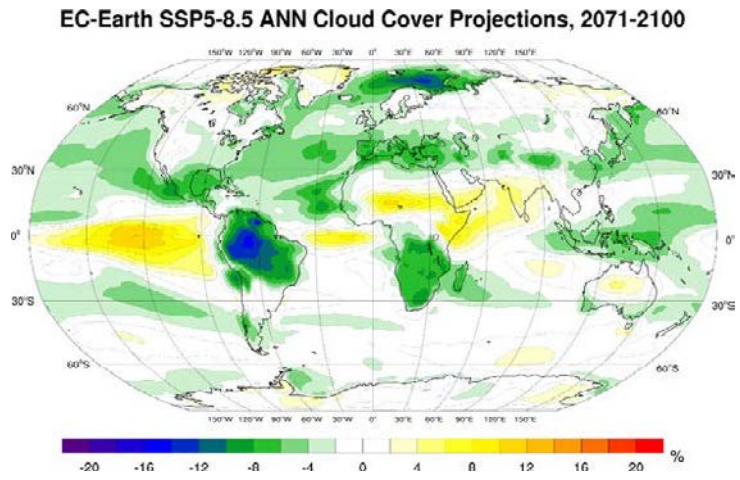

Figure 3.34. EC-Earth annual total cloud cover projections (2071-2100 vs 1981-2010, \% difference): (a) SSP1-2.6, (b) SSP2-4.5, (c) SSP3-7.0 and (d) SSP5-8.5. In each case, an average is taken of the ensemble members r6i1p1f1, r9i1p1f1, r11i1p1f1, r13i1p1f1 and r15i1p1f1. The anomalies (\%) are calculated as "future (\%) minus past (\%)" as opposed to a percentage change. 
(a)

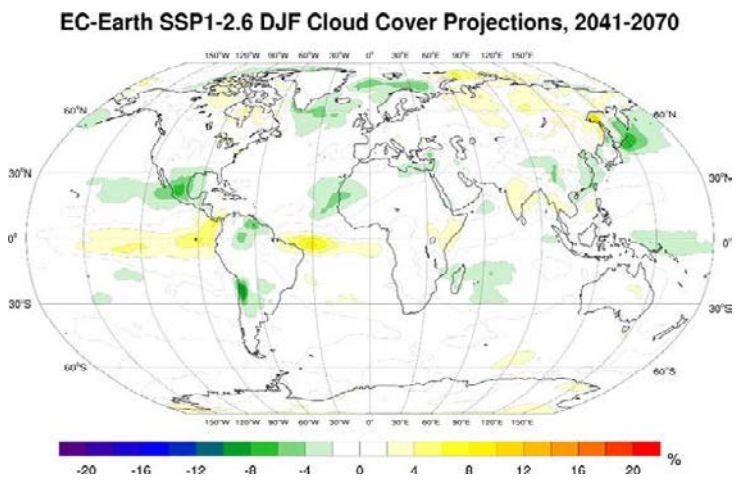

(c)

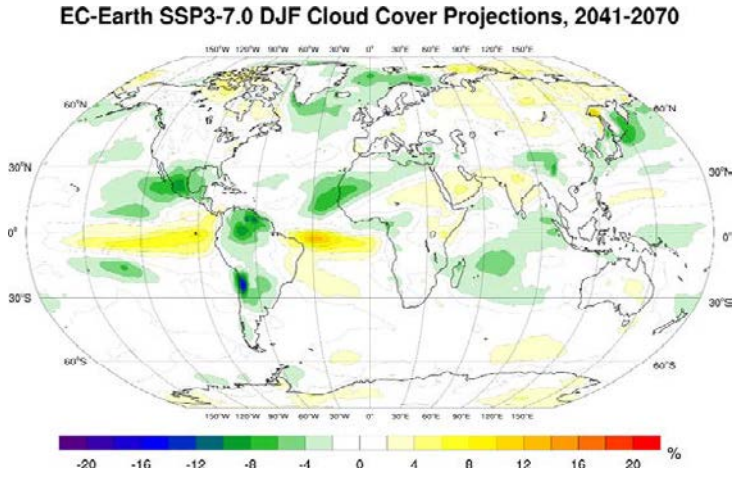

(b)

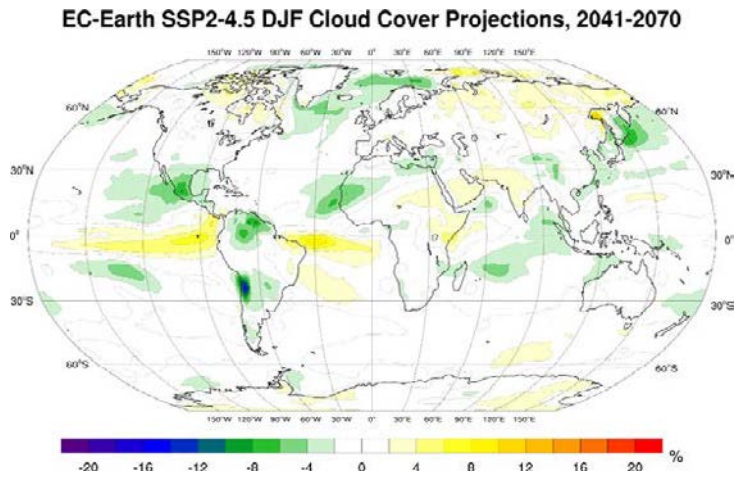

(d)

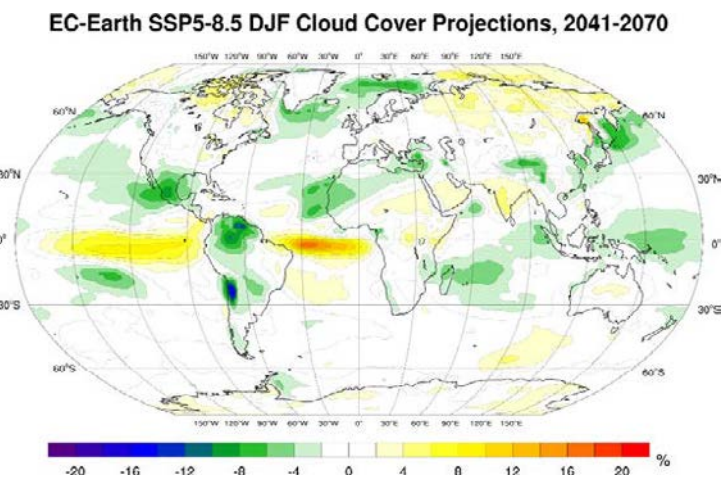

Figure 3.35. EC-Earth DJF total cloud cover projections (2041-2070 vs 1981-2010, \% difference): (a) SSP1-2.6, (b) SSP2-4.5, (c) SSP3-7.0 and (d) SSP5-8.5. In each case, an average is taken of the ensemble members r6i1p1f1, r9i1p1f1, r11i1p1f1, r13i1p1f1 and r15i1p1f1. The anomalies (\%) are calculated as "future (\%) minus past (\%)" as opposed to a percentage change.

(a)

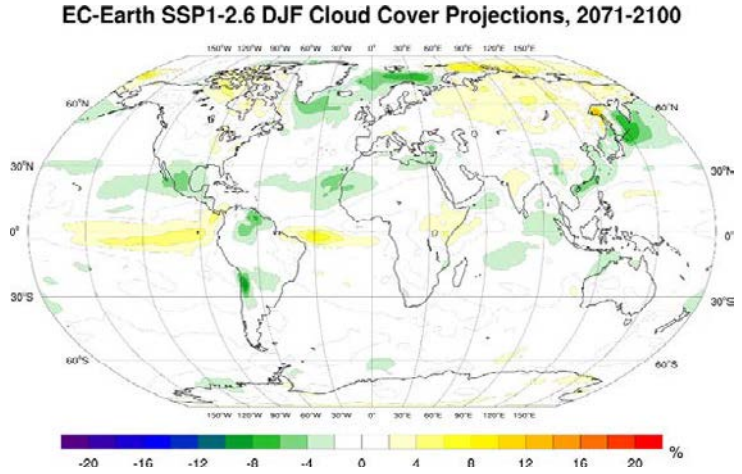

(c)

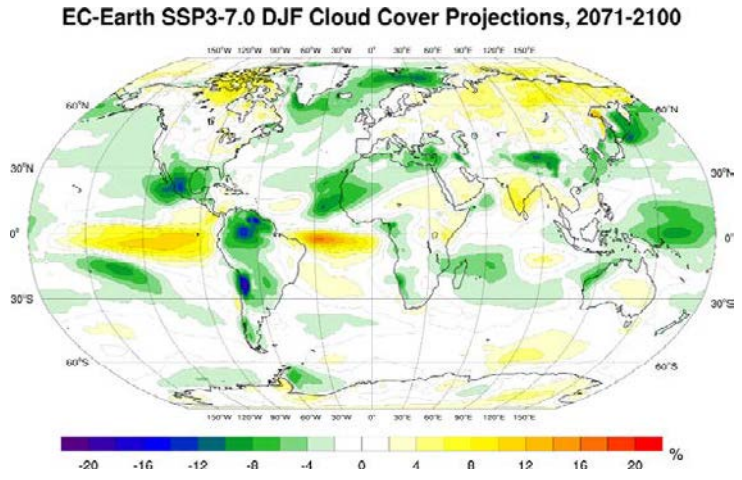

(b)

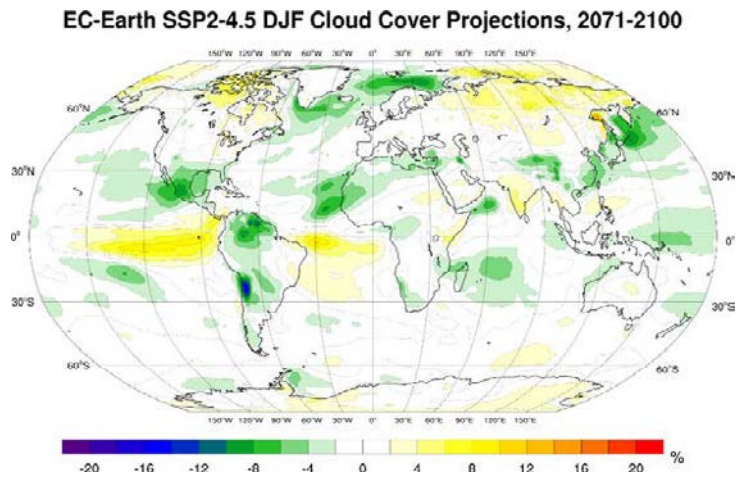

(d)

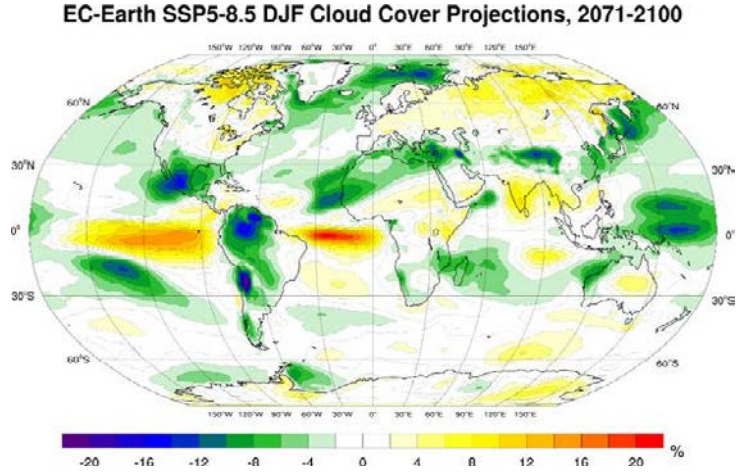

Figure 3.36. EC-Earth DJF total cloud cover projections (2071-2100 vs $1981-2010$, \% difference): (a) SSP1-2.6, (b) SSP2-4.5, (c) SSP3-7.0 and (d) SSP5-8.5. In each case, an average is taken of the ensemble members r6i1p1f1, r9i1p1f1, r11i1p1f1, r13i1p1f1 and r15i1p1f1. The anomalies (\%) are calculated as "future (\%) minus past (\%)" as opposed to a percentage change. 
(a)

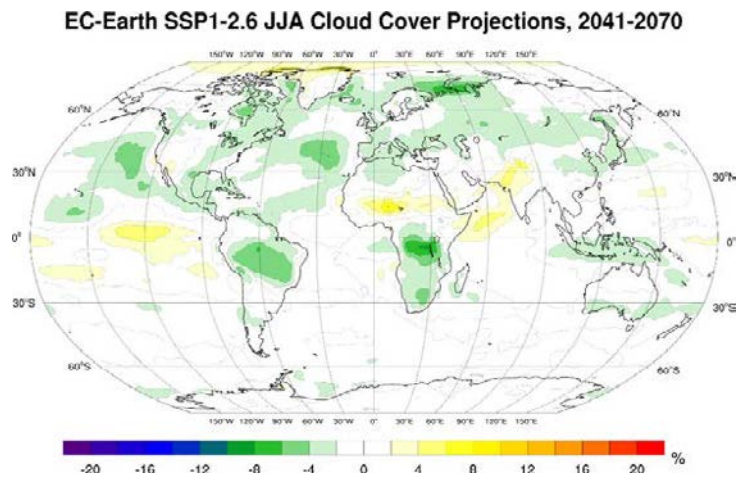

(c)

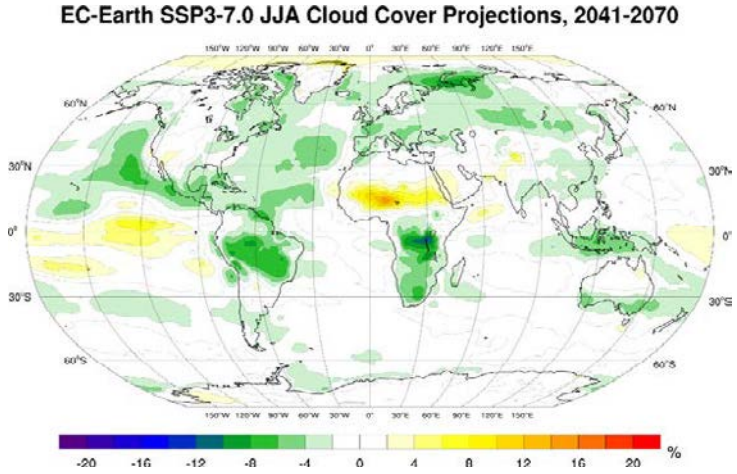

(b)

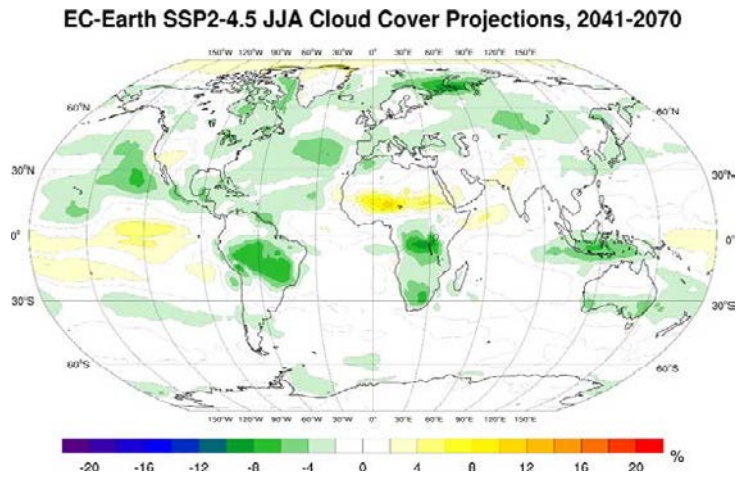

(d)

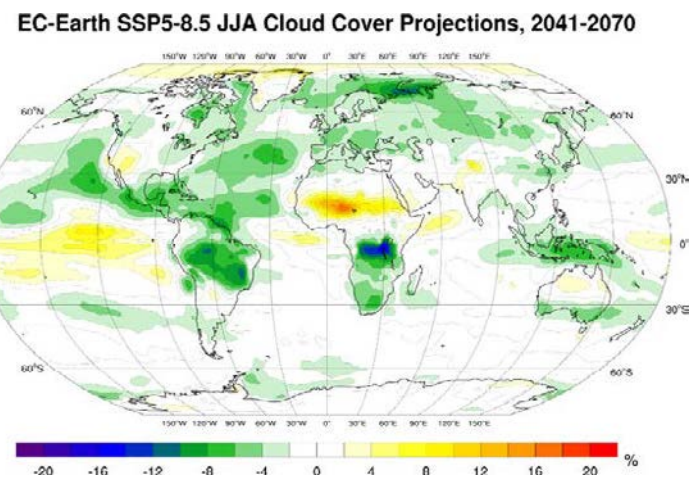

Figure 3.37. EC-Earth JJA total cloud cover projections (2041-2070 vs 1981-2010, \% difference): (a) SSP1-2.6, (b) SSP2-4.5, (c) SSP3-7.0 and (d) SSP5-8.5. In each case, an average is taken of the ensemble members r6i1p1f1, r9i1p1f1, r11i1p1f1, r13i1p1f1 and r15i1p1f1. The anomalies (\%) are calculated as "future (\%) minus past (\%)" as opposed to a percentage change.

(a)

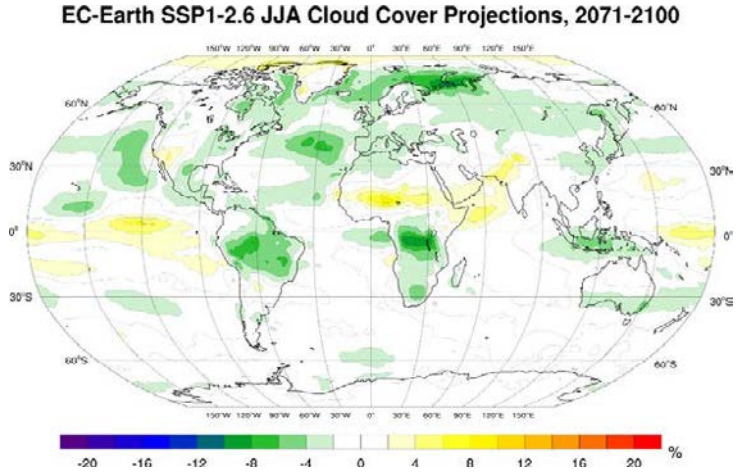

(c)

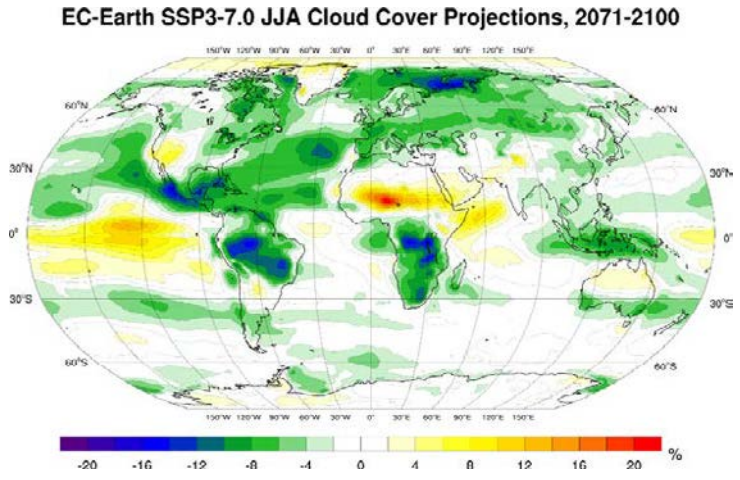

(b)

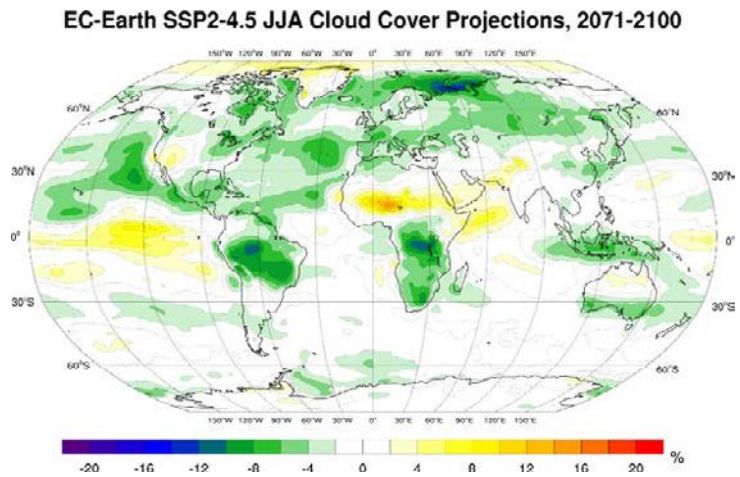

(d)

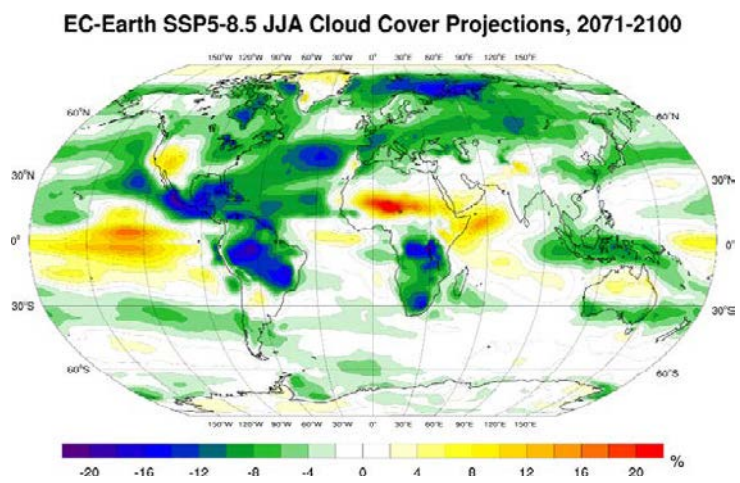

Figure 3.38. EC-Earth JJA total cloud cover projections (2071-2100 vs $1981-2010$, \% difference): (a) SSP1-2.6, (b) SSP2-4.5, (c) SSP3-7.0 and (d) SSP5-8.5. In each case, an average is taken of the ensemble members r6i1p1f1, r9i1p1f1, r11i1p1f1, r13i1p1f1 and r15i1p1f1. The anomalies (\%) are calculated as "future (\%) minus past (\%)" as opposed to a percentage change. 
The mean global annual total cloud cover anomalies (relative to 1981-2010) for all five historical simulations (1850-2014) and 20 SSPs (2015-2100) are presented in Figure 3.39. The bold lines represent the ensemble means. All ensemble members show a small steady decrease in total cloud cover from around 2000. Although a small divergence between the SSPs is evident from around 2060, the differences are small. By the end of the century, global mean total cloud cover is projected to decrease by approximately $0.5 \%, 1 \%, 1.5 \%$ and $2 \%$ for SSP $1-2.6$, SSP $2-4.5$,
SSP3-7.0 and SSP5-8.5, respectively. Figure 3.40, which presents the standard deviation of each SSP ensemble of climate projections, shows high agreement between ensemble members. The North Atlantic and tropical Pacific regions exhibit the largest disagreements. In these regions, the annual cloud projections (see Figure 3.34) should be viewed with caution as the magnitude of change is less than (or equal to) the spread (standard deviation) between ensemble member projections (Figure 3.40).

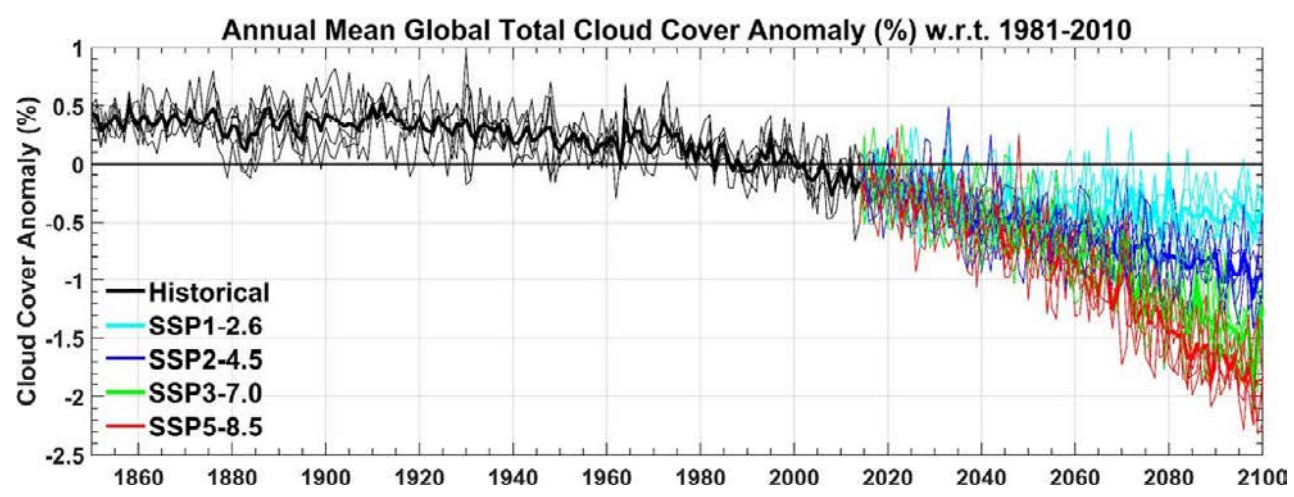

Figure 3.39. Global annual total cloud cover anomalies (\%) with respect to the 30-year period 1981-2010: EC-Earth ensemble members r6i1p1f1, r9i1p1f1, r11i1p1f1, r13i1p1f1 and r15i1p1f1. The bold lines represent the ensemble means. The anomalies (\%) are calculated as "future (\%) minus past (\%)" as opposed to a percentage change.

(a)

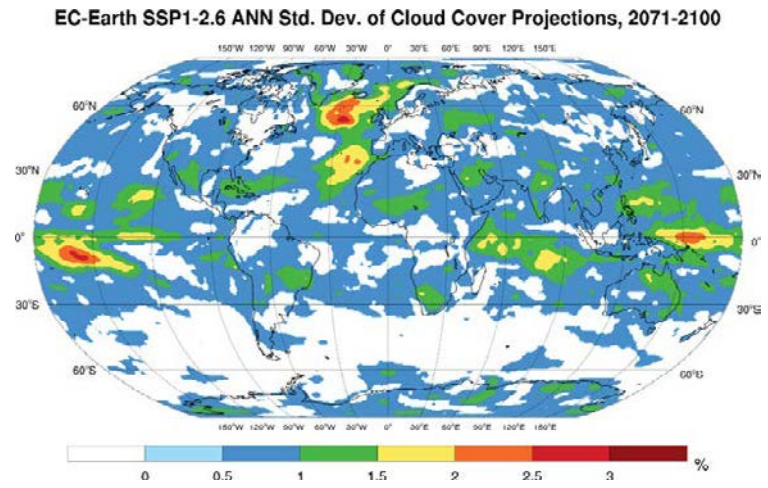

(c)

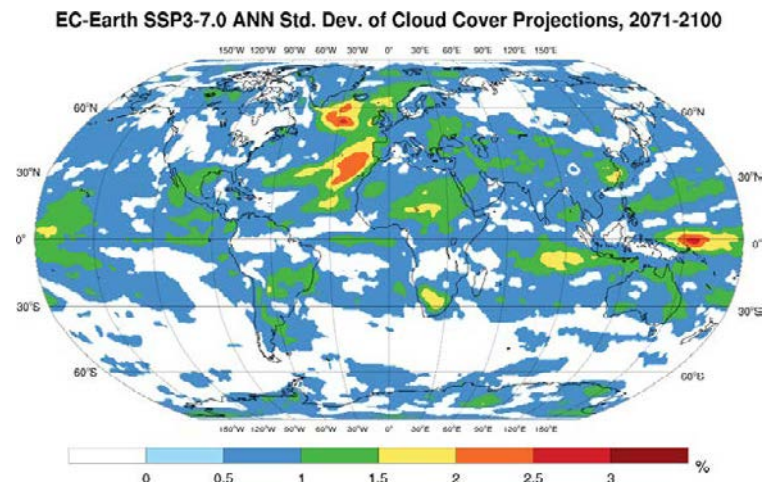

(b)

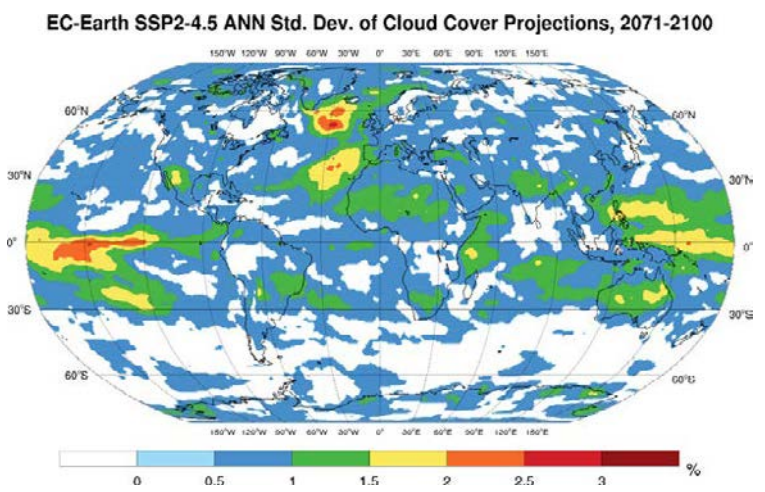

(d)

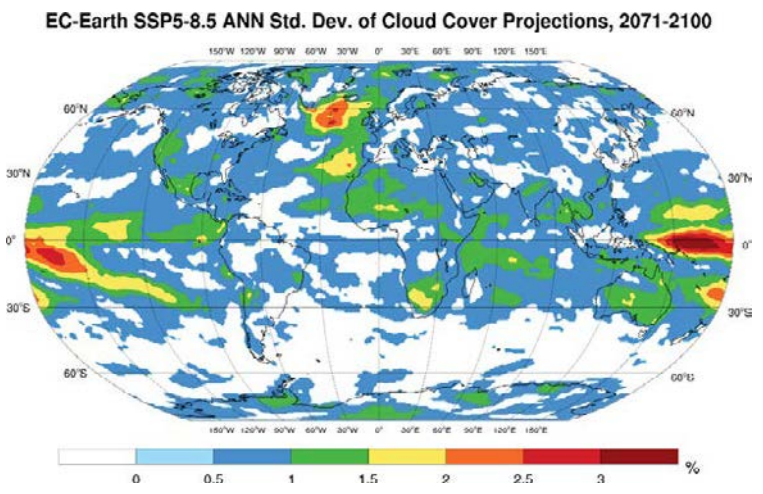

Figure 3.40. Standard deviation of the ensemble of annual total cloud cover projections (2071-2100): (a) SSP1-2.6, (b) SSP2-4.5, (c) SSP3-7.0 and (d) SSP5-8.5. 


\subsection{Snowfall Projections}

Figures 3.41 and 3.42 present the spatial distribution of annual projections of snowfall for each of the four SSPs for the 2041-2070 and 2071-2100 periods, respectively. The general trend is for large decreases in snowfall for all regions except Antarctica, northern Russia and Greenland. The DJF projections (Figures 3.43 and 3.44) are similar except that the trends are greatly enhanced in the Northern Hemisphere and the increase in snowfall extends over the Arctic. For JJA, snowfall is projected to decrease in all regions except for central Greenland and Antarctica (Figures 3.45 and 3.46). In general, the snowfall trends are enhanced for the 2071-2100 period and the higher SSPs.

The mean global annual snowfall anomalies (\% change relative to 1981-2010) for all five historical simulations (1850-2014) and 20 SSPs (2015-2100) are presented in Figure 3.47a. The bold lines represent the ensemble means. All ensemble members show a steady decrease in snowfall from around 2020. By the end of the century, global mean snowfall is projected to decrease by approximately $3 \%$, $7 \%, 8 \%$ and $10 \%$ for SSP $1-2.6$, SSP $2-4.5$, SSP $3-7.0$ and SSP5-8.5, respectively. The Northern Hemisphere annual snowfall anomalies, presented in Figure $3.47 \mathrm{~b}$, are substantially larger, with end-of-century projected decreases ranging from 7\% (SSP1-2.6) to 18\% (SSP5-8.5). The Southern Hemisphere annual snowfall anomalies (Figure 3.47c) exhibit no trend.

Figure 3.48 shows the standard deviation of each SSP ensemble of climate projections; there is a high level of agreement between ensemble members for all regions except the north-west Atlantic. An analysis of the individual ensemble members shows that the disagreement in the North Atlantic region is the result of a difference between two groups - the r6i1p1f1 and r11i1p1f1 ensemble members project larger decreases in snowfall than the r9i1p1f1, r13i1p1f1 and r15i1p1f1 ensemble members. Note that this result is similar (and probably related) to the spread of 2-m temperature projections discussed in section 3.1.

Future work will fully address this issue by extending the analysis to include the full ensemble of EC-Earth CMIP6 simulations produced by the consortium. 
(a)

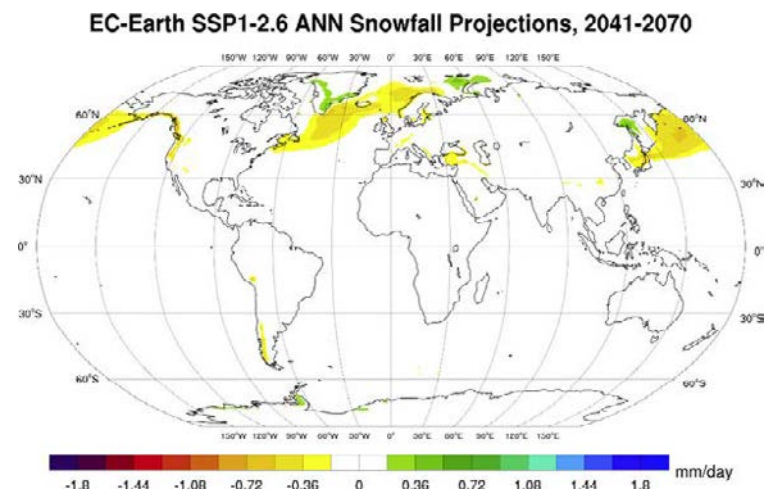

(c)

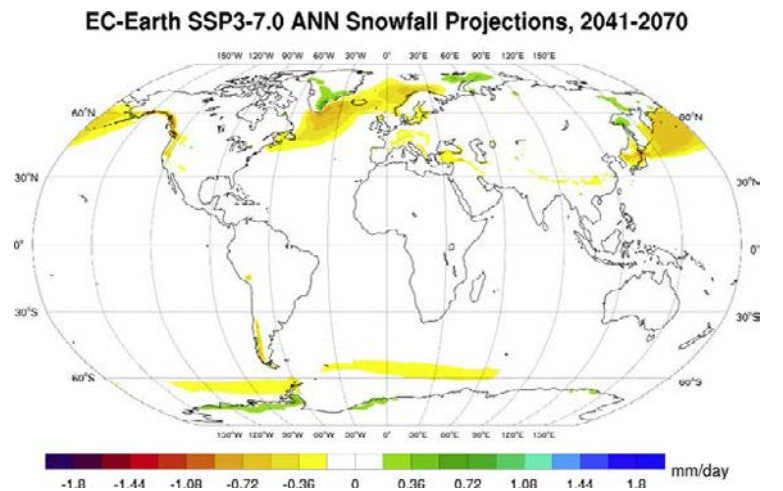

(b)

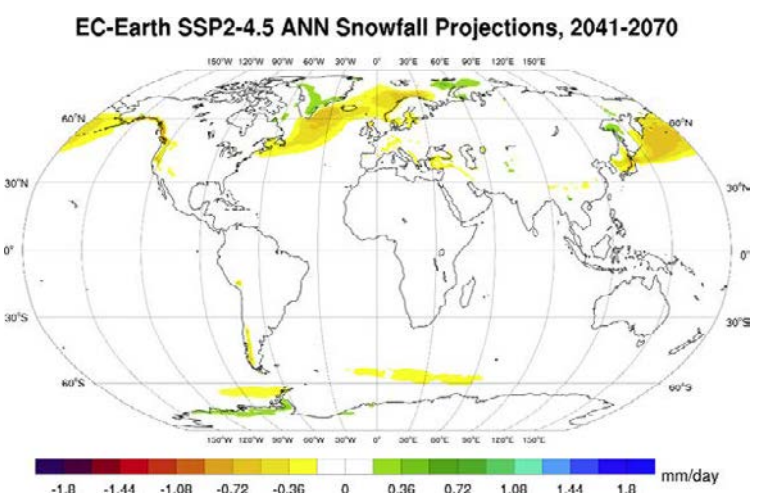

(d)

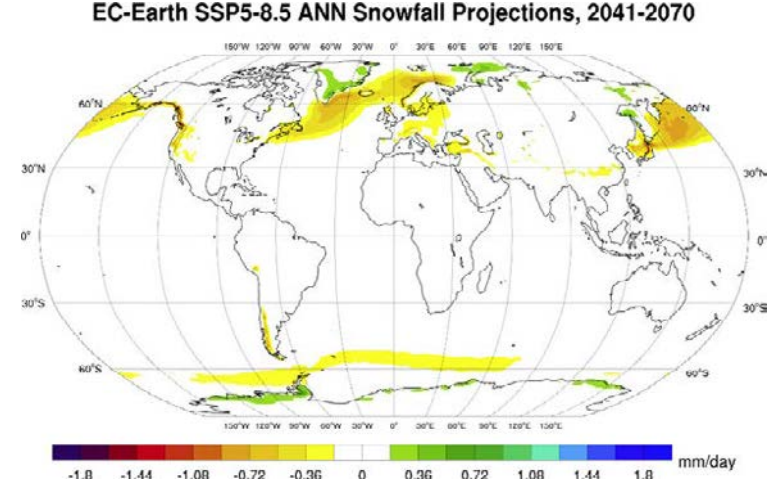

Figure 3.41. EC-Earth annual snowfall projections (2041-2070 vs 1981-2010, $\mathrm{mm} /$ day difference): (a) SSP1-2.6, (b) SSP2-4.5, (c) SSP3-7.0 and (d) SSP5-8.5. In each case, an average is taken of the ensemble members r6i1p1f1, r9i1p1f1, r11i1p1f1, r13i1p1f1 and r15i1p1f1.

(a)

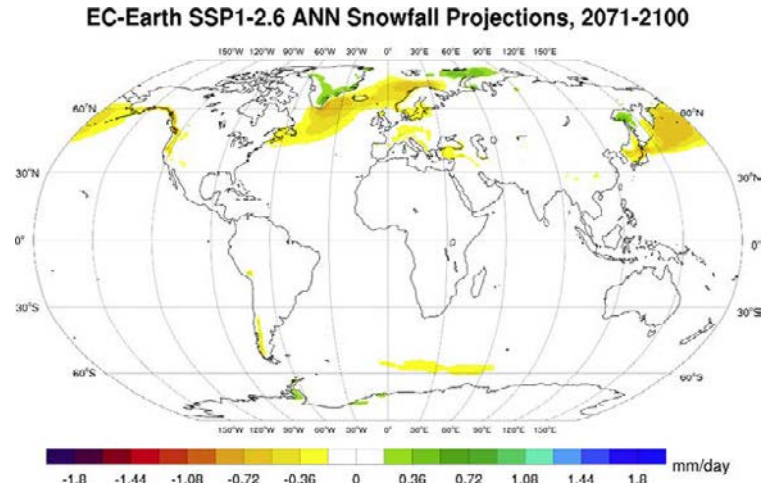

(c)

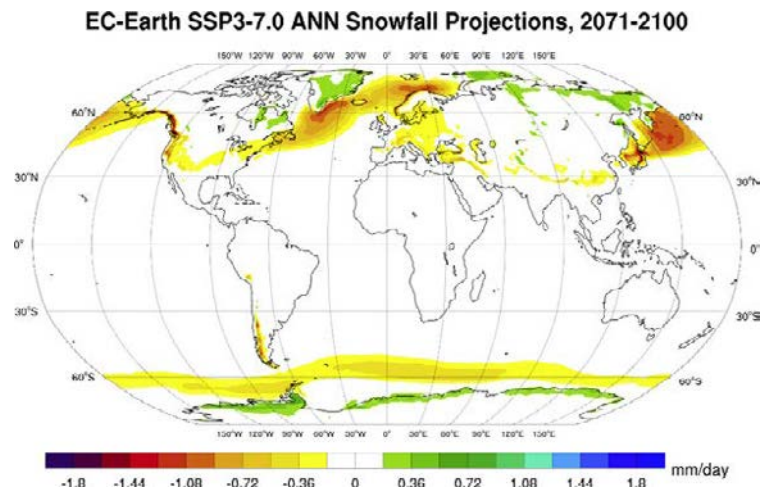

(b)

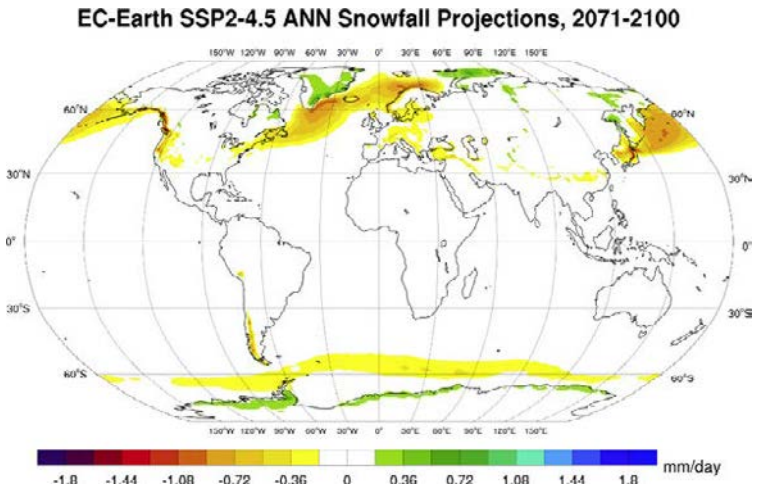

(d)

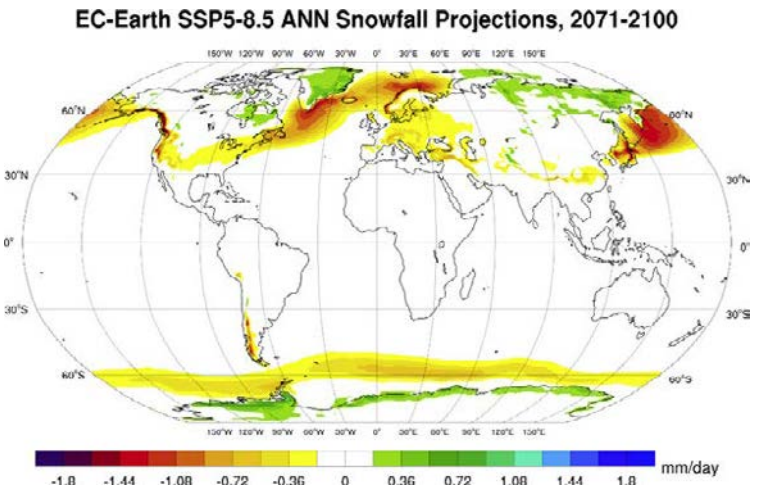

Figure 3.42. EC-Earth annual snowfall projections $(2071-2100$ vs $1981-2010, \mathrm{~mm} /$ day difference): (a) SSP1-2.6, (b) SSP2-4.5, (c) SSP3-7.0 and (d) SSP5-8.5. In each case, an average is taken of the ensemble members r6i1p1f1, r9i1p1f1, r11i1p1f1, r13i1p1f1 and r15i1p1f1. 
(a)

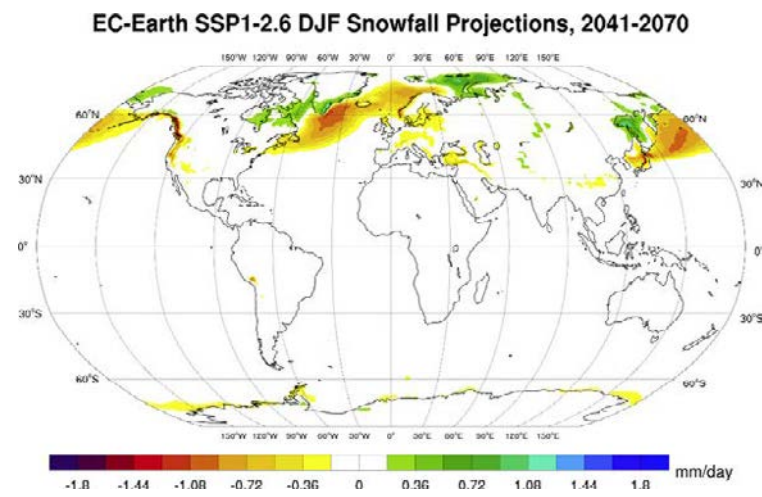

(c)

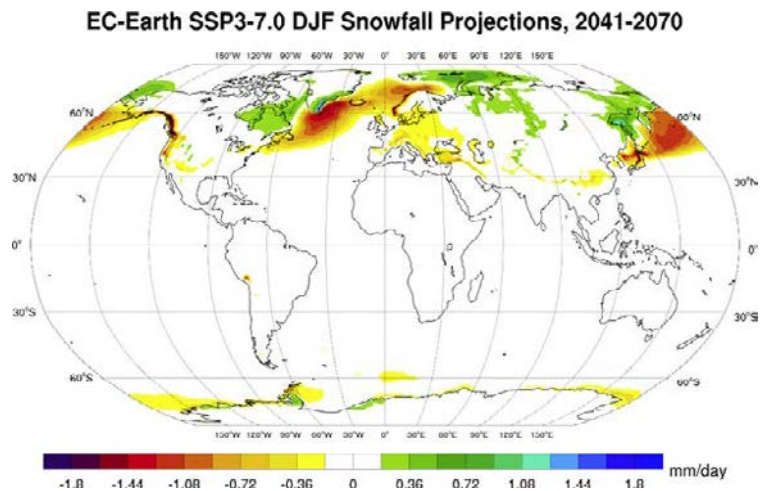

(b)

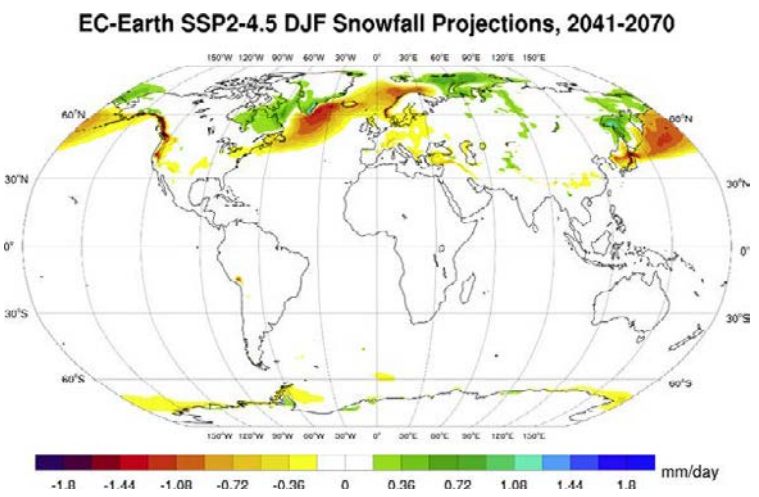

(d)

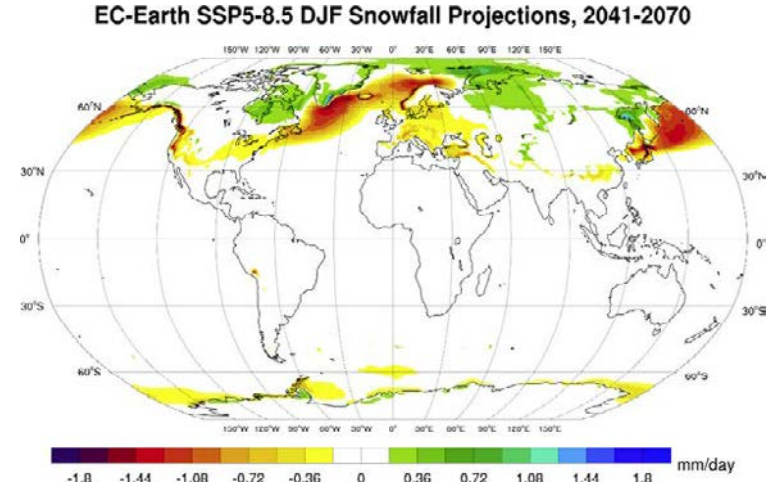

Figure 3.43. EC-Earth DJF snowfall projections (2041-2070 vs 1981-2010, $\mathrm{mm} /$ day difference): (a) SSP1-2.6, (b) SSP2-4.5, (c) SSP3-7.0 and (d) SSP5-8.5. In each case, an average is taken of the ensemble members r6i1p1f1, r9i1p1f1, r11i1p1f1, r13i1p1f1 and r15i1p1f1.

(a)

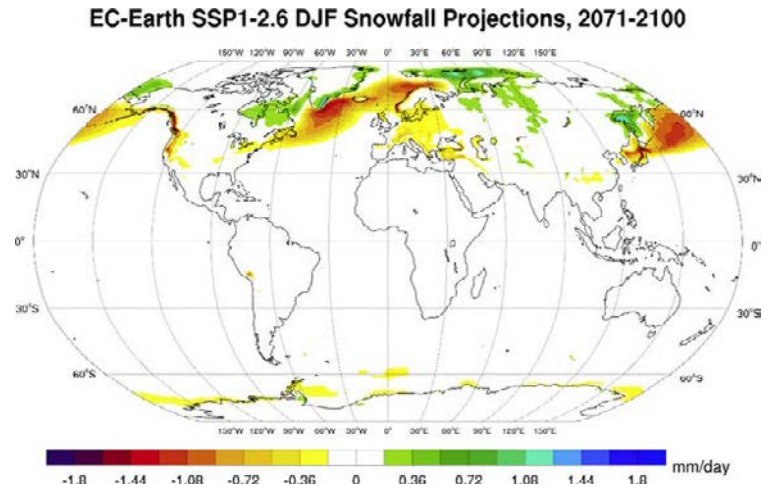

(c)

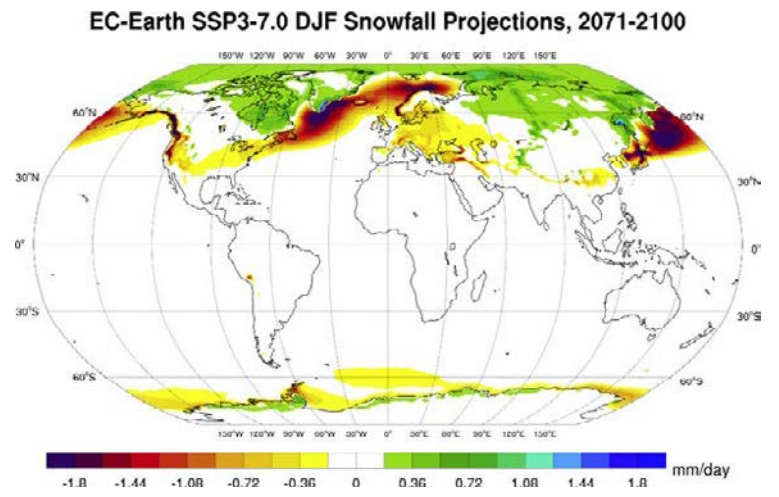

(b)

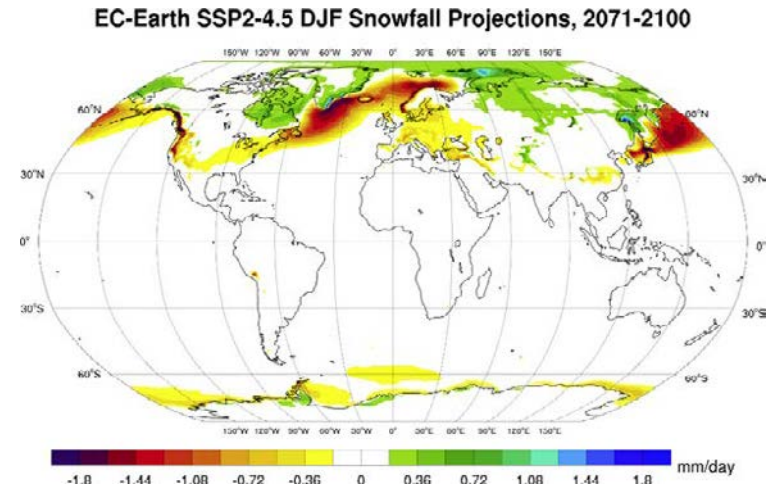

(d)

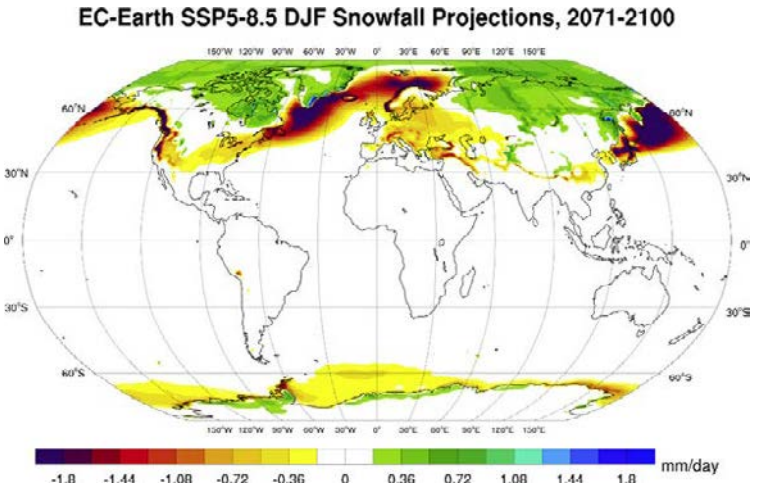

Figure 3.44. EC-Earth DJF snowfall projections (2071-2100 vs $1981-2010, \mathrm{~mm} /$ day difference):

(a) SSP1-2.6, (b) SSP2-4.5, (c) SSP3-7.0 and (d) SSP5-8.5. In each case, an average is taken of the ensemble members r6i1p1f1, r9i1p1f1, r11i1p1f1, r13i1p1f1 and r15i1p1f1. 
(a)

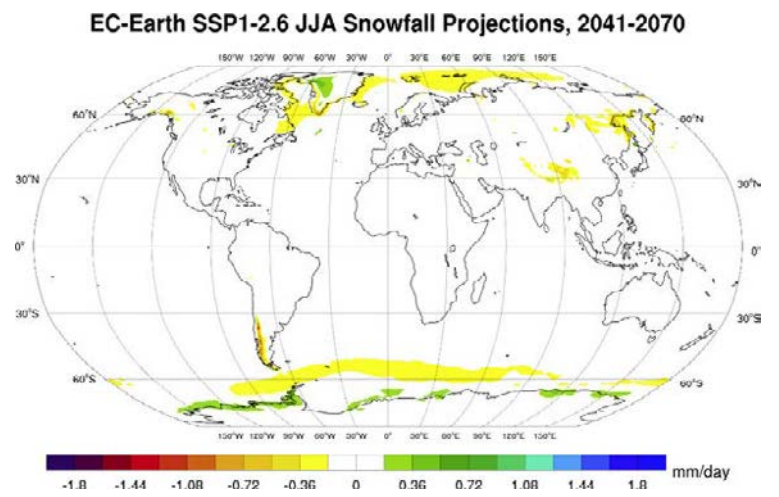

(c)

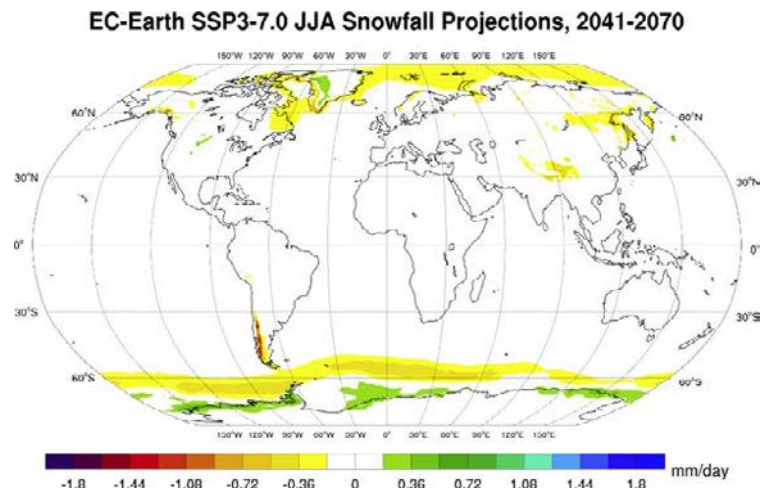

(b)

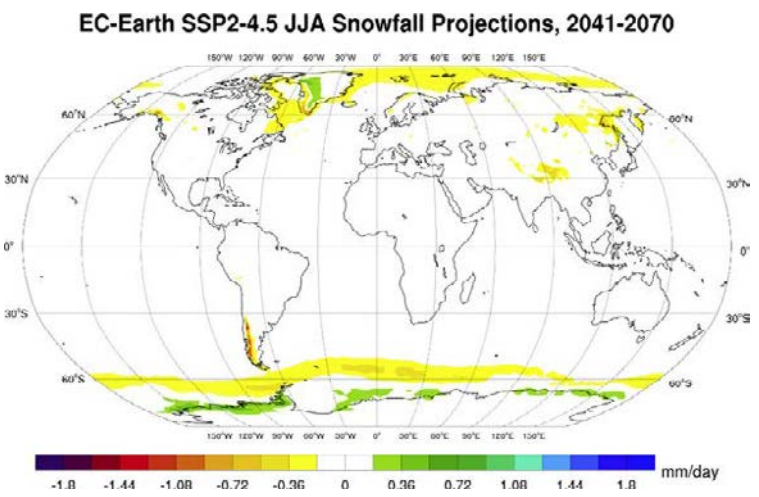

(d)

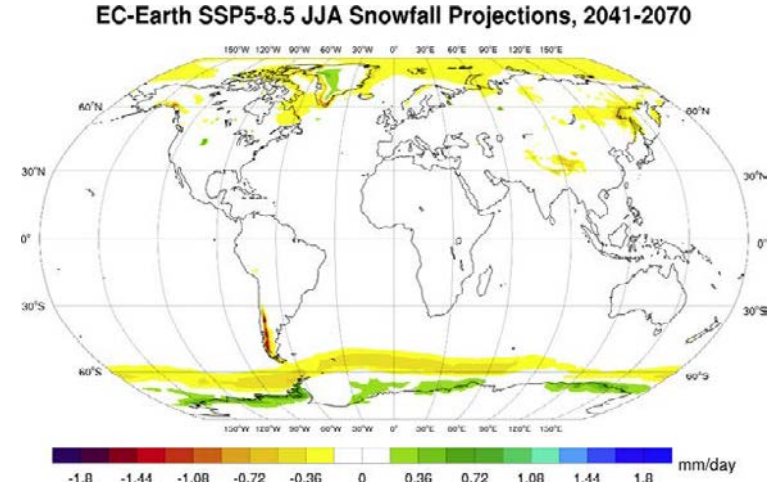

Figure 3.45. EC-Earth JJA snowfall projections (2041-2070 vs 1981-2010, $\mathrm{mm} /$ day difference):

(a) SSP1-2.6, (b) SSP2-4.5, (c) SSP3-7.0 and (d) SSP5-8.5. In each case, an average is taken of the ensemble members r6i1p1f1, r9i1p1f1, r11i1p1f1, r13i1p1f1 and r15i1p1f1.

(a)

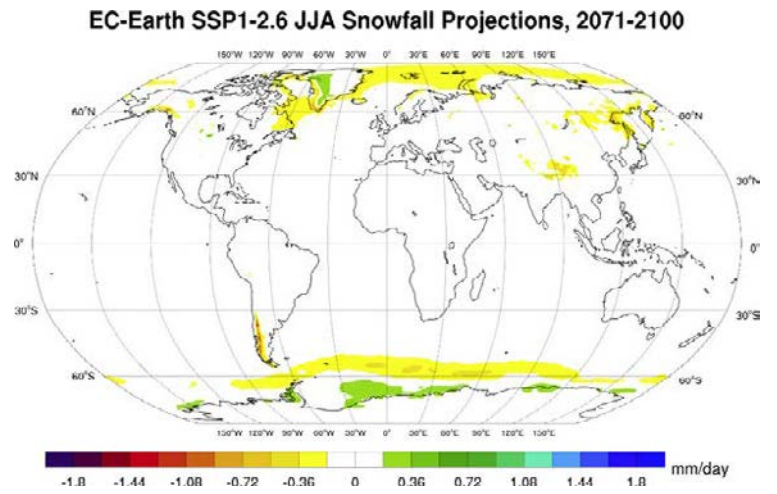

(c)

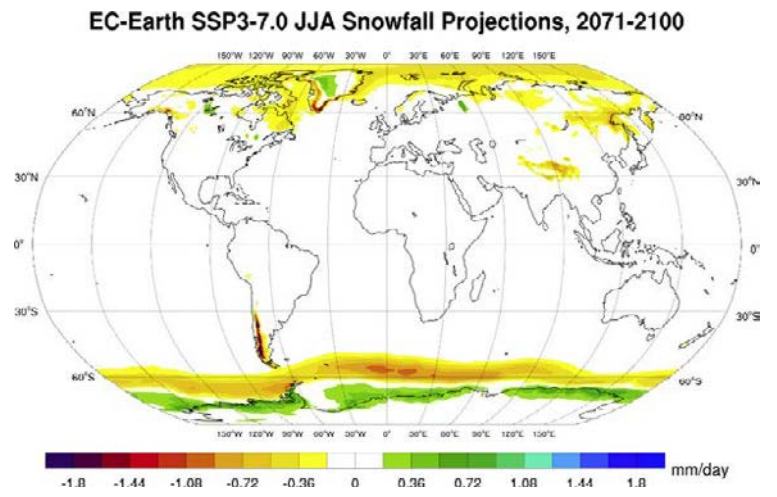

(b)

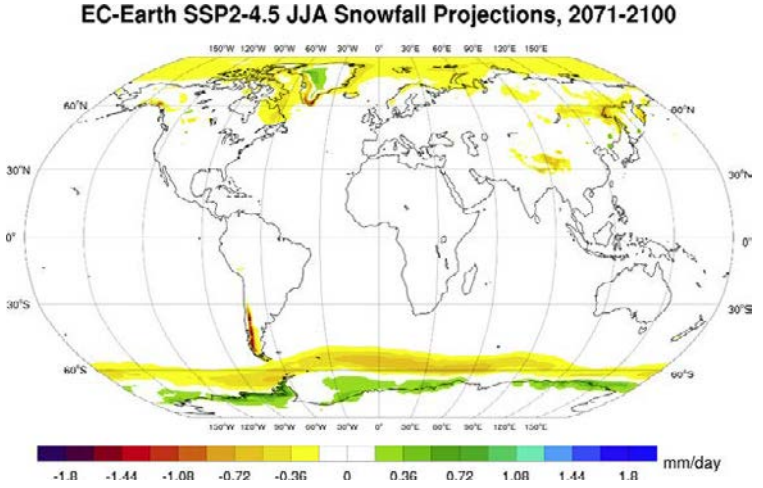

(d)

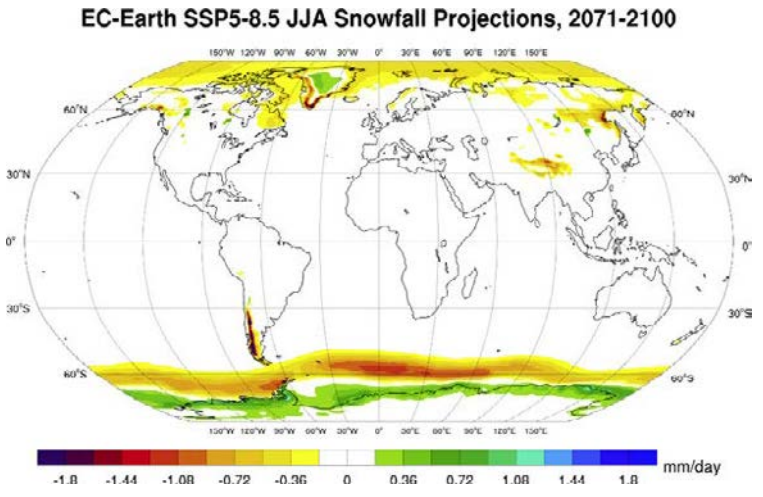

Figure 3.46. EC-Earth JJA snowfall projections (2071-2100 vs $1981-2010, \mathrm{~mm} /$ day difference):

(a) SSP1-2.6, (b) SSP2-4.5, (c) SSP3-7.0 and (d) SSP5-8.5. In each case, an average is taken of the ensemble members r6i1p1f1, r9i1p1f1, r11i1p1f1, r13i1p1f1 and r15i1p1f1. 
(a)

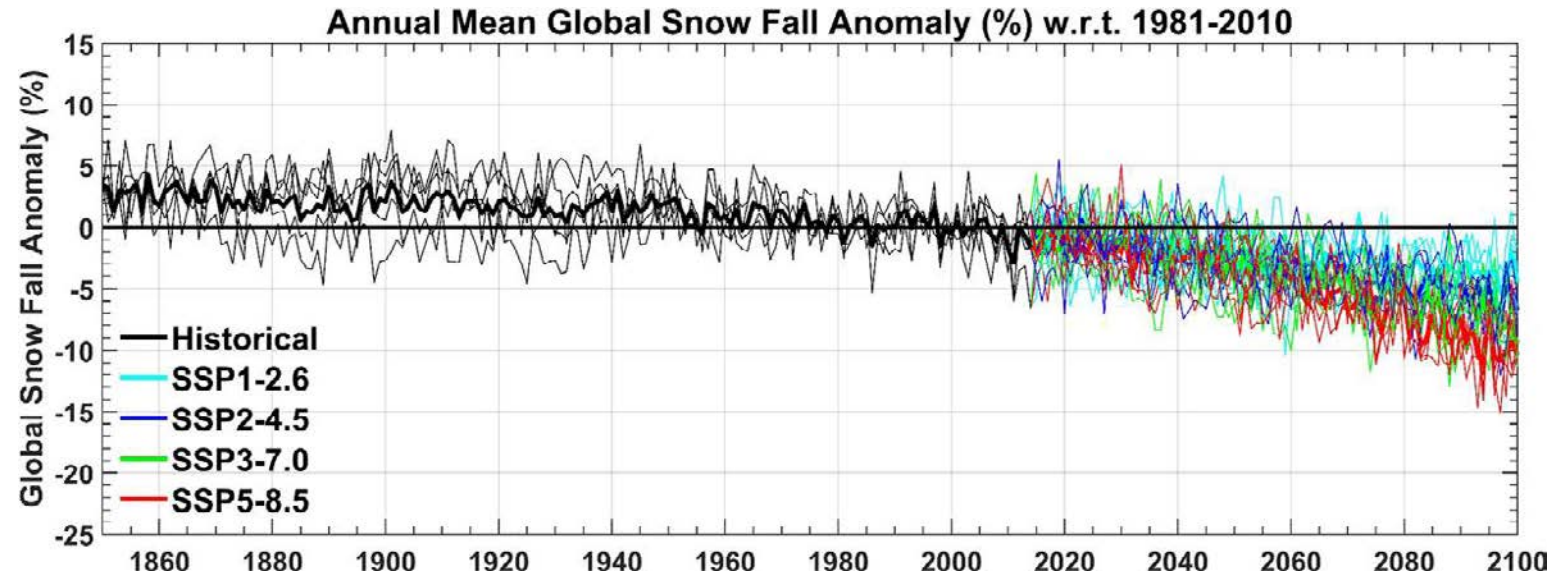

(b)

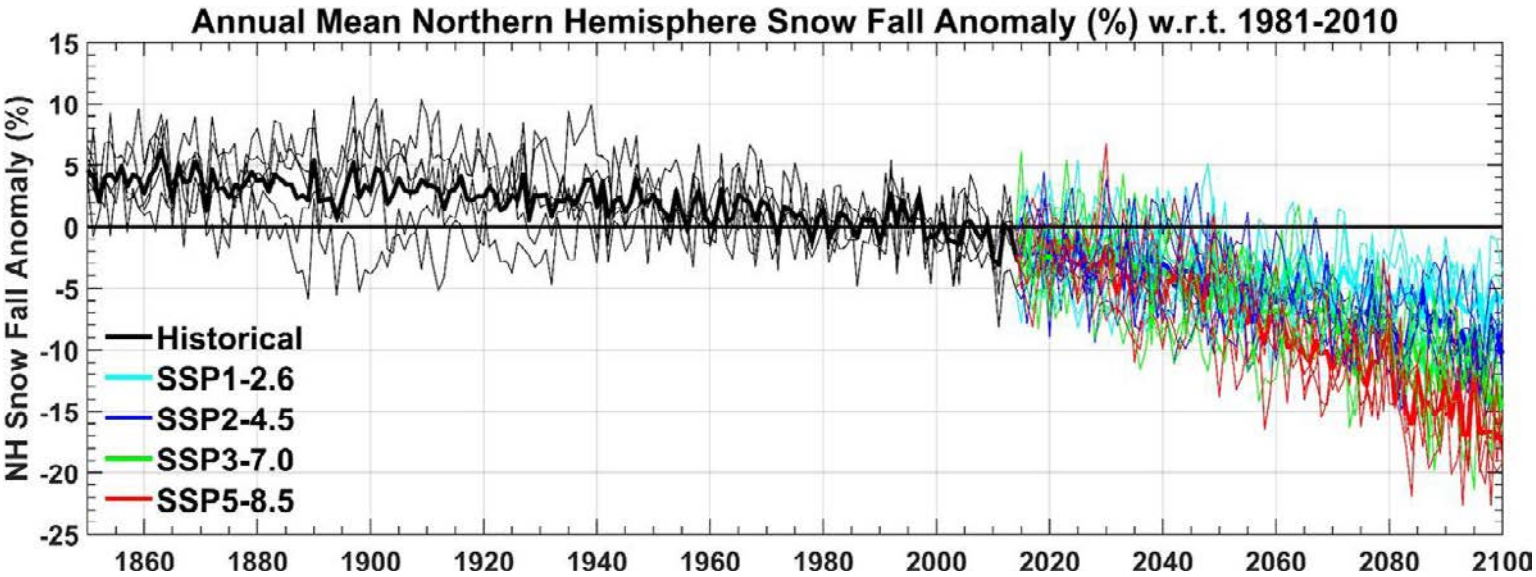

(c)

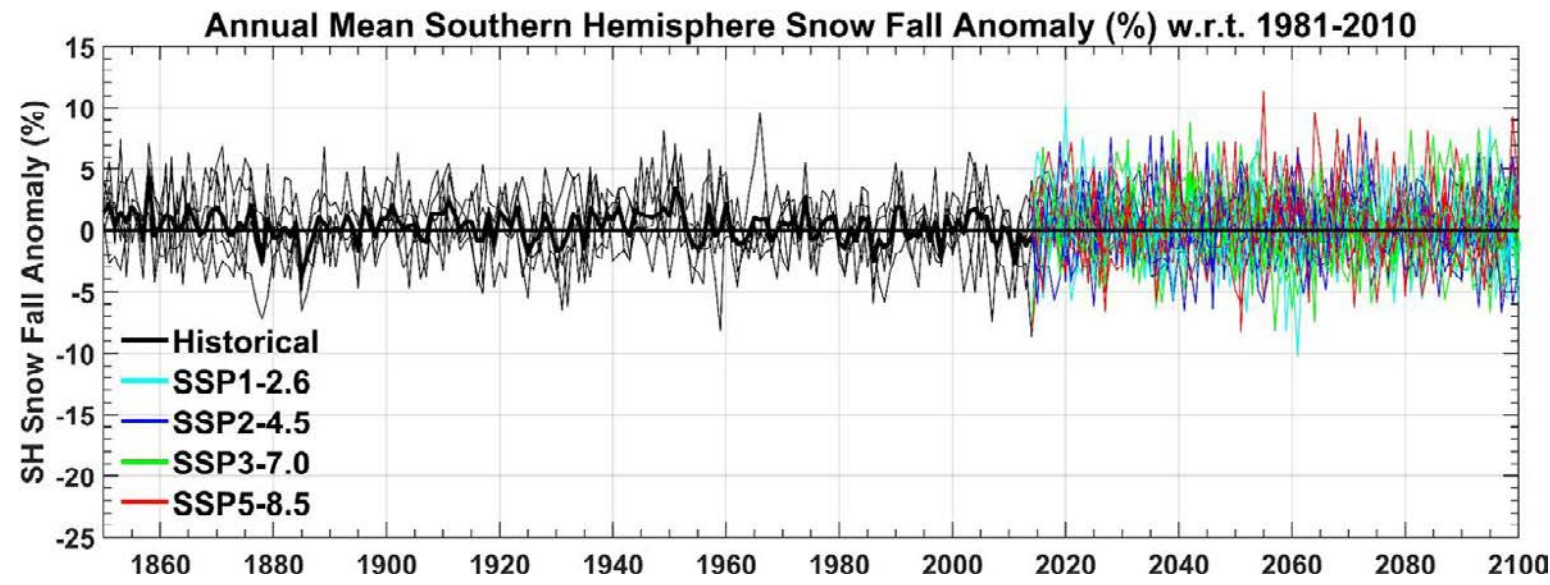

Figure 3.47. Annual snowfall anomalies (\%) with respect to the 30-year period 1981-2010: EC-Earth ensemble members r6i1p1f1, r9i1p1f1, r11i1p1f1, r13i1p1f1 and r15i1p1f1 - (a) global, (b) Northern Hemisphere and (c) Southern Hemisphere. The bold lines represent the ensemble means. 
(a)

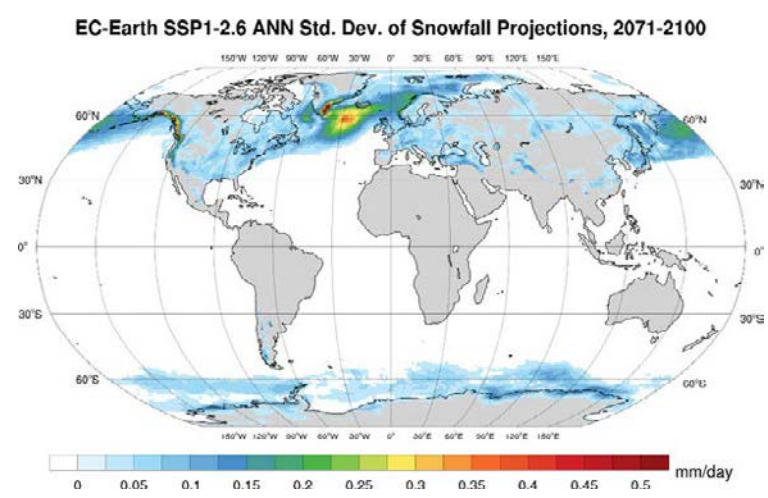

(c)

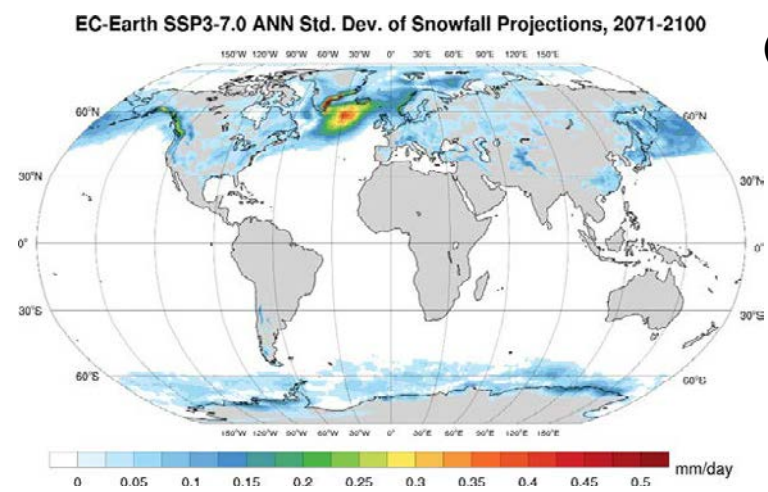

(b)

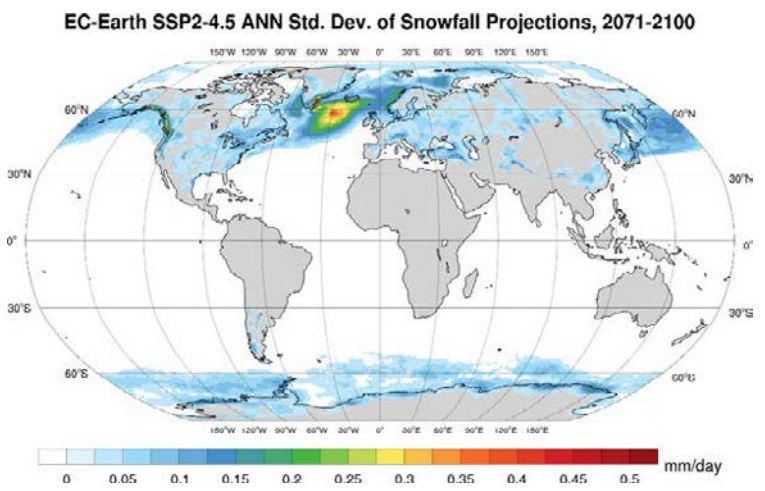

(d)

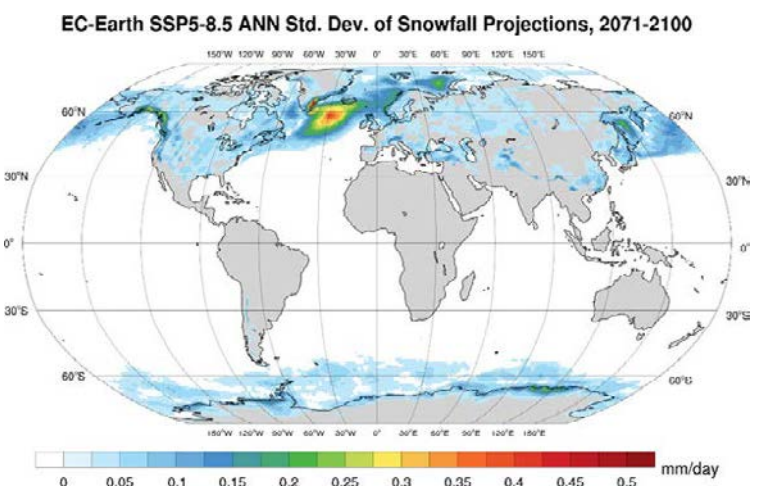

Figure 3.48. Standard deviation of the ensemble of annual snowfall projections (2071-2100): (a) SSP1-2.6, (b) SSP2-4.5, (c) SSP3-7.0 and (d) SSP5-8.5.

\subsection{Sea Surface Temperature Projections}

Figures 3.49 and 3.50 present the spatial distribution of annual SST projections for each of the four SSPs for 2041-2070 and 2071-2100, respectively. The largest increases in temperatures are noted over the northern latitudes, in particular over the Arctic region. Projections for DJF (Figures 3.51 and 3.52) follow a similar trend to the annual projections except that increases in SST over the Southern (Northern) Hemisphere are enhanced (diminished). The projections for JJA (Figures 3.53 and 3.54) also follow a similar trend to the annual projections except that increases in SST over the northern latitudes are enhanced. In all plots, a "dipole" feature is noted in the North Atlantic, where SST projections are enhanced (diminished) north (south) of Iceland. Projections of SST range from $-1^{\circ} \mathrm{C}$ for the region south of Iceland for DJF 2041-2070 SSP1-2.6 (Figure 3.51a) to $+10^{\circ} \mathrm{C}$ over the Arctic region for JJA 2071-2100 SSP5-8.5 (Figure 3.54d).

The mean global annual SST anomalies (relative to 1981-2010) for all five historical simulations (1850-2014) and 20 SSPs (2015-2100) are presented in Figure 3.55. The bold lines represent the ensemble means. All ensemble members show a steady increase in temperature from around 2000 with a noticeable divergence between the SSPs from around 2050. By the year 2100, the global mean SST is projected to increase by approximately $1^{\circ} \mathrm{C}, 2^{\circ} \mathrm{C}$, $3^{\circ} \mathrm{C}$ and $4^{\circ} \mathrm{C}$ for SSP $1-2.6$, SSP2-4.5, SSP3-7.0 and SSP5-8.5, respectively. The small spread between the individual ensemble members demonstrates a high level of agreement and adds a measure of confidence to the projections. This is confirmed in Figure 3.56, which shows the standard deviation of each SSP ensemble of climate projections; a high level of agreement between ensembles is noted for all regions except those south of Greenland and north of Iceland. An analysis of the individual ensemble members showed that the disagreement over the region south of Greenland is the result of a difference between two groups - the r6i1p1f1 and r11i1p1f1 ensemble members project larger increases in SST than the r9i1p1f1, r13i1p1f1 and r15i1p1f1 ensemble members. This result is similar (and very probably related) to the spread of 2-m temperature projections discussed in section 3.1. Future work will fully address this issue by extending the analysis to include the full ensemble of EC-Earth CMIP6 simulations produced by the consortium. 
(a)

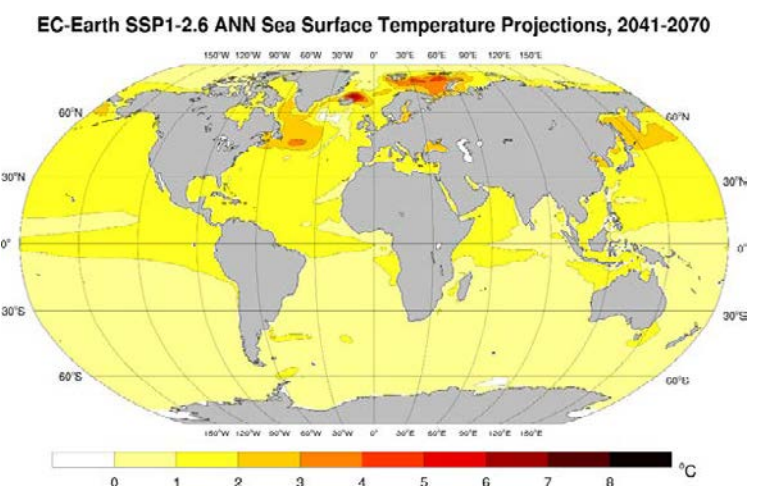

(c)

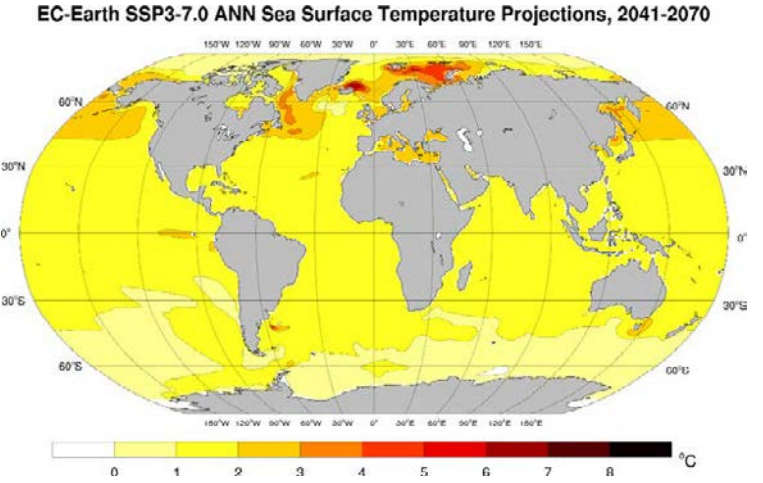

(b)

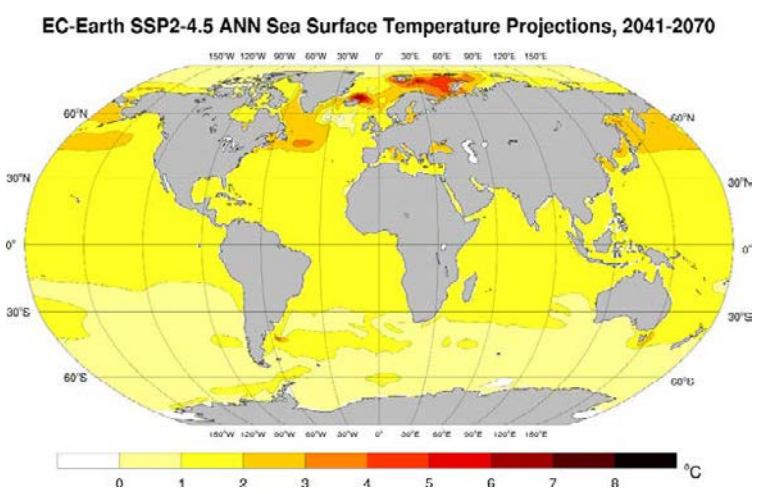

(d)

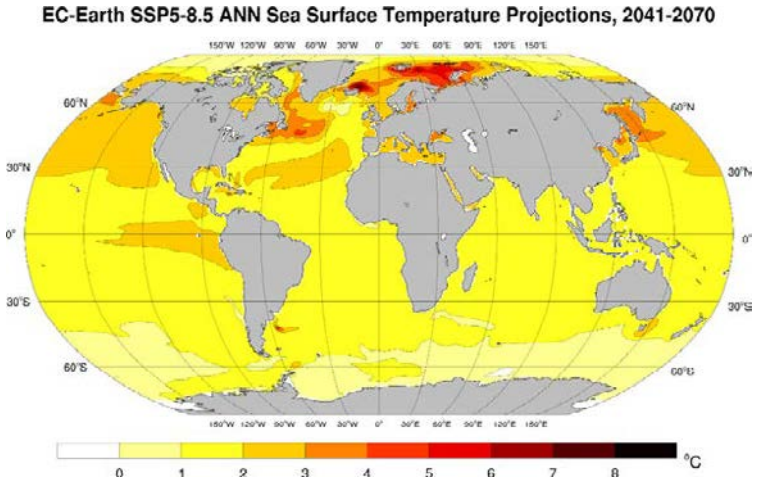

Figure 3.49. EC-Earth annual SST projections (2041-2070 vs $1981-2010,{ }^{\circ} \mathrm{C}$ change): (a) SSP1-2.6, (b) SSP2-4.5, (c) SSP3-7.0 and (d) SSP5-8.5. In each case, an average is taken of the ensemble members r6i1p1f1, r9i1p1f1, r11i1p1f1, r13i1p1f1 and r15i1p1f1.

(a)

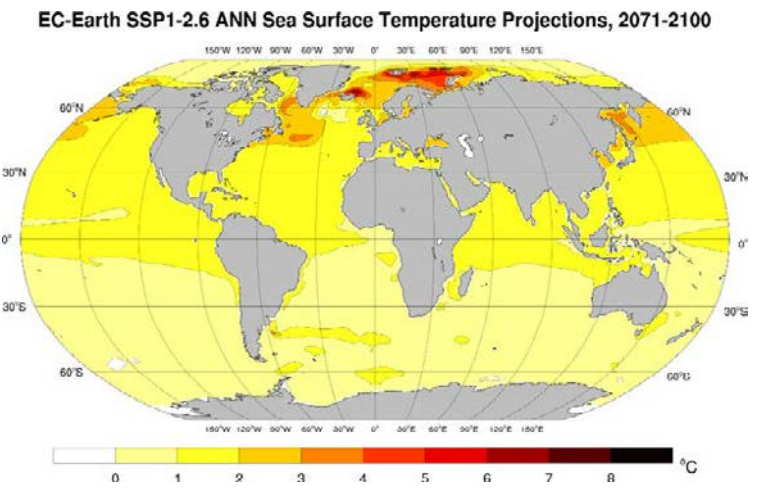

(c)

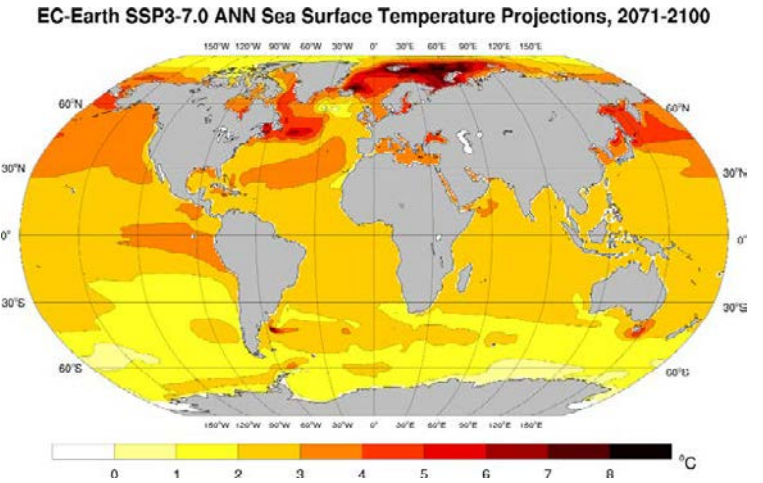

(b)

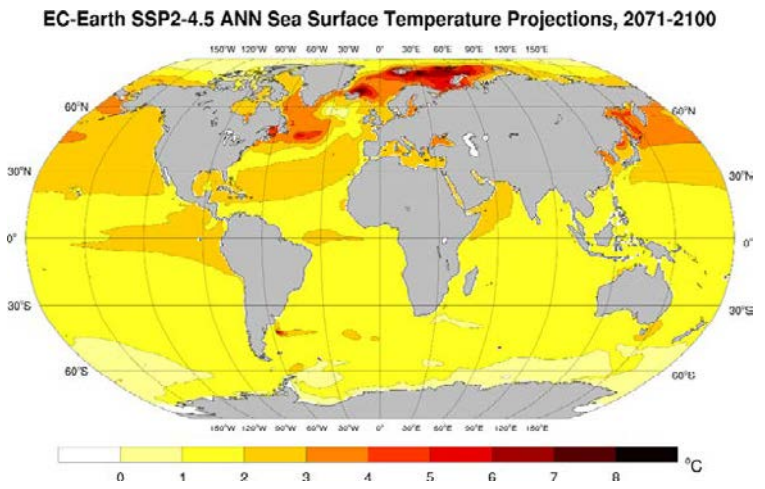

(d)

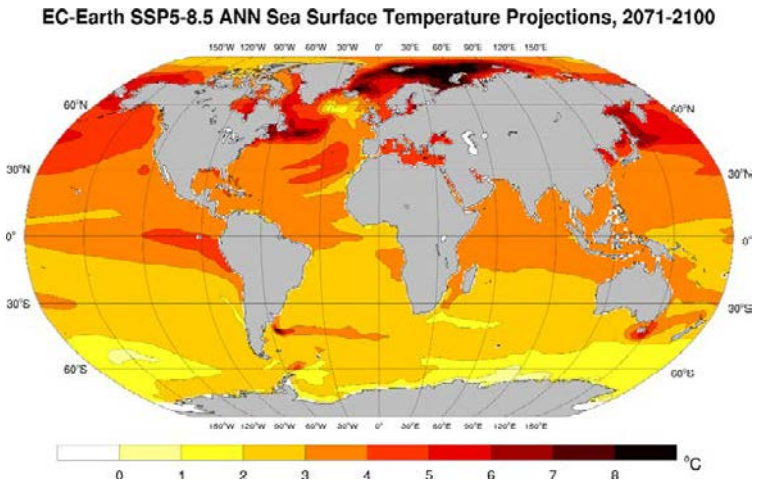

Figure 3.50. EC-Earth annual SST projections (2071-2100 vs $1981-2010,{ }^{\circ} \mathrm{C}$ change): (a) SSP1-2.6, (b) SSP2-4.5, (c) SSP3-7.0 and (d) SSP5-8.5. In each case, an average is taken of the ensemble members r6i1p1f1, r9i1p1f1, r11i1p1f1, r13i1p1f1 and r15i1p1f1 
(a)

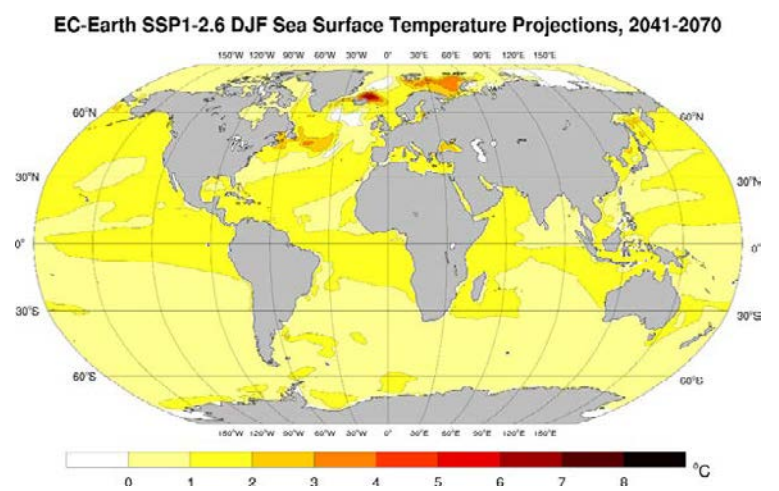

(c)

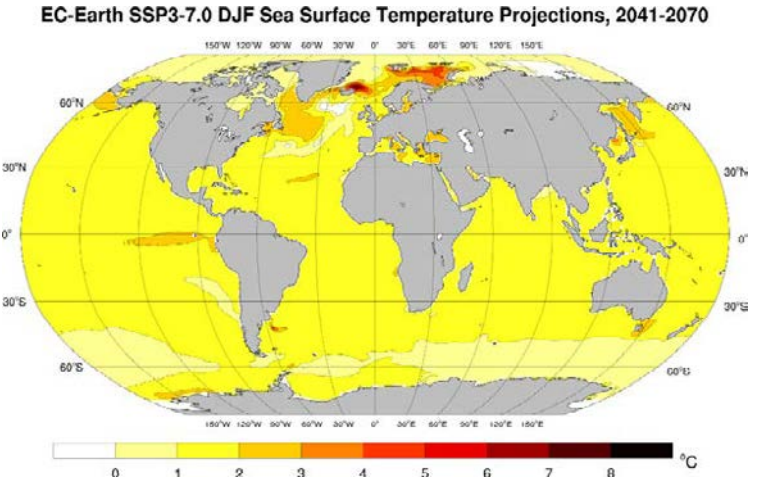

(b)

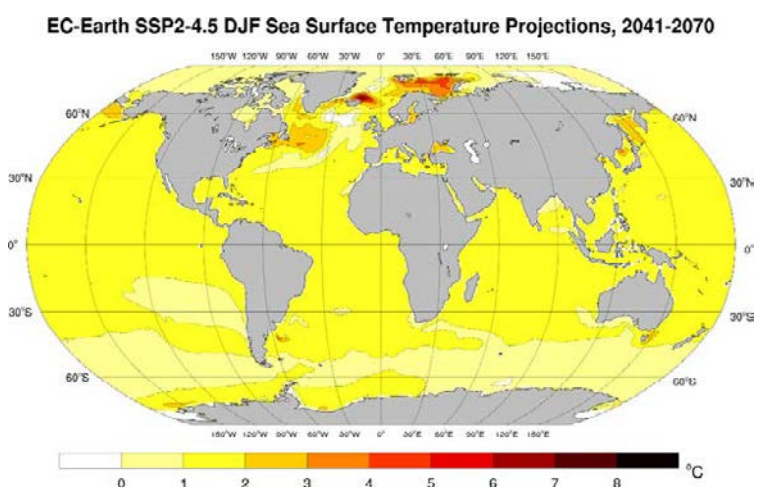

(d)

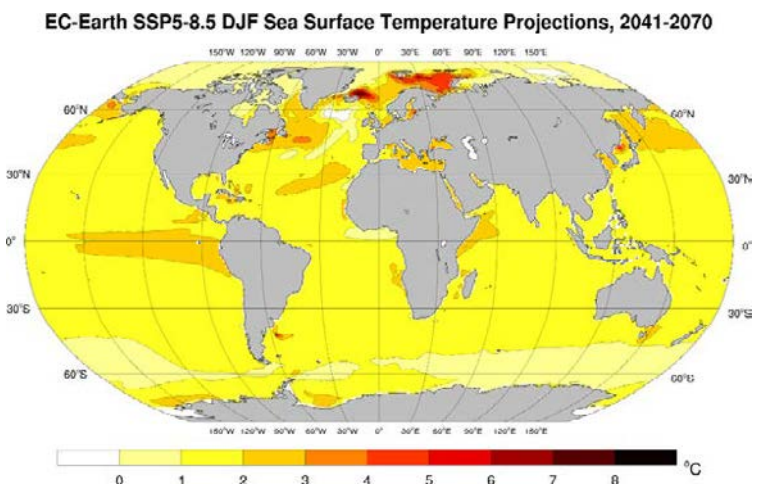

Figure 3.51. EC-Earth DJF SST projections (2041-2070 vs $1981-2010,{ }^{\circ} \mathrm{C}$ change): (a) SSP1-2.6, (b) SSP2-4.5, (c) SSP3-7.0 and (d) SSP5-8.5. In each case, an average is taken of the ensemble members r6i1p1f1, r9i1p1f1, r11i1p1f1, r13i1p1f1 and r15i1p1f1.

(a)

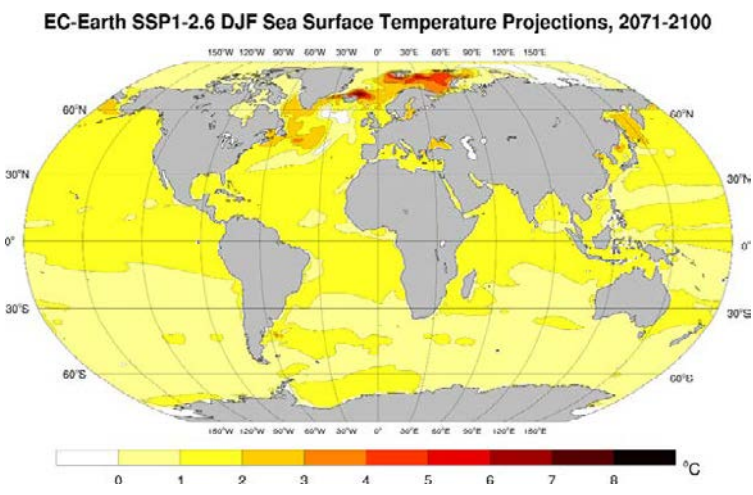

(c)

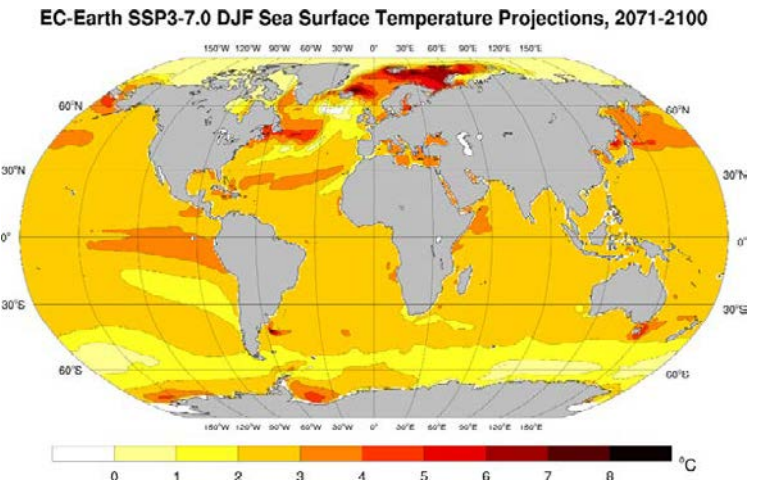

(b)

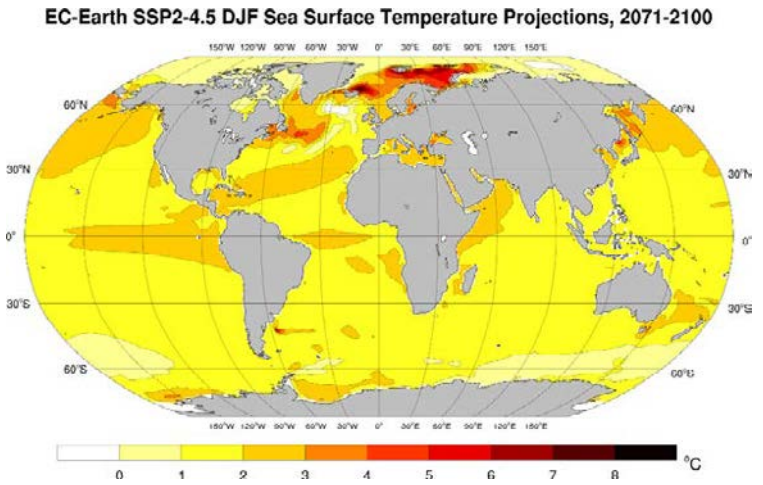

(d)

EC-Earth SSP5-8.5 DJF Sea Surface Temperature Projections, 2071-2100

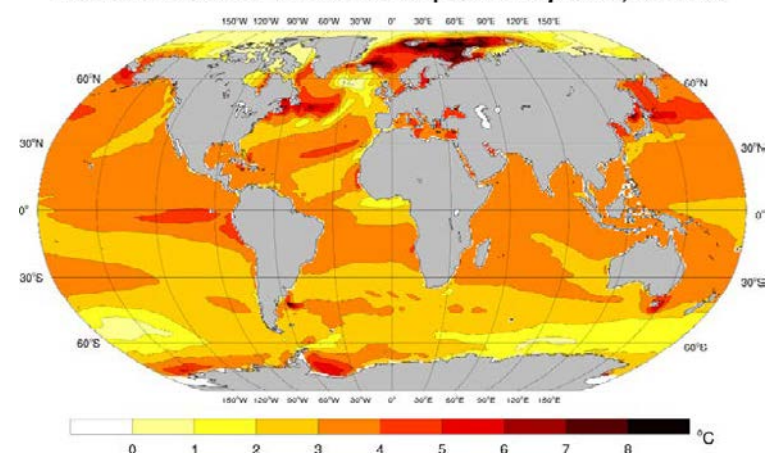

Figure 3.52. EC-Earth DJF SST projections (2071-2100 vs $1981-2010,{ }^{\circ} \mathrm{C}$ change): (a) SSP1-2.6,

(b) SSP2-4.5, (c) SSP3-7.0 and (d) SSP5-8.5. In each case, an average is taken of the ensemble members r6i1p1f1, r9i1p1f1, r11i1p1f1, r13i1p1f1 and r15i1p1f1. 
(a)

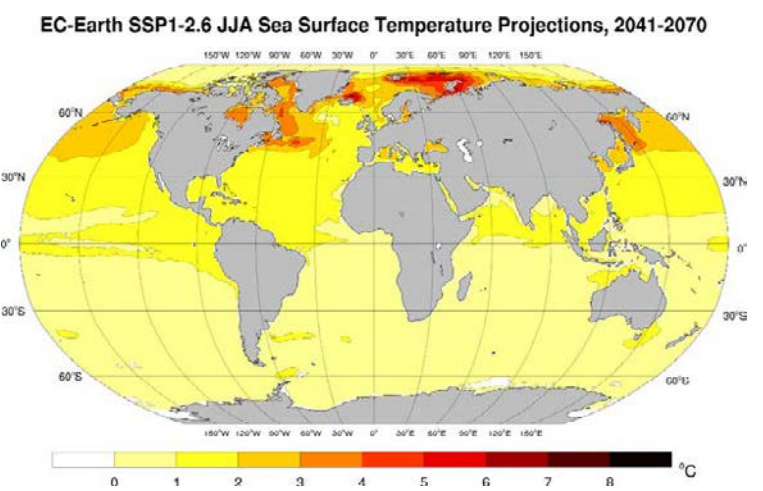

(c)

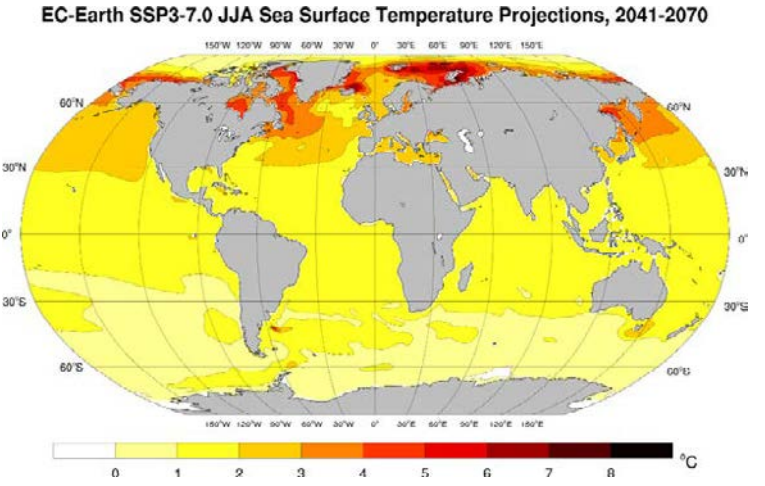

(b)

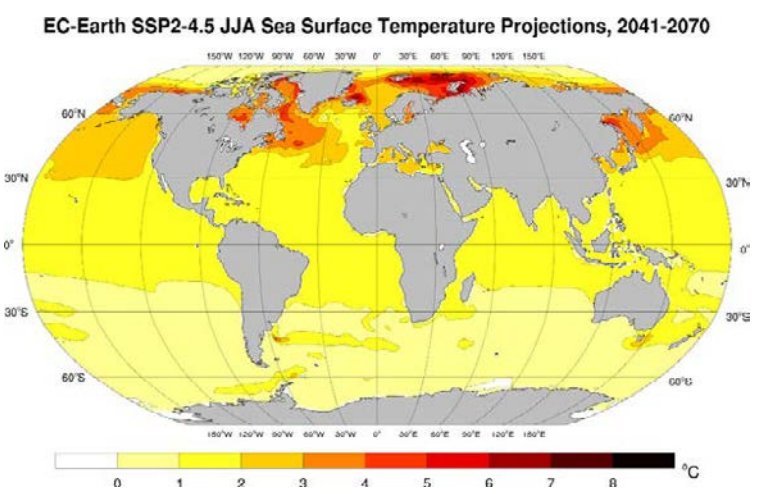

(d)

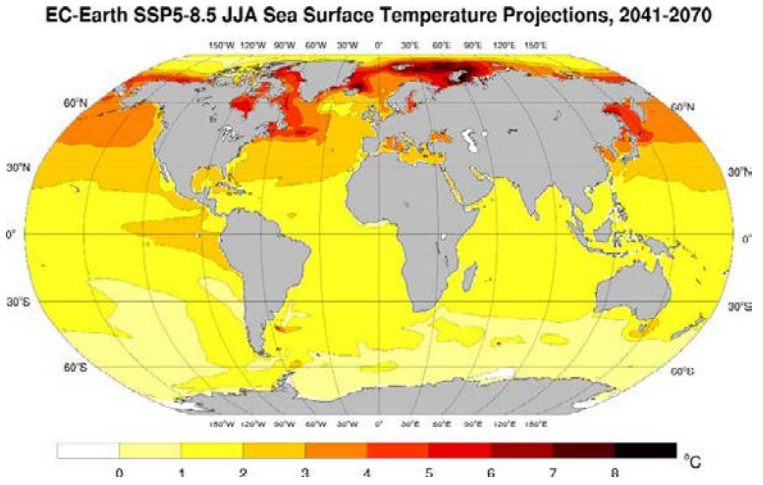

Figure 3.53. EC-Earth JJA SST projections (2041-2070 vs $1981-2010,{ }^{\circ} \mathrm{C}$ change); (a) SSP1-2.6, (b) SSP2-4.5, (c) SSP3-7.0 and (d) SSP5-8.5. In each case, an average is taken of the ensemble members r6i1p1f1, r9i1p1f1, r11i1p1f1, r13i1p1f1 and r15i1p1f1.

(a)

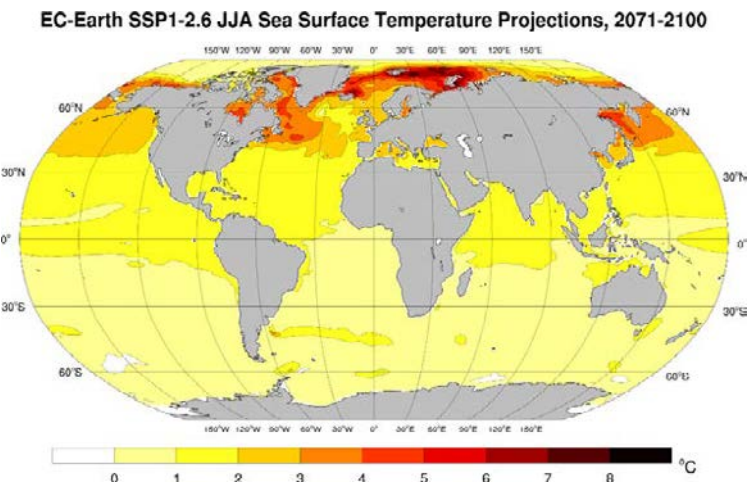

(c)

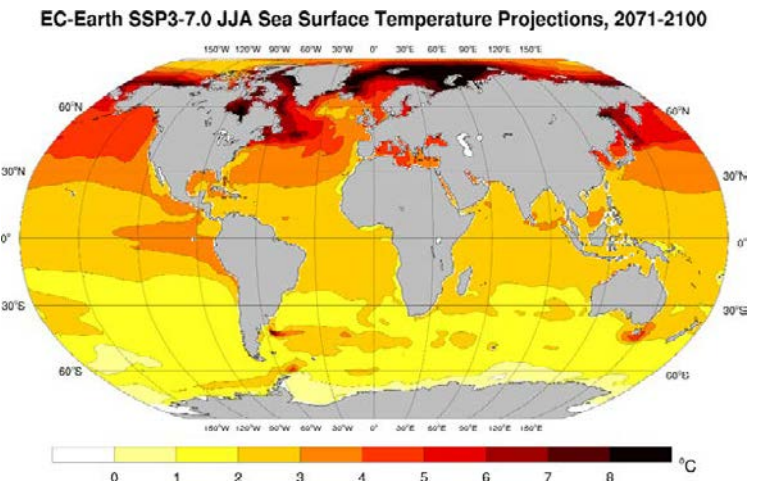

(b)

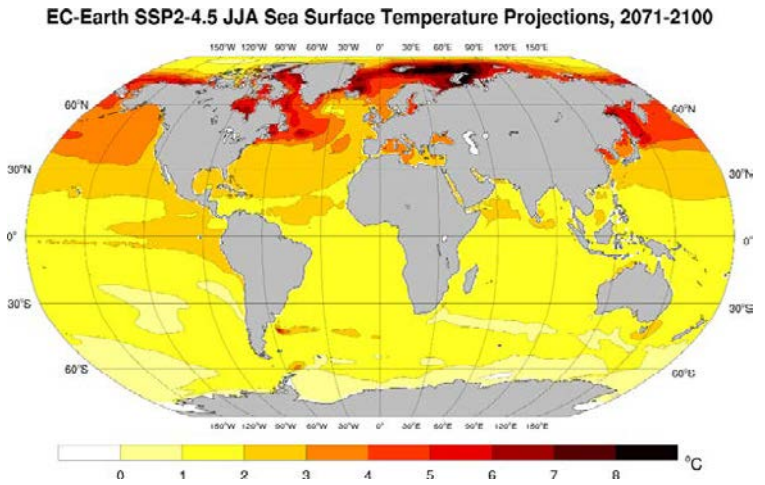

(d)

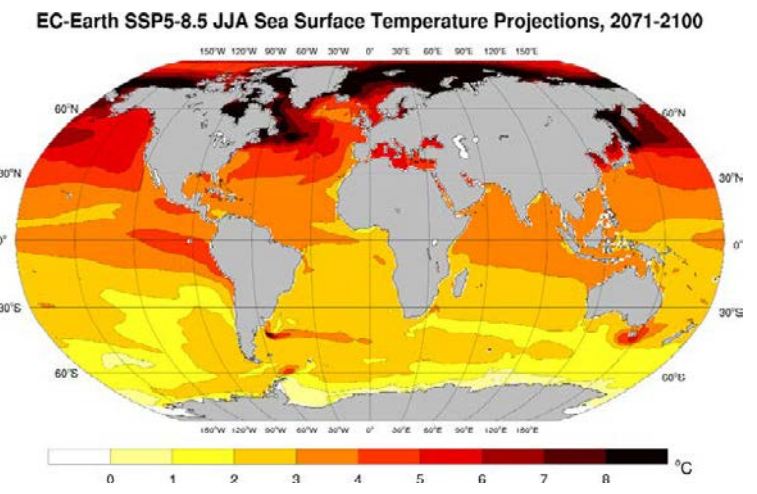

Figure 3.54. EC-Earth JJA SST projections (2071-2100 vs $1981-2010{ }^{\circ} \mathrm{C}$ change): (a) SSP1-2.6, (b) SSP2-4.5, (c) SSP3-7.0 and (d) SSP5-8.5. In each case, an average is taken of the ensemble members r6i1p1f1, r9i1p1f1, r11i1p1f1, r13i1p1f1 and r15i1p1f1. 


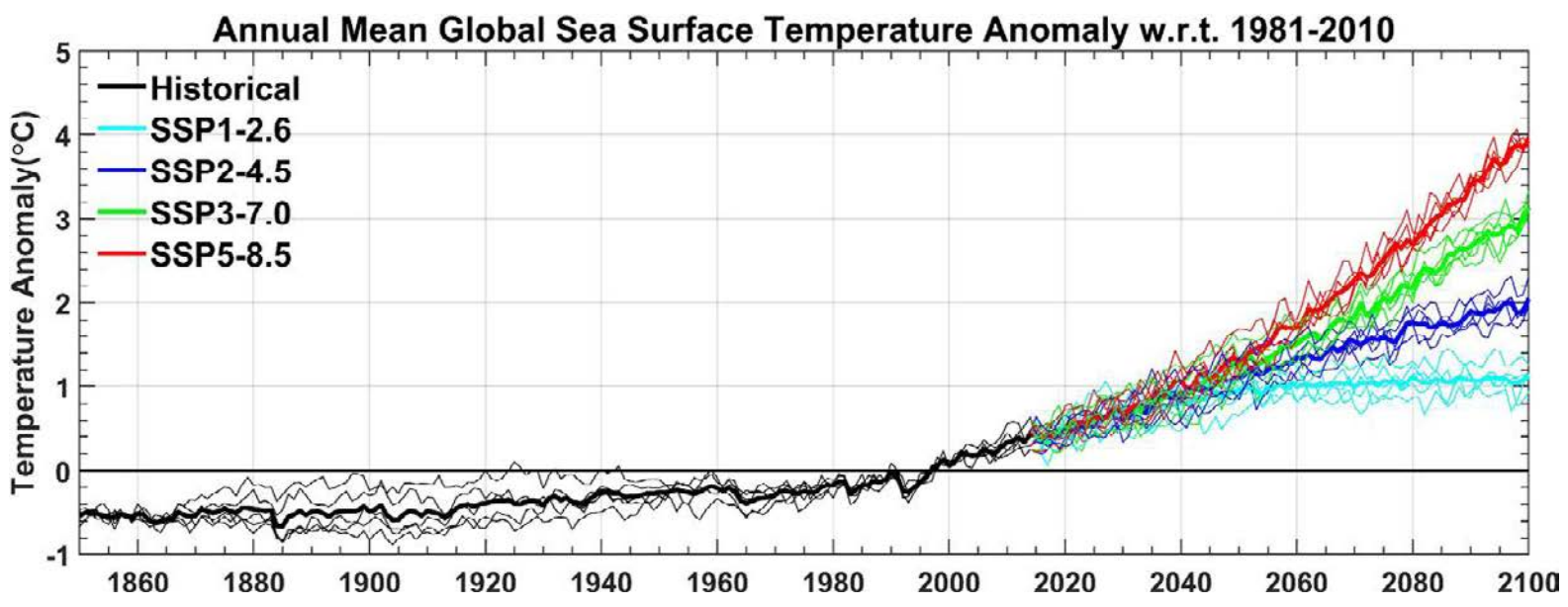

Figure 3.55. Global annual SST anomalies with respect to the 30-year period 1981-2010: EC-Earth ensemble members r6i1p1f1, r9i1p1f1, r11i1p1f1, r13i1p1f1 and r15i1p1f1. The bold lines represent the ensemble mean.

(a)

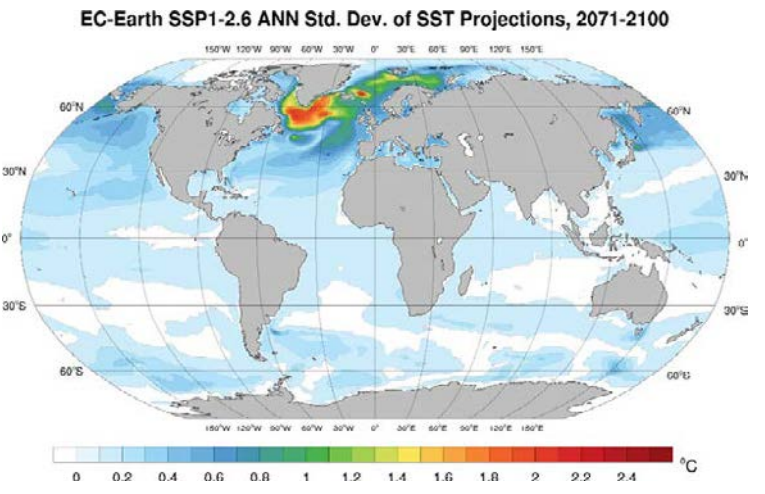

(c)

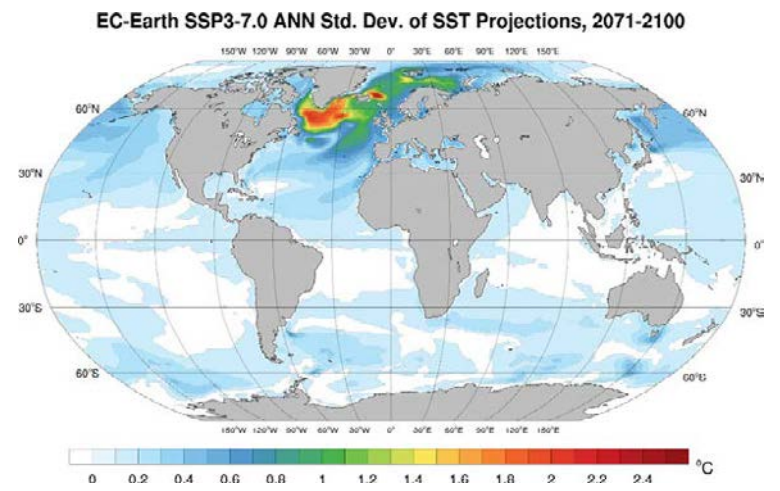

(b)

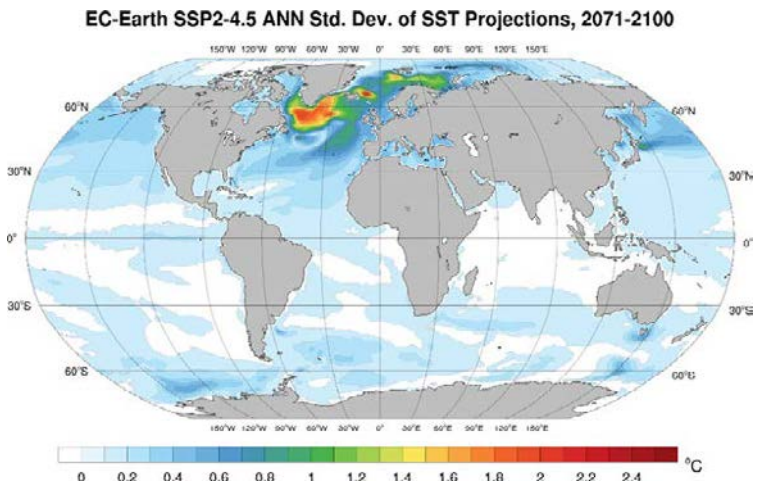

(d)

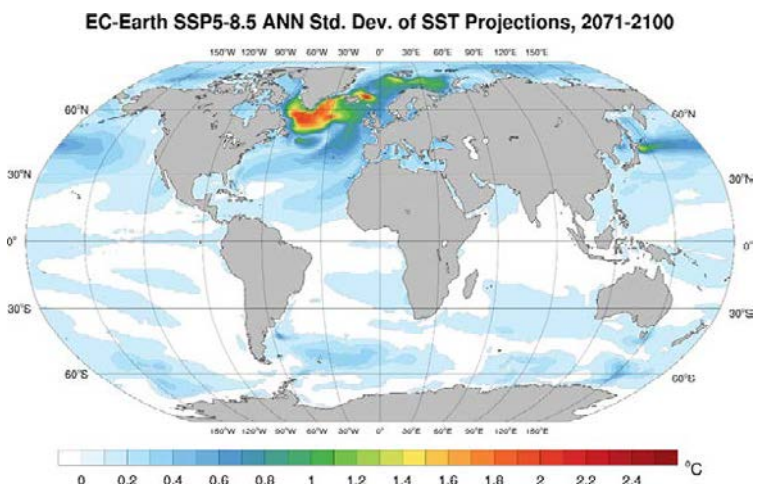

Figure 3.56. Standard deviation of the ensemble of annual SST projections (2071-2100): (a) SSP1-2.6, (b) SSP2-4.5, (c) SSP3-7.0 and (d) SSP5-8.5. 


\subsection{Sea Ice Projections}

\subsubsection{Northern Hemisphere sea ice projections}

Figure 3.57 presents the Northern Hemisphere annual mean sea ice fraction for the historical ensemble (1981-2010) and each of the four SSPs (2041-2070). The projected annual anomalies (\%) for 2041-2070 are presented in Figure 3.58. The corresponding data for 2071-2100 are presented in Figures 3.59 and 3.60. The results show large projected decreases in sea ice in terms of both extent and fraction.

The corresponding results for the Northern Hemisphere March sea ice fraction are presented in Figure 3.61 (sea ice fraction; historical ensemble
1981-2010 and 2041-2070), Figure 3.62 (sea ice anomalies; 2041-2070), Figure 3.63 (sea ice fraction; 2071-2100) and Figure 3.64 (sea ice anomalies; 2071-2100). Although substantial decreases are projected for the March Northern Hemisphere sea ice extent, the changes are smaller than those in the annual (and September) projections.

The projected changes for the Northern Hemisphere September sea ice fraction are substantial.

Figures 3.65 and 3.66 show large decreases for all SSPs, with the Arctic projected to be nearly ice free under SSP3-7.0 and SSP5-8.5 by 2041-2070. For the period 2071-2100 all SSPs except SSP1-2.6 (Figure 3.67) project an ice-free Arctic during September. 
(a)

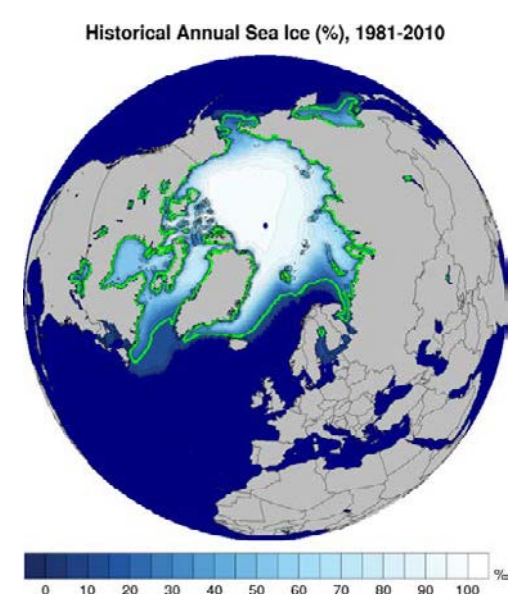

(c)

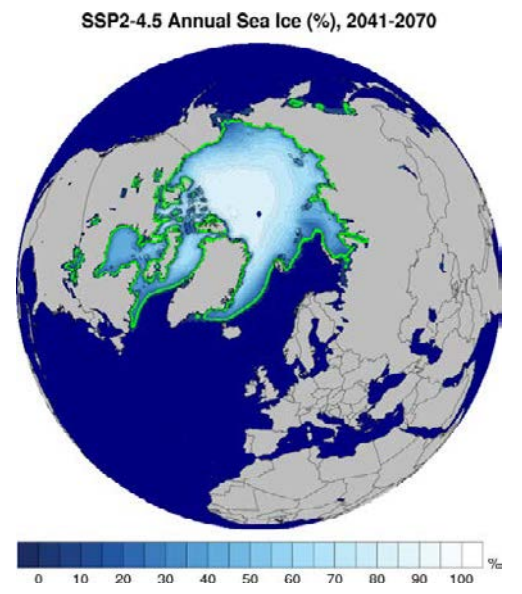

(e)

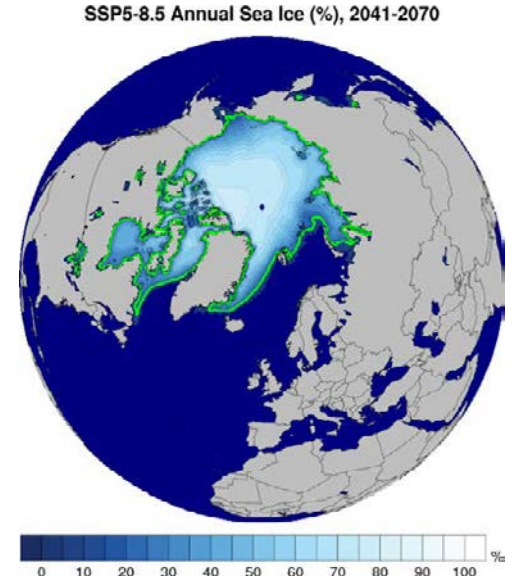

(b)
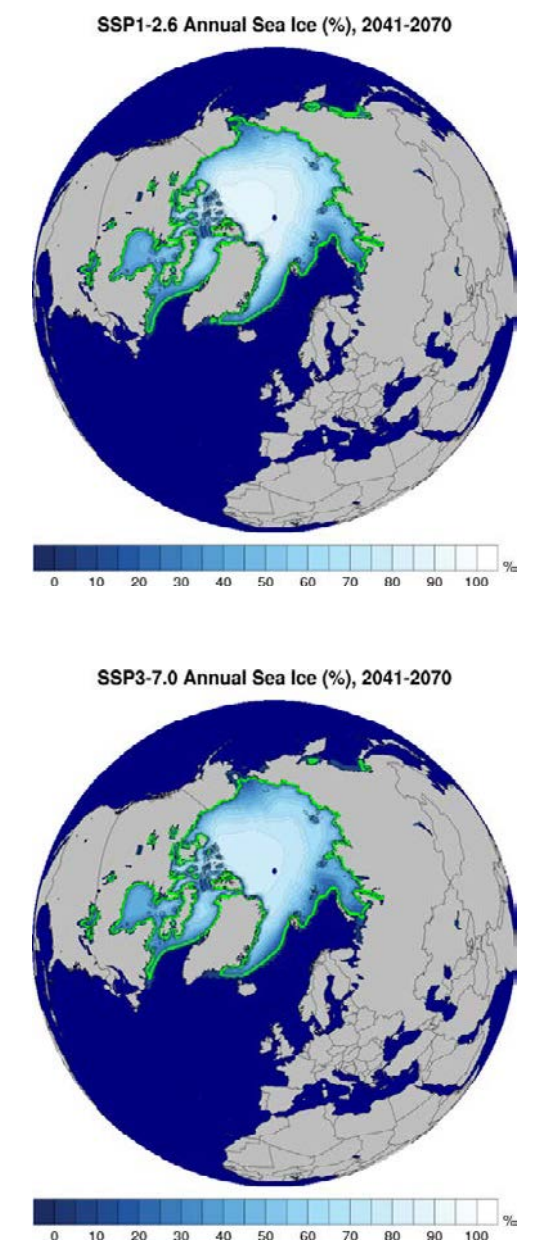

(b)

(d) 
(a)

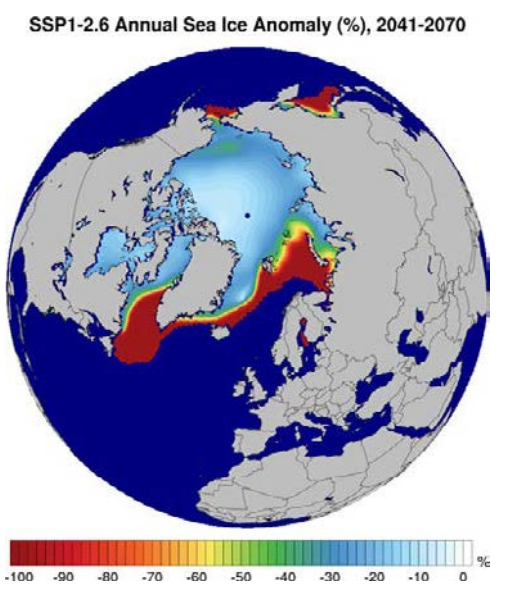

(c)

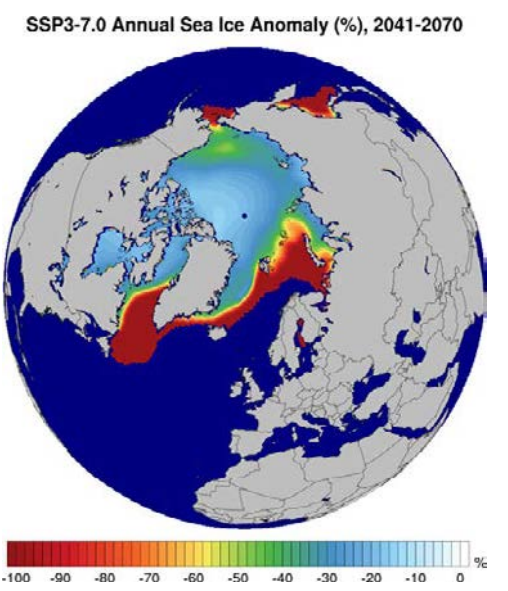

(b)

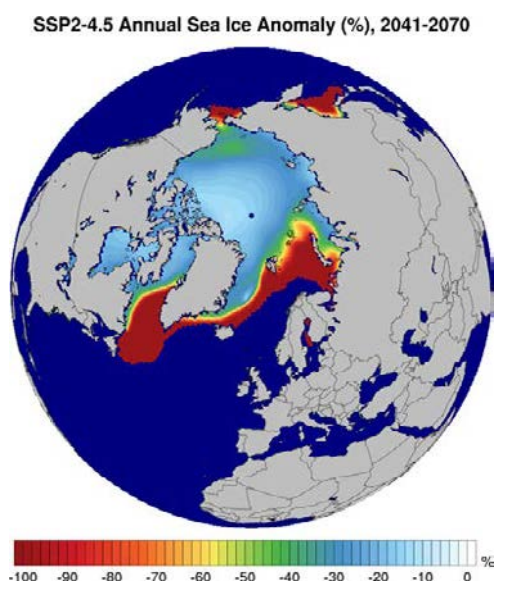

(d)

SSP5-8.5 Annual Sea Ice Anomaly (\%), 2041-2070

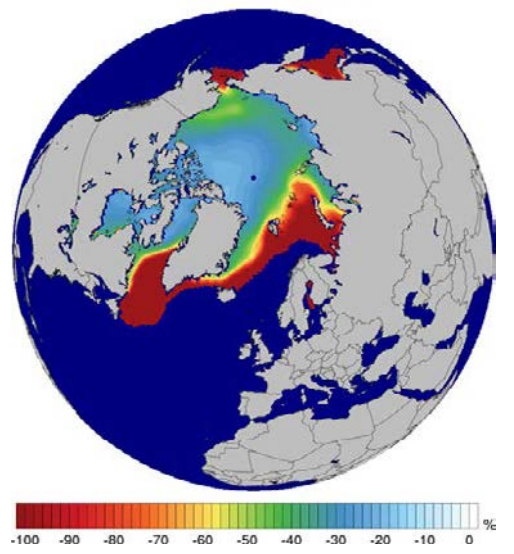

Figure 3.58. Annual Northern Hemisphere sea ice projections (\% change) (2041-2070 vs 1981-2010): (a) SSP1-2.6, (b) SSP2-4.5, (c) SSP3-7.0 and (d) SSP5-8.5. In each case, an average is taken of the ensemble members r6i1p1f1, r9i1p1f1, r11i1p1f1, r13i1p1f1 and r15i1p1f1. 
(a)

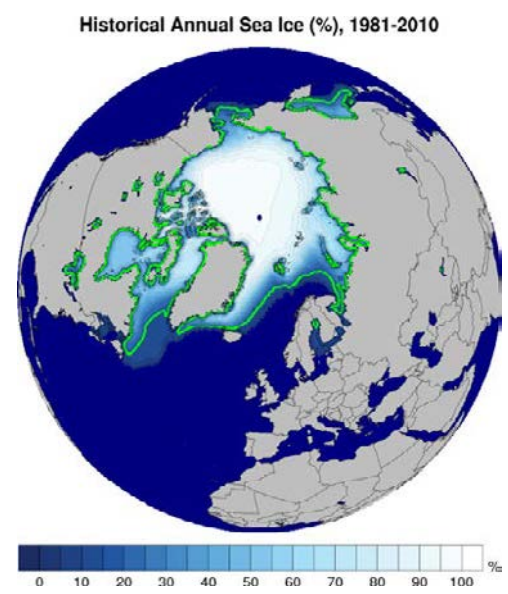

(c)

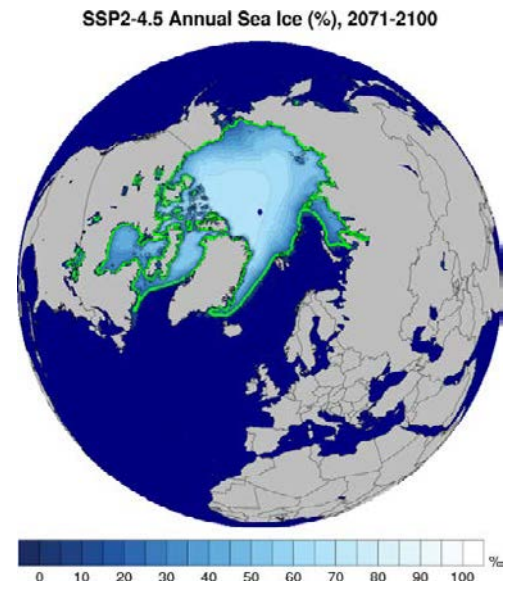

(e)

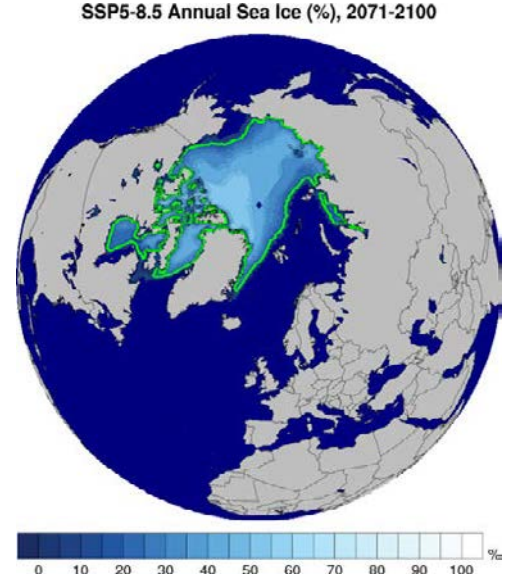

(b)

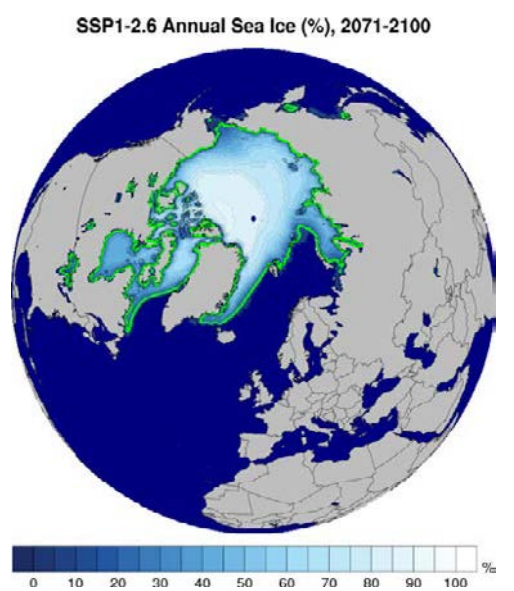

(d)

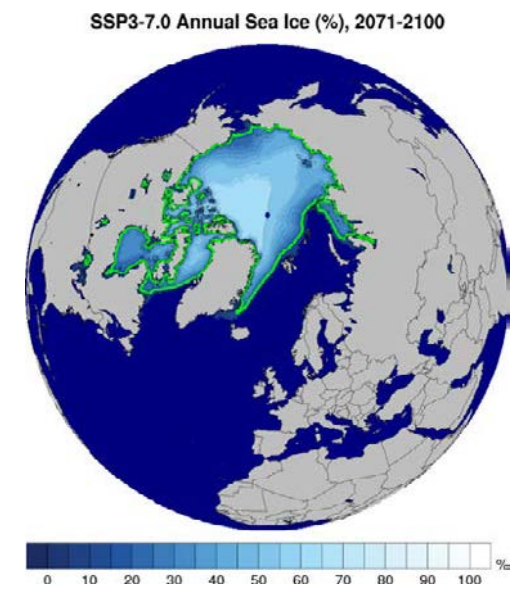

Figure 3.59. Annual mean Northern Hemisphere sea ice fraction (\%): (a) historical ensemble for the period 1981-2010 and (b) SSP1-2.6, (c) SSP2-4.5, (d) SSP3-7.0 and (e) SSP5-8.5 for the period 2071-2100. In each case, an average is taken of the ensemble members r6i1p1f1, r9i1p1f1, r11i1p1f1, r13i1p1f1 and r15i1p1f1. The green line shows the $15 \%$ contour line of the sea ice fraction. 
(a)

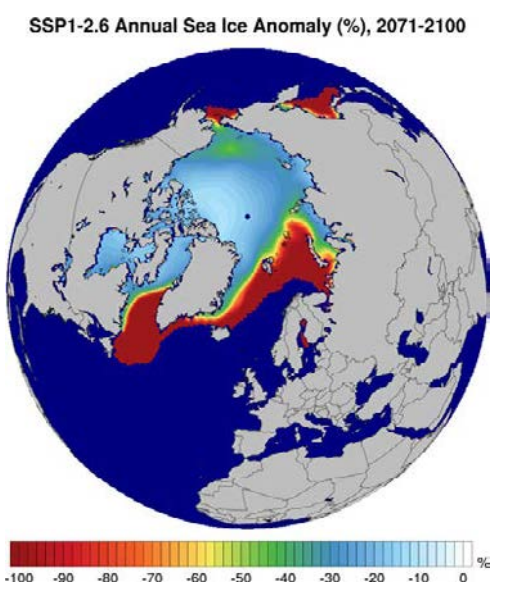

(c)

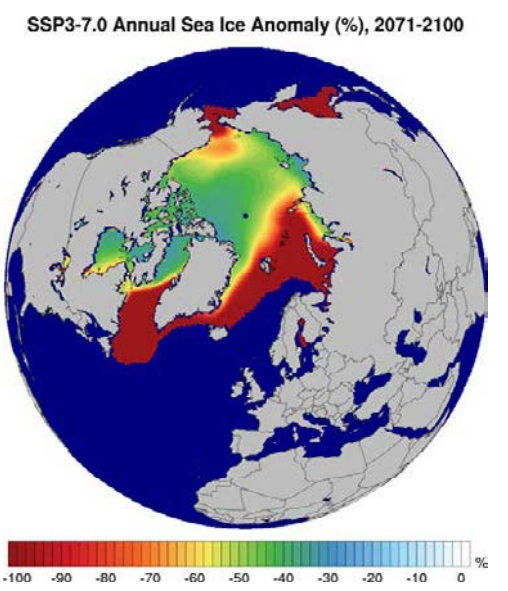

(b)

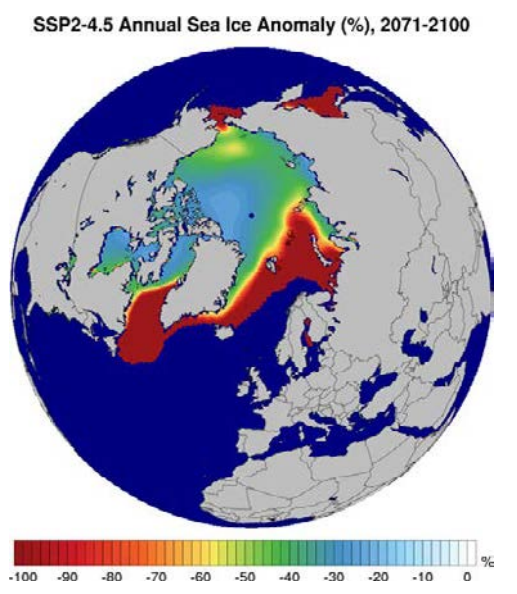

(d)

SSP5-8.5 Annual Sea Ice Anomaly (\%), 2071-2100

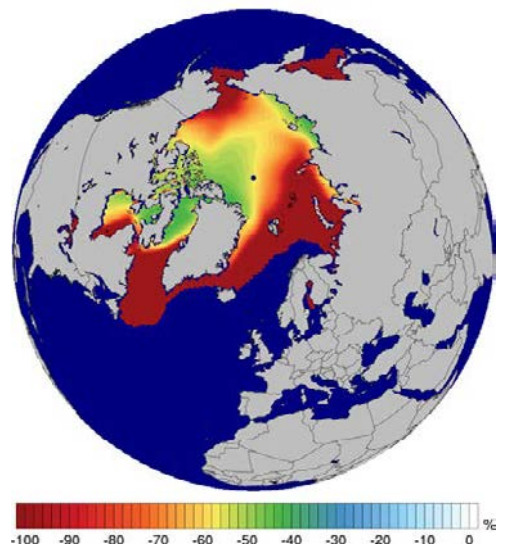

Figure 3.60. Annual Northern Hemisphere sea ice projections (\% change) (2071-2100 vs 1981-2010): (a) SSP1-2.6, (b) SSP2-4.5, (c) SSP3-7.0 and (d) SSP5-8.5. In each case, an average is taken of the ensemble members r6i1p1f1, r9i1p1f1, r11i1p1f1, r13i1p1f1 and r15i1p1f1. 
(a)

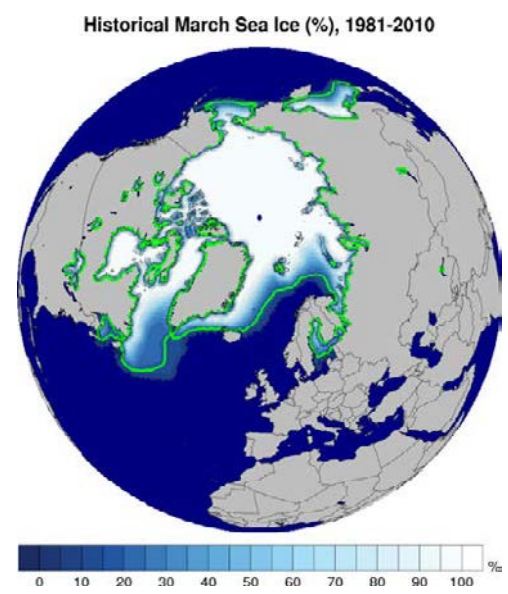

(c)

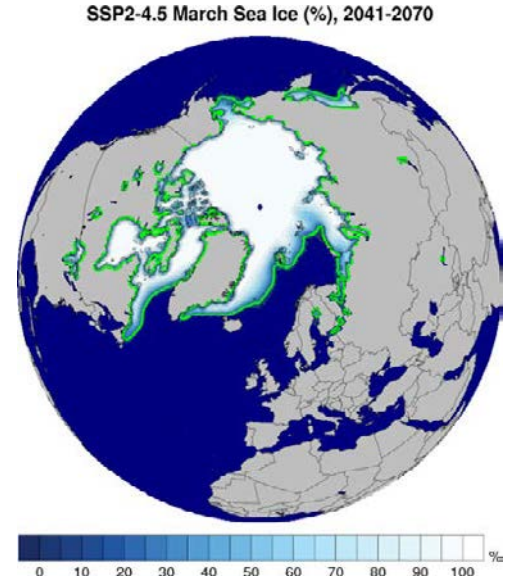

(e)

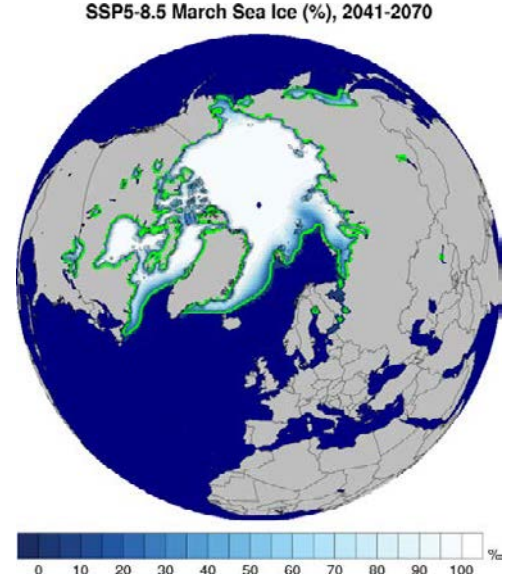

(b)

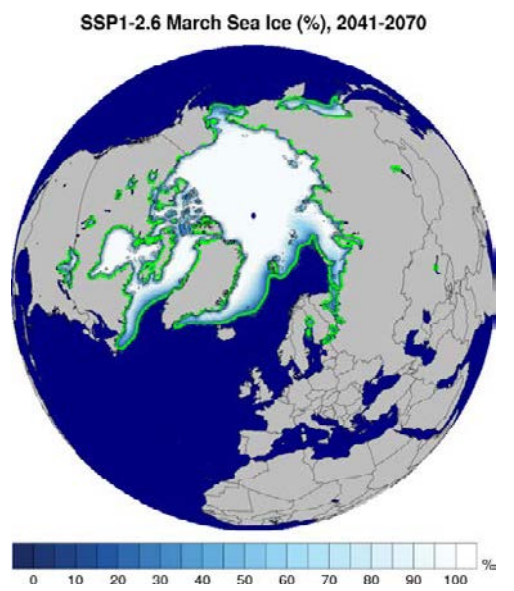

(d)

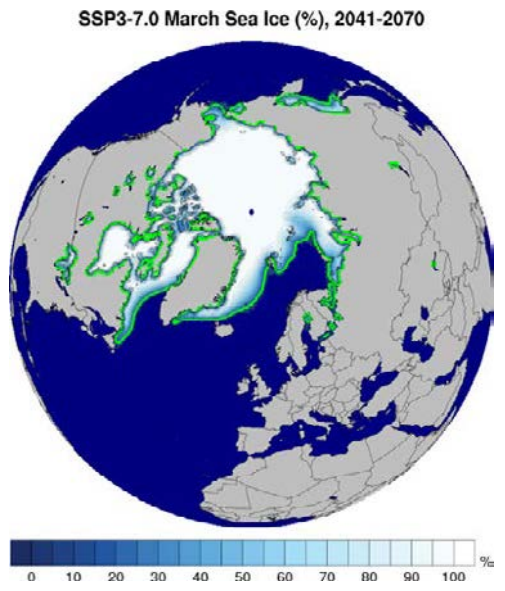

Figure 3.61. Mean Northern Hemisphere March sea ice fraction (\%): (a) historical ensemble for the period 1981-2010 and (b) SSP1-2.6, (c) SSP2-4.5, (d) SSP3-7.0 and (e) SSP5-8.5 for the period 2041-2070. In each case, an average is taken of the ensemble members r6i1p1f1, r9i1p1f1, r11i1p1f1, r13i1p1f1 and r15i1p1f1. The green line shows the $15 \%$ contour line of the sea ice fraction. 
(a)

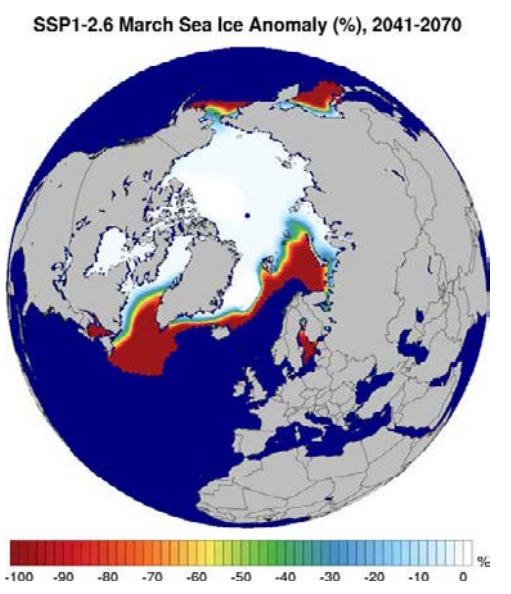

(c)

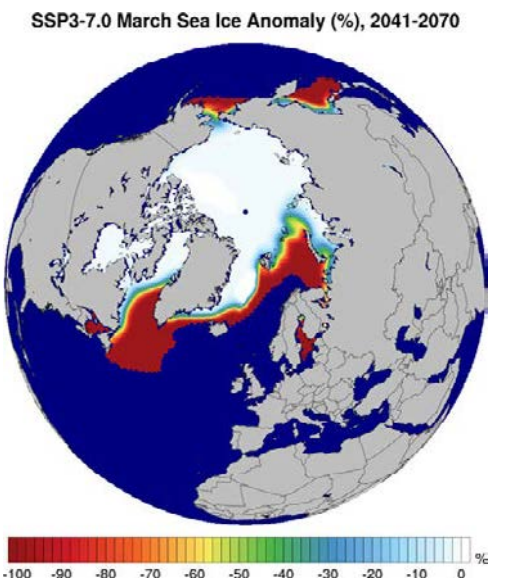

(b)

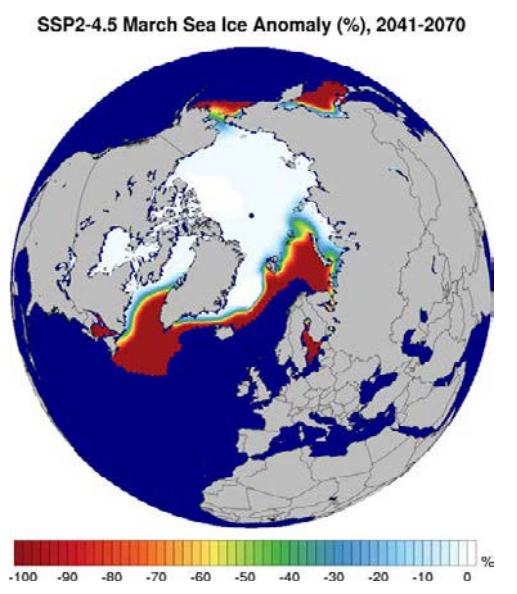

(d)

SSP5-8.5 March Sea Ice Anomaly (\%), 2041-2070

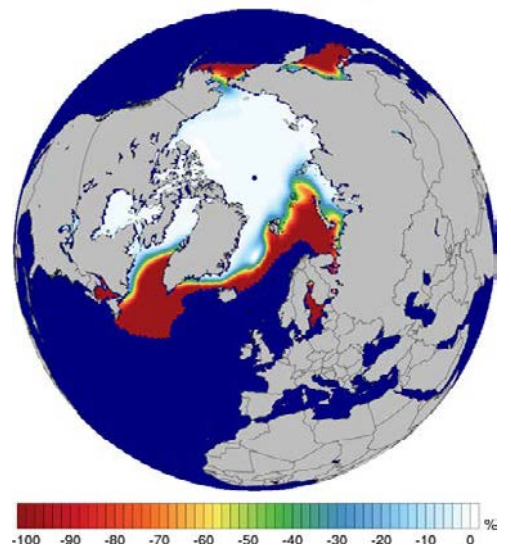

Figure 3.62. Northern Hemisphere March sea ice projections (\% change) (2041-2070 vs 1981-2010): (a) SSP1-2.6, (b) SSP2-4.5, (c) SSP3-7.0 and (d) SSP5-8.5. In each case, an average is taken of the ensemble members r6i1p1f1, r9i1p1f1, r11i1p1f1, r13i1p1f1 and r15i1p1f1. 
(a)

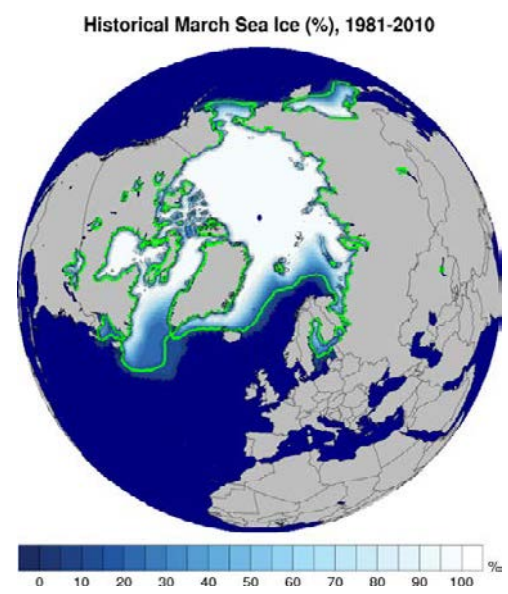

(c)

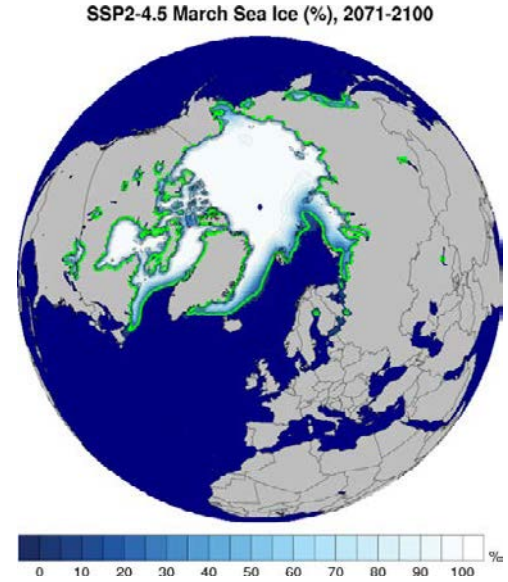

(e)

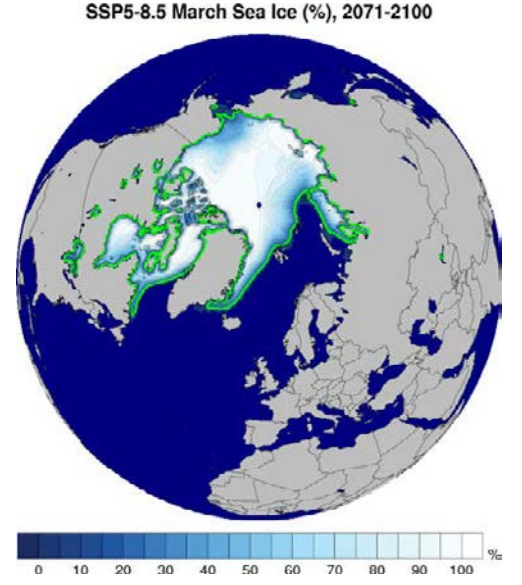

(b)

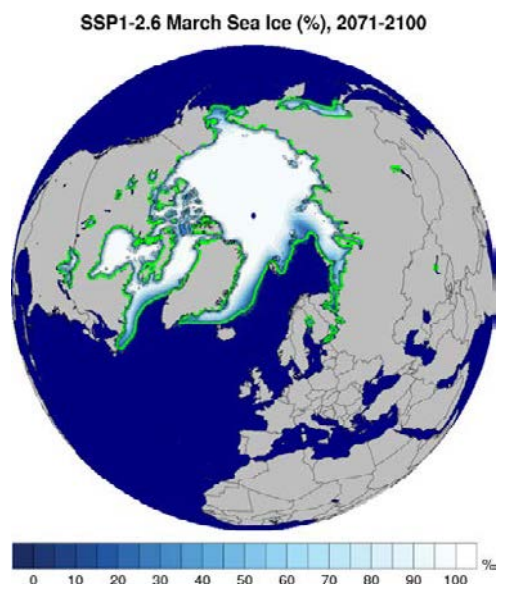

(d)

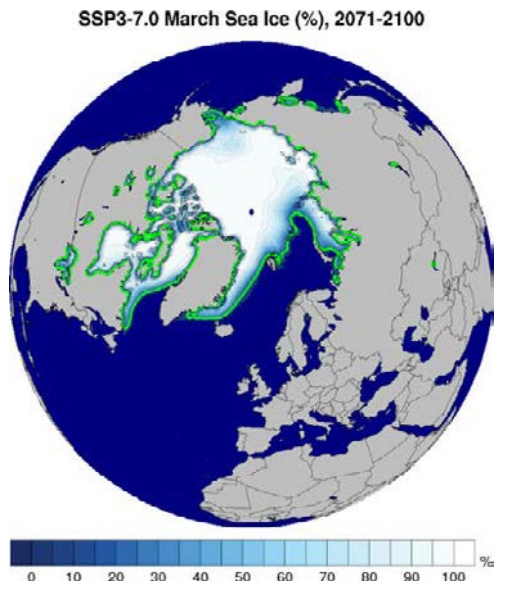

Figure 3.63. Mean Northern Hemisphere March sea ice fraction (\%): (a) historical ensemble for the period 1981-2010 and (b) SSP1-2.6, (c) SSP2-4.5, (d) SSP3-7.0 and (e) SSP5-8.5 for the period 2071-2100. In each case, an average is taken of the ensemble members r6i1p1f1, r9i1p1f1, r11ip1f1, r13i1p1f1 and r15i1p1f1. The green line shows the $15 \%$ contour line of the sea ice fraction. 
(a)

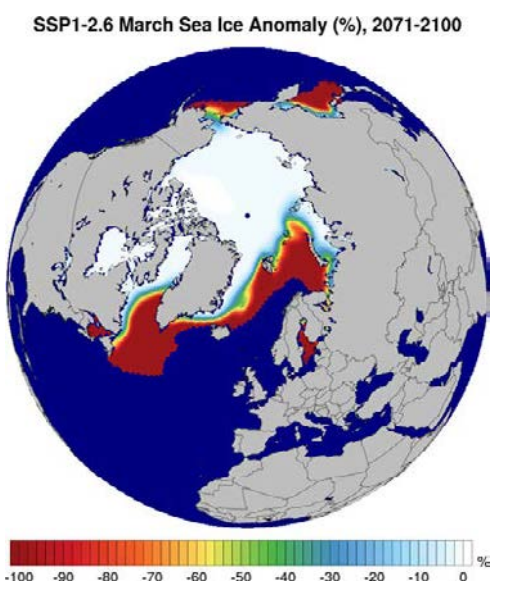

(c)

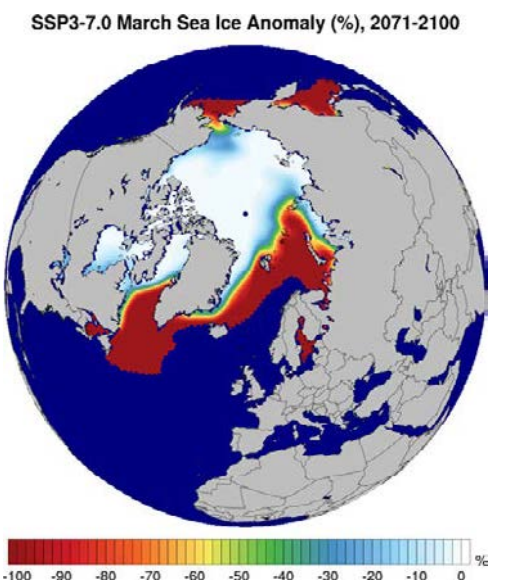

(b)

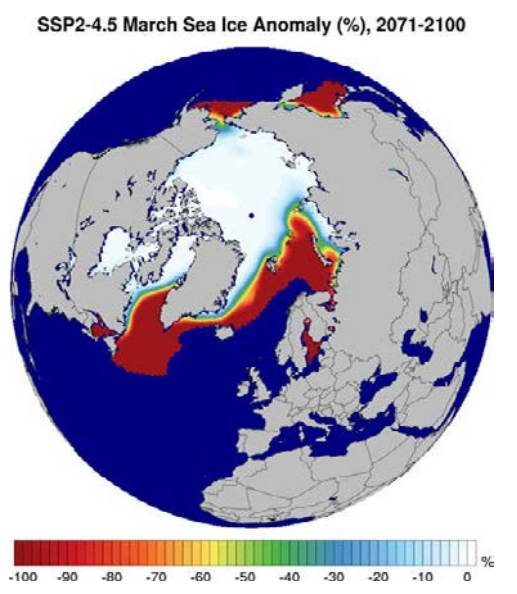

(d)

SSP5-8.5 March Sea Ice Anomaly (\%), 2071-2100

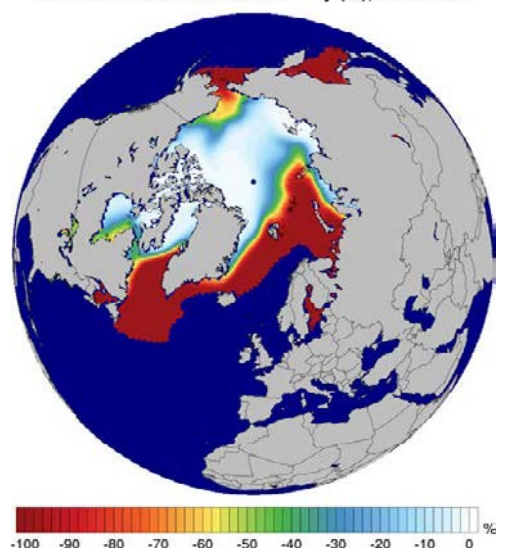

Figure 3.64. Northern Hemisphere March sea ice projections (\% change) (2071-2100 vs 1981-2010): (a) SSP1-2.6, (b) SSP2-4.5, (c) SSP3-7.0 and (d) SSP5-8.5. In each case, an average is taken of the ensemble members r6i1p1f1, r9i1p1f1, r11i1p1f1, r13i1p1f1 and r15i1p1f1. 
(a)

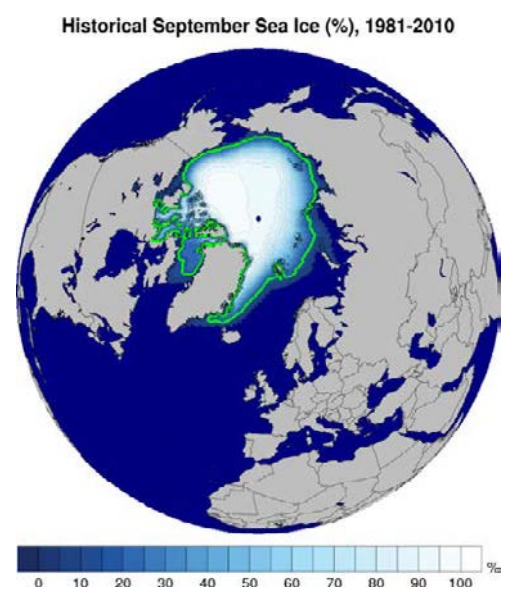

(c)

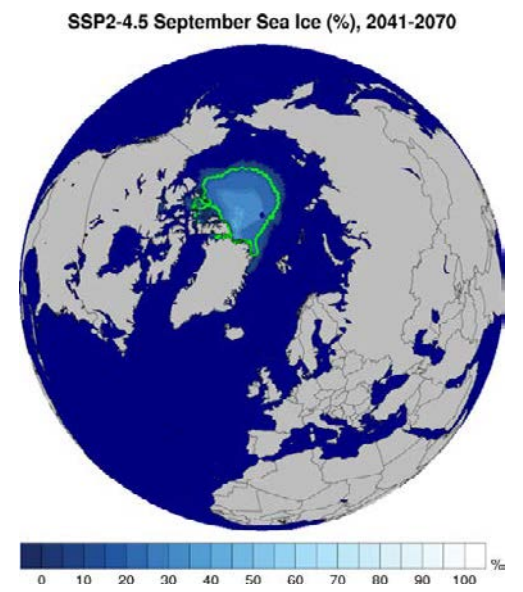

(e)

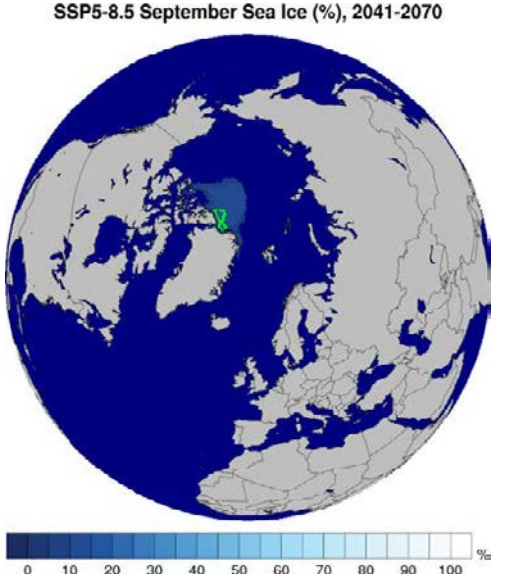

(b)

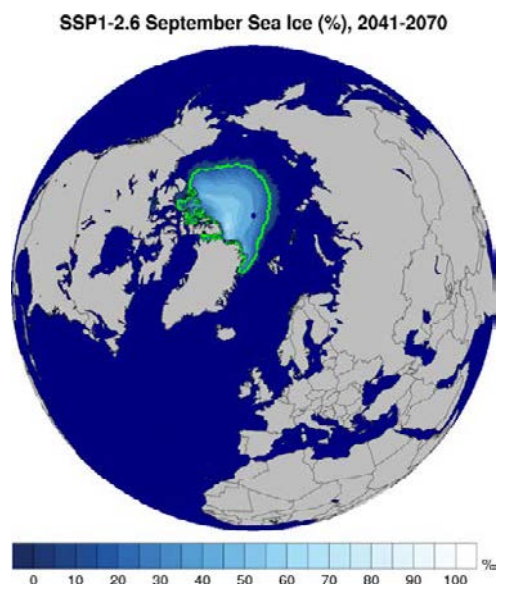

(d)

SSP3-7.0 September Sea Ice (\%), 2041-2070

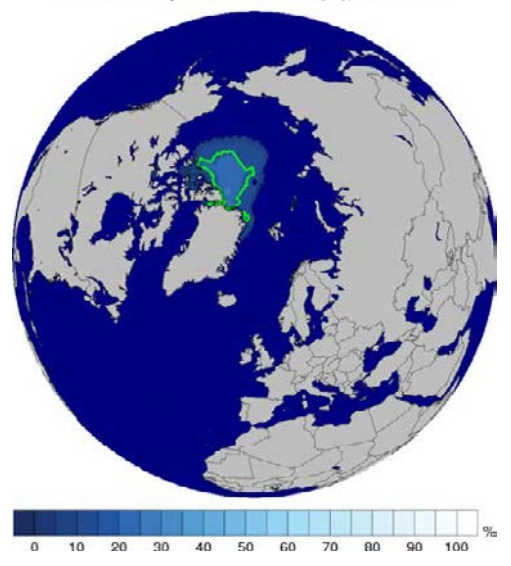

Figure 3.65. Mean Northern Hemisphere September sea ice fraction (\%): (a) historical ensemble for the period 1981-2010 and (b) SSP1-2.6, (c) SSP2-4.5, (d) SSP3-7.0 and (e) SSP5-8.5 for the period 20412070. In each case, an average is taken of the ensemble members r6i1p1f1, r9i1p1f1, r11i1p1f1, r13i1p1f1 and $\mathrm{r} 15 \mathrm{i} 1 \mathrm{p} 1 \mathrm{f} 1$. The green line shows the $15 \%$ contour line of the sea ice fraction. 
(a)

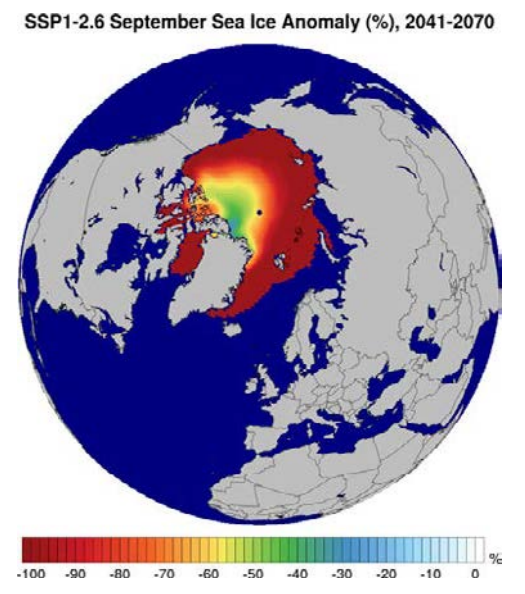

(c)

SSP3-7.0 September Sea Ice Anomaly (\%), 2041-2070

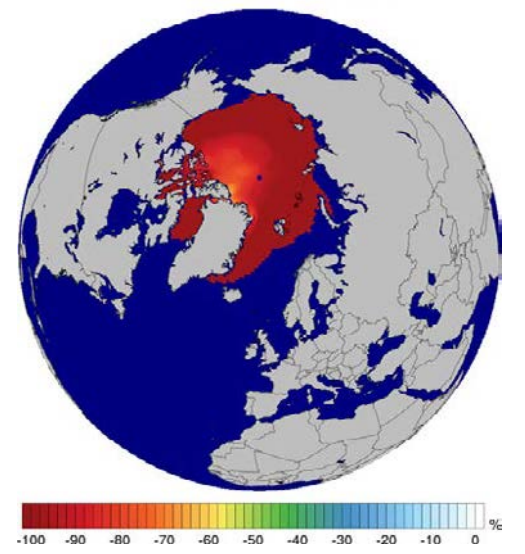

(b)

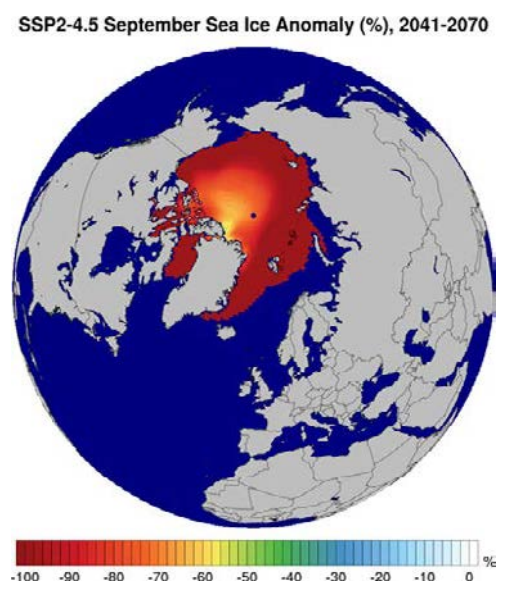

(d)

SSP5-8.5 September Sea Ice Anomaly (\%), 2041-2070

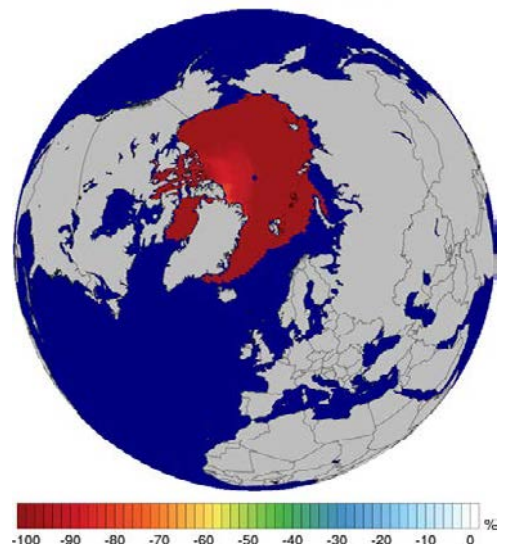

Figure 3.66. Northern Hemisphere September sea ice projections (\% change) (2041-2070 vs 1981-2010): (a) SSP1-2.6, (b) SSP2-4.5, (c) SSP3-7.0 and (d) SSP5-8.5. In each case, an average is taken of the ensemble members r6i1p1f1, r9i1p1f1, r11i1p1f1, r13i1p1f1 and r15i1p1f1. 
(a)

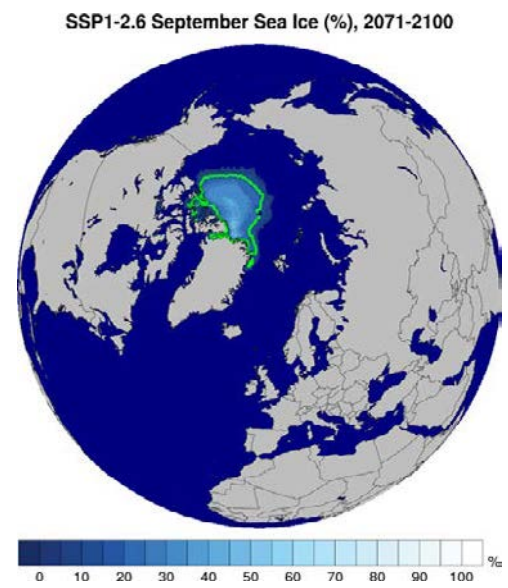

(b)

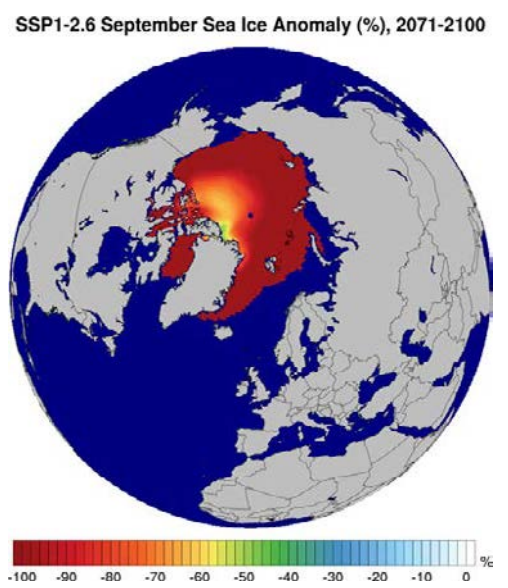

Figure 3.67. Northern Hemisphere September sea ice projections, 2071-2100: (a) SSP1-2.6 sea ice fraction (\%) with green line showing the $15 \%$ contour line and (b) SSP1-2.6 anomaly relative to 1981-2010 (\% change). In each case, an average is taken of the ensemble members r6i1p1f1, r9i1p1f1, r11i1p1f1, r13i1p1f1 and r15i1p1f1.

\subsubsection{Southern Hemisphere sea ice projections}

Similarly, Southern Hemisphere sea ice fraction projections are presented in Figures 3.68-3.78. In summary, these projections show substantial decreases in sea ice fraction over the full year (Figures 3.68-3.71). By 2071-2100, the Southern Ocean is projected to be nearly ice free during March under SSP1-2.6 and SSP2-4.5 (Figure 3.74) and completely ice free under SSP3-7.0 and SSP5-8.5. Although substantial decreases are projected for the Southern Hemisphere September sea ice extent (Figures 3.75-3.78), the changes are smaller than those in the annual and March projections.

\subsubsection{Sea ice projection annual time series}

The mean global annual sea ice anomalies (relative to 1981-2010) for all five historical simulations (1850-2014) and 20 SSPs (2015-2100) are presented in Figure 3.79a. The bold lines represent the ensemble means. All ensemble members show a steady decrease in global sea ice from around 2000. By the end of the century, global mean sea ice is projected to decrease by approximately $30 \%, 50 \%, 70 \%$ and $85 \%$ for SSP1-2.6, SSP2-4.5, SSP3-7.0 and SSP5-8.5, respectively. The Northern and Southern Hemisphere annual sea ice anomalies (Figures 3.79b and 3.79c, respectively) are similar to the global trend, although a larger spread between ensemble members is evident in the Southern Hemisphere. 
(a)

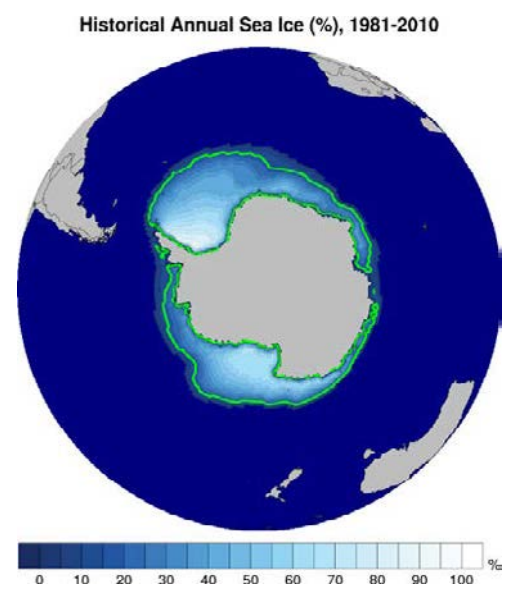

(c)

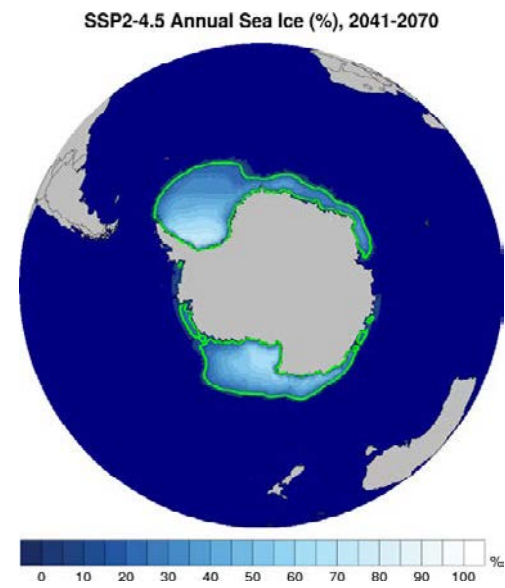

(e)

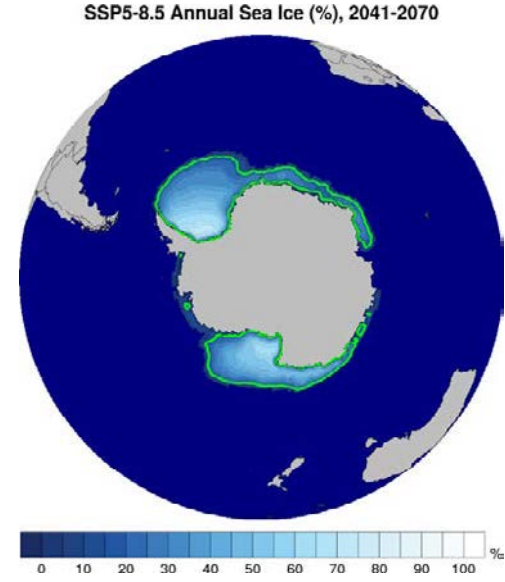

(b)

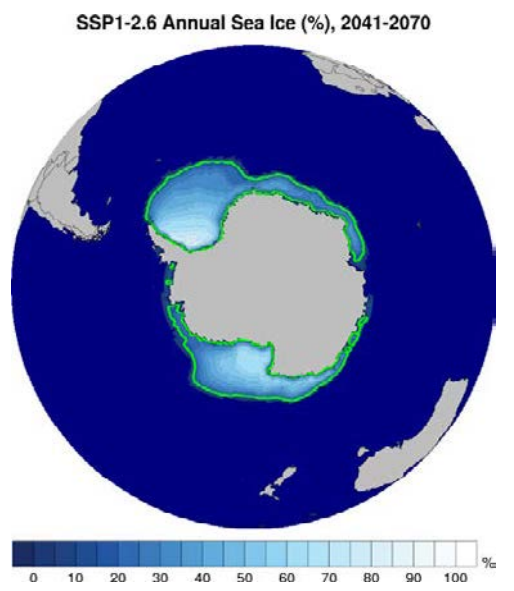

(d)

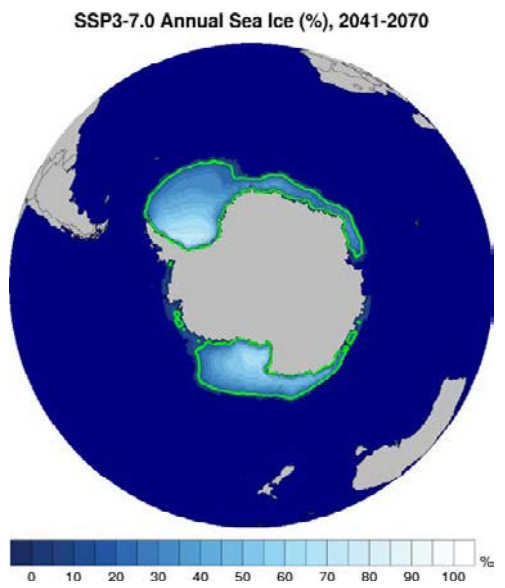

Figure 3.68. Annual mean Southern Hemisphere sea ice fraction (\%): (a) historical ensemble for the period 1981-2010 and (b) SSP1-2.6, (c) SSP2-4.5, (d) SSP3-7.0 and (e) SSP5-8.5 for the period 20412070. In each case, an average is taken of the ensemble members r6i1p1f1, r9i1p1f1, r11i1p1f1, r13i1p1f1 and $\mathrm{r} 15 \mathrm{i} 1 \mathrm{p} 1 \mathrm{f} 1$. The green line shows the $15 \%$ contour line of the sea ice fraction. 
(a)

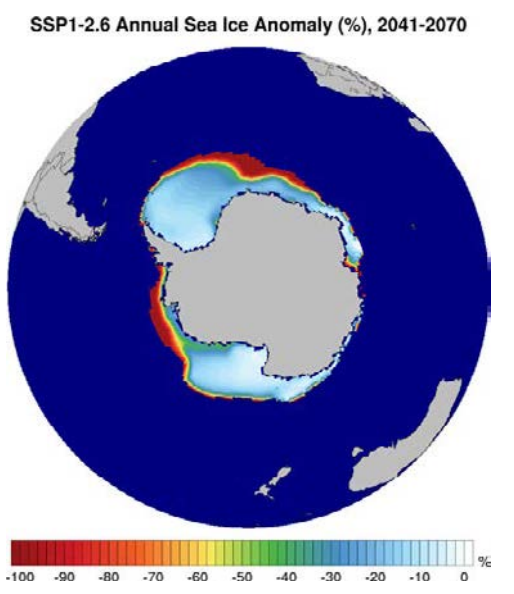

(c)

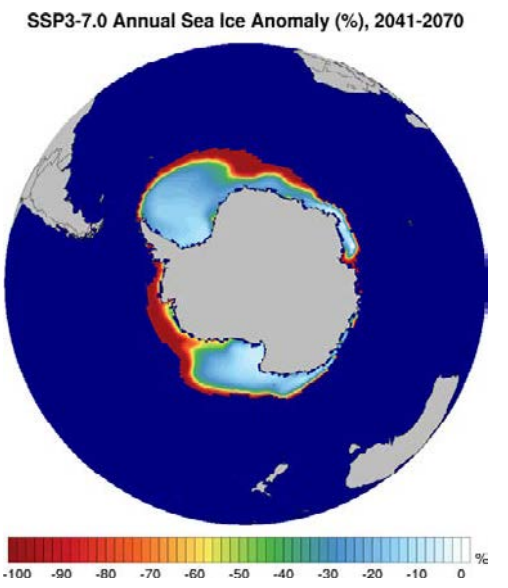

(b)

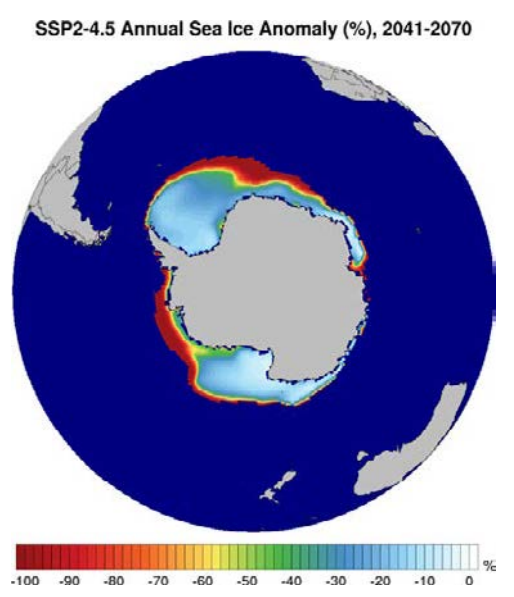

(d)

SSP5-8.5 Annual Sea Ice Anomaly (\%), 2041-2070

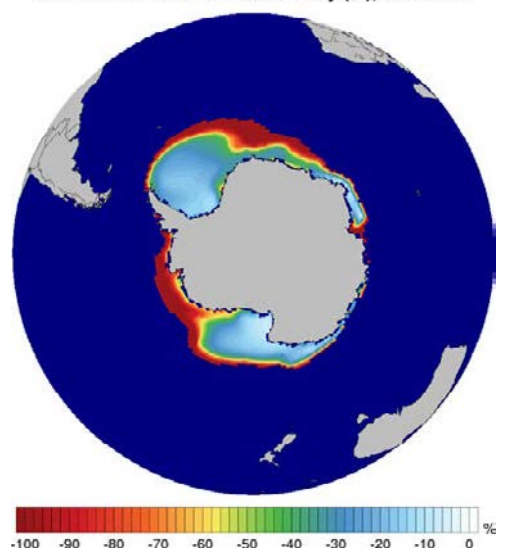

Figure 3.69. Annual Southern Hemisphere sea ice projections (\% change) (2041-2070 vs 1981-2010): (a) SSP1-2.6, (b) SSP2-4.5, (c) SSP3-7.0 and (d) SSP5-8.5. In each case, an average is taken of the ensemble members r6i1p1f1, r9i1p1f1, r11i1p1f1, r13i1p1f1 and r15i1p1f1. 
(a)

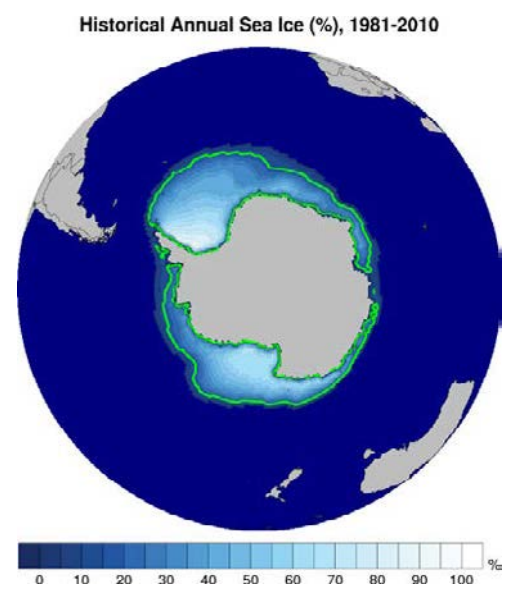

(c)

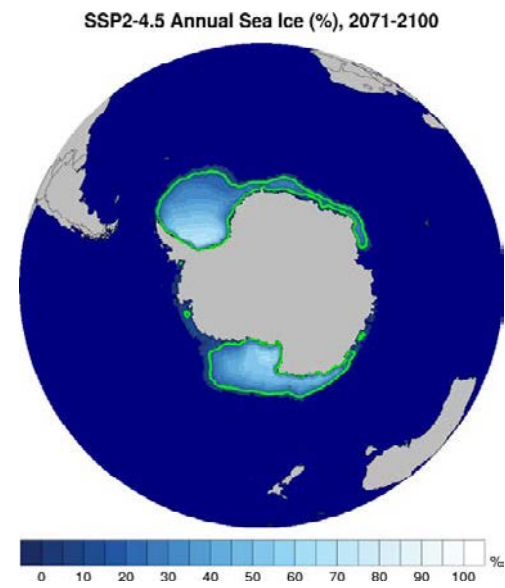

(e)

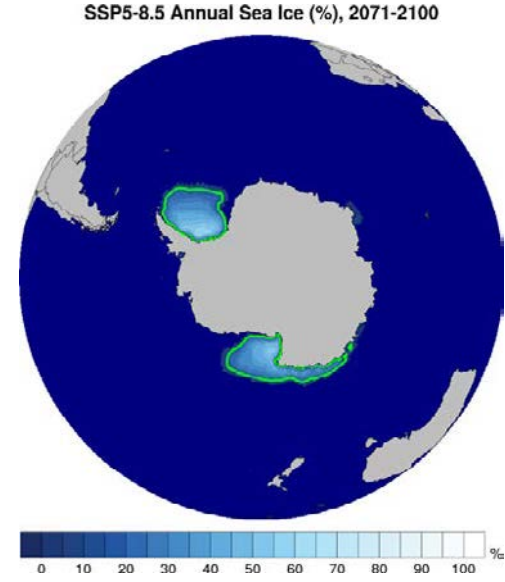

(b)

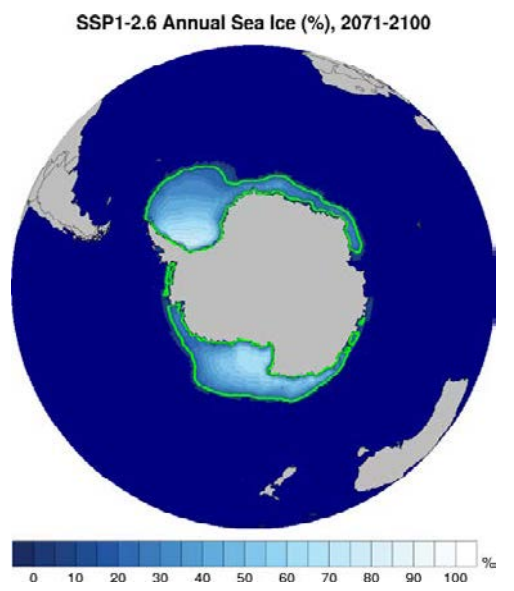

(d)

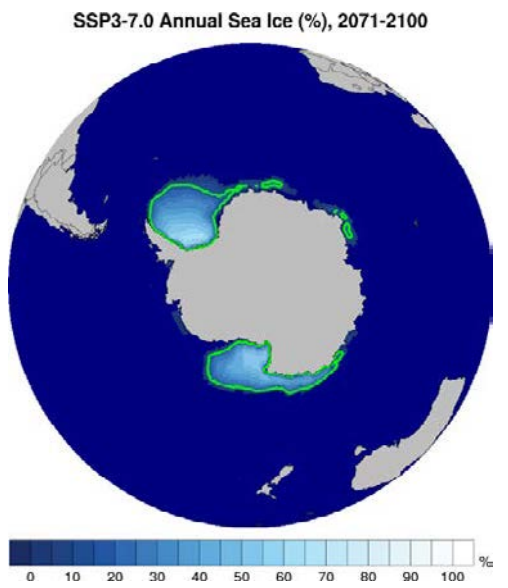

Figure 3.70. Annual mean Southern Hemisphere sea ice fraction (\%): (a) historical ensemble for the period 1981-2010 and (b) SSP1-2.6, (c) SSP2-4.5, (d) SSP3-7.0 and (e) SSP5-8.5 for the period 20712100. In each case, an average is taken of the ensemble members r6i1p1f1, r9i1p1f1, r11i1p1f1, r13i1p1f1 and $\mathrm{r} 15 \mathrm{i} 1 \mathrm{p} 1 \mathrm{f} 1$. The green line shows the $15 \%$ contour line of the sea ice fraction. 
(a)

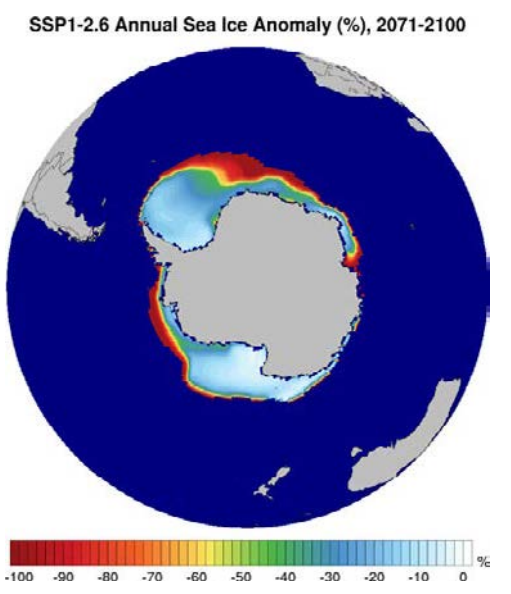

(c)

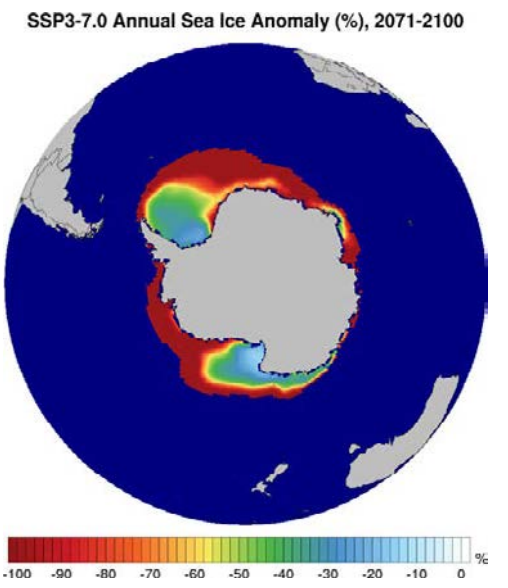

(b)

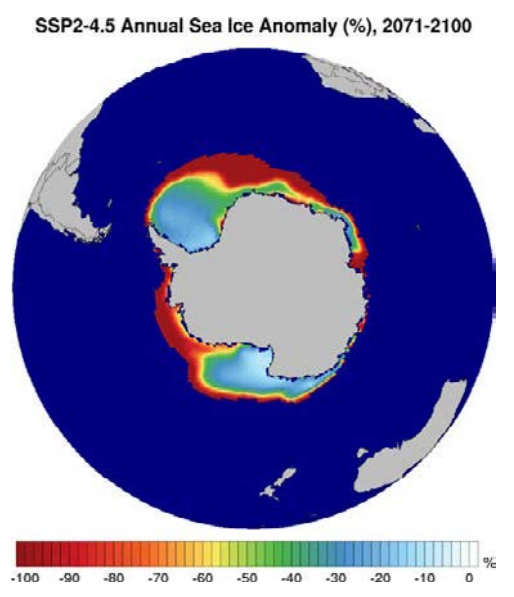

(d)

SSP5-8.5 Annual Sea Ice Anomaly (\%), 2071-2100

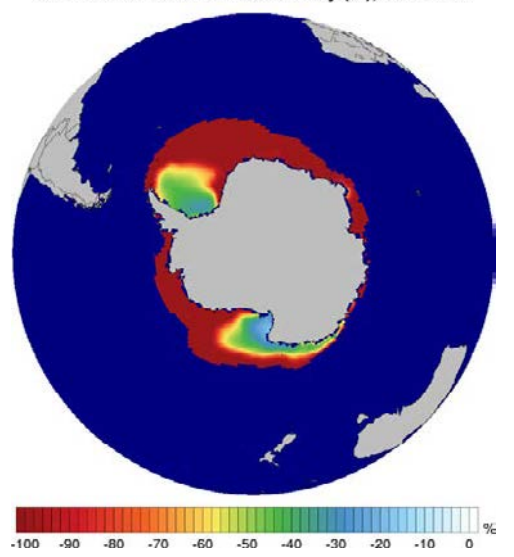

Figure 3.71. Annual Southern Hemisphere sea ice projections (\% change) (2071-2100 vs 1981-2010): (a) SSP1-2.6, (b) SSP2-4.5, (c) SSP3-7.0 and (d) SSP5-8.5. In each case, an average is taken of the ensemble members r6i1p1f1, r9i1p1f1, r11i1p1f1, r13i1p1f1 and r15i1p1f1. 
(a)

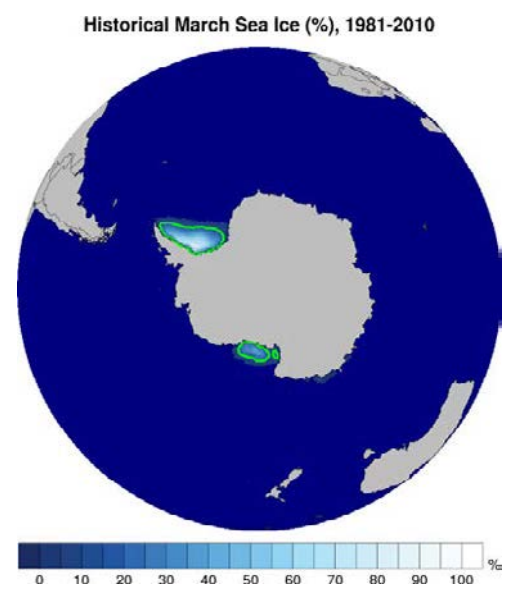

(c)

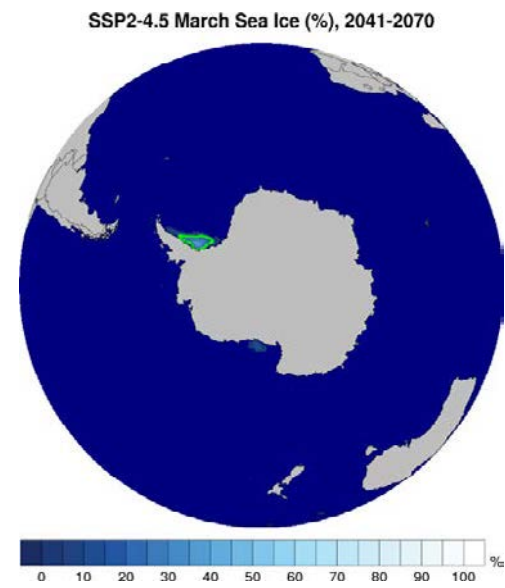

(e)

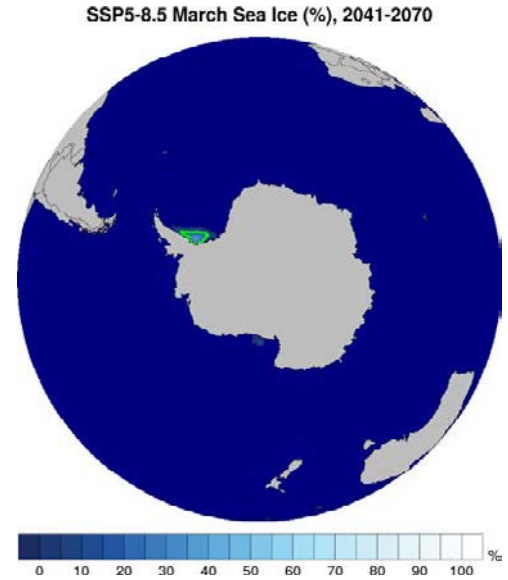

(b)

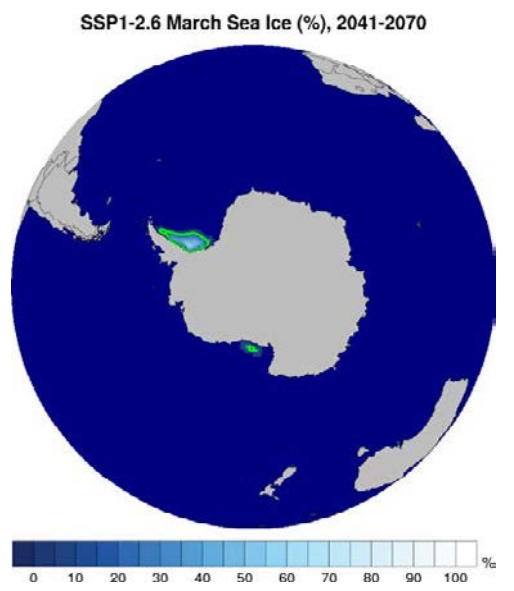

(d)

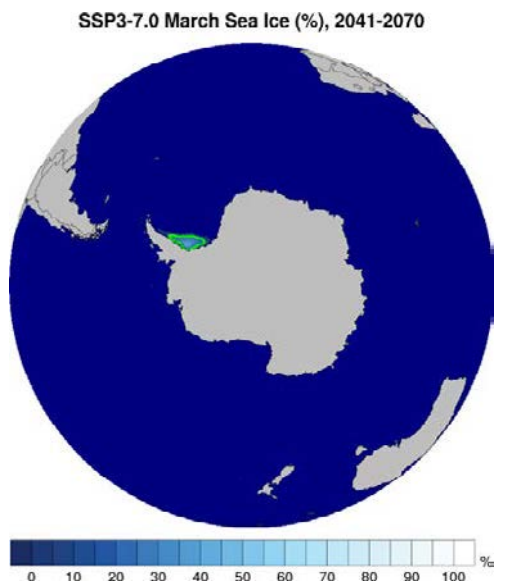

Figure 3.72. Mean Southern Hemisphere March sea ice fraction (\%): (a) historical ensemble for the period 1981-2010 and (b) SSP1-2.6, (c) SSP2-4.5, (d) SSP3-7.0 and (e) SSP5-8.5 for the period 2041-2070. In each case, an average is taken of the ensemble members r6i1p1f1, r9i1p1f1, r11i1p1f1, r13i1p1f1 and r15i1p1f1. The green line shows the $15 \%$ contour line of sea ice fraction. 
(a)

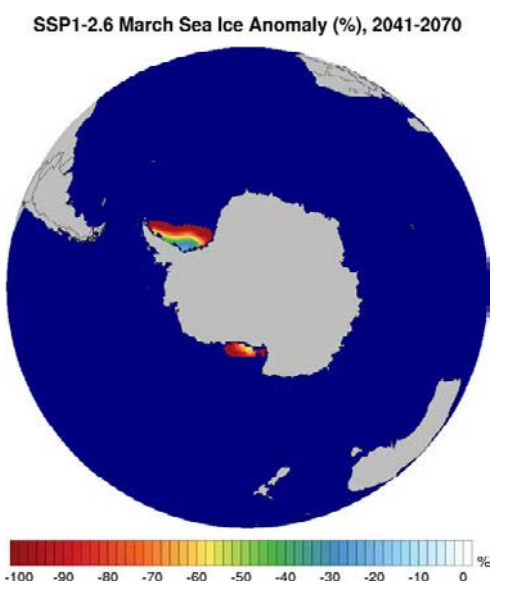

(c)

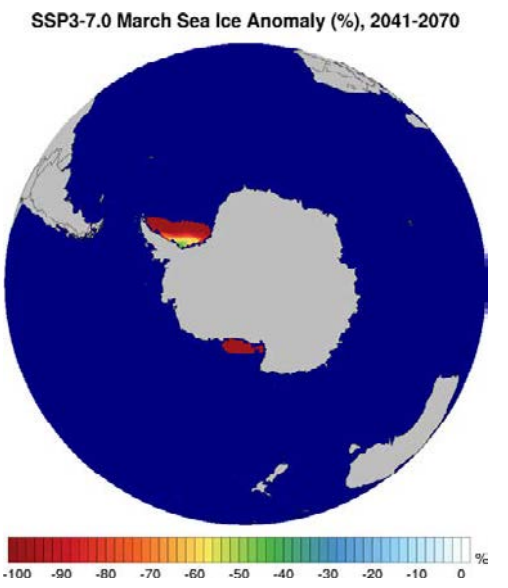

(b)

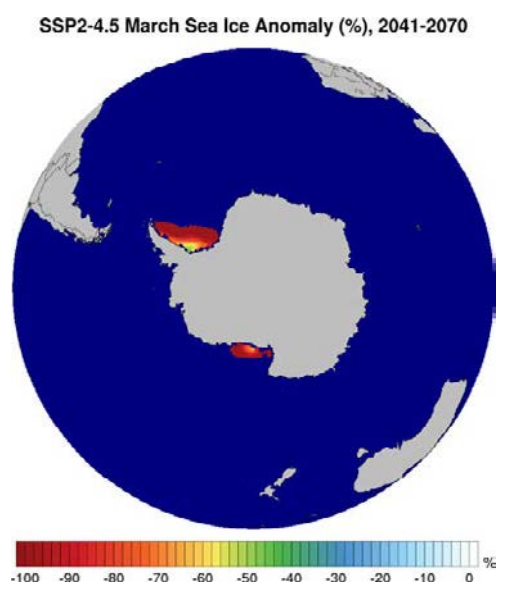

(d)

SSP5-8.5 March Sea Ice Anomaly (\%), 2041-2070

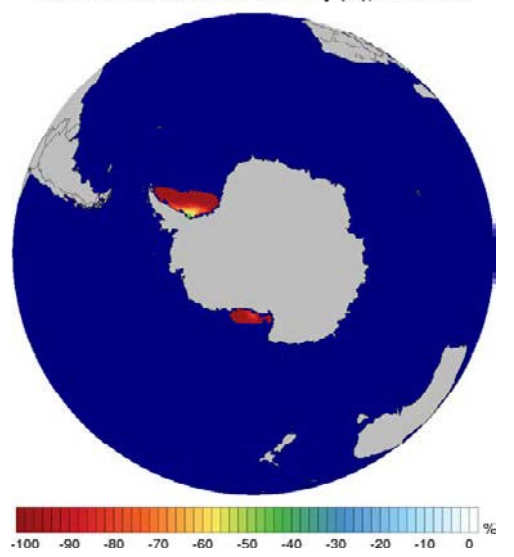

Figure 3.73. Southern Hemisphere March sea ice projections (\% change) (2041-2070 vs 1981-2010): (a) SSP1-2.6, (b) SSP2-4.5, (c) SSP3-7.0 and (d) SSP5-8.5. In each case, an average is taken of the ensemble members r6i1p1f1, r9i1p1f1, r11i1p1f1, r13i1p1f1 and r15i1p1f1. 
(a)

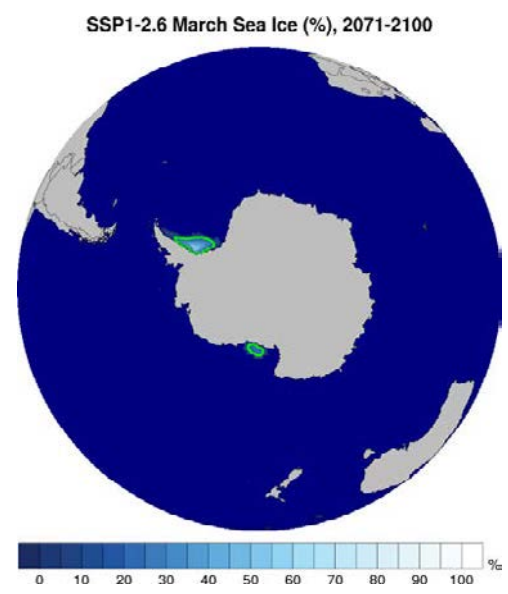

(c)

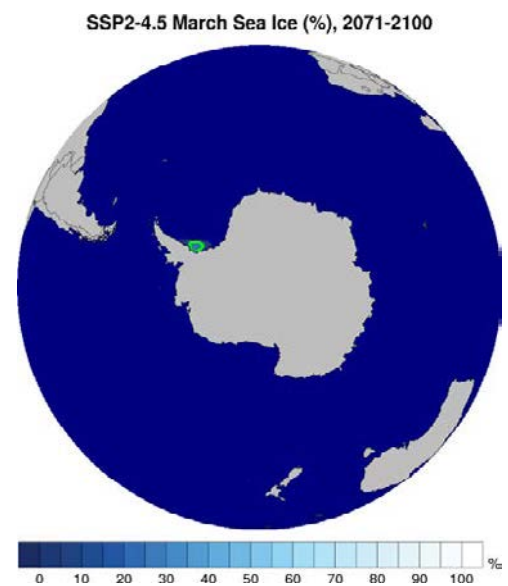

(b)

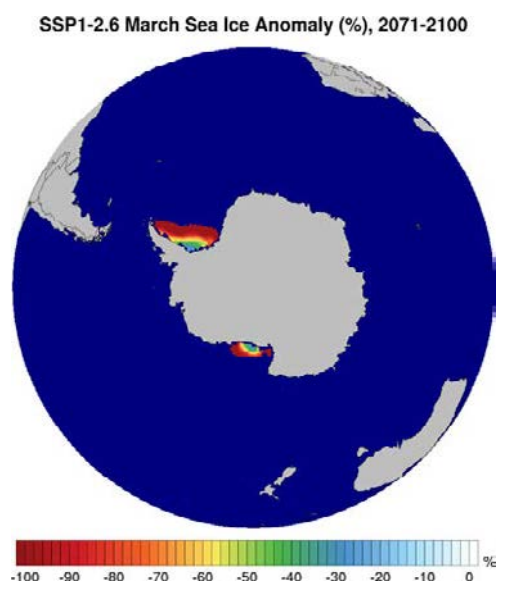

(d)

SSP2-4.5 March Sea Ice Anomaly (\%), 2071-2100

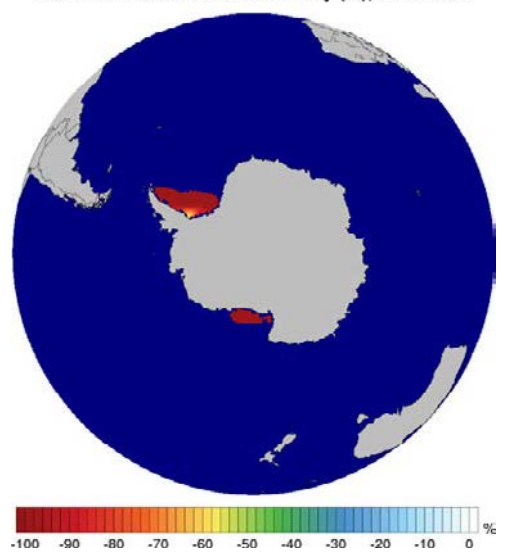

Figure 3.74. Mean Southern Hemisphere March sea ice projections, 2071-2100: (a) SSP1-2.6 sea ice fraction (\%) with green line showing the 15\% contour line, (b) SSP1-2.6 anomaly relative to 1981-2010 (\% change), (c) SSP2-4.5 sea ice fraction (\%) with green line showing the 15\% contour line, (d) SSP2-4.5 anomaly relative to $1981-2010$ (\% change). In each case, an average is taken of the ensemble members r6i1p1f1, r9i1p1f1, r11i1p1f1, r13i1p1f1 and r15i1p1f1. 
(a)

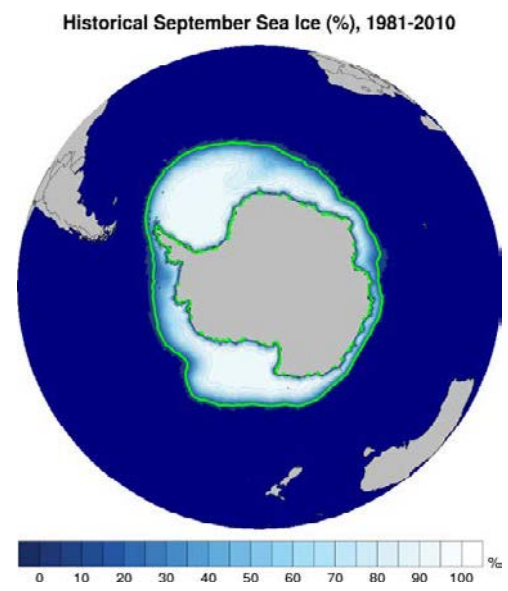

(c)

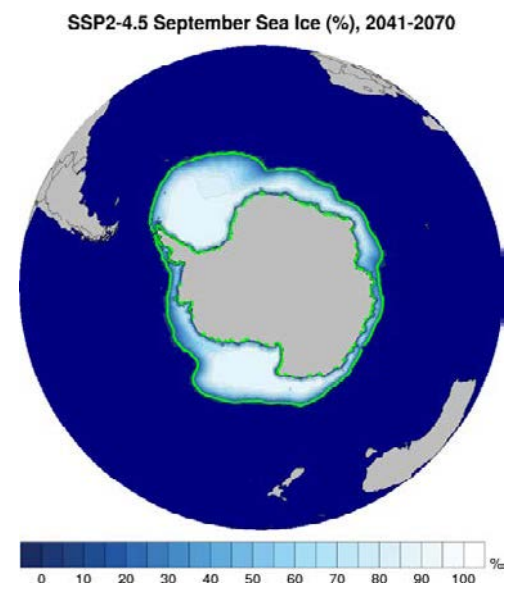

(e)

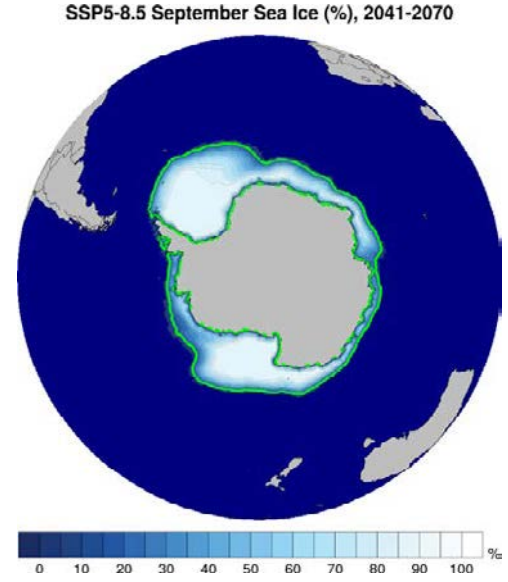

(b)

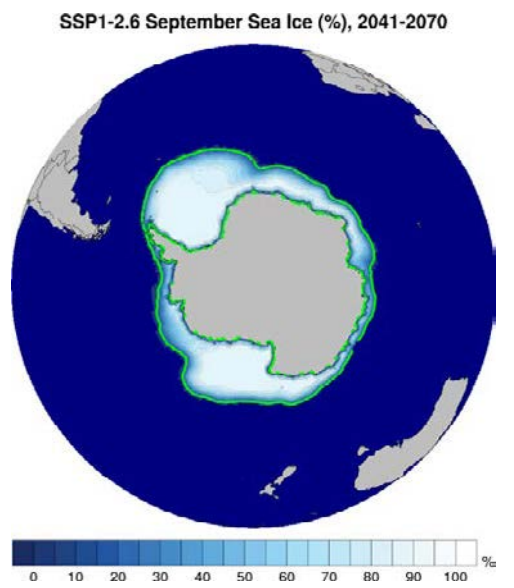

(d)

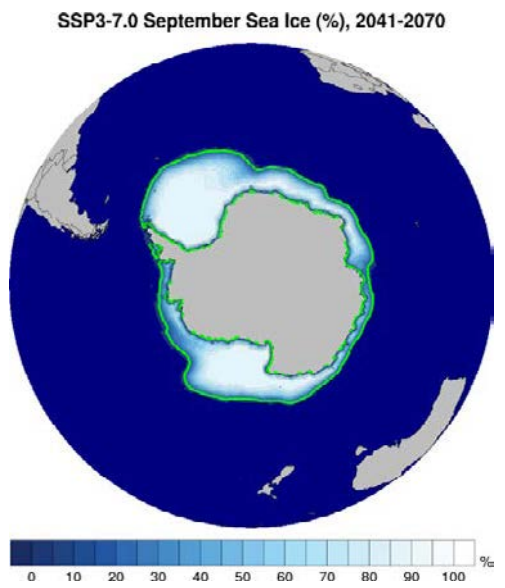

Figure 3.75. Mean Southern Hemisphere September sea ice fraction (\%): (a) historical ensemble for the period 1981-2010 and (b) SSP1-2.6, (c) SSP2-4.5, (d) SSP3-7.0 and (e) SSP5-8.5 for the period 20412070. In each case, an average is taken of the ensemble members r6i1p1f1, r9i1p1f1, r11i1p1f1, r13i1p1f1 and $\mathrm{r} 15 \mathrm{i} 1 \mathrm{p} 1 \mathrm{f} 1$. The green line shows the $15 \%$ contour line of sea ice fraction. 
(a)

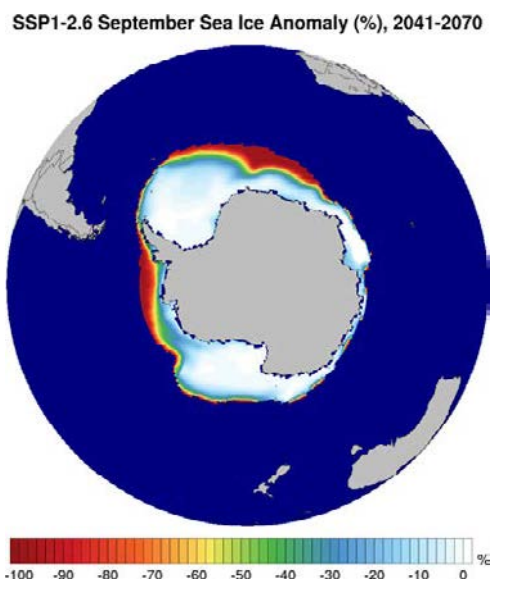

(c)

SSP3-7.0 September Sea Ice Anomaly (\%), 2041-2070

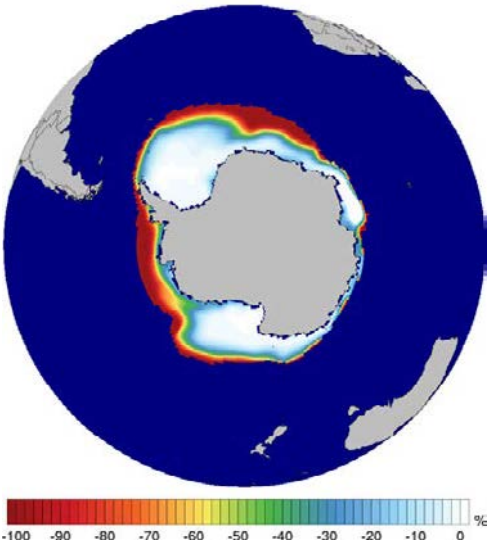

(b)

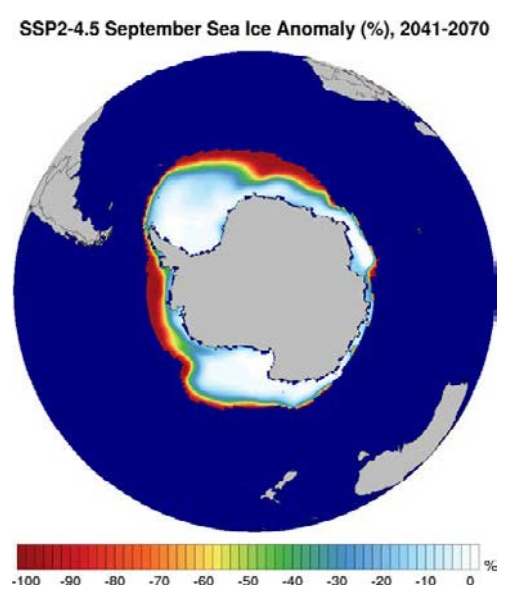

(d)

SSP5-8.5 September Sea Ice Anomaly (\%), 2041-2070

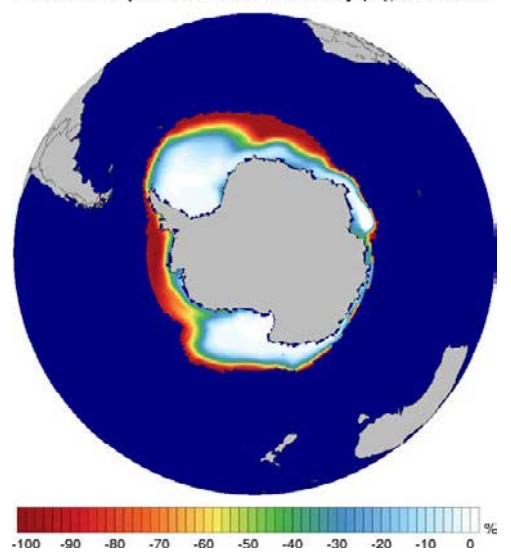

Figure 3.76. Southern Hemisphere September sea ice projections (\% change) (2041-2070 vs 1981-2010): (a) SSP1-2.6, (b) SSP2-4.5, (c) SSP3-7.0 and (d) SSP5-8.5. In each case, an average is taken of the ensemble members r6i1p1f1, r9i1p1f1, r11i1p1f1, r13i1p1f1 and r15i1p1f1. 
(a)

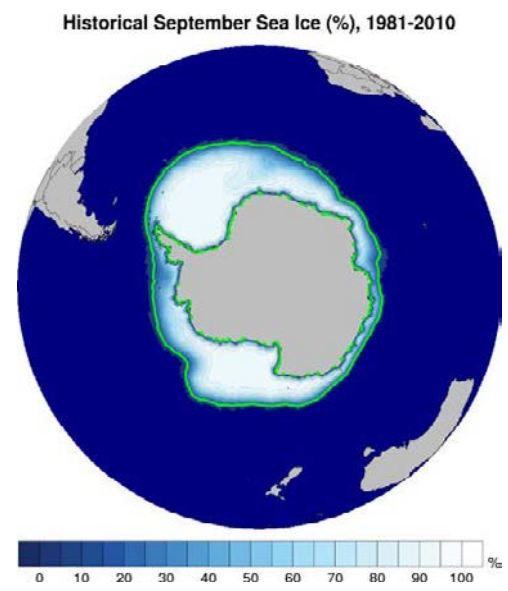

(c)

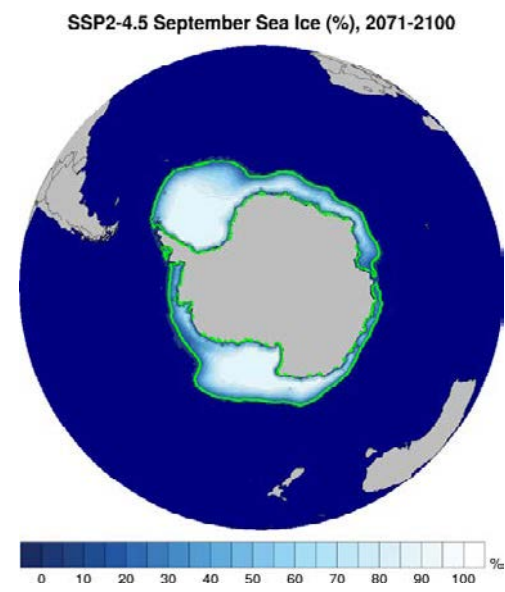

(e)

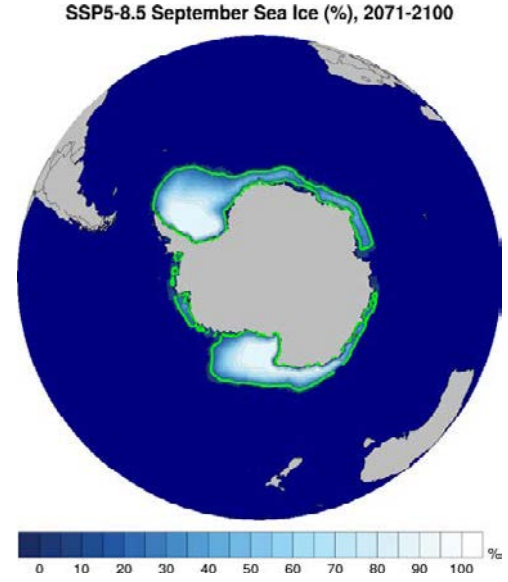

(b)

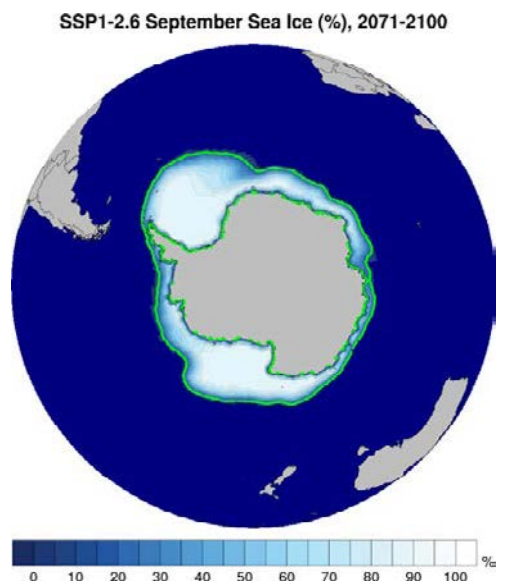

(d)

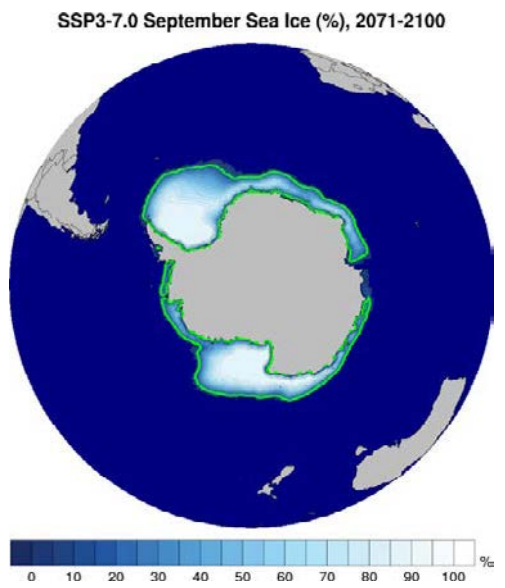

Figure 3.77. Mean Southern Hemisphere September sea ice fraction (\%): (a) historical ensemble for the period 1981-2010 and (b) SSP1-2.6, (c) SSP2-4.5, (d) SSP3-7.0 and (e) SSP5-8.5 for the period 2071-2100. In each case, an average is taken of the ensemble members r6i1p1f1, r9i1p1f1, r11i1p1f1, r13i1p1f1 and r15i1p1f1. The green line shows the $15 \%$ contour line of sea ice fraction. 
(a)

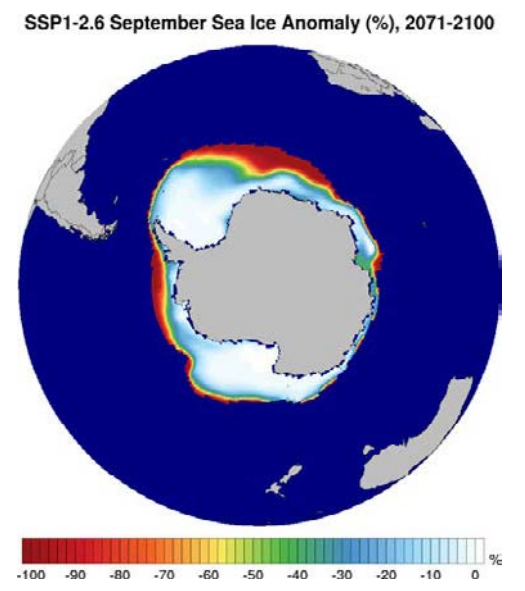

(c)

SSP3-7.0 September Sea Ice Anomaly (\%), 2071-2100

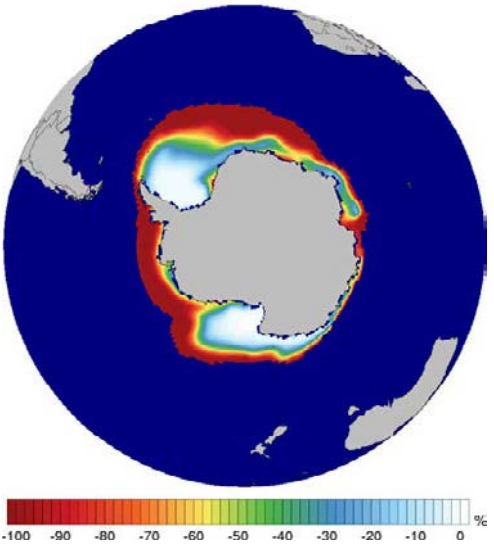

(b)

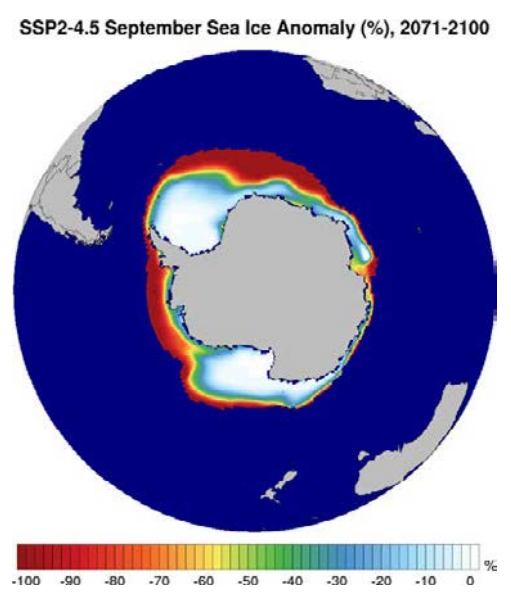

(d)

SSP5-8.5 September Sea Ice Anomaly (\%), 2071-2100

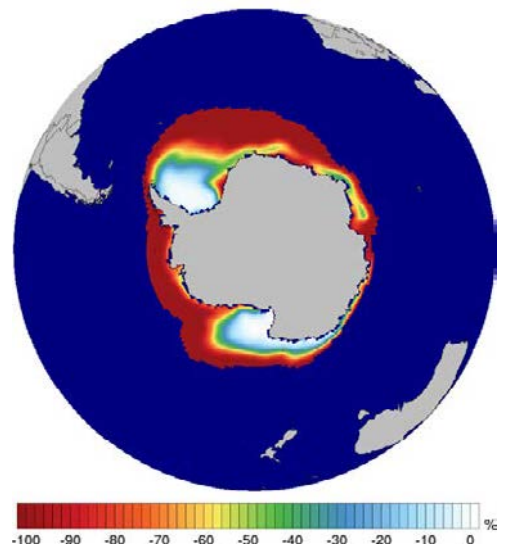

Figure 3.78. Southern Hemisphere September sea ice projections (\% change) (2071-2100 vs 1981-2010): (a) SSP1-2.6, (b) SSP2-4.5, (c) SSP3-7.0 and (d) SSP5-8.5. In each case, an average is taken of the ensemble members r6i1p1f1, r9i1p1f1, r11i1p1f1, r13i1p1f1 and r15i1p1f1. 
(a)

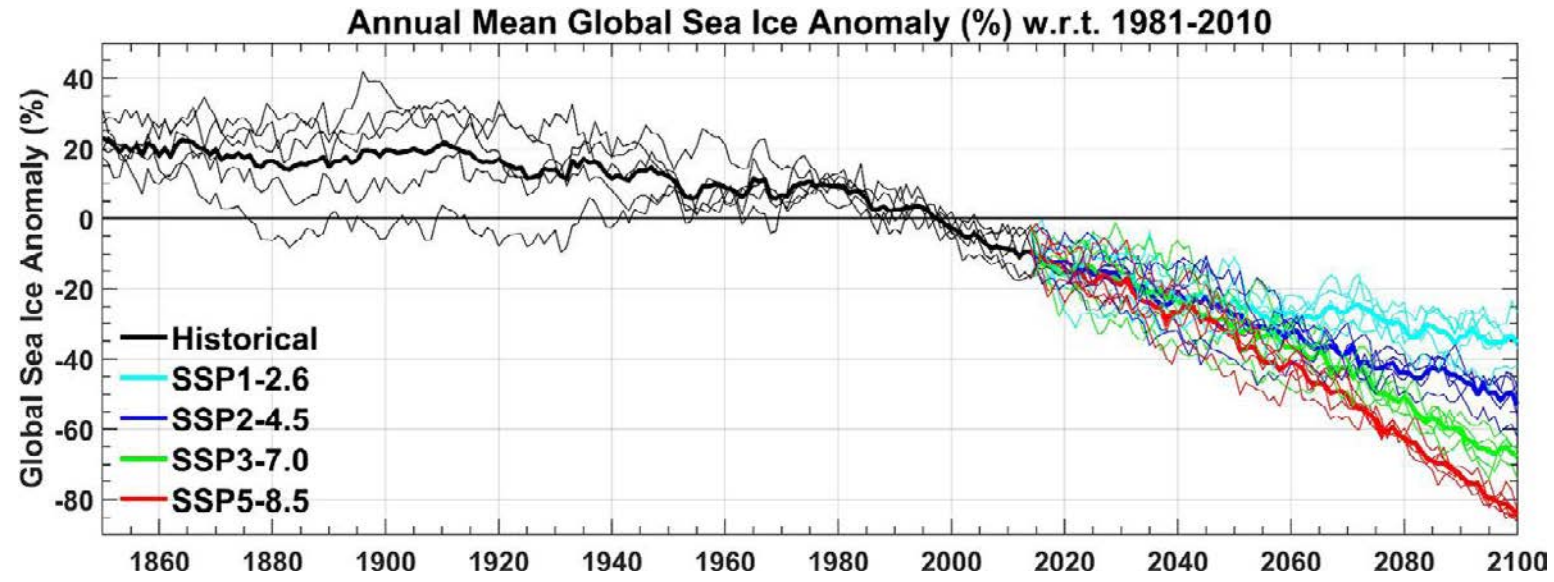

(b)

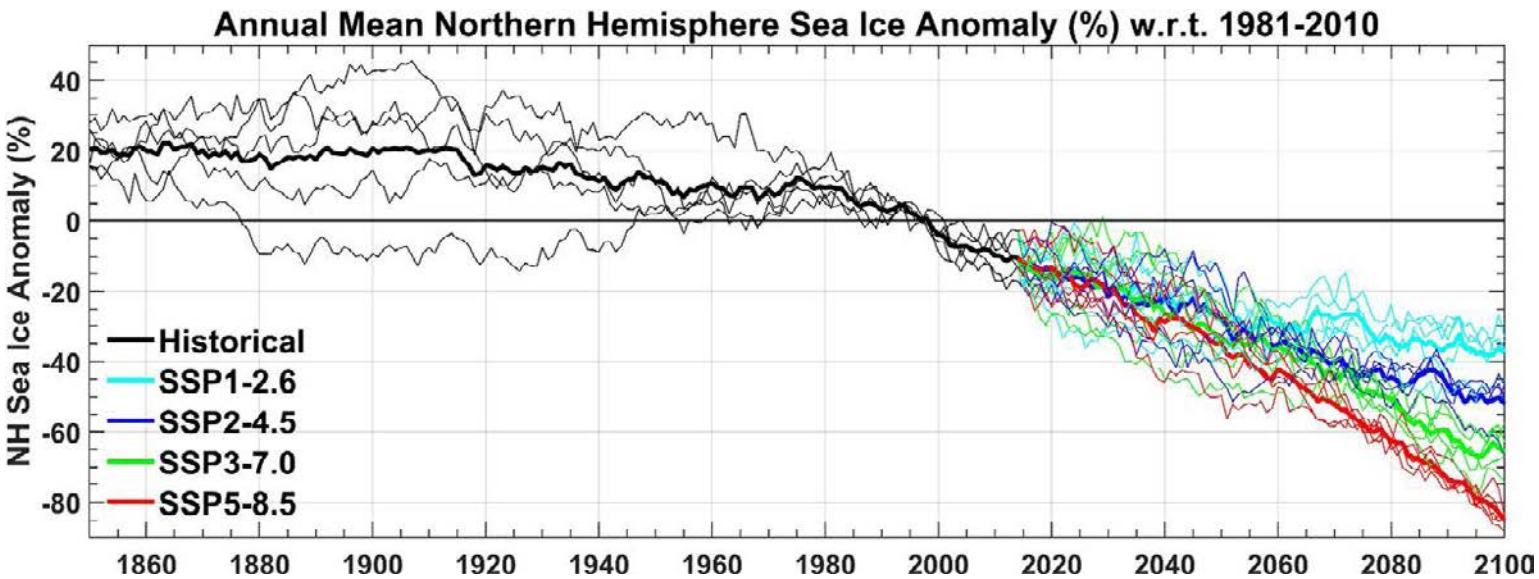

(c)

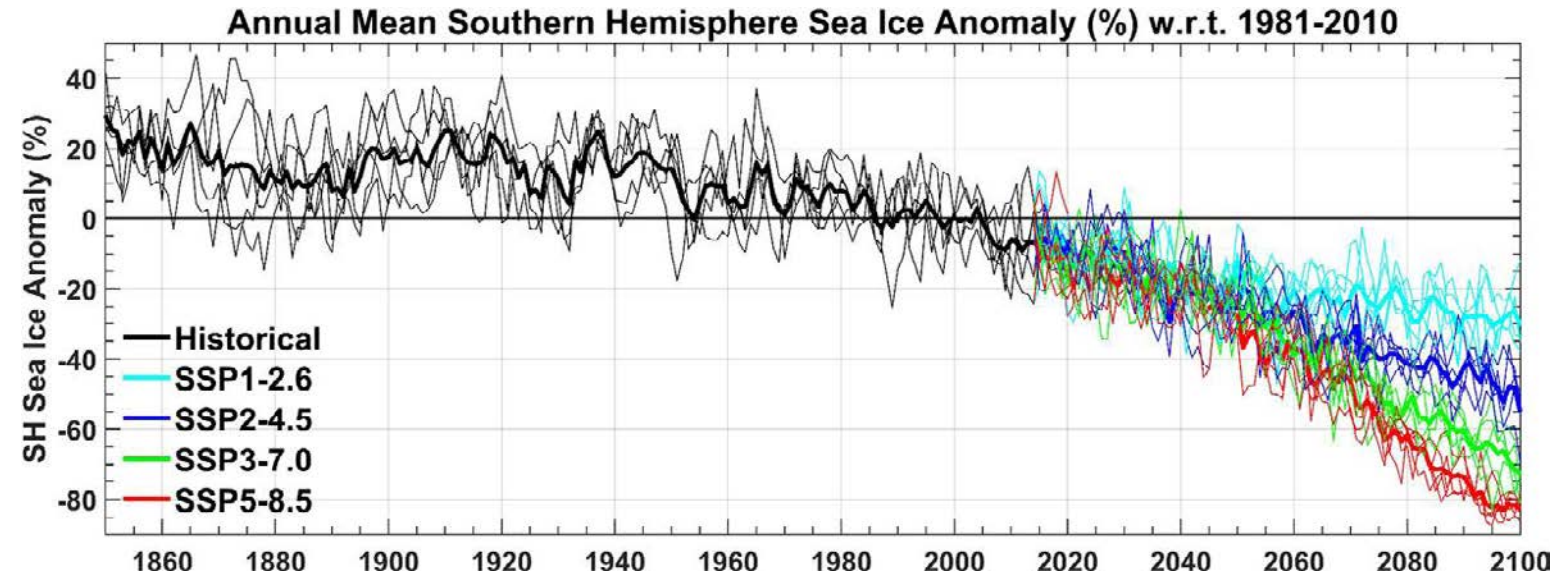

Figure 3.79. Annual sea ice anomalies (\% change) with respect to the 30-year period 1981-2010: EC-Earth ensemble members r6i1p1f1, r9i1p1f1, r11i1p1f1, r13i1p1f1 and r15i1p1f1 - (a) global, (b) Northern Hemisphere and (c) Southern Hemisphere. The bold lines represent the ensemble means. 


\section{$4 \quad$ Recommendations}

The research presented in this report focuses on Ireland's contribution to CMIP6. To date, CMIP6 participation is in the form of EC-Earth DECK CMIP (historical) and ScenarioMIP contributions. Specifically, the following CMIP6 EC-Earth contributions were run:

- five T255L91-ORCA1L75 AOGCM CMIP6 historical simulations, 1850-2014;

- 20 ScenarioMIP simulations: five T255L91ORCA1L75 AOGCM CMIP6 for all four ScenarioMIP "tier 1" SSPs (SSP1-2.6, SSP2-4.5, SSP3-7.0 and SSP5-8.5).

It is recommended that future national CMIP contributions involve participation in the following MIPs: high-resolution (T511L91-ORCA025L75) HighResMIP and additional historical/ScenarioMIP simulations using the EC-Earth-Veg interactive vegetation configuration. Currently, the authors are managing EC-Earth-Veg contributions, comprising:

- two T255L91-ORCA1L75 EC-Earth-Veg historical simulations, 1850-2014;

- eight ScenarioMIP simulations, 2015-2100: two T255L91-ORCA1L75 EC-Earth-Veg simulations for all four ScenarioMIP "tier 1" SSPs (SSP1-2.6, SSP2-4.5, SSP3-7.0 and SSP5-8.5).

These EC-Earth-Veg simulations are complete. The data are currently being post-processed ("cmorised") and will be hosted on the ICHEC ESGF node in March 2020. The project team will commence a number of high-resolution experiments in the near future after consultation with the EC-Earth community.

To evaluate the impact of improved models, additional earth system components and increased resolution, it is recommended that a study be undertaken to compare EC-Earth CMIP5 data with CMIP6 data. In particular, the impact on the accuracy of simulated precipitation amounts, extreme events such as heavy rain and high temperature, and storm tracks should be assessed. Preliminary validations, carried out by the EC-Earth community and the project team, confirm that the CMIP6 EC-Earth model outperforms the CMIP5 model in the simulation of the historical climate for the majority of variables analysed. A detailed analysis of the relative capability of CMIP6 compared with CMIP5 EC-Earth data is an important and necessary next step. This work is currently being carried out by the EC-Earth consortium and the results will be presented in a peer-reviewed publication.

The analysis described in the current report should be extended to include (1) the full ensemble of EC-Earth CMIP6 simulations produced by the consortium and (2) the full CMIP6 dataset produced by the international community. This will be possible as international CMIP6 datasets become available via the ESGF over the coming months. Analysing a large ensemble will allow a robust quantification of climate projection uncertainty and a measure of confidence to be assigned to the projections. Moreover, analysis of a large ensemble will allow the construction of a probability density function of climate projections. Likelihood values can then be assigned to the projected changes. In addition, the validation and climate projection analysis should be extended to include an assessment of sea level rise, extreme events, storm tracks and derived variables, such as frost/ice days, the growing season, drought index, heavy precipitation days and evapotranspiration.

Even with modern supercomputers, running large ensembles of global climate simulations is currently feasible only with horizontal resolutions of $\sim 50 \mathrm{~km}$ or coarser (the atmospheric component of the EC-Earth simulations of the current report is limited to $\sim 79-\mathrm{km}$ spatial resolution). As climate fields such as precipitation, wind speed and temperature are closely correlated with the local topography, this is inadequate to simulate the detail and pattern of climate change and its effects on the future climate of Ireland. The RCM method dynamically downscales the coarse information provided by the global models and provides high-resolution information on a subdomain covering Ireland. The computational cost of running the RCM, for a given resolution, is considerably less than that of running a global model. Numerous studies have demonstrated that high-resolution RCMs improve the simulation of fields such as precipitation (Kendon et al., 2012, 2014; Lucas-Picher et al., 2012; Bieniek et al., 2015; Nolan et al., 2017) and 
topography-influenced phenomena and extremes with relatively small spatial or short temporal character (Feser et al., 2011; Feser and Barcikowska, 2012; Shkol'nik et al., 2012; Flato et al., 2013). An additional advantage is that the physically based RCMs explicitly resolve more small-scale atmospheric features and provide a better representation of convective precipitation (Rauscher et al., 2010) and extreme precipitation (Kanada et al., 2008). Other examples of the added value of RCMs include improved simulation of near-surface temperatures (Feser, 2006; Di Luca et al., 2016), European storm damage (Donat et al., 2010), strong mesoscale cyclones (Cavicchia and von Storch, 2011), North Atlantic tropical cyclone tracks (Daloz et al., 2015) and near-surface wind speeds (e.g. Kanamaru and Kanamitsu, 2007), particularly in coastal areas with complex topography (Feser et al., 2011; Winterfeldt et al., 2011). The IPCC has concluded that there is "high confidence that downscaling adds value to the simulation of spatial climate detail in regions with highly variable topography (e.g., distinct orography, coastlines) and for mesoscale phenomena and extremes" (Flato et al., 2013). Current RCM research carried out by the project team aims to reduce climate change projection uncertainty and provide sharper estimates of expected climate change in the decades ahead. This is being achieved as follows:

- A large ensemble of high-resolution downscaled simulations will be run using the most up-to-date
RCMs (both standard and coupled atmosphereocean-wave models), CMIP6 GCMs and all four "tier-1" SSPs (SSP1-2.6, SSP2-4.5, SSP3-7.0 and SSP5-8.5) for the period 1979-2100.

- Additionally, the accuracy and usefulness of the model predictions will be enhanced by increasing the model resolution $(<4 \mathrm{~km})$.

- Furthermore, the RCM work will contribute to the CORDEX project by running the required outer nested domain of the RCM simulations on the Euro-CORDEX domains, conforming to the CORDEX standards and extending the simulation period to 1950-2100.

It should be noted that the EC-Earth ensemble member that was archived for RCM downscaling (r11ip1f1) was shown to exhibit large biases compared with the other ensemble members in the current report. The biases were particularly evident over the North Atlantic, a region where the boundary data for downscaling over Ireland (and Europe) will be derived. To address this issue, the project team is also archiving model-level data for downscaling from the currently running EC-Earth-Veg ensemble members. Both the r11i1p1f1 and EC-Earth-Veg datasets will be downscaled over the coming months. Furthermore, it is recommended that additional CMIP6 datasets (both EC-Earth datasets and datasets provided by international research groups) are sourced for downscaling. 


\section{References}

Aumont, O., Éthé, C., Tagliabue, A., Bopp, L. and Gehlen, M., 2015. PISCES-v2: an ocean biogeochemical model for carbon and ecosystem studies. Geoscientific Model Development Discussions 8: 2465-2513.

Balsamo. G., Viterbo, P., Beljaars, A.C.M., van den Hurk, B.J.J., Hirschi, M., Betts, A.K. and Scipal, K., 2009. A revised hydrology for the ECMWF model: verification from field site to terrestrial water storage and impact in the Integrated Forecast System. Journal of Hydrometeorology 10: 623-643. https://doi. org/10.1175/2008JHM1068.1.

Bieniek, P.A., Bhatt, U.S., Walsh, J.E., Rupp, T.S., Zhang, J., Krieger, J.R. and Lader, R., 2015. Dynamical downscaling of ERA-Interim temperature and precipitation for Alaska. Journal of Applied Meteorology and Climatology 55: 635-654. https://doi.org/10.1175/ JAMC-D-15-0153.1.

C3S (Copernicus Climate Change Service), 2017. ERA5: fifth generation of ECMWF atmospheric reanalyses of the global climate. Copernicus Climate Change Service Climate Data Store (CDS). Available online: https://confluence.ecmwf.int/display/CKB/ERA5\%3 $\mathrm{A}+$ data+documentation\#ERA5:datadocumentationHowtociteERA5 (accessed 11 February 2020).

Camargo, S., 2013. Global and regional aspects of tropical cyclone activity in the CMIP5 models. Journal of Climate 26: 9880-9902. https://doi.org/10.1175/ JCLI-D-12-00549.1

Cavicchia, L. and von Storch, H., 2011. The simulation of medicanes in a high-resolution regional climate model. Climate Dynamics 39: 2273-2290.

Chassignet, E.P. and Marshall, D.P., 2008. Gulf stream separation in numerical ocean models. In Hecht, M. and Hasumi, $\mathrm{H}$. (eds), Ocean Modelling in an Eddying Regime, vol. 177. American Geophysical Union, Washington, DC, pp. 39-62.

Church, J.A., Clark, P.U., Cazenave, A., Gregory, J.M., Jevrejeva, S., Levermann, A., Merrifield, M.A., Milne, G.A., Nerem, R.S., Nunn, P.D. and Payne, A.J., 2013. Sea level change. In Stocker, T.F., Qin, D., Plattner, G.-K., Tignor, M., Allen, S.K., Boschung, J., Nauels, A., Xia, Y., Bex, V. and Midgley, P.M. (eds), Climate Change 2013: The Physical Science Basis. Contribution of Working Group I to the Fifth Assessment Report of the Intergovernmental Panel on Climate Change. Cambridge University Press, Cambridge, pp. 1137-1216.
Cinquini, L., Crichton, D., Mattmann, C., Harney, J. Shipman, G., Wang, F., Ananthakrishnan, R., Miller, N., Denvil, S., Morgan, M. and Pobre, Z., 2014. The Earth System Grid Federation: an open infrastructure for access to distributed geospatial data. Future Generation Computer Systems 36: 400-417.

Daloz, A.S., Camargo, S.J., Kossin, J.P., Emanuel, K., Horn, M., Jonas, J.A., Kim, D., LaRow, T., Lim, Y.K., Patricola, C.M. and Roberts, M., 2015. Cluster analysis of downscaled and explicitly simulated North Atlantic tropical cyclone tracks. Journal of Climate 28: 1333-1361.

DCCAE (Department of Communications, Climate Action and Environment (DCCAE), 2018. National Adaptation Framework. Planning for a Climate Resilient Ireland. Available online: https://dccae.gov.ie/en-ie/ climate-action/publications/Documents/10/FINAL\%20 National\%20Adaptation\%20Framework-Planning $\% 20$ for $\% 20 a \% 20$ Climate $\% 20$ Resilient $\% 20$ Ireland.pdf (accessed 11 February 2020).

Demory, M.E., Vidale, P.L., Roberts, M.J., Berrisford, P., Strachan, J., Schiemann, R. and Mizielinski, M.S., 2014. The role of horizontal resolution in simulating drivers of the global hydrological cycle. Climate Dynamics 42: 2201-2225.

Di Luca, A., Argüeso, D., Evans, J.P., de Elía, R. and Laprise, R., 2016. Quantifying the overall added value of dynamical downscaling and the contribution from different spatial scales. Journal of Geophysical Research Atmospheres 121: 1575-1590. https://doi. org/10.1002/2015JD024009.

Donat, M., Leckebusch, G., Wild, S. and Ulbrich, U., 2010. Benefits and limitations of regional multi-model ensembles for storm loss estimations. Climate Research 44: 211-225.

Ebi, K.L., Hallegatte, S., Kram, T., Arnell, N.W., Carter, T.R., Edmonds, J., Kriegler, E., Mathur, R., O’Neill, B.C., Riahi, K. and Winkler, H., 2014. A new scenario framework for climate change research: background, process, and future directions. Climatic Change 122: 363-372.

ES-DOC (Earth System Documentation), n.d. Earth System Documentation (ES-DOC). Available online: https://www.earthsystemcog.org/projects/es-docmodels/ (accessed 11 February 2020).

ESGF-DOCKER, n.d. Contents. Available online: https:// esgf.github.io/esgf-docker/ (accessed 11 February 2020). 
Eyring, V., Bony, S., Meehl, G.A., Senior, C.A., Stevens, B., Stouffer, R.J. and Taylor, K.E., 2015. Overview of the Coupled Model Intercomparison Project Phase 6 (CMIP6) experimental design and organization. Geoscientific Model Development 9: 1937-1958.

Fealy, R., Bruyere, C. and Duffy, C., 2018. Regional Climate Model Simulations for Ireland for the 21st Century. Environmental Protection Agency, Johnstown Castle, Ireland.

Ferraro, R., Waliser, D.E., Gleckler, P., Taylor, K.E. and Eyring, V., 2015. Evolving Obs4MIPs to support phase 6 of the Coupled Model Intercomparison Project (CMIP6). Bulletin of the American Meteorological Society 96: ES131-ES133.

Feser, F., 2006. Enhanced detectability of added value in limited-area model results separated into different spatial scales. Monthly Weather Review 134: 2180-2190.

Feser, F. and Barcikowska, M., 2012. The influence of spectral nudging on typhoon formation in regional climate models. Environmental Research Letters 7: 014024.

Feser, F., Rockel, B., von Storch, H., Winterfeldt, J. and Zahn, M., 2011. Regional climate models add value to global model data: a review and selected examples. Bulletin of the American Meteorological Society 92: 1181-1192.

Flato, G., Marotzke, J., Abiodun, B., Braconnot, P., Chou, S.C., Collins, W., Cox, P., Driouech, F., Emori, S., Eyring, V. and Forest, C. (eds), 2013. Climate Change 2013: The Physical Science Basis. Contribution of Working Group I to the Fifth Assessment Report of the Intergovernmental Panel on Climate Change. Cambridge University Press, Cambridge.

Gleeson, E., McGrath R. and Treanor M., 2013. Ireland's Climate: The Road Ahead. Met Éireann, Dublin.

Haarsma, R.J., Hazeleger, W., Severijns, C., de Vries, H., Sterl, A., Bintanja, R., van Oldenborgh, G.J. and van den Brink, H.W., 2013. More hurricanes to hit Western Europe due to global warming. Geophysical Research Letters 40: 1783-1788. https://doi.org/10.1002/ grl.50360.

Harris, I.P.D.J., Jones, P.D., Osborn, T.J. and Lister, D.H., 2014. Updated high-resolution grids of monthly climatic observations - the CRU TS3.10 Dataset. International Journal of Climatology 34: 623-642.

Hodges, K.I., Lee, R.W. and Bengtsson, L., 2011. A comparison of extratropical cyclones in recent re-analyses ERA-Interim, NASA MERRA, NCEP CFSR, JRA-25. Journal of Climate 24: 4888-4906.
Huijnen, V., Williams, J.E., van Weele, M., Van Noije, T.P.C., Krol, M.C., Dentener, F.J., Segers, A., Houweling, S., Peters, W., de Laat, J. and Boersma, F., 2010. The global chemistry transport model TM5: description and evaluation of the tropospheric chemistry version 3.0. Geoscientific Model Development 3: 445-473.

IPCC (Intergovernmental Panel on Climate Change), 2013a. Summary for policymakers. In Stocker, T.F., Qin, D., Plattner, G.-K., Tignor, M., Allen, S.K., Boschung, J., Nauels, A., Xia, Y., Bex, V. and Midgley, P.M. (eds), Climate Change 2013: The Physical Science Basis. Contribution of Working Group I to the Fifth Assessment Report of the Intergovernmental Panel on Climate Change. Cambridge University Press, Cambridge.

IPCC (Intergovernmental Panel on Climate Change), 2013b. Climate Change 2013: The Physical Science Basis. Contribution of Working Group I to the Fifth Assessment Report of the Intergovernmental Panel on Climate Change. Stocker, T.F., Qin, D., Plattner, G.-K., Tignor, M., Allen, S.K., Boschung, J., Nauels, A., Xia, Y., Bex, V. and Midgley, P.M. (eds). Cambridge University Press, Cambridge.

IPCC (Intergovernmental Panel on Climate Change), 2013c. Annex I: atlas of global and regional climate projections. van Oldenborgh, G.J., Collins, M., Arblaster, J., Christensen, J.H., Marotzke, J., Power, S.B., Rummukainen, M. and Zhou, T. (eds). In Stocker, T.F., Qin, D., Plattner, G.-K., Tignor, M., Allen, S.K., Boschung, J., Nauels, A., Xia, Y., Bex, V. and Midgley, P.M. (eds), Climate Change 2013: The Physical Science Basis. Contribution of Working Group I to the Fifth Assessment Report of the Intergovernmental Panel on Climate Change. Cambridge University Press, Cambridge.

IPCC (Intergovernmental Panel on Climate Change), 2018. Summary for policymakers. In MassonDelmotte, V., Zhai, P., Pörtner, H.-O., Roberts, D., Skea, J., Shukla, P.R., Pirani, A., Moufouma-Okia, W., Péan, C., Pidcock, R., Connors, S., Matthews, J.B.R., Chen, Y., Zhou, X., Gomis, M.I., Lonnoy, E., Maycock, T., Tignor, M. and Waterfield, T. (eds), Global Warming of $1.5^{\circ} \mathrm{C}$. An IPCC Special Report on the Impacts of Global Warming of $1.5^{\circ} \mathrm{C}$ above Preindustrial Levels and Related Global Greenhouse Gas Emission Pathways, in the Context of Strengthening the Global Response to the Threat of Climate Change, Sustainable Development, and Efforts to Eradicate Poverty. World Meteorological Organization, Geneva. 
IPCC (Intergovernmental Panel on Climate Change), 2019. Summary for policymakers. In Pörtner, H.-O., Roberts, D.C., Masson-Delmotte, V., Zhai, P., Tignor, M., Poloczanska, E., Mintenbeck, K., Alegría, A., Nicolai, M., Okem, A., Petzold, J., Rama, B. and Weyer, N.M. (eds), IPCC Special Report on the Ocean and Cryosphere in a Changing Climate. Available online: https://www.ipcc.ch/site/assets/uploads/ sites/3/2019/12/02_SROCC_FM_FINAL.pdf (accessed 1 March 2020).

Jung, T., Miller, M.J., Palmer, T.N., Towers, P., Wedi, N., Achuthavarier, D., Adams, J.M., Altshuler, E.L., Cash, B.A., Kinter III, J.L. and Marx, L., 2012. High-resolution global climate simulation with the ECMWF model in project Athena: experimental design, model climate, and seasonal forecast skill. Journal of Climate 25: 3155-3172.

Kanada, S., Nakano, M., Hayashi, S., Kato, T., Nakamura, M., Kurihara, K. and Kitoh, A., 2008. Reproducibility of maximum daily precipitation amount over Japan by a high-resolution non-hydrostatic model. Sola 4: 105-108.

Kanamaru, H. and Kanamitsu, M., 2007. Fifty-seven-year California reanalysis downscaling at $10 \mathrm{~km}$ (CaRD10). Part II: comparison with North American regional reanalysis. Journal of Climate 20: 5572-5592.

Kendon, E., Roberts, N., Senior, C. and Roberts, M., 2012. Realism of rainfall in a very high-resolution regional climate model. Journal of Climate 25: 5791-5806.

Kendon, E.J., Roberts, N.M., Fowler, H.J., Roberts, M.J., Chan, S.C. and Senior, C.A., 2014. Heavier summer downpours with climate change revealed by weather forecast resolution model. Nature Climate Change 4: 570-576.

Kriegler, E., Edmonds, J., Hallegatte, S., Ebi, K.L., Kram, T., Riahi, K., Winkler, H. and Van Vuuren, D.P., 2014. A new scenario framework for climate change research: the concept of shared climate policy assumptions. Climatic Change 122: 401-414

Kuwano-Yoshida, A., Minobe, S. and Xie, S.P., 2010. Precipitation response to the gulf stream in an atmospheric GCM. Journal of Climate 23: 3676-3698.

Lucas-Picher, P., Wulff-Nielsen, M., Christensen, J.H., Ađalgeirsdóttir, G., Mottram, R. and Simonsen, S.B., 2012. Very high resolution regional climate model simulations over Greenland: identifying added value. Journal of Geophysical Research 117: D02108.

Madec, G. and the NEMO team, 2008. NEMO ocean engine. Note du Pôle de modélisation de Institut Pierre-Simon Laplace, No. 27. Available online: https:// www.nemo-ocean.eu/doc/ (accessed 13 February 2020).
Marvel, K., Cook, B.I., Bonfils. C.J.W., Durack, P.J., Smerdon, J.E. and Park Williams, A., 2019. Twentiethcentury hydroclimate changes consistent with human influence. Nature 569: 59-65.

Massonnet, F., Ménegoz, M., Acosta, M., YepesArbós, X., Exarchou, E. and Doblas-Reyes, F.J., 2019. Replicability of the EC-Earth3 Earth System Model under a change in computing environment. Geoscientific Model Development. https://doi. org/10.5194/gmd-2019-91

Molteni, F., Stockdale, T., Balmaseda, M., Balsamo, G., Buizza, R., Ferranti, L., Magnusson, L., Mogensen, K., Palmer, T. and Vitart, F., 2011. The New ECMWF Seasonal Forecast System (System 4), vol. 49. European Centre for Medium-Range Weather Forecasts, Reading.

Moss, R.H., Edmonds, J.A., Hibbard, K.A., Manning, M.R., et al., 2010. The next generation of scenarios for climate change research and assessment. Nature 463: 747-756

Nolan, P., 2015. Ensemble of Regional Climate Model Projections for Ireland. Environmental Protection Agency, Johnstown Castle, Ireland.

Nolan, P., Lynch, P. and Sweeney, C., 2014. Simulating the future wind energy resource of Ireland using the COSMO-CLM model. Wind Energy 17: 19-37.

Nolan, P., O'Sullivan, J. and McGrath, R., 2017. Impacts of climate change on mid-twenty-first-century rainfall in Ireland: a high-resolution regional climate model ensemble approach. International Journal of Climatology 37: 4347-4363.

O’Neill, B.C., Kriegler, E., Riahi, K., Ebi, K.L., Hallegatte, S., Carter, T.R., Mathur, R. and van Vuuren, D.P., et al., 2014. A new scenario framework for climate change research: the concept of shared socioeconomic pathways. Climatic Change 122: 387-400.

O'Neill, B.C., Tebaldi, C., van Vuuren, D.P., Eyring, V., Friedlingstein, P., Hurtt, G., Knutti, R., Kriegler, E., Lamarque, J.F., Lowe, J. and Meehl, G.A., 2016. The scenario model intercomparison project (ScenarioMIP) for CMIP6. Geoscientific Model Development 9: 3461-3482.

O'Sullivan J., Sweeney C., Nolan P. and Gleeson E., 2015. A high-resolution, multi-model analysis of Irish temperatures for the mid 21st-century. International Journal of Climatology 36: 1256-1267.

Rauscher, S.A., Coppola, E., Piani, C. and Giorgi, F., 2010. Resolution effects on regional climate model simulations of seasonal precipitation over Europe. Climate Dynamics 35: 685-711. 
Riahi, K., Van Vuuren, D.P., Kriegler, E., Edmonds, J., O’Neill, B.C., Fujimori, S., Bauer, N., Calvin, K., Dellink, R., Fricko, O. and Lutz, W., 2017. The shared socioeconomic pathways and their energy, land use, and greenhouse gas emissions implications: an overview. Global Environmental Change 42: 153-168.

Roberts, M.J., Clayton, A., Demory, M.E., Donners, J., Vidale, P.L., Norton, W., Shaffrey, L., Stevens, D.P., Stevens, I., Wood, R.A. and Slingo, J., 2009. Impact of resolution on the tropical pacific circulation in a matrix of coupled models. Journal of Climate 22: 2541-2556.

Rousset, C., Vancoppenolle, M., Madec, G., Fichefet, T., Flavoni, S., Barthélemy, A., Benshila, R., Chanut, J., Lévy, C., Masson, S. and Vivier, F., 2015. The Louvain-la-Neuve sea ice model LIM3. 5: global and regional capabilities. Geoscientific Model Development 8: 3403-3441.

Shaffrey, L., Stevens, I., Norton, W.A., Roberts, M.J., Vidale, P.L., Harle, J.D., Jrrar, A., Stevens, D.P., Woodage, M.J., Demory, M.E. and Donners, J.B.C.D., 2009. UK HiGEM: the new UK High-Resolution Global Environment Model - model description and basic evaluation. Journal of Climate 22: 1861-1896.

Shkol'nik, I., Meleshko, V., Efimov, S. and Stafeeva E., 2012. Changes in climate extremes on the territory of Siberia by the middle of the 21 st century: an ensemble forecast based on the MGO regional climate model. Russian Meteorology and Hydrology 37: 71-84.

Sillmann, J., Kharin, V.V., Zhang, X., Zwiers, F.W. and Bronaugh, D., 2013. Climate extremes indices in the CMIP5 multimodel ensemble: part 1. Model evaluation in the present climate. Journal of Geophysical Research Atmospheres 118: 1716-1733.

Small, C. and Nichols, R.J., 2003. A global analysis of human settlement in coastal zones. Journal of Coastal Research 19: 584-599.

Smith, B., Wårlind, D., Arneth, A., Hickler, T., Leadley, P., Siltberg, J. and Zaehle, S., 2014. Implications of incorporating $\mathrm{N}$ cycling and $\mathrm{N}$ limitations on primary production in an individual-based dynamic vegetation model. Biogeosciences 11: 2027-2054.

Taylor, K.E., Stouffer, R.J. and Meehl, G.A., 2012. An overview of CMIP5 and the experiment design. Bulletin of the American Meteorological Society 93: 485-498.
Taylor, K.E., Juckes, M., Balaji, V., Cinquini, L., Denvil, S., Durack, P.J., Elkington, M., Guilyardi, E., Kharin, S., Lautenschlager, M., Lawrence, B., Nadeau, D. and Stockhause, M., 2018. CMIP6 global attributes, DRS, filenames, directory structure, and CV's. Document version 6.2.7. Available online: https://goo.gl/v1drZl (accessed 13 February 2020).

van Vuuren, D.P., Edmonds, J., Kainuma, M., Riahi, K., Thomson, A., Hibbard, K., Hurtt, G.C., Kram, T., Krey, V., Lamarque, J.F. and Masui, T., 2011. The representative concentration pathways: an overview. Climatic Change 109: 5.

van Vuuren, D.P., Kriegler, E., O’Neill, B.C., Ebi, K.L., Riahi, K., Carter, T.R., Edmonds, J., Hallegatte, S., Kram, T., Mathur, R. and Winkler, H., 2014. A new scenario framework for climate change research: scenario matrix architecture. Climatic Change 122: 373-386.

Vousdoukas, M.I., Mentaschi, L., Voukouvalas, E., Bianchi, A., Dottori F. and Feyen, L., 2018. Climatic and socioeconomic controls of future coastal flood risk in Europe. Nature Climate Change 8: 776-780.

Wehner, M.F., Smith, R.L., Bala, G. and Duffy, P., 2010. The effect of horizontal resolution on simulation of very extreme us precipitation events in a global atmosphere model. Climate Dynamics 24: 241-247.

Winterfeldt, J., Geyer, B. and Weisse, R., 2011. Using QuikSCAT in the added value assessment of dynamically downscaled wind speed. International Journal of Climatology 31: 1028-1039.

WMO (World Meteorological Organization), 2013. The Global Climate 2001-2010: A Decade of Climate Extremes: Summary Report. Available online: https:// library.wmo.int/doc_num.php?explnum_id=7802 (accessed 11 February 2020).

Zappa G., Shaffrey, L.C. and Hodges, K.I., 2013. The ability of CMIP5 models to simulate North Atlantic extratropical cyclones. Journal of Climate 26: 5379-5396.

Zhao, M., Held, I.M., Lin, S.J. and Vecchi, G.A., 2009. Simulations of global hurricane climatology, interannual variability, and response to global warming using a $50 \mathrm{~km}$ resolution GCM. Journal of Climate 33: 6653-6678. 


\section{Abbreviations}

\begin{tabular}{|c|c|}
\hline AOGCM & Atmosphere-Ocean General Circulation Model \\
\hline AR5 & Fifth Assessment Report \\
\hline AR6 & Sixth Assessment Report \\
\hline CMIP & Coupled Model Intercomparison Project \\
\hline CORDEX & Coordinated Regional Downscaling Experiment \\
\hline CRU & Climatic Research Unit \\
\hline DCPP & Decadal Climate Prediction Project \\
\hline DECK & Diagnostic, Evaluation and Characterization of Klima \\
\hline DJF & December, January and February \\
\hline DKRZ & German Climate Computing Centre \\
\hline ECMWF & European Centre for Medium-Range Weather Forecast \\
\hline ELPiN & Exclude Land Processes in NEMO \\
\hline ESGF & Earth System Grid Federation \\
\hline ESM & Earth system model \\
\hline GCM & Global climate model \\
\hline GMSL & Global mean sea level \\
\hline GSLR & Global sea level rise \\
\hline HPC & High-performance computing \\
\hline ICHEC & Irish Centre for High-End Computing \\
\hline IFS & Integrated Forecast System \\
\hline IPCC & Intergovernmental Panel on Climate Change \\
\hline ITCZ & Intertropical Convergence Zone \\
\hline JJA & June, July and August \\
\hline KNMI & Royal Netherlands Meteorological Institute \\
\hline LIM3 & Louvain-la-Neuve sea ice model \\
\hline LPJ-GUESS & Lund-Potsdam-Jena General Ecosystem Simulator \\
\hline MAE & Mean absolute error \\
\hline MAM & March, April and May \\
\hline MIP & Model Intercomparison Project \\
\hline MSLP & Mean sea level pressure \\
\hline NEMO & Nucleus for European Modelling of the Ocean \\
\hline $\mathbf{P} 2 \mathbf{P}$ & Peer-to-Peer \\
\hline PISCES & Pelagic Interactions Scheme for Carbon and Ecosystem Studies \\
\hline RCM & Regional climate model \\
\hline RCP & Representative concentration pathway \\
\hline SON & September, October and November \\
\hline SSP & Shared socioeconomic pathway \\
\hline SST & Sea surface temperature \\
\hline TM5 & Tracer Model version 5 \\
\hline
\end{tabular}


AN GHNÍOMHAIREACHT UM CHAOMHNÚ COMHSHAOIL

Tá an Ghníomhaireacht um Chaomhnú Comhshaoil (GCC) freagrach as an gcomhshaol a chaomhnú agus a fheabhsú mar shócmhainn luachmhar do mhuintir na hÉireann. Táimid tiomanta do dhaoine agus don chomhshaol a chosaint ó éifeachtaí díobhálacha na radaíochta agus an truaillithe.

\section{Is féidir obair na Gníomhaireachta a roinnt ina trí phríomhréimse:}

Rialú: Déanaimid córais éifeachtacha rialaithe agus comhlíonta comhshaoil a chur i bhfeidhm chun torthai maithe comhshaoil a sholáthar agus chun diriú orthu siúd nach gcloionn leis na córais sin.

Eolas: Soláthraímid sonrai, faisnéis agus measúnú comhshaoil atá ar ardchaighdeán, spriocdhírithe agus tráthuiil chun bonn eolais a chur faoin gcinnteoireacht ar gach leibhéal.

Tacaíocht: Bímid ag saothrú i gcomhar le grúpaí eile chun tacú le comhshaol atá glan, táirgiúil agus cosanta go maith, agus le hiompar a chuirfidh le comhshaol inbhuanaithe.

\section{Ár bhFreagrachtaí}

\section{Ceadúnú}

Déanaimid na gníomhaíochtaí seo a leanas a rialú ionas nach ndéanann siad dochar do shláinte an phobail ná don chomhshaol:

- saoráidí dramhaíola (m.sh. láithreáin líonta talún, loisceoirí, stáisiúin aistrithe dramhaiola);

- gníomhaíochtaí tionsclaíocha ar scála mór (m.sh. déantúsaíocht cógaisíochta, déantúsaiocht stroighne, stáisiúin chumhachta);

- an diantalmhaíocht (m.sh. muca, éanlaith);

- úsáid shrianta agus scaoileadh rialaithe Orgánach Géinmhodhnaithe (OGM);

- foinsí radaíochta ianúcháin (m.sh. trealamh x-gha agus radaiteiripe, foinsí tionsclaiocha);

- áiseanna móra stórála peitril;

- scardadh dramhuisce;

- gníomhaíochtaí dumpála ar farraige.

\section{Forfheidhmiú Náisiúnta i leith Cúrsaí Comhshaoil}

- Clár náisiúnta iniúchtaí agus cigireachtaí a dhéanamh gach bliain ar shaoráidí a bhfuil ceadúnas ón nGníomhaireacht acu.

- Maoirseacht a dhéanamh ar fhreagrachtaí cosanta comhshaoil na n-údarás áitiúil.

- Caighdeán an uisce óil, arna sholáthar ag soláthraithe uisce phoiblí, a mhaoirsiú.

- Obair le húdaráis áitiúla agus le gníomhaireachtaí eile chun dul i ngleic le coireanna comhshaoil trí chomhordú a dhéanamh ar líonra forfheidhmiúcháin náisiúnta, trí dhíriú ar chiontóirí, agus trí mhaoirsiú a dhéanamh ar leasúchán.

- Cur i bhfeidhm rialachán ar nós na Rialachán um Dhramhthrealamh Leictreach agus Leictreonach (DTLL), um Shrian ar Shubstaintí Guaiseacha agus na Rialachán um rialú ar shubstaintí a ídíonn an ciseal ózóin.

- An dlí a chur orthu siúd a bhriseann dlí an chomhshaoil agus a dhéanann dochar don chomhshaol.

\section{Bainistíocht Uisce}

- Monatóireacht agus tuairisciú a dhéanamh ar cháilíocht aibhneacha, lochanna, uiscí idirchriosacha agus cósta na hÉireann, agus screamhuiscí; leibhéil uisce agus sruthanna aibhneacha a thomhas.

- Comhordú náisiúnta agus maoirsiú a dhéanamh ar an gCreatTreoir Uisce.

- Monatóireacht agus tuairisciú a dhéanamh ar Cháilíocht an Uisce Snámha.

\section{Monatóireacht, Anailís agus Tuairisciú ar}

\section{an gComhshaol}

- Monatóireacht a dhéanamh ar cháilíocht an aeir agus Treoir an AE maidir le hAer Glan don Eoraip (CAFÉ) a chur chun feidhme.

- Tuairisciú neamhspleách le cabhrú le cinnteoireacht an rialtais náisiúnta agus na n-údarás áitiúil (m.sh. tuairisciú tréimhsiúil ar staid Chomhshaol na hÉireann agus Tuarascálacha ar Tháscairí).

\section{Rialú Astaíochtaí na nGás Ceaptha Teasa in Éirinn}

- Fardail agus réamh-mheastacháin na hÉireann maidir le gáis cheaptha teasa a ullmhú.

- An Treoir maidir le Trádáil Astaíochtaí a chur chun feidhme i gcomhair breis agus 100 de na táirgeoirí dé-ocsaíde carbóin is mó in Éirinn.

\section{Taighde agus Forbairt Comhshaoil}

- Taighde comhshaoil a chistiú chun brúnna a shainaithint, bonn eolais a chur faoi bheartais, agus réitigh a sholáthar i réimsí na haeráide, an uisce agus na hinbhuanaitheachta.

\section{Measúnacht Straitéiseach Timpeallachta}

- Measúnacht a dhéanamh ar thionchar pleananna agus clár beartaithe ar an gcomhshaol in Éirinn (m.sh. mórphleananna forbartha).

\section{Cosaint Raideolaíoch}

- Monatóireacht a dhéanamh ar leibhéil radaíochta, measúnacht a dhéanamh ar nochtadh mhuintir na hÉireann don radaíocht ianúcháin.

- Cabhrú le pleananna náisiúnta a fhorbairt le haghaidh éigeandálaí ag eascairt as taismí núicléacha.

- Monatóireacht a dhéanamh ar fhorbairtí thar lear a bhaineann le saoráidí núicléacha agus leis an tsábháilteacht raideolaíochta.

- Sainseirbhísí cosanta ar an radaíocht a sholáthar, nó maoirsiú a dhéanamh ar sholáthar na seirbhísí sin.

Treoir, Faisnéis Inrochtana agus Oideachas

- Comhairle agus treoir a chur ar fáil d'earnáil na tionsclaíochta agus don phobal maidir le hábhair a bhaineann le caomhnú an chomhshaoil agus leis an gcosaint raideolaíoch.

- Faisnéis thráthúil ar an gcomhshaol ar a bhfuil fáil éasca a chur ar fáil chun rannpháirtíocht an phobail a spreagadh sa chinnteoireacht i ndáil leis an gcomhshaol (m.sh. Timpeall an Ti, léarscáileanna radóin).

- Comhairle a chur ar fáil don Rialtas maidir le hábhair a bhaineann leis an tsábháilteacht raideolaíoch agus le cúrsaí práinnfhreagartha.

- Plean Náisiúnta Bainistíochta Dramhaíola Guaisí a fhorbairt chun dramhaíl ghuaiseach a chosc agus a bhainistiú.

\section{Múscailt Feasachta agus Athrú Iompraíochta}

- Feasacht chomhshaoil níos fearr a ghiniúint agus dul i bhfeidhm ar athrú iompraíochta dearfach trí thacú le gnóthais, le pobail agus le teaghlaigh a bheith níos éifeachtúla ar acmhainní.

- Tástáil le haghaidh radóin a chur chun cinn i dtithe agus in ionaid oibre, agus gníomhartha leasúcháin a spreagadh nuair is gá.

\section{Bainistíocht agus struchtúr na Gníomhaireachta um Chaomhnú Comhshaoil}

Tá an ghníomhaíocht á bainistiú ag Bord lánaimseartha, ar a bhfuil Ard-Stiúrthóir agus cúigear Stiúrthóirí. Déantar an obair ar fud cúig cinn d'Oifigí:

- An Oifig um Inmharthanacht Comhshaoil

- An Oifig Forfheidhmithe i leith cúrsaí Comhshaoil

- An Oifig um Fianaise is Measúnú

- Oifig um Chosaint Radaíochta agus Monatóireachta Comhshaoil

- An Oifig Cumarsáide agus Seirbhísí Corparáideacha

Tá Coiste Comhairleach ag an nGníomhaireacht le cabhrú léi. Tá dáréag comhaltaí air agus tagann siad le chéile go rialta le plé a dhéanamh ar ábhair imní agus le comhairle a chur ar an mBord. 


\section{EPA Research Report 310}

\section{EC-Earth Global Climate Simulations - Ireland's Contributions to CMIP6}

Authors: Paul Nolan and Alastair McKinstry

This report provides an overview of future global climate projections as simulated by the EC-Earth Earth system model. In total, five historical (1850-2014) and 20 simulations of future climate across a full range of emissions pathways (2015-2100) were run and analysed. Model-level data were archived allowing for regional downscaling using regional climate models. The simulations comprise Ireland's contribution to the Coupled Model Intercomparison Project (phase 6) (CMIP6) and will be included for assessment in the United Nations Intergovernmental Panel on Climate Change (IPCC) Sixth Assessment (AR6) reports.

\section{Identifying Pressures}

It is now accepted beyond doubt that historical and future greenhouse gas emissions and changing land use had and will have a significant effect on the Earth's climate. The IPCC Fifth Assessment (AR5) report concluded that "warming of the climate system is unequivocal, and since the 1950s, many of the observed changes are unprecedented over decades to millennia". Furthermore, it is extremely likely (95-100\% probability) that human influence was the dominant cause of global warming between 1951 and 2010. The United Nations has declared that the world experienced more unprecedented high-impact climate extremes in the first decade of the 21st century than in any previous decade. Understanding of the potential for additional climate change needs to be continually refined, improved and updated to reflect the best available science and emerging understanding of global social and economic development and exploit the advances in information technologies.

\section{Informing Policy}

Accurate climate projections, produced by climate models, can assist policymakers to plan for and adapt to the adverse effects of climate change. The EC-Earth CMIP6 data, produced as part of this report, provide sharper and more accurate projections of the future global climate and will lead to a better understanding not only of the physical climate system but also of the climate impact on societies. The EC-Earth data will assist in addressing all three of the CMIP6 broad scientific questions and a number of the grand challenges of the World Climate Research Programme. The datasets will enhance the overall understanding of anthropogenic climate change on a global scale and will assist in presenting a case for a follow on to national targets, such as the United Nations COP21 Paris Agreement. This study ensures that Ireland remains at the forefront of global climate change research and continues its involvement with the Coordinated Regional Climate Downscaling Experiment (CORDEX) and CMIP6 and IPCC AR6 reports.

\section{Developing Solutions}

All CMIP6 EC-Earth data were published on the Irish Centre for High-End Computing (ICHEC) Earth System Grid Federation (ESGF) node. ESGF is an international collaboration between climate centres with a mission to support CMIP6 and future IPCC assessments. The data produced as part of the current report will be analysed by the international research community for inclusion in the upcoming IPCC AR6 reports. In addition, the EC-Earth simulation data will be used as a basis for more focused regional climate impact studies, such as national downscaling projects and the international CORDEX research community. 